\title{
AGC-2 Specimen Post- Irradiation Data Package Report
}

\author{
William E. Windes \\ W. David Swank \\ David T. Rohrbaugh \\ David L. Cottle
}

August 2015

The INL is a

U.S. Department of Energy

National Laboratory

operated by

Battelle Energy Alliance

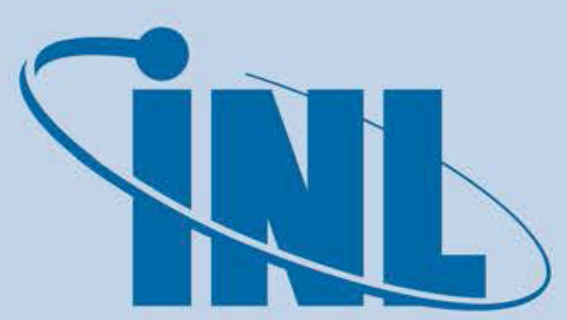

Idaho National Laboratory

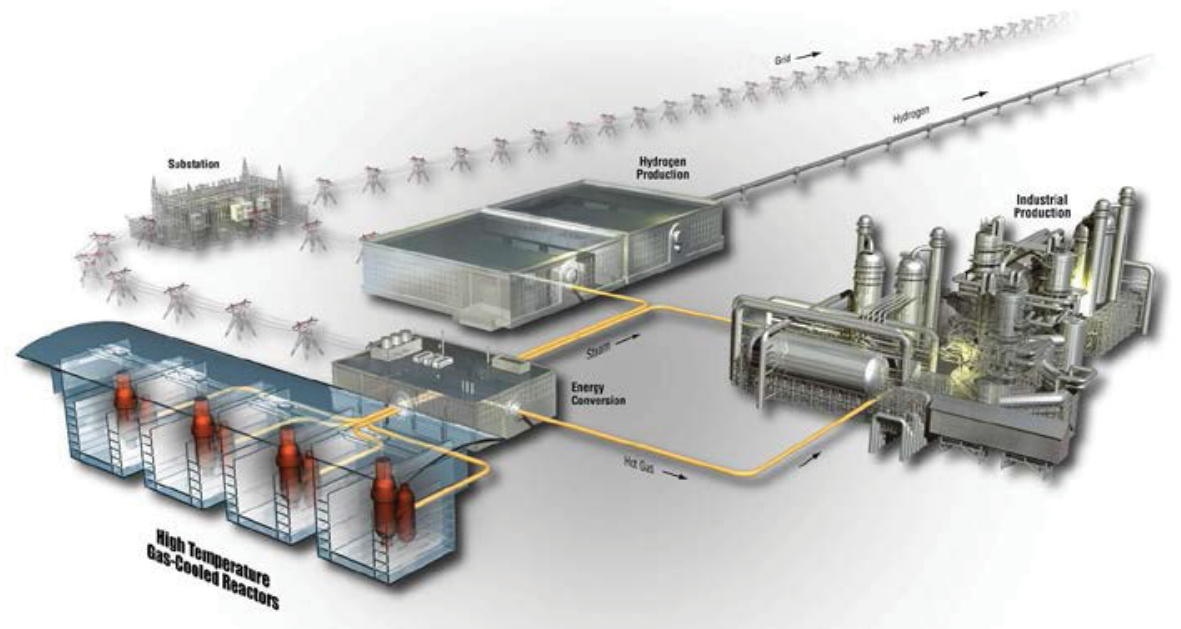




\section{DISCLAIMER}

This information was prepared as an account of work sponsored by an agency of the U.S. Government. Neither the U.S. Government nor any agency thereof, nor any of their employees, makes any warranty, expressed or implied, or assumes any legal liability or responsibility for the accuracy, completeness, or usefulness, of any information, apparatus, product, or process disclosed, or represents that its use would not infringe privately owned rights. References herein to any specific commercial product, process, or service by trade name, trade mark, manufacturer, or otherwise, does not necessarily constitute or imply its endorsement, recommendation, or favoring by the U.S. Government or any agency thereof. The views and opinions of authors expressed herein do not necessarily state or reflect those of the U.S. Government or any agency thereof. 
INL/EXT-15-36244

Revision 0

\title{
AGC-2 Specimen Post-Irradiation Data Package Report
}

\author{
William E. Windes \\ W. David Swank \\ David T. Rohrbaugh \\ David L. Cottle
}

August 2015

\begin{abstract}
Idaho National Laboratory
INL ART TDO Program

Idaho Falls, Idaho 83415
\end{abstract}

http://www.inl.gov

Prepared for the

U.S. Department of Energy

Office of Nuclear Energy

Under DOE Idaho Operations Office

Contract DE-AC07-05ID14517 



\title{
INL ART TDO Program
}

\section{AGC-2 Specimen Post-Irradiation Data Package Report}

\author{
INL/EXT-15-36244
}

Revision 0

August 2015

\section{Approved by:}

William $E$. Windes

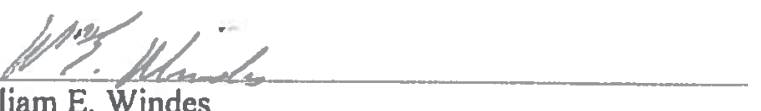

Author/INL ART TDO Graphite R\&D Technical Lead

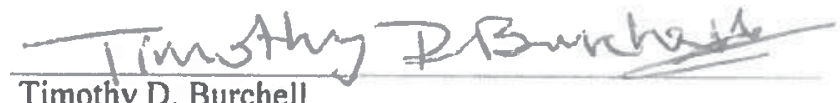

Timothy D. Burchell

ORNL Graphite Technical Peer Reviewer
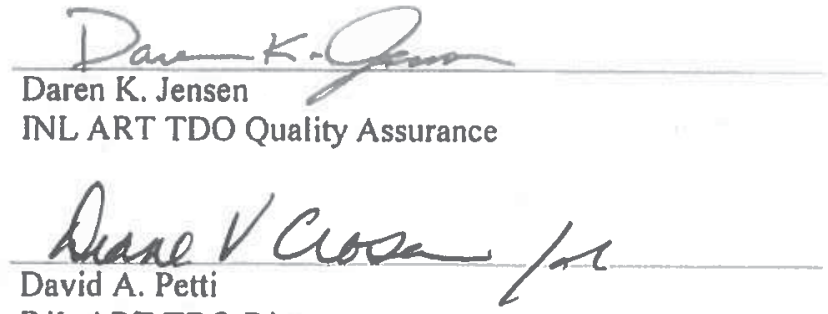
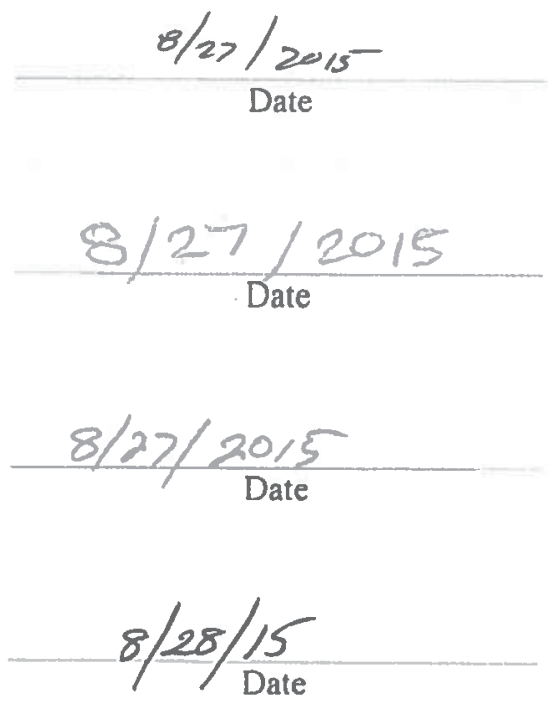



\section{SUMMARY}

This report documents results of the post-irradiation examination material property testing of the creep, control, and piggyback specimens from the irradiation creep capsule Advanced Graphite Creep (AGC)-2 are reported. This is the second of a series of six irradiation test trains planned as part of the AGC experiment to fully characterize the neutron irradiation effects and radiation creep behavior of current nuclear graphite grades. The AGC-2 capsule was irradiated in the Idaho National Laboratory Advanced Test Reactor at a nominal temperature of $600^{\circ} \mathrm{C}$ and to a peak dose of $5 \mathrm{dpa}$ (displacements per atom). One half of the creep specimens were subjected to mechanical stresses (an applied stress of either 13.8, 17.2, or 20.7 MPa) to induce irradiation creep. All postirradiation testing and measurement results are reported with the exception of the irradiation mechanical strength testing, which is the last destructive testing stage of the irradiation testing program. Material property tests were conducted on specimens from 15 nuclear graphite grades using a similar loading configuration as the first AGC capsule (AGC-1) to provide easy comparison between the two capsules. However, AGC-2 contained an increased number of specimens (i.e., 487 total specimens irradiated) and replaced specimens of the minor grade 2020 with the newer grade 2114 . The data reported include specimen dimensions for both stressed and unstressed specimens to establish the irradiation creep rates, mass and volume data necessary to derive density, elastic constants (Young's modulus, shear modulus, and Poisson's ratio) from ultrasonic time of flight velocity measurements, Young's modulus from the fundamental frequency of vibration, electrical resistivity, and thermal diffusivity and thermal expansion data from $100-500^{\circ} \mathrm{C}$. No data outliers were determined after all measurements were completed.

A brief statistical analysis was performed on the irradiated data and a limited comparison between pre- and post-irradiation properties is presented. A more complete evaluation of trends in the material property changes, as well as irradiation-induced creep due to irradiation, temperature, and applied load on specimens will be discussed in later AGC-2 post-irradiation examination analysis reports. 


\section{CONTENTS}

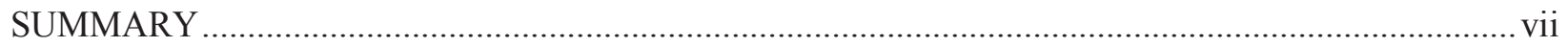

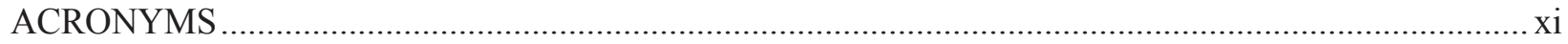

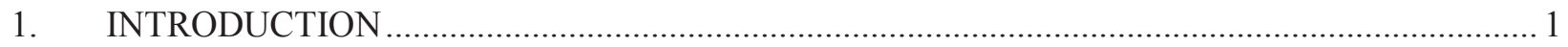

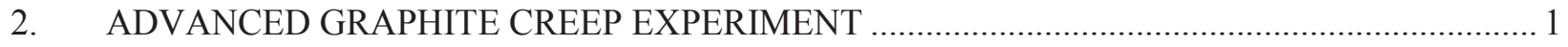

2.1 Design Parameters of AGC Experiment .......................................................................... 2

2.2 AGC Graphite Grades and Specimen Dimensions ........................................................... 4

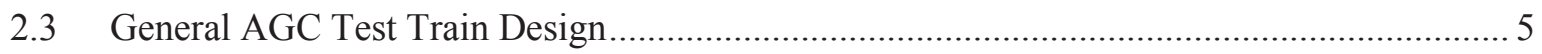

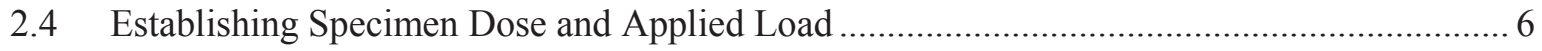

2.5 Physical Positions of Creep Specimens in the Stacks ....................................................... 8

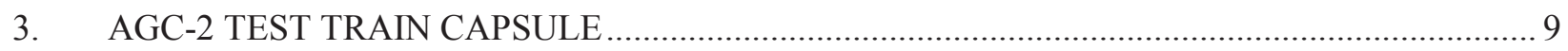

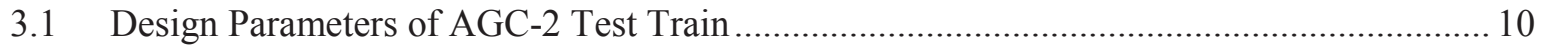

3.2 AGC-2 Graphite Grades and Changes to Dimensions ......................................................... 10

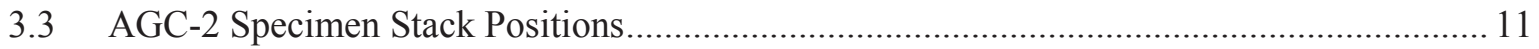

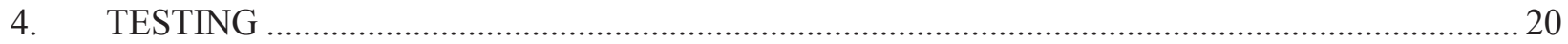

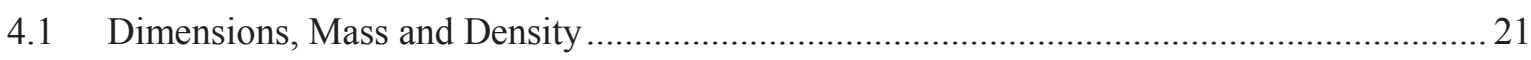

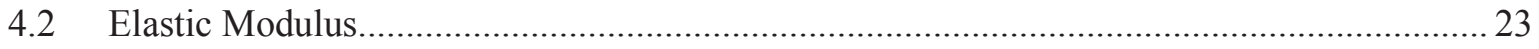

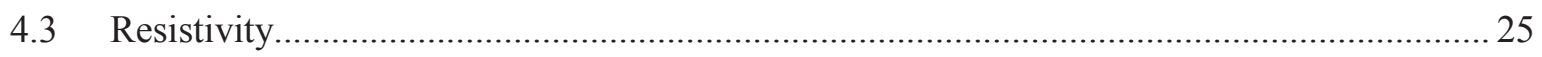

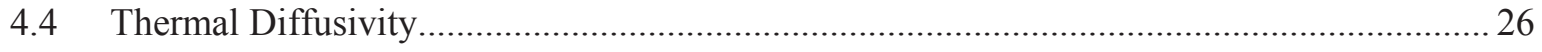

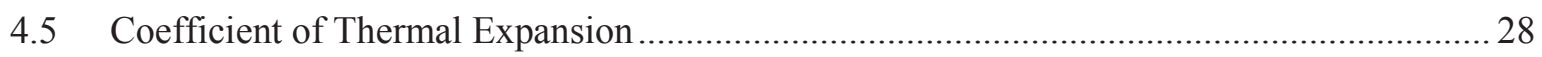

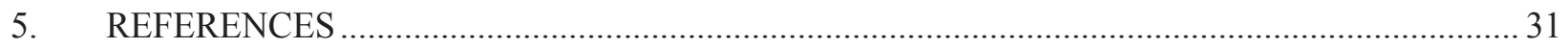

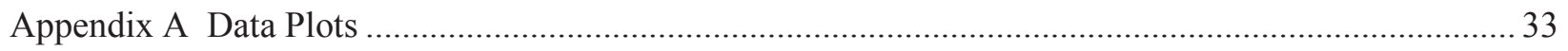

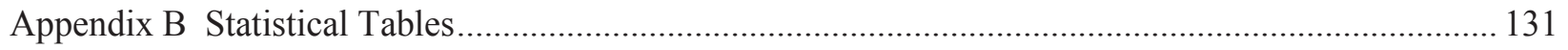

\section{FIGURES}

Figure 1. Irradiation dose and temperature parameters for the AGC experiment..................................... 3

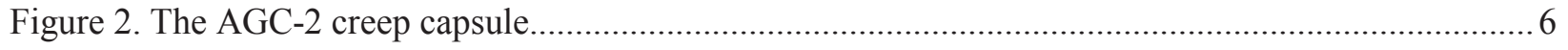

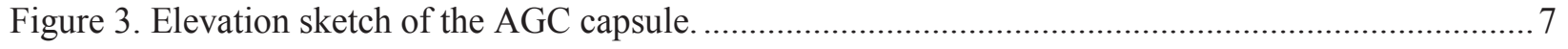

Figure 4. A typical dose profile for creep graphite specimens utilizing similar applied stress levels in matched stacks.

Figure 5. Volume decrease due to irradiation creep for six major grades of graphite. The dimensional change dependency on irradiation dose and irradiation temperature is not presented. 
Figure 6. Density increase due to volume shrinkage for six major grades of graphite and four stress conditions. The density change dependency on irradiation dose and irradiation temperature is not presented.

Figure 7. Young's modulus derived from the measurement of fundamental frequency for six grades of graphite and four different stress conditions. The Young's modulus dependency on irradiation dose and irradiation temperature is not presented.

Figure 8. Young's modulus derived from the measurement of ultrasonic velocity for six grades of graphite and four different stress conditions. The Young's modulus dependency on irradiation dose and irradiation temperature is not presented.

Figure 9. Electrical resistivity for six grades of graphite and four different stress conditions. The electrical resistivity dependency on irradiation dose and irradiation temperature is not presented.

Figure 10. Ratio of irradiated diffusivity to unirradiated diffusivity for specimens of IG-110 at $500^{\circ} \mathrm{C}$ as a function of irradiation dose. Thermal diffusivity dependency on irradiation temperature is not presented.

Figure 11. Ratio of irradiated diffusivity to unirradiated diffusivity for specimens of IG-110 at $500^{\circ} \mathrm{C}$ as a function of irradiation temperature. Thermal diffusivity dependency on irradiation dose is not presented.

Figure 12. Ratio of irradiated diffusivity to unirradiated diffusivity as a function of specimen temperature for seven grades of graphite. Changes to thermal diffusivity resulting from irradiation dose and irradiation temperature are assumed to have been saturated allowing all graphite grades to be grouped together. The assumption that diffusivity change saturates will be validated within the subsequent AGC-2 analysis report

Figure 13. Percent increase in CTE at $500^{\circ} \mathrm{C}$ for graphite grade IG-110 as a function of irradiation dose for four different stress levels. CTE dependency on irradiation temperature is not presented.

Figure 14. Percent increase in $\mathrm{CTE}$ at $500^{\circ} \mathrm{C}$ for graphite grade IG-110 as a function of irradiation temperature for four different stress levels. CTE dependency on irradiation dose is not presented.

Figure 15. Percent increase in CTE for six different grades of graphite as a function of temperature for stressed and unstressed conditions. Changes to CTE resulting from irradiation dose and temperature are assumed to have been saturated allowing all graphite grades to be grouped together. The assumption that CTE change saturates will be validated within the subsequent AGC-2 analysis report.

\section{TABLES}

Table 1. Major, minor, alternate, and experimental graphite grades within the AGC-2 capsule.

Table 2. Total number of irradiated-creep specimens in the AGC-2 test series capsule.

Table 3. AGC-2 loading order for Stacks 1 and 2.

Table 4. AGC-2 loading order for Stacks 3 and 4.

Table 5. AGC-2 loading order for Stacks 5 and $6 .$.

Table 6. AGC-2 center channel loading order. 


\section{ACRONYMS}

AG against grain

AGC Advanced Graphite Creep

ATR Advanced Test Reactor

CCL Carbon Characterization Laboratory

COV coefficient of variance

CTE coefficient of thermal expansion

HOPG Highly Ordered Pyrolitic Graphite

HTR high temperature reactor

INL Idaho National Laboratory

IQR interquartile range

NGNP Next Generation Nuclear Plant

PIE post-irradiation examination

WG with grain 


\section{INTRODUCTION}

The Advanced Reactor Technologies Graphite research and development program is conducting an extensive graphite irradiation program to provide data for licensing of a high temperature reactor (HTR) design. In past applications, graphite has been used effectively as a structural and moderator material in both research and commercial high temperature gas-cooled reactor designs. ${ }^{[1,2]}$ Nuclear graphite H-451, used previously in the United States for nuclear reactor graphite components, is no longer available. New nuclear graphite grades have been developed and are considered suitable candidates for new HTR reactor designs. To support the design and licensing of HTR core components within a commercial reactor, a complete properties database must be developed for these current grades of graphite. Quantitative data on in-service material performance are required for the physical, mechanical, and thermal properties of each graphite grade with a specific emphasis on data accounting for the life-limiting effects of irradiation creep on key physical properties of the HTR candidate graphite grades. Further details on the research and development activities and associated rationale required to qualify nuclear-grade graphite for use within the HTR are documented in the graphite technology research and development plan. ${ }^{[3]}$

Based on experience with previous graphite core components, the phenomenon of irradiation-induced creep within the graphite has been shown to be critical to the total useful lifetime of graphite components. Irradiation-induced creep occurs under the simultaneous application of high temperatures, neutron irradiation, and applied stresses within the graphite components. Significant internal stresses within the graphite components can result from a second phenomenon - irradiation-induced dimensional changewhere the graphite physically changes (i.e., first shrinking and then expanding with increasing neutron dose). This disparity in material volume change can induce significant internal stresses within graphite components. Irradiation-induced creep relaxes these large internal stresses, thus reducing the risk of crack formation and component failure. Obviously, higher irradiation creep levels tend to relieve more internal stress, thus allowing the components longer useful lifetimes within the core. Determining the irradiation creep rates of nuclear graphite grades is critical for determining the useful lifetime of graphite components and is a major component of the Advanced Graphite Creep (AGC) experiment.

The AGC experiment is currently underway to determine the in-service behavior of these new graphite grades for HTR. This test series will examine the properties and behaviors of nuclear-grade graphite over a large spectrum of temperatures, irradiation fluence, and applied stress levels that are expected to induce irradiation creep strains within an HTR graphite component. Irradiation data are provided through the AGC test series, which comprises six planned capsules irradiated in the Advanced Test Reactor (ATR) in a large flux trap at Idaho National Laboratory (INL). The AGC irradiation conditions are similar to the anticipated environment within a high temperature core design. Each irradiation capsule is comprised of over 400 graphite specimens that are characterized before and after irradiation to determine the irradiation-induced material properties changes and life-limiting irradiation creep rate for each graphite grade.

The data and information produced in this document and the referenced documents within were generated under the approved Quality Assurance programs for the respective organizations including INL and ORNL in compliance with the appropriate NQA-1 requirements. It is anticipated that all data will be robust enough to stand up to a review by the Nuclear Regulatory Commission as support for a graphite reactor design selection.

\section{ADVANCED GRAPHITE CREEP EXPERIMENT}

The AGC test series is designed to establish the data necessary to determine the safe operating envelope of graphite core components for an HTR by measuring the irradiated material property changes and the behavior of several new nuclear graphite grades over a large range of temperatures, neutron fluence, and mechanical compressive loads. The experiment consists of three interrelated stages: 
pre-irradiation characterization of the graphite specimens, the irradiation test series (designated as six separate irradiation test train capsules), and post-irradiation examination (PIE) and analysis of the graphite specimens after irradiation. Separate reports for each distinct stage are prepared after each individual activity is completed.

The pre-irradiation examination report details the total number of graphite grades and individual specimens, the specimen loading configuration designed to expose all specimens to the entire range of irradiation conditions, and the pre-irradiation material property testing data and results. The as-run irradiation report details the irradiation history of each capsule while in reactor, noting any changes from the technical and functional specifications for each specific test series capsule and identifying the possible improvements to the next test series capsule design. The disassembly report details specimen recovery from the irradiation capsule, noting any damage to the specimens and providing an inventory of recovered specimens for PIE testing. The PIE data report details the changes in specimen dimensional measurements as well as irradiated material properties upon exposure to neutron irradiation. Finally, the PIE analysis report analyzes the irradiation results reported within the data package reports, utilizing the irradiation conditions recorded within the as-run irradiation report. The PIE analysis report(s) determine the irradiation-induced creep rates for the major grades of graphite and assess any changes to the material properties in all graphite grades. The PIE analysis report(s) interpret the irradiation behavior of graphite to assist in determining a credible, safe operating envelope for graphite core components in an HTR design and licensing application. This report is an AGC PIE data report and provides the results and data from post-irradiation testing for AGC-2 specimens. A brief statistical analysis is performed here along with a limited comparison to the pre- and post-irradiation data. In this way, the consistency and soundness of the data is initially tested. A more detailed analysis and trends in the data will be reported within the subsequent AGC analysis report(s).

\subsection{Design Parameters of AGC Experiment}

The AGC test series is designed to measure changes in key thermal, physical, and mechanical material properties over the anticipated range of HTR operating conditions. By comparing the material properties of each specimen before and after irradiation, the experiment generates quantitative material property change data and irradiation creep data that will be used to predict the in-service behavior and operating performance of the current nuclear graphite grades for HTR designs. Specific emphasis is placed on data that account for the life-limiting effects of irradiation creep on graphite components and the effects creep may have on key irradiated material properties of several candidate graphite grades for use in an HTR design.

The critical component of the experiment is the irradiation test series, which irradiates the graphite specimens after pre-irradiation examination characterization has been completed. The AGC test series is comprised of six planned irradiation test trains that are irradiated in ATR in a large flux trap, as described in "Graphite Technology Development Plan." ${ }^{\text {[3] }}$ The test series exposes test specimens of select nuclear graphite grades to temperatures and the initial range of irradiation dose that are expected within an HTR design. Specifically, graphite specimens will be exposed to a fast neutron dose ranging from 1 to $7 \mathrm{dpa}$ and temperatures of 600,800 , and $1100^{\circ} \mathrm{C}$, as shown in Figure 1. The first and second AGC capsules, AGC-1 and AGC-2, were designed to be irradiated within the ATR's South Flux Trap. ${ }^{[4]}$ All other AGC capsules will be irradiated within ATR's East Flux Trap. Generally, irradiations within the South Flux Trap require approximately 175 effective full-power days to provide a nominal fast neutron dose range (in graphite) of approximately 0.5-3.5 dpa. For those capsules requiring a large dose range of approximately 3.5-7.0 dpa, the irradiation capsule (containing the graphite specimens) is irradiated for twice as long inside the ATR, approximately 350 effective full-power days. 


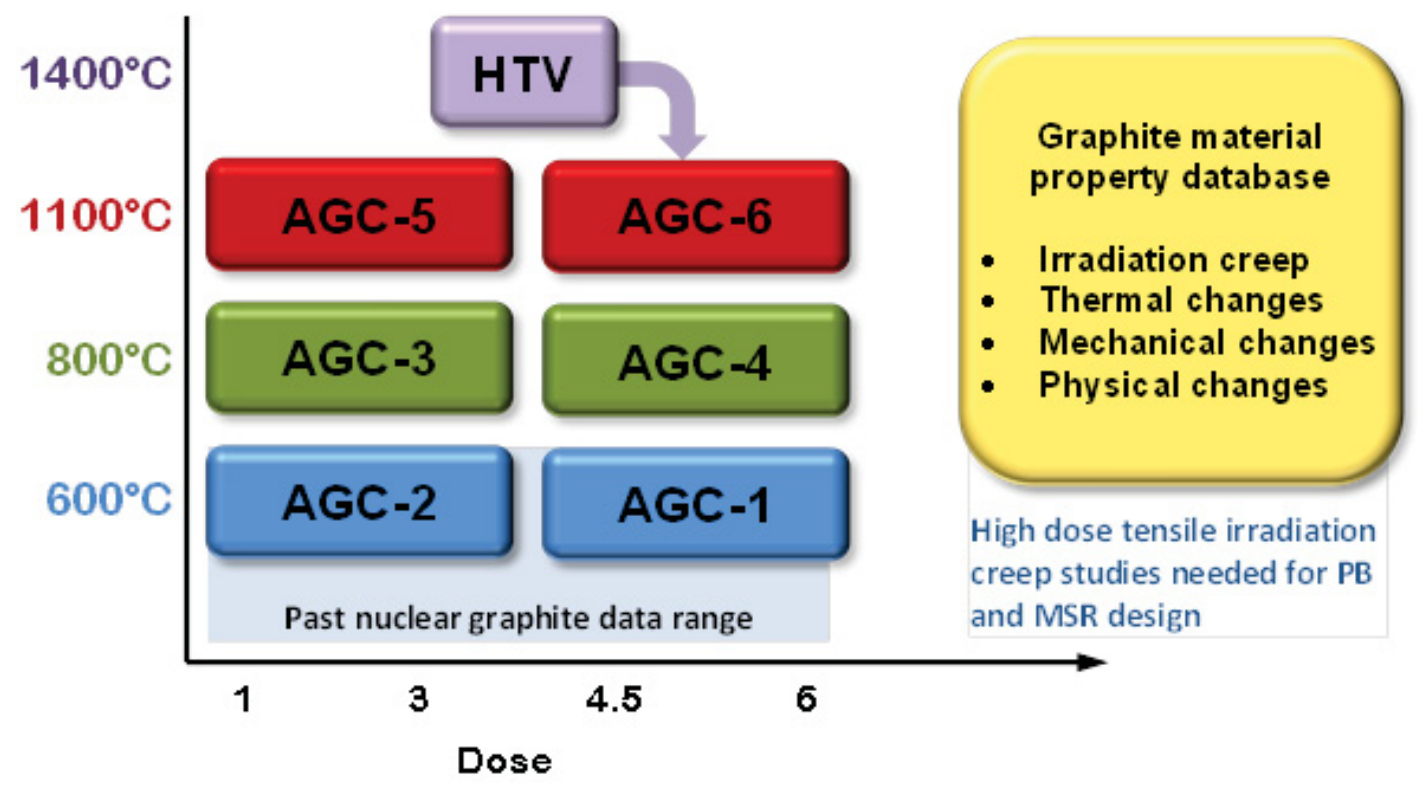

Figure 1. Irradiation dose and temperature parameters for the AGC experiment.

In addition to determining the irradiation-induced changes to the material properties of selected nuclear graphite grades, the AGC experiment dedicates a significant amount of scope to determining rates of irradiation-induced creep for different nuclear graphite grades. The traditional method for measuring irradiation-induced creep is to apply a significant mechanical load (inducing a mechanical stress within the graphite) to half the specimens during irradiation while leaving the remaining half of the specimens unloaded (unstressed). The resulting difference in dimensional change between the loaded and unloaded specimens (assuming that temperature and dose levels are the same) provides the amount of irradiation-induced strain for each "matched pair" of graphite specimens. From this strain level, a creep rate for each graphite grade can be calculated as a function of dose if both specimens were irradiated at the same constant temperature and dose level. Thus, each capsule is designed to be irradiated at a constant temperature, allowing only the dose and applied mechanical load to vary within the test train of each test-series capsule. With all graphite specimens at a constant temperature, only the applied stress level and dose will affect the calculated creep rate of each graphite grade within a test series capsule.

The AGC experiment is designed to measure the constant creep strain rate (secondary creep) of the various grades. The experiment assumes that the induced creep strain for all specimens is within the secondary creep regime and therefore behaves linearly with respect to received neutron dose. ${ }^{[3]}$ This assumption is valid provided the specimens do not go beyond their turn-around dose where creep strain can no longer be expected to be linear with irradiation dose (the onset of tertiary creep). Once the specimens begin to reach turnaround, the creep strain response becomes non-linear with received dose. To ensure specimens remain within the constant linear creep strain regime within AGC-5 and AGC-6 (the highest temperature and dose) test trains, a High Temperature Irradiation Vessel will be used to measure the dimensional changes of the graphite grades at a high temperature $\left(1400^{\circ} \mathrm{C}\right)$ and moderate dose (4.5 dpa). Results from this high temperature dimensional change study will be utilized in the AGC-5 and AGC-6 designs, which constitute the upper bounds of the AGC experiment, Figure 1. In addition, if any graphite grade achieves turnaround within the High Temperature Irradiation Vessel, it will be considered to be outside the upper bounds of the AGC experiment and will be eliminated from the AGC- 6 test train. 
While the effects from applied mechanical stresses and neutron dose can be determined within each irradiation capsule, the temperature dependency of any irradiation-induced material property changes within the graphite grades is achieved by comparing the measured values of the specimens between irradiation capsules. Since each test train is irradiated at a constant temperature (either $600^{\circ} \mathrm{C}, 800^{\circ} \mathrm{C}$, or $1100^{\circ} \mathrm{C}$ ), the temperature induced/enhanced material property changes must be determined by comparing specimens in different capsules exposed to similar dose and applied mechanical load levels. All AGC capsules are designed to have the same specimen stacking patterns. Thus, if specimens of identical graphite grades are located in similar positions within each capsule, a similar dose and load level will be imposed on a consistent grade of graphite. Maintaining consistent specimen positions for each grade within the six different capsules will allow the determination of temperature-induced changes for irradiation creep and material properties across the AGC experiment.

\subsection{AGC Graphite Grades and Specimen Dimensions}

The AGC experiment is designed to ascertain the irradiation behavior of currently available nuclear graphite grades within the anticipated operating parameters of an HTR design. By exposing a variety of nuclear graphite grades representing the range of fabrication parameters (grain size, fabrication processes, and raw source material) to the expected operating conditions for an HTR design $\left(600^{\circ} \mathrm{C}-1100^{\circ} \mathrm{C}\right.$ and 0.5-7 dpa dose) a comprehensive understanding of the irradiation response and behavior of graphite components in general can be achieved. This will limit the need for additional research in the future if the current graphite grades are altered (i.e., new raw material sources are used) or new grades are utilized in future reactors.

The AGC experiment utilizes a variety of current graphite grades to envelope the major fabrication parameters believed to be responsible for the irradiation behavior of nuclear graphite. ${ }^{[1]}$ This range of fabrication parameters are represented by AGC major grades, which were deemed to be production-ready grades that could be used in current or future HTR designs. Major graphite grades are one type of sample within the AGC irradiation capsule. In addition, four other sample types are designated within the AGC experiment. The five AGC sample types are categorized as follows ${ }^{[5]}$ :

1. Major Grades (Irradiation Creep and Control Specimens)

These graphite grades are current reactor candidates for the core structures of an HTR design as well as historical (reference) grades. Due to their fabrication maturity and consistency, HTR core components are most likely to be formed from these major grades and are thus expected to receive reasonably large neutron doses in their lifetime. Only major grade specimens were used to determine the critical irradiation-induced creep strain rate.

2. Minor Grades (Piggyback Specimens)

These grades are HTR-relevant grades that are not yet production ready or are most likely to be used in low neutron dose regions of the core (e.g., the permanent structure of the prismatic block HTR design).

3. Alternate Grades (Piggyback Specimens)

Grades that current HTR vendors have identified as being of interest as alternate graphite grades for certain components within the reactor.

4. Experimental Grades (Piggyback Specimens)

Experimental graphite grades are included in AGC to assess the viability of new graphite grades whose manufacturing processes and raw materials are such that they may offer superior irradiation stability. Additionally, other carbonaceous materials such as fuel compact matrix materials, 
carbon-carbon composites, silicon-carbide composites, or other experimental materials that could offer superior performance within the extreme environment of an HTR core are included.

5. Single Crystal Graphite (Piggyback Specimens)

Samples of Highly Ordered Pyrolytic Graphite (HOPG) are included in AGC to assess the fundamental irradiation response of single crystal graphite. These specimens offer specific dimensional change behavior of graphite, which is particularly significant to the behavior of polycrystalline (polygranular) graphite grades.

To provide all necessary material property tests in the AGC experiments, each test series capsule contains two primary specimens: (1) "creep" specimens, providing irradiation creep-rate value as well as mechanical properties and (2) "piggyback" specimens, providing thermal material property changes to the graphite. Creep specimens are fabricated only from major grade graphite types. ${ }^{[2,6]}$ Piggyback specimens are fabricated from major, minor, and experimental grade graphite. The piggyback specimens are not mechanically loaded and are subjected only to neutron irradiation at high operating temperatures to assess the effects of a reactor environment on the specific graphite grade.

All specimens are $12.7 \mathrm{~mm}$ in diameter, with the larger creep specimens being $25.4 \mathrm{~mm}$ long and the button-sized piggyback specimens being $6 \mathrm{~mm}$ long. Small graphite containers that are $12.7 \mathrm{~mm}$ in diameter by $6 \mathrm{~mm}$ long contain the thin wafer HOPG specimens. The large creep specimens provide accurate dimensional change, elastic modulus, thermal expansion, electrical resistivity, and mechanical strength measurements. However, the longer creep specimens make them unsuitable for thermal diffusivity measurements. The small piggyback specimens permitted only dimensional measurements, density, and thermal diffusivity testing to be performed. Together, both types of specimens provide the changes in material properties for stressed and unstressed graphite grades. ${ }^{[7]}$

\subsection{General AGC Test Train Design}

All AGC test trains and irradiation capsules have the same general physical configuration to provide consistent dose and applied mechanical stresses on specimens of similar graphite grades. While there are key machining and structural differences between capsules to change the irradiation temperature for the different capsules, the majority of the AGC design is identical for all capsules. A schematic of the AGC-2 test train is shown in Figure 2. ${ }^{[8]}$ 


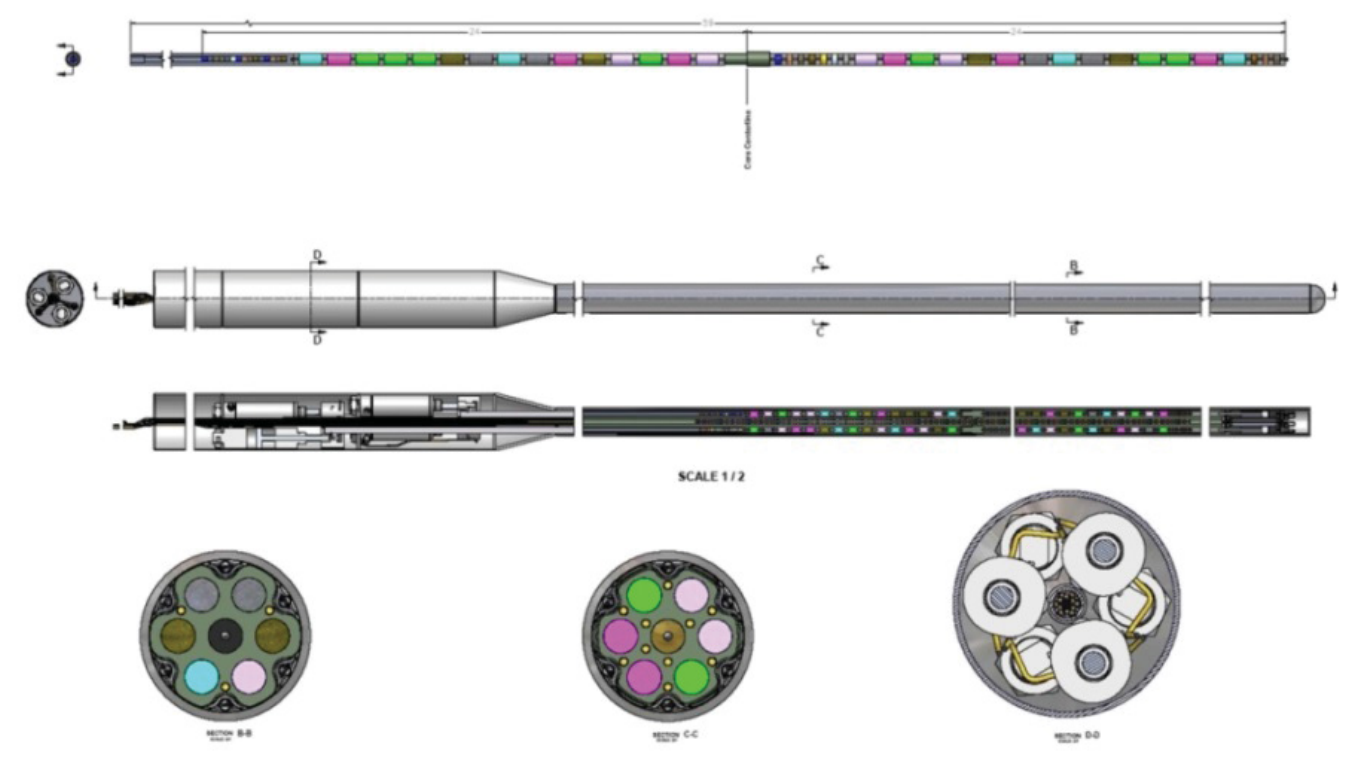

Figure 2. The AGC-2 creep capsule.

All irradiation capsules have six channels located on the outer perimeter of the graphite specimen holder body and a center channel. All channels are $12.9 \mathrm{~mm}(0.51 \mathrm{inch})$ in diameter and are designed to hold all types of AGC specimens. The upper (top) half the outer channels has had mechanical loads applied to the specimens. However, the lower (bottom) half for these channels has had no mechanical load applied to the specimens in these locations. Due to the neutron flux profile in ATR, matched pairs with similar neutron fluence and temperatures are achieved by pre-ordering the specimen axial locations. Specimens in the upper half of the channels were stressed by the applied mechanical load while their matched pair received a similar dose in an unstressed state. Three stress levels-13.8MPa, 17.2MPa, and 20.7 MPa (2.0 ksi, $2.5 \mathrm{ksi}$ and $3.0 \mathrm{ksi})$ nominal — are applied in all AGC capsules to provide a known stress upon the graphite specimens during irradiation. These induced stress levels are high enough to produce irradiation-induced creep strain with the graphite specimens.

Temperature values within all AGC capsules are calculated based upon thermocouple readings at select positions within the capsule. Specimen temperature is calculated with a Finite Element Model that has been calibrated to predict the known thermocouple readings in the capsule. Dose levels are calculated using Monte Carlo N-Particle Transport Code models and operating conditions in the ATR core and are corroborated from flux wire data.

\subsection{Establishing Specimen Dose and Applied Load}

To achieve the desired irradiation dose levels and applied mechanical loads to the specific specimens, an exact specimen loading order is critical. Because irradiation creep is usually determined by the difference in dimensional change occurring within specimens that have an applied load and those that do not, these "matched pair" specimens are assumed to have the same irradiation dose and irradiation temperature values. The AGC test train designs utilize the symmetric flux profile generated within the ATR to achieve these similar irradiation conditions for "matched pairs."

Specimens within the upper half of the capsule have a mechanical load applied to them via a pneumatic ram system. Specimens within the lower half remain unloaded and thus have no applied stress. The ATR specimens in the upper half of the capsule are located in positions that receive a dose level similar to their "matched pair" specimens in the lower half of the capsule. A careful specimen loading 
order within the irradiation capsule is required to assure similar dose levels for each "matched pair."[9,10] Other considerations included the size of each creep specimen, the need for periodically placed spacers containing flux wires, and the space requirements in the top of the stacks for the pneumatic push rods. The core flux mid-plane, in relation to the capsule arrangement, was established so that the reactor neutron flux field could be correlated to the physical elevations and positions in the capsule to yield accurate "match pair" irradiation dose levels, Figure 3. ${ }^{[1]}$



Figure 3. Elevation sketch of the AGC capsule. 
Irradiation dose values, as a function of distance from the reactor core centerline, are calculated from the total calculated fluence using standard conversion factors for carbon in a fast neutron irradiation field $(\mathrm{E}>0.1 \mathrm{MeV}) .{ }^{[12]}$ There is a neutron flux gradient across the capsule thickness requiring the capsule to be rotated 180 degrees at the irradiation mid-point. This rotation results in a uniform neutron-fluence profile for all stacks, regardless of their position within the capsule.

As described in previous reports, ${ }^{[5]}$ the ATR neutron flux profile is not completely symmetrical along the vertical axis. Thus, to produce matched-pair specimens that have similar dose profiles both above and below the core mid-plane, an offset position from the mid-plane is required. An offset distance of $1.25 \mathrm{in}$. from the core mid-plane for the bottom creep specimens produces the closest dose matches between specimens. While it was impossible to exactly match the dose levels for both the upper and lower specimens, the dose levels for each specimen pair were fairly close, ranging from $0-2 \%{ }^{[13]}$

\subsection{Physical Positions of Creep Specimens in the Stacks}

Once the specimen-position offset is established for the bottom half of the specimens, the number of total creep specimens for each grade of graphite is determined. It should be noted that the specimen stacking order for subsequent AGC irradiation capsules was changed from that initially established for the AGC-1 test train. In the initial AGC capsule design, AGC-1 utilized 0.25-in.-long NBG-25 graphite spacers between all creep specimens to separate them from each other. It was determined that this was not necessary, and most of the 0.25 -in.-long NBG-25 graphite spacers were eliminated. This decision to eliminate the spacers increased the total number of creep specimens in the AGC capsules to 216 total specimens (or 36 matched pairs in each outer perimeter channel). This allowed more specimens per graphite grade to be irradiated within the AGC-2 capsule.

As discussed, the six outer stacks in the capsule allow the specimens in two of the channel stacks to be loaded at 13.8 MPa, while the other two pairs of channels are loaded at 17.2 and $20.7 \mathrm{MPa}$, respectively. Because two stacks are at similar applied stress levels, the specimen loading order can be shifted between the two stacks, allowing the same grade of graphite to be mechanically loaded over a broader neutron dose range, as illustrated in Figure 4. Assuming that both stacks will have the same applied stress level, receive similar dose levels per position, and have a constant temperature allows this shifting of the specimens and, consequently, a more uniform, smoother dose profile for each graphite grade. 


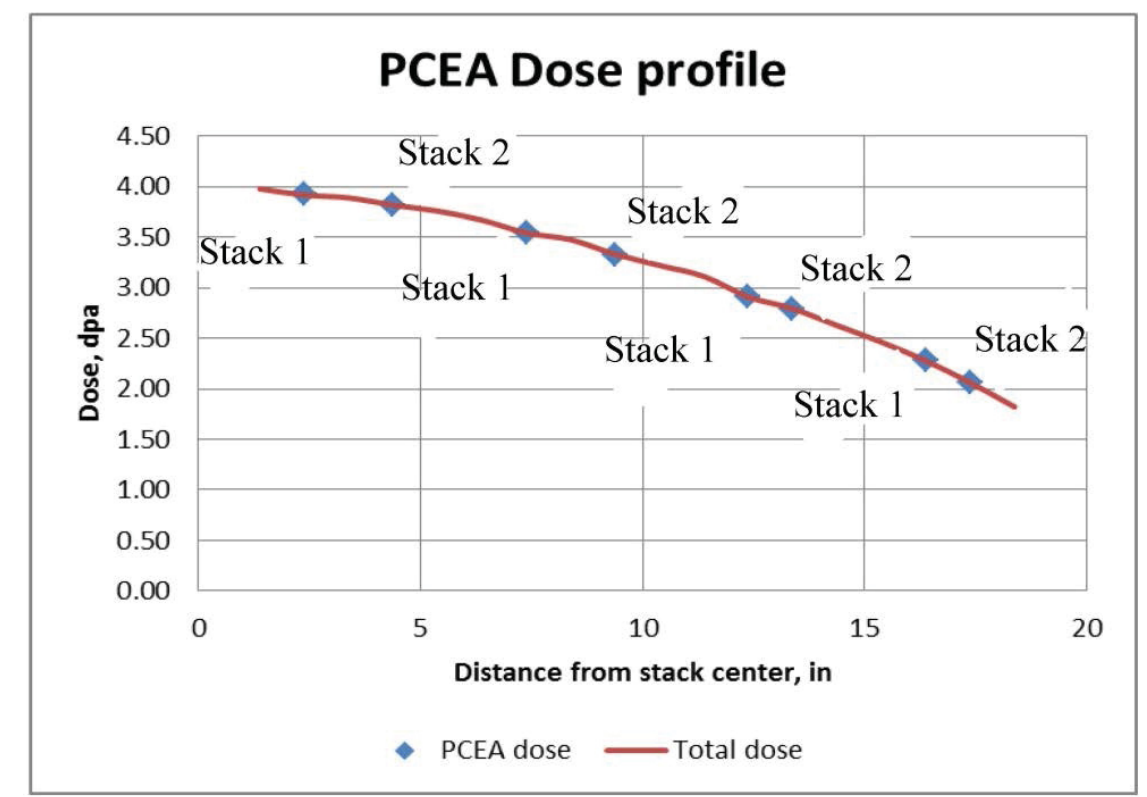

Figure 4. A typical dose profile for creep graphite specimens utilizing similar applied stress levels in matched stacks.

A final consideration when establishing the specimen loading positions is the grain orientation of the specimens. All AGC capsules attempt to account for the grain orientation to irradiation behavior. For extruded graphite grades, the against-grain (AG) and with-grain (WG) directions are obviously perpendicular and parallel to the extrusion direction, respectively. Isomolded grades have little to no grain direction and there is no consideration for their orientation. However, in the case of the vibration-molded graphites (i.e., NBG-17 and -18), there are actually two WG directions and one AG direction as a consequence of the fabrication process. The total number of WG and AG specimens is dependent upon the particular AGC capsule pair (i.e., AGC-1 and AGC-2 have the same number of specimens with similar orientation).

Once these considerations are accounted for, the dose-level profiles are determined for each graphite grade within each channel stack. It should be noted that due to the elimination of the majority of the NBG-25 spacers from the AGC-1 design, the dose-level profiles for each graphite grade have been altered for the succeeding AGC capsules. ${ }^{[14,15,16]}$ However, the changes are modest, allowing nearly direct comparison between AGC-1 and the subsequent AGC capsules.

\section{AGC-2 TEST TRAIN CAPSULE}

The AGC-2 capsule was irradiated in the ATR beginning with Cycle 149A on April 12, 2011, and ending with ATR Cycle 151B on May 5, 2012. ${ }^{[17]}$ The average estimated irradiation temperature for all samples was $600^{\circ} \mathrm{C}$ with a standard deviation of $65^{\circ} \mathrm{C}$ and a range of $705^{\circ} \mathrm{C}$ to $399^{\circ} \mathrm{C}$. The average radiation dose of all specimens was $3 \mathrm{dpa}$ with a standard deviation of $1 \mathrm{dpa}$ and a range of $5 \mathrm{dpa}$ to $1 \mathrm{dpa}$. While the temperature range was much better than the prototype AGC-1 irradiation capsule, the irradiation temperature range exceeded the design $\left(600 \pm 40^{\circ} \mathrm{C}\right) \cdot{ }^{[18]}$ Estimates of AGC-2 dose, average temperature, and stress conditions are summarized in this report. Specific individual dose levels, temperature ranges, and stress conditions for all specimens contained within the irradiation capsule will be reported in the following AGC-2 analysis report. AGC-2 design documents and drawings pertinent to the AGC-2 graphite specimens have been reported in the previous AGC-2 graphite pre-irradiation data package, INL/EXT-10-19588. ${ }^{[19]}$ 
The AGC-2 irradiation capsule is the companion capsule for AGC-1. The original AGC-2 capsule design was intended to be a mirror of AGC-1 but would be irradiated within ATR for twice as long to achieve the higher dose levels. However, due to the large and unintended temperature variation across the AGC-1 capsule, it was decided that AGC-1 would be exposed to the higher neutron dose (i.e., 3.5-7 dpa) to provide additional high dose data. Subsequently, the AGC-2 design was modified to provide irradiation data across the range of $1.5-5 \mathrm{dpa}^{[18]}$ The ATR irradiation cycles were chosen to achieve this new dose range.

While some significant modifications were made to the AGC-2 irradiation samples and layout (e.g., the elimination of the majority of NBG-25 spacers to provide more space for creep specimens), the overall design of AGC-2 was kept as close to AGC-1 as possible. All minor and experimental graphite grades were retained while only one alternate graphite grade was replaced with a new nuclear grade. This allows results from both capsules to be compared and combined into a larger data population.

\subsection{Design Parameters of AGC-2 Test Train}

It was decided that $\mathrm{AGC}-2$ and subsequent capsules did not require the addition of $\mathrm{SiC}$ temperature monitors to provide independent temperature verification during capsule irradiation. ${ }^{[20]}$ Consequently, the central hole in each of the central piggyback specimens was not machined to accommodate the SiC temperature monitors. By eliminating the $\mathrm{SiC}$ monitors, the independent temperature monitoring capability for the AGC-2 capsule was excluded, but it was determined that being able to measure the changes to the thermal diffusivity at high temperatures was of more importance. Central holes in all subsequent AGC piggyback specimens for the remaining AGC irradiation capsules were eliminated. ${ }^{[7]}$

As discussed previously, a majority of the 0.25 -in.-long NBG-25 graphite spacers between all AGC-1 creep specimens was eliminated. Four spacers were left in each channel (two in the upper section of the channel and two in the lower section) to allow flux wires to be placed within them. Eliminating most of the spacers allowed 12 additional creep specimens to be tested within the AGC-2 capsule. The total number of creep specimens in the AGC-2 capsule was increased to 216 total specimens (or 36 matched pairs in each outer perimeter channel). ${ }^{[19]}$ This stacking configuration with minimal spacers will be utilized with subsequent AGC irradiation capsules (i.e., AGC-3, AGC-4, etc.).

\subsection{AGC-2 Graphite Grades and Changes to Dimensions}

One of the graphite grades in AGC-2 was changed by request of the graphite vendor (Mersen, USA). Piggyback specimens of graphite grade 2114 were directly substituted for graphite grade 2020 and irradiated within the AGC-2 central stack (axial spine of capsule). All other graphite grades irradiated within AGC-2 were similar to the graphite grades in AGC-1. However, the sample numbers for two major grades, H-451 and IG-430, were reduced to allow for more creep test specimens in the remaining major grade graphite.

Graphite grades used in AGC-2 were categorized as follows:

The major grades of the nuclear graphite to be tested in AGC-2 are similar to AGC-2 major grades and include NBG-17, NBG-18, PCEA, IG-110, H-451, and IG-430. Minor, alternate, and experimental grades of graphite are presented below.

1. Major graphite grades: NBG-17, NBG-18, PCEA, IG-110, H-451, and IG-430

2. Minor graphite grades: NBG-25, NBG-10, HLM, PGX

3. Alternate grades: PPEA, PCIB, and 2114 (replaced grade 2020)

4. Experimental grades: BAN and A3-3/A3-27 fuel compact matrix material

5. Single crystal graphite: HOPG. 
A more complete description of all of the graphite samples included in capsule AGC-2 is given in Table 1. ${ }^{[19]}$

Table 1. Major, minor, alternate, and experimental graphite grades within the AGC-2 capsule.

\begin{tabular}{|c|c|c|c|}
\hline $\begin{array}{c}\text { Graphite } \\
\text { Grade }\end{array}$ & Forming Method & Intended Purpose & $\begin{array}{c}\text { AGC Code } \\
\text { Letter }\end{array}$ \\
\hline NBG-17 & Vibrational molded & $\begin{array}{l}\text { AREVA Next Generation Nuclear } \\
\text { Plant design }\end{array}$ & A \\
\hline NBG-18 & Vibrational molded & $\begin{array}{l}\text { Pebble Bed Modular Reactor (not } \\
\text { currently being pursued) }\end{array}$ & $\mathrm{B}$ \\
\hline H-451 & Extruded & Historical grade (Reference grade) & $\mathrm{C}$ \\
\hline PCEA & Extruded & $\begin{array}{l}\text { AREVA Next Generation Nuclear } \\
\text { Plant design }\end{array}$ & $\mathrm{D}$ \\
\hline IG-110 & Isostatically pressed & $\begin{array}{l}\text { High Temperature Reactor - Pebble- } \\
\text { Bed Module (China) }\end{array}$ & $\mathrm{E}$ \\
\hline IG-430 & Isostatically pressed & Candidate graphite & $\mathrm{F}$ \\
\hline HOPG & Vapor deposited & Fundamental studies & $\mathrm{G}$ \\
\hline A3 matrix & Hot pressed & Fuel matrix material & $\mathrm{H}$ \\
\hline HLM & Molded & Low dose core component & I \\
\hline PGX & Extruded & Low dose core component & $\mathrm{J}$ \\
\hline PPEA & Extruded & Alternate candidate & $\mathrm{L}$ \\
\hline NBG-25 & Isostatically pressed & Low dose core component & M \\
\hline PCIB & Isostatically pressed & Alternate candidate & $\mathrm{P}$ \\
\hline BAN & $\begin{array}{l}\text { Isostatically pressed and } \\
\text { extruded }\end{array}$ & Experimental graphite & $\mathrm{R}$ \\
\hline NBG-10 & Isostatically pressed & Low dose core component & $\mathrm{S}$ \\
\hline 2114 & Isostatically pressed & Candidate graphite & $\mathrm{T}$ \\
\hline
\end{tabular}

For grades NBG-17, NBG-18, and PCEA (codes A, B, and D) both WG and AG specimen orientations are included in the capsule.

\subsection{AGC-2 Specimen Stack Positions}

The final loading configuration for the outer channel/stacks was determined for each graphite grade to optimize the number of specimens for each grade, create a smooth irradiation profile for the creep and piggyback specimens, and to assure the proper position of creep specimens to create the matched-pairs.

A further decision was made to increase the creep specimen number population for the newer graphite grades because little-to-no irradiation data are available on these grades. Specifically, more specimens of graphite grades NBG-18 and PCEA were chosen to be irradiated instead of the IG-110, IG-430, and NBG-17 graphite grades ${ }^{[19]}$. NBG-18 and PCEA were determined to have 16 specimens per applied stress level for a total of 48 specimens within AGC-2. Graphite grades IG-110 and IG-430 were represented by 
only 12 specimens per applied stress level, for a total of 36 specimens within AGC-2. Table 2 lists the total number of specimens irradiated per major graphite grade.

Table 2. Total number of irradiated-creep specimens in the AGC-2 test series capsule.

\begin{tabular}{|c|c|}
\hline Graphite Grade & $\begin{array}{c}\text { Number of } \\
\text { Creep Specimens }\end{array}$ \\
\hline PCEA & 48 \\
\hline NBG-18 & 48 \\
\hline IG-110 & 36 \\
\hline IG-430 & 36 \\
\hline NBG-17 & 24 \\
\hline H-451 & 24 \\
\hline \hline Total Creep & 216 \\
\hline
\end{tabular}

A final consideration before establishing the final loading configuration is the grain orientation of the specimens. A decision was made to have approximately $75 \%$ of the specimens be orientated in the WG direction and $25 \%$ of the specimens be AG. However, in the case of the vibration-molded graphite grades (i.e., NBG-17 and -18), there are actually two WG directions and one AG direction as a consequence of the fabrication process. ${ }^{[2]}$ As such, it was logical to split the WG and AG specimens evenly (i.e., 50/50 ratio) rather than following the 75/25 ratio established for the other specimens.

The orientation of the specimen is designated by the second digit in the sample identification number. Specimen identification numbers possessing a "W" in the second digit are specimens in the WG orientation (e.g., CW101). Specimen identification numbers possessing an "A" in the second digit are specimens machined from an AG orientation (e.g., DA402). As discussed previously, vibrationally molded grades are designated with an "L" or "P" for the two WG orientations (e.g., BP402 for an NBG-17 grade specimen).

The final loading configuration for AGC-2, including creep specimen matched-pair positions (above and below the capsule mid-plane), piggyback order, lower stack offset, and flux wire spacers, was mapped for each graphite specimen as it was loaded into the AGC-2 irradiation capsule during assembly (Table 3 through Table 6). ${ }^{[19,21]}$ 
Table 3. AGC-2 loading order for Stacks 1 and 2.

\begin{tabular}{|c|c|c|c|}
\hline S-1, Compressed & & \\
\hline $\begin{array}{c}\text { Work } \\
\text { Order } \\
137268 \\
\text { Loading } \\
\text { Order }\end{array}$ & $\begin{array}{c}\text { Work } \\
\text { Order } \\
137268 \\
\text { ID } \\
\text { Number }\end{array}$ & $\begin{array}{c}\text { Initial } \\
\text { Graphite } \\
\text { Type }\end{array}$ & $\begin{array}{c}\text { Specimen } \\
\text { COM } \\
\text { Elevation } \\
\text { (in) }\end{array}$ \\
\hline 23 & CW101 & H451 & 19.500 \\
\hline 22 & 1A & Flux Monitor & 18.875 \\
\hline 21 & DW101 & PCEA & 18.250 \\
\hline 20 & BW101 & NBG-18 & 17.250 \\
\hline 19 & EW0102 & IG-110 & 16.250 \\
\hline 18 & FW0101 & IG-430 & 15.250 \\
\hline 17 & DW102 & PCEA & 14.250 \\
\hline 16 & AY & Flux Monitor & 13.625 \\
\hline 15 & BW102 & NBG-18 & 13.000 \\
\hline 14 & FW0102 & IG-430 & 12.000 \\
\hline 13 & EW0104 & IG-110 & 11.000 \\
\hline 12 & DW1001 & PCEA & 10.000 \\
\hline 11 & BW103 & NBG-18 & 9.000 \\
\hline 10 & FW0103 & IG-430 & 8.000 \\
\hline 9 & 1H & Flux Monitor & 7.375 \\
\hline 8 & CW102 & H451 & 6.750 \\
\hline 7 & EW0201 & IG-110 & 5.750 \\
\hline 6 & DW1002 & PCEA & 4.750 \\
\hline 5 & BW1001 & NBG-18 & 3.750 \\
\hline 4 & FW0104 & IG-430 & 2.750 \\
\hline 3 & 8 H & Flux Monitor & 2.125 \\
\hline 2 & AW101 & NBG-17 & 1.500 \\
\hline & & & \\
\hline
\end{tabular}

\begin{tabular}{|c|c|c|c|}
\hline \multicolumn{2}{|c|}{ S-2, Compressed } & \multirow{6}{*}{$\begin{array}{c}\text { Graphite } \\
\text { Type }\end{array}$} & \multirow{6}{*}{$\begin{array}{c}\text { Initial } \\
\text { Specimen } \\
\text { COM } \\
\text { Elevation } \\
\text { (in) }\end{array}$} \\
\hline Work & Work & & \\
\hline Order & Order & & \\
\hline 137268 & 137268 & & \\
\hline Loading & ID & & \\
\hline Order & Number & & \\
\hline 23 & EW0301 & IG-110 & 19.500 \\
\hline 22 & AW1707 & NBG-17 & 18.875 \\
\hline 21 & FW0301 & IG-430 & 18.250 \\
\hline 20 & DA402 & PCEA & 17.250 \\
\hline 19 & BP402 & NBG-18 & 16.250 \\
\hline 18 & AW103 & NBG-17 & 15.250 \\
\hline 17 & EW0302 & IG-110 & 14.250 \\
\hline 16 & $2 \mathrm{~B}$ & Flux Monitor & 13.625 \\
\hline 15 & DW1103 & PCEA & 13.000 \\
\hline 14 & BW1103 & NBG-18 & 12.000 \\
\hline 13 & CW1003 & $\mathrm{H} 451$ & 11.000 \\
\hline 12 & AW1001 & NBG-17 & 10.000 \\
\hline 11 & EW0303 & IG-110 & 9.000 \\
\hline 10 & DA403 & PCEA & 8.000 \\
\hline 9 & AW1708 & NBG-17 & 7.375 \\
\hline 8 & BP403 & NBG-18 & 6.750 \\
\hline 7 & FW0302 & IG430 & 5.750 \\
\hline 6 & AP402 & NBG-17 & 4.750 \\
\hline 5 & CW1101 & $\mathrm{H} 451$ & 3.750 \\
\hline 4 & DW1104 & PCEA & 2.750 \\
\hline 3 & 37 & Flux Monitor & 2.125 \\
\hline 2 & BW1201 & NBG-18 & 1.500 \\
\hline
\end{tabular}

\section{S-1, Uncompressed}

\begin{tabular}{|c|c|c|c|c|c|c|c|}
\hline \multicolumn{3}{|l|}{1, Un } & & \multicolumn{3}{|c|}{ mpressed } & \multirow[b]{2}{*}{$\begin{array}{c}\text { Initial } \\
\text { Specimen } \\
\text { COM } \\
\text { Elevation } \\
\text { (in) }\end{array}$} \\
\hline $\begin{array}{c}\text { Work } \\
\text { Order } \\
137268 \\
\text { Loading } \\
\text { Order }\end{array}$ & $\begin{array}{c}\text { Work } \\
\text { Order } \\
137268 \\
\text { ID } \\
\text { Number }\end{array}$ & $\begin{array}{c}\text { Graphite } \\
\text { Type }\end{array}$ & $\begin{array}{c}\text { Initial } \\
\text { Specimen } \\
\text { cOM } \\
\text { Elevation } \\
\text { (in) }\end{array}$ & $\begin{array}{c}\text { Work } \\
\text { Order } \\
137268 \\
\text { Loading } \\
\text { Order }\end{array}$ & $\begin{array}{c}\text { Work } \\
\text { Order } \\
137268 \\
\text { ID } \\
\text { Number }\end{array}$ & $\begin{array}{c}\text { Graphite } \\
\text { Type }\end{array}$ & \\
\hline 36 & AW102 & NBG-17 & -1.750 & 36 & BW1202 & NBG-18 & -1.750 \\
\hline 35 & $\mathrm{AO}$ & Flux Monitor & -2.375 & 35 & AW1709 & NBG-17 & -2.375 \\
\hline 34 & FW0201 & IG-430 & -3.000 & 34 & DW1201 & PCEA & -3.000 \\
\hline 33 & BW1002 & NBG-18 & -4.000 & 33 & CW1102 & $\mathrm{H} 451$ & -4.000 \\
\hline 32 & DW1003 & PCEA & -5.000 & 32 & AP403 & NBG-17 & -5.000 \\
\hline 31 & EW0202 & IG-110 & -6.000 & 31 & FW0303 & IG-430 & -6.000 \\
\hline 30 & CW103 & $\mathrm{H} 451$ & -7.000 & 30 & BP501 & NBG-18 & -7.000 \\
\hline 29 & AL1402 & NBG-17 & -7.625 & 29 & EW1509 & IG-110 & -7.625 \\
\hline 28 & FW0202 & IG-430 & -8.250 & 28 & DA501 & PCEA & -8.250 \\
\hline 27 & BW1003 & NBG-18 & -9.250 & 27 & EW0304 & IG-110 & -9.250 \\
\hline 26 & DW1004 & PCEA & -10.250 & 26 & AW1002 & NBG-17 & -10.250 \\
\hline 25 & EW0203 & IG-110 & -11.250 & 25 & CW1103 & $\mathrm{H} 451$ & -11.250 \\
\hline 24 & FW0203 & IG-430 & -12.250 & 24 & BW1203 & NBG-18 & -12.250 \\
\hline 23 & BW1101 & NBG-18 & -13.250 & 23 & DW1202 & PCEA & -13.250 \\
\hline 22 & $8 \mathrm{U}$ & Flux Monitor & -13.875 & 22 & $7 D$ & Flux Monitor & -13.875 \\
\hline 21 & DW1101 & PCEA & -14.500 & 21 & EW0401 & IG-110 & -14.500 \\
\hline 20 & FW0204 & IG-430 & -15.500 & 20 & AW1003 & NBG-17 & -15.500 \\
\hline 19 & EW0204 & IG-110 & -16.500 & 19 & BP502 & NBG-18 & -16.500 \\
\hline 18 & BW1102 & NBG-18 & -17.500 & 18 & DA502 & PCEA & -17.500 \\
\hline 17 & DW1102 & PCEA & -18.500 & 17 & FW0304 & IG-430 & -18.500 \\
\hline 16 & $\mathrm{AE}$ & Flux Monitor & -19.125 & 16 & EW1510 & IG-110 & -19.125 \\
\hline 15 & CW1002 & $\mathrm{H} 451$ & -19.750 & 15 & EW0402 & IG-110 & -19.750 \\
\hline 14 & BP7 06 & NBG-18 & -20.375 & 14 & $\mathrm{~J} 207$ & HLM & -20.375 \\
\hline 13 & L2 08 & PPEA & -20.625 & 13 & RW2 10 & BAN & -20.625 \\
\hline 12 & K2 09 & PGX & -20.875 & 12 & $\mathrm{H} 571$ & Compact Matrix & -20.875 \\
\hline 11 & $\mathrm{P} 2-08$ & $\mathrm{PCIB}$ & -21.125 & 11 & TP19 & 2114 & -21.125 \\
\hline 10 & DW18 03 & PCEA & -21.375 & 10 & BP7 07 & NBG-18 & -21.375 \\
\hline 9 & M2 07 & NBG-25 & -21.625 & 9 & L2 09 & PPEA & -21.625 \\
\hline 8 & S2 07 & NBG-10 & -21.875 & 8 & K2 10 & $\mathrm{PGX}$ & -21.875 \\
\hline 7 & FW15 01 & IG-430 & -22.125 & 7 & P2-09 & $\mathrm{PCIB}$ & -22.125 \\
\hline 6 & EW14 01 & IG-110 & -22.375 & 6 & DW18 04 & PCEA & -22.375 \\
\hline 5 & $\mathrm{~J} 206$ & HLM & -22.625 & 5 & $\mathrm{M} 2-08$ & NBG-25 & -22.625 \\
\hline 4 & RW2 09 & BAN & -22.875 & 4 & S2 08 & NBG-10 & -22.875 \\
\hline 3 & H562 & Compact Matrix & -23.125 & 3 & FW15 02 & IG-430 & -23.125 \\
\hline 2 & TP 18 & 2114 & -23.375 & 2 & EW14 02 & IG-110 & -23.375 \\
\hline
\end{tabular}


Table 4. AGC-2 loading order for Stacks 3 and 4.

\begin{tabular}{|c|c|c|c|}
\hline \multicolumn{2}{|c|}{ S-3, Compressed } & \multirow{6}{*}{$\begin{array}{c}\text { Graphite } \\
\text { Type }\end{array}$} & \multirow{6}{*}{$\begin{array}{c}\text { Initial } \\
\text { Specimen } \\
\text { COM } \\
\text { Elevation } \\
\text { (in) }\end{array}$} \\
\hline Work & & & \\
\hline Order & Work Order & & \\
\hline 137268 & 137268 & & \\
\hline Loading & ID & & \\
\hline Order & Number & & \\
\hline 23 & CW1202 & $\mathrm{H} 451$ & 19.500 \\
\hline 22 & TP 27 & 2114 & 18.875 \\
\hline 21 & DW1203 & PCEA & 18.250 \\
\hline 20 & BW1301 & NBG-18 & 17.250 \\
\hline 19 & EW0403 & IG-110 & 16.250 \\
\hline 18 & FW0401 & IG-430 & 15.250 \\
\hline 17 & DW1204 & PCEA & 14.250 \\
\hline 16 & $2 U$ & Flux Monitor & 13.625 \\
\hline 15 & BW1302 & NBG-18 & 13.000 \\
\hline 14 & FW0402 & IG-430 & 12.000 \\
\hline 13 & EW0404 & IG-110 & 11.000 \\
\hline 12 & DW1301 & PCEA & 10.000 \\
\hline 11 & BW1303 & NBG-18 & 9.000 \\
\hline 10 & FW0404 & IG-430 & 8.000 \\
\hline 9 & TP 12 & 2114 & 7.375 \\
\hline 8 & CW1203 & $\mathrm{H} 451$ & 6.750 \\
\hline 7 & EW0501 & IG-110 & 5.750 \\
\hline 6 & DW1302 & PCEA & 4.750 \\
\hline 5 & BW1401 & NBG-18 & 3.750 \\
\hline 4 & FW0501 & IG-430 & 2.750 \\
\hline 3 & $8 Y$ & Flux Monitor & 2.125 \\
\hline 2 & AW1101 & NBG-17 & 1.500 \\
\hline
\end{tabular}

S-4, Compressed
\begin{tabular}{|c|c|c|c|}
\hline $\begin{array}{c}\text { Work } \\
\text { Order } \\
137268 \\
\text { Loading } \\
\text { Order }\end{array}$ & $\begin{array}{c}\text { Work } \\
\text { Order } \\
137268 \\
\text { ID } \\
\text { Number }\end{array}$ & $\begin{array}{c}\text { Graphite } \\
\text { Type }\end{array}$ & $\begin{array}{c}\text { Initial } \\
\text { Specimen } \\
\text { COM } \\
\text { Elevation } \\
\text { (in) }\end{array}$ \\
\hline 23 & EW0601 & IG-110 & 19.500 \\
\hline 22 & AX & Flux Monitor & 18.875 \\
\hline 21 & FW0602 & IG-430 & 18.250 \\
\hline 20 & DA503 & PCEA & 17.250 \\
\hline 19 & BP503 & NBG-18 & 16.250 \\
\hline 18 & AW1103 & NBG-17 & 15.250 \\
\hline 17 & EW0602 & IG-110 & 14.250 \\
\hline 16 & 18 & Flux Monitor & 13.625 \\
\hline 15 & DW1403 & PCEA & 13.000 \\
\hline 14 & BW1503 & NBG-18 & 12.000 \\
\hline 13 & CW1303 & H451 & 11.000 \\
\hline 12 & AW1201 & NBG-17 & 10.000 \\
\hline 11 & EW0603 & IG-110 & 9.000 \\
\hline 10 & DA601 & PCEA & 8.000 \\
\hline 9 & AR & Flux Monitor & 7.375 \\
\hline 8 & BP601 & NBG-18 & 6.750 \\
\hline 7 & FW0603 & IG-430 & 5.750 \\
\hline 6 & AP501 & NBG-17 & 4.750 \\
\hline 5 & CW201 & H451 & 3.750 \\
\hline 4 & DW1404 & PCEA & 2.750 \\
\hline 3 & $3 F$ & Flux Monitor & 2.125 \\
\hline 2 & BW1601 & NBG-18 & 1.500 \\
\hline & & & \\
\hline
\end{tabular}

\section{S-3, Uncompressed}

\begin{tabular}{|c|c|c|c|}
\hline $\begin{array}{c}\text { Work } \\
\text { Order } \\
137268 \\
\text { Loading } \\
\text { Order } \\
\end{array}$ & \begin{tabular}{|c|} 
Work Order \\
137268 \\
ID \\
Number \\
\end{tabular} & $\begin{array}{c}\text { Graphite } \\
\text { Type }\end{array}$ & $\begin{array}{c}\text { Initial } \\
\text { Specimen } \\
\text { COM } \\
\text { Elevation } \\
\text { (in) }\end{array}$ \\
\hline 36 & AW1102 & NBG-17 & -1.750 \\
\hline 35 & TP24 & 2114 & -2.375 \\
\hline 34 & FW0502 & IG-430 & -3.000 \\
\hline 33 & BW1402 & NBG-18 & -4.000 \\
\hline 32 & DW1303 & PCEA & -5.000 \\
\hline 31 & EW0502 & IG-110 & -6.000 \\
\hline 30 & CW1301 & $\mathrm{H} 451$ & -7.000 \\
\hline 29 & TP25 & 2114 & -7.625 \\
\hline 28 & FW0503 & IG-430 & -8.250 \\
\hline 27 & BW1403 & NGB-18 & -9.250 \\
\hline 26 & DW1304 & PCEA & -10.250 \\
\hline 25 & EW0503 & IG-110 & -11.250 \\
\hline 24 & FW0504 & IG-430 & -12.250 \\
\hline 23 & BW1501 & NGB-18 & -13.250 \\
\hline 22 & $1 Y$ & Flux Monitor & -13.875 \\
\hline 21 & DW1401 & PCEA & -14.500 \\
\hline 20 & FW0601 & IG-430 & -15.500 \\
\hline 19 & EW0504 & IG-110 & -16.500 \\
\hline 18 & BW1502 & NBG-18 & -17.500 \\
\hline 17 & DW1402 & PCEA & -18.500 \\
\hline 16 & TP26 & 2114 & -19.125 \\
\hline 15 & CW1302 & $\mathrm{H} 451$ & -19.750 \\
\hline 14 & DW18 05 & PCEA & -20.375 \\
\hline 13 & M2-09 & NBG-25 & -20.625 \\
\hline 12 & S2 09 & NBG-10 & -20.875 \\
\hline 11 & FW1503 & IG-430 & -21.125 \\
\hline 10 & EW1403 & IG-110 & -21.375 \\
\hline 9 & $\mathrm{~J} 208$ & HLM & -21.625 \\
\hline 8 & RW4 01 & BAN & -21.875 \\
\hline 7 & $\mathrm{H} 572$ & Compact Matrix & -22.125 \\
\hline 6 & TP 20 & 2114 & -22.375 \\
\hline 5 & L3 04 & PPEA & -22.625 \\
\hline 4 & L2 10 & PPEA & -22.875 \\
\hline 3 & K3 01 & PGX & -23.125 \\
\hline 2 & P2-10 & $\mathrm{PClB}$ & -23.375 \\
\hline
\end{tabular}

S-4, Uncompressed

\begin{tabular}{|c|c|c|c|}
\hline $\begin{array}{c}\text { Work } \\
\text { Order } \\
137268 \\
\text { Loading } \\
\text { Order }\end{array}$ & $\begin{array}{c}\text { Work } \\
\text { Order } \\
137268 \\
\text { ID } \\
\text { Number }\end{array}$ & $\begin{array}{c}\text { Graphite } \\
\text { Type }\end{array}$ & $\begin{array}{c}\text { Initial } \\
\text { Specimen } \\
\text { COM } \\
\text { Elevation } \\
\text { (in) }\end{array}$ \\
\hline 36 & BW1602 & NBG-18 & -1.750 \\
\hline 35 & $5 \mathrm{~B}$ & Flux Monitor & -2.375 \\
\hline 34 & DW1502 & PCEA & -3.000 \\
\hline 33 & CW202 & H451 & -4.000 \\
\hline 32 & AP502 & NBG-17 & -5.000 \\
\hline 31 & FW0604 & IG-430 & -6.000 \\
\hline 30 & BP602 & NBG-18 & -7.000 \\
\hline 29 & AL1403 & NBG-17 & -7.625 \\
\hline 28 & DA602 & PCEA & -8.250 \\
\hline 27 & EW0604 & IG-110 & -9.250 \\
\hline 26 & AW1202 & NBG-17 & -10.250 \\
\hline 25 & CW203 & $\mathrm{H} 451$ & -11.250 \\
\hline 24 & BW1603 & NBG-18 & -12.250 \\
\hline 23 & DW1503 & PCEA & -13.250 \\
\hline 22 & $2 Y$ & Flux Monitor & -13.875 \\
\hline 21 & EW0701 & IG-110 & -14.500 \\
\hline 20 & AW1203 & NBG-17 & -15.500 \\
\hline 19 & BP603 & NBG-18 & -16.500 \\
\hline 18 & DA701 & PCEA & -17.500 \\
\hline 17 & FW0701 & IG-430 & -18.500 \\
\hline 16 & $8 Z$ & Flux Monitor & -19.125 \\
\hline 15 & EW0702 & IG-110 & -19.750 \\
\hline 14 & L3 05 & PPEA & -20.375 \\
\hline 13 & L3 01 & PPEA & -20.625 \\
\hline 12 & K3 02 & PGX & -20.875 \\
\hline 11 & P3-01 & $\mathrm{PCIB}$ & -21.125 \\
\hline 10 & DW18 06 & PCEA & -21.375 \\
\hline 9 & M2-10 & NBG-25 & -21.625 \\
\hline 8 & S2 10 & NBG-10 & -21.875 \\
\hline 7 & P3-06 & $\mathrm{PCIB}$ & -22.125 \\
\hline 6 & K3 05 & PGX & -22.375 \\
\hline 5 & $\mathrm{~J} 209$ & HLM & -22.625 \\
\hline 4 & RW4 02 & BAN & -22.875 \\
\hline 3 & H581 & Compact Matrix & -23.125 \\
\hline 2 & TP21 & 2114 & -23.375 \\
\hline
\end{tabular}


Table 5. AGC-2 loading order for Stacks 5 and 6.

\begin{tabular}{|c|c|c|c|}
\hline \multicolumn{2}{|c|}{ S-5, Compressed } & \multirow{6}{*}{$\begin{array}{c}\text { Graphite } \\
\text { Type }\end{array}$} & \multirow{6}{*}{$\begin{array}{c}\text { Initial } \\
\text { Specimen } \\
\text { COM } \\
\text { Elevation } \\
\text { (in) }\end{array}$} \\
\hline Work & Work & & \\
\hline Order & Order & & \\
\hline 137268 & 137268 & & \\
\hline Loading & ID & & \\
\hline Order & Number & & \\
\hline 23 & CW301 & $\mathrm{H} 451$ & 19.500 \\
\hline 22 & EW1511 & IG-110 & 18.875 \\
\hline 21 & DW1504 & PCEA & 18.250 \\
\hline 20 & BW201 & NBG-18 & 17.250 \\
\hline 19 & EW0703 & IG-110 & 16.250 \\
\hline 18 & FW0703 & IG-430 & 15.250 \\
\hline 17 & DW1601 & PCEA & 14.250 \\
\hline 16 & 57 & Flux Monitor & 13.625 \\
\hline 15 & BW202 & NBG-18 & 13.000 \\
\hline 14 & FW0704 & IG-430 & 12.000 \\
\hline 13 & EW0704 & IG-110 & 11.000 \\
\hline 12 & DW1602 & PCEA & 10.000 \\
\hline 11 & BW203 & NGB-18 & 9.000 \\
\hline 10 & FW0801 & IG-430 & 8.000 \\
\hline 9 & AW1710 & NGB-17 & 7.375 \\
\hline 8 & CW302 & $\mathrm{H} 451$ & 6.750 \\
\hline 7 & EW0801 & IG-110 & 5.750 \\
\hline 6 & DW1603 & PCEA & 4.750 \\
\hline 5 & BW301 & NGB-18 & 3.750 \\
\hline 4 & FW0802 & IG-430 & 2.750 \\
\hline 3 & $\mathrm{AL}$ & Flux Monitor & 2.125 \\
\hline 2 & AW1301 & NGB-17 & 1.500 \\
\hline
\end{tabular}

\begin{tabular}{|c|c|c|c|}
\hline \multicolumn{2}{|c|}{ S-6, Compressed } & \multirow{6}{*}{$\begin{array}{l}\text { Graphite } \\
\text { Type }\end{array}$} & \multirow{6}{*}{$\begin{array}{c}\text { Initial } \\
\text { Specimen } \\
\text { COM } \\
\text { Elevation } \\
\text { (in) }\end{array}$} \\
\hline Work & Work & & \\
\hline Order & Order & & \\
\hline 137268 & 137268 & & \\
\hline Loading & ID & & \\
\hline Order & Number & & \\
\hline 23 & EW0901 & IG-110 & 19.500 \\
\hline 22 & TP16 & 2114 & 18.875 \\
\hline 21 & FW0903 & IG-430 & 18.250 \\
\hline 20 & DA303 & PCEA & 17.250 \\
\hline 19 & BP401 & NBG-18 & 16.250 \\
\hline 18 & AW1303 & NBG-17 & 15.250 \\
\hline 17 & EW0902 & IG-110 & 14.250 \\
\hline 16 & $5 \mathrm{~F}$ & Flux Monitor & 13.625 \\
\hline 15 & DW201 & PCEA & 13.000 \\
\hline 14 & BW403 & NBG-18 & 12.000 \\
\hline 13 & CW402 & $\mathrm{H} 451$ & 11.000 \\
\hline 12 & AW1401 & NBG-17 & 10.000 \\
\hline 11 & EA902 & IG-110 & 9.000 \\
\hline 10 & DA302 & PCEA & 8.000 \\
\hline 9 & TP17 & 2114 & 7.375 \\
\hline 8 & BP303 & NBG-18 & 6.750 \\
\hline 7 & FW0904 & IG-430 & 5.750 \\
\hline 6 & AP503 & NBG-17 & 4.750 \\
\hline 5 & CW403 & $\mathrm{H} 451$ & 3.750 \\
\hline 4 & DW202 & PCEA & 2.750 \\
\hline 3 & $5 \mathrm{H}$ & Flux Monitor & 2.125 \\
\hline 2 & BW501 & NBG-18 & 1.500 \\
\hline
\end{tabular}

S-5, Uncompressed

\begin{tabular}{|c|c|c|c|}
\hline $\begin{array}{c}\text { Work } \\
\text { Order } \\
137268 \\
\text { Loading } \\
\text { Order }\end{array}$ & $\begin{array}{c}\text { Work } \\
\text { Order } \\
137268 \\
\text { ID } \\
\text { Number }\end{array}$ & $\begin{array}{c}\text { Graphite } \\
\text { Type }\end{array}$ & $\begin{array}{c}\text { Initial } \\
\text { Specimen } \\
\text { coM } \\
\text { Elevation } \\
\text { (in) }\end{array}$ \\
\hline 36 & AW1302 & NGB-17 & -1.750 \\
\hline 35 & EW1508 & IG-110 & -2.375 \\
\hline 34 & FW0803 & IG-430 & -3.000 \\
\hline 33 & BW302 & NGB-18 & -4.000 \\
\hline 32 & DW1604 & PCEA & -5.000 \\
\hline 31 & EW0802 & IG-110 & -6.000 \\
\hline 30 & CW303 & $\mathrm{H} 451$ & -7.000 \\
\hline 29 & EW1507 & IG-110 & -7.625 \\
\hline 28 & FW0804 & IG-430 & -8.250 \\
\hline 27 & BW303 & NGB-18 & -9.250 \\
\hline 26 & DW1701 & PCEA & -10.250 \\
\hline 25 & EW0803 & IG-110 & -11.250 \\
\hline 24 & FW0901 & IG-430 & -12.250 \\
\hline 23 & BW401 & NBG-18 & -13.250 \\
\hline 22 & $7 Z$ & Flux Monitor & -13.875 \\
\hline 21 & DW1702 & PCEA & -14.500 \\
\hline 20 & FW0902 & IG-430 & -15.500 \\
\hline 19 & EW0804 & IG-110 & -16.500 \\
\hline 18 & BW402 & NBG-18 & -17.500 \\
\hline 17 & DW1704 & PCEA & -18.500 \\
\hline 16 & EW1506 & IG-110 & -19.125 \\
\hline 15 & CW401 & $\mathrm{H} 451$ & -19.750 \\
\hline 14 & $\mathrm{~J} 210$ & HLM & -20.375 \\
\hline 13 & RW4 03 & BAN & -20.625 \\
\hline 12 & H582 & Compact Matrix & -20.875 \\
\hline 11 & TP 22 & 2114 & -21.125 \\
\hline 10 & L3 06 & PPEA & -21.375 \\
\hline 9 & L3 02 & PPEA & -21.625 \\
\hline 8 & K3 03 & PGX & -21.875 \\
\hline 7 & P3-02 & $\mathrm{PCIB}$ & -22.125 \\
\hline 6 & DW18 07 & PCEA & -22.375 \\
\hline 5 & M2-11 & NBG-25 & -22.625 \\
\hline 4 & S2 11 & NBG-10 & -22.875 \\
\hline 3 & P3-05 & $\mathrm{PClB}$ & -23.125 \\
\hline 2 & EW14 05 & IG-110 & -23.375 \\
\hline
\end{tabular}

\section{S-6, Uncompressed}

\begin{tabular}{|c|c|c|c|}
\hline $\begin{array}{c}\text { Work } \\
\text { Order } \\
137268 \\
\text { Loading } \\
\text { Order }\end{array}$ & $\begin{array}{c}\text { Work } \\
\text { Order } \\
137268 \\
\text { ID } \\
\text { Number }\end{array}$ & $\begin{array}{c}\text { Graphite } \\
\text { Type }\end{array}$ & $\begin{array}{c}\text { Initial } \\
\text { Specimen } \\
\text { COM } \\
\text { Elevation } \\
\text { (in) }\end{array}$ \\
\hline 36 & BW502 & NBG-18 & -1.750 \\
\hline 35 & TP13 & 2114 & -2.375 \\
\hline 34 & DW203 & PCEA & -3.000 \\
\hline 33 & CW501 & $\mathrm{H} 451$ & -4.000 \\
\hline 32 & AP601 & NBG-17 & -5.000 \\
\hline 31 & FW1001 & IG-430 & -6.000 \\
\hline 30 & BP302 & NGB-18 & -7.000 \\
\hline 29 & TP14 & 2114 & -7.625 \\
\hline 28 & DA203 & PCEA & -8.250 \\
\hline 27 & EW0904 & IG-110 & -9.250 \\
\hline 26 & AW1402 & NBG-17 & -10.250 \\
\hline 25 & CW503 & $\mathrm{H} 451$ & -11.250 \\
\hline 24 & BW503 & NBG-18 & -12.250 \\
\hline 23 & DW204 & PCEA & -13.250 \\
\hline 22 & $7 Y$ & Flux Monitor & -13.875 \\
\hline 21 & EW1001 & IG-110 & -14.500 \\
\hline 20 & AW1403 & NBG-17 & -15.500 \\
\hline 19 & BP301 & NBG-18 & -16.500 \\
\hline 18 & DA202 & PCEA & -17.500 \\
\hline 17 & FW1002 & IG-430 & -18.500 \\
\hline 16 & TP15 & 2114 & -19.125 \\
\hline 15 & EW1002 & IG-110 & -19.750 \\
\hline 14 & CW14 06 & $\mathrm{H} 451$ & -20.375 \\
\hline 13 & M2-12 & NBG-25 & -20.625 \\
\hline 12 & S2 12 & NBG-10 & -20.875 \\
\hline 11 & K306 & $\mathrm{PGX}$ & -21.125 \\
\hline 10 & EW14 06 & IG-110 & -21.375 \\
\hline 9 & $J 211$ & HLM & -21.625 \\
\hline 8 & RW4 04 & BAN & -21.875 \\
\hline 7 & H591 & Compact Matrix & -22.125 \\
\hline 6 & TP23 & 2114 & -22.375 \\
\hline 5 & P3-04 & $\mathrm{PClB}$ & -22.625 \\
\hline 4 & L3 03 & PPEA & -22.875 \\
\hline 3 & K3 04 & $P G X$ & -23.125 \\
\hline 2 & P3-03 & $\mathrm{PClB}$ & -23.375 \\
\hline
\end{tabular}


Table 6. AGC-2 center channel loading order.

\begin{tabular}{|c|c|c|c|}
\hline \multicolumn{4}{|c|}{ S-7, Uncompressed } \\
\hline $\begin{array}{c}\text { Work Order } 137268 \\
\text { Loading Order }\end{array}$ & $\begin{array}{c}\text { Work Order } 137268 \\
\text { ID No. }\end{array}$ & $\begin{array}{l}\text { Graphite } \\
\text { Type }\end{array}$ & $\begin{array}{l}\text { Initial Specimen COM } \\
\text { Elevation } \\
\text { (in) }\end{array}$ \\
\hline 170 & A3P43Z12 & A3 matrix & 18.375 \\
\hline 169 & J1 11 & HLM & 18.125 \\
\hline 168 & K2 02 & PGX & 17.875 \\
\hline 167 & L2 01 & PPEA & 17.625 \\
\hline 166 & M1-12 & NBG-25 & 17.375 \\
\hline 165 & TP 11 & 2114 & 17.125 \\
\hline 164 & $\mathrm{P} 2-01$ & PCIB & 16.875 \\
\hline 163 & RW2 02 & BAN & 16.625 \\
\hline 162 & S1 11 & NBG-10 & 16.375 \\
\hline 161 & CPB101 & $\mathrm{H} 451$ & 16.125 \\
\hline 160 & BP7 08 & NBG-18 & 15.875 \\
\hline 159 & DW18 08 & PCEA & 15.625 \\
\hline 158 & BP7 09 & NBG-18 & 15.375 \\
\hline 157 & FW1504 & IG-430 & 15.125 \\
\hline 156 & EW14 04 & IG-110 & 14.875 \\
\hline 155 & CW14 05 & $\mathrm{H} 451$ & 14.625 \\
\hline 154 & A3H08Z19 & A3 matrix & 14.375 \\
\hline 153 & $\mathrm{~J} 110$ & HLM & 14.125 \\
\hline 152 & K2 01 & PGX & 13.875 \\
\hline 151 & L1 10 & PPEA & 13.625 \\
\hline 150 & M1-11 & NBG-25 & 13.375 \\
\hline 149 & TP-10 & 2114 & 13.125 \\
\hline 148 & $\mathrm{P} 1-10$ & PCIB & 12.875 \\
\hline 147 & RW2 01 & BAN & 12.625 \\
\hline 146 & S1 10 & NBG-10 & 12.375 \\
\hline 145 & CPB91 & $\mathrm{H} 451$ & 12.125 \\
\hline 144 & BP7 10 & NBG-18 & 11.875 \\
\hline 143 & DA8 05 & PCEA & 11.625 \\
\hline 142 & AP7 08 & NBG-17 & 11.375 \\
\hline 141 & FW15 05 & IG-430 & 11.125 \\
\hline 140 & EW15 03 & IG-110 & 10.875 \\
\hline 139 & CW14 04 & $\mathrm{H} 451$ & 10.625 \\
\hline 138 & H521 & Compact matrix & 10.375 \\
\hline 137 & J1 09 & HLM & 10.125 \\
\hline 136 & K1 10 & PGX & 9.875 \\
\hline 135 & L1 09 & PPEA & 9.625 \\
\hline 134 & M1-10 & NBG-25 & 9.375 \\
\hline
\end{tabular}


Table 6. (continued).

\begin{tabular}{|c|c|c|c|}
\hline \multicolumn{4}{|c|}{ S-7, Uncompressed } \\
\hline $\begin{array}{c}\text { Work Order } 137268 \\
\text { Loading Order }\end{array}$ & $\begin{array}{c}\text { Work Order } 137268 \\
\text { ID No. }\end{array}$ & $\begin{array}{l}\text { Graphite } \\
\text { Type }\end{array}$ & $\begin{array}{l}\text { Initial Specimen COM } \\
\text { Elevation } \\
\text { (in) }\end{array}$ \\
\hline 133 & TP 09 & 2114 & 9.125 \\
\hline 132 & P1-09 & PCIB & 8.875 \\
\hline 131 & RW1 10 & BAN & 8.625 \\
\hline 130 & S1 09 & NBG-10 & 8.375 \\
\hline 129 & CPB81 & $\mathrm{H} 451$ & 8.125 \\
\hline 128 & BW17 01 & NBG-18 & 7.875 \\
\hline 127 & DA8 04 & PCEA & 7.625 \\
\hline 126 & AP7 09 & NBG-17 & 7.375 \\
\hline 125 & FW15 06 & IG-430 & 7.125 \\
\hline 124 & EW15 02 & IG-110 & 6.875 \\
\hline 123 & CW14 03 & $\mathrm{H} 451$ & 6.625 \\
\hline 122 & $\mathrm{H} 512$ & Compact matrix & 6.375 \\
\hline 121 & $\mathrm{~J} 108$ & HLM & 6.125 \\
\hline 120 & K1 09 & PGX & 5.875 \\
\hline 119 & L1 08 & PPEA & 5.625 \\
\hline 118 & M1-09 & NBG-25 & 5.375 \\
\hline 117 & ТР 08 & 2114 & 5.125 \\
\hline 116 & P1-08 & PCIB & 4.875 \\
\hline 115 & RW1 09 & BAN & 4.625 \\
\hline 114 & S1 08 & NBG-10 & 4.375 \\
\hline 113 & CPB71 & $\mathrm{H} 451$ & 4.125 \\
\hline 112 & BW17 09 & NBG-18 & 3.875 \\
\hline 111 & DA8 03 & PCEA & 3.625 \\
\hline 110 & AP7 10 & NBG-17 & 3.375 \\
\hline 109 & FW16 01 & IG-430 & 3.125 \\
\hline 108 & EW15 01 & IG-110 & 2.875 \\
\hline 107 & CPB151 & $\mathrm{H} 451$ & 2.625 \\
\hline 106 & A3P33Z09 & A3 matrix & 2.375 \\
\hline 105 & J1 07 & HLM & 2.125 \\
\hline 104 & K1 08 & PGX & 1.875 \\
\hline 103 & L1 07 & PPEA & 1.625 \\
\hline 102 & M1-08 & NBG-25 & 1.375 \\
\hline 101 & ТР 07 & 2114 & 1.125 \\
\hline 100 & P1-07 & PCIB & 0.875 \\
\hline 99 & RW1 08 & BAN & 0.625 \\
\hline 98 & S1 07 & NBG-10 & 0.375 \\
\hline 97 & CPB61 & $\mathrm{H} 451$ & 0.125 \\
\hline 96 & BW17 08 & NBG-18 & -0.125 \\
\hline
\end{tabular}


Table 6. (continued).

\begin{tabular}{|c|c|c|c|}
\hline \multicolumn{4}{|c|}{ S-7, Uncompressed } \\
\hline $\begin{array}{c}\text { Work Order } 137268 \\
\text { Loading Order }\end{array}$ & $\begin{array}{c}\text { Work Order } 137268 \\
\text { ID No. }\end{array}$ & $\begin{array}{l}\text { Graphite } \\
\text { Type }\end{array}$ & $\begin{array}{l}\text { Initial Specimen COM } \\
\text { Elevation } \\
\text { (in) }\end{array}$ \\
\hline 95 & DA8 02 & PCEA & -0.375 \\
\hline 94 & AW17 01 & NBG-17 & -0.625 \\
\hline 93 & FW15 12 & IG-430 & -0.875 \\
\hline 92 & EW14 12 & IG-110 & -1.125 \\
\hline 91 & CPB141 & $\mathrm{H} 451$ & -1.375 \\
\hline 90 & A3H08Z07 & A3 matrix & -1.625 \\
\hline 89 & $\mathrm{~J} 106$ & HLM & -1.875 \\
\hline 88 & K1 07 & PGX & -2.125 \\
\hline 87 & L1 06 & PPEA & -2.375 \\
\hline 86 & M1-07 & NBG-25 & -2.625 \\
\hline 85 & TP 06 & 2114 & -2.875 \\
\hline 84 & $\mathrm{P} 1-06$ & PCIB & -3.125 \\
\hline 83 & RW1 07 & BAN & -3.375 \\
\hline 82 & S1 06 & NBG-10 & -3.625 \\
\hline 81 & CPB51 & $\mathrm{H} 451$ & -3.875 \\
\hline 80 & BW17 07 & NBG-18 & -4.125 \\
\hline 79 & DA8 01 & PCEA & -4.375 \\
\hline 78 & AW17 02 & NBG-17 & -4.625 \\
\hline 77 & FW15 11 & IG-430 & -4.875 \\
\hline 76 & EW14 11 & IG-110 & -5.125 \\
\hline 75 & CPB131 & $\mathrm{H} 451$ & -5.375 \\
\hline 74 & $\mathrm{~A} 3 \mathrm{P} 43 \mathrm{Z} 03$ & A3 matrix & -5.625 \\
\hline 73 & $\mathrm{~J} 105$ & HLM & -5.875 \\
\hline 72 & K1 06 & PGX & -6.125 \\
\hline 71 & L1 05 & PPEA & -6.375 \\
\hline 70 & M1-06 & NBG-25 & -6.625 \\
\hline 69 & TP 05 & 2114 & -6.875 \\
\hline 68 & $\mathrm{P} 1-05$ & PCIB & -7.125 \\
\hline 67 & RW1 06 & BAN & -7.375 \\
\hline 66 & S1 05 & NBG-10 & -7.625 \\
\hline 65 & CPB41 & $\mathrm{H} 451$ & -7.875 \\
\hline 64 & BW17 05 & NBG-18 & -8.125 \\
\hline 63 & DW18 12 & PCEA & -8.375 \\
\hline 62 & AW17 03 & NBG-17 & -8.625 \\
\hline 61 & FW15 10 & IG-430 & -8.875 \\
\hline 60 & EW14 10 & IG-110 & -9.125 \\
\hline 59 & CPB121 & $\mathrm{H} 451$ & -9.375 \\
\hline 58 & $\mathrm{H} 491$ & Compact matrix & -9.625 \\
\hline
\end{tabular}


Table 6. (continued).

\begin{tabular}{|c|c|c|c|}
\hline \multicolumn{4}{|c|}{ S-7, Uncompressed } \\
\hline $\begin{array}{c}\text { Work Order } 137268 \\
\text { Loading Order }\end{array}$ & $\begin{array}{c}\text { Work Order } 137268 \\
\text { ID No. }\end{array}$ & $\begin{array}{l}\text { Graphite } \\
\text { Type }\end{array}$ & $\begin{array}{l}\text { Initial Specimen COM } \\
\text { Elevation } \\
\text { (in) }\end{array}$ \\
\hline 57 & $\mathrm{~J} 104$ & HLM & -9.875 \\
\hline 56 & K1 05 & PGX & -10.125 \\
\hline 55 & L1 04 & PPEA & -10.375 \\
\hline 54 & M1-05 & NBG-25 & -10.625 \\
\hline 53 & TP 04 & 2114 & -10.875 \\
\hline 52 & $\mathrm{P} 1-04$ & PCIB & -11.125 \\
\hline 51 & RW1 05 & BAN & -11.375 \\
\hline 50 & S1 04 & NBG-10 & -11.625 \\
\hline 49 & CPB31 & $\mathrm{H} 451$ & -11.875 \\
\hline 48 & BW17 04 & NBG-18 & -12.125 \\
\hline 47 & DW18 11 & PCEA & -12.375 \\
\hline 46 & AW17 06 & NBG-17 & -12.625 \\
\hline 45 & FW15 09 & IG-430 & -12.875 \\
\hline 44 & EW14 09 & IG-110 & -13.125 \\
\hline 43 & CPB111 & $\mathrm{H} 451$ & -13.375 \\
\hline 42 & $\mathrm{H} 482$ & Compact matrix & -13.625 \\
\hline 41 & $\mathrm{~J} 103$ & HLM & -13.875 \\
\hline 40 & K1 04 & PGX & -14.125 \\
\hline 39 & L1 03 & PPEA & -14.375 \\
\hline 38 & M1-04 & NBG-25 & -14.625 \\
\hline 37 & TP-03 & 2114 & -14.875 \\
\hline 36 & P1-03 & PCIB & -15.125 \\
\hline 35 & RW1 04 & BAN & -15.375 \\
\hline 34 & S1 03 & NBG-10 & -15.625 \\
\hline 33 & СРB21 & $\mathrm{H} 451$ & -15.875 \\
\hline 32 & BW17 03 & NBG-18 & -16.125 \\
\hline 31 & DW18 10 & PCEA & -16.375 \\
\hline 30 & AW17 05 & NBG-17 & -16.625 \\
\hline 29 & FW1508 & IG-430 & -16.875 \\
\hline 28 & EW14 08 & IB-110 & -17.125 \\
\hline 27 & CA11 02 & $\mathrm{H} 451$ & -17.375 \\
\hline 26 & A3P33Z20 & A3 matrix & -17.625 \\
\hline 25 & $\mathrm{~J} 102$ & HLM & -17.875 \\
\hline 24 & K1 03 & PGX & -18.125 \\
\hline 23 & L1 02 & PPEA & -18.375 \\
\hline 22 & M1-02 & NBG-25 & -18.625 \\
\hline 21 & TP 02 & 2114 & -18.875 \\
\hline 20 & P1-02 & PCIB & -19.125 \\
\hline
\end{tabular}


Table 6. (continued).

\begin{tabular}{|c|c|c|c|}
\hline \multicolumn{4}{|c|}{ S-7, Uncompressed } \\
\hline $\begin{array}{c}\text { Work Order } 137268 \\
\text { Loading Order }\end{array}$ & $\begin{array}{c}\text { Work Order } 137268 \\
\text { ID No. }\end{array}$ & $\begin{array}{l}\text { Graphite } \\
\text { Type }\end{array}$ & $\begin{array}{l}\text { Initial Specimen COM } \\
\text { Elevation } \\
\text { (in) }\end{array}$ \\
\hline 19 & RW1 03 & BAN & -19.375 \\
\hline 18 & $\mathrm{~S} 102$ & NBG-10 & -19.625 \\
\hline 17 & CPB11 & $\mathrm{H} 451$ & -19.875 \\
\hline 16 & BW17 02 & NBG-18 & -20.125 \\
\hline 15 & DW18 09 & PCEA & -20.375 \\
\hline 14 & AW17 04 & NBG-17 & -20.625 \\
\hline 13 & FW15 07 & IG-430 & -20.875 \\
\hline 12 & EW14 07 & IG-110 & -21.125 \\
\hline 11 & CA11 01 & H451 & -21.375 \\
\hline 10 & $\mathrm{H} 472$ & Compact matrix & -21.625 \\
\hline 9 & $\mathrm{~J} 101$ & HLM & -21.875 \\
\hline 8 & K1 01 & PGX & -22.125 \\
\hline 7 & L1 01 & PPEA & -22.375 \\
\hline 6 & M1-01 & NBG-25 & -22.625 \\
\hline 5 & TP 01 & 2114 & -22.875 \\
\hline 4 & $\mathrm{P} 1-01$ & PCIB & -23.125 \\
\hline 3 & RW1 02 & BAN & -23.375 \\
\hline 2 & CPB1 & $\mathrm{H} 451$ & -23.625 \\
\hline 1 & S101 & NBG-10 & -23.875 \\
\hline
\end{tabular}

Following irradiation in the ATR at INL, the AGC-2 capsule was disassembled. ${ }^{[22,23]}$ All specimens recovered from disassembly were visually inspected and physically measured within the INL Carbon Characterization Laboratory (CCL) before storage within the irradiated graphite storage vault. It should be noted that NBG-25 specimen S101 was lost during AGC-2 disassembly activities. After accounting for all recovered specimens from the AGC-2 capsule, PIE and testing were performed for each specimen at the INL Carbon Characterization Laboratory.

\section{TESTING}

A significant level of preparation was needed to meet Nuclear Quality Assurance Level 2 quality requirements prior to actual material property testing. An approved characterization plan was developed that was dependent upon the two graphite specimen geometries and on the material properties to be measured. ${ }^{[23]}$ In general, all testing was performed through American Society for Testing and Materials approved standards; however, due to the small size of the graphite specimens, some methods required modification and/or variation of the testing standards. Details of these testing standard variations, along with equipment calibration, personnel training on testing methodology, and data acquisition, are specified in a specimen characterization plan. 
Thermal diffusivity, coefficient of thermal expansion (CTE), electrical resistivity, elastic modulus, and mass and dimensions measurements were performed on the AGC-2 irradiated specimens. These measurements were performed per "AGC-2 Graphite Specimen Postirradiation Characterization Plan," PLN-4657. This plan describes in detail the measurement techniques, equipment, and standards used to gather the data presented here.

Data gathered for the characterization of AGC-2 specimens are contained in the appendices of this report. Appendix A contains plots of the individual data points for each specimen. Shown by the dashed lines in each plot are the upper and lower limits of the interquartile range (IQR). These limits are established by either the least or greatest value in the data or by multiplying the IQR by 1.5 and adding or subtracting this value from the third and first quartile. Any datum value outside of these established limits is considered a suspected outlier of the established pattern. However, it is important to note that these outlying values are not only subject to measurement variability but also material and dose variability and, therefore, cannot necessarily be discarded.

These outlying values are examined in the context of the entire data set, including pre-irradiation data. Other statistical parameters are calculated and presented in the tables of Appendix B. The mean, standard deviation, and coefficient of variance (COV) are all calculated for the various measurement data sets and graphite types. Upper and lower limits called out in the tables of Appendix B are the IQR limits described above. Raw, tabulated data can be found in the Nuclear Data Management and Analysis System database, including parameters specified by the applicable American Society for Testing and Materials standard (e.g., dates, performer identifier, and room conditions).

There are many ways to combine and compare the data presented here. In doing so, the validity of the data is exercised and scrutinized. First, the data sets are evaluated independently, utilizing the statistical analysis described above. Additionally, a limited comparison of pre- and post-irradiation values is made for each graphite grade to illustrate the effects of irradiation and applied stress (where applicable). Note that pre-irradiation properties were measured using the same techniques, equipment, and standards per PLN-3267, "AGC-2 Characterization Plan." Pre-irradiation data for the AGC-2 specimens can be found in INL/EXT-10-19588, "AGC-2 Graphite Preirradiation Data Package.” These initial comparisons provide an initial examination of the data for trends and correlations that are intuitive and logical. In this way, the physical and thermal post-irradiation property measurements are vetted.

The data presented here include all AGC-2 specimens that were irradiated. Variables of irradiation temperature and dose are not considered for any property measurement in Figure 5Figure 9. The data are presented simply to illustrate the initial trends and correlations in irradiation induced material property changes. Data presented in Figure 10Figure 11Figure 13, Figure 14 demonstrate the induced changes as a function of either received irradiation dose or irradiation temperature. A more detailed analysis and trends in the data will be reported within the subsequent AGC-2 analysis report. From initial estimates of the as-run AGC-2 irradiation and temperature analysis, the average irradiation temperature is $600^{\circ} \mathrm{C}$ with a standard deviation of $65^{\circ} \mathrm{C}$ and a range of $705^{\circ} \mathrm{C}$ to $399^{\circ} \mathrm{C}$. The average radiation dose of all specimens is 3 dpa with a standard deviation of $1 \mathrm{dpa}$ and a range of 5 dpa to $1 \mathrm{dpa}$.

\subsection{Dimensions, Mass and Density}

Specimens are weighed and dimensionally measured at room temperature ${ }^{[23]}$. Plots of the measured mass, dimensions, and density for all AGC-2 irradiated specimens are shown in Figures A-1 through A-84 (see Appendix A). Prior to irradiation, the freshly machined surfaces produced consistent dimensional measurements with a COV of less than $0.05 \%$ for all specimens. Following irradiation, the surfaces are more irregular and inconsistent, resulting in a COV between $0.25 \%$ and $0.96 \%$. (Table B-1 and B-2). Similar distributions in the pre- and post-irradiation mass data were observed with no difference 
in mass observed between pre- and post-irradiation measurements outside of the measurement uncertainty.

Figure 5 illustrates the overall volume decrease for the irradiated specimens as a function of graphite type and stress. Detailed volume dependency on irradiation dose and irradiation temperature will be addressed within the subsequent AGC-2 analysis report. As expected, all graphite types experienced an overall shrinkage due to neutron irradiation. Shrinkage for all graphite types increased as the specimen stress increased. This is consistent with trends observed in the literature. ${ }^{[24,25]}$

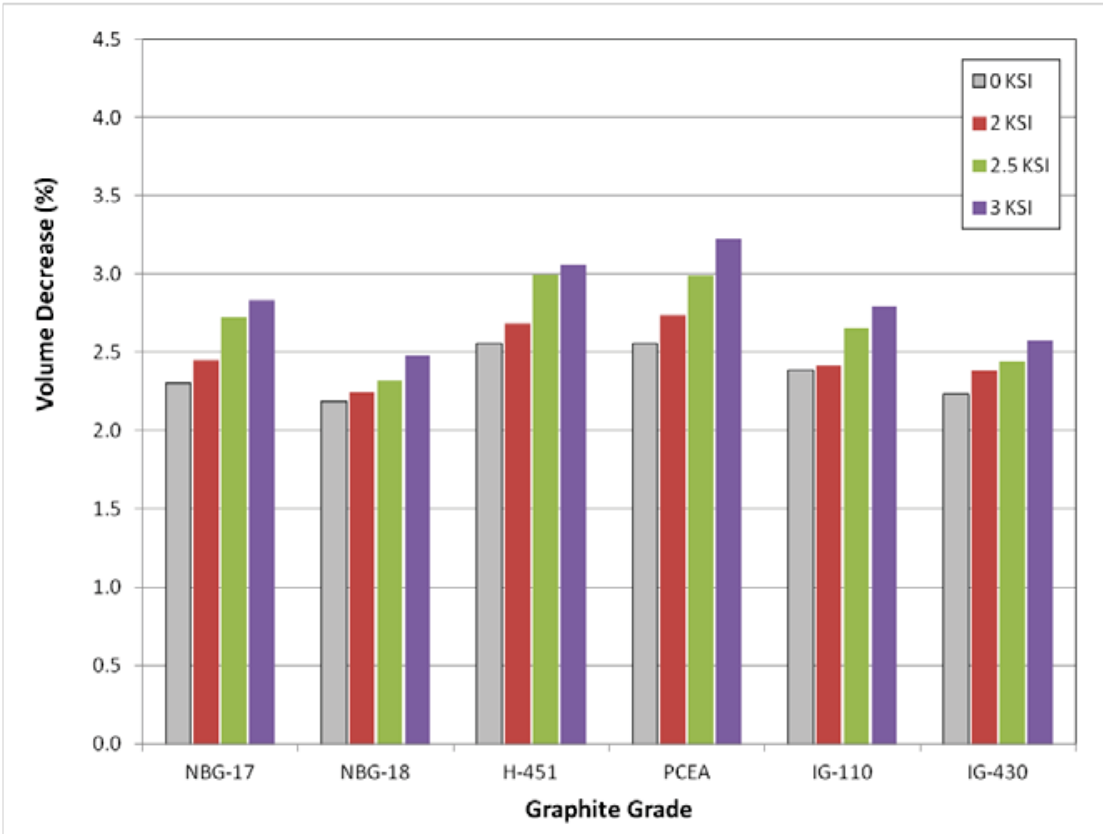

Figure 5. Volume decrease due to irradiation creep for six major grades of graphite. The dimensional change dependency on irradiation dose and irradiation temperature is not presented.

Naturally, a reduction in volume with no change in mass will result in an increase in density, Figure 6. As expected, density changes in a linear fashion with the decrease in volume and therefore demonstrates a general increase between $2.2 \%$ and $3.2 \%$. The COV for density values of irradiated specimens of the individual graphite types was below $0.85 \%$, Table B-4. Detailed density analysis and trends will be addressed within the subsequent AGC-2 analysis report. 


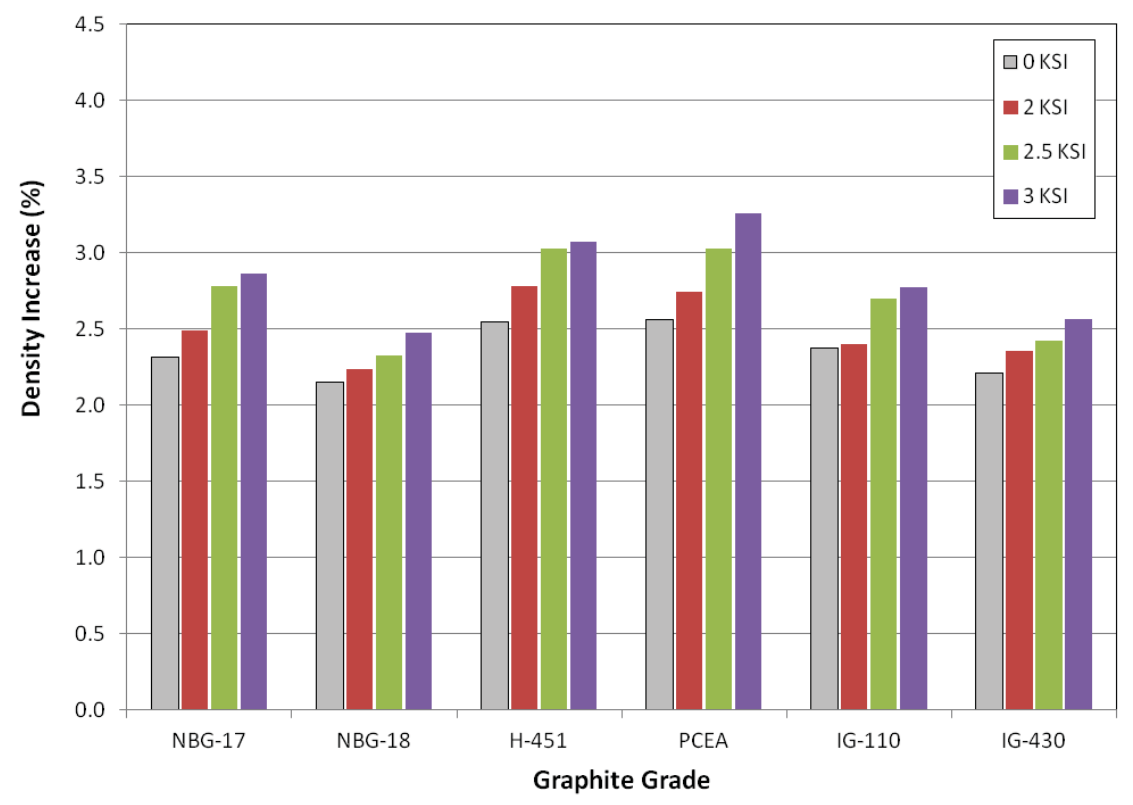

Figure 6. Density increase due to volume shrinkage for six major grades of graphite and four stress conditions. The density change dependency on irradiation dose and irradiation temperature is not presented.

\subsection{Elastic Modulus}

Elastic properties were measured with two dynamic nondestructive testing techniques at room temperature. ${ }^{[23]}$ In the fundamental frequency testing technique, Young's modulus is derived from the natural frequency of the creep specimens oscilating in the flexural mode. Due to the geometry of the specimens, the shear modulus can not be determined using this technique. However, both Young's modulus and shear modulus can be determined using the sonic velocity technique. In this case, an ultrasonic longitudinal or shear wave is sent through the specimen. The time it takes for this wave to pass through the specimen and the length of the specimen are used to determine the sonic velocity. Young's modulus and shear modulus can then be calculated from this time-of-flight measurement.

Young's modulus, determined by measurement of fundamental frequency, is plotted in Figures A-109 through A-114, and the statistical data are contained in Table B-8. Statistically, these data are well-behaved, with the IQR analysis showing no significant outliers. Figures A-121 through A-132 contain plots of Young's and shear moduli determined from the measurement of sonic velocity. Statistical parameters are shown in Tables B-10 and B-11 for Young's and shear moduli, respectively, and again the IQR analysis does not reveal any inconsistency or significant outliers. The spread in all modulus data is reasonable with the COV $\sim 5 \%$. The relatively high $10.8 \%$ COV value achieved in the PCEA graphite grade is due to the increased flaw and crack distribution within the microstructure of the graphite material.

It should be noted that for AGC-2 specimens the dynamic Young's modulus values determined by sonic velocity testing techniques are generally 2-3 GPa higher than the measurements obtained by the fundamental frequency testing technique (Appendix A). This difference between modulus measurements between the two standards has been noted for a number of years and was finally addressed in the latest version of the sonic velocity standard (ASTM-769-09). ${ }^{[26]}$ In this latest ASTM standard version the Young's modulus value is calculated from the time-of-flight measurement utilizing a correction factor 
designated as the Poisson's Factor $\left(\mathrm{C}_{\mathrm{v}}\right)$ which is normally valued at 0.9 . Calculating the modulus using the Poisson's Factor effectively lowers the value by $10 \%$, bringing the measured sonic velocity values to values arrived at by the fundamental frequency test method.

However, AGC-2 pre-irradiation testing was performed under the previous ASTM sonic velocity standard, ASTM-769-98 (reapproved 2005), ${ }^{[27]}$ which does not use the Poisson Factor to calculate the modulus values. The AGC-2 Characterization Plan (PLN-4657) specifies that the testing standards used to measure the material properties for the graphite specimens must be the same version before and after irradiation. Using the same ASTM test method version assures the unirradiated and irradiated measurements will be consistent and comparable without testing method bias.

Since only the measured data is being corrected by the Poisson's Factor, and not the actual testing technique itself, the pre- and post-irradiation AGC-2 sonic velocity data can be re-calculated.

Recalculating the data will be considered within the subsequent AGC-2 analysis report to provide a more accurate modulus value from the sonic velocity testing method.

While the absolute Young's modulus values between sonic velocity and fundamental frequency may be different for the AGC-2 measurement, when making relative comparisons between pre- and postirradiation measurements, the difference is consistent between the two techniques. Dynamic Young's modulus generally increases when pre- and post-irradiation Young's modulus values (as a percentage) are compared. This increase is shown to be similar for the fundamental frequency and sonic velocity testing techniques, Figure 7 and Figure 8. Also notable is the modulus increase correlation with specimens tested in compressive stress. In general, the overall irradiated modulus increase is less for higher compressive stressed specimens. Detailed Young's modulus dependency on irradiation dose and irradiation temperature will be addressed within the subsequent AGC-2 analysis report.

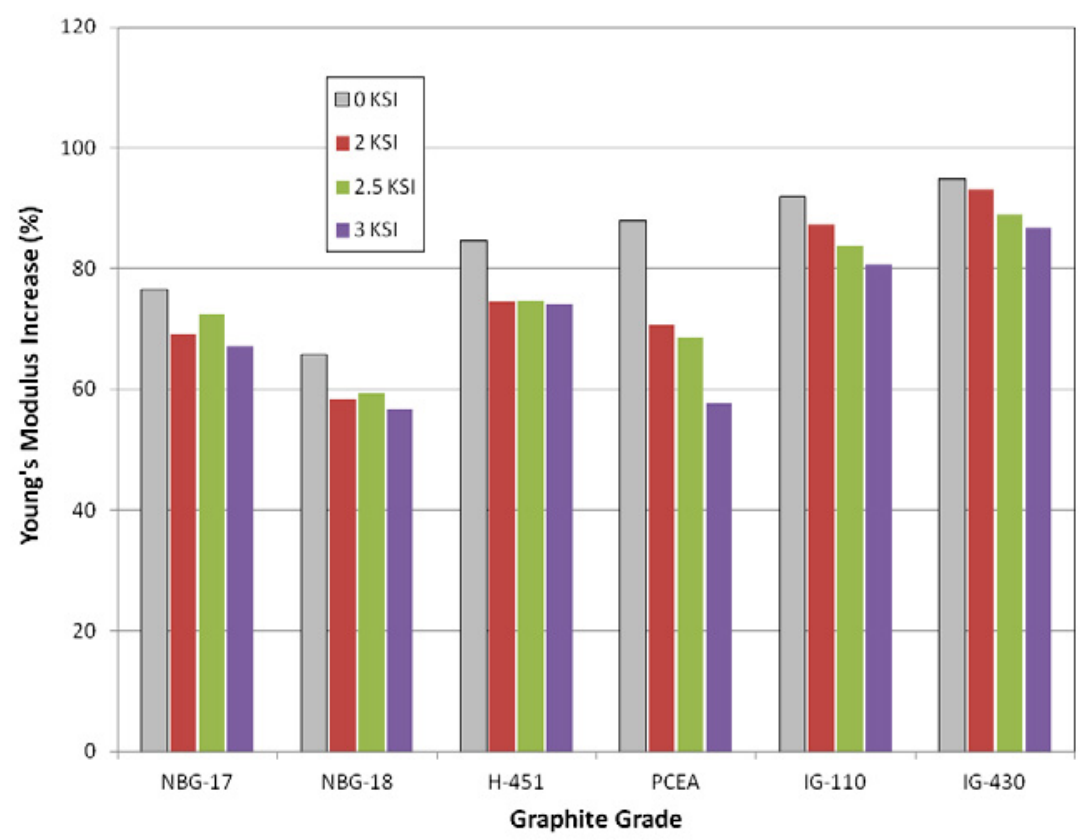

Figure 7. Young's modulus derived from the measurement of fundamental frequency for six grades of graphite and four different stress conditions. The Young's modulus dependency on irradiation dose and irradiation temperature is not presented. 


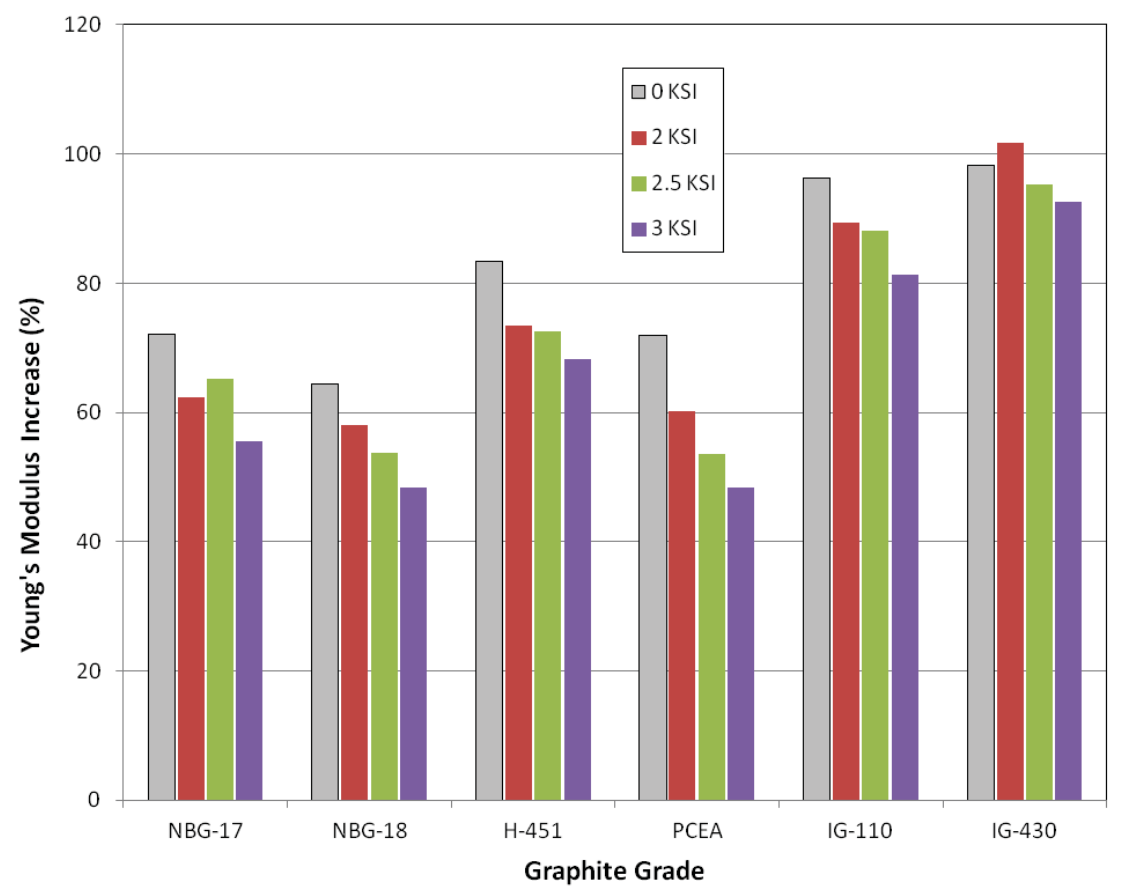

Figure 8. Young's modulus derived from the measurement of ultrasonic velocity for six grades of graphite and four different stress conditions. The Young's modulus dependency on irradiation dose and irradiation temperature is not presented.

\subsection{Resistivity}

Plots of electrical resistivity are shown in Figures A-115 through A-120 for graphite grades of PCEA, NBG-18, H-451, IG-110, IG-430, and NBG-17. All resistivity measurements were performed only on creep specimens. ${ }^{[23]}$ Statistical parameters can be found in Table B-9. The resistivity data are well $\backslash$ behaved with no outliers and a COV ranging from $2.7 \%$ to $4.8 \%$.

Specimen resistivity is measured at room temperature using a four point testing method. A constant current is passed through the long axis of the specimen, and voltage is measured at a fixed distance along the specimen axis. Resistivity is calculated from these values and the specimen geometry. Figure 9 indicates an overall general increase in resistivity after neutron irradiation. This increase is believed to occur primarily from atomic level defects that disrupt the flow of electrons. It is interesting to note that stress may initially have an effect on electrical resistivity but the effects appear to saturate with only limited changes with increasing the stress. Detailed electrical resistivity analysis including irradiation dose and irradiation temperature effects will be addressed within the subsequent AGC-2 analysis report. 


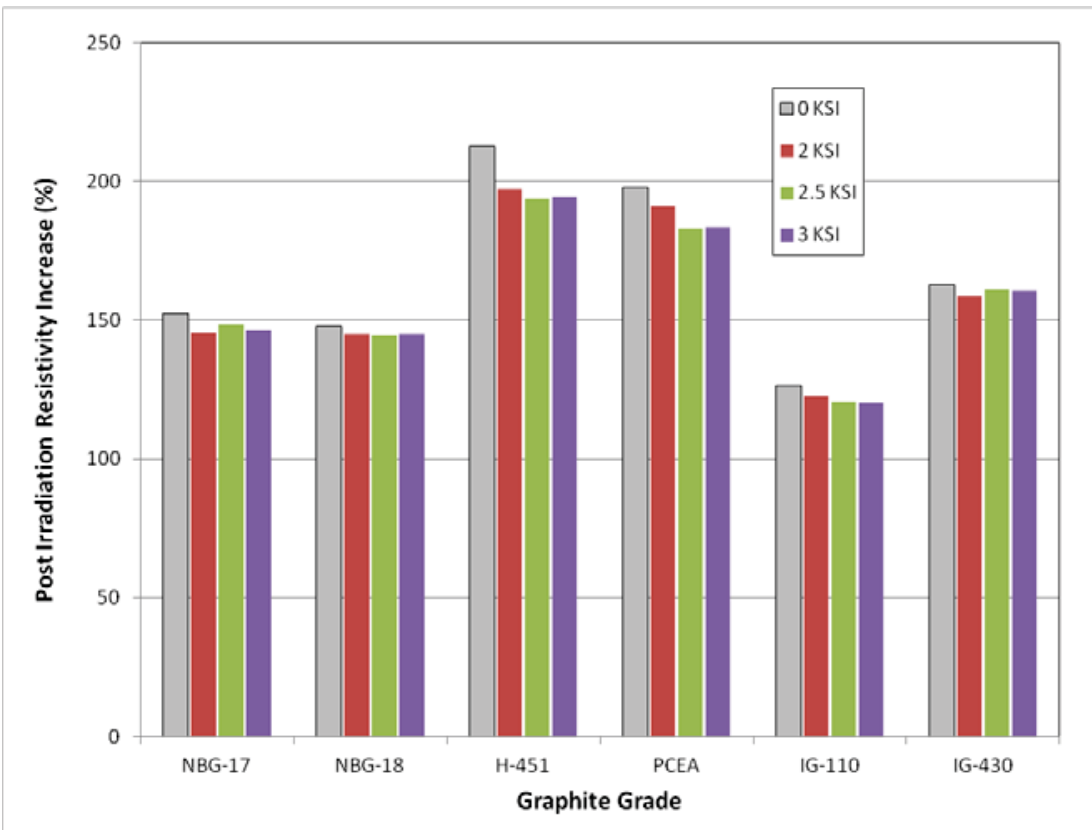

Figure 9. Electrical resistivity for six grades of graphite and four different stress conditions. The electrical resistivity dependency on irradiation dose and irradiation temperature is not presented.

\subsection{Thermal Diffusivity}

Thermal diffusivity was measured using a laser flash technique ${ }^{[23]}$ Because annealing of irradiation damage begins to take place at temperatures above the irradiation temperature, measurements of thermal diffusivity were limited to $500^{\circ} \mathrm{C}\left(100^{\circ} \mathrm{C}\right.$ below the average AGC-2 specimen irradiation temperature).

Plots of thermal diffusivity are shown in Figures A-133 through A-192. Discrete temperatures of 100, 300 , and $500^{\circ} \mathrm{C}$ were statistically evaluated. Tables B-16a through B-18c contain values of the mean, standard deviation, and COV. Considering the complexities associated with measuring the thermal diffusivity of irradiated specimens, the COVs are reasonable, ranging between $\sim 4 \%$ and $\sim 8 \%$. The high $\mathrm{COV}$ for the A3 matrix material may be explained from the fabrication history. A3 fuel compact matrix material is fabricated from a composite mixture of graphitic flake material and phenolic resins. In addition, the AGC-2 A3 matrix specimens were fabricated from a small, non-production sized batch that mixed the resins and graphitic flake material and individually cold pressed the mixture into solid specimen compacts. These compacts are then baked to a relatively low temperature of $1800^{\circ} \mathrm{C}$, which is not high enough to induce graphitization of the mixture (a minimum of $2200^{\circ} \mathrm{C}$ is needed to begin graphitization). With inconsistencies due to the limited batch size, mixing, and/or individual die pressing of the mixture, it is not surprising that these specimens have larger thermal diffusivity variation. In addition, the higher variation in diffusivity of PCEA is most likely due to the higher level of microstructure defects from fabrication (increased porosity).

As stated earlier, the material property change dependence on dose and irradiation temperature have not been evaluated up to this point. Figure 10 and Figure 11 illustrate the ratio of irradiated to unirradiated thermal diffusivity as a function of dose and as a function of irradiation temperature, respectively, at $500^{\circ} \mathrm{C}$ for graphite type IG-110. Both plots for IG-110 and the other graphite types indicate that the general change in diffusivity is neither a function of dose or irradiation temperature. However, due to the singular nature of the specimen location in the experiment and the dose and temperature profiles along the axis of the experiment, it is not possible to separate the two. Specimens located in the center of the 
AGC-2 irradiation capsule experienced the highest radiation dose and temperature while those further from the centerline correspondingly received a lower dose at a lower irradiation temperature. With this correspondence between dose and temperature, there is no set of specimens with a variation in temperature for a constant dose and vice-versa. Therefore, the constant delta in diffusivity displayed in Figure 10 and Figure 11 is possibly a function of both temperature and dose. However, because they do display relatively constant values of diffusivity change (i.e., the property change has saturated), it may be possible to average all specimens of a particular type of graphite to evaluate other variables, as was done in Figure 12. Here the ratio of irradiated to unirradiated diffusivity is shown as a function of specimen temperature for seven graphite types. The plot shows that the greatest change in diffusivity occurs at lower temperatures. All graphite grades follow this similar trend. Detailed diffusivity dependency analysis including irradiation dose and irradiation temperature dependency will be addressed within the subsequent AGC-2 analysis report.

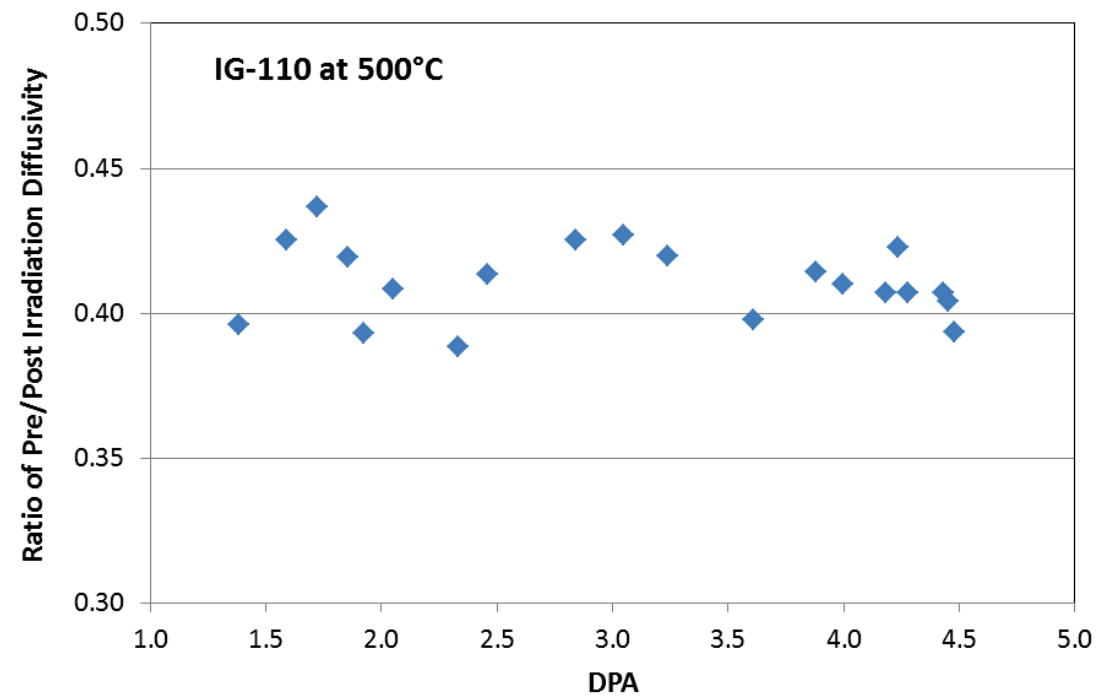

Figure 10. Ratio of irradiated diffusivity to unirradiated diffusivity for specimens of IG-110 at $500^{\circ} \mathrm{C}$ as a function of irradiation dose. Thermal diffusivity dependency on irradiation temperature is not presented. 


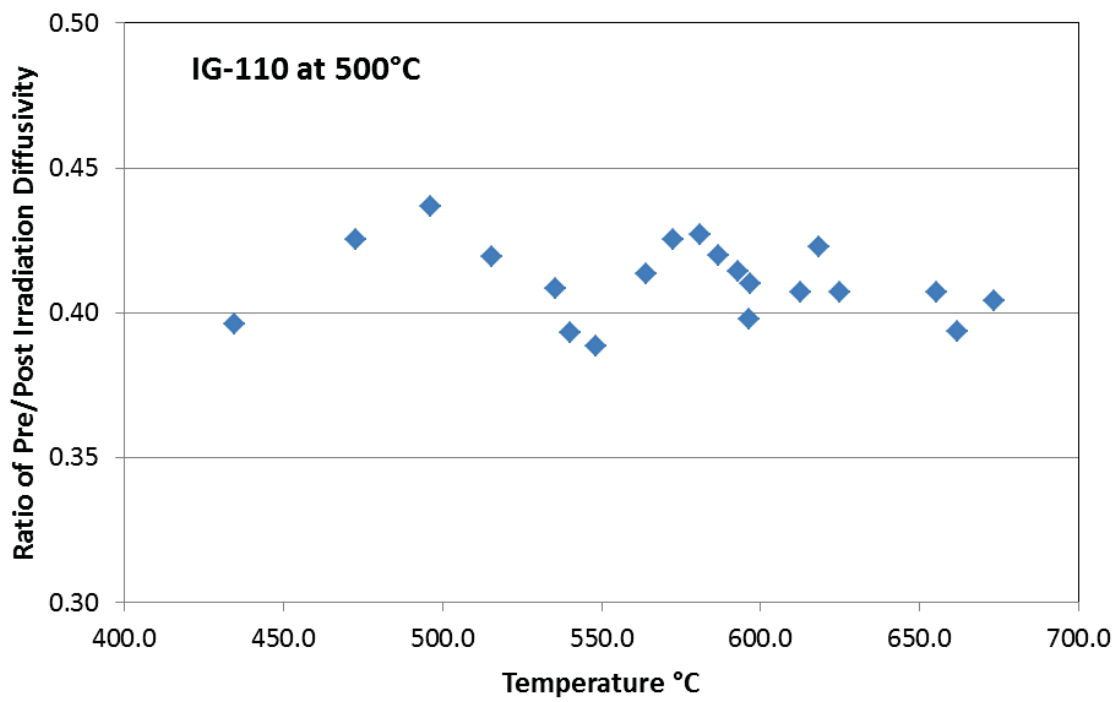

Figure 11. Ratio of irradiated diffusivity to unirradiated diffusivity for specimens of IG- 110 at $500^{\circ} \mathrm{C}$ as a function of irradiation temperature. Thermal diffusivity dependency on irradiation dose is not presented.

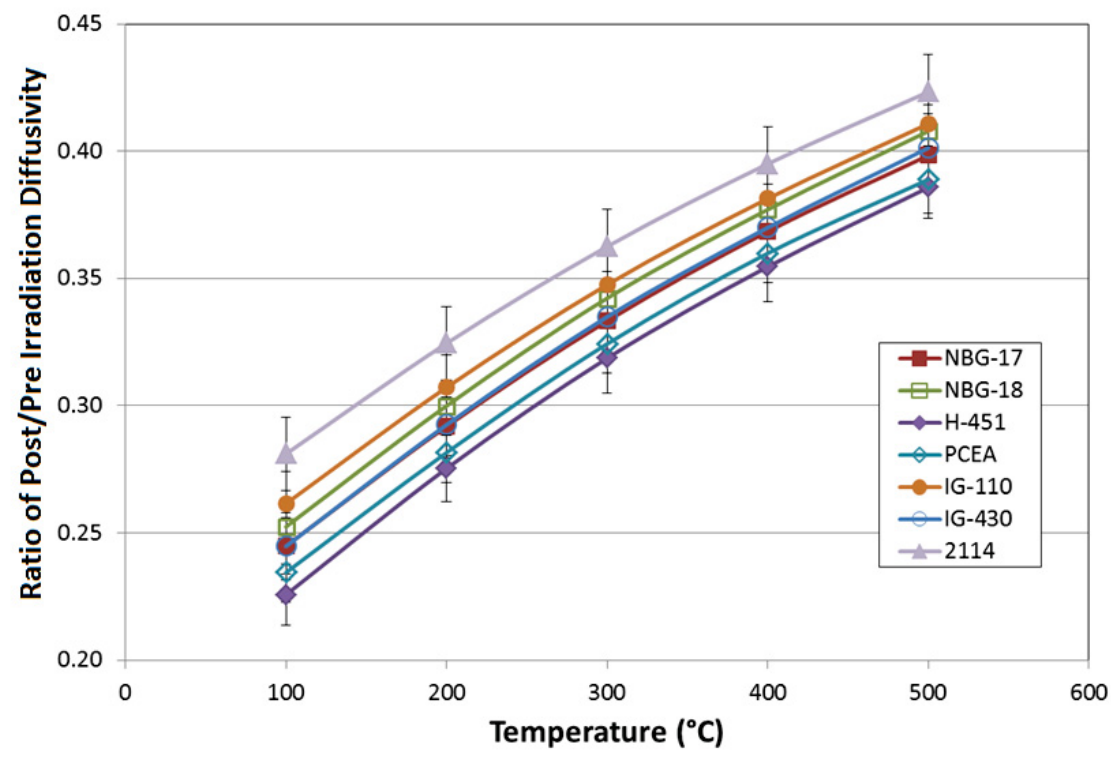

Figure 12. Ratio of irradiated diffusivity to unirradiated diffusivity as a function of specimen temperature for seven grades of graphite. Changes to thermal diffusivity resulting from irradiation dose and irradiation temperature are assumed to have been saturated allowing all graphite grades to be grouped together. The assumption that diffusivity change saturates will be validated within the subsequent AGC-2 analysis report.

\subsection{Coefficient of Thermal Expansion}

CTE was measured using a linear dilatometer technique. ${ }^{[23]}$ Because annealing of irradiation damage begins to take place at temperatures above the irradiation temperature, measurements of thermal expansion were limited to $500^{\circ} \mathrm{C}\left(100^{\circ} \mathrm{C}\right.$ below the average AGC-2 specimen irradiation temperature). 
Mean CTE data are plotted in Figures A-85 through A-108. A statistical evaluation of the CTE data was performed at three discrete temperatures (i.e., 100,300 , and $500^{\circ} \mathrm{C}$ ) for each graphite type. Again, the dashed lines in these plots indicate the upper and lower IQR limits. There are no outliers to consider.

Tables B-5 through B-7 (see Appendix B) list the mean, standard deviation, and COV values for data evaluated at the discrete temperatures. As shown, COV values range from $\sim 8 \%$ to $16 \%$. This relatively large scatter in the data may be a result of the variation in stress levels of the different specimens.

Figure 13 and Figure 14 illustrate an increase in CTE as a function of dose and irradiation temperature, respectively, for graphite type IG- 110 at $500^{\circ} \mathrm{C}$. As is common in all the data, there is a near singular relationship between the irradiation temperature and dose for a given location in the experiment. Therefore, specimens at a given temperature will have a very narrow range of doses, and specimens at a given dose will have a very narrow range of temperatures. Because there are only a few (2-6) specimens at similar channel positions within the capsule, it is difficult to separate the variables of dose and irradiation temperature. Fortunately, the general trend for CTE change seems to demonstrate very little dependence on increasing dose or irradiation temperature for all specimens tested, as seen in Figure 13 and Figure 14.

While the change to CTE appears to saturate with increasing dose and irradiation temperature, it can be observed that stress appears to increase CTE values rather markedly in irradiated specimens. As applied stress is increased, significant changes in CTE are noted. Detailed CTE dependency on irradiation dose and irradiation temperature will be addressed within the subsequent AGC-2 analysis report.

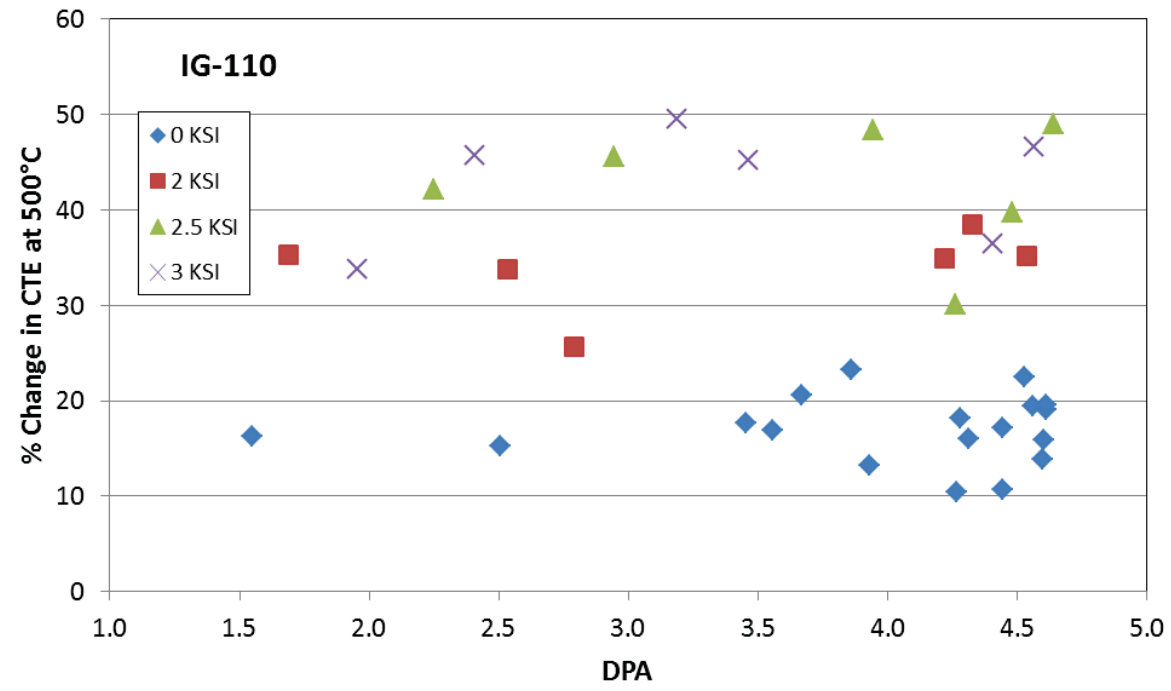

Figure 13. Percent increase in CTE at $500^{\circ} \mathrm{C}$ for graphite grade IG-110 as a function of irradiation dose for four different stress levels. CTE dependency on irradiation temperature is not presented. 


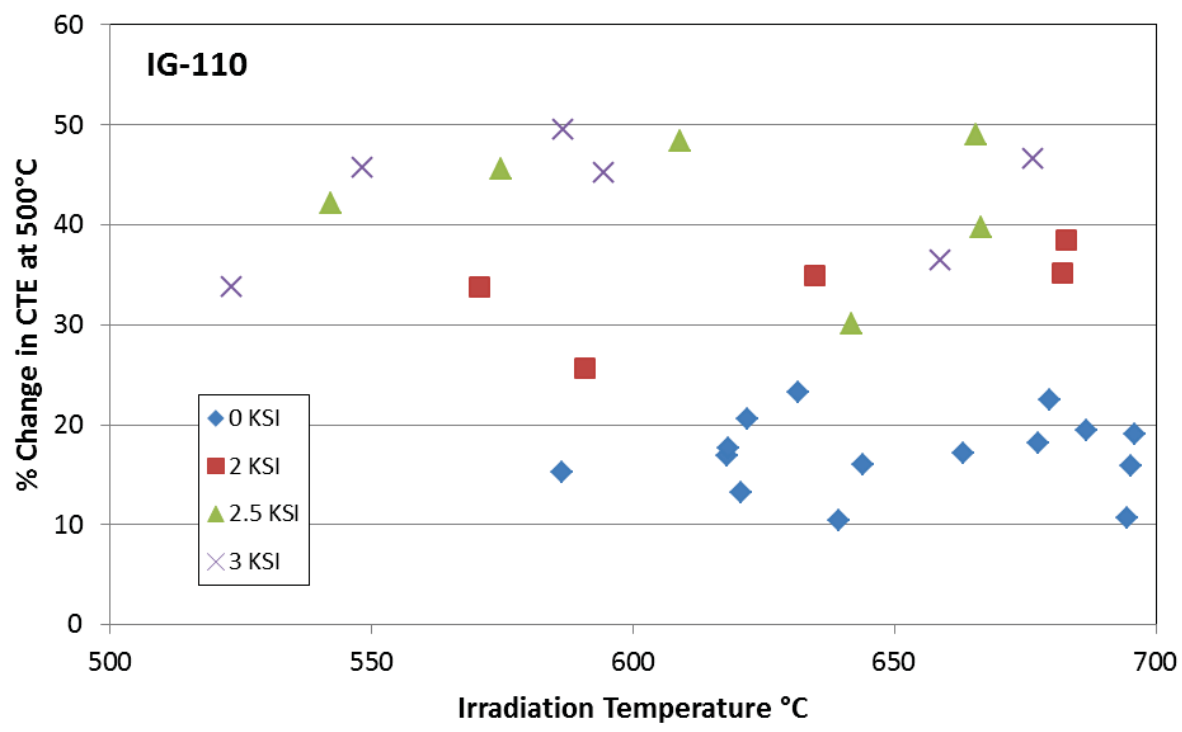

Figure 14. Percent increase in $\mathrm{CTE}$ at $500^{\circ} \mathrm{C}$ for graphite grade $\mathrm{IG}-110$ as a function of irradiation temperature for four different stress levels. CTE dependency on irradiation dose is not presented.

Figure 15 illustrates the average increase in CTE as a function of temperature for stressed and unstressed specimens of the six major graphite grades. Each data point is averaged over all specimen doses and irradiation temperatures for each graphite grade. Specimens that were mechanically loaded are averaged over stress values of 13.8 $\mathrm{MPa}, 17.2 \mathrm{MPa}$, or $20.7 \mathrm{MPa}(2 \mathrm{ksi}, 2.5 \mathrm{ksi}$ and $3 \mathrm{ksi}$, respectively). The measured CTE values for all stressed creep specimens can be seen to be considerably higher than the unstressed control specimens of the same grade. This indicates that stress has a significant effect on the CTE of irradiated graphite and will be analyzed further in the subsequent AGC-2 analysis report.

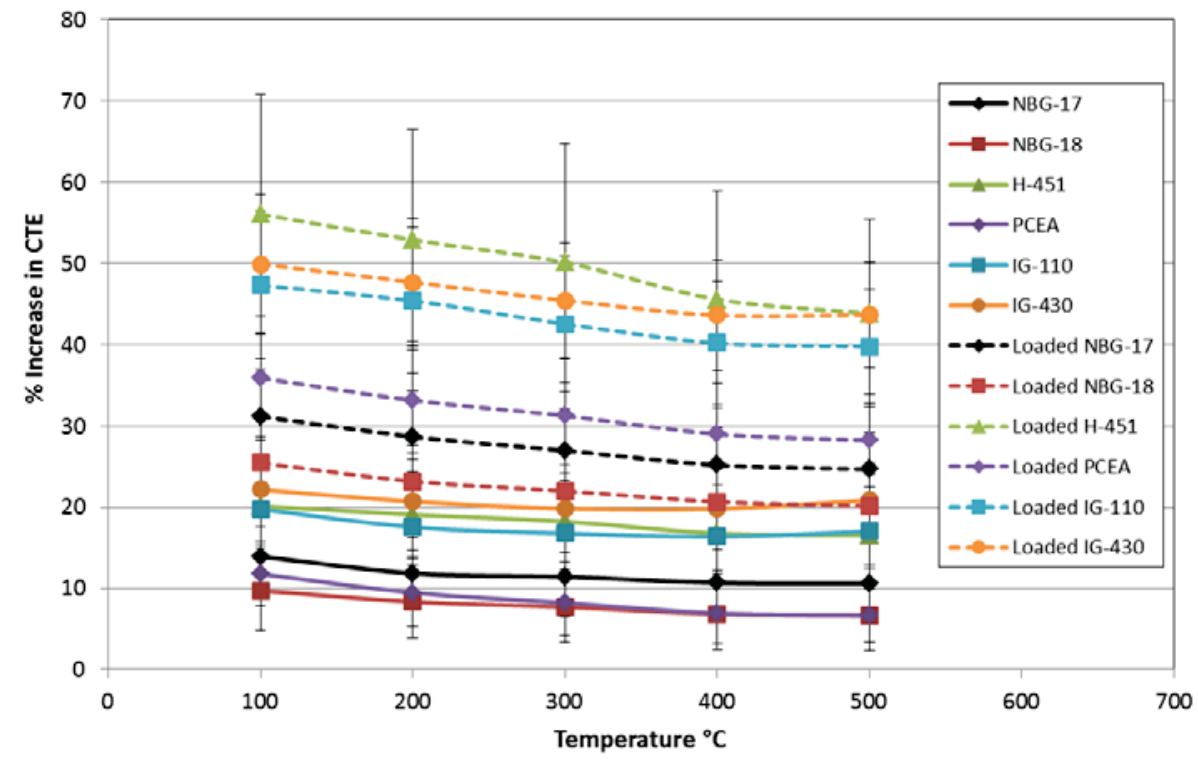

Figure 15. Percent increase in CTE for six different grades of graphite as a function of temperature for stressed and unstressed conditions. Changes to CTE resulting from irradiation dose and temperature are 
assumed to have been saturated allowing all graphite grades to be grouped together. The assumption that CTE change saturates will be validated within the subsequent AGC-2 analysis report.

\section{REFERENCES}

1. T. Burchell, R. Bratton, and W. Windes, "NGNP Graphite Selection and Acquisition Strategy," ORNL/TM-2007/153, September 2007.

2. R. L. Bratton and T. D. Burchell, 2005, "NGNP Graphite Testing and Qualification Specimen Selection Strategy,” INL/EXT-05-00269, Idaho National Laboratory, May 2005.

3. PLN-2497, 2010, Graphite Technology Development Plan, Rev. 1, Idaho National Laboratory, October 2010.

4. TFR-645, 2010, Advanced Graphite Capsule AGC-2 Experiment Test Train, Rev. 0, Idaho National Laboratory, July 2010.

5. T. Burchell and R. Bratton, 2005, "Graphite Irradiation Creep Capsule AGC-1 Experimental Plan," ORNL/TM-2005/505, Oak Ridge National Laboratory, May 2005.

6. INL Drawing 600786, 2009, “ATR Advanced Graphite Capsule (AGC-2) Graphite Specimen Machining Details," Rev. 1, Idaho National Laboratory, March 2009.

7. INL Drawing 600786, 2012, “ATR Advanced Graphite Capsule (AGC-2) Graphite Specimen Machining Details," Rev. 2, Idaho National Laboratory, July 2012.

8. INL Drawing 600787, 2010, "ATR Advanced Graphite Capsule (AGC-2) Experiment Graphite Specimen Cut-Out Diagrams,” Rev. 3, Idaho National Laboratory, July 2010.

9. DWG 601266, 2012, ATR Advanced Graphite Capsule Number 2 (AGC-2) Capsule Facility Assemblies, Rev. 2, Idaho National Laboratory, July 2012.

10. DWG 601258, 2012, ATR Advanced Graphite Capsule 2 (AGC-2) Graphite Specimen Holder Assemblies and Details, Rev. 1, Idaho National Laboratory, July 2012.

11. DWG 600001, 2009, ATR TMIST-1 Oxidation Experiment In-Vessel Installation, Rev. 2, Idaho National Laboratory, March 2009.

12. J. R. Parry, 2010, "Engineering Calculations and Analysis Report: Reactor Physics Projections for the AGC-2 Experiment Irradiated in the ATR South Flux Trap," ECAR-1050, Rev. 0, Idaho National Laboratory, October 13, 2010.

13. T. Burchell, 2009, “A Revised AGC-1 Creep Capsule Layout,” ORNL/TM-2009/009, Oak Ridge National Laboratory, January 2009.

14. T. Burchell, J. Strizak, and M. Williams, 2011, "AGC-1 Specimen Preirradiation Data Report," ORNL/TM-2010/285, Oak Ridge National Laboratory, August 2011.

15. T. Reed, 2012, "AGC-1 Individual Fluence, Temperature, and Load Calculation and Tabulation," ECAR-1943, Idaho National Laboratory, September 2012.

16. T. Reed, 2012, “AGC-1 As Run Thermal Results,” ECAR-1944, Idaho National Laboratory, September 2012.

17. L. Hull, 2012, “AGC-2 Irradiation Data Qualification Final Report,” INL/EXT-12-26248, July 2012. 
18. TFR-645, 2010, Advanced Graphite Capsule AGC-2 Experiment Test Train, Rev. 0, Idaho National Laboratory, July 2010.

19. D. Swank, 2010, “AGC-2 Graphite Preirradiation Data Package,” INL/EXT-10-19588, Rev. 0, Idaho National Laboratory, August 2010.

20. R. G. Ambrosek, “Thermal Projections for AGC-2,” ECAR-1161, Idaho National Laboratory.

21. M. Davenport, 2010, Engineering Work Instructions for Assembling the AGC-2 Experiment, Quality Assurance Record: QA\# 137268, October 2010.

22. W. Windes, 2014, “AGC-2 Disassembly Report,” INL/EXT-14-32060, May 2014.

23. D. Swank, AGC-2 Graphite Specimen Postirradiation Characterization Plan," PLN-4657, Rev. 0, Idaho National Laboratory,

24. J. E. Brocklehurst and R. G. Brown, "Constant Stress Irradiation Creep Experiments on Graphite In Br-2" Carbon, Volume 7, Issue 4, August 1969.

25. W. J. Gray, "Constant Stress Irradiation-Induced Compressive Creep of Graphite at High Fluences," Carbon, Volume 11, Issue 4, August 1973.

26. ASTM 769-09, Standard Test Method for Sonic Velocity in Manufactured Carbon and Graphite Materials for Use in Obtaining Young's Modulus, American Society for Testing and Materials International, 2009

27. ASTM-769-98 (reapproved 2005), Standard Test Method for Sonic Velocity in Manufactured Carbon and Graphite Materials for Use in Obtaining Young's Modulus, American Society for Testing and Materials International, 2005 
Appendix A

\section{Data Plots}




\section{Appendix A AGC-2 Post Irradiation Data Plots}

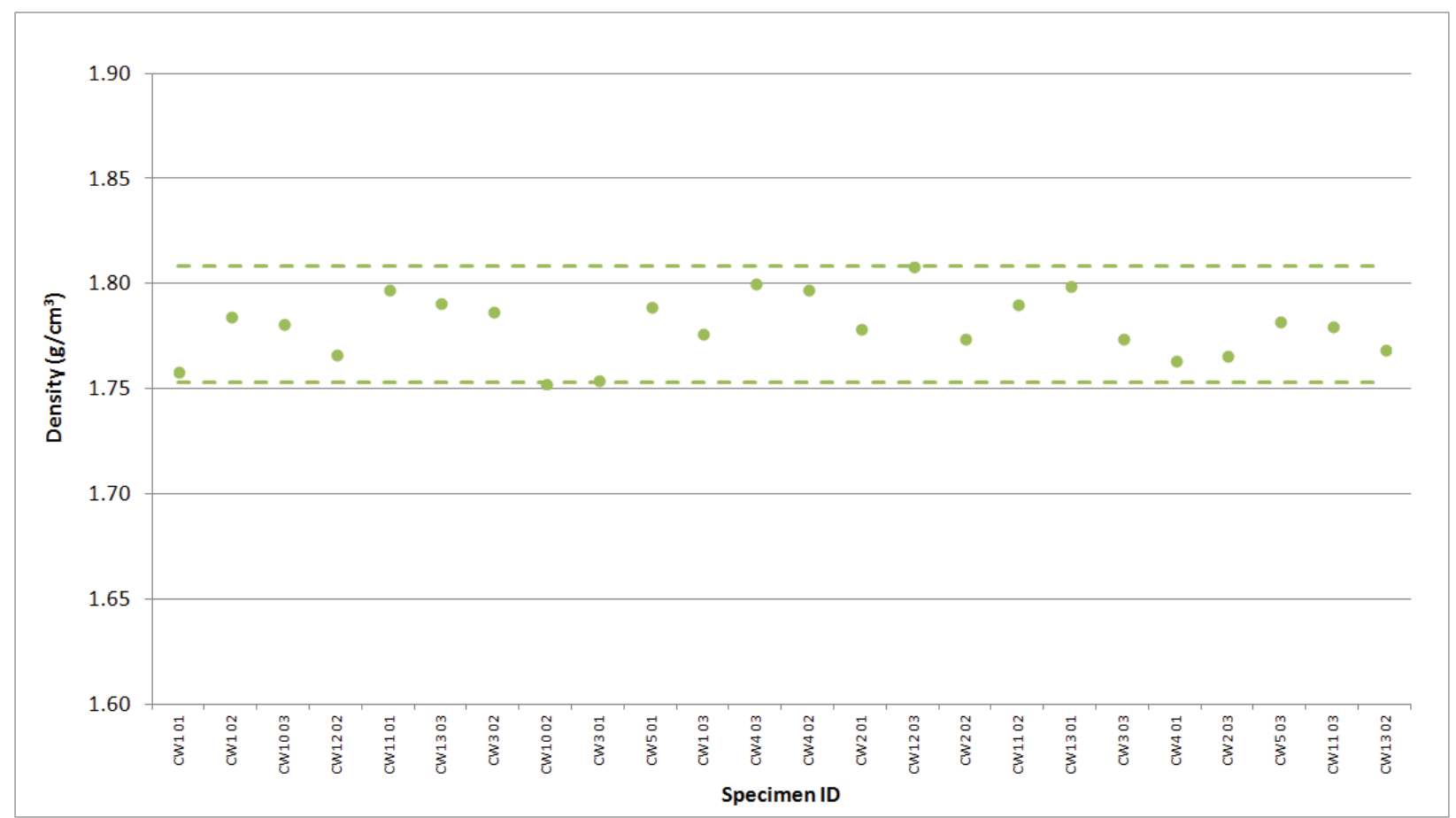

Figure A-1. H-451 Creep Density.

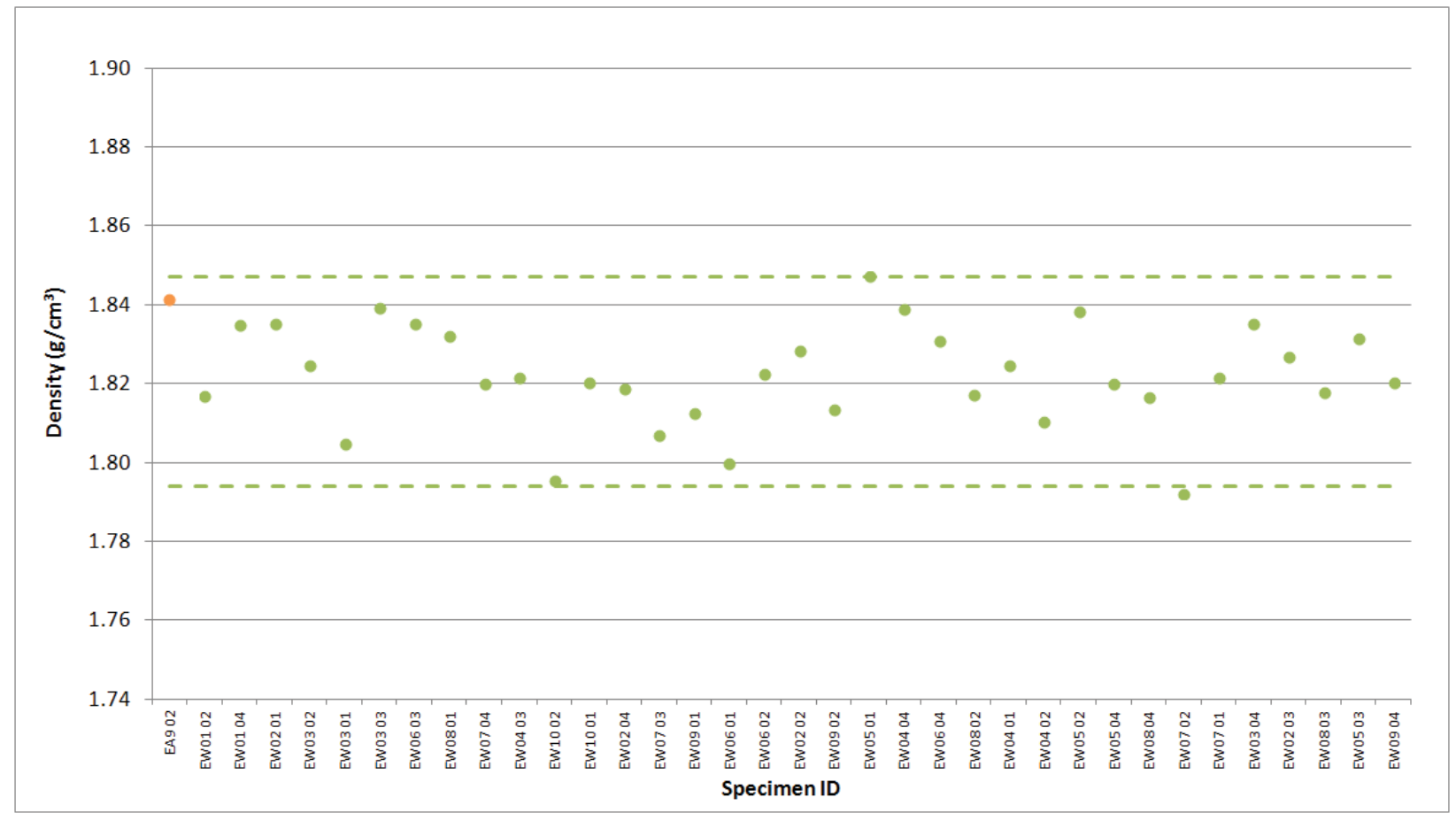

Figure A-2. IG-110 Creep Density. 




Figure A-3. IG-430 Creep Density.

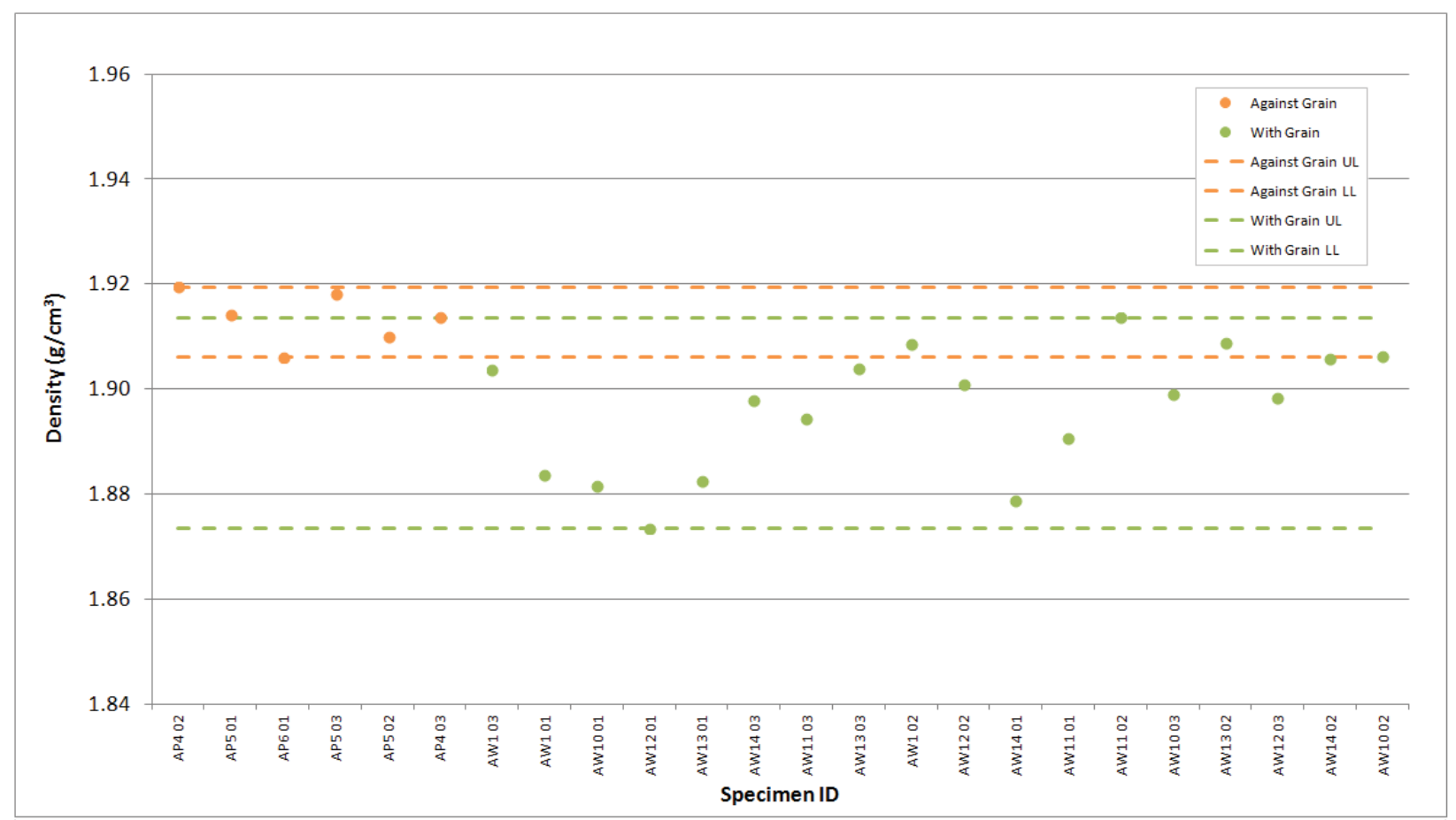

Figure A-4. NBG-17 Creep Density. 


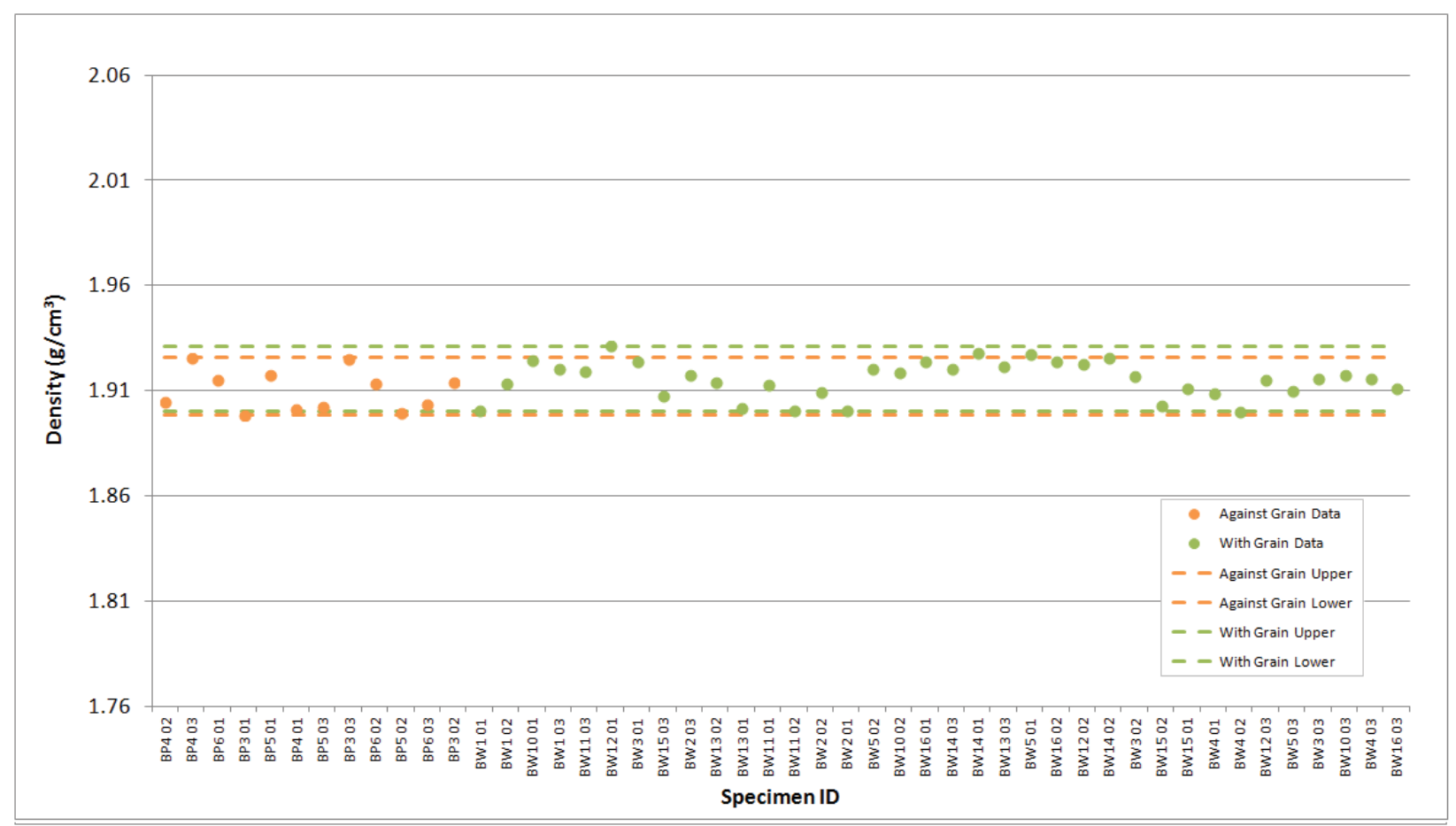

Figure A-5. NBG-18 Creep Density.

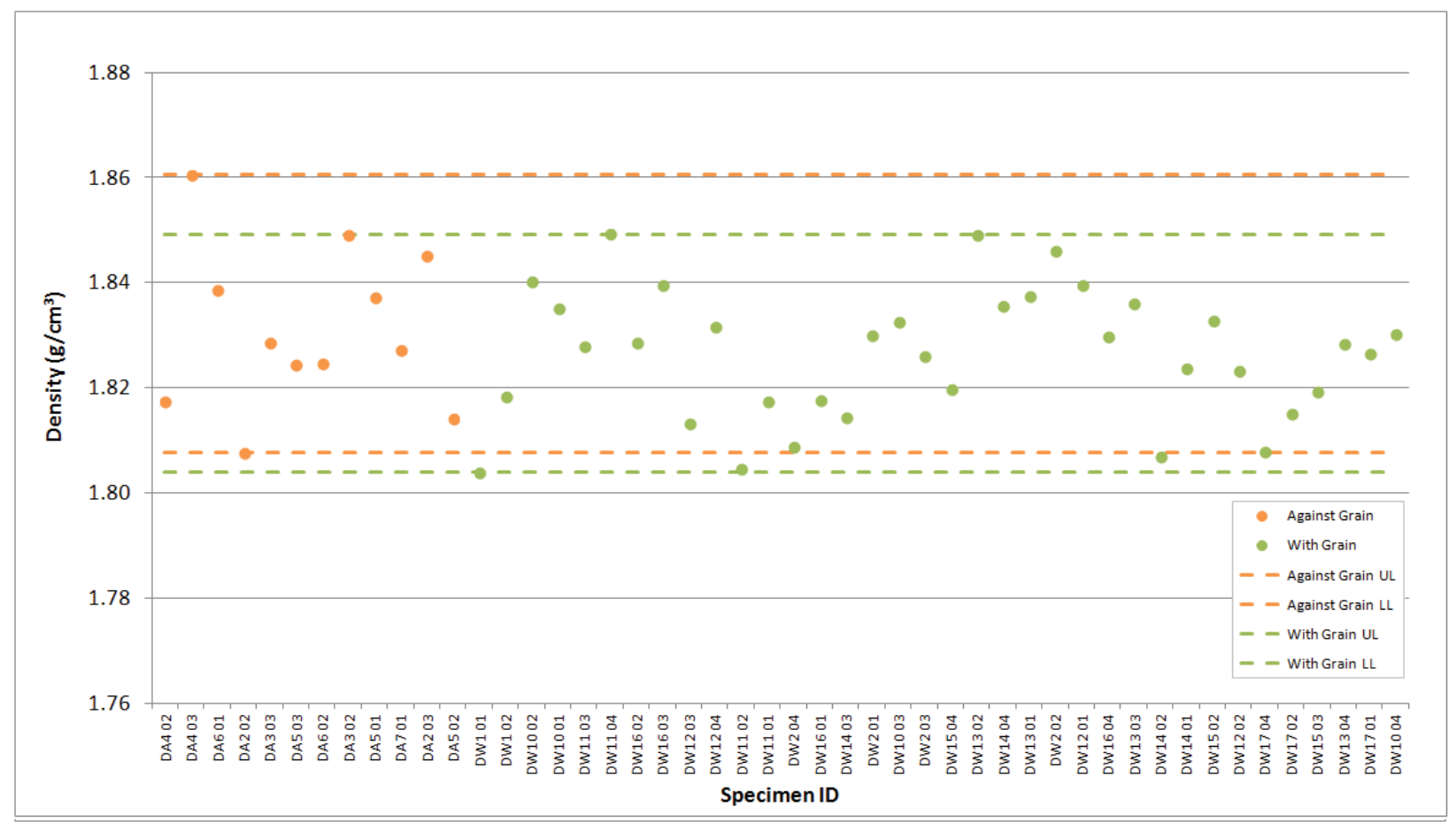

Figure A-6. PCEA Creep Density. 


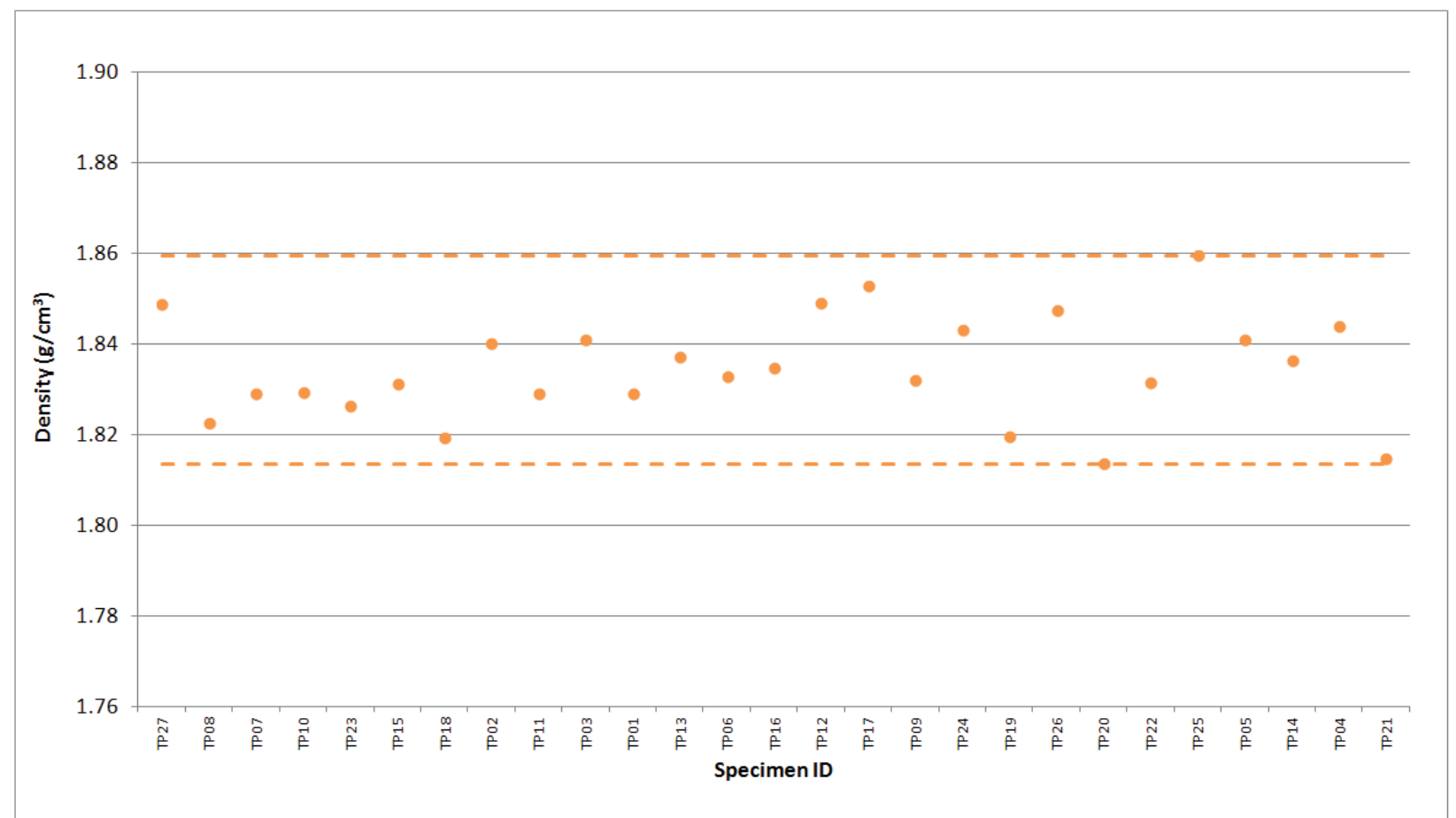

Figure A-7. 2114 Piggyback Density.

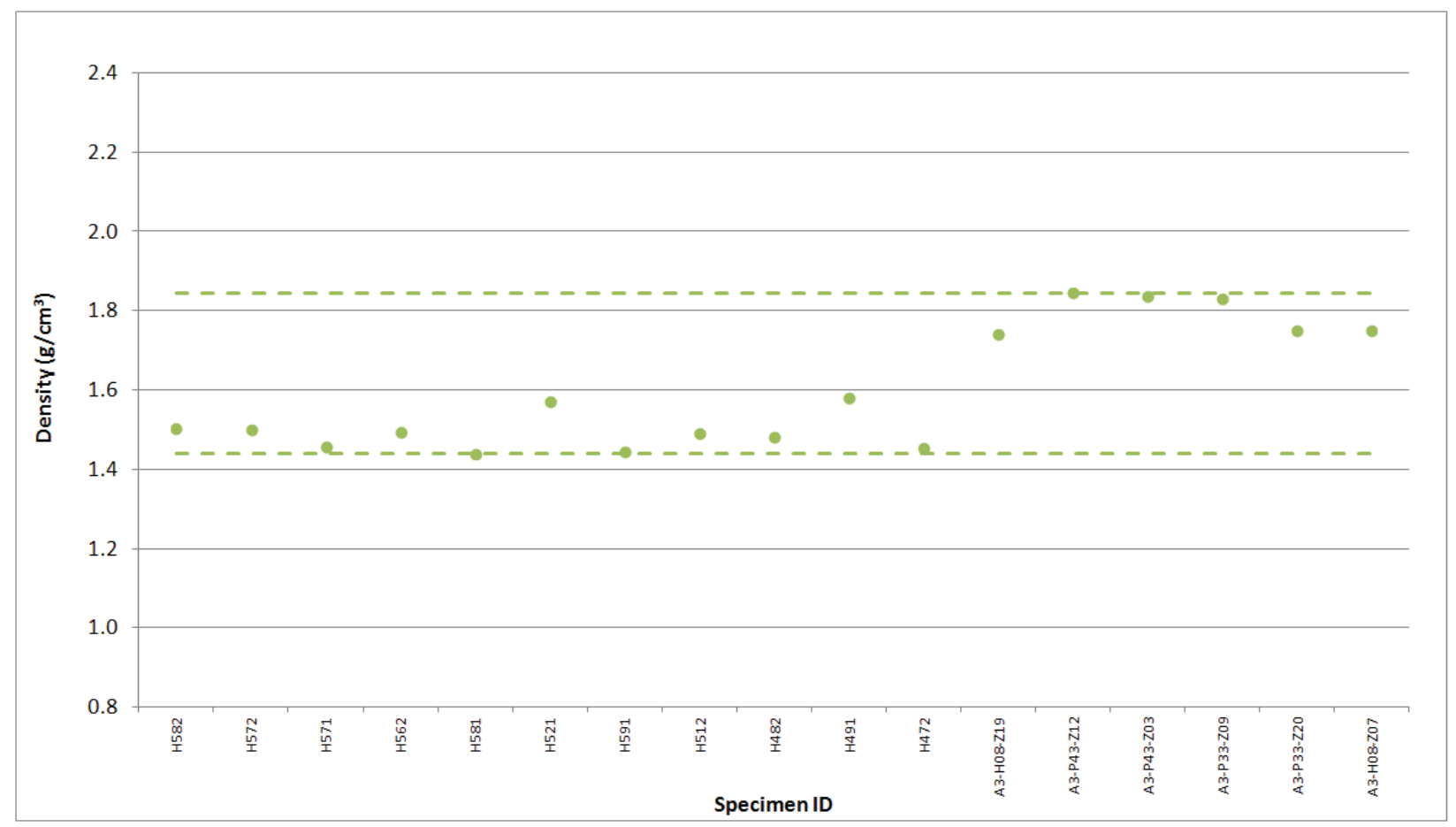

Figure A-8. A3 Piggyback Density. 


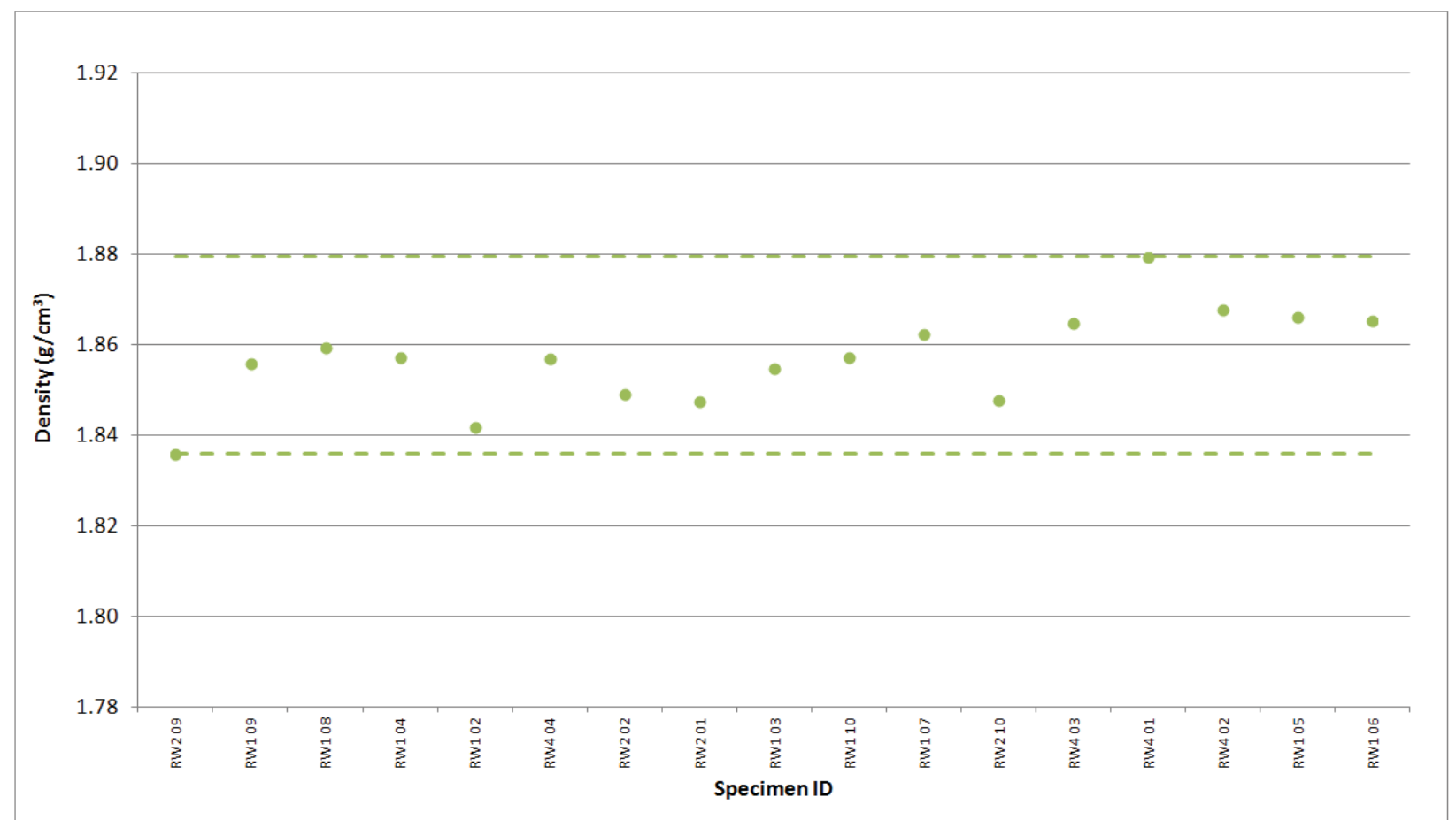

Figure A-9. BAN Piggyback Density.

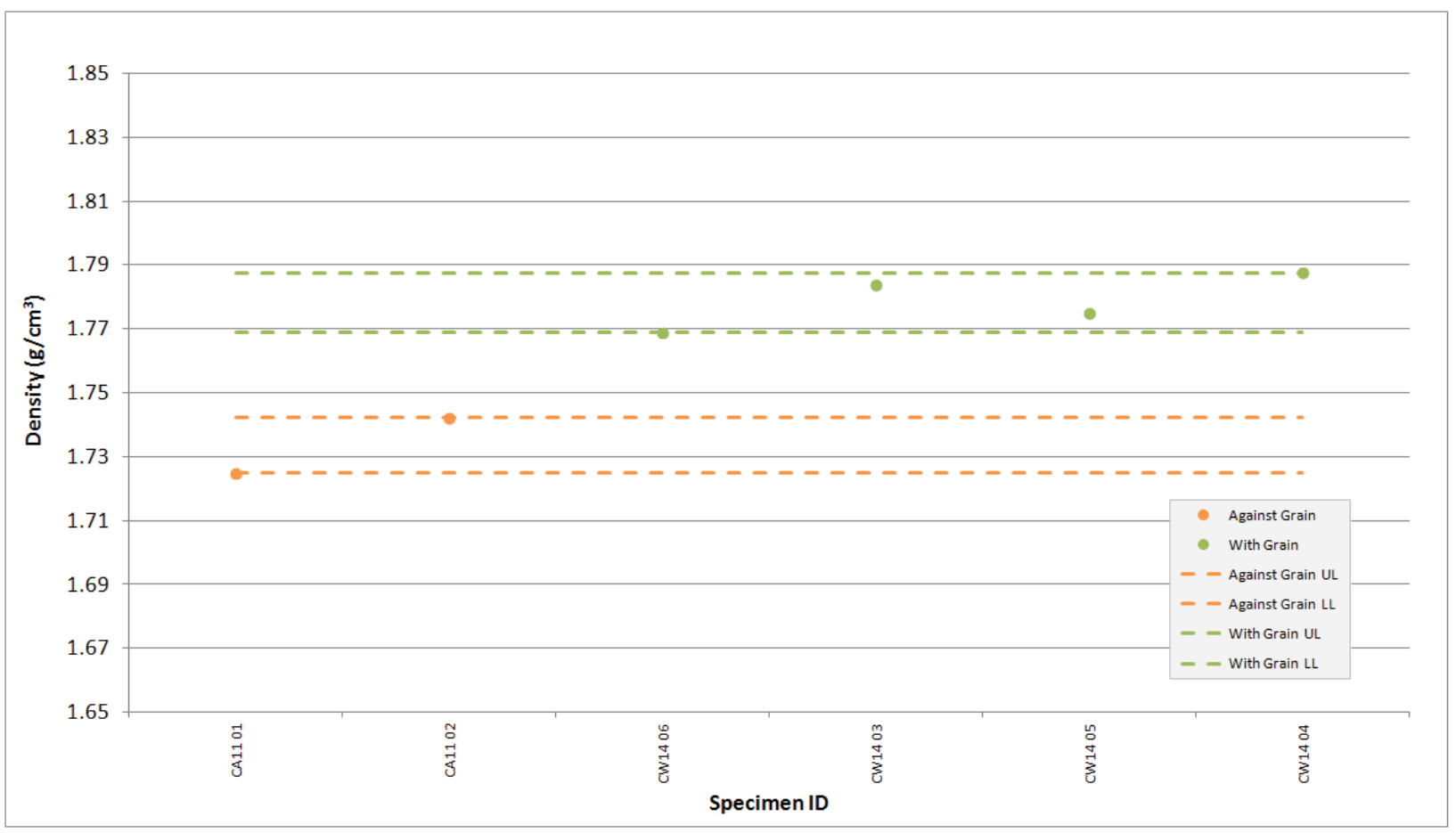

Figure A-10. H-451 Piggyback Density. 


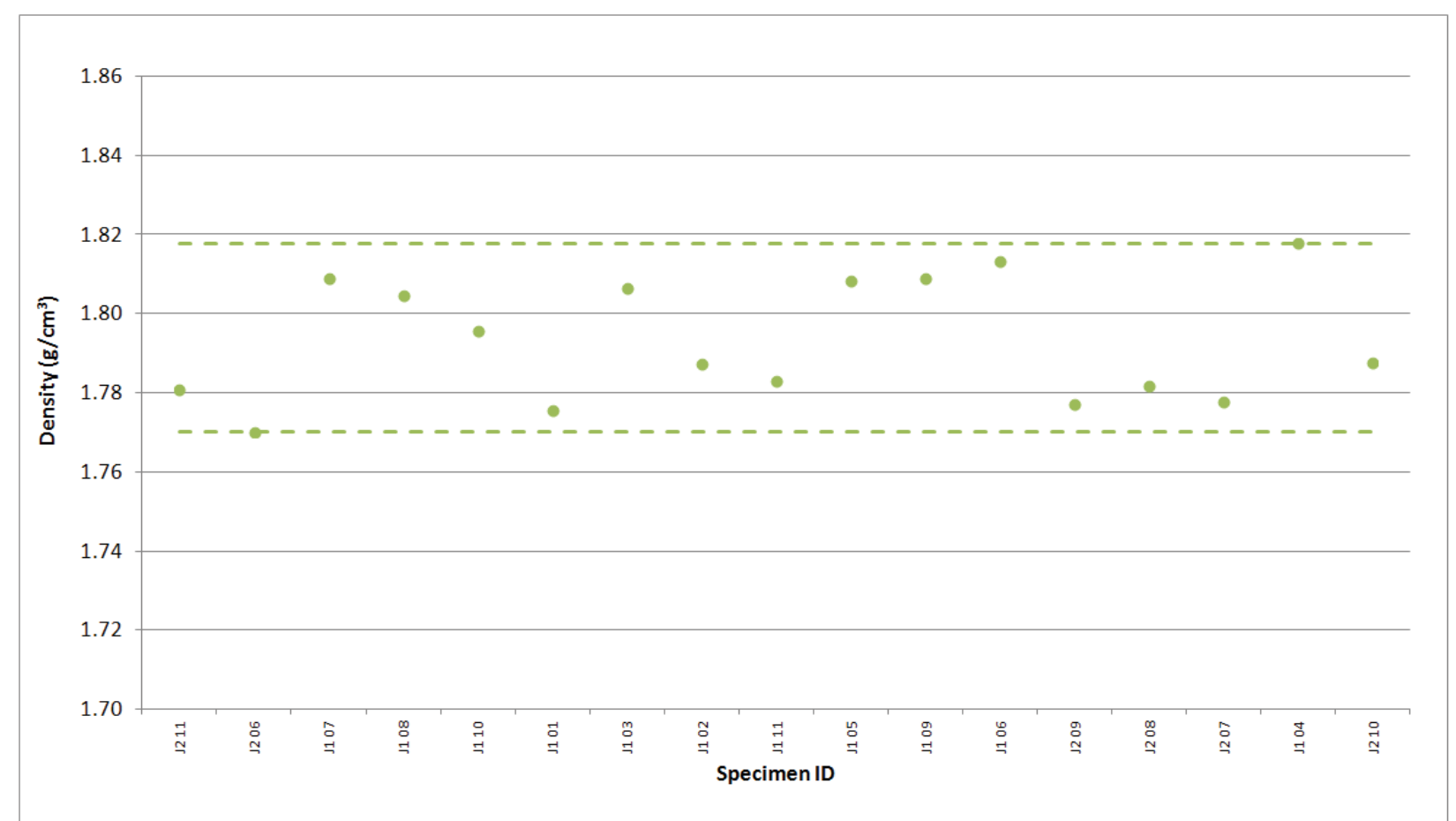

Figure A-11. HLM Piggyback Density.

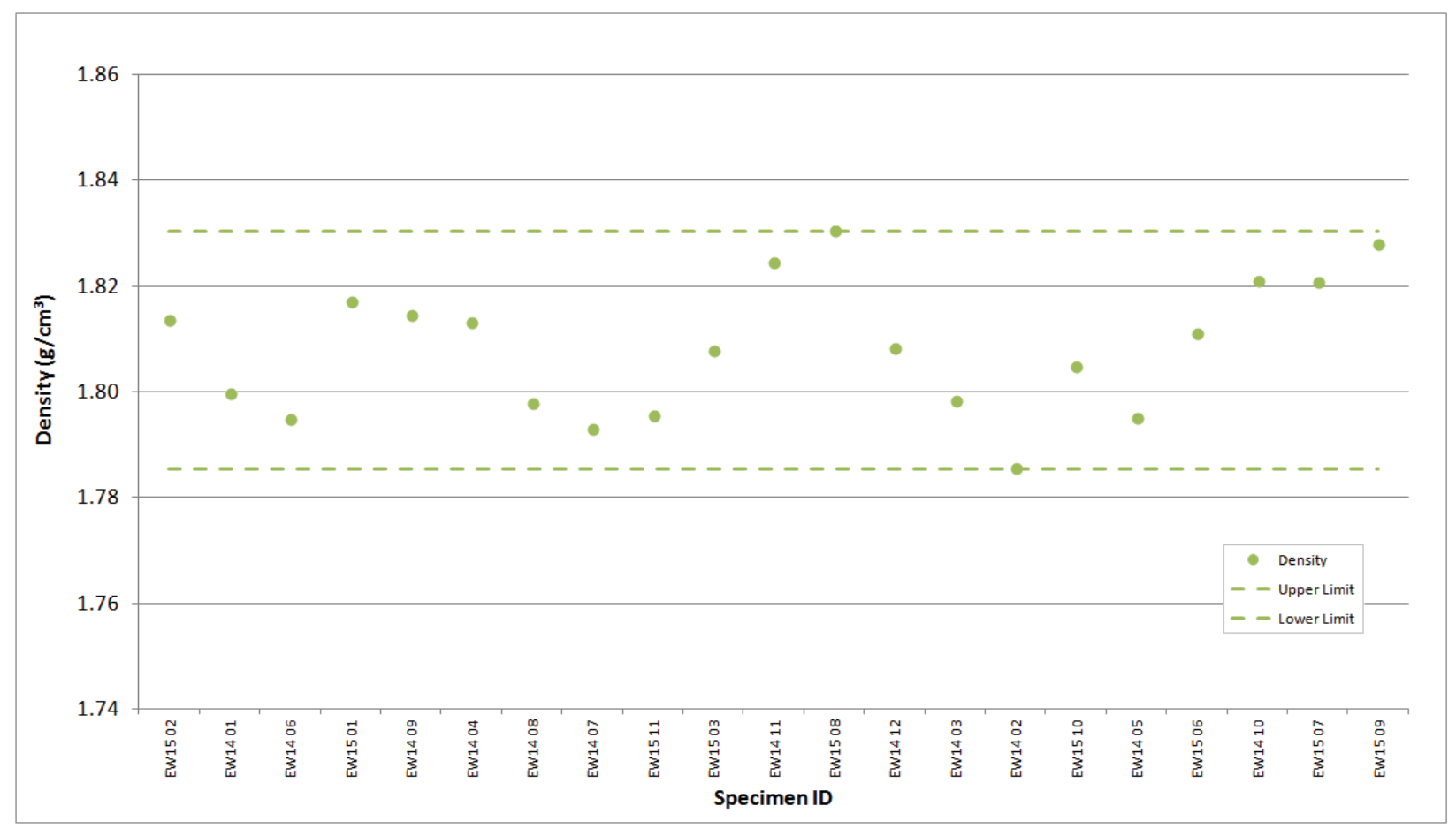

Figure A-12. IG-110 Piggyback Density. 


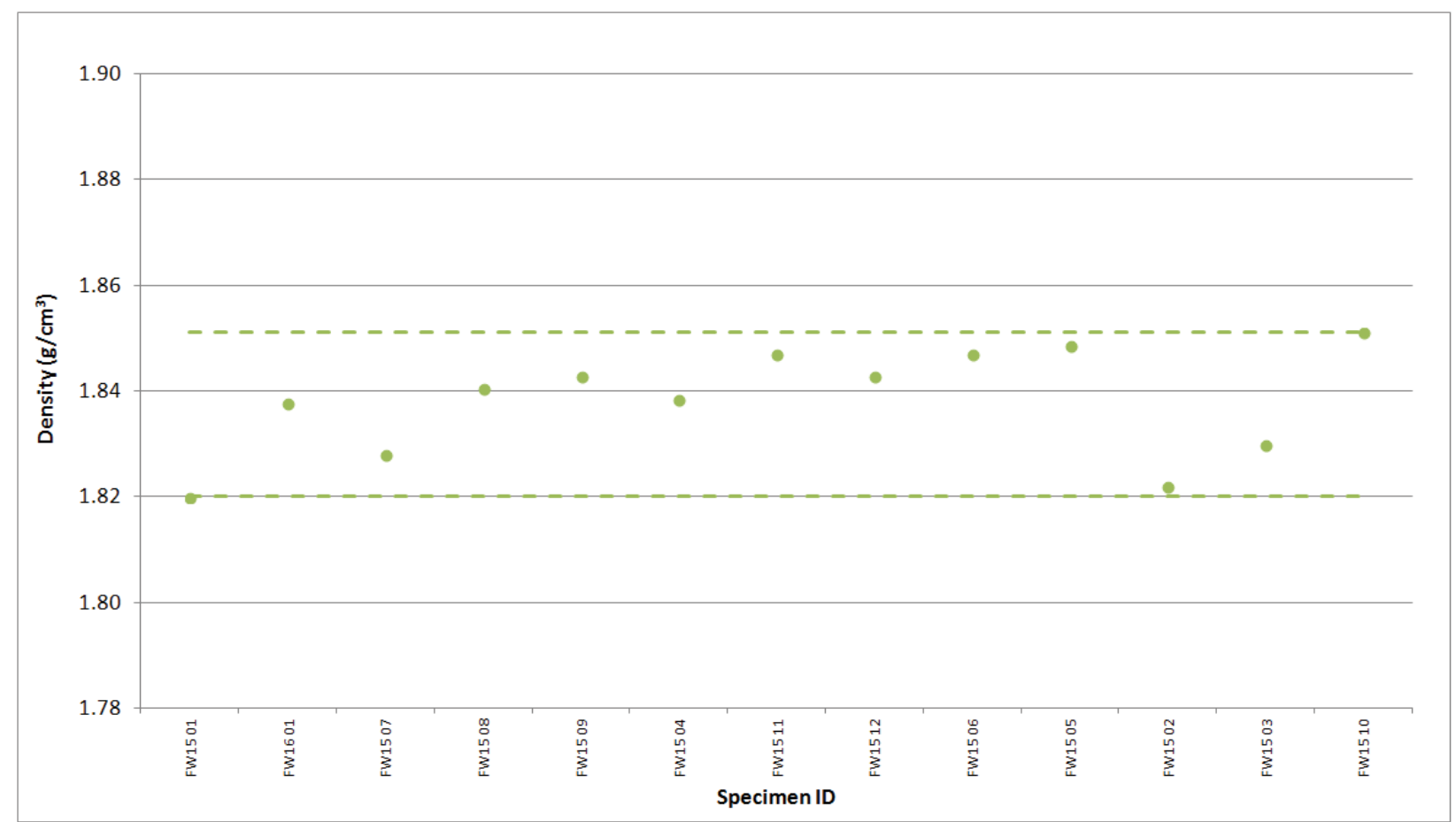

Figure A-13. IG-430 Piggyback Density.

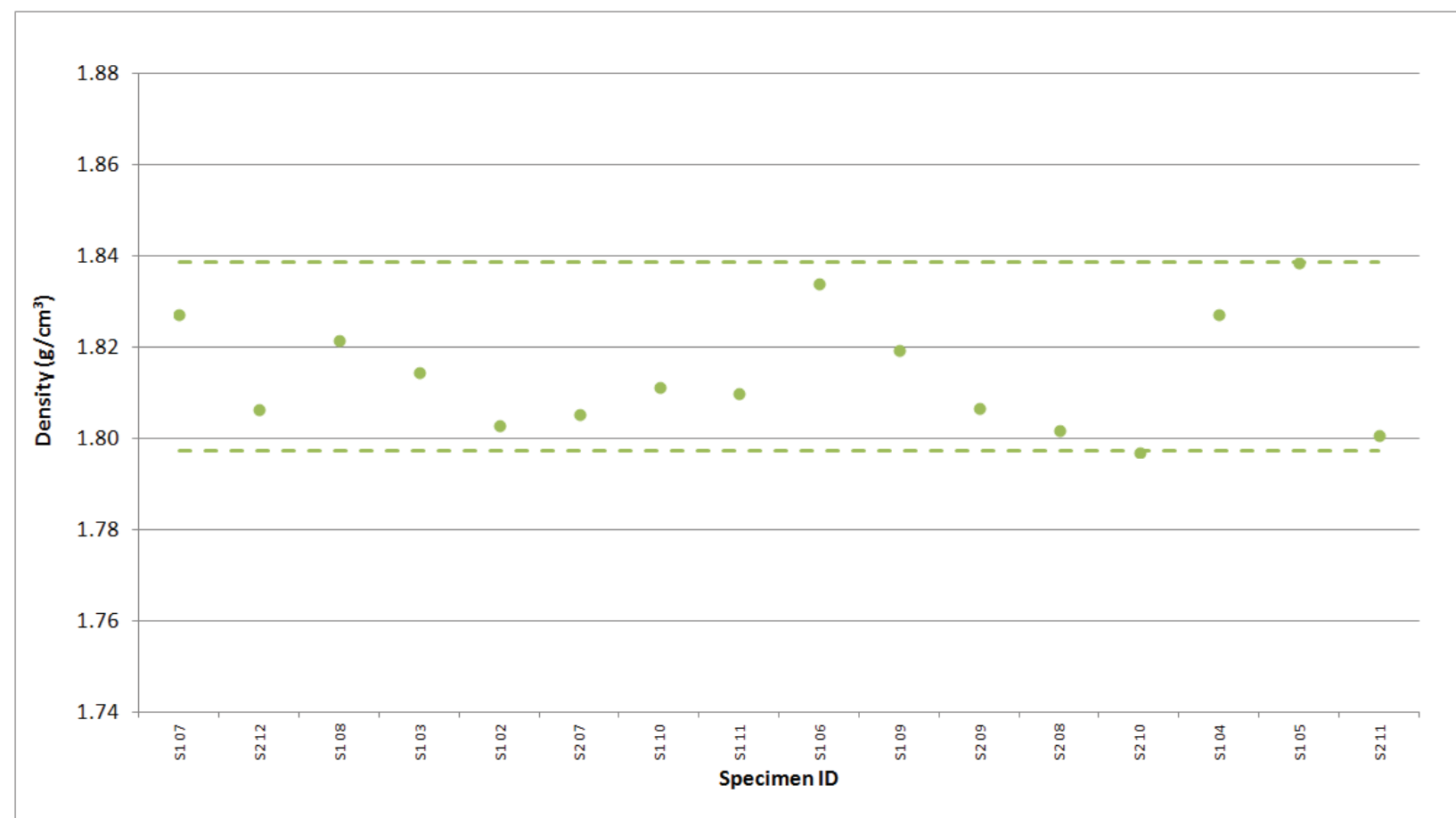

Figure A-14. NBG-10 Piggyback Density. 


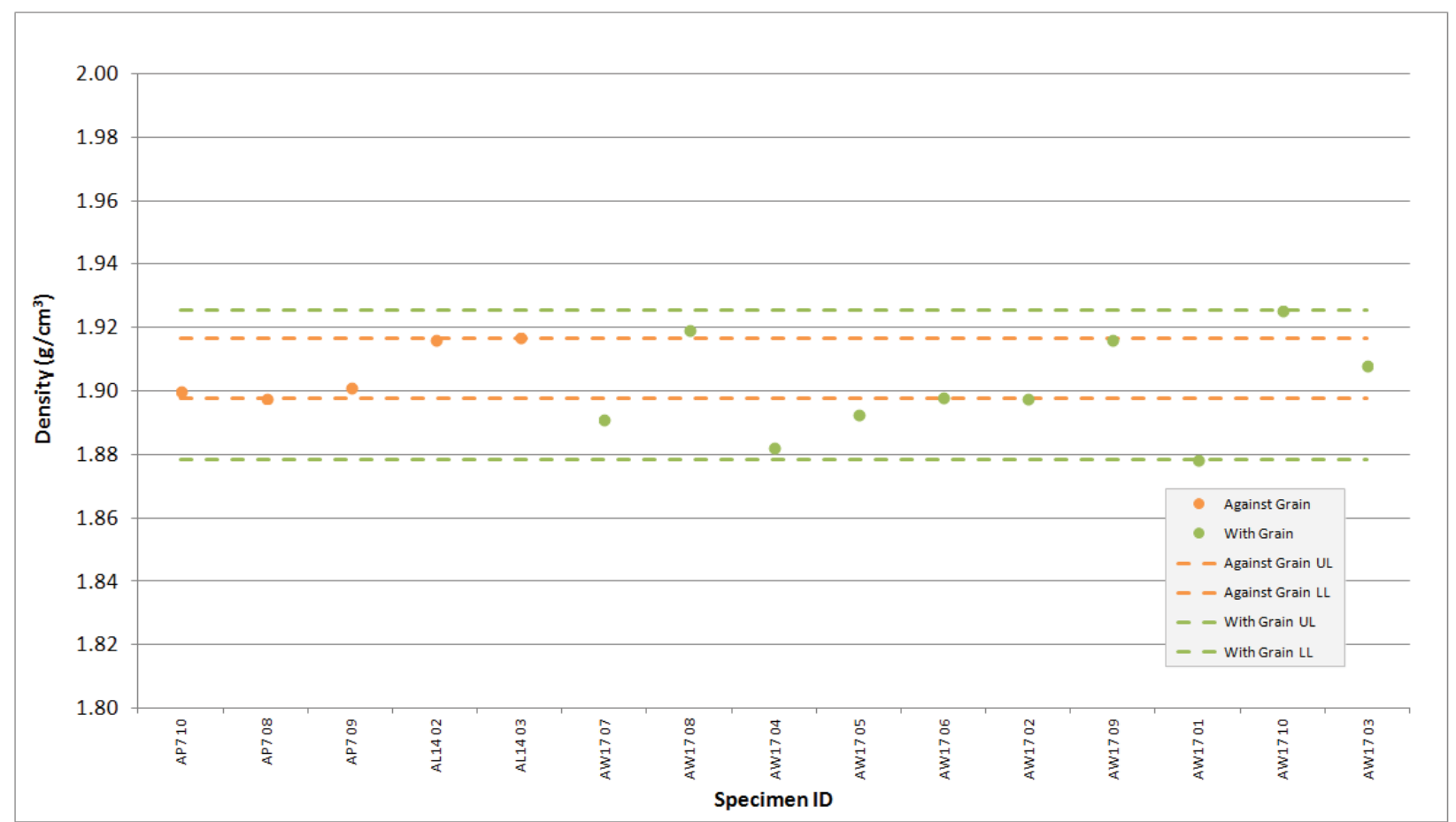

Figure A-15. NBG-17 Piggyback Density.

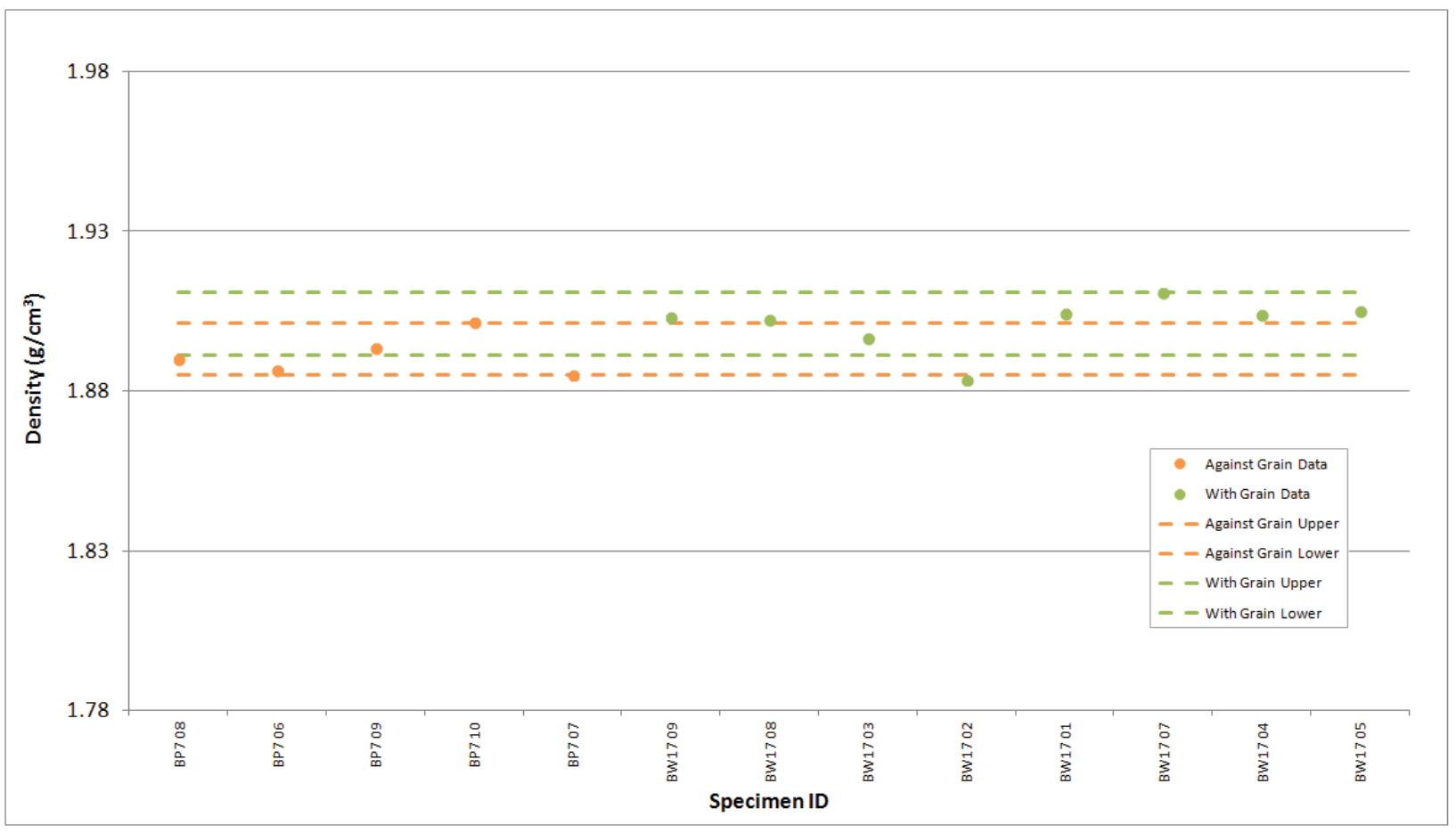

Figure A-16. NBG-18 Piggyback Density. 


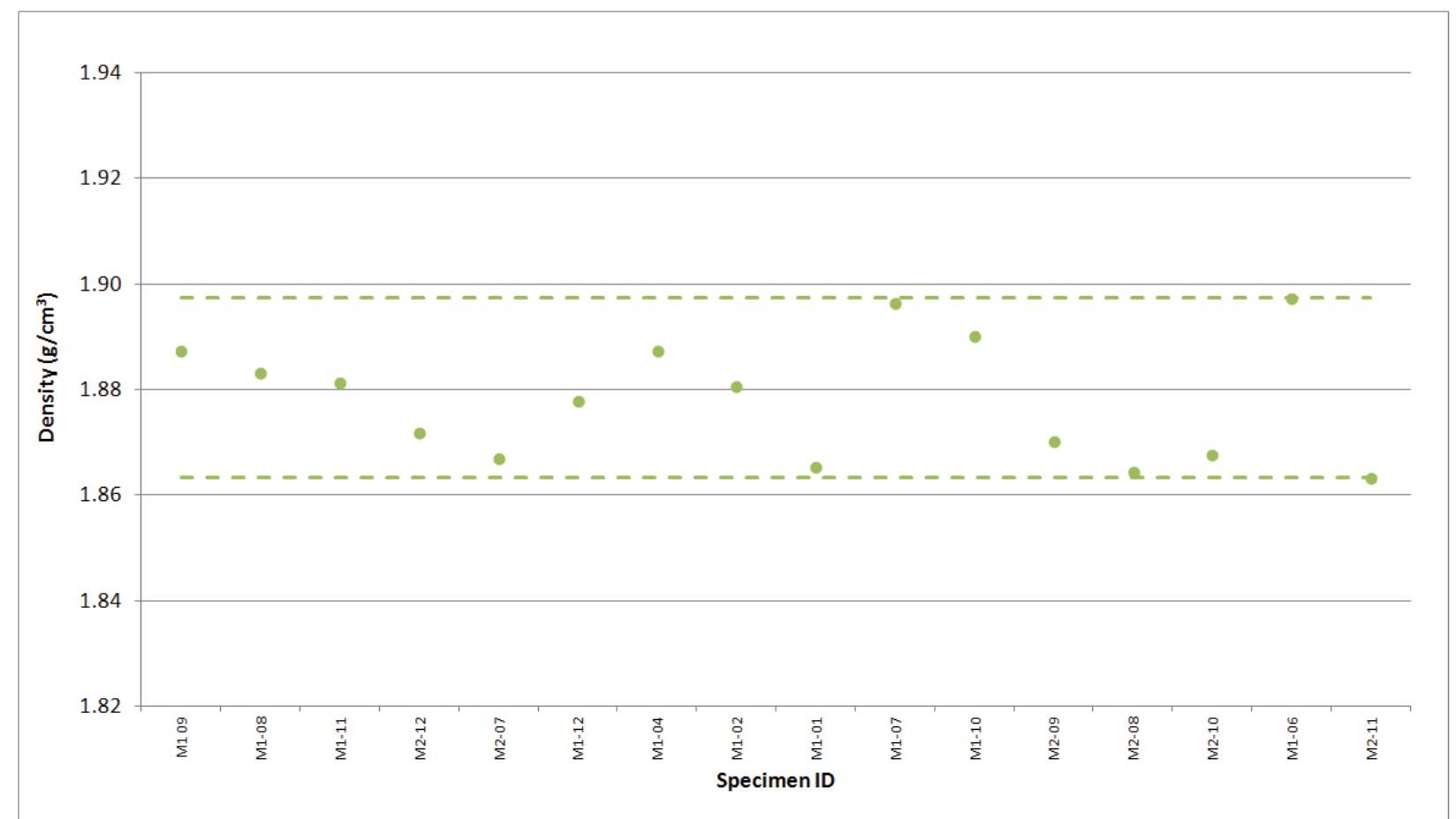

Figure A-17. NBG-25 Piggyback Density.

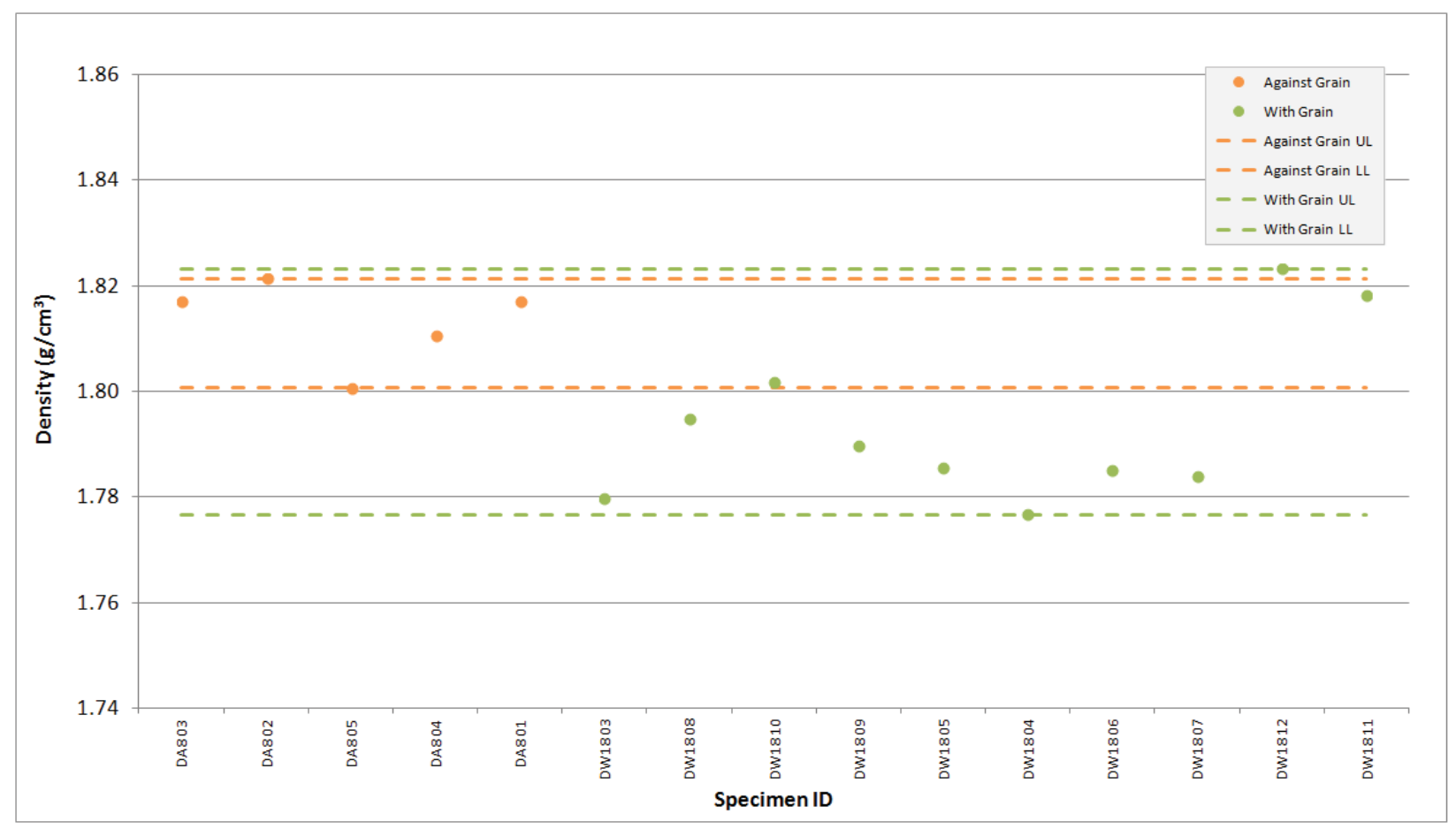

Figure A-18. PCEA Piggyback Density. 


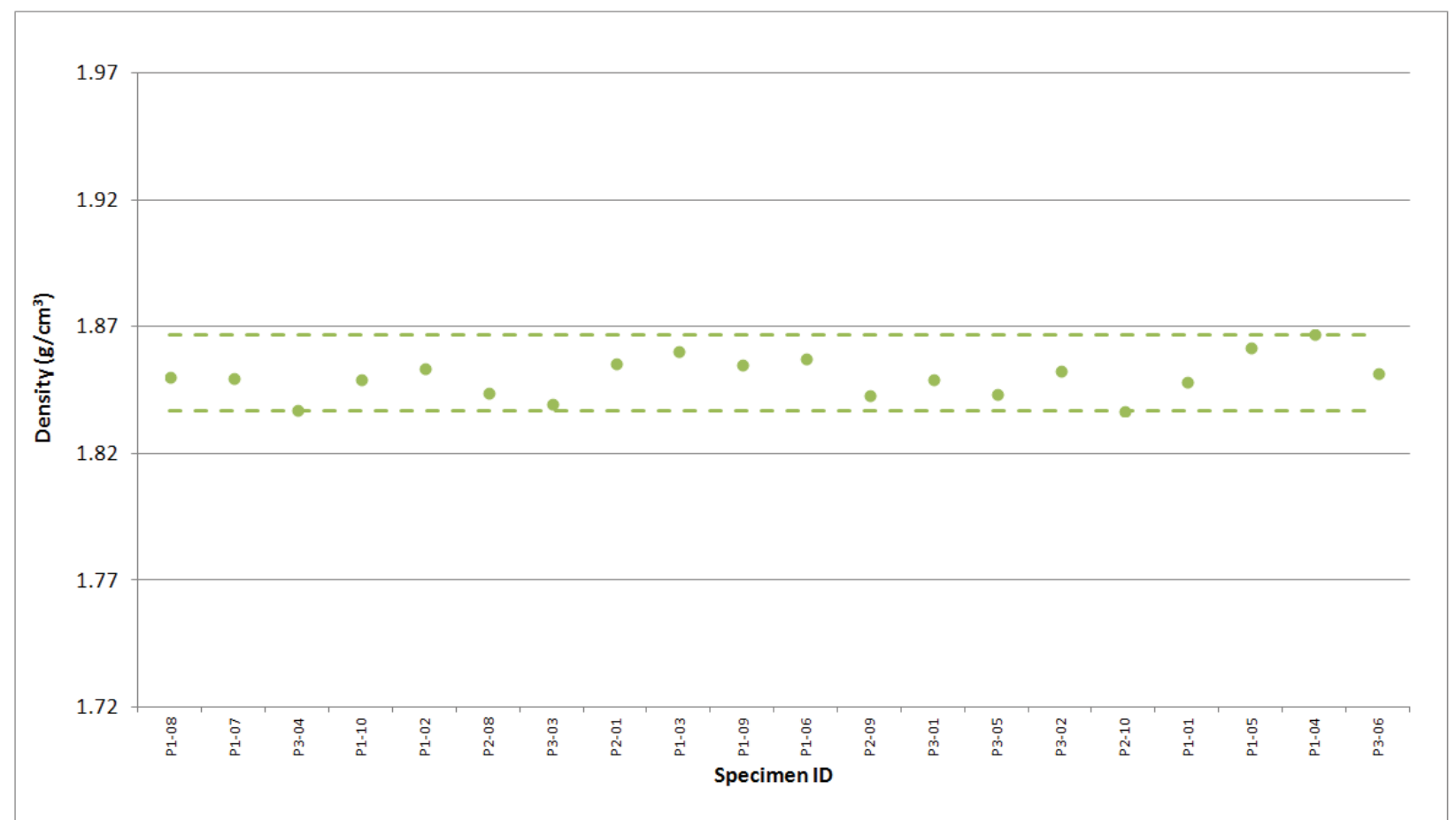

Figure A-19. PCIB Piggyback Density.



Figure A-20. PGX Piggyback Density. 


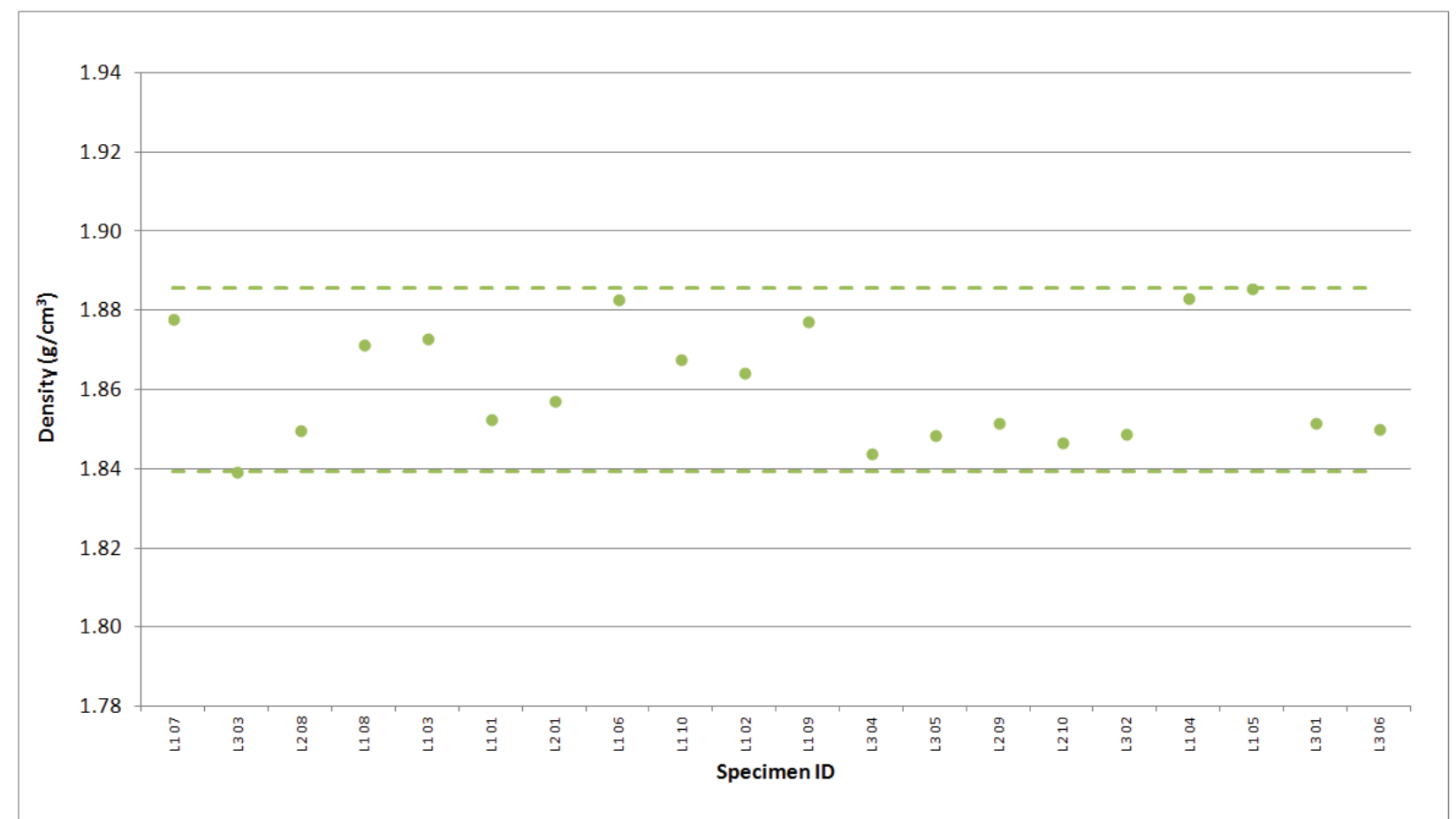

Figure A-21. PPEA Piggyback Density.

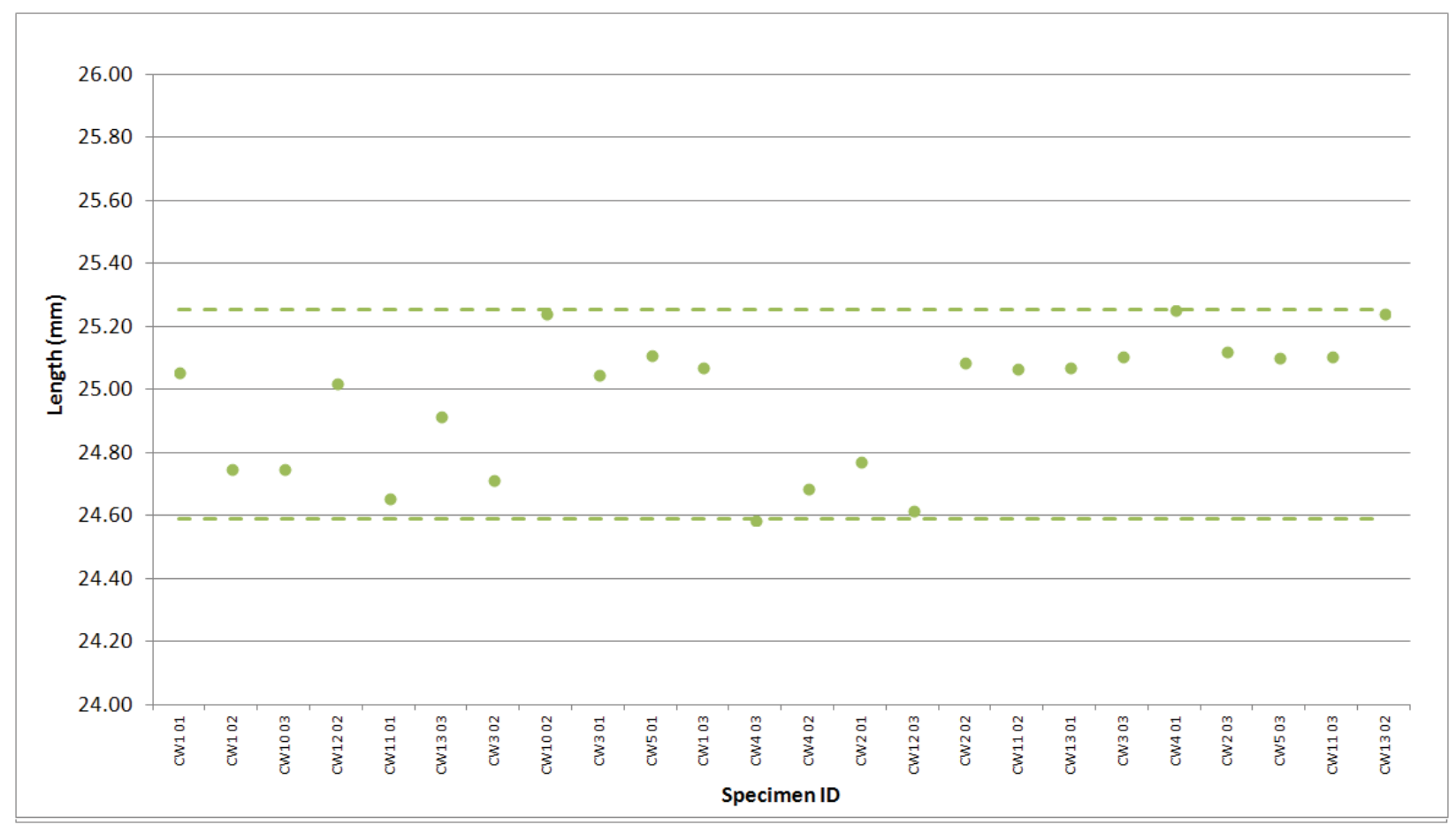

Figure A-22. H-451 Creep Length. 


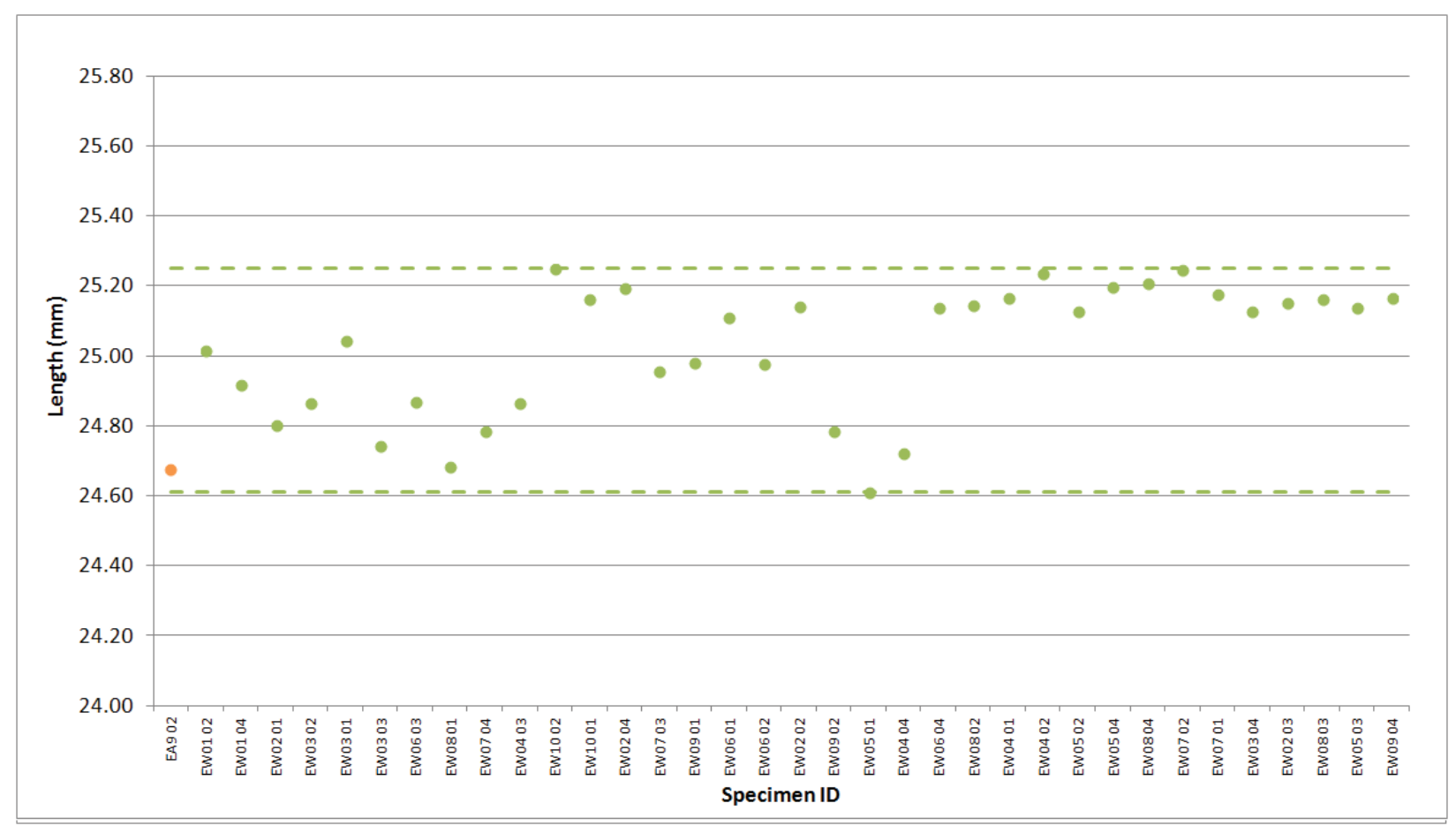

Figure A-23. IG-110 Creep Length.

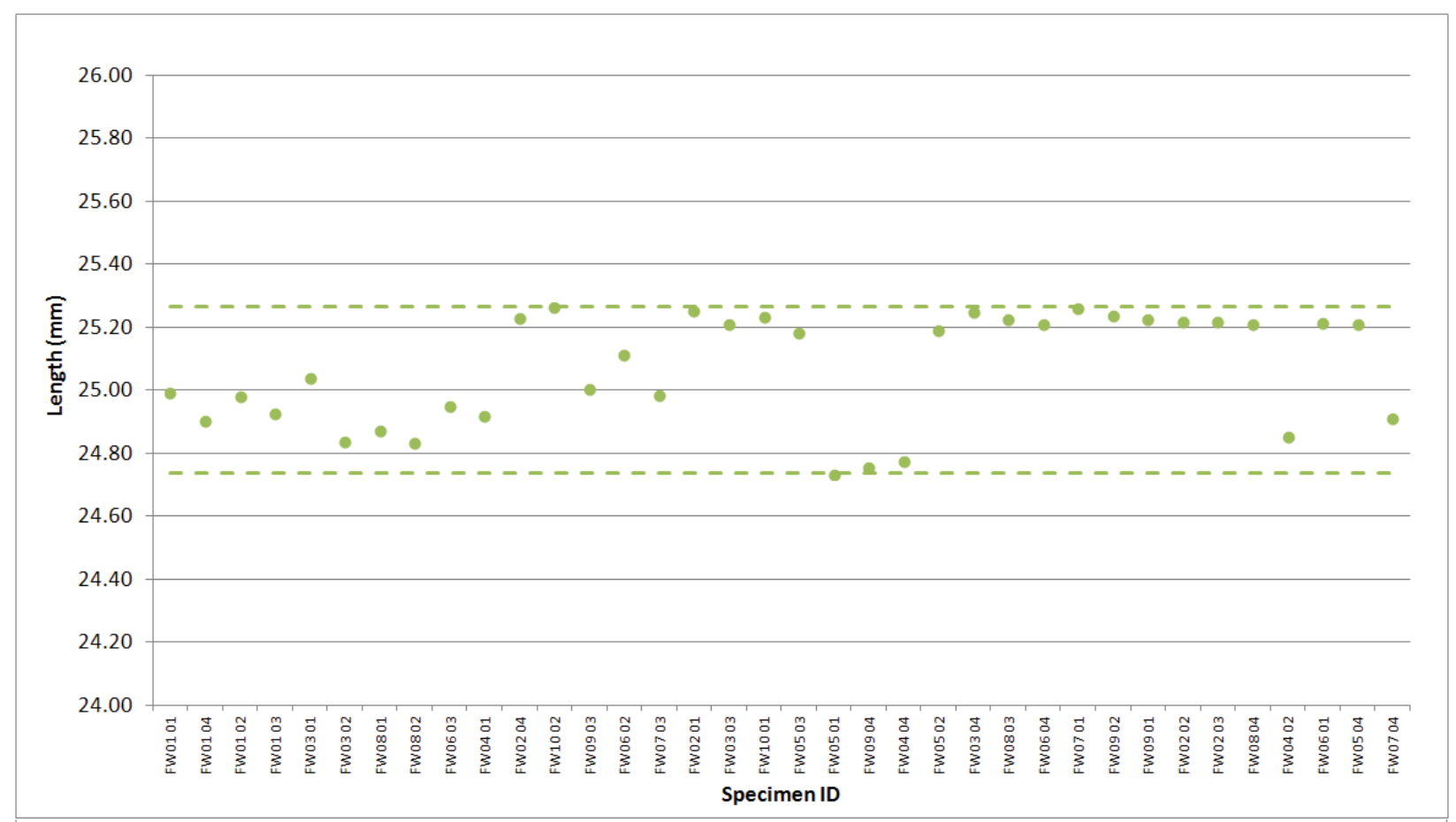

Figure A-24. IG-430 Creep Length. 


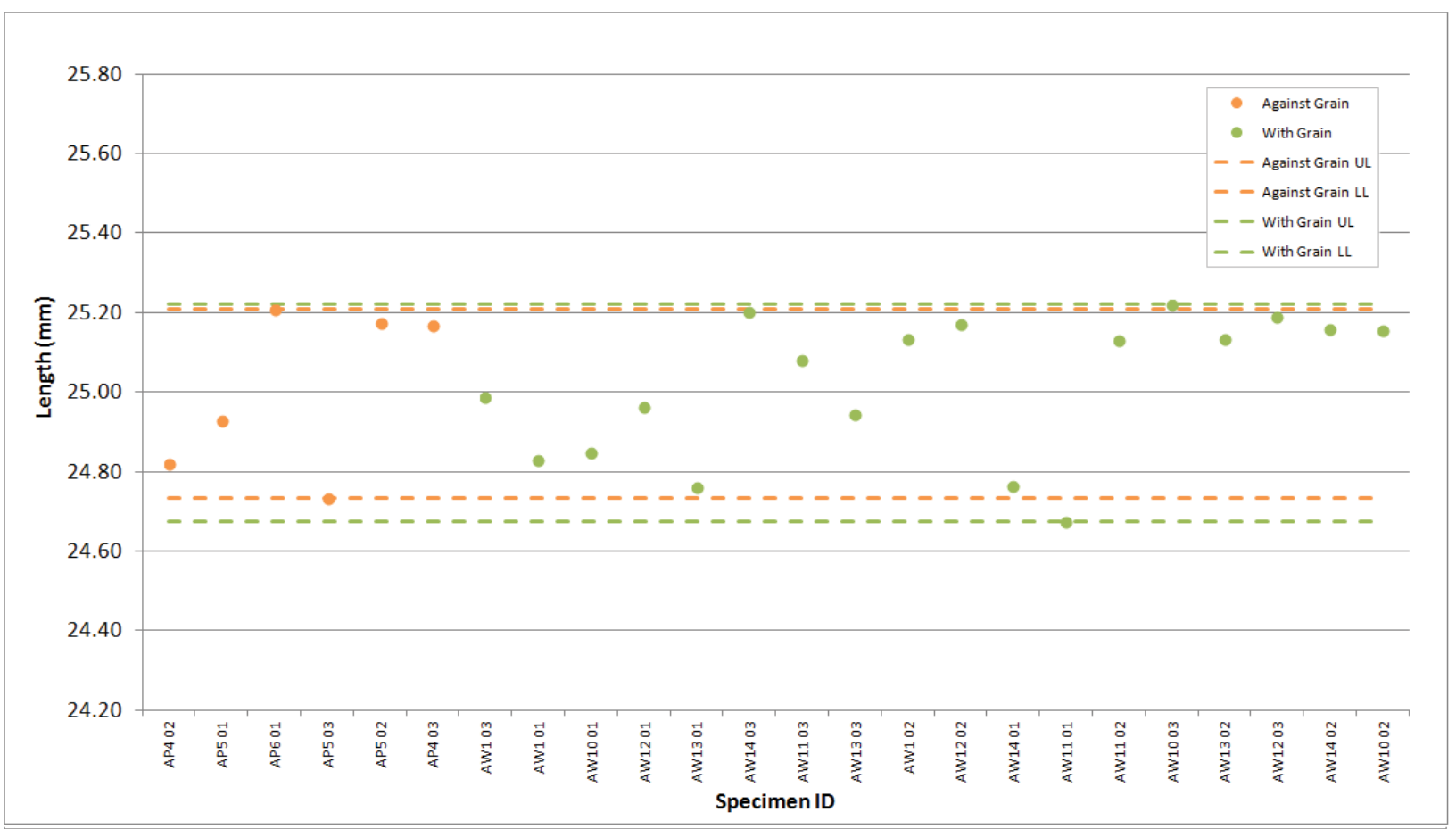

Figure A-25. NBG-17 Creep Length.

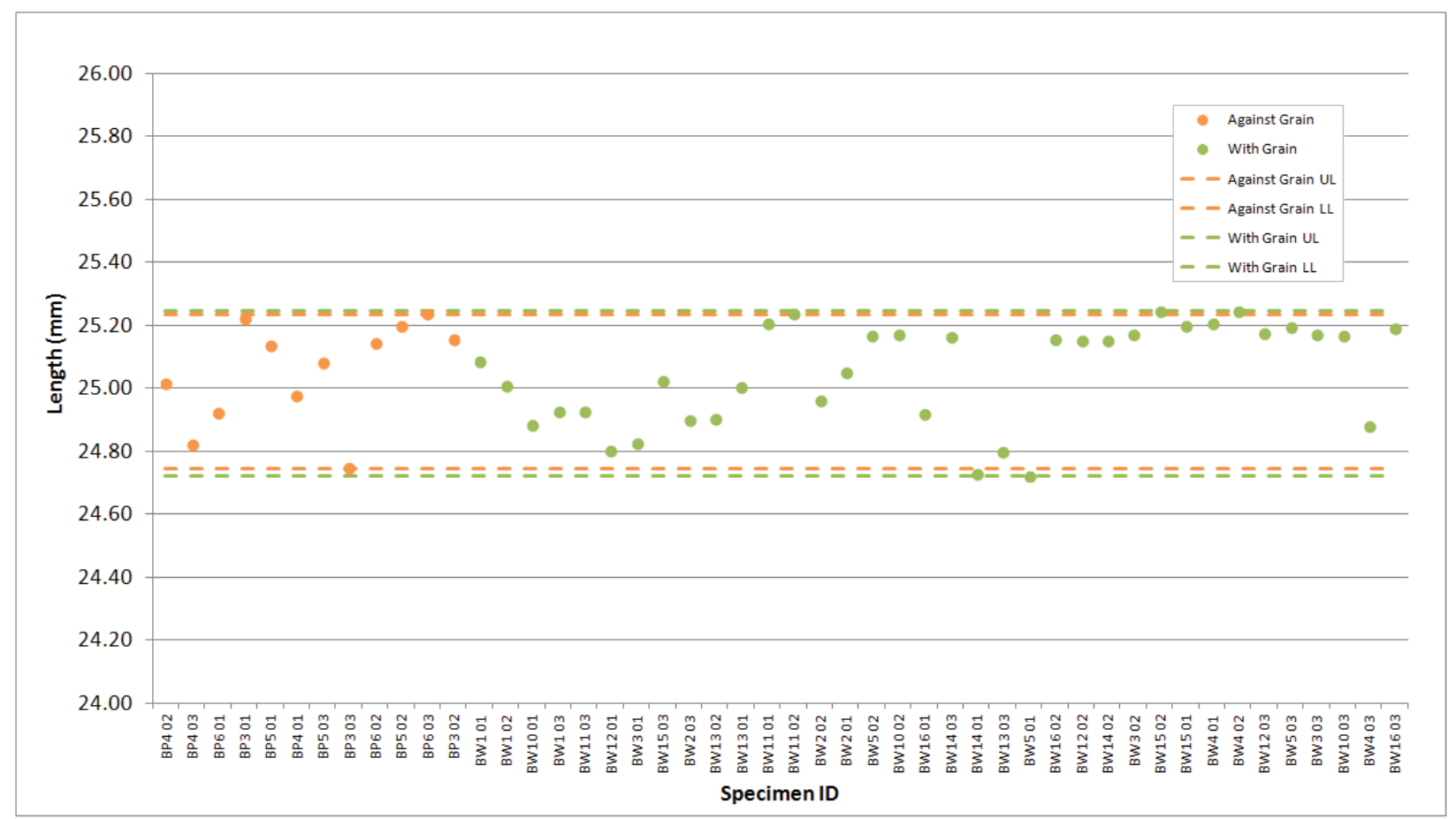

Figure A-26. NBG-18 Creep Length. 




Figure A-27. PCEA Creep Length.

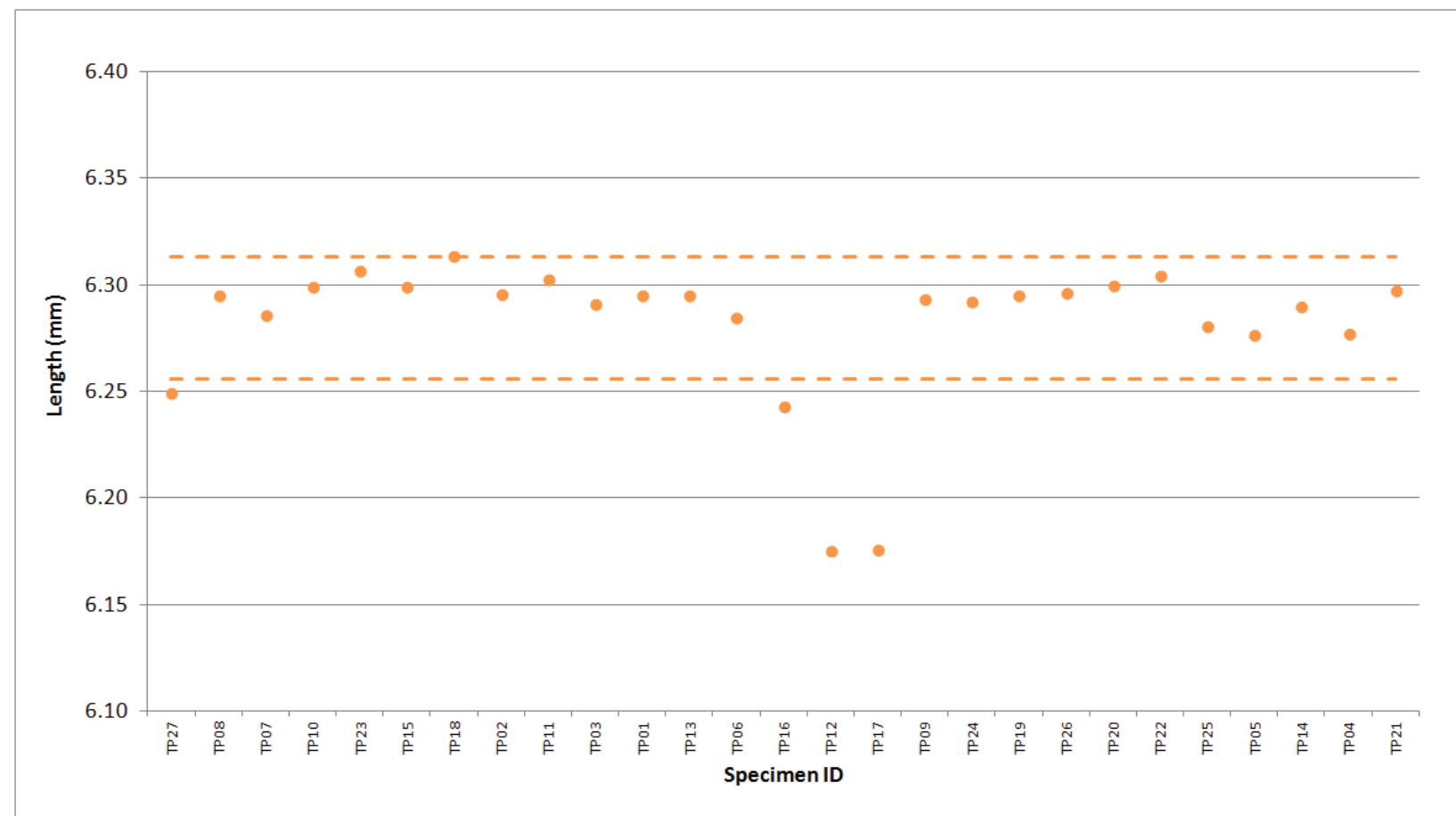

Figure A-28. 2114 Piggyback Length. 


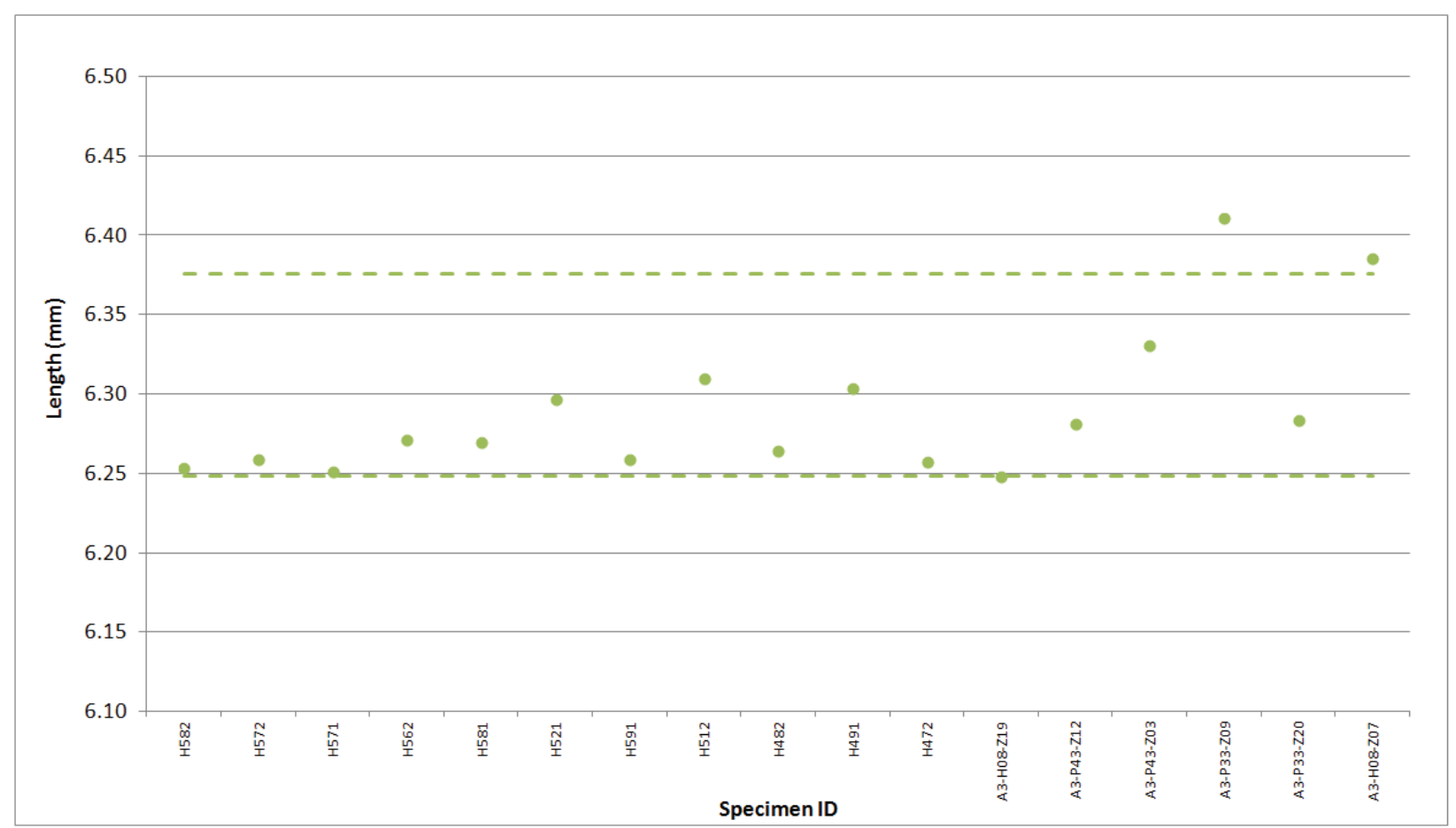

Figure A-29. A3 Piggyback Length.



Figure A-30. BAN Piggyback Length. 


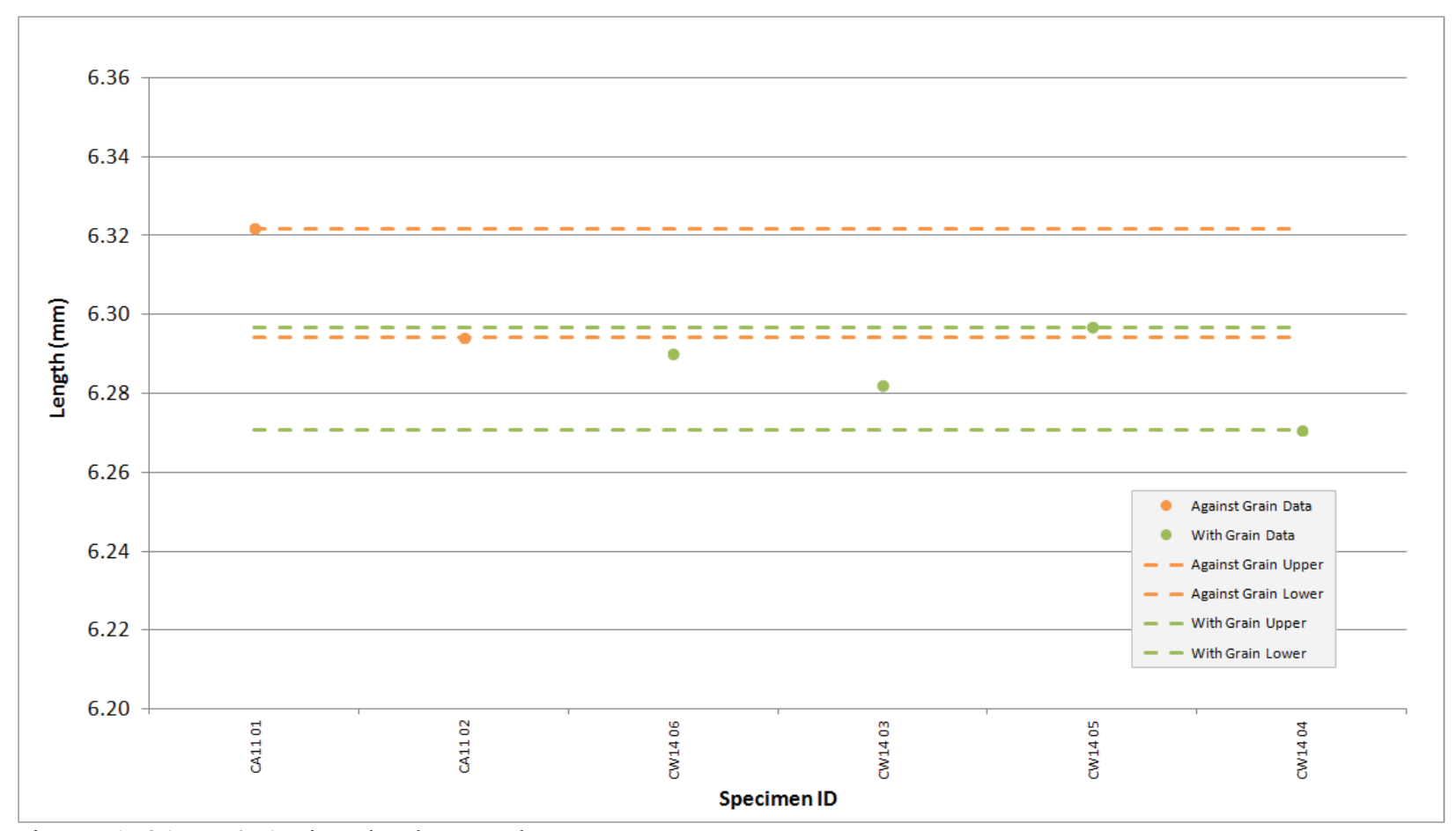

Figure A-31. H-451 Piggyback Length.

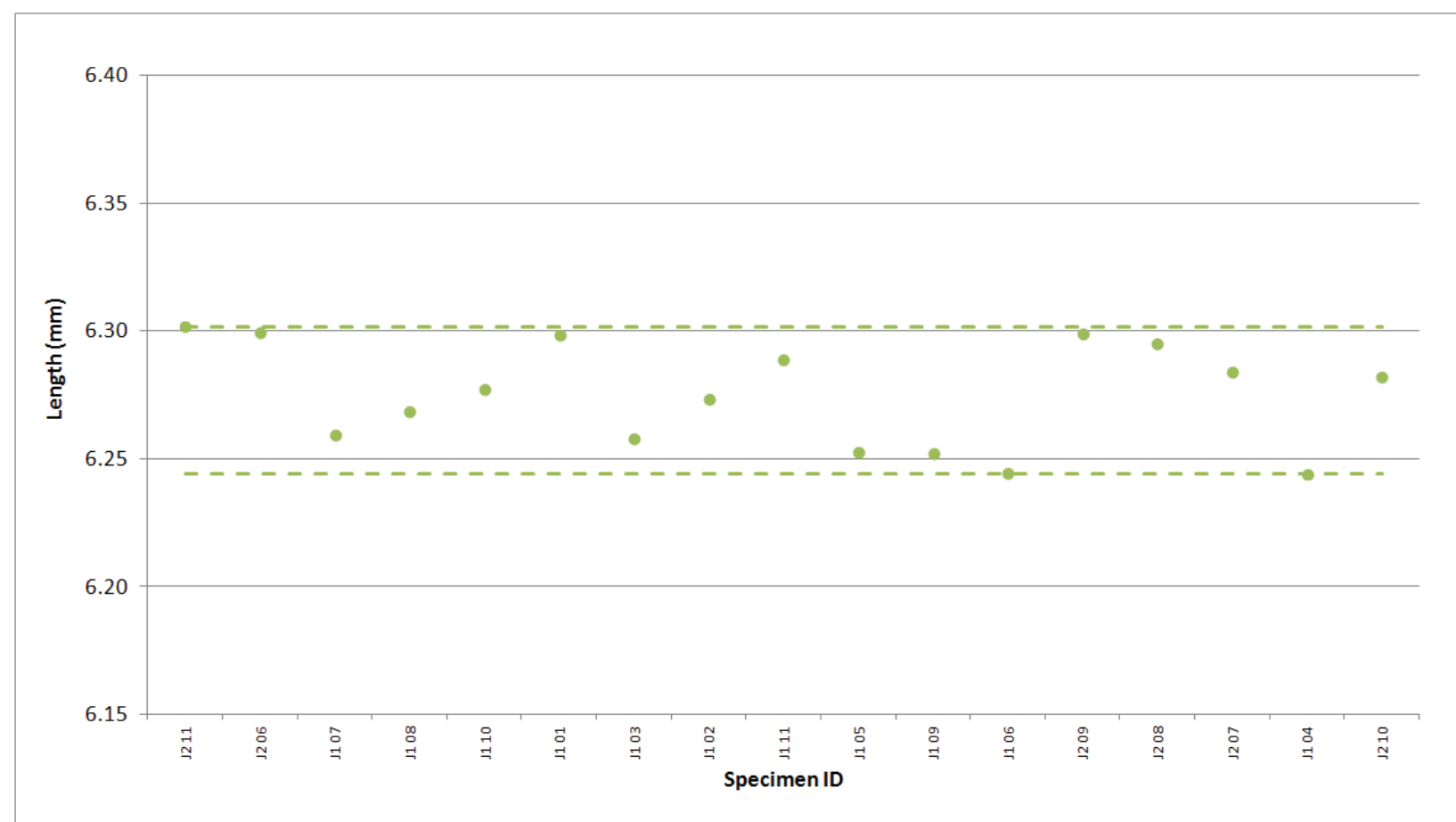

Figure A-32. HLM Piggyback Length. 


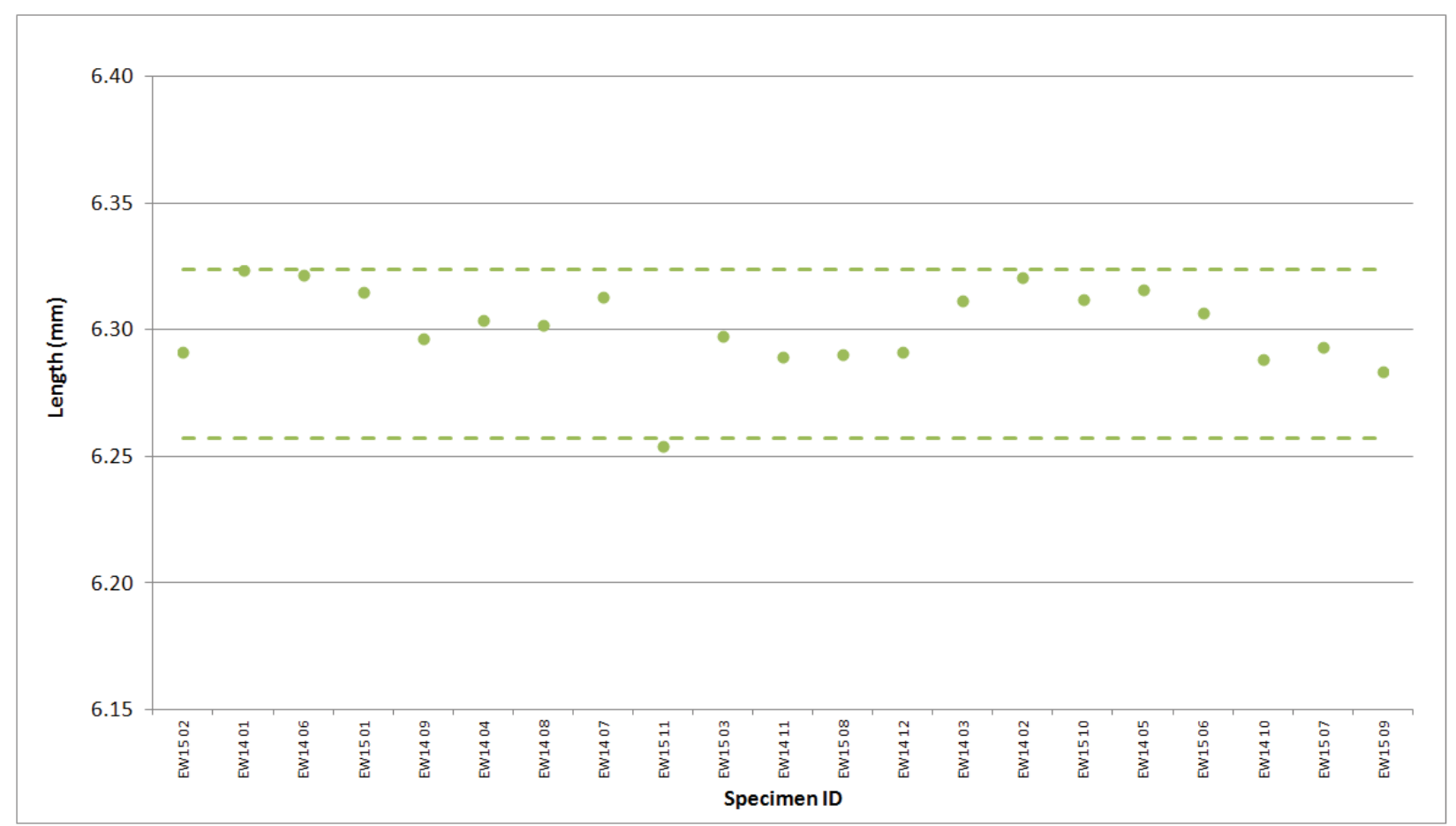

Figure A-33. IG-110 Piggyback Length.

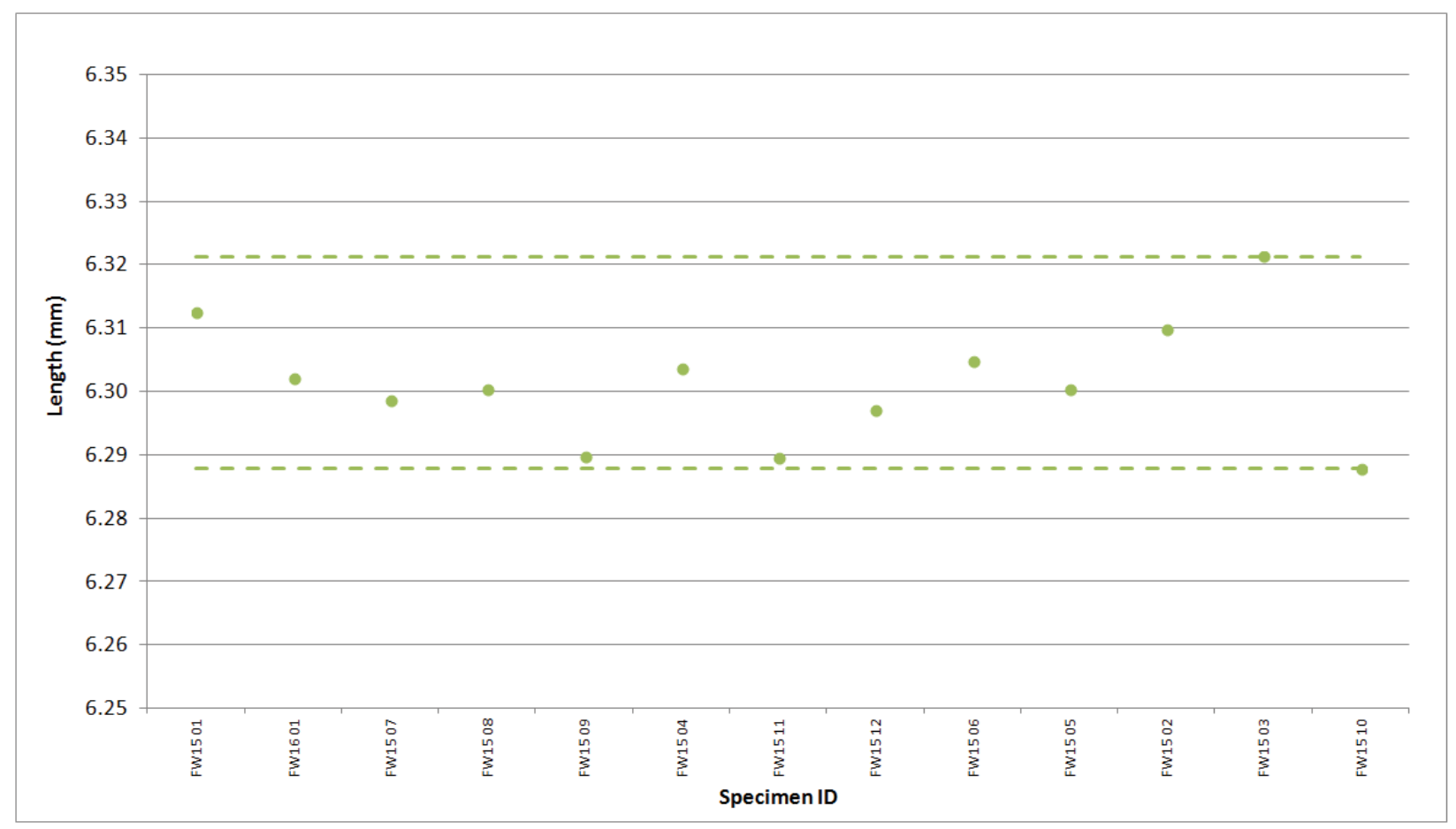

Figure A-34. IG-430 Piggyback Length. 


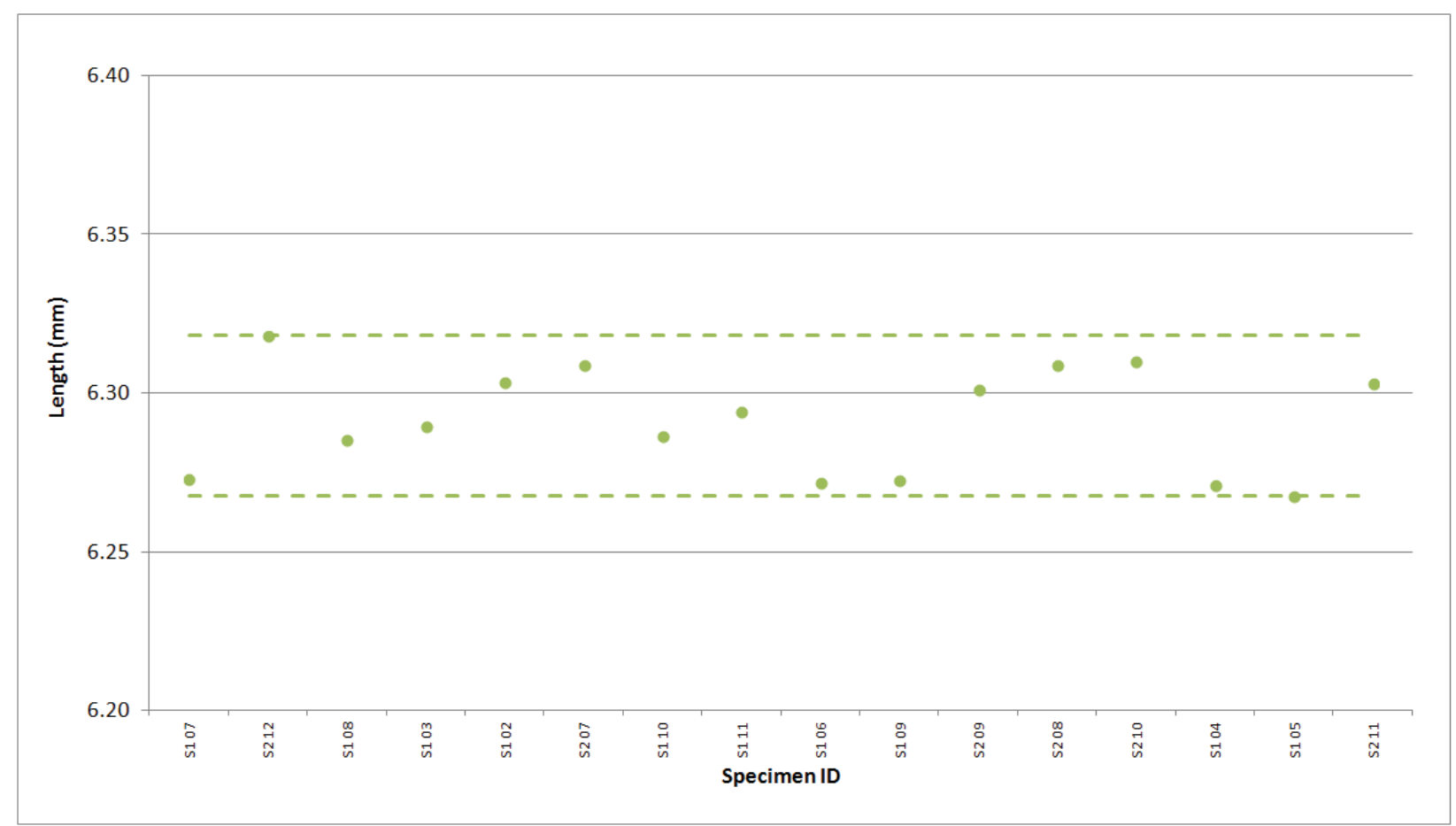

Figure A-35. NBG-10 Piggyback Length.

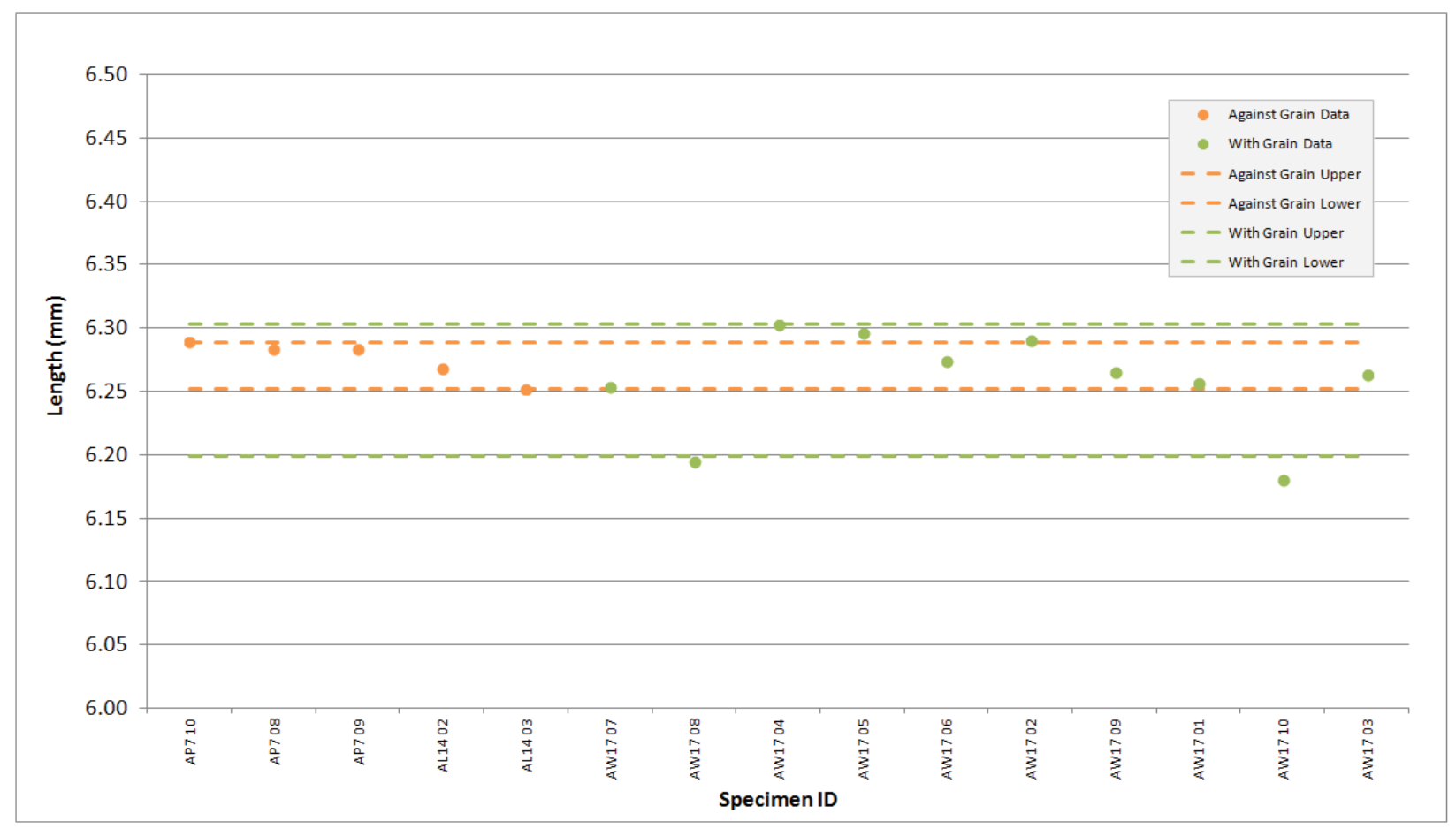

Figure A-36. NBG-17 Piggyback Length. 


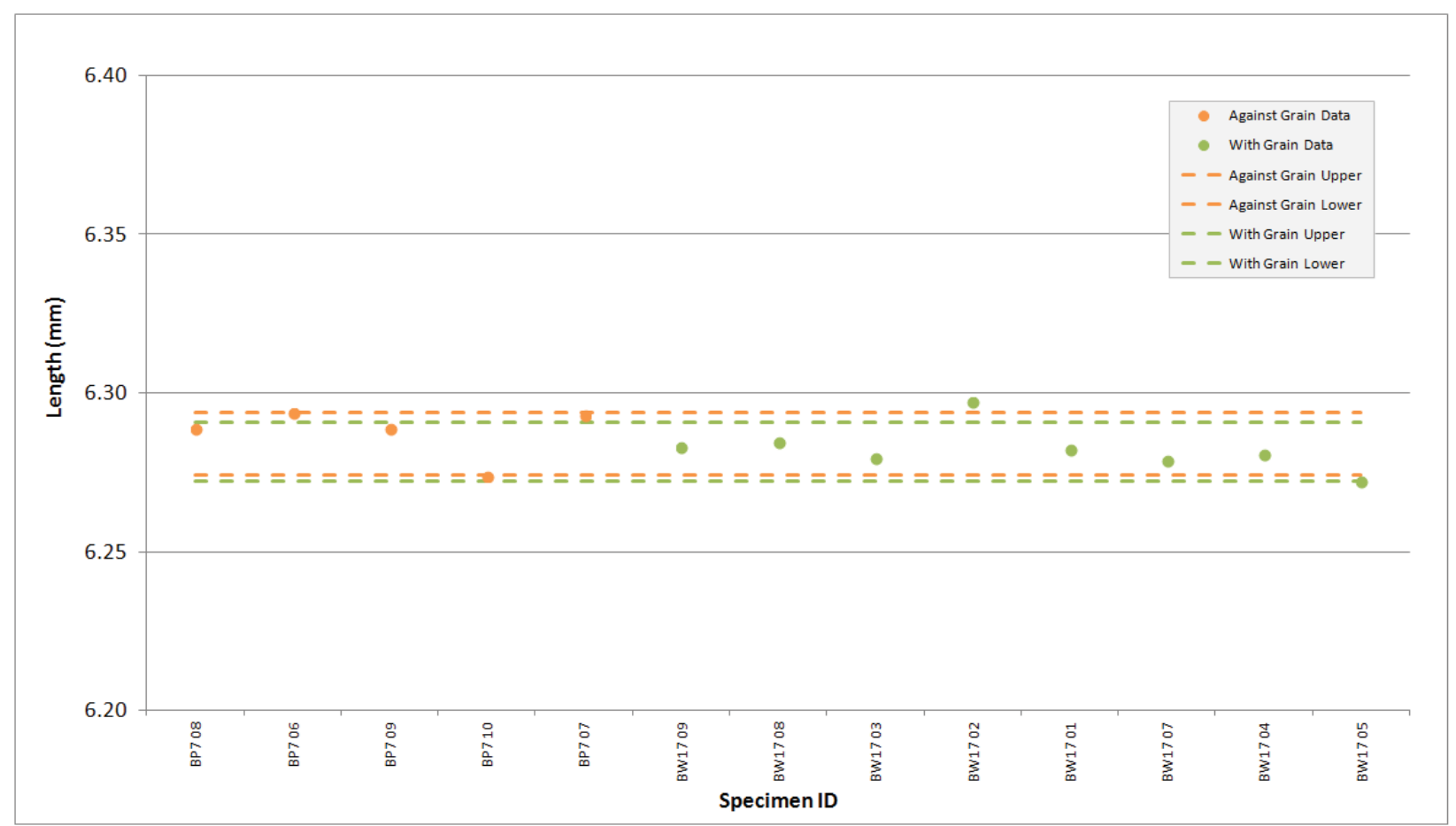

Figure A-37. NBG-18 Piggyback Length.

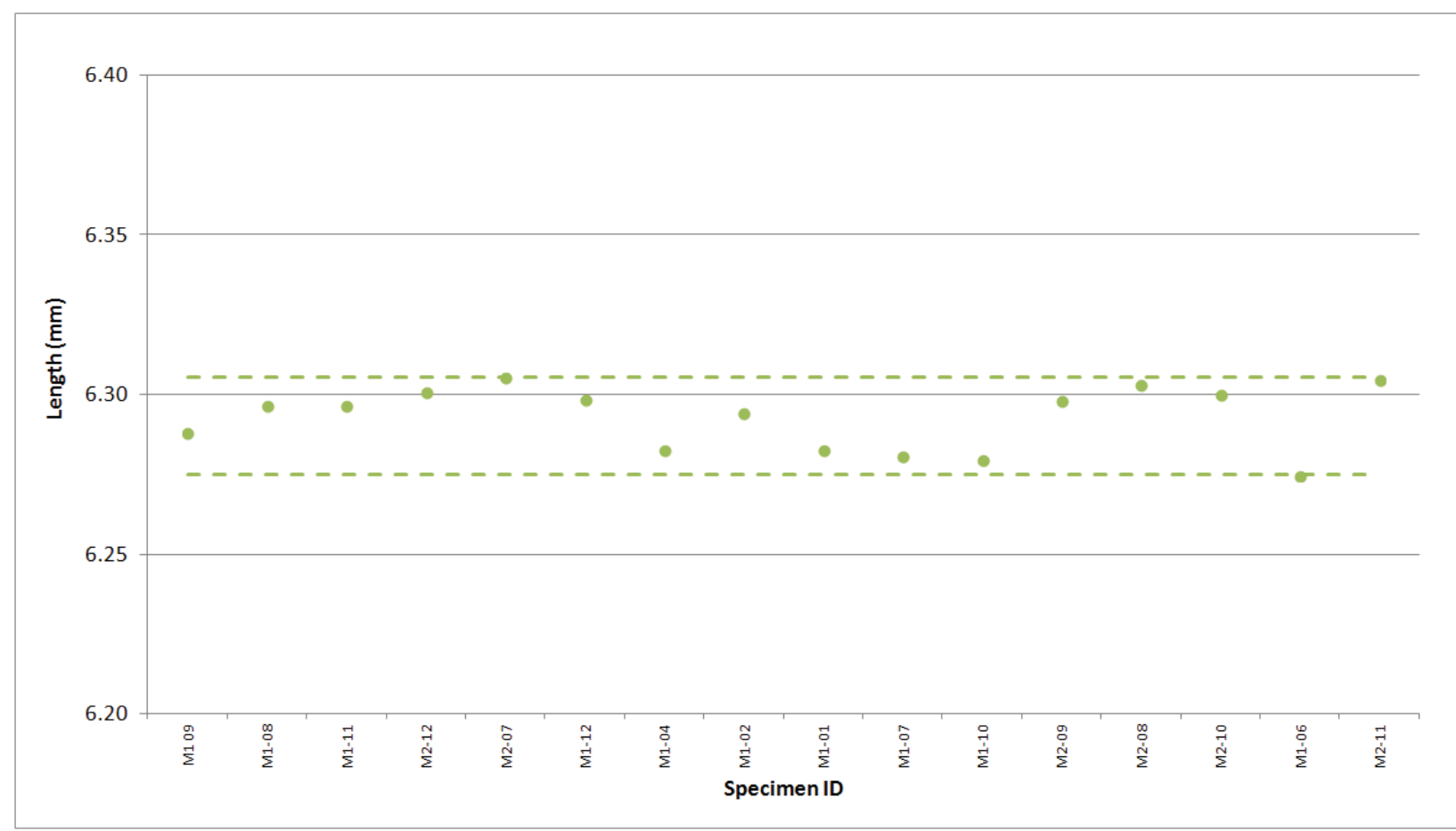

Figure A-38. NBG-25 Piggyback Length. 


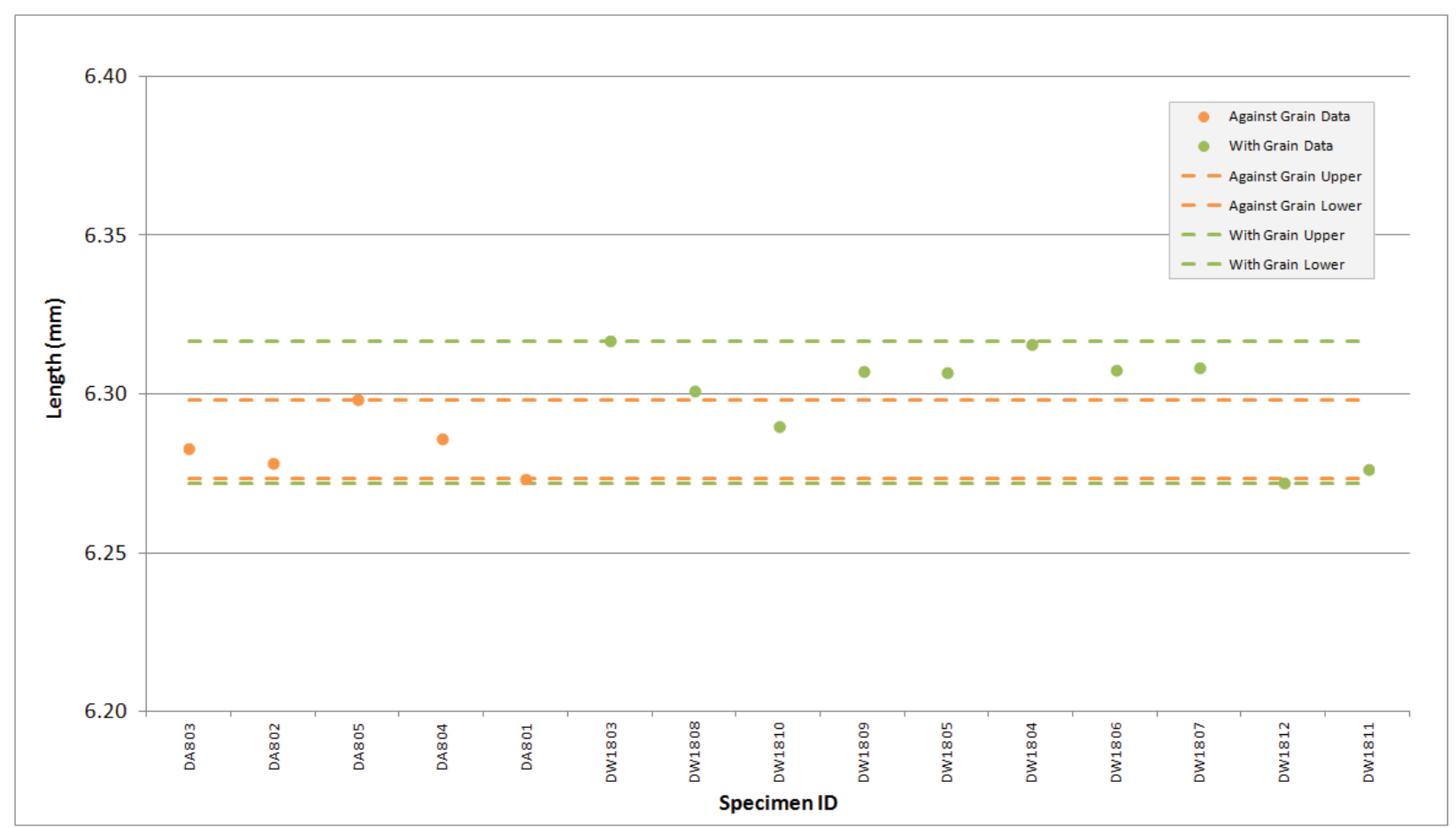

Figure A-39. PCEA Piggyback Length.

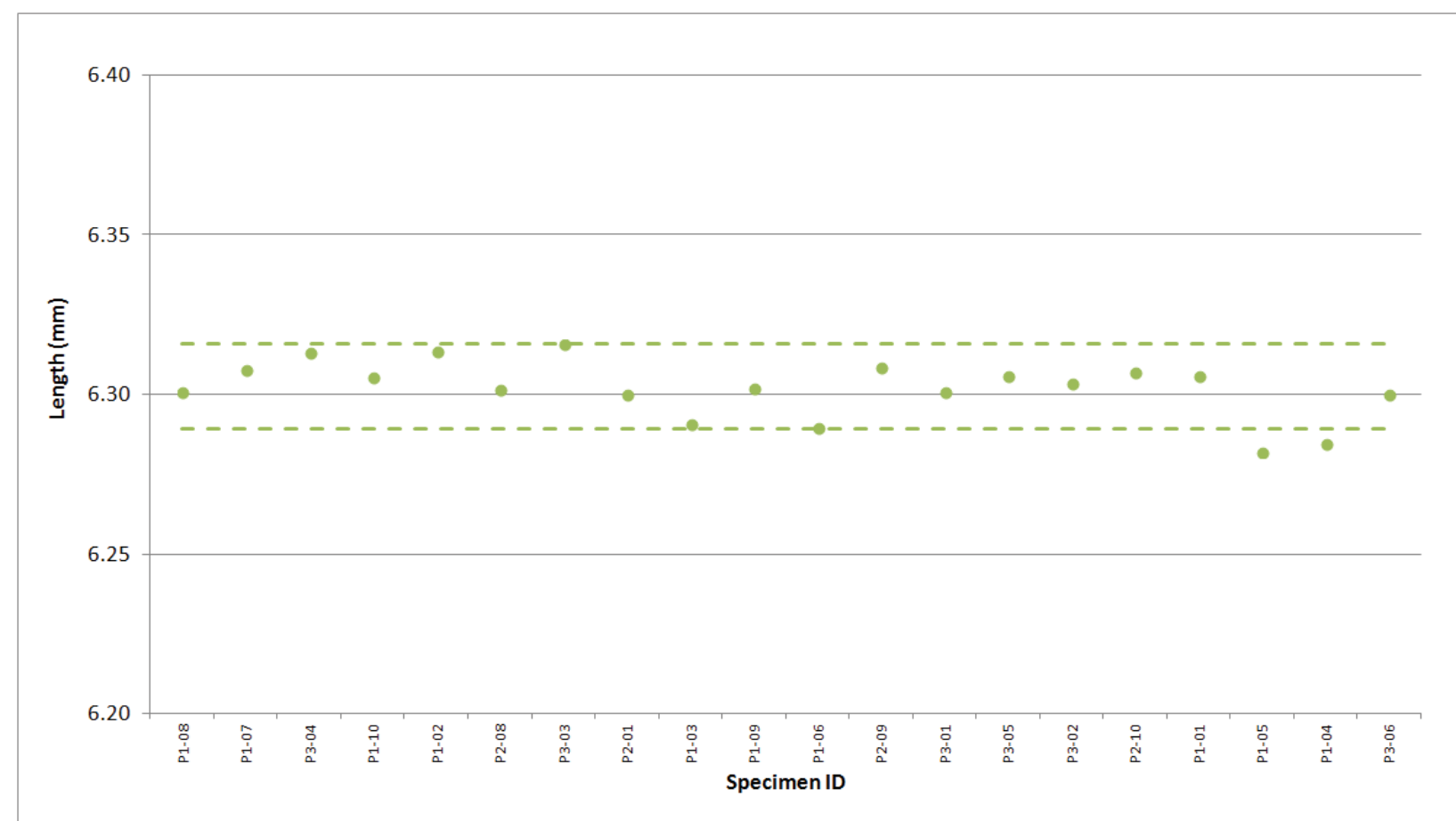

Figure A-40. PCIB Piggyback Length. 


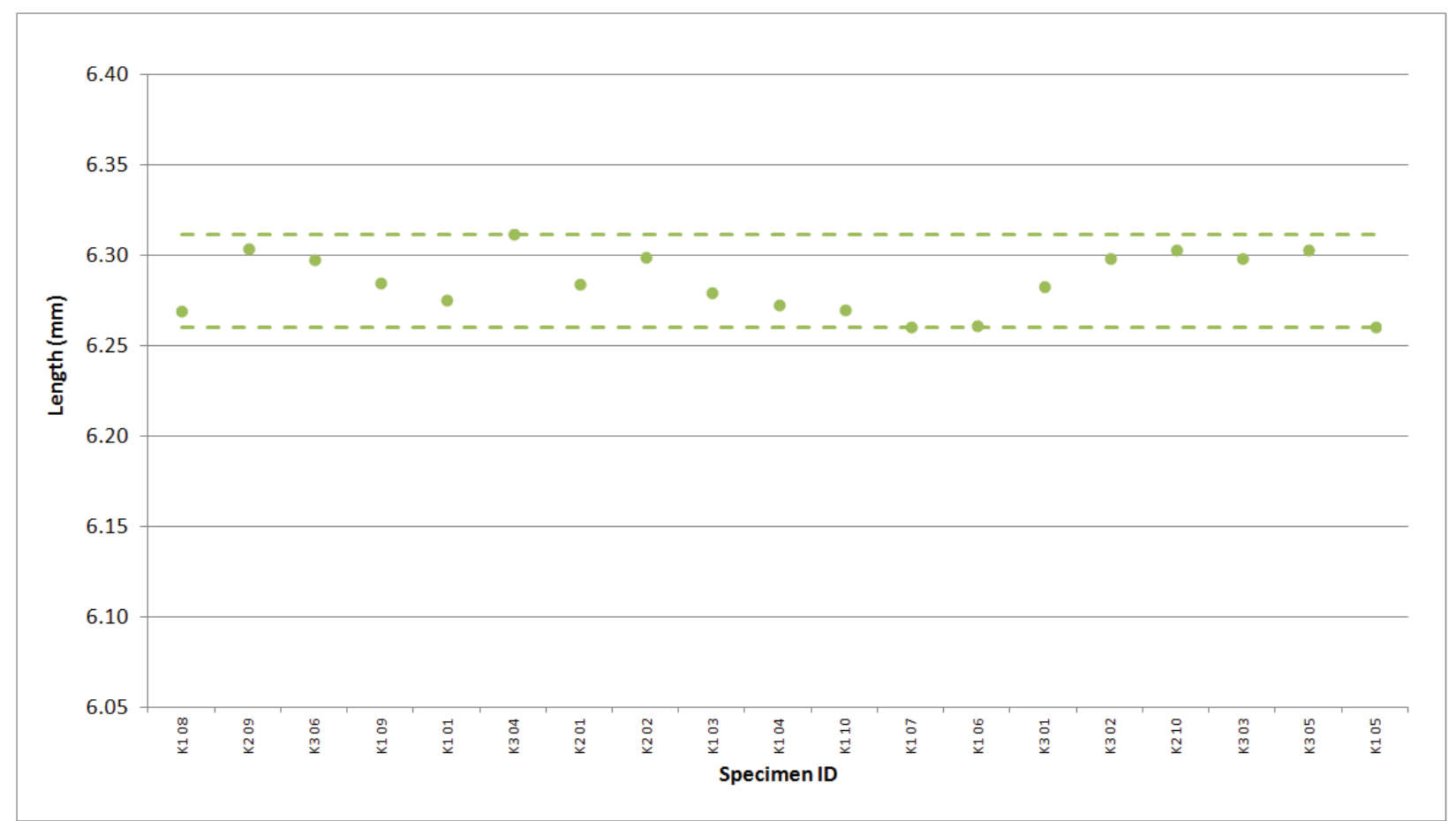

Figure A-41. PGX Piggyback Length.

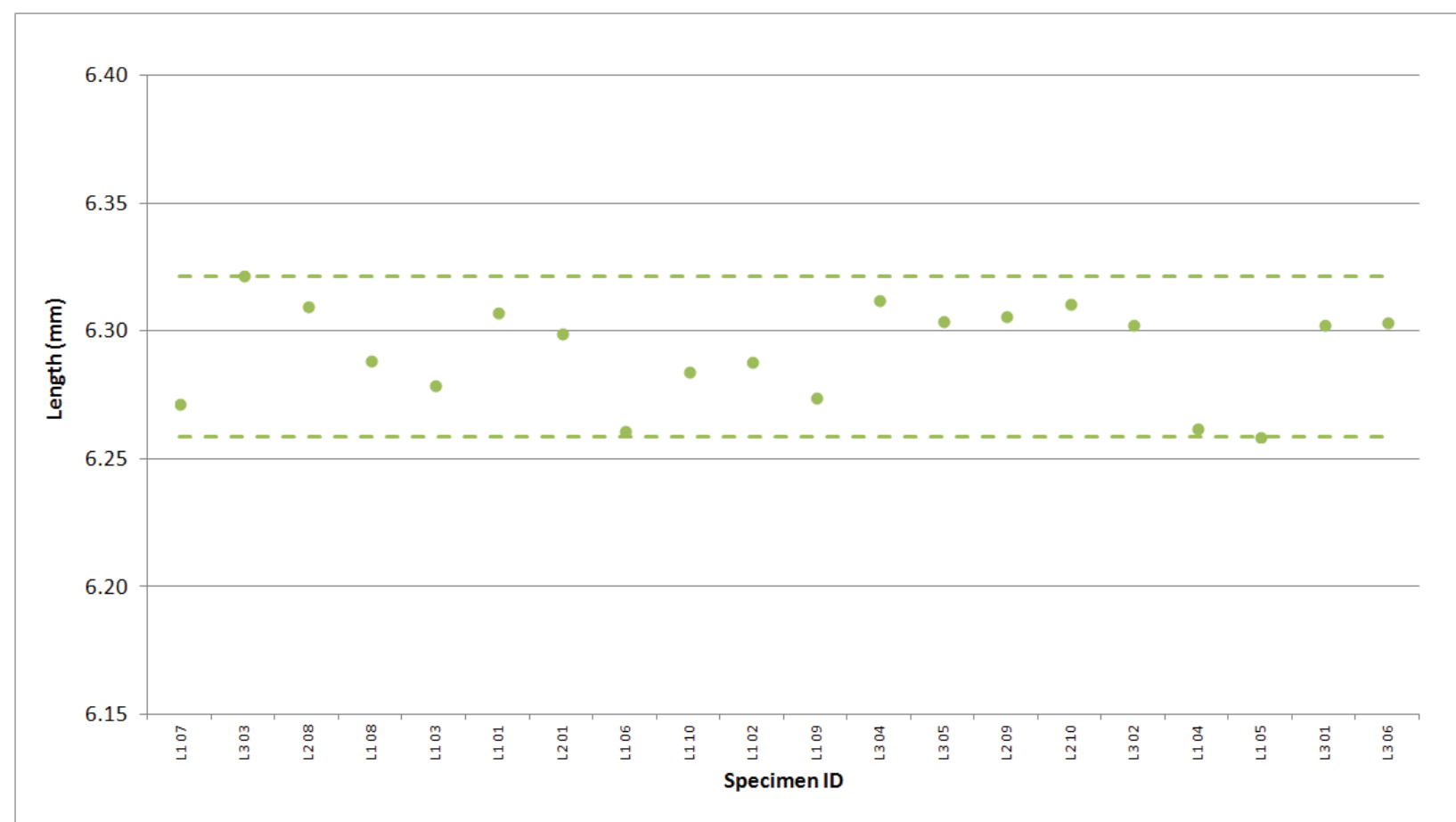

Figure A-42. PPEA Piggyback Length. 


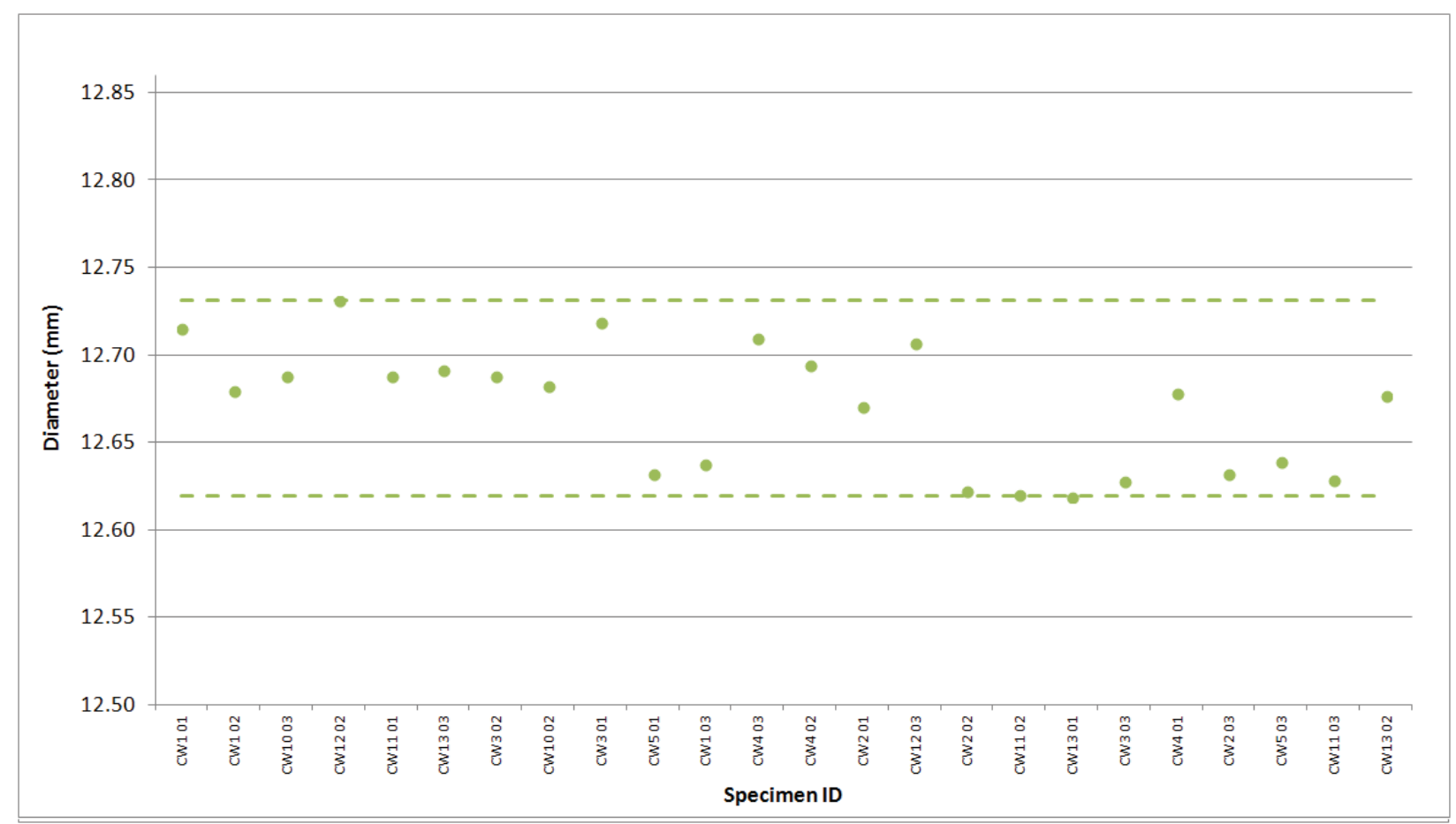

Figure A-43. H-451 Creep Diameter.

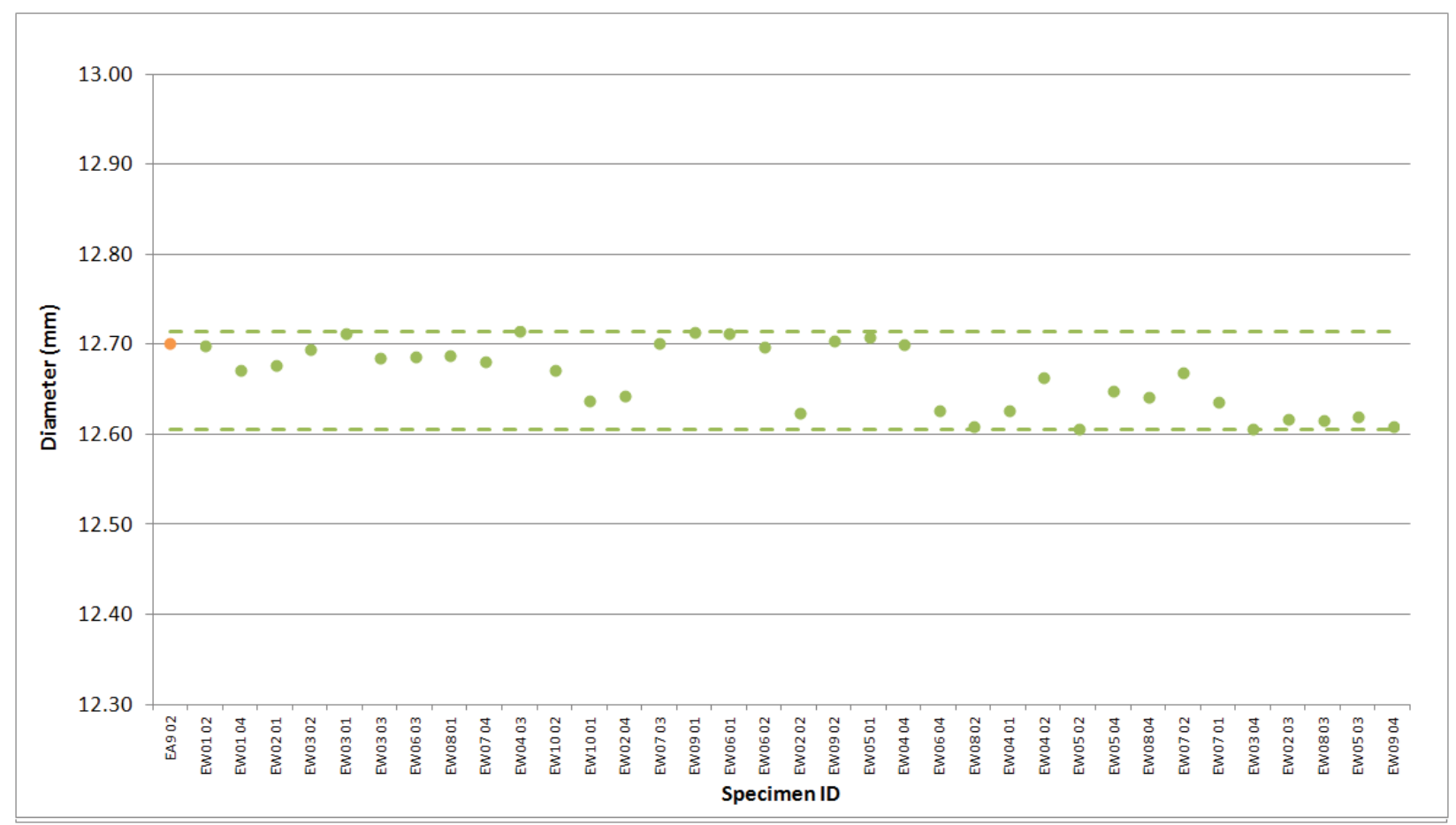

Figure A-44. IG-110 Creep Diameter. 




Figure A-45. IG-430 Creep Diameter.

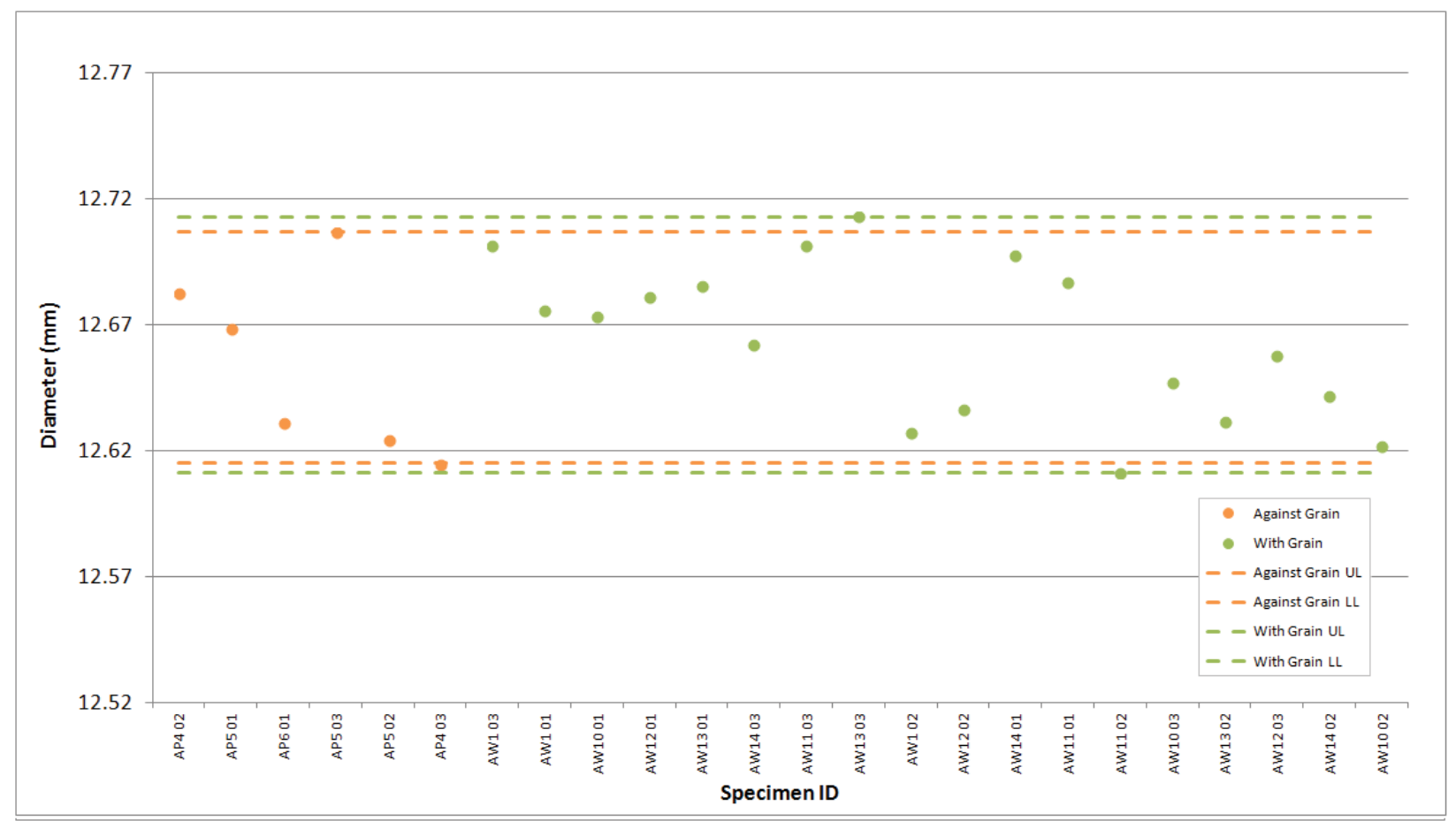

Figure A-46. NBG-17 Creep Diameter. 


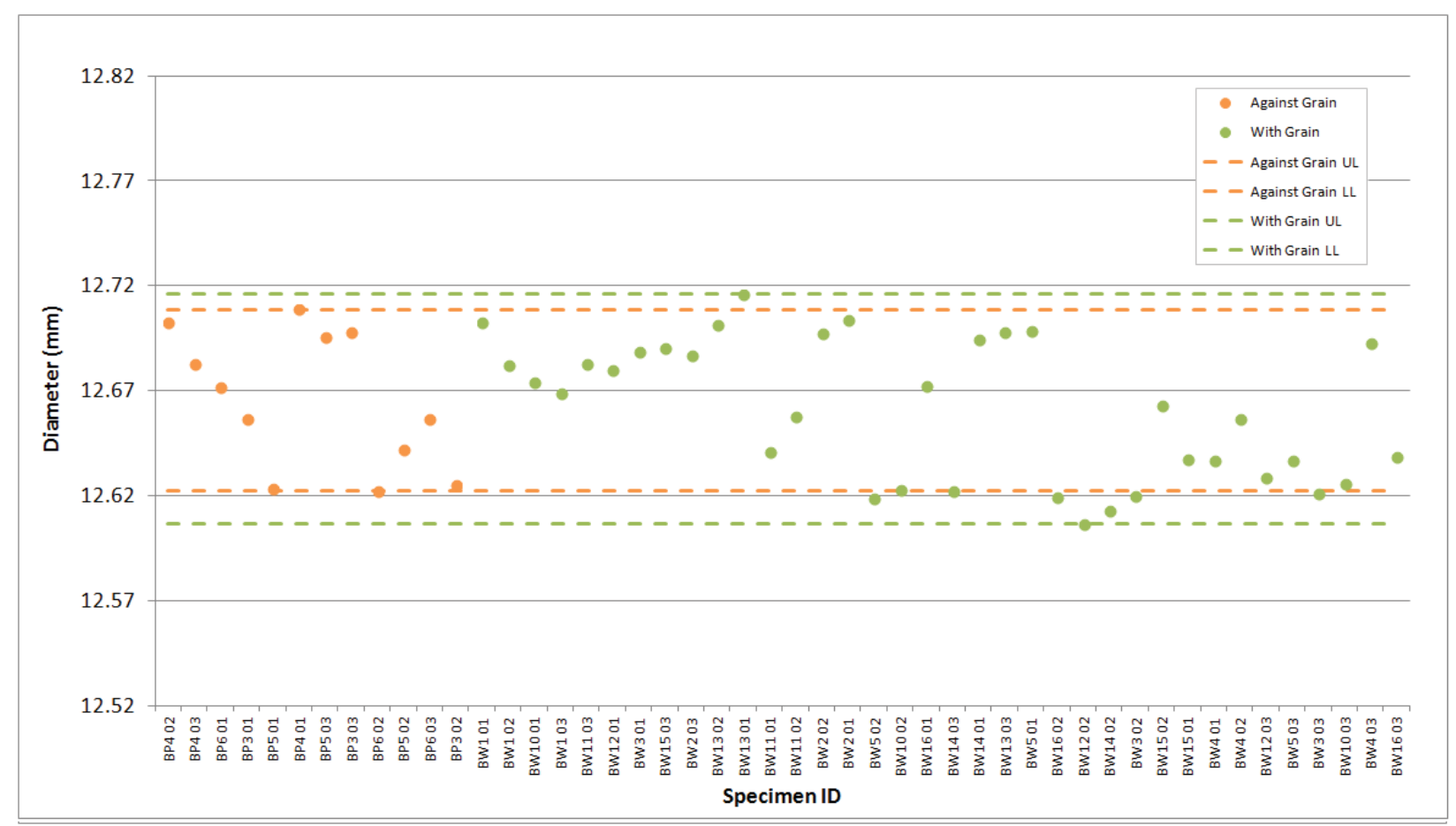

Figure A-47. NBG-18 Creep Diameter.

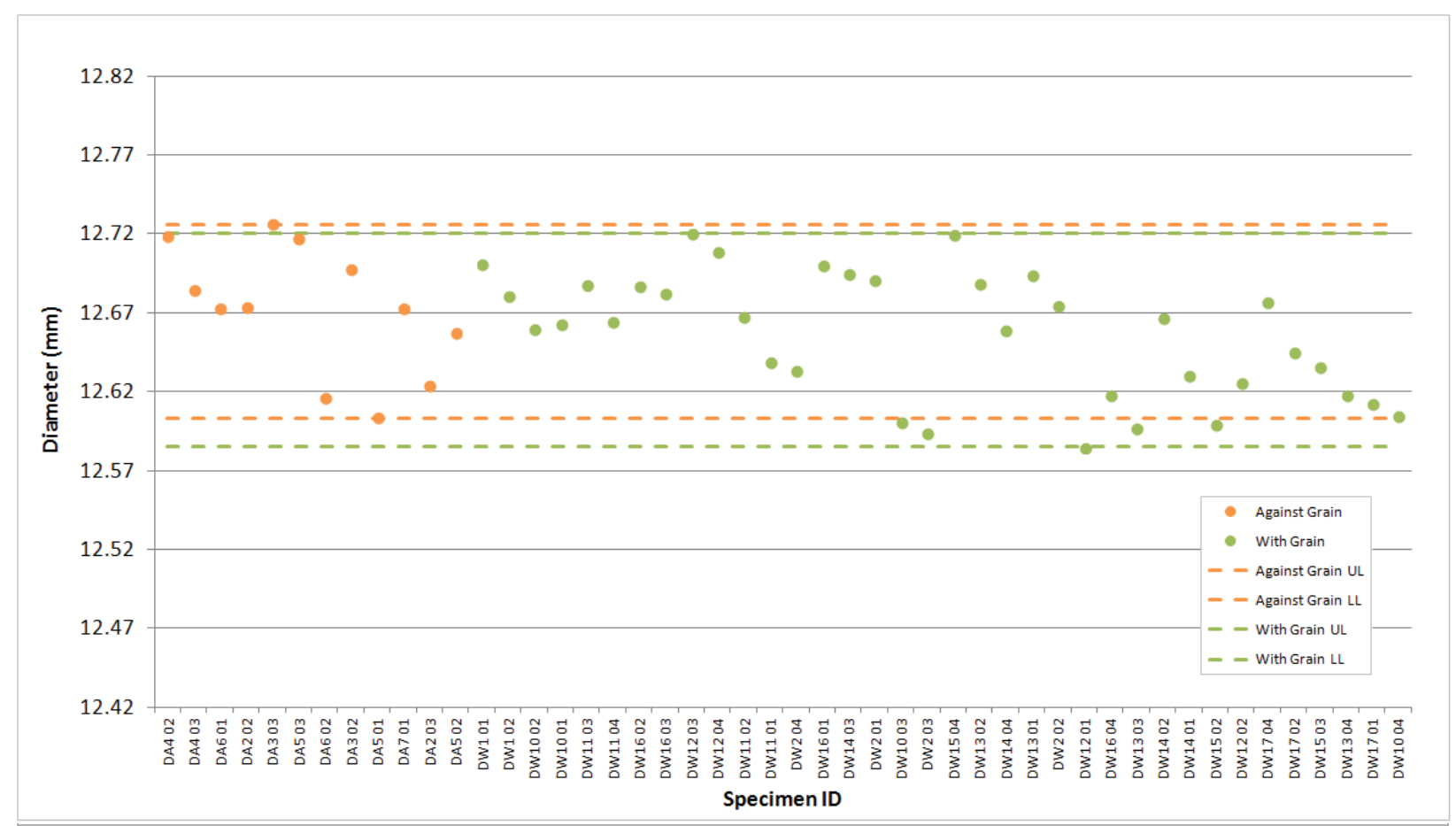

Figure A-48. PCEA Creep Diameter. 


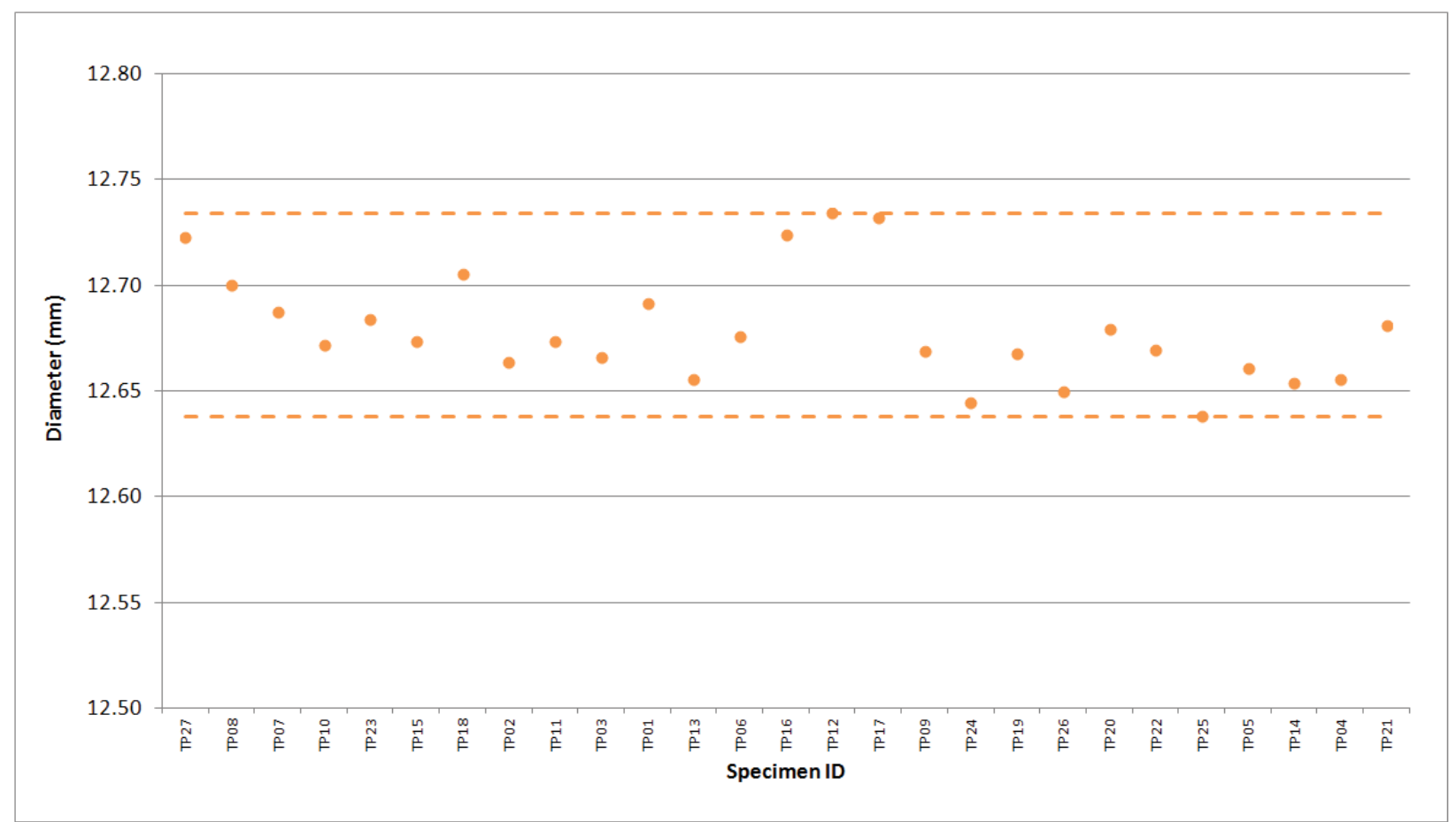

Figure A-49. 2114 Piggyback Diameter.

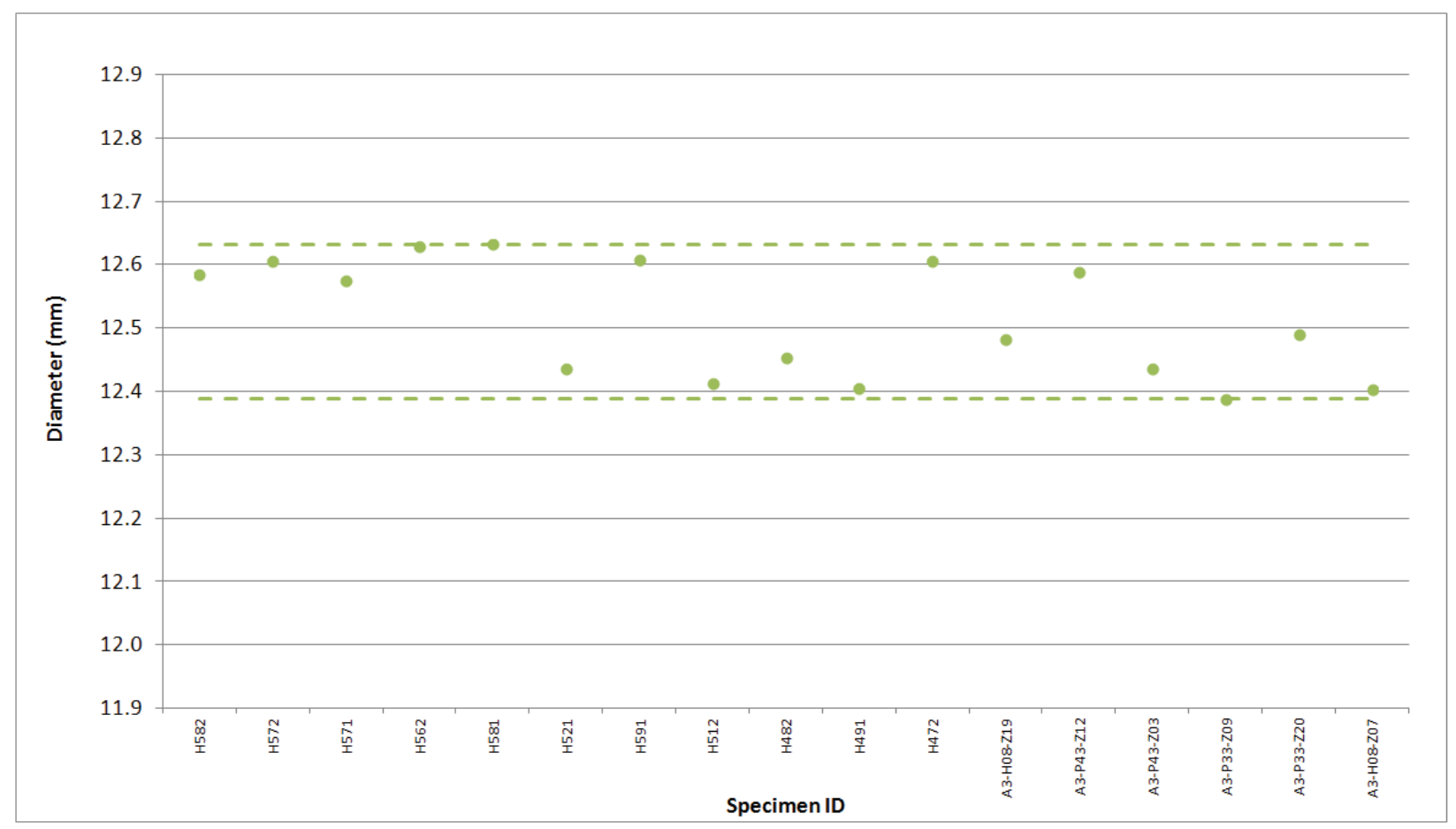

Figure A-50. A3 Piggyback Diameter. 


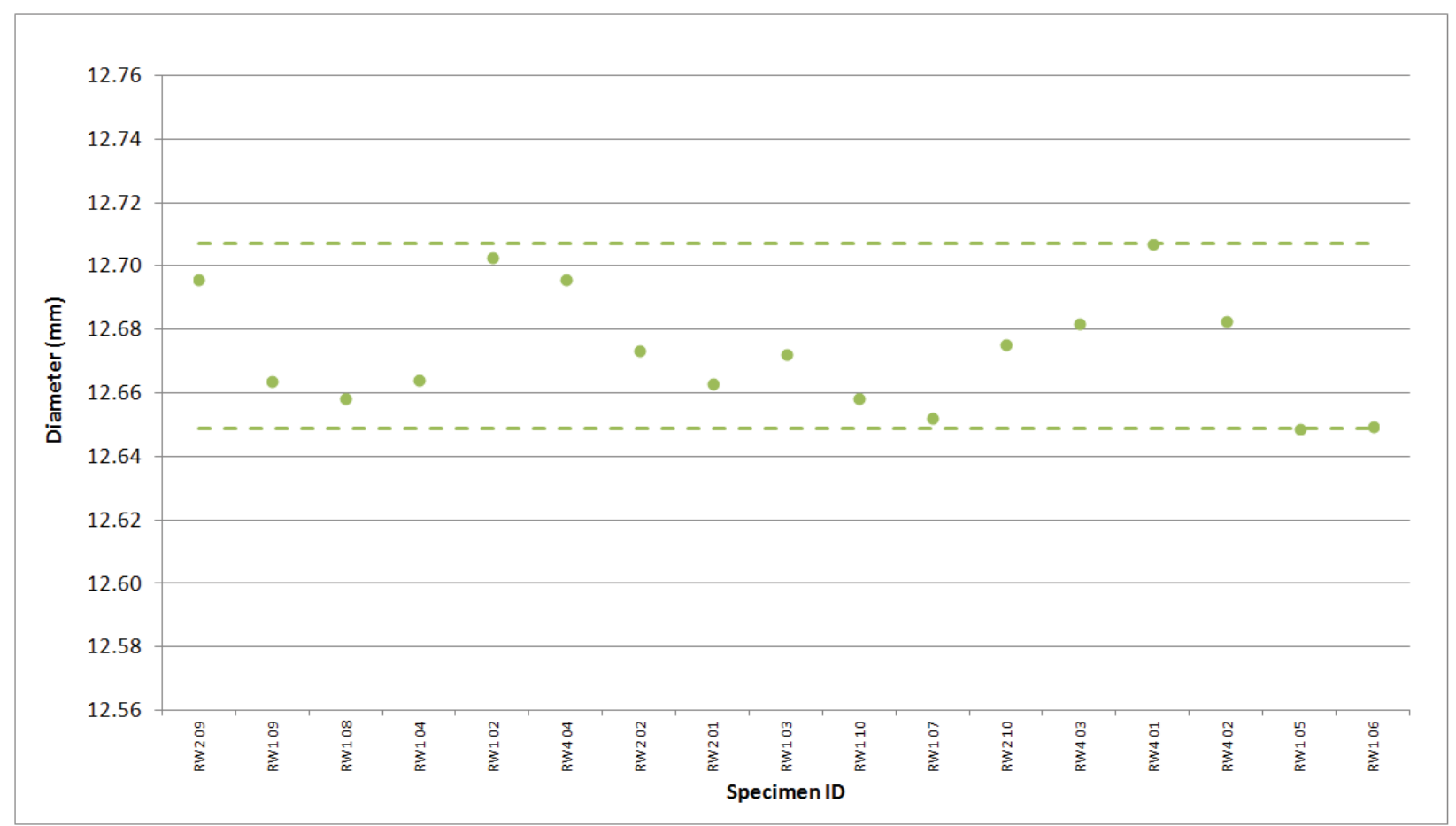

Figure A-51. BAN Piggyback Diameter.

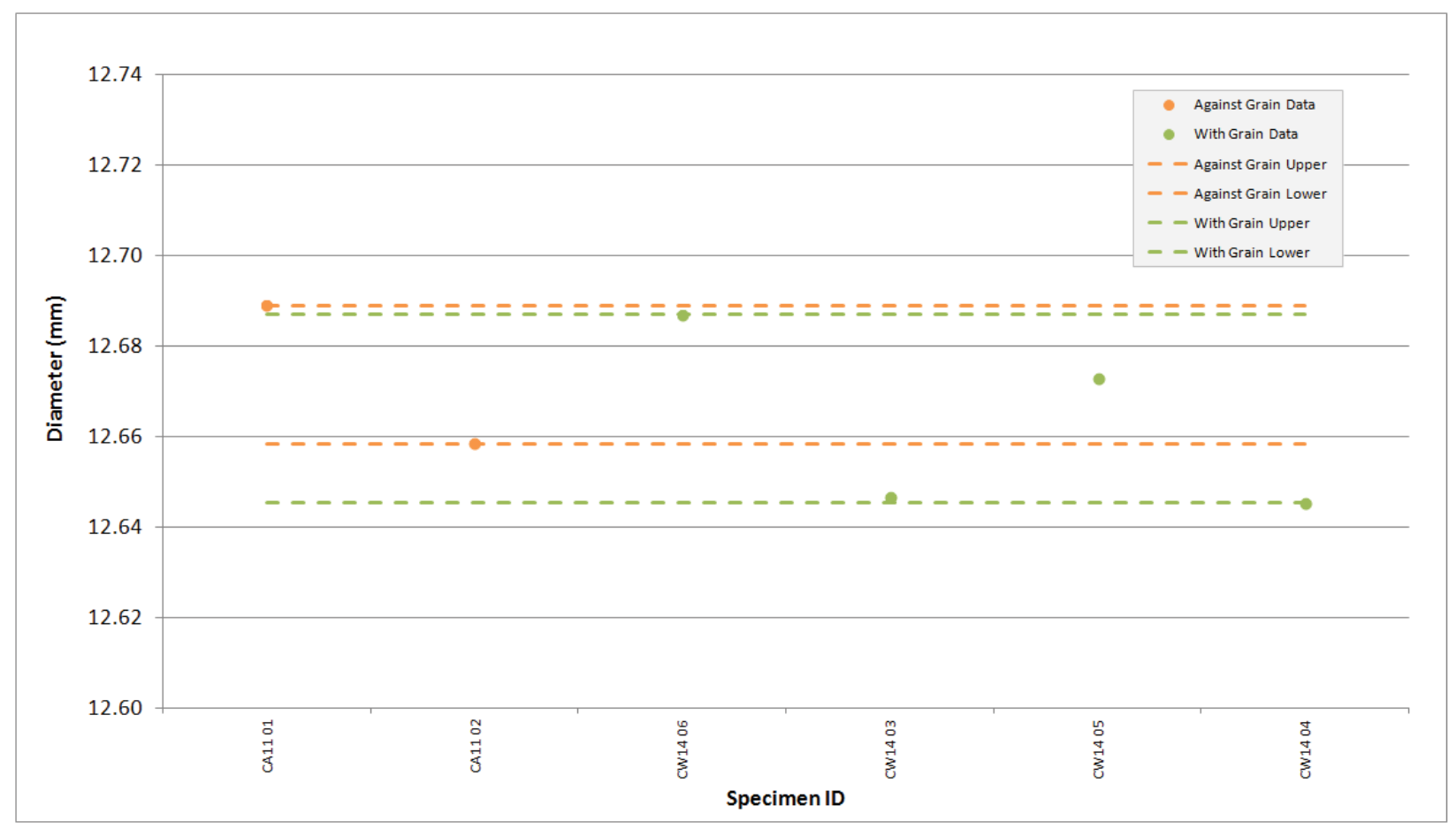

Figure A-52. H-451 Piggyback Diameter. 


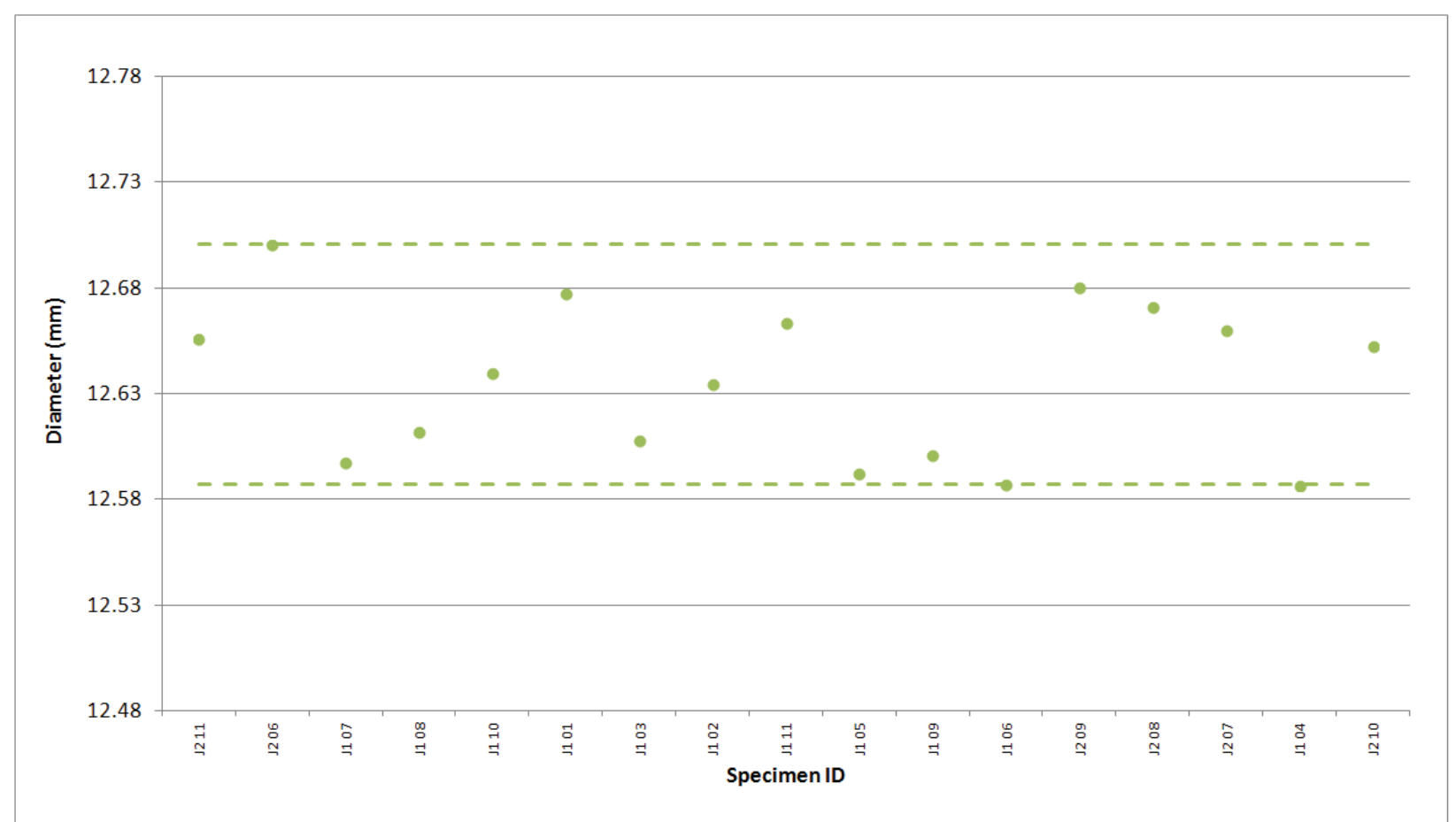

Figure A-53. HLM Piggyback Diameter.

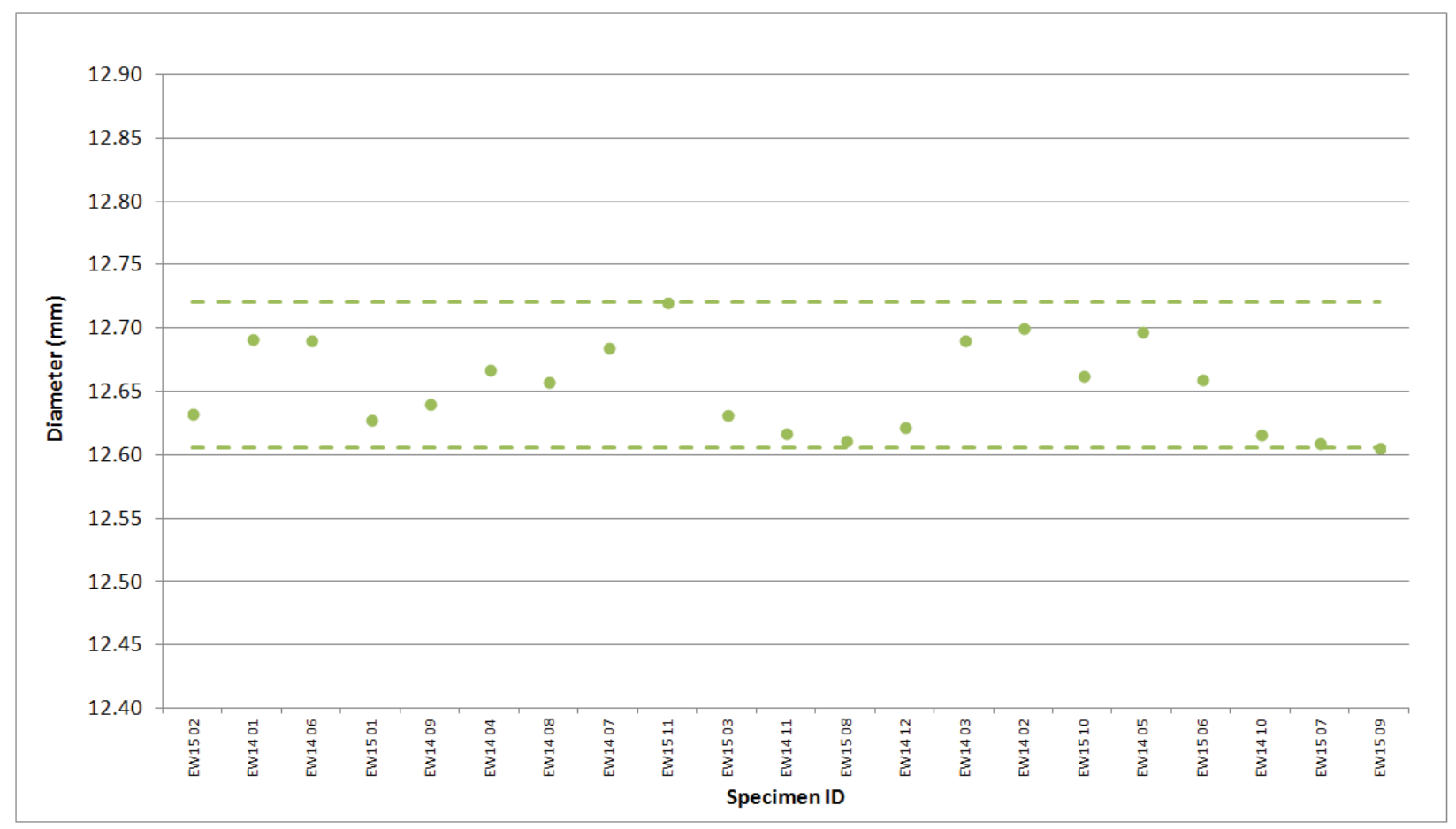

Figure A-54. IG-110 Piggyback Diameter. 


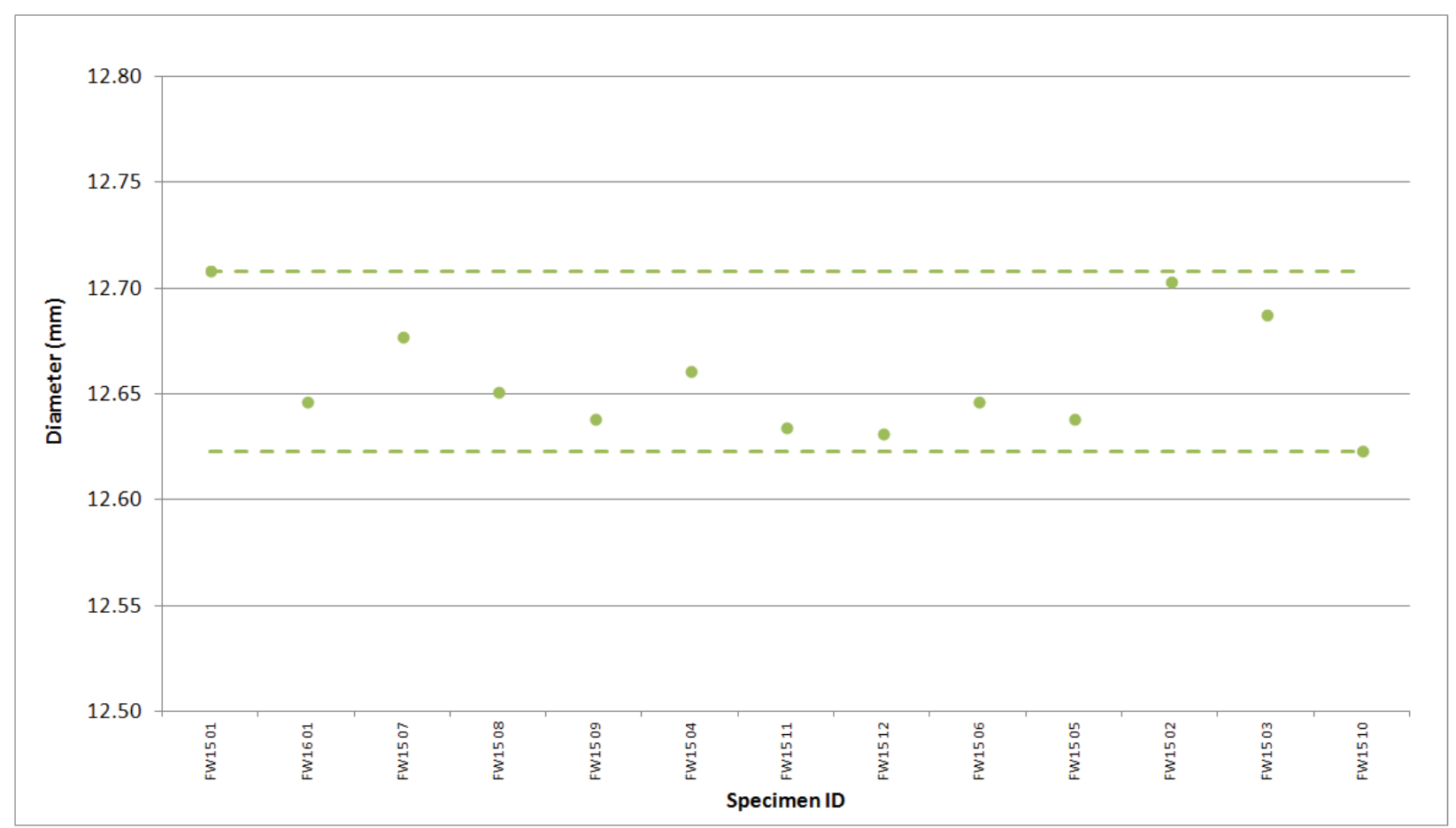

Figure A-55. IG-430 Piggyback Diameter.

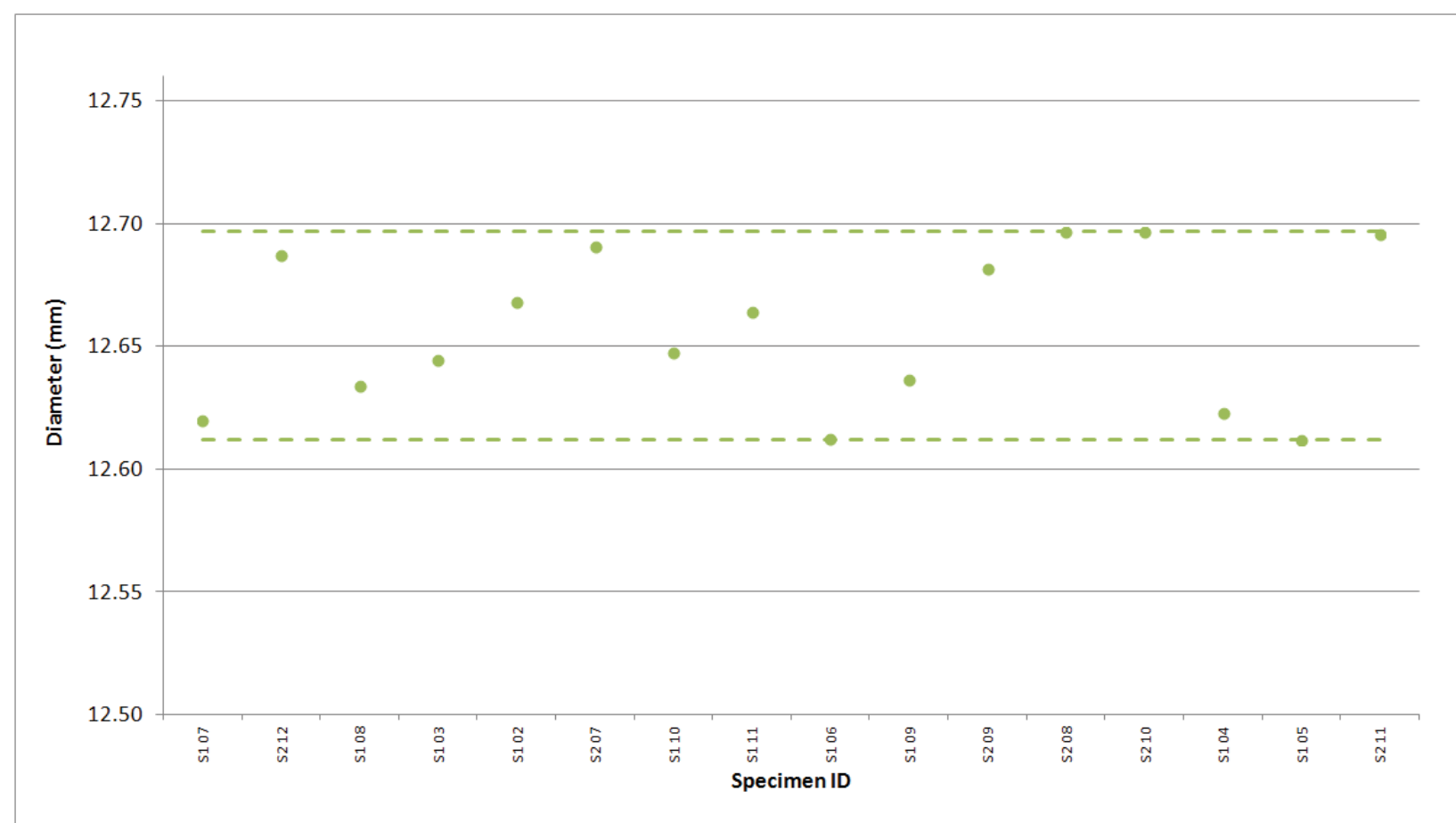

Figure A-56. NBG-10 Piggyback Diameter. 


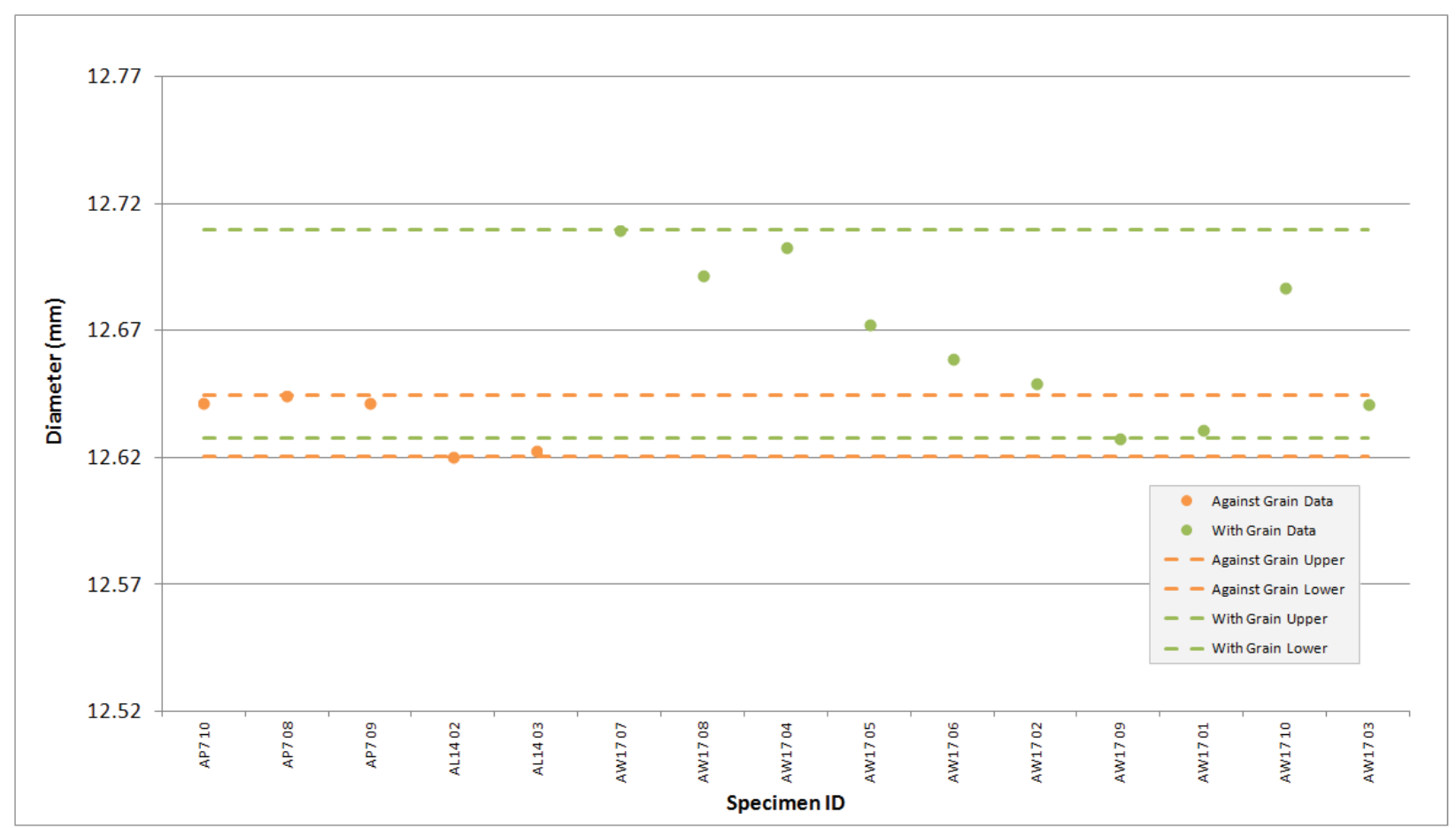

Figure A-57. NBG-17 Piggyback Diameter.

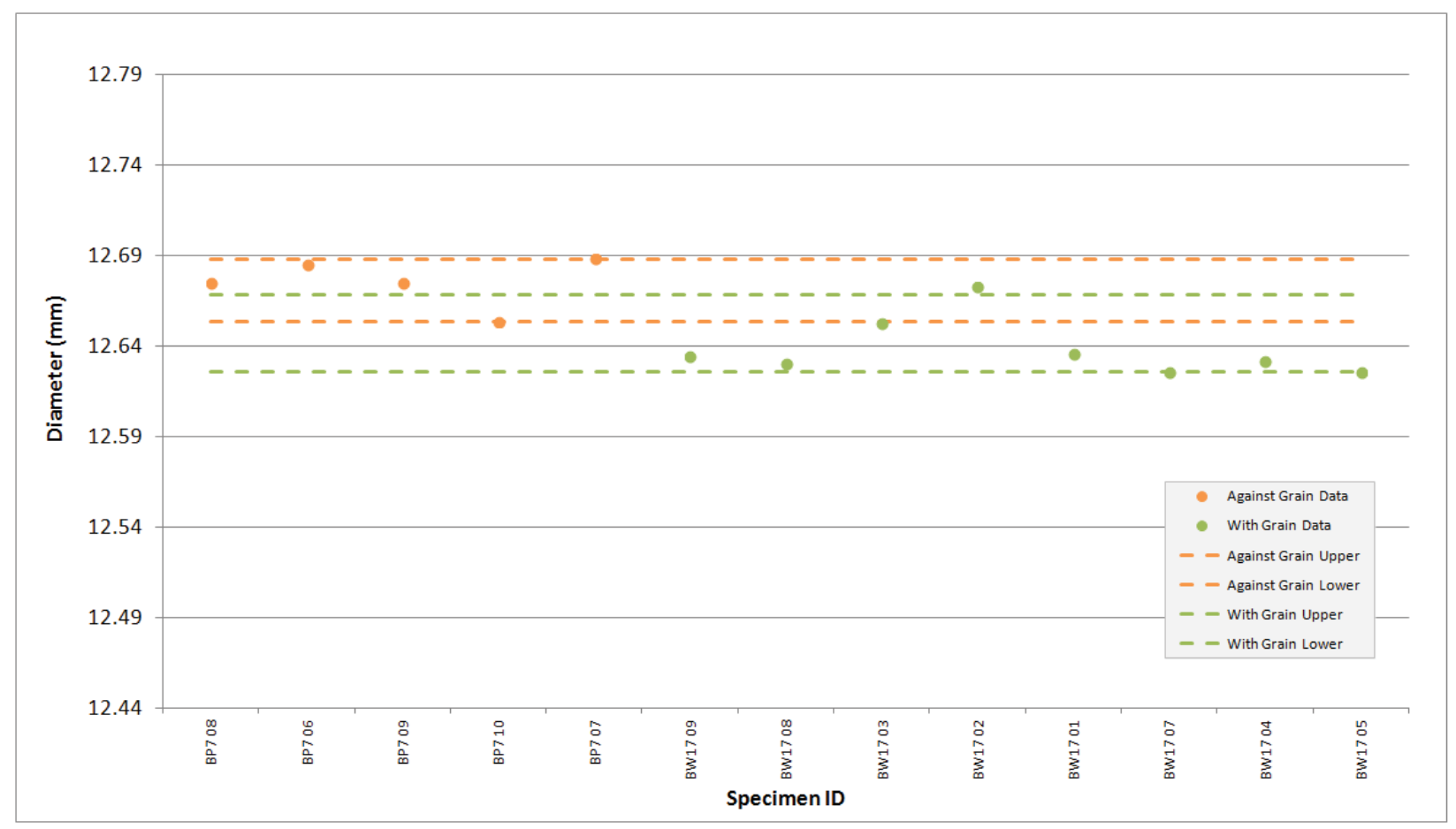

Figure A-58. NBG-18 Piggyback Diameter. 


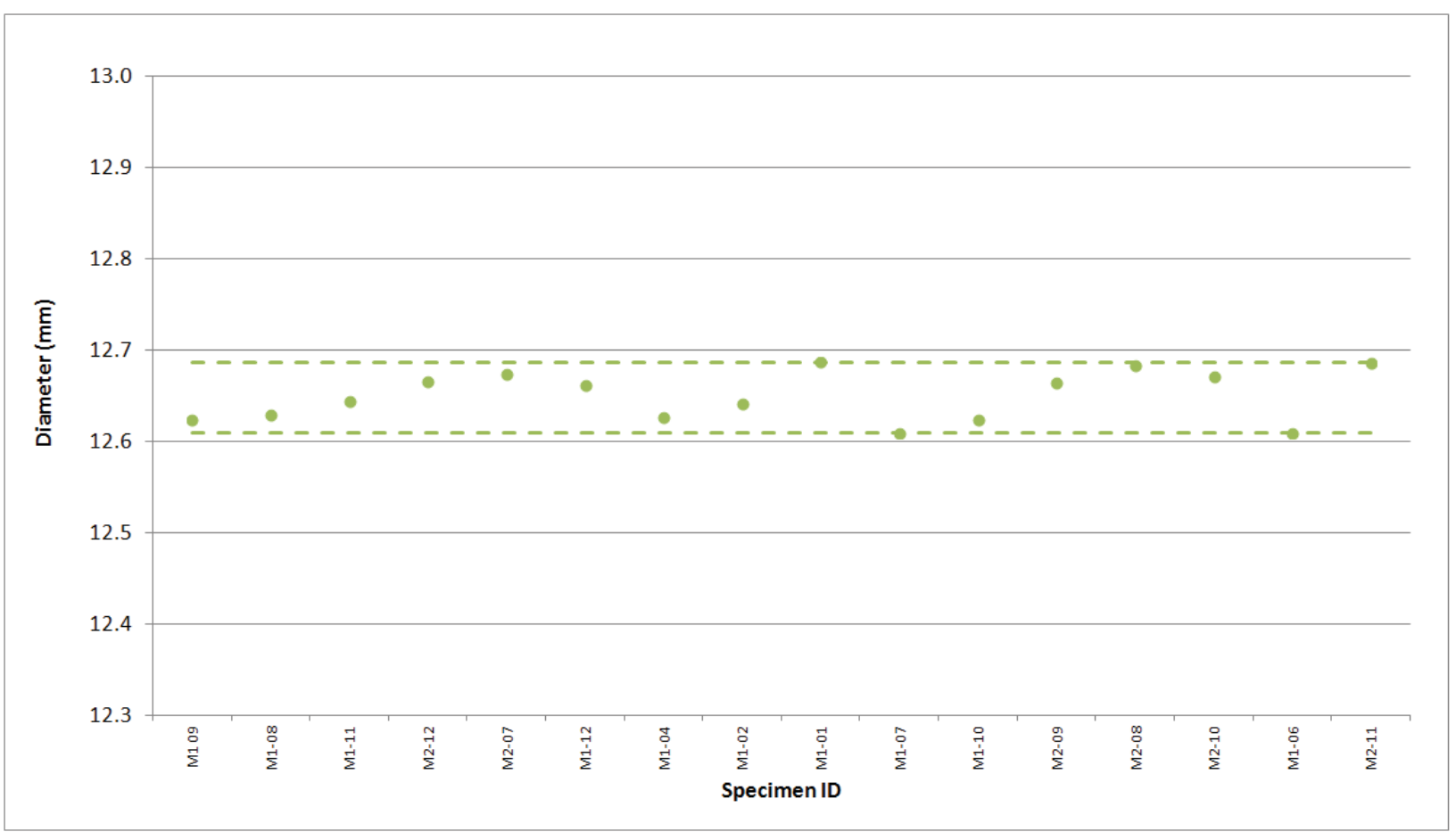

Figure A-59. NBG-25 Piggyback Diameter.

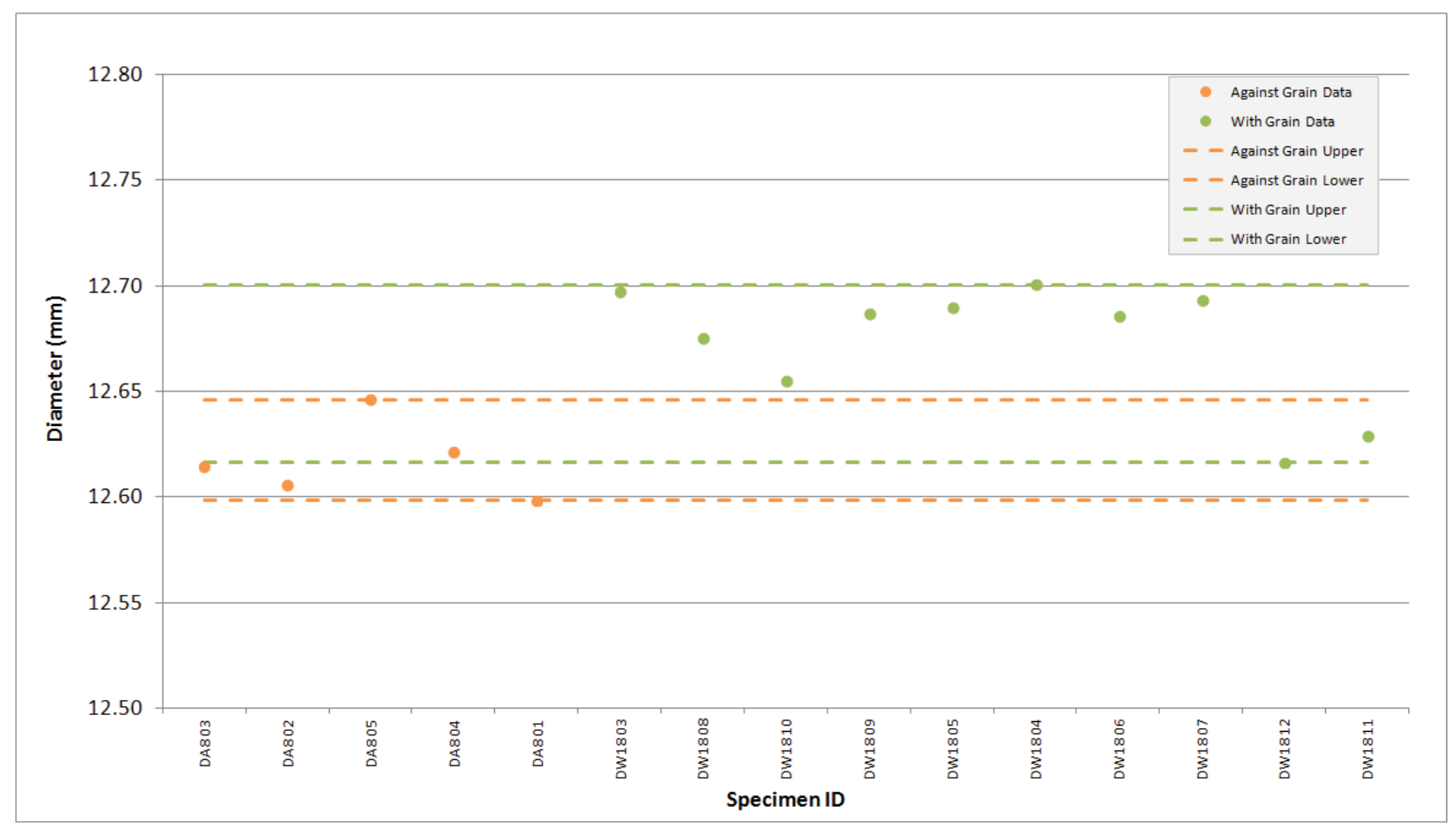

Figure A-60. PCEA Piggyback Diameter. 




Figure A-61. PCIB Piggyback Diameter.

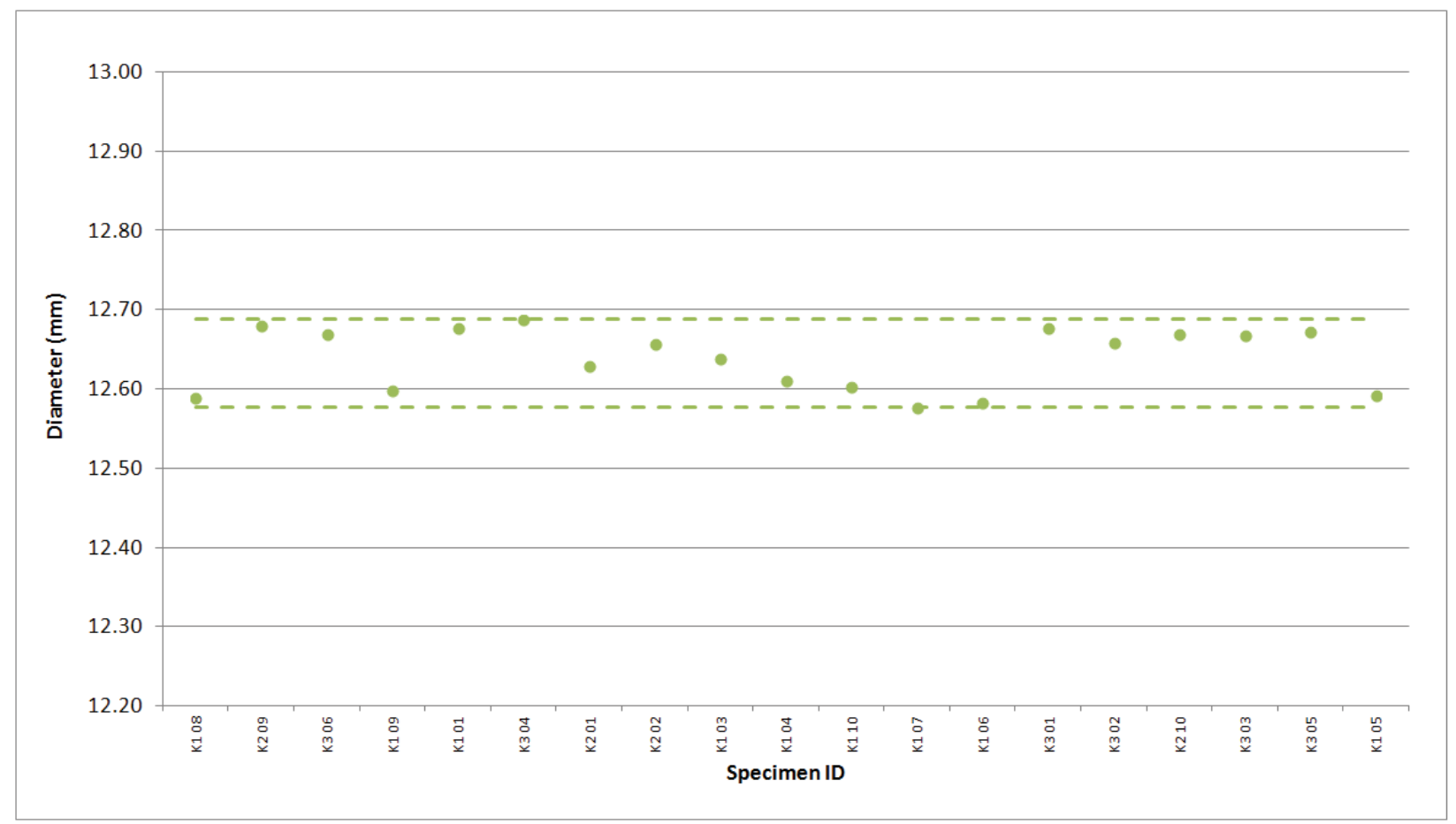

Figure A-62. PGX Piggyback Diameter. 


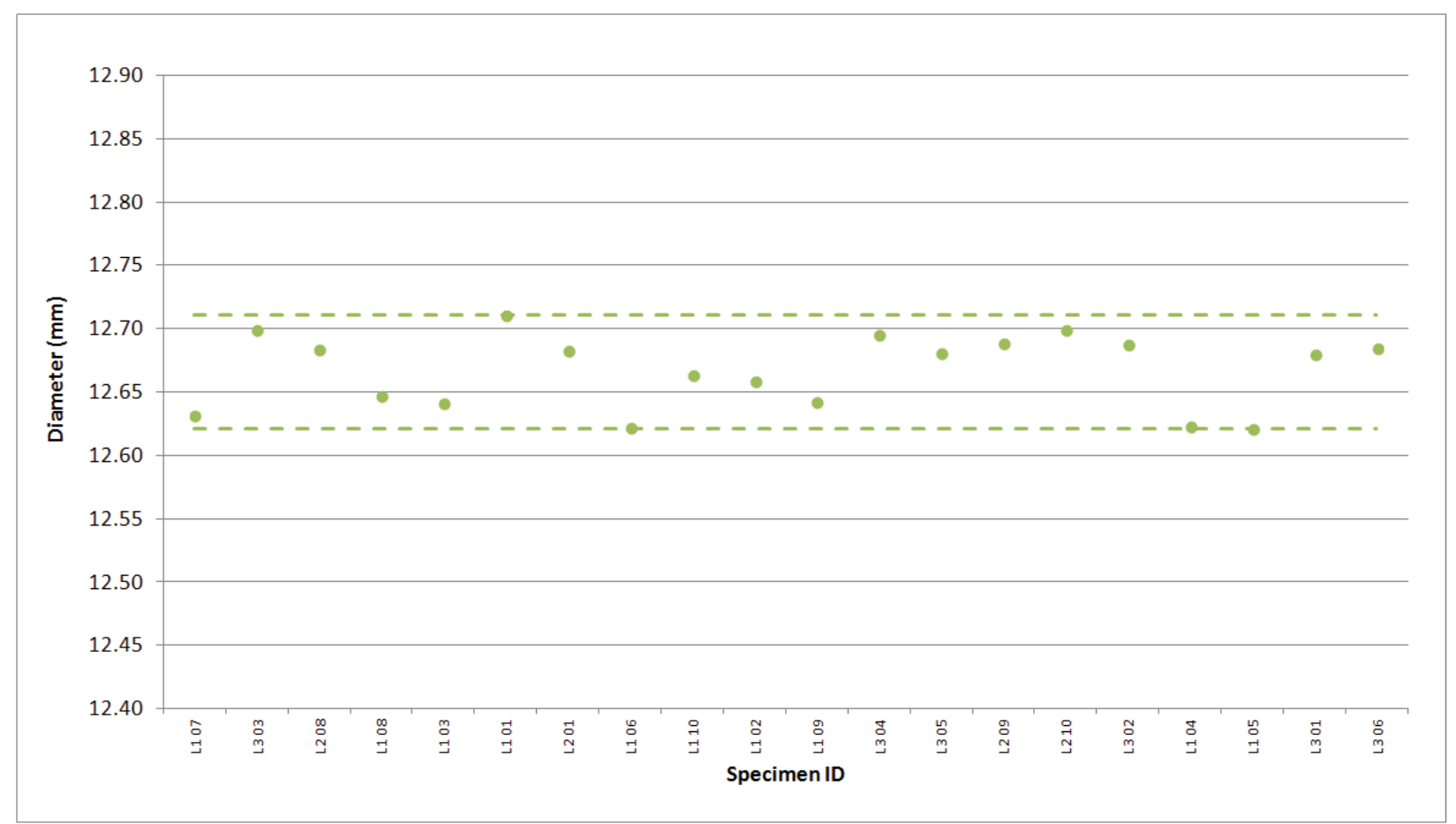

Figure A-63. PPEA Piggyback Diameter.

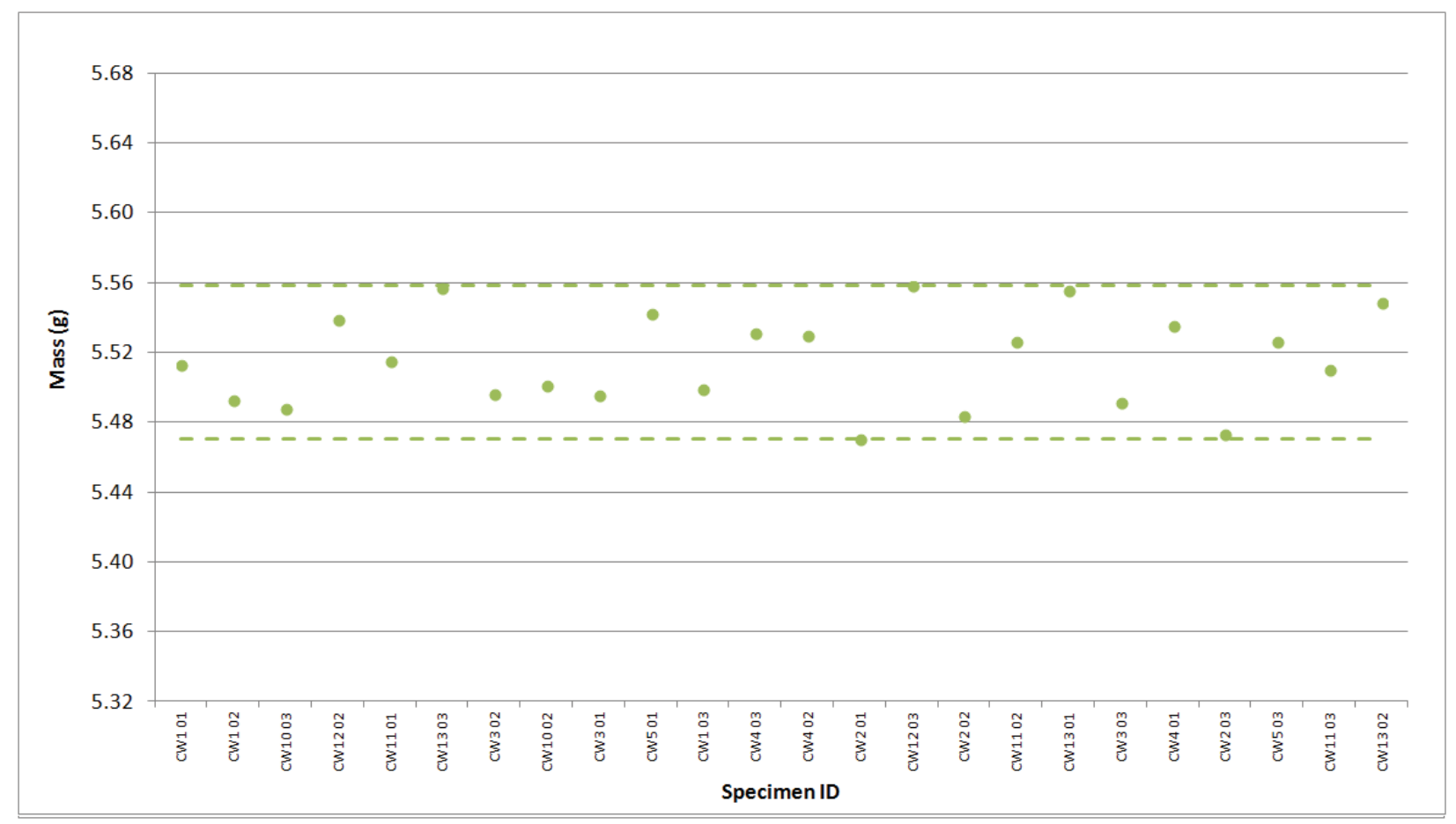

Figure A-64. H-451 Creep Mass. 


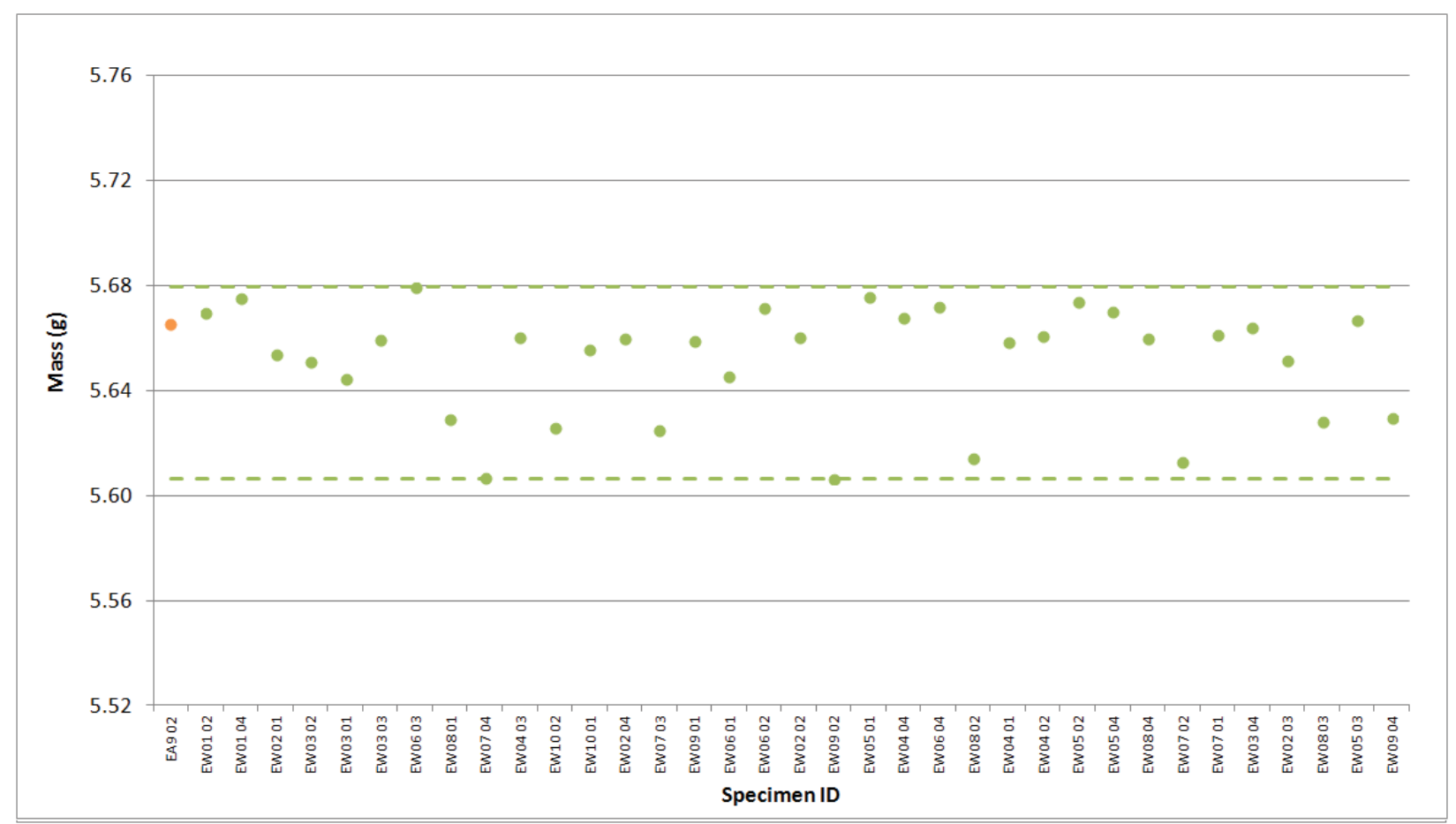

Figure A-65. IG-110 Creep Mass.

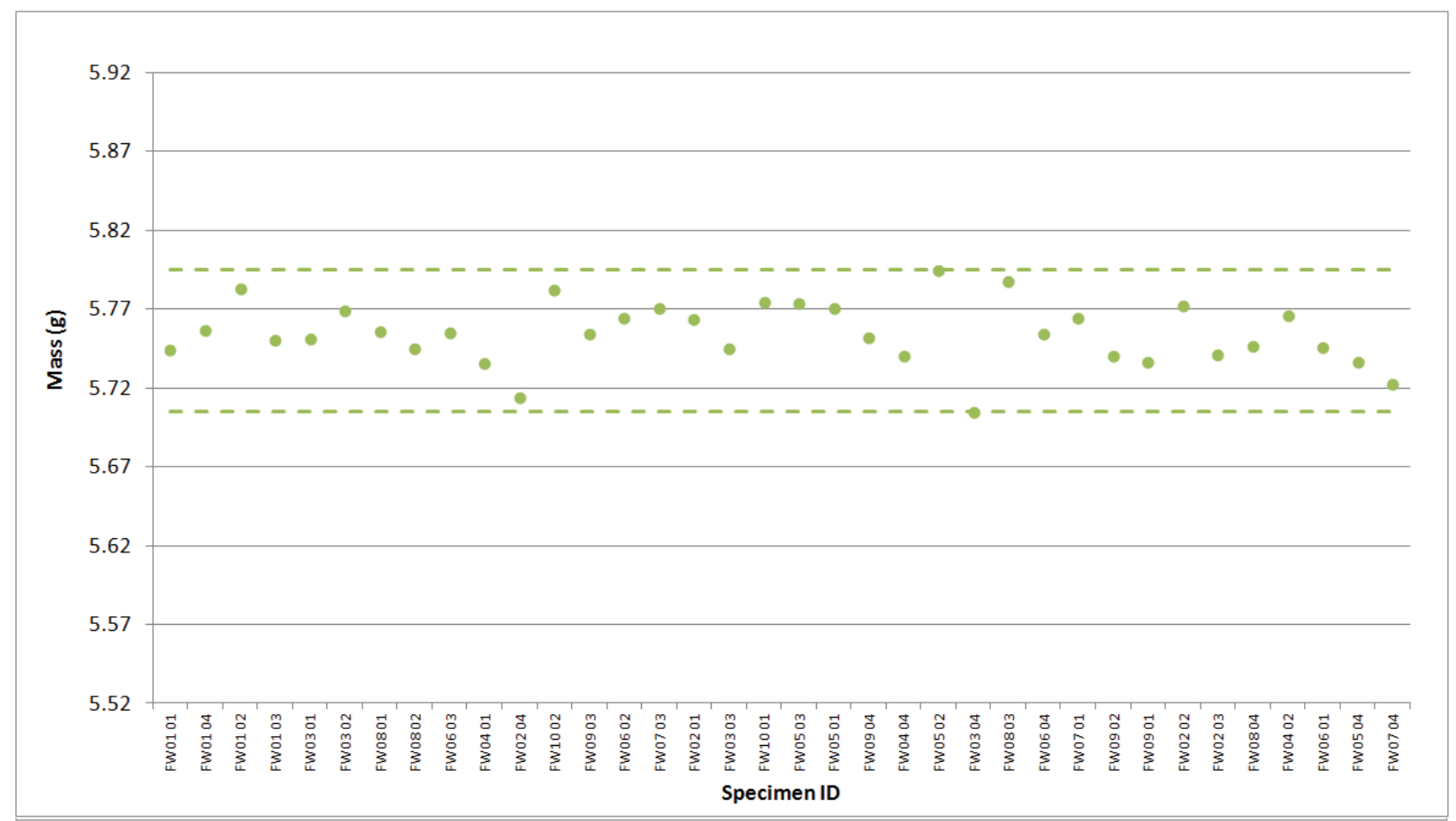

Figure A-66. IG-430 Creep Mass. 


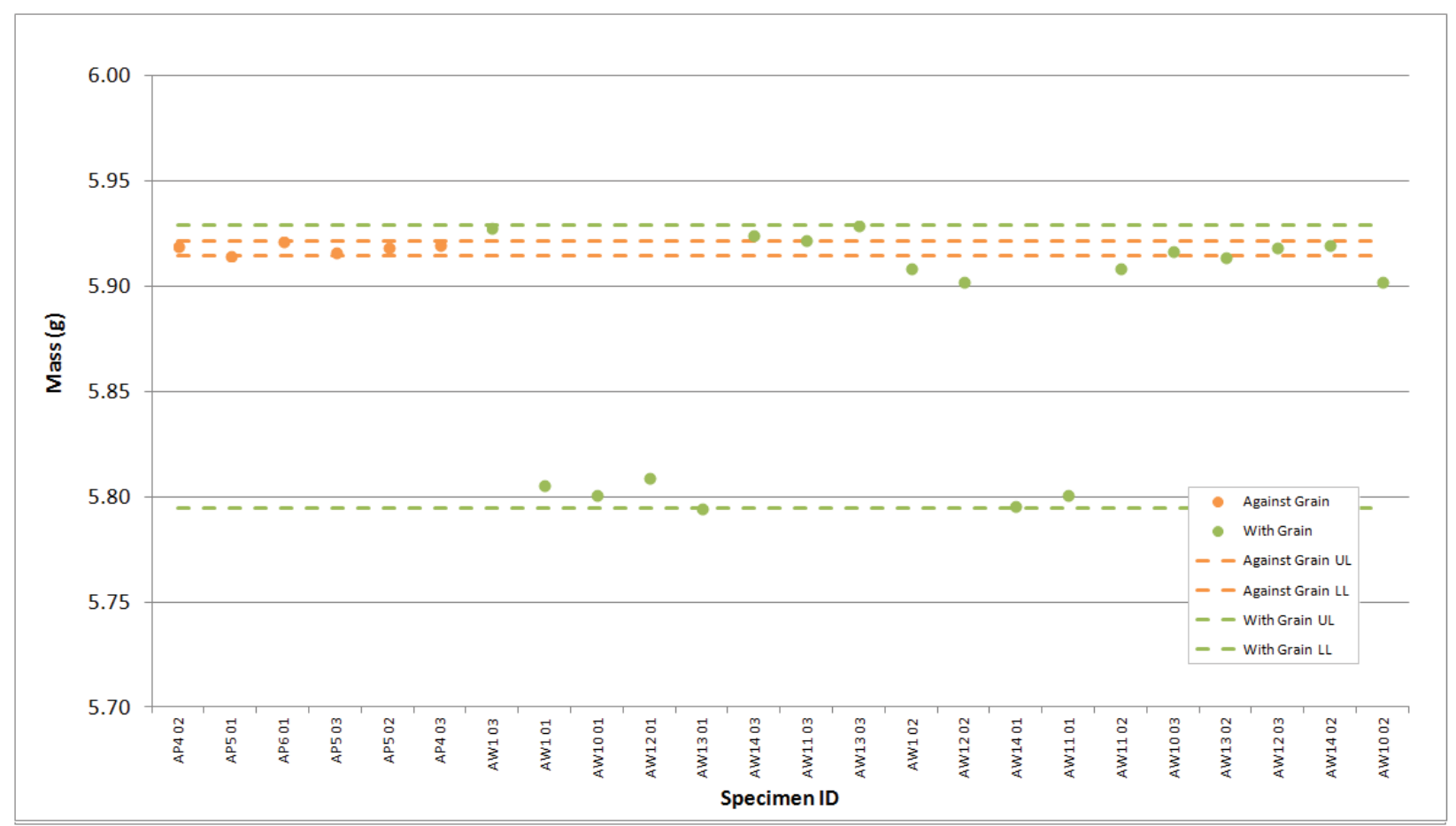

Figure A-67. NBG-17 Creep Mass.

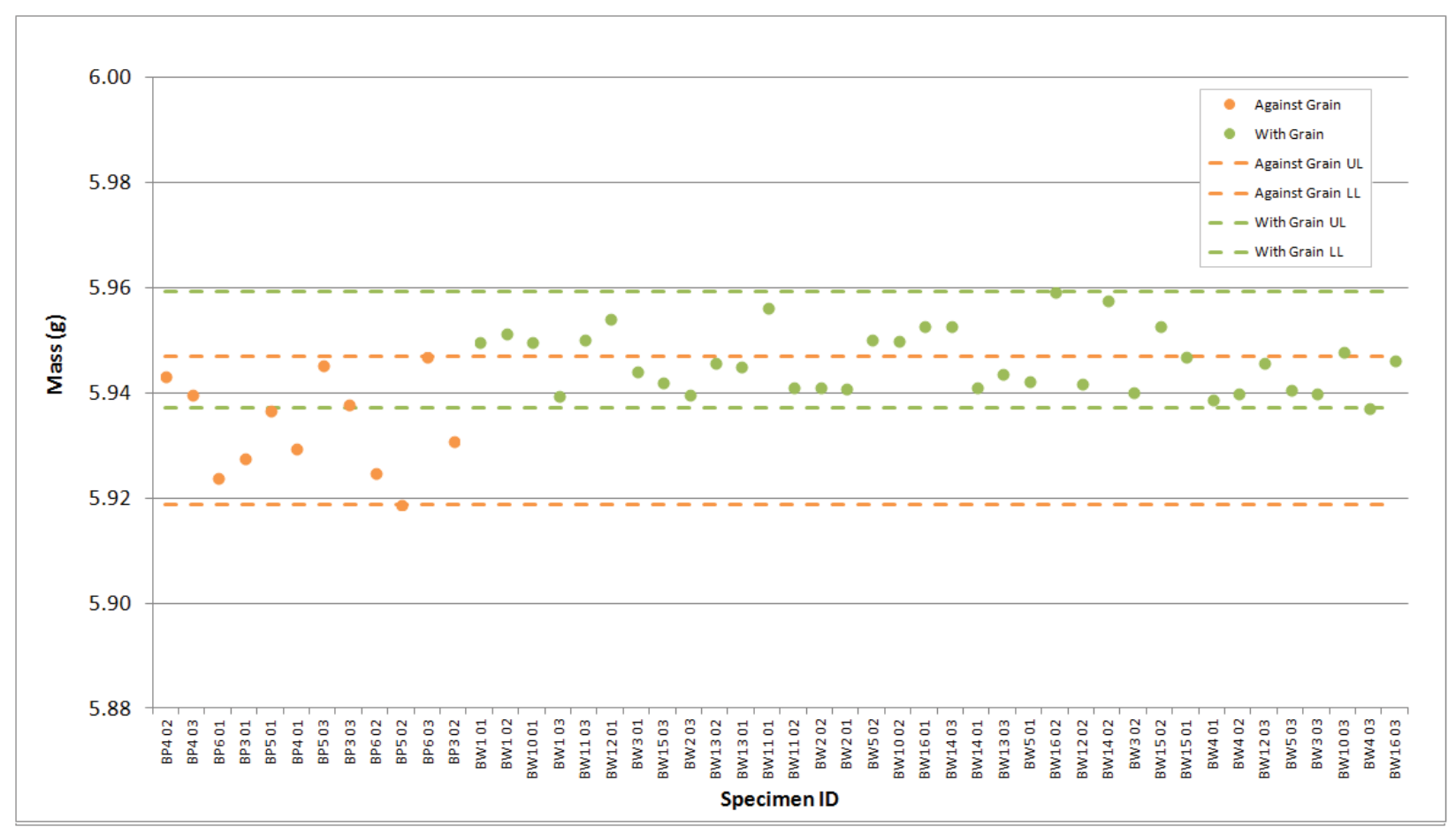

Figure A-68. NBG-18 Creep Mass. 


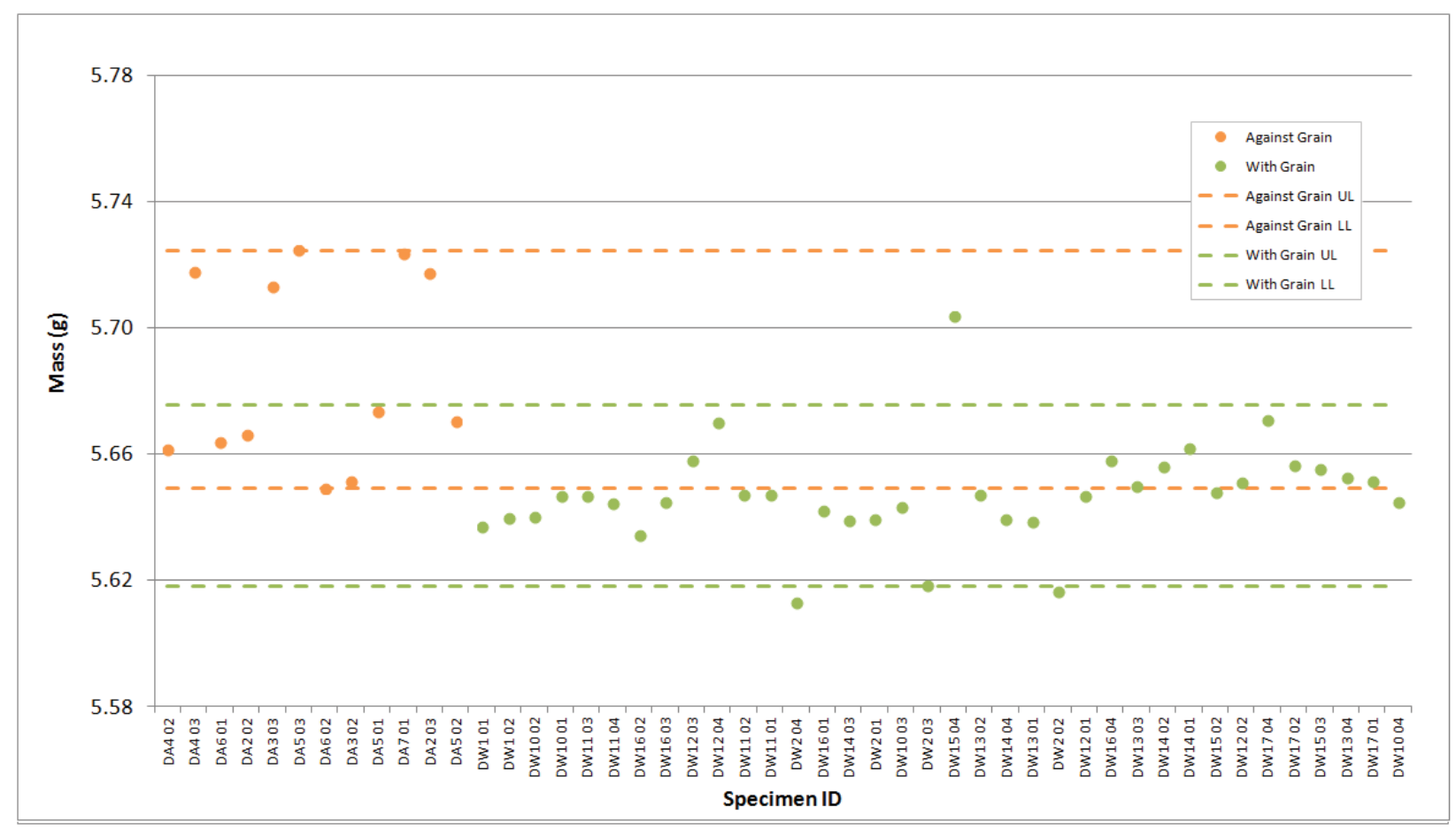

Figure A-69. PCEA Creep Mass.

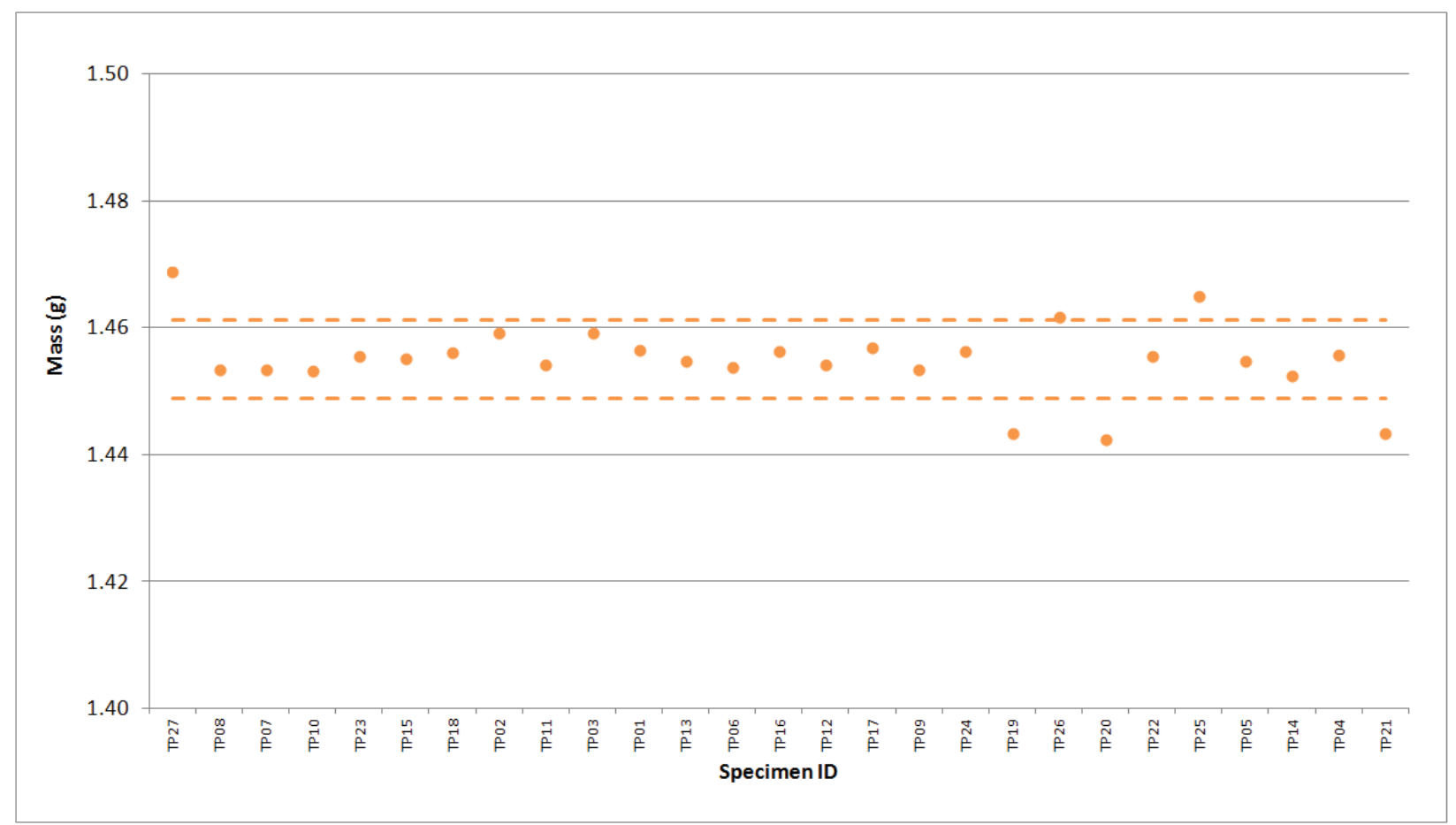

Figure A-70. 2114 Piggyback Mass. 


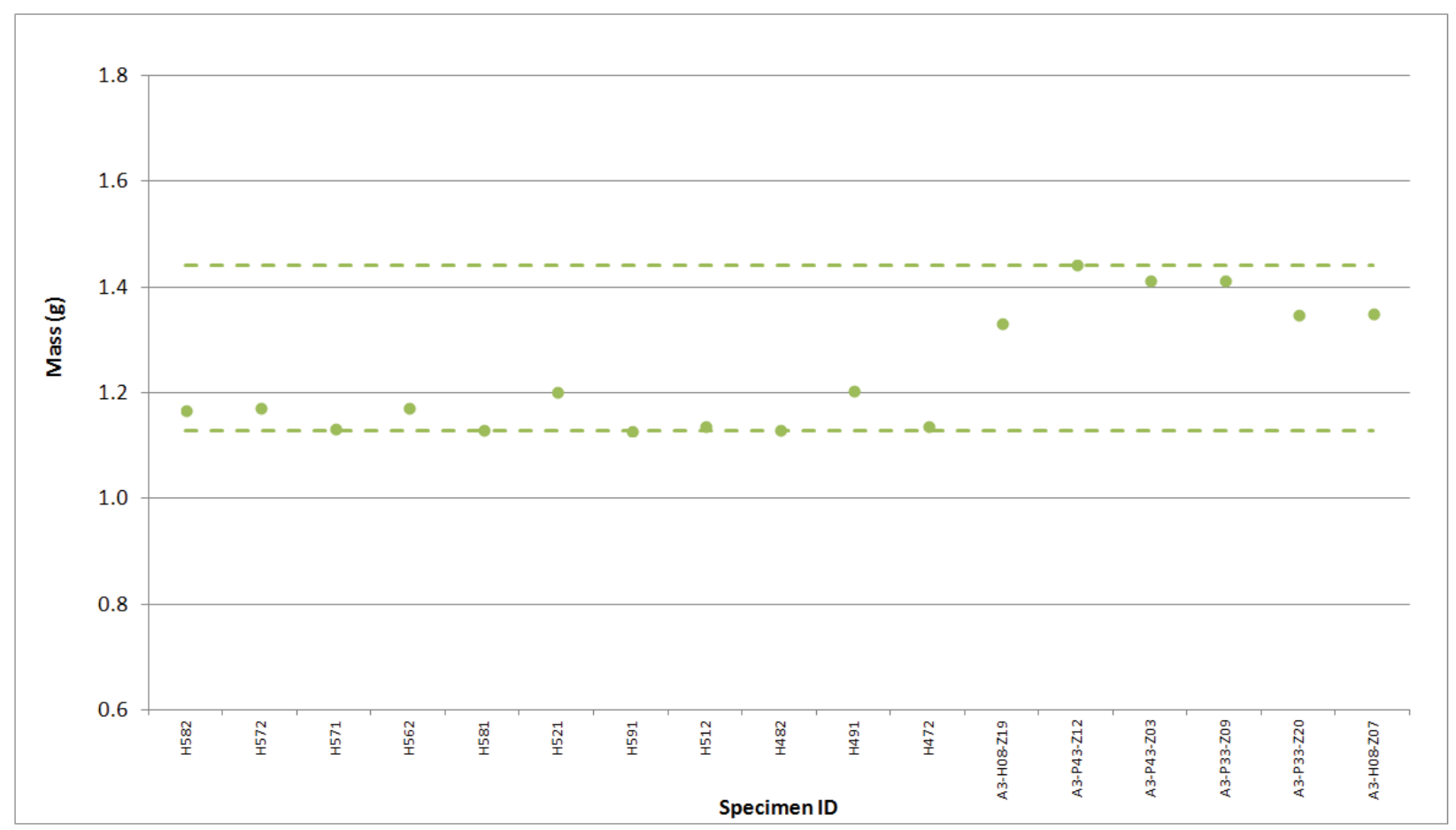

Figure A-71. A3 Piggyback Mass.

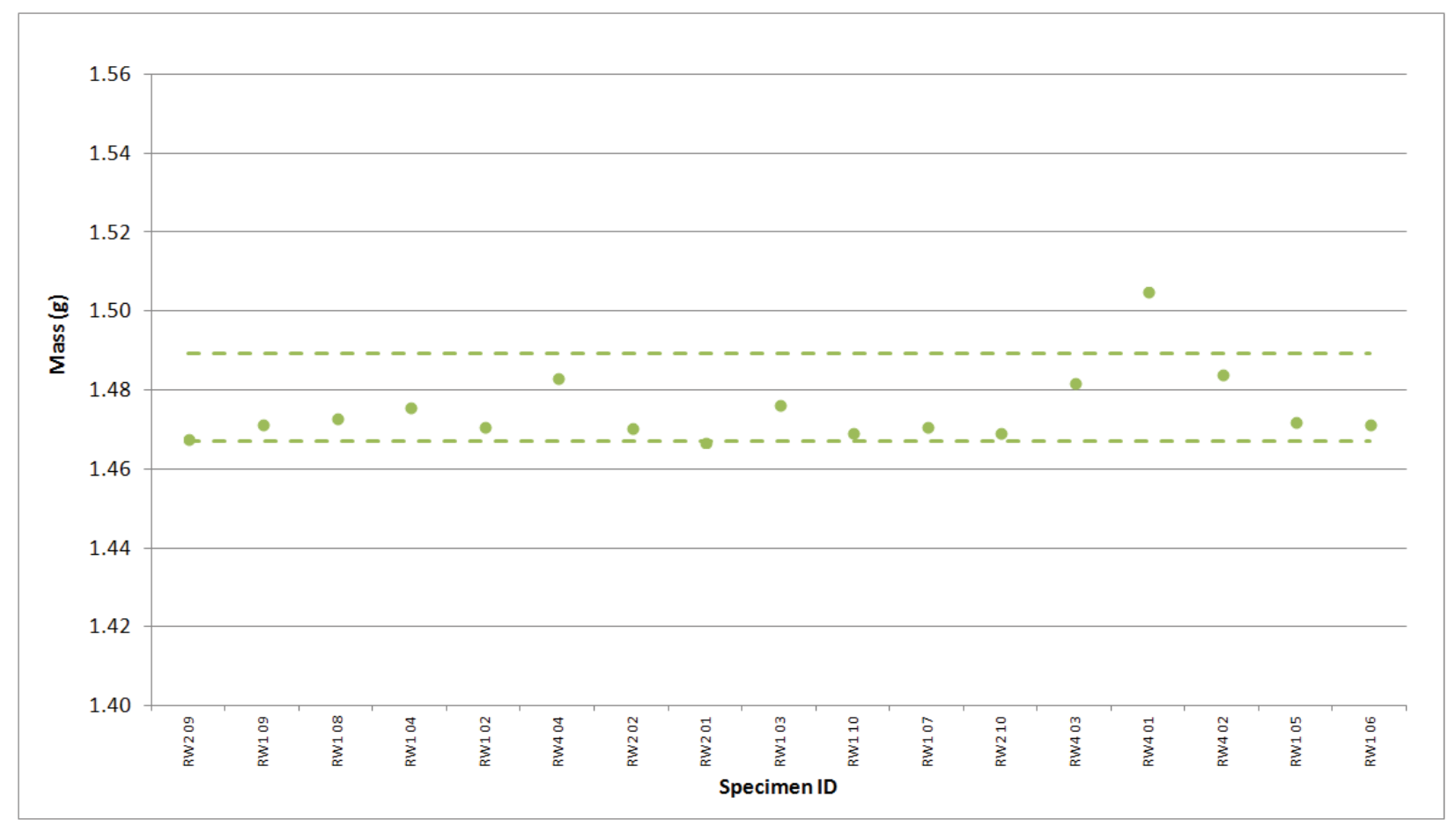

Figure A-72. BAN Piggyback Mass. 


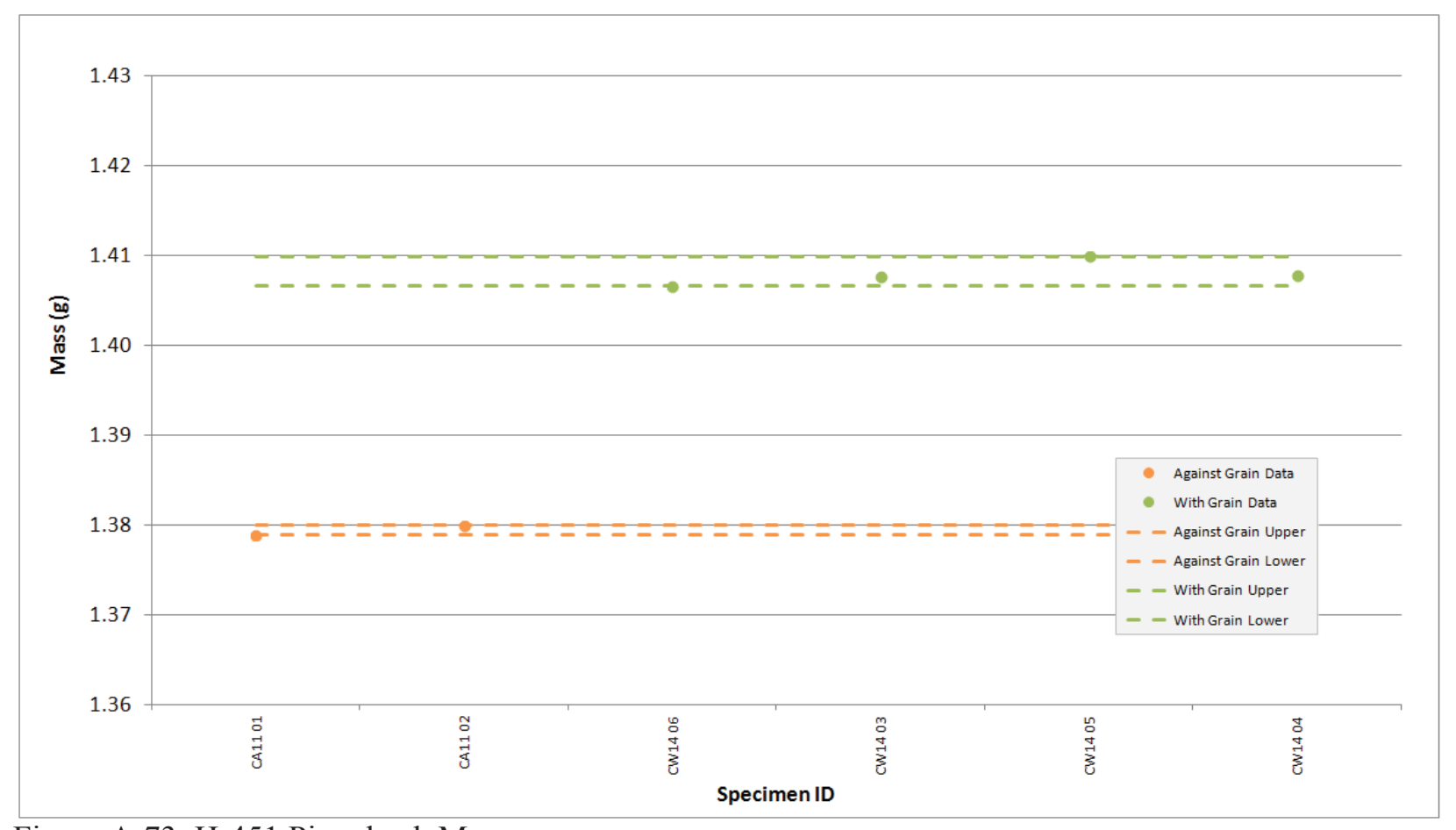

Figure A-73. H-451 Piggyback Mass.

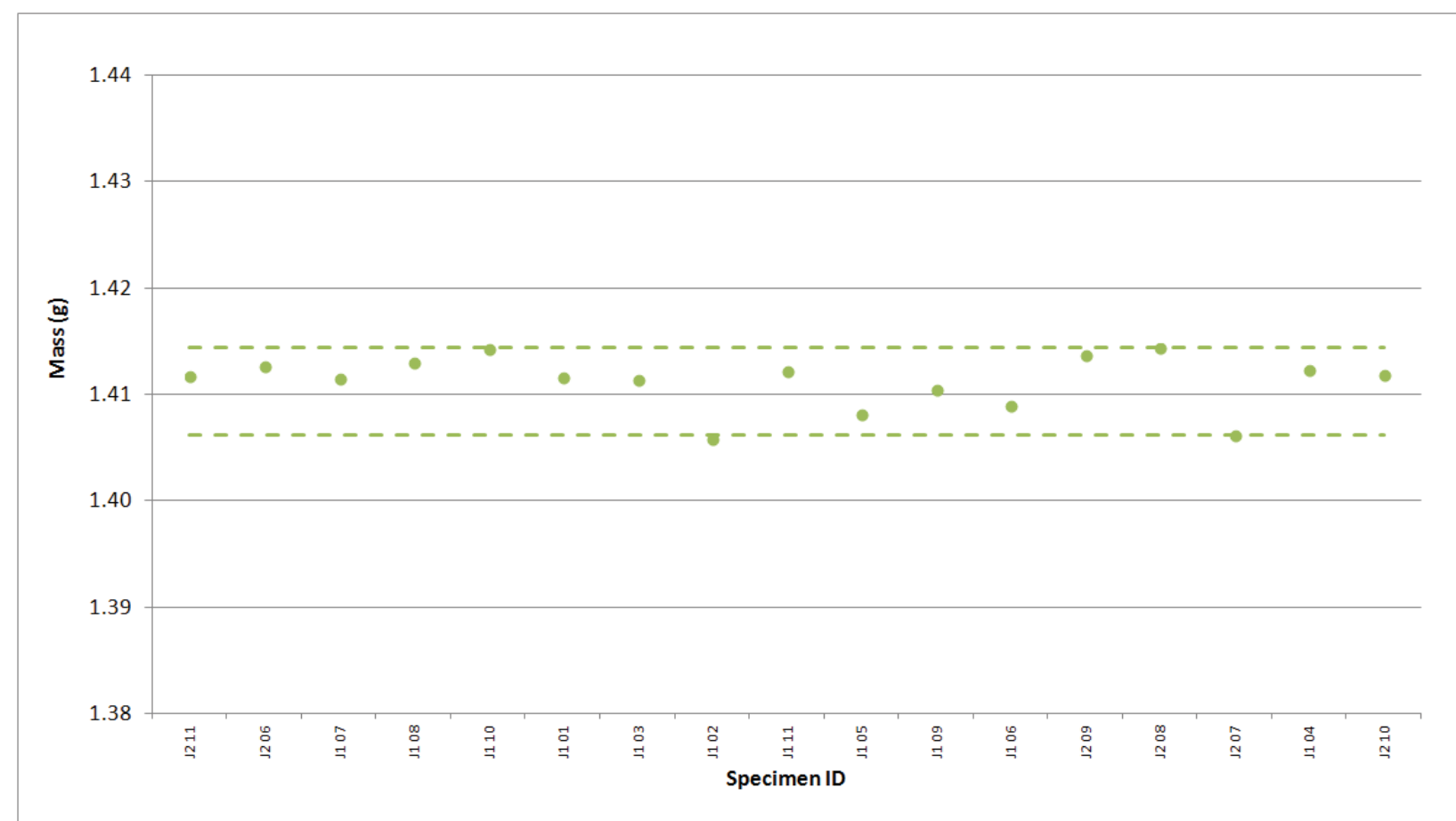

Figure A-74. HLM Piggyback Mass. 


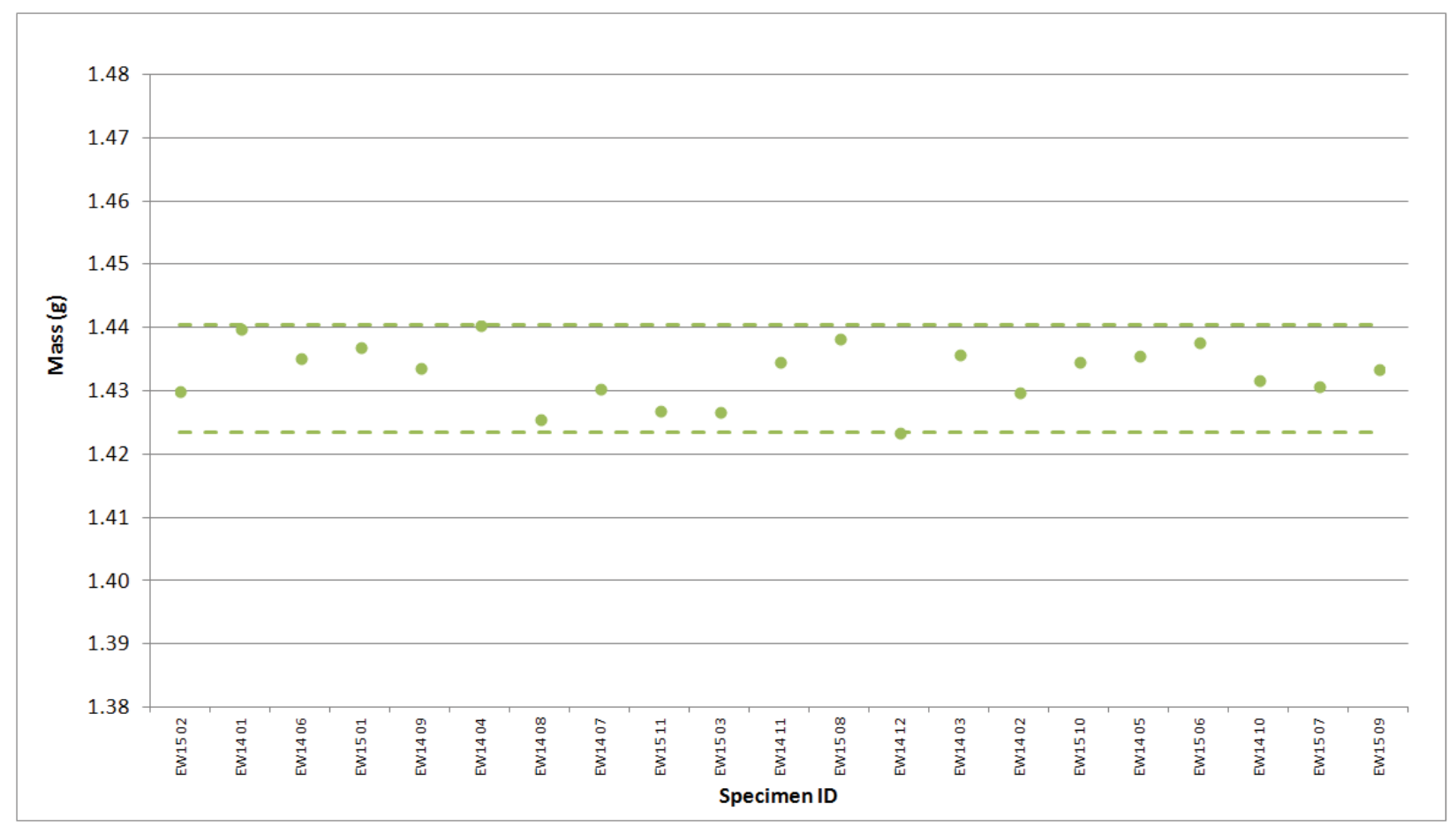

Figure A-75. IG-110 Piggyback Mass.

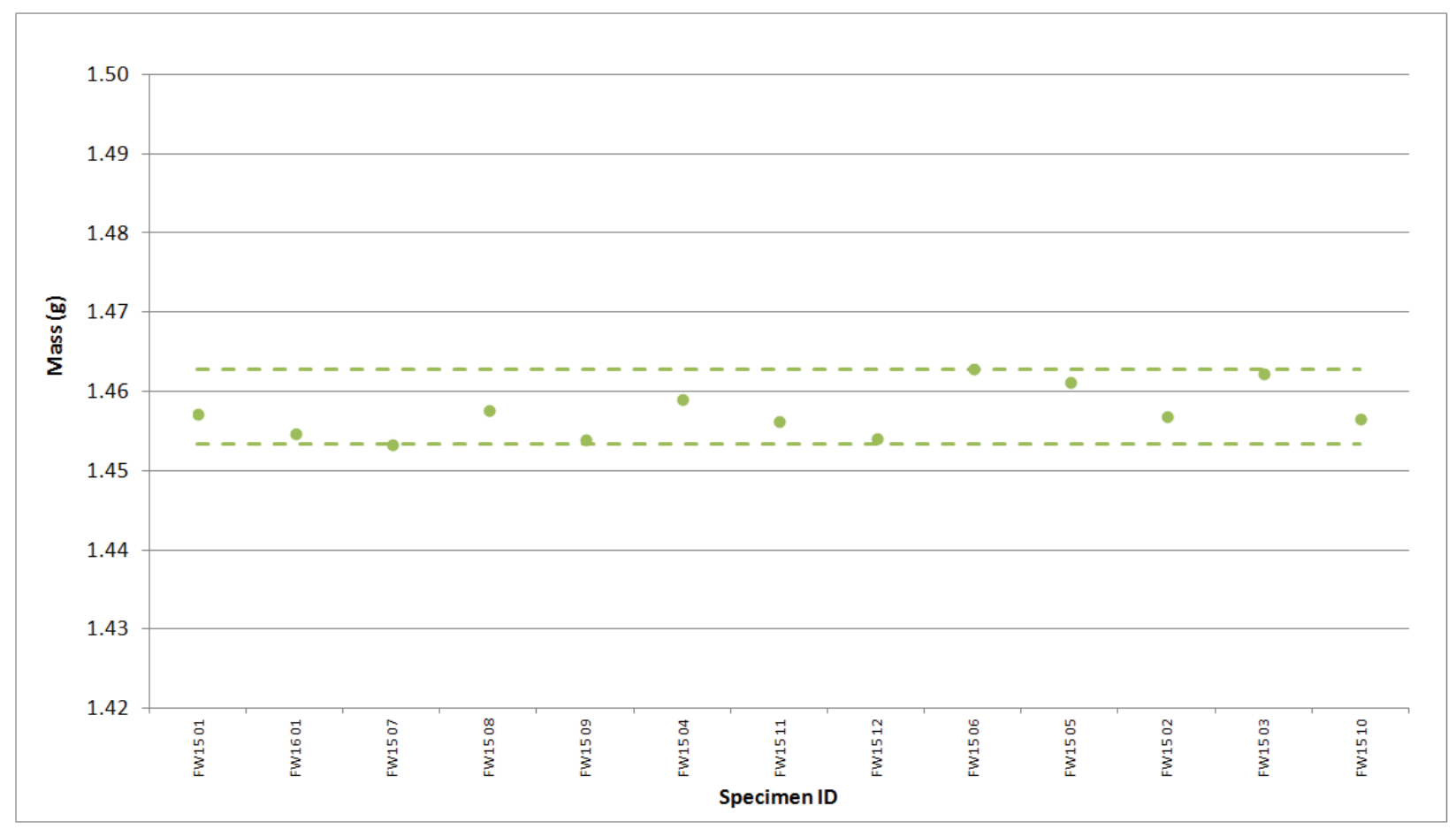

Figure A-76. IG-430 Piggyback Mass. 


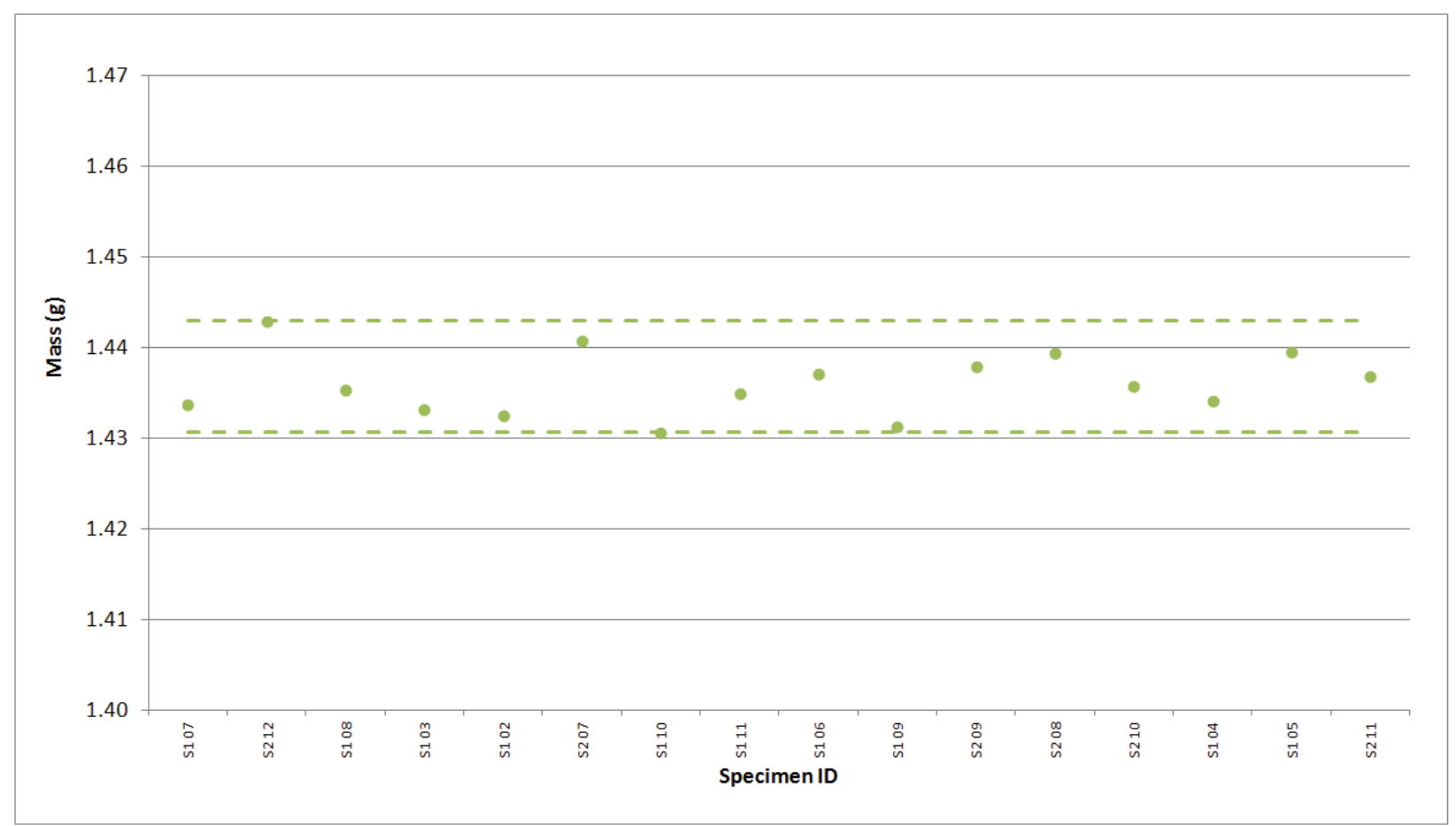

Figure A-77. NBG-10 Piggyback Mass.

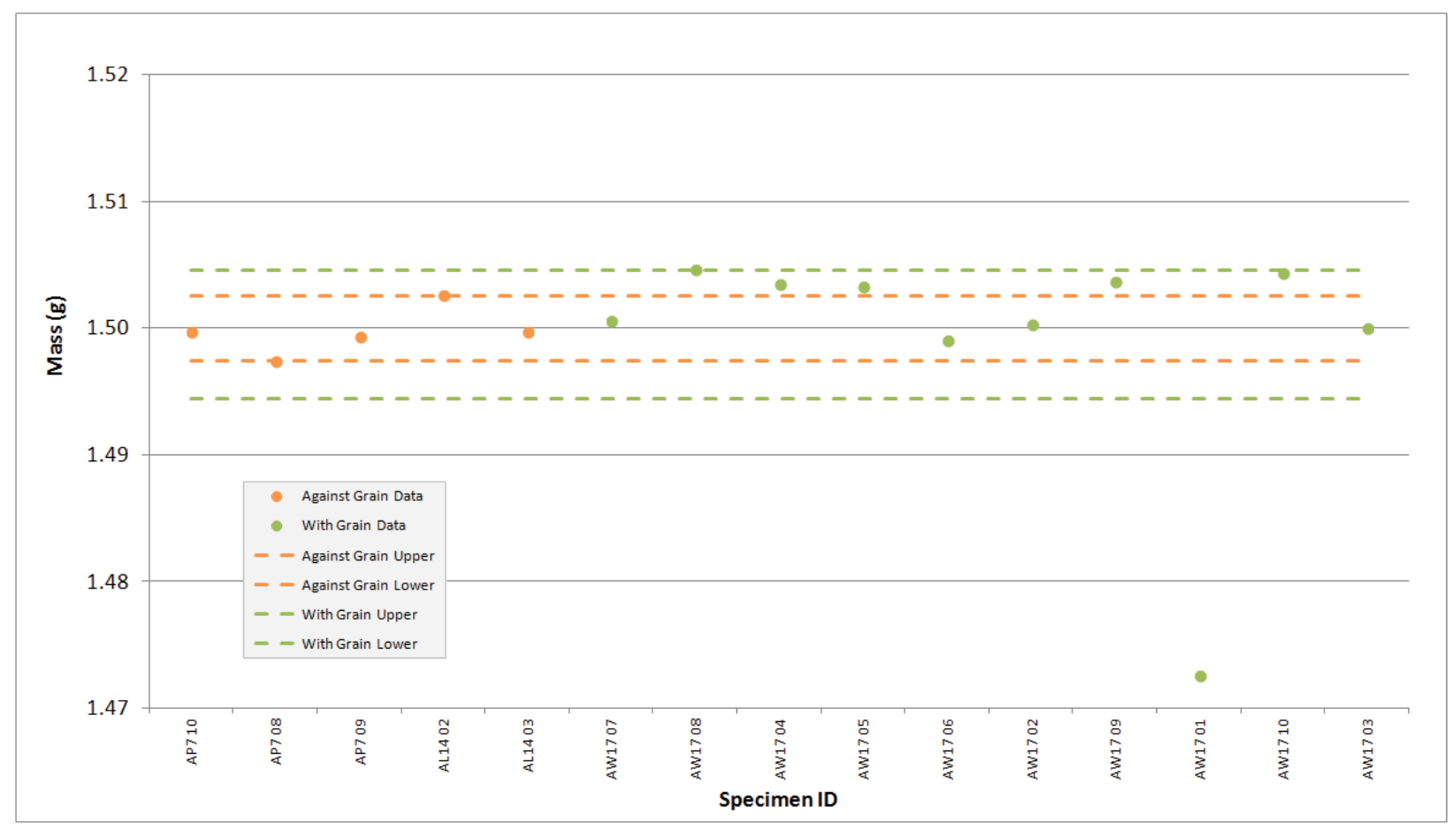

Figure A-78. NBG-17 Piggyback Mass. 


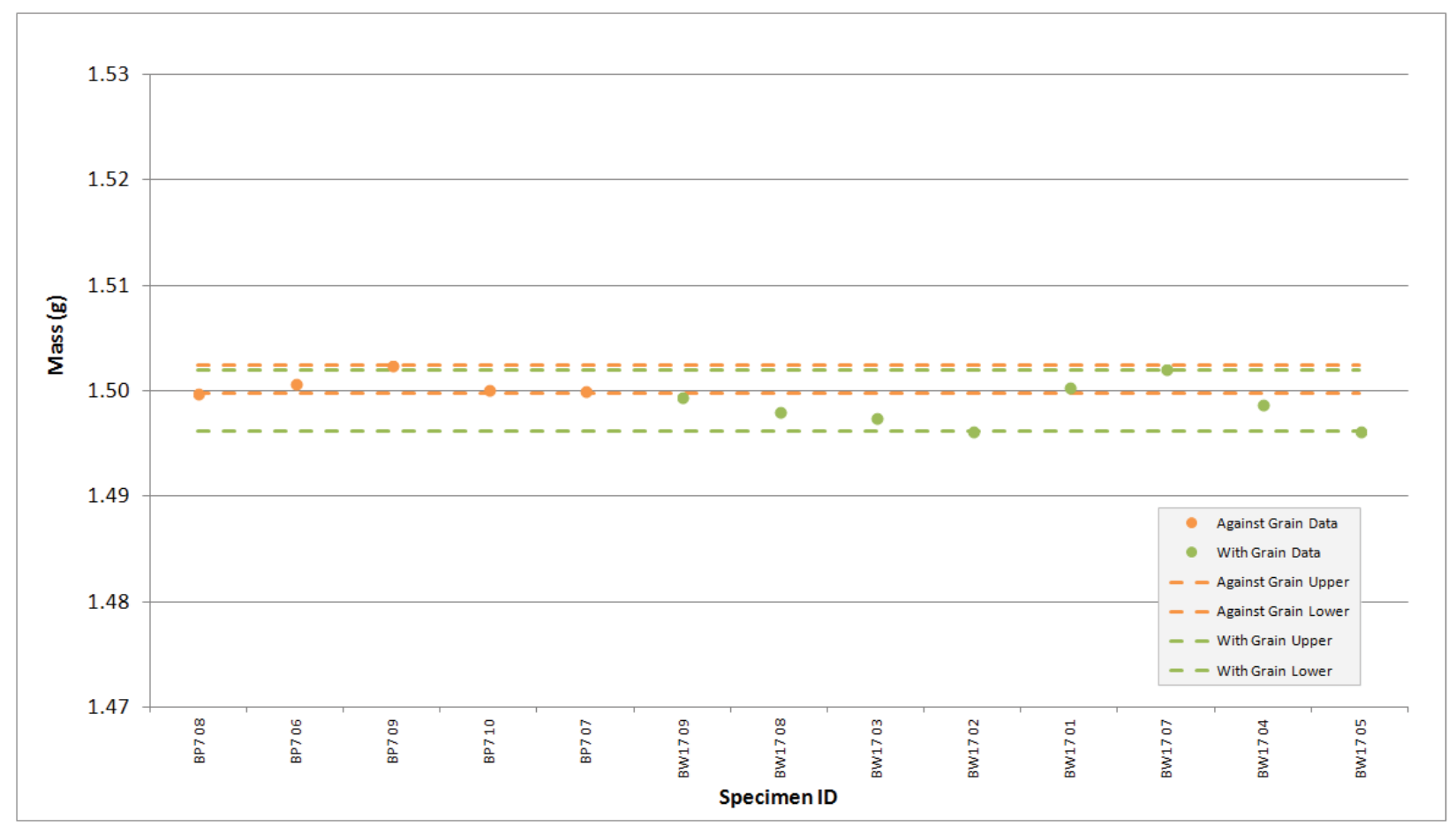

Figure A-79. NBG-18 Piggyback Mass.

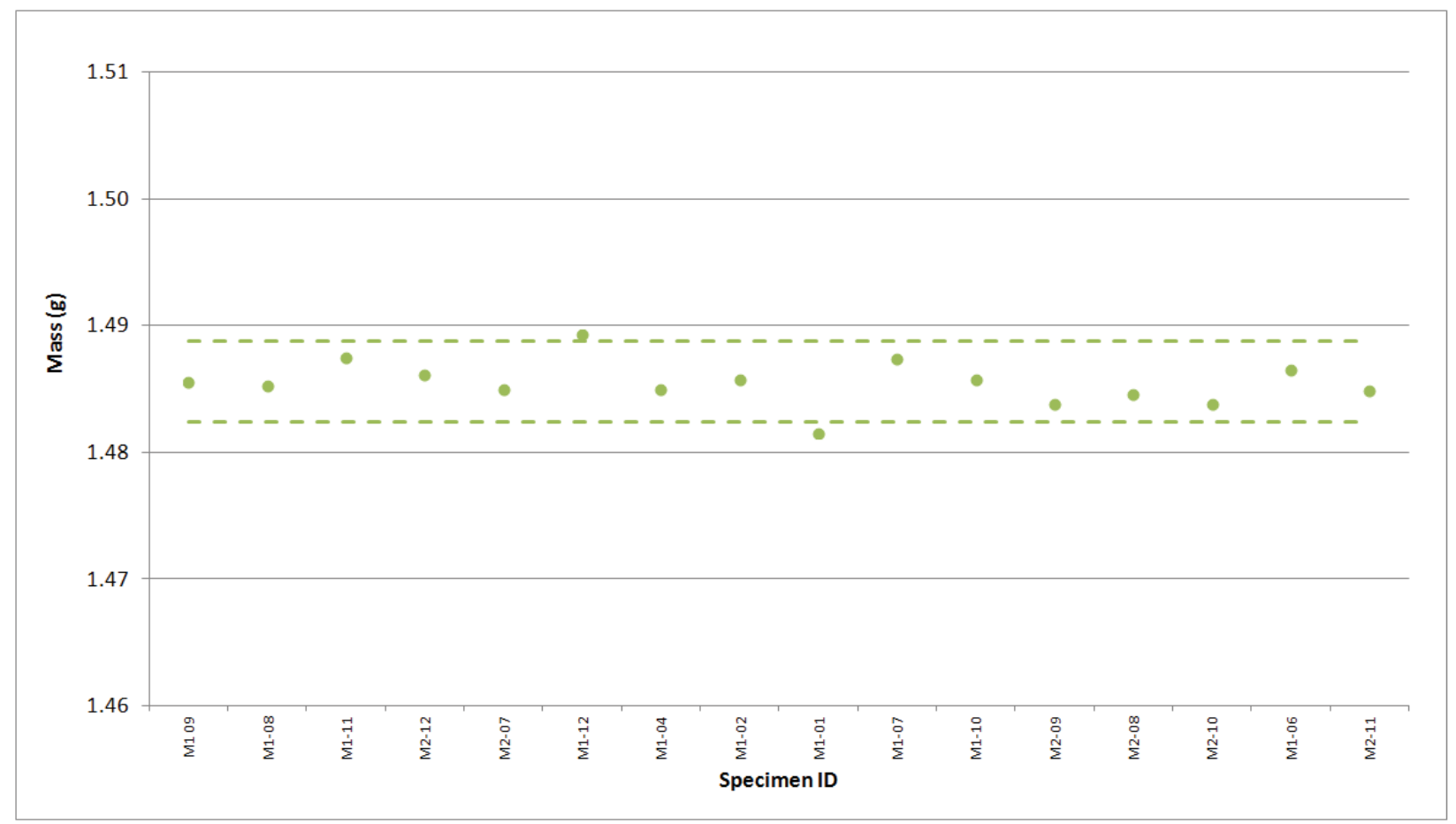

Figure A-80. NBG-25 Piggyback Mass. 


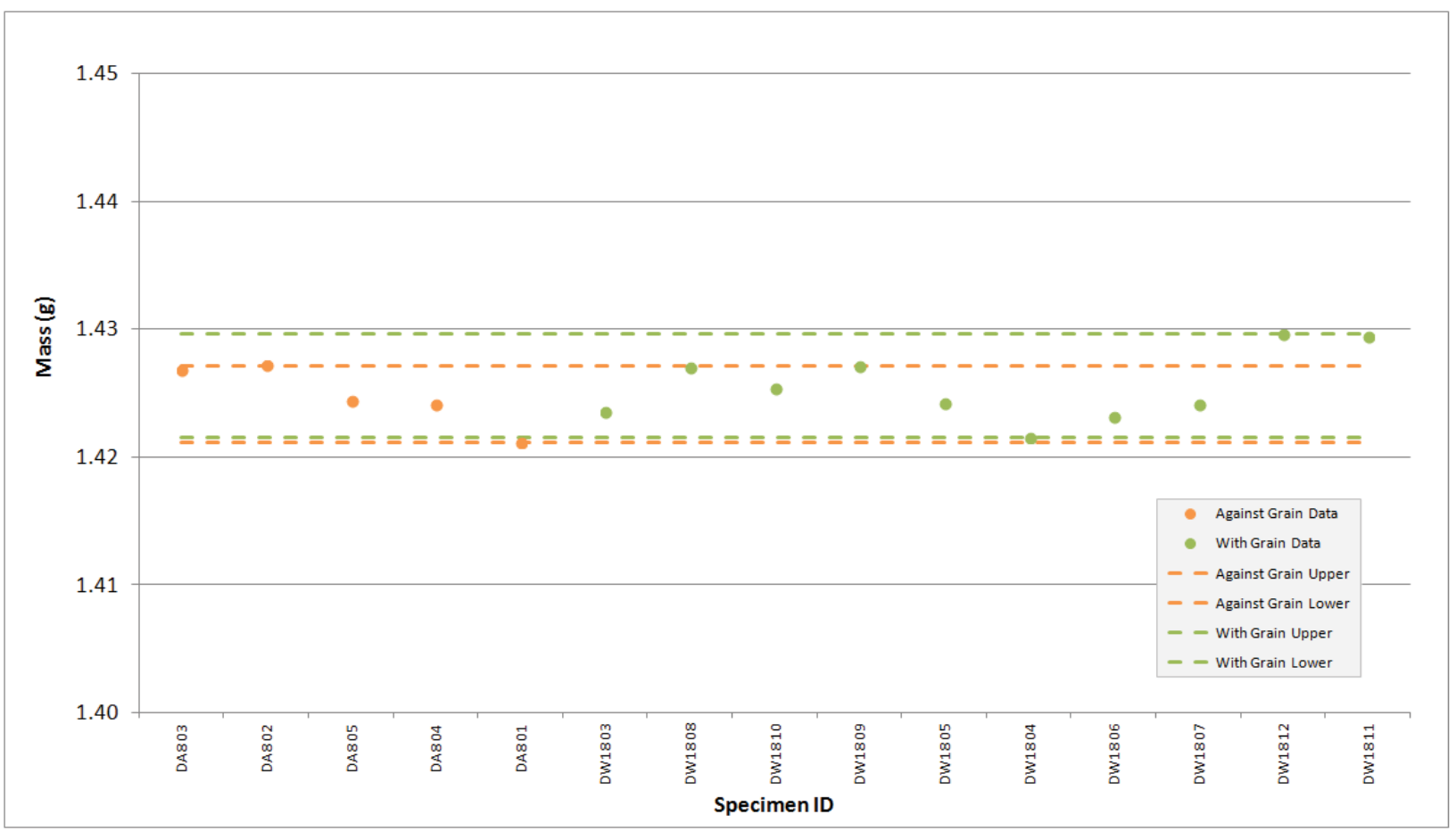

Figure A-81. PCEA Piggyback Mass.

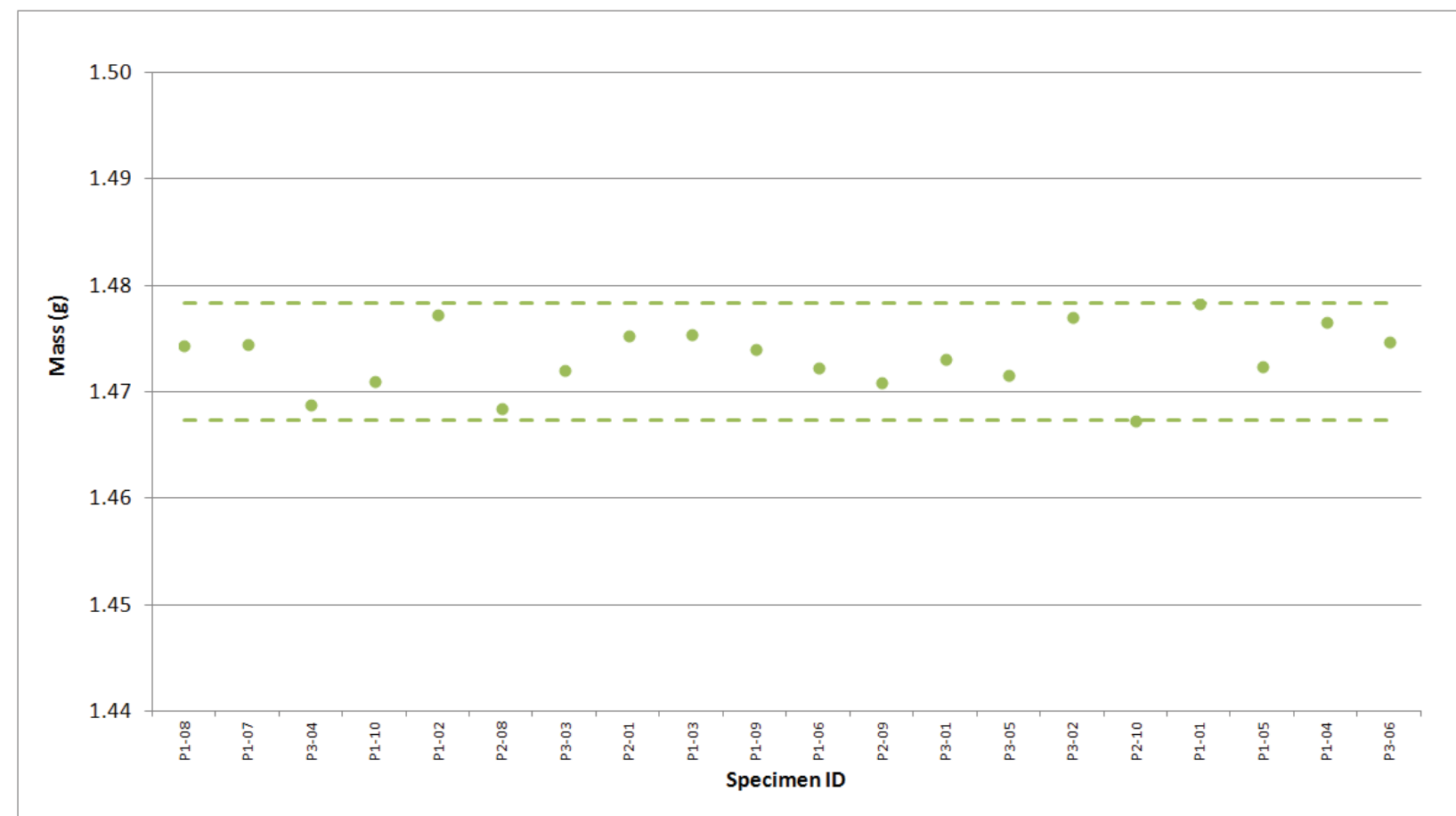

Figure A-82. PCIB Piggyback Mass. 


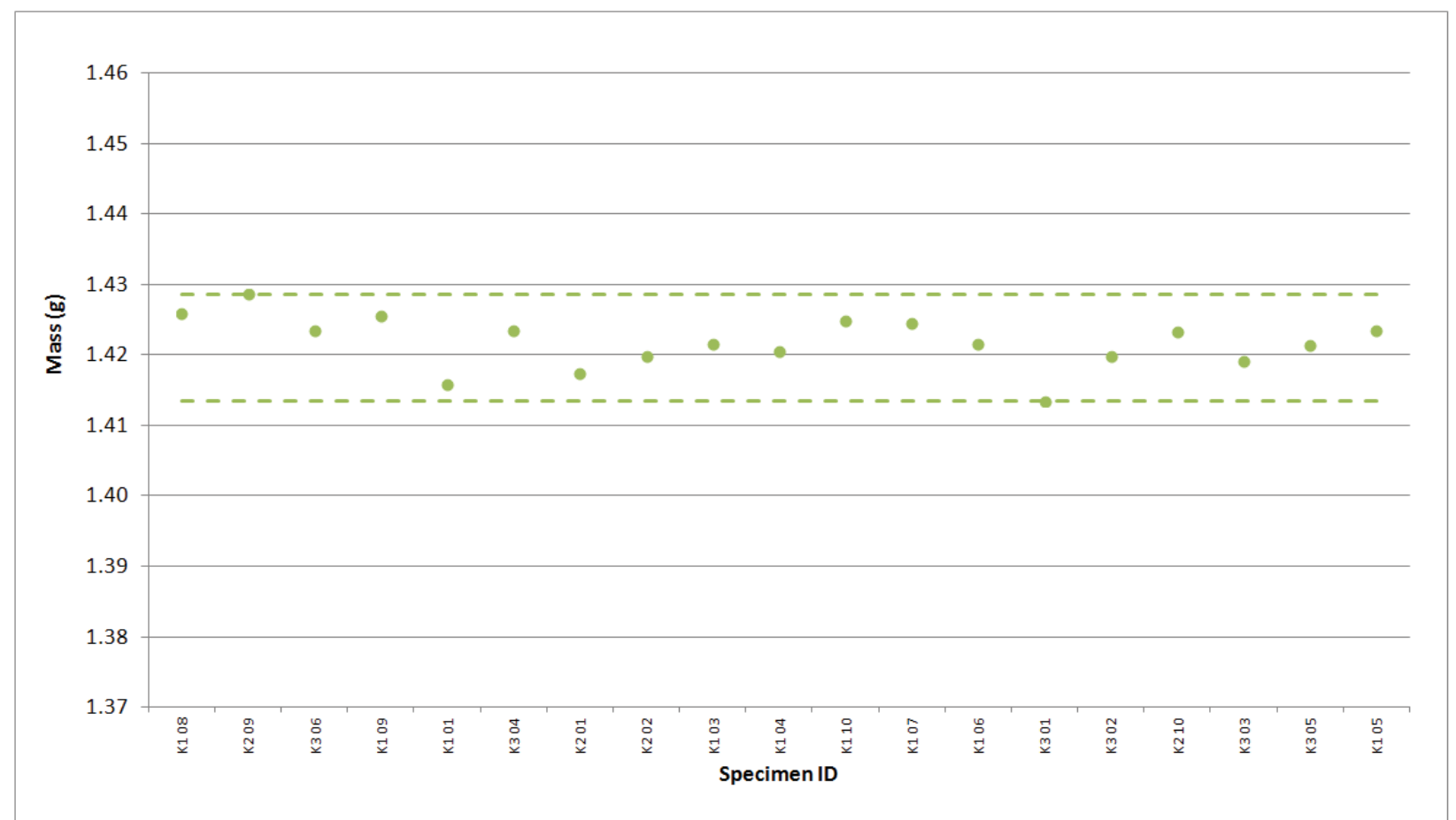

Figure A-83. PGX Piggyback Mass.

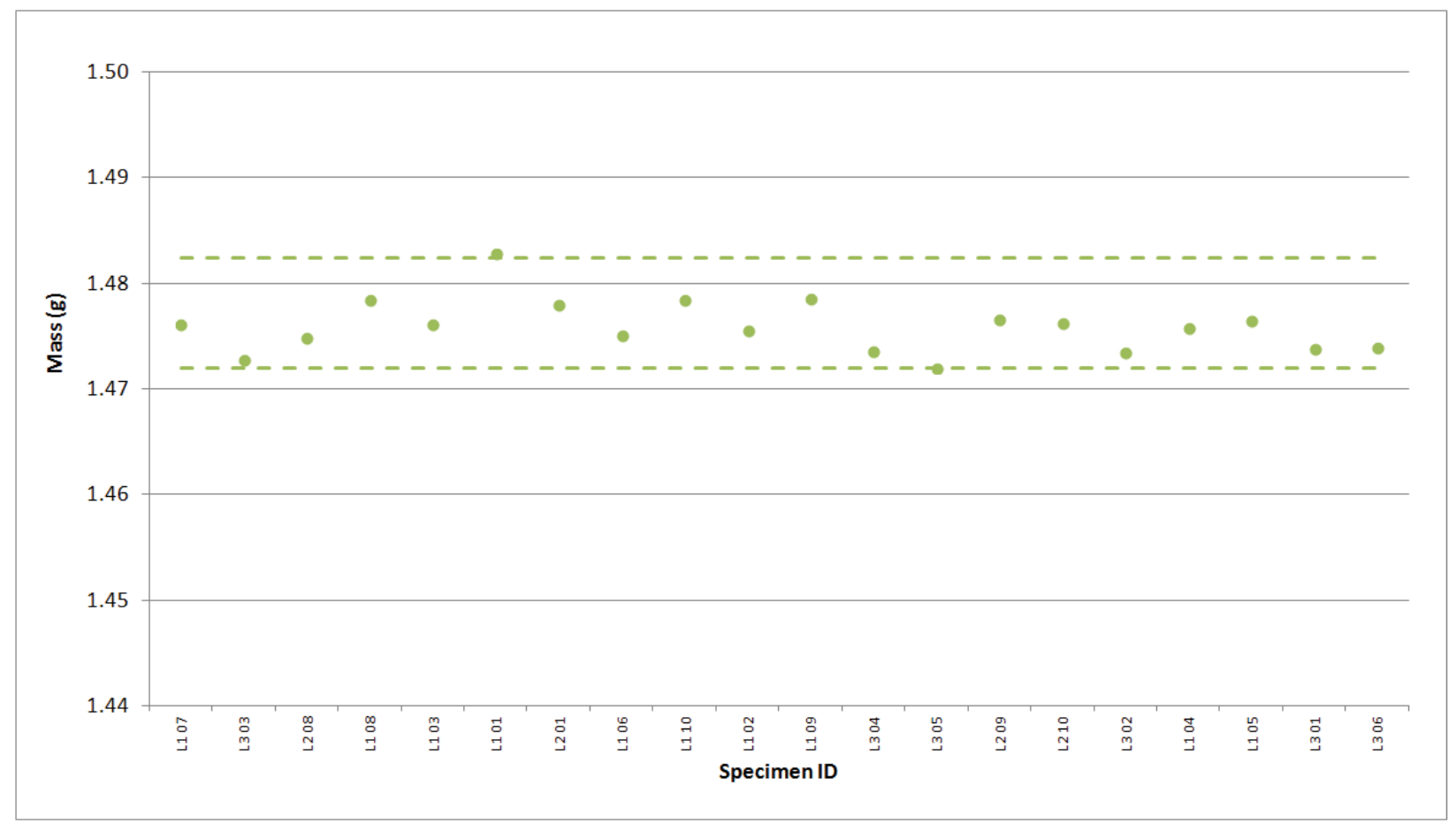

Figure A-84. PPEA Piggyback Mass. 


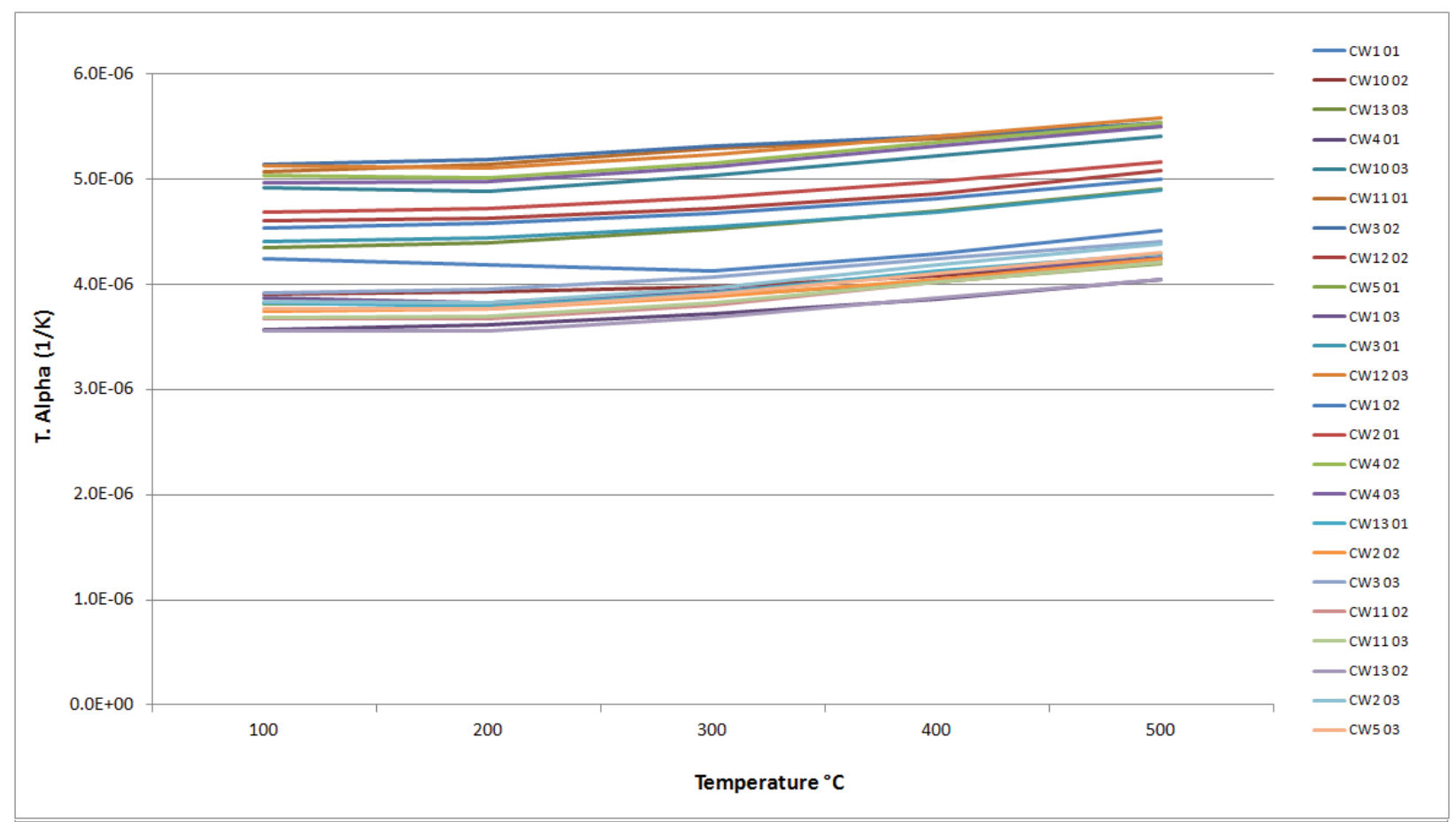

Figure A-85. H-451 Creep Coefficient of Thermal Expansion.

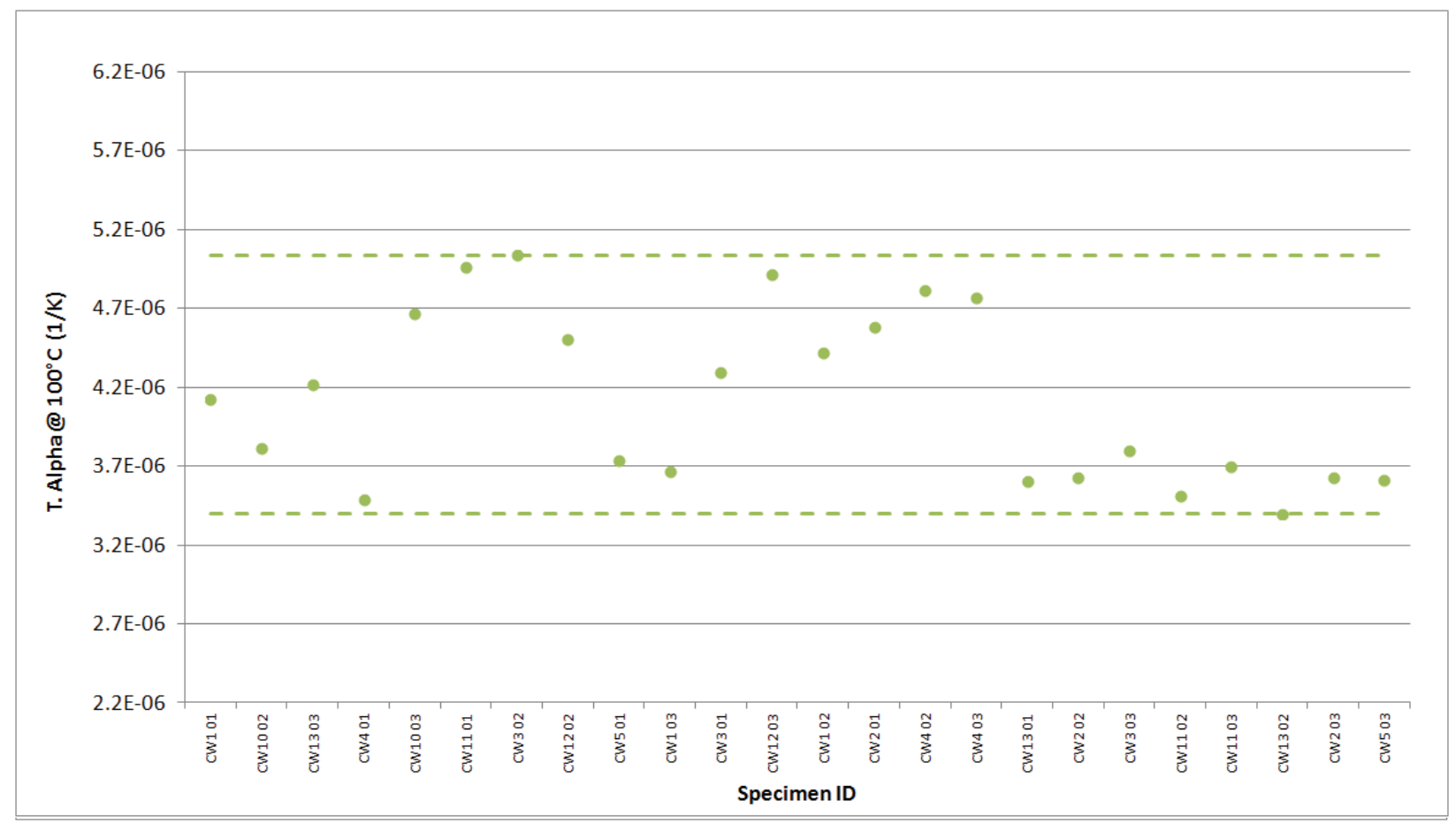

Figure A-86. H-451 Creep Coefficient of Thermal Expansion@100². 


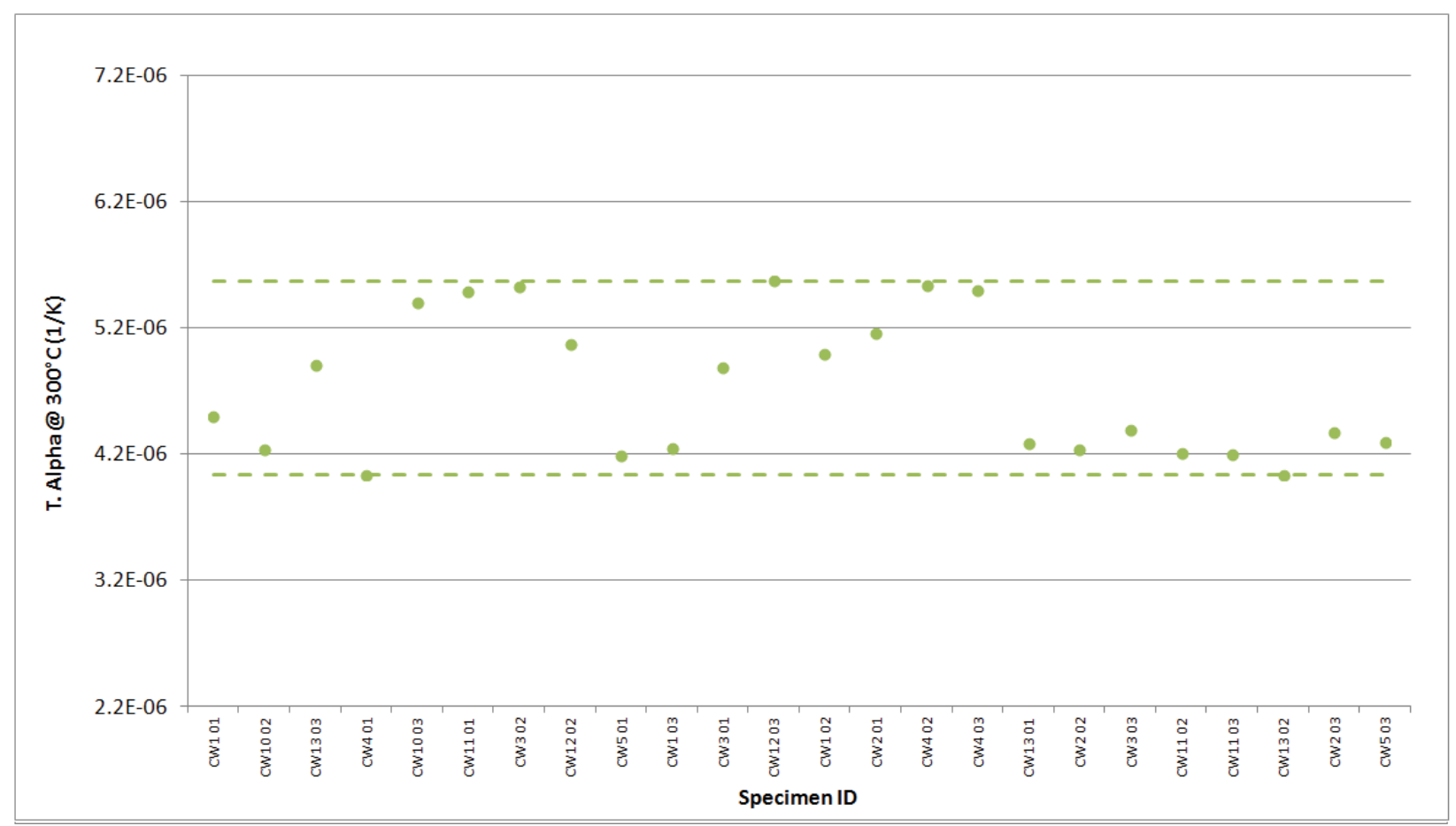

Figure A-87. H-451 Creep Coefficient of Thermal Expansion@ @ 300 C.

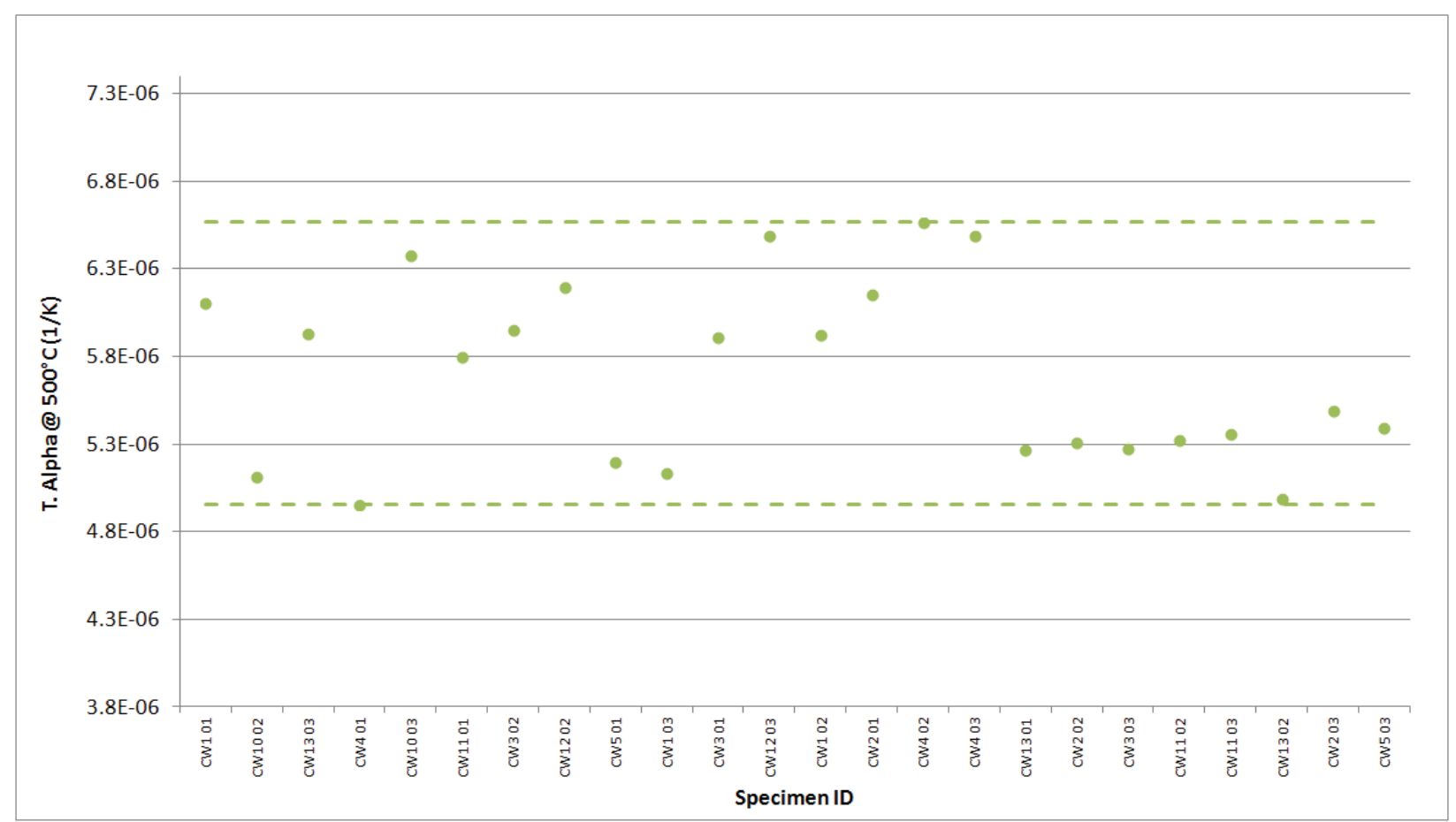

Figure A-88. H-451 Creep Coefficient of Thermal Expansion@ @ 500 ${ }^{\circ}$. 


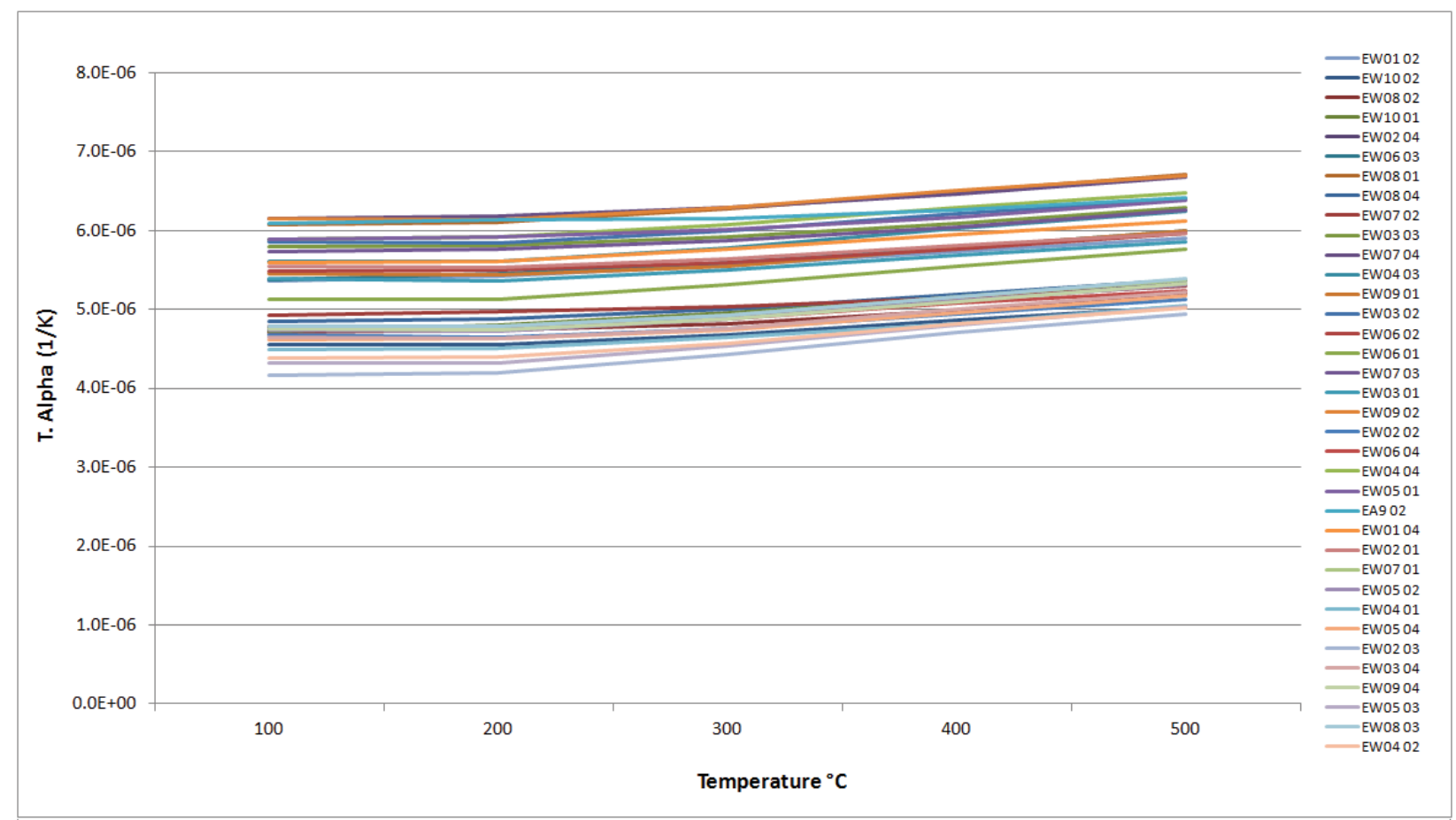

Figure A-89. IG-110 Creep Coefficient of Thermal Expansion.

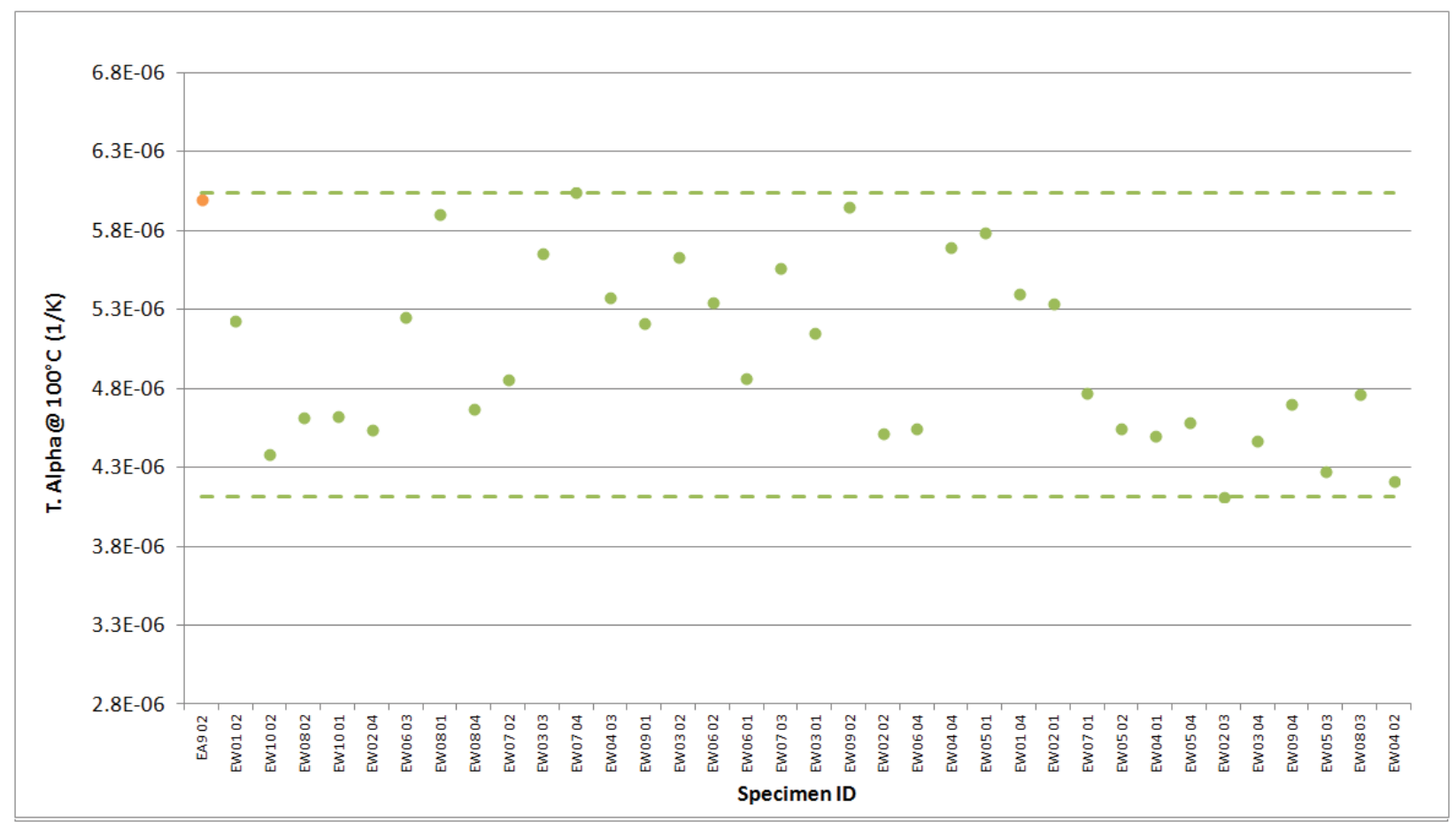

Figure A-90. IG-110 Creep Coefficient of Thermal Expansion @ $100^{\circ} \mathrm{C}$. 


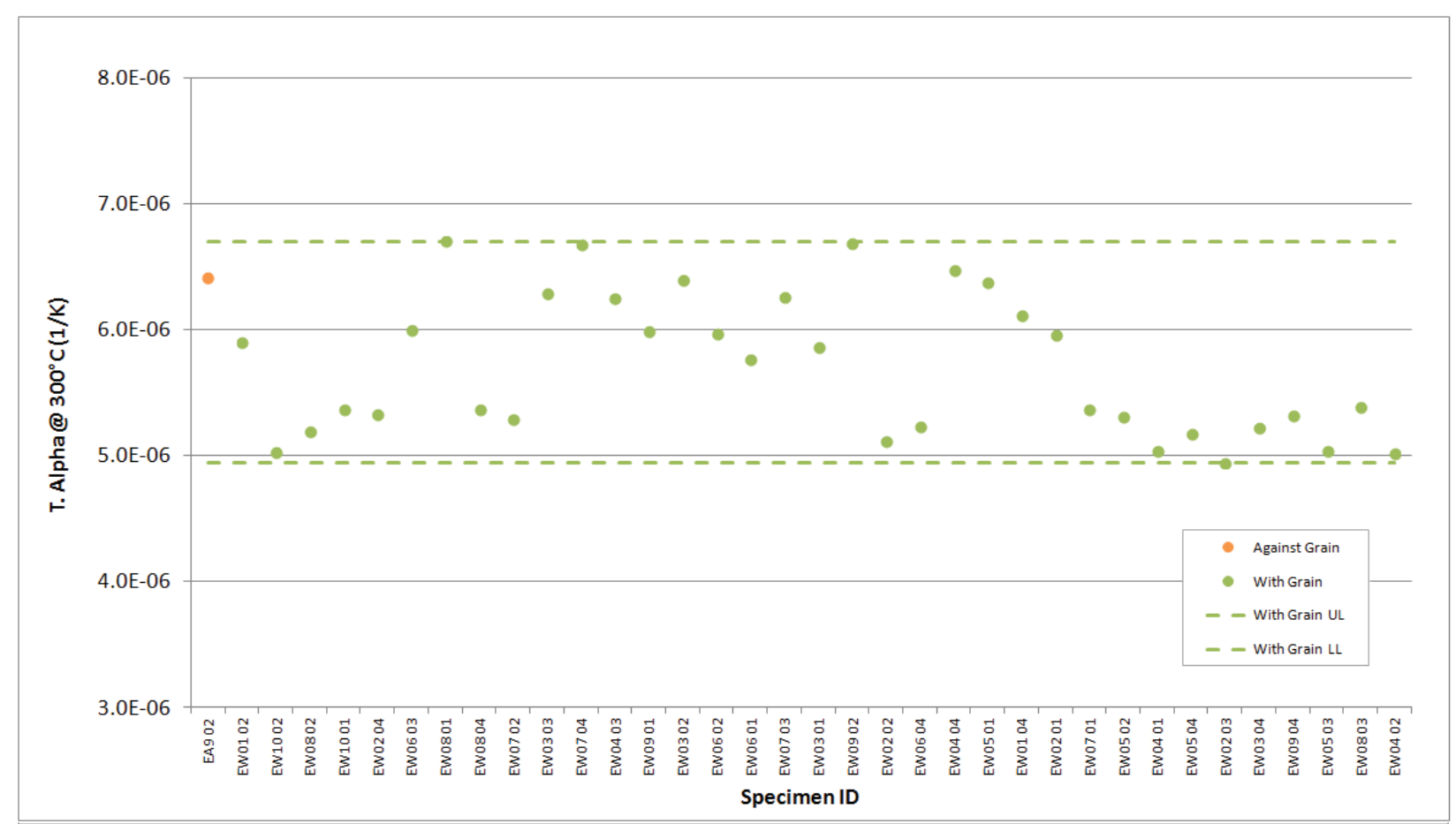

Figure A-91. IG-110 Creep Coefficient of Thermal Expansion @ $300^{\circ} \mathrm{C}$.

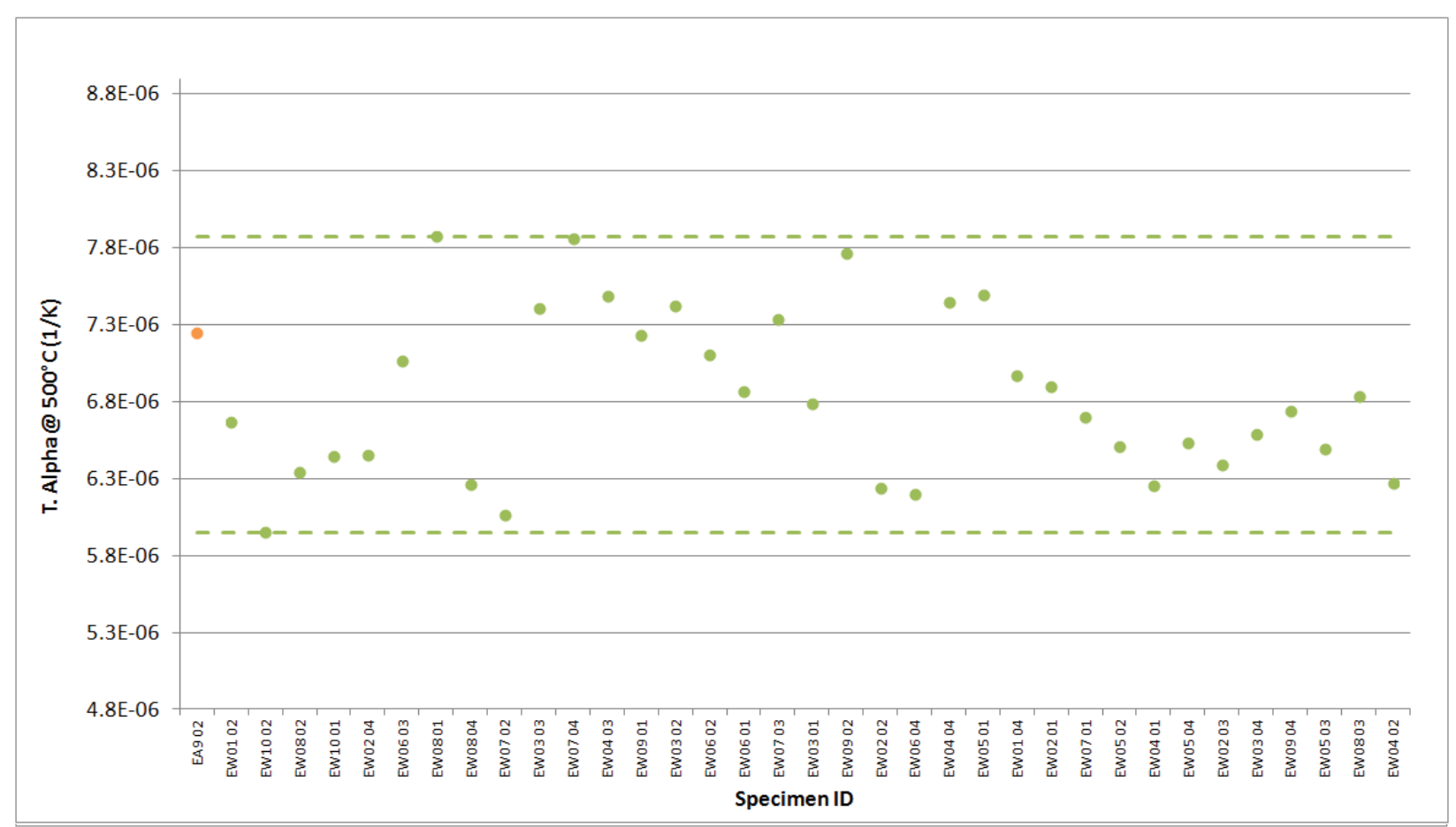

Figure A-92. IG-110 Creep Coefficient of Thermal Expansion @ $500^{\circ} \mathrm{C}$. 


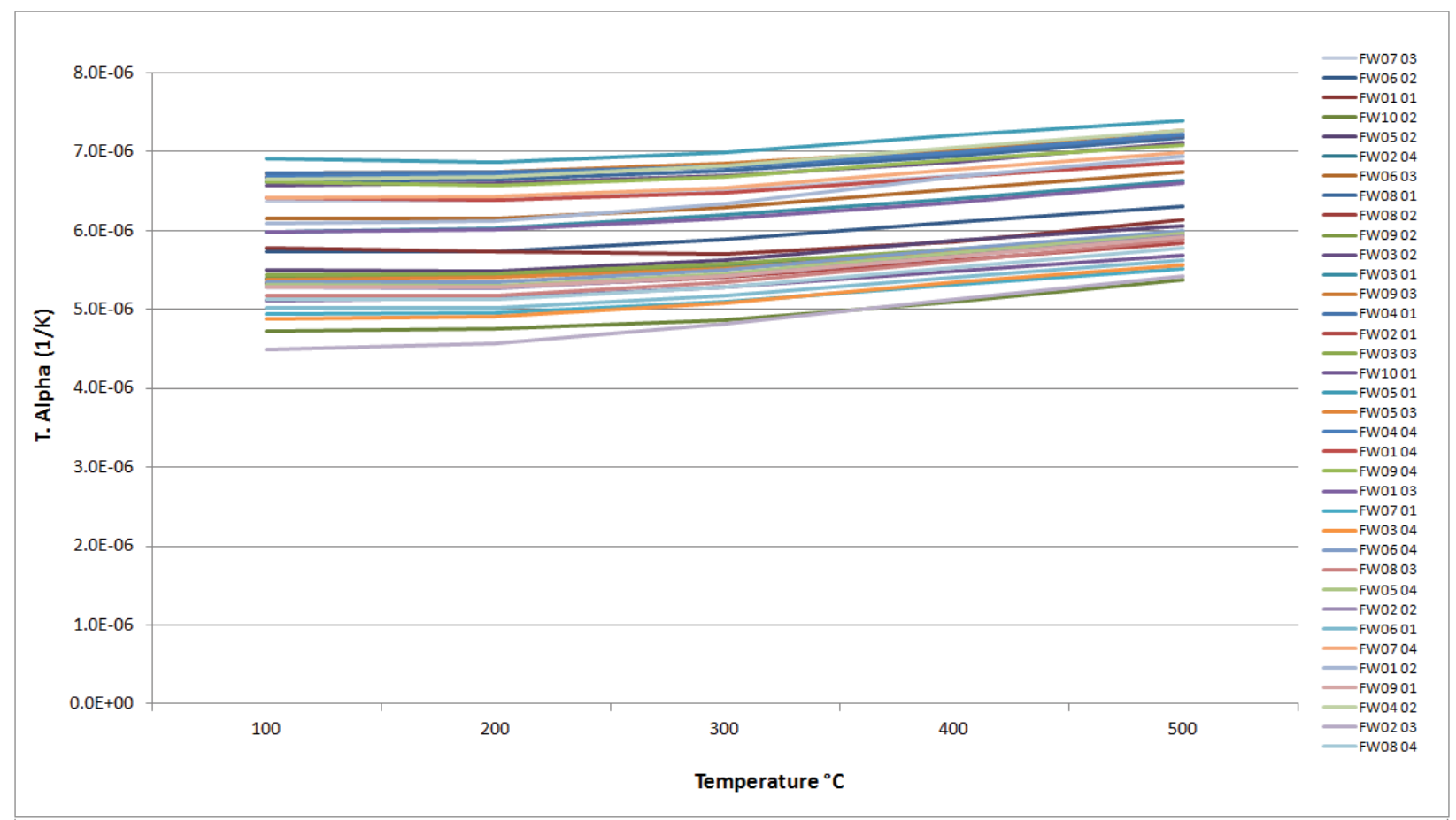

Figure A-93. IG-430 Creep Coefficient of Thermal Expansion.

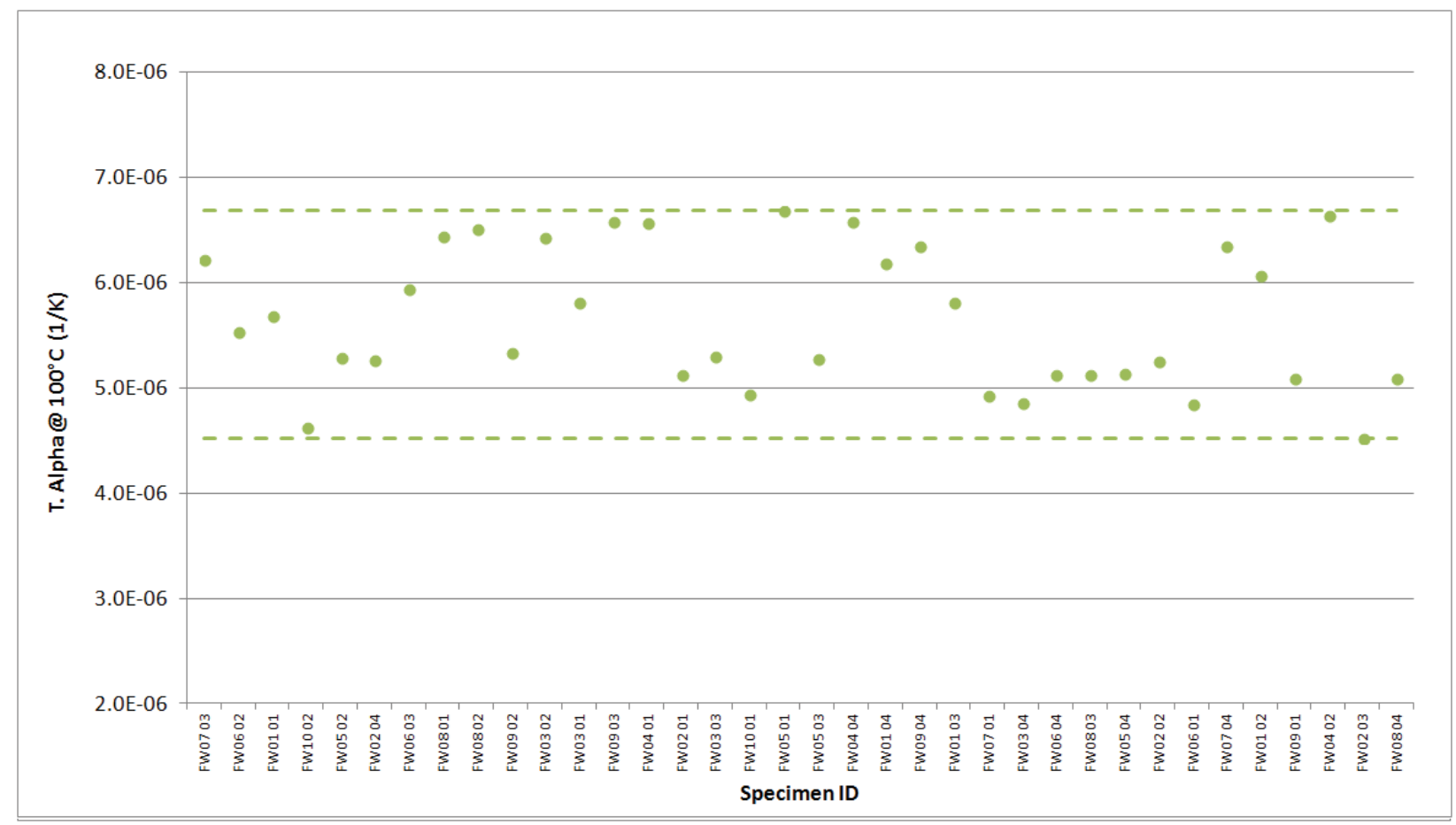

Figure A-94. IG-430 Creep Coefficient of Thermal Expansion @ $100^{\circ} \mathrm{C}$. 


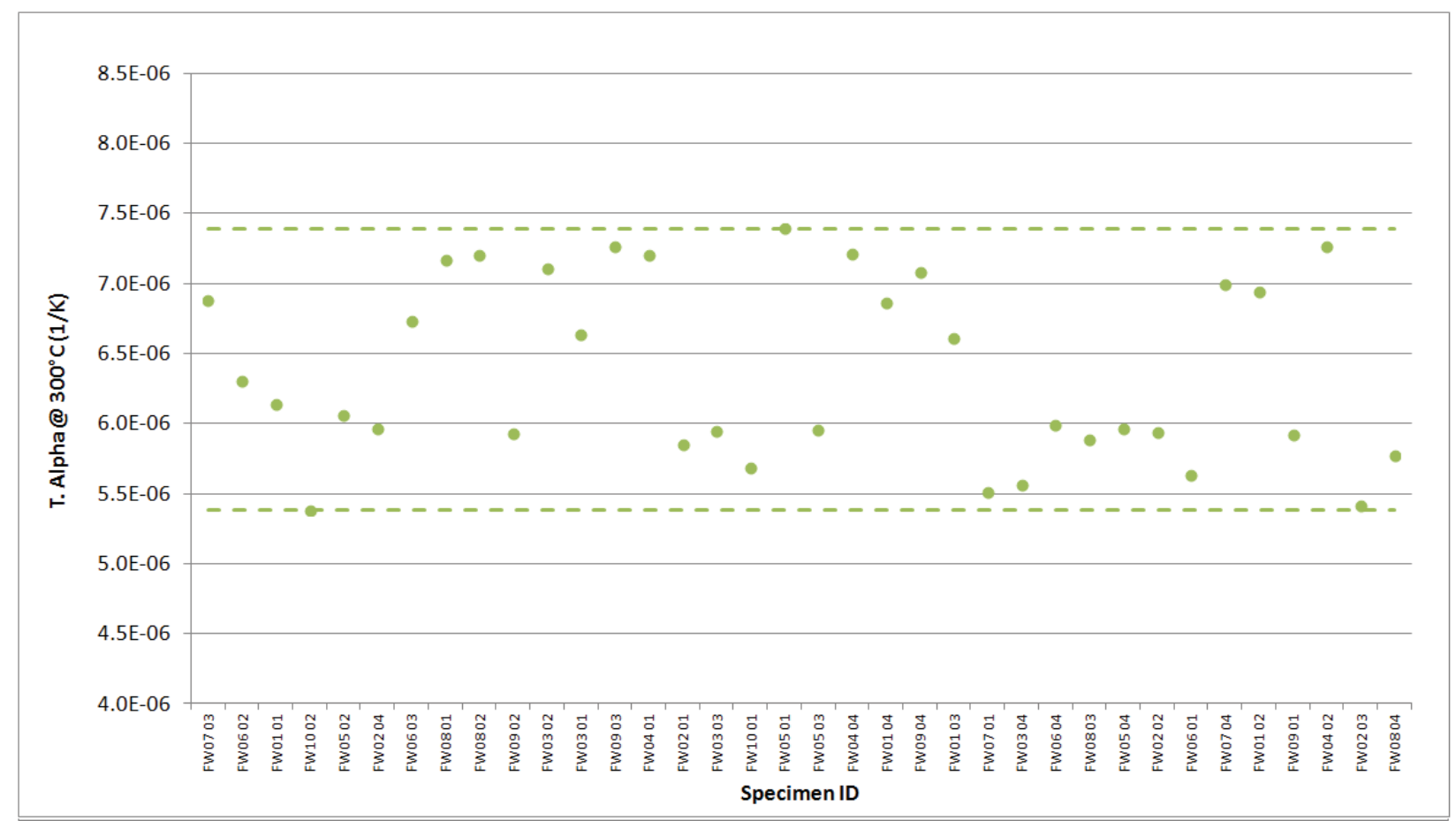

Figure A-95. IG-430 Creep Coefficient of Thermal Expansion @ $300^{\circ} \mathrm{C}$.

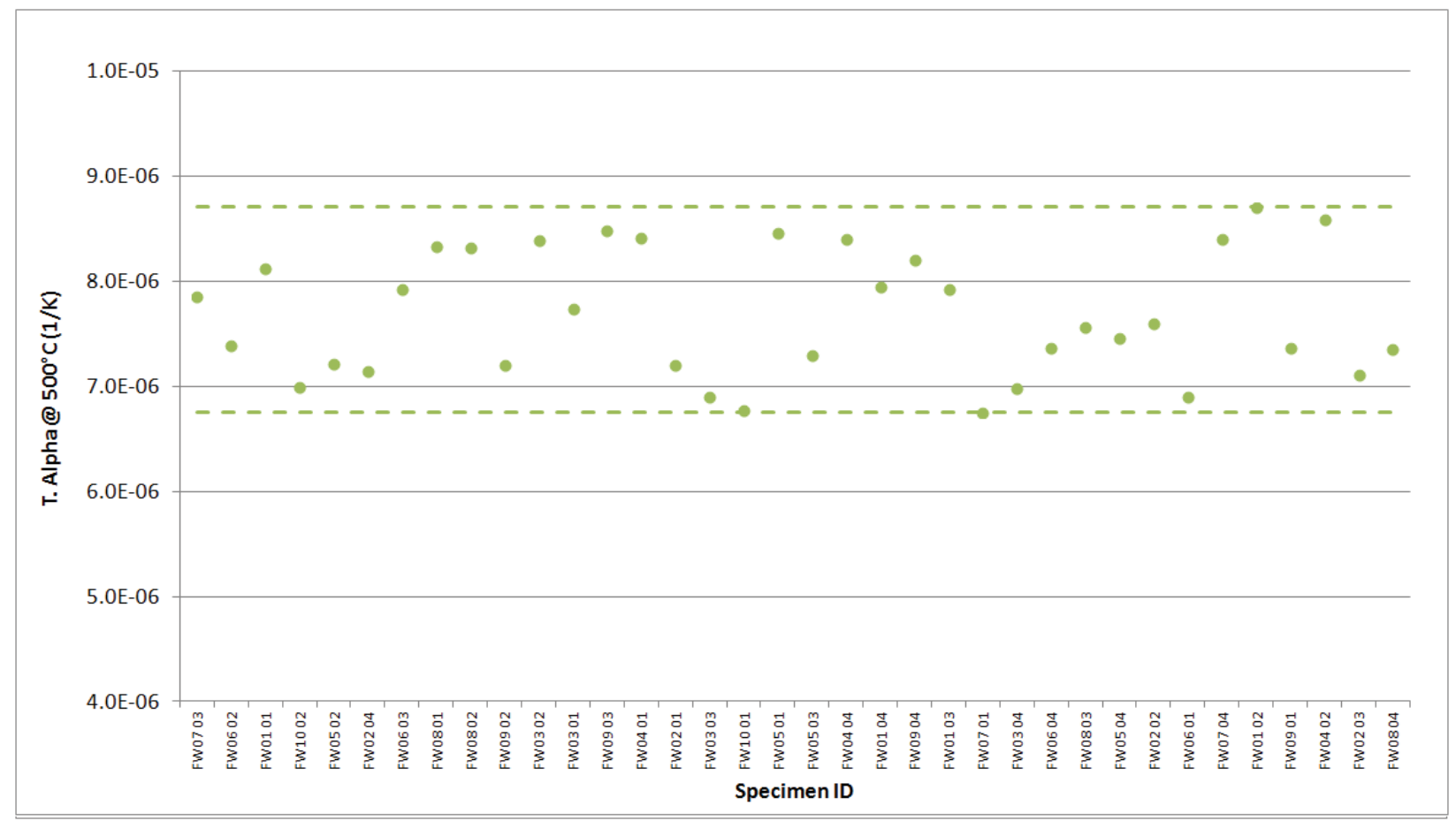

Figure A-96. IG-430 Creep Coefficient of Thermal Expansion@ @ 500 ${ }^{\circ}$. 


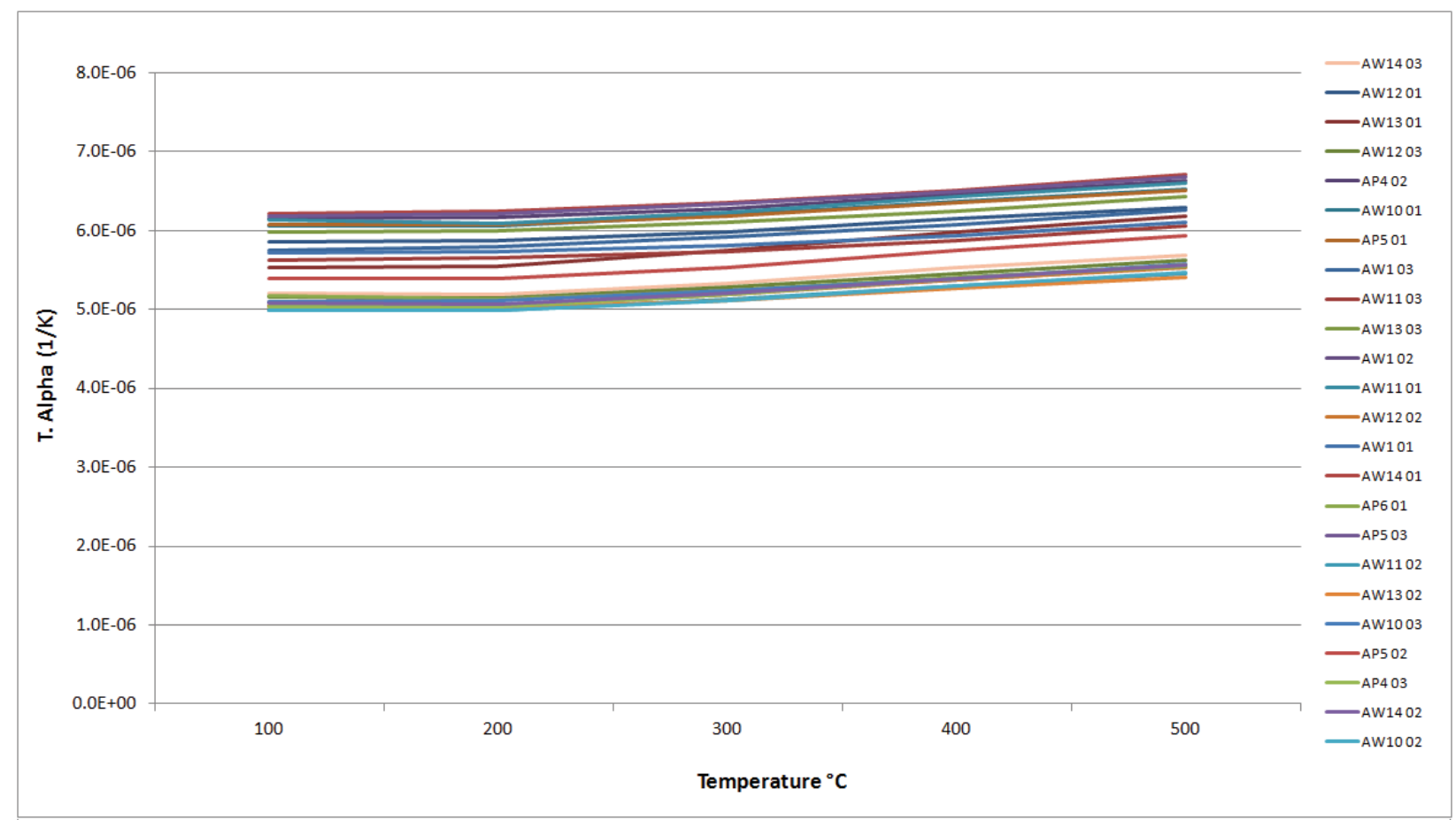

Figure A-97. NBG-17 Creep Coefficient of Thermal Expansion.

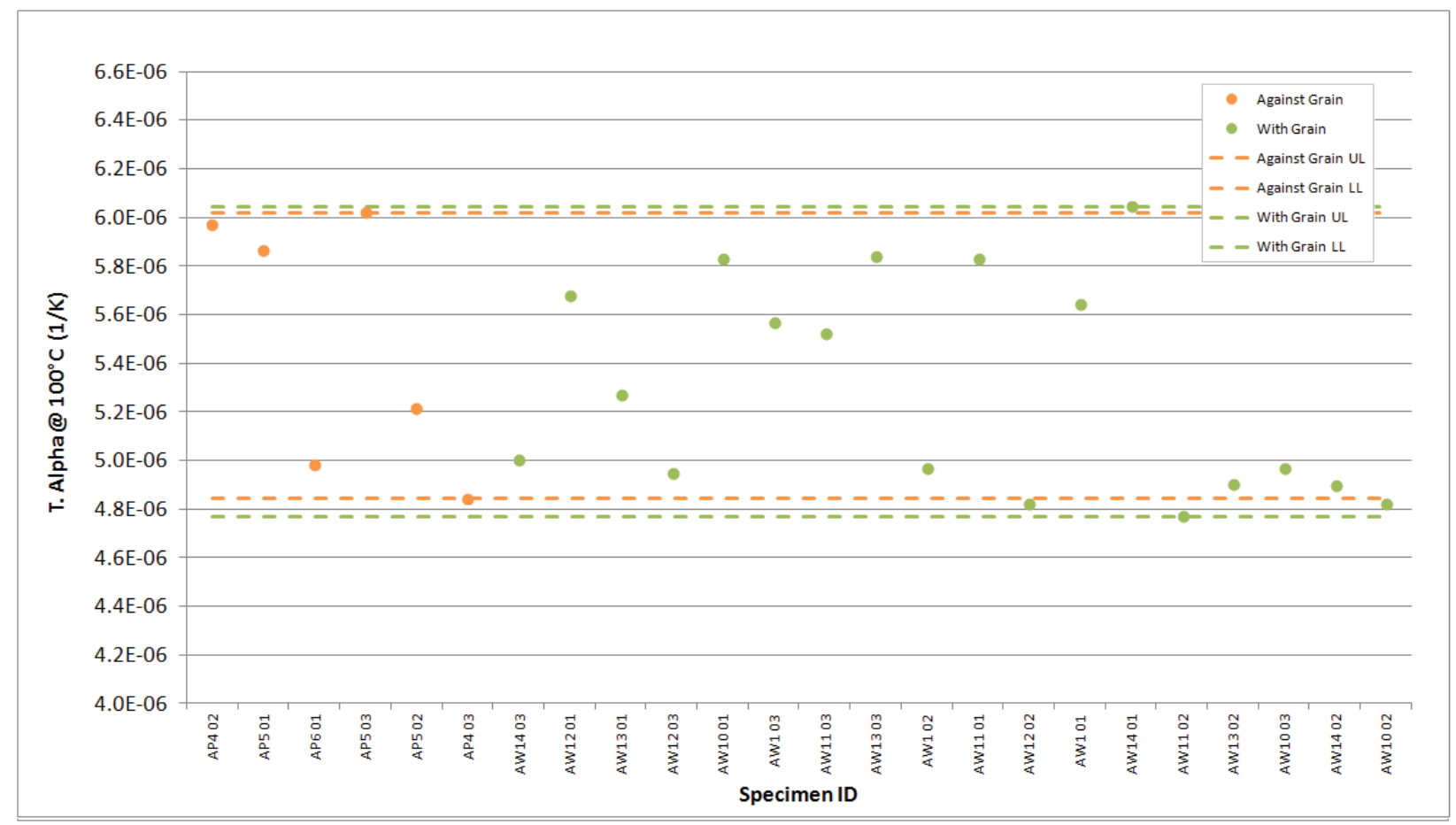

Figure A-98. NBG-17 Creep Coefficient of Thermal Expansion @ $100^{\circ} \mathrm{C}$. 


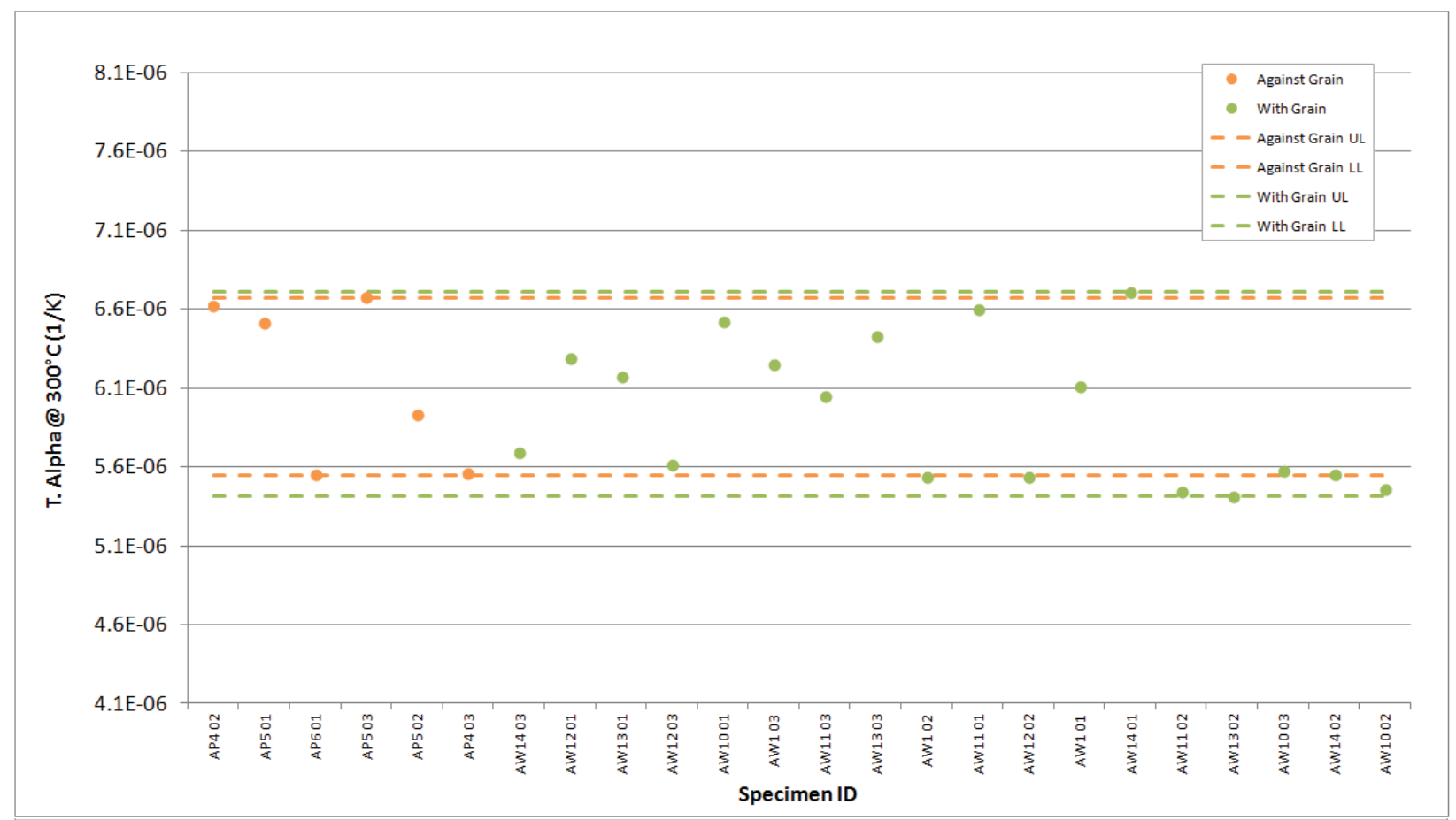

Figure A-99. NBG-17 Creep Coefficient of Thermal Expansion @ 300 $\mathrm{C}$.

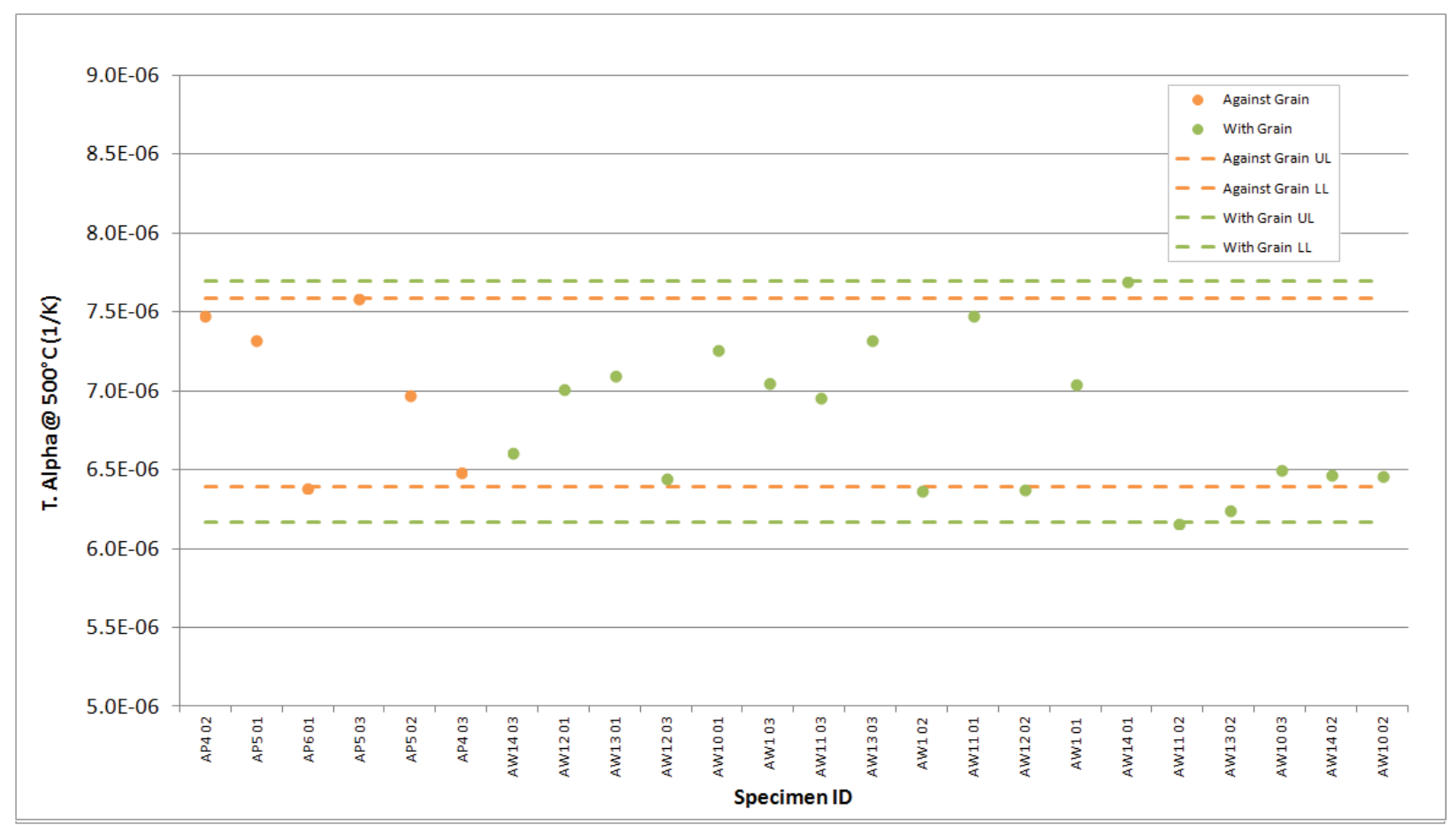

Figure A-100. NBG-17 Creep Coefficient of Thermal Expansion @ $500^{\circ} \mathrm{C}$. 




Figure A-101. NBG-18 Creep Coefficient of Thermal Expansion.

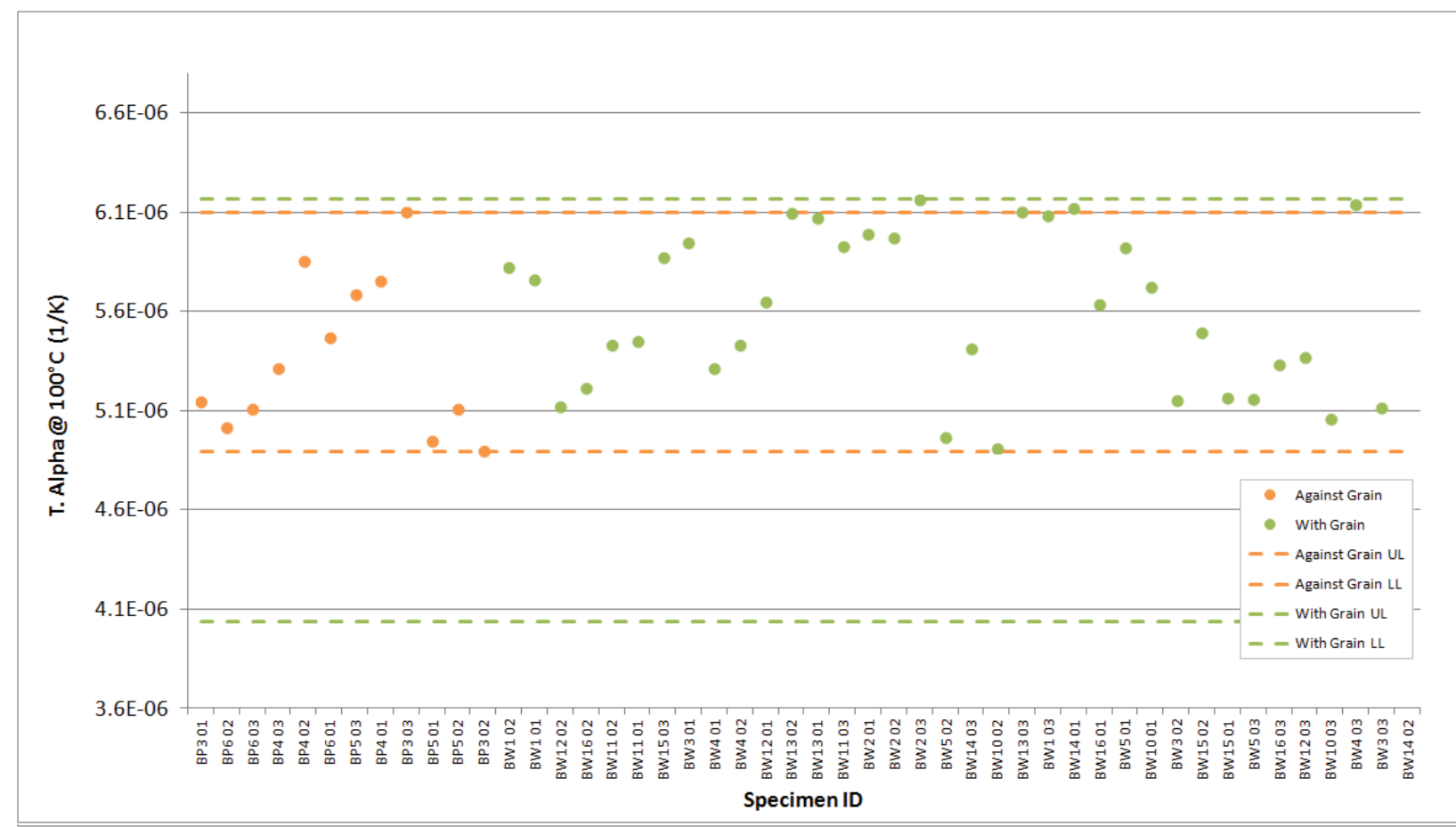

Figure A-102. NBG-18 Creep Coefficient of Thermal Expansion @ $100^{\circ} \mathrm{C}$. 


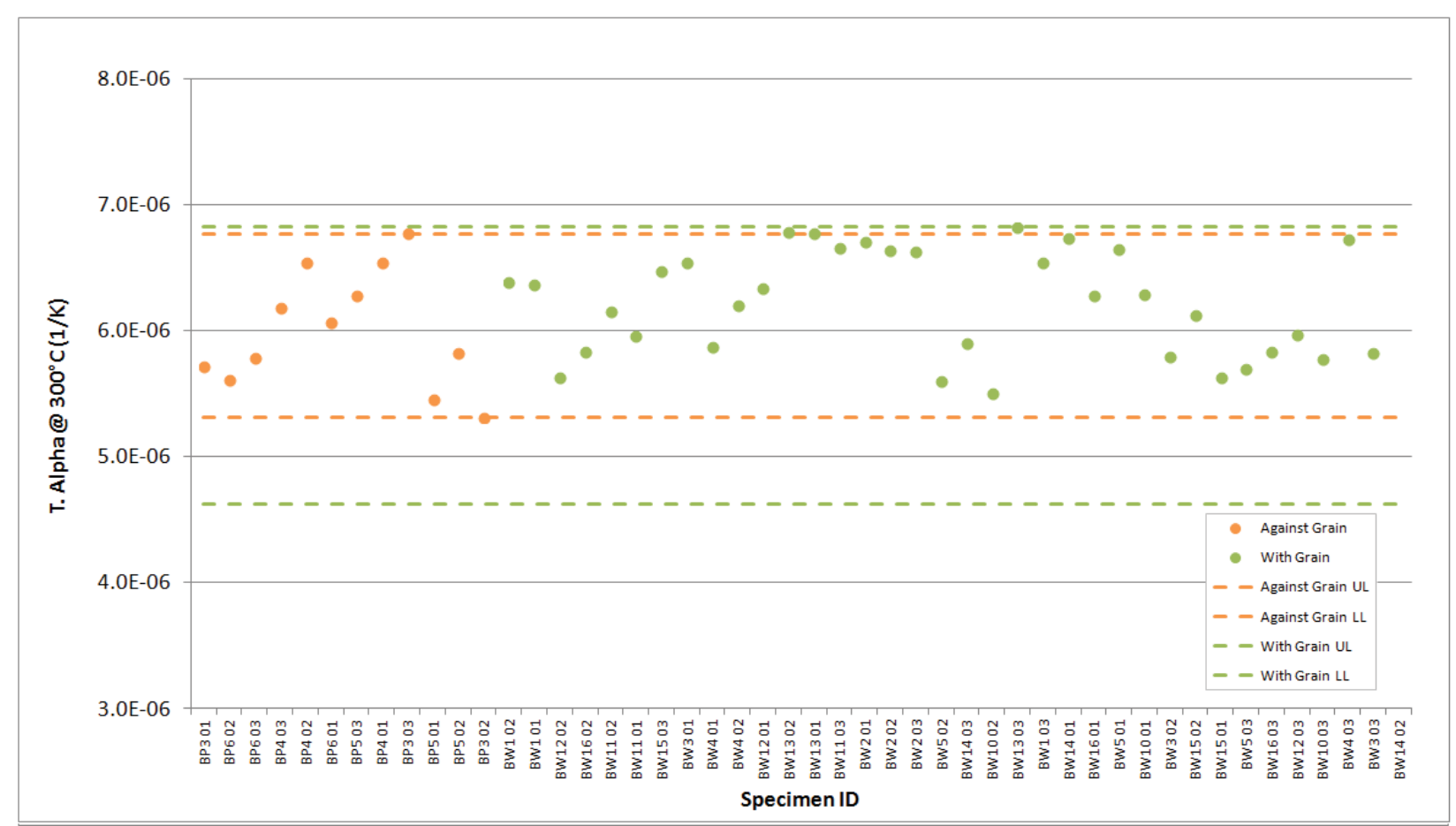

Figure A-103. NBG-18 Creep Coefficient of Thermal Expansion @ $300^{\circ} \mathrm{C}$.

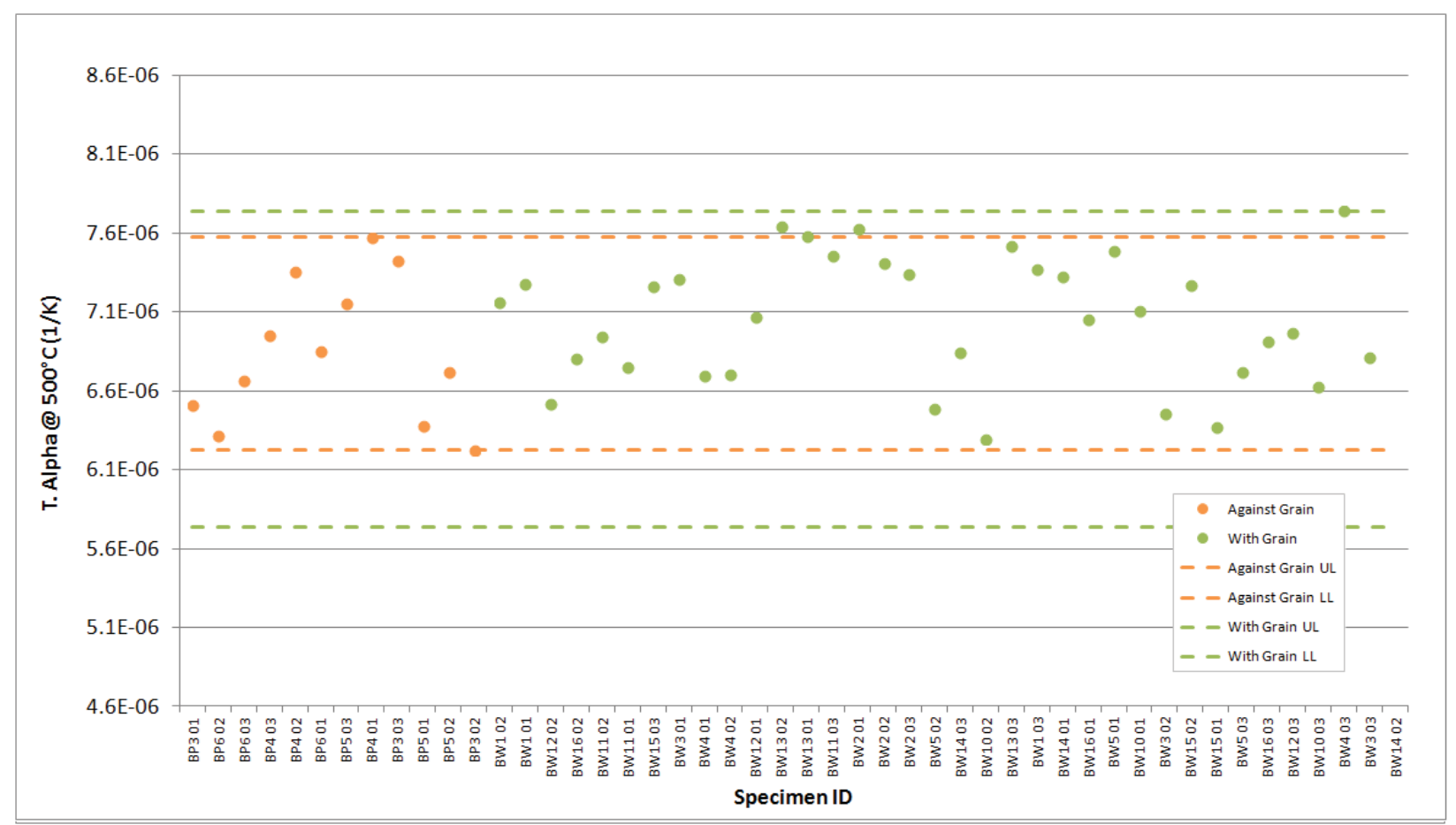

Figure A-104. NBG-18 Creep Coefficient of Thermal Expansion @ $500^{\circ} \mathrm{C}$. 


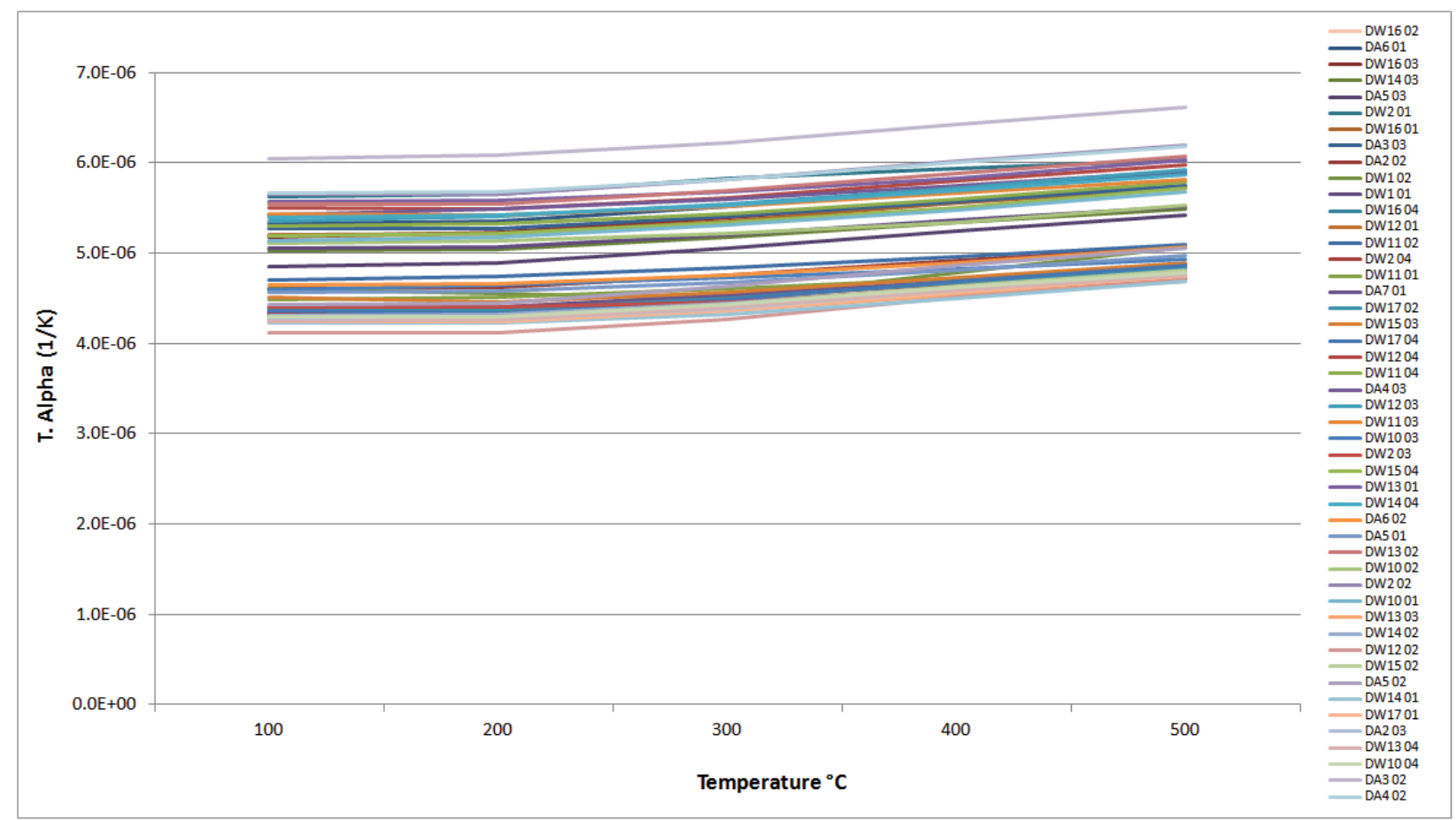

Figure A-105. PCEA Creep Coefficient of Thermal Expansion.

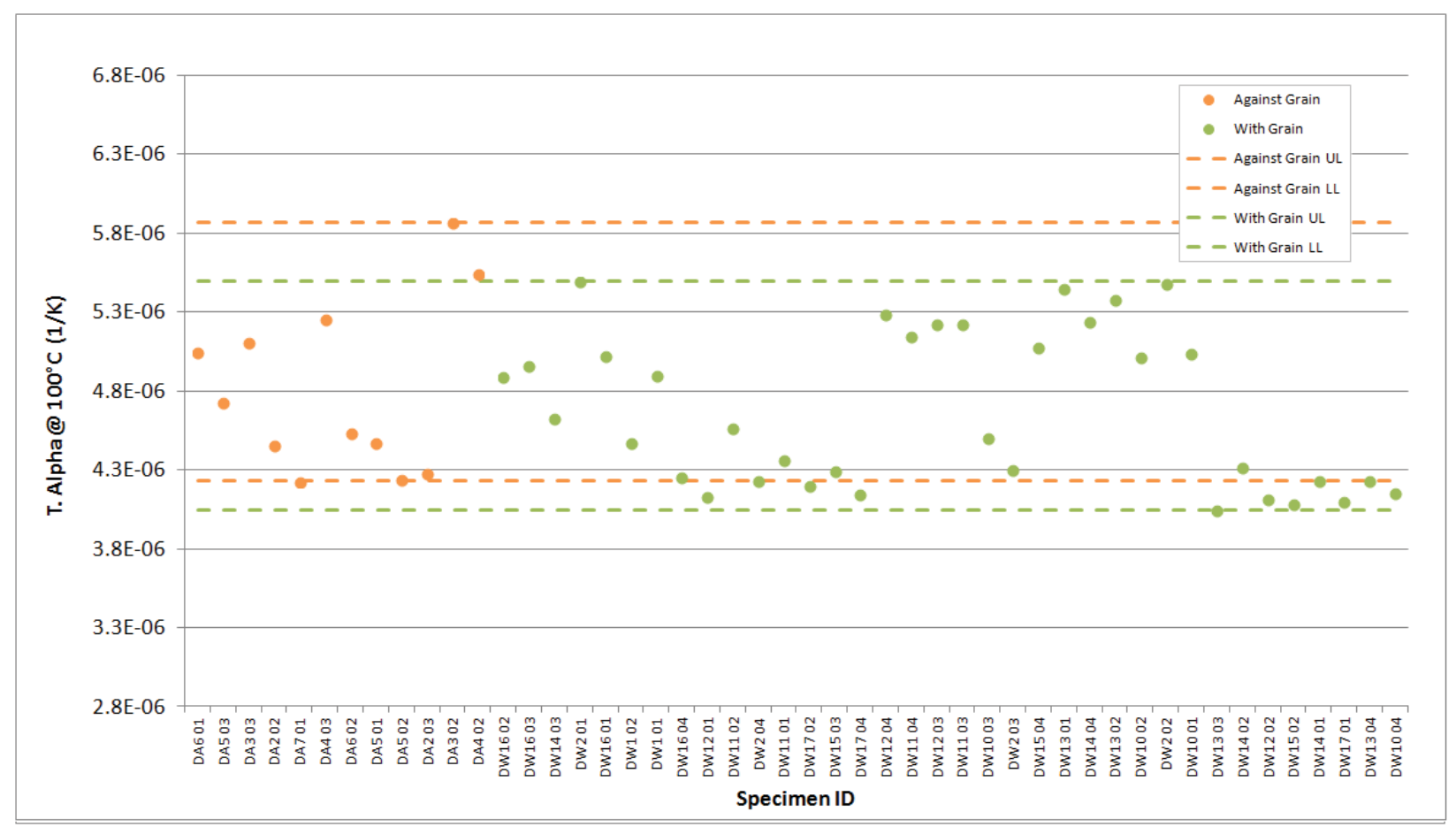

Figure A-106. PCEA Creep Coefficient of Thermal Expansion @ $100^{\circ} \mathrm{C}$. 


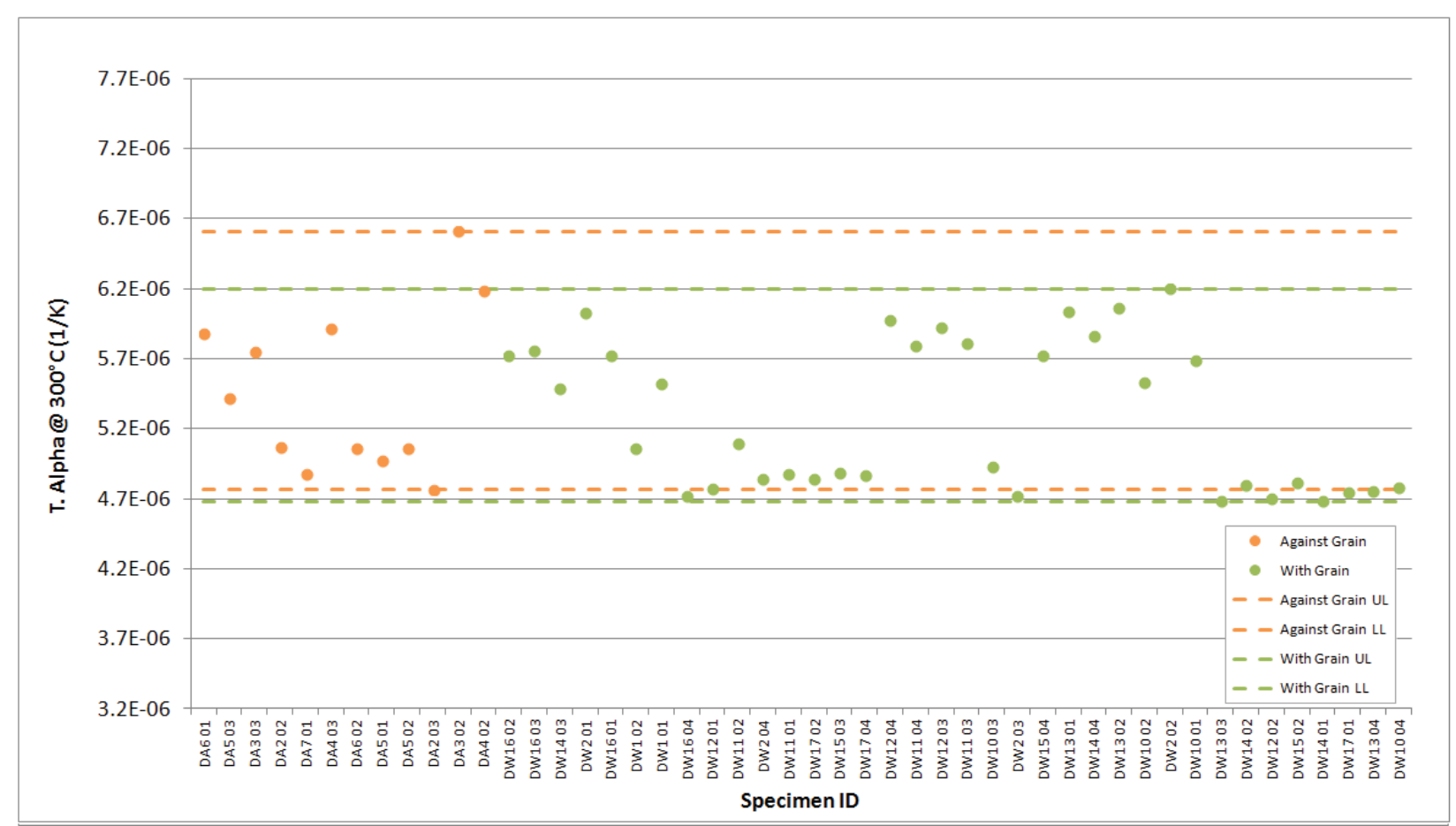

Figure A-107. PCEA Creep Coefficient of Thermal Expansion @ $300^{\circ} \mathrm{C}$.

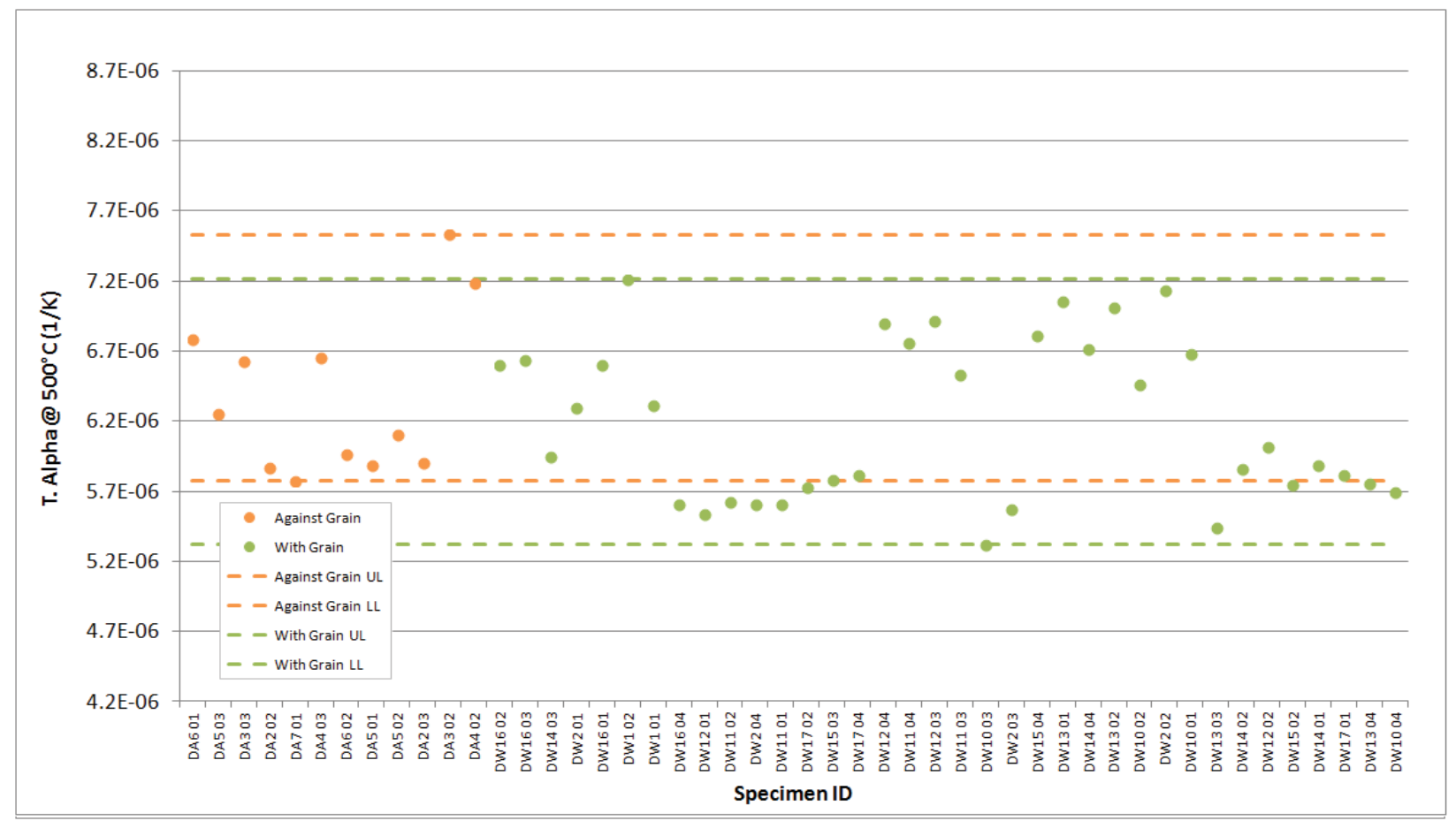

Figure A-108. PCEA Creep Coefficient of Thermal Expansion @ $500^{\circ} \mathrm{C}$. 


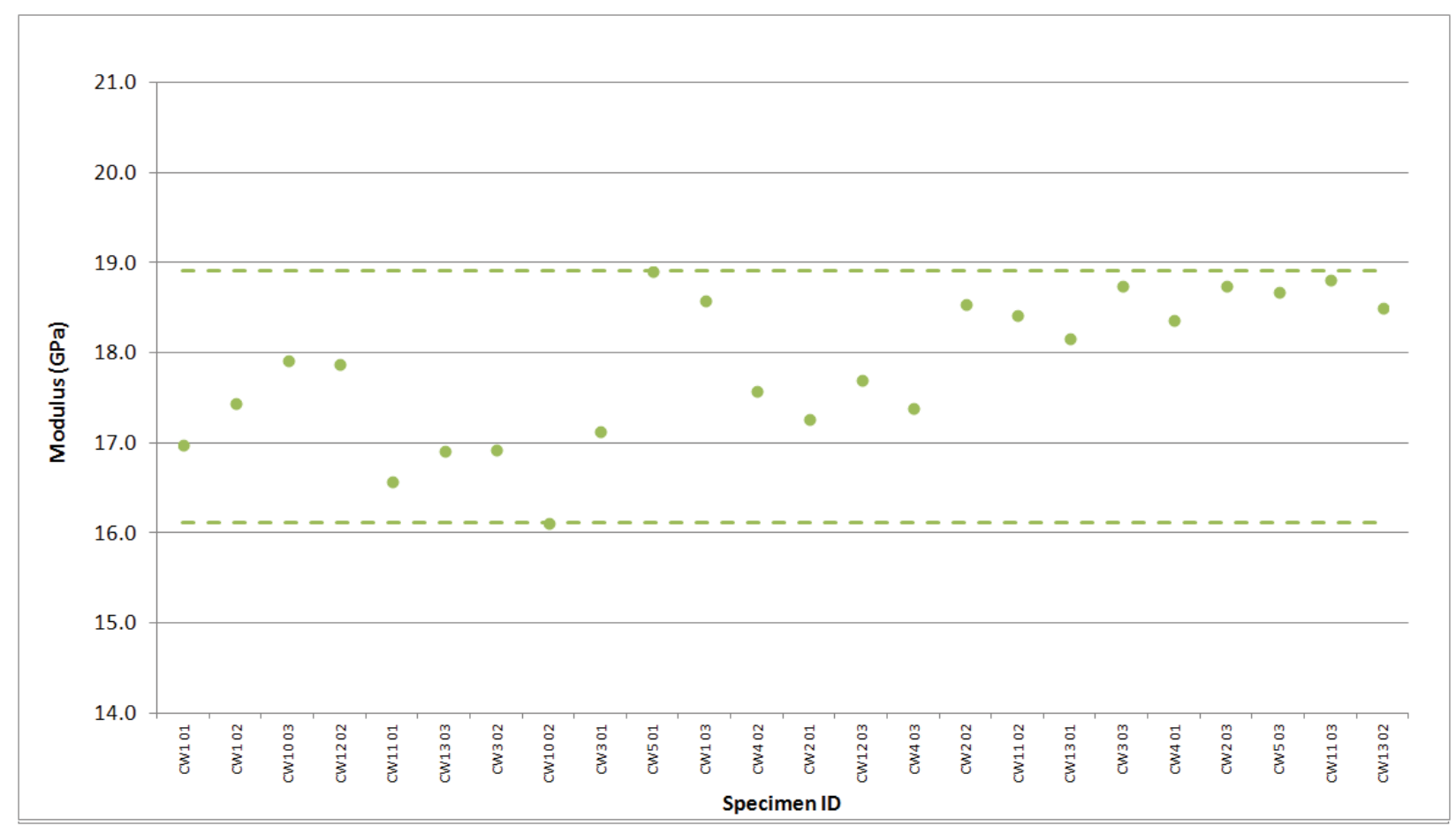

Figure A-109. H-451 Creep Modulus by Sonic Resonance.

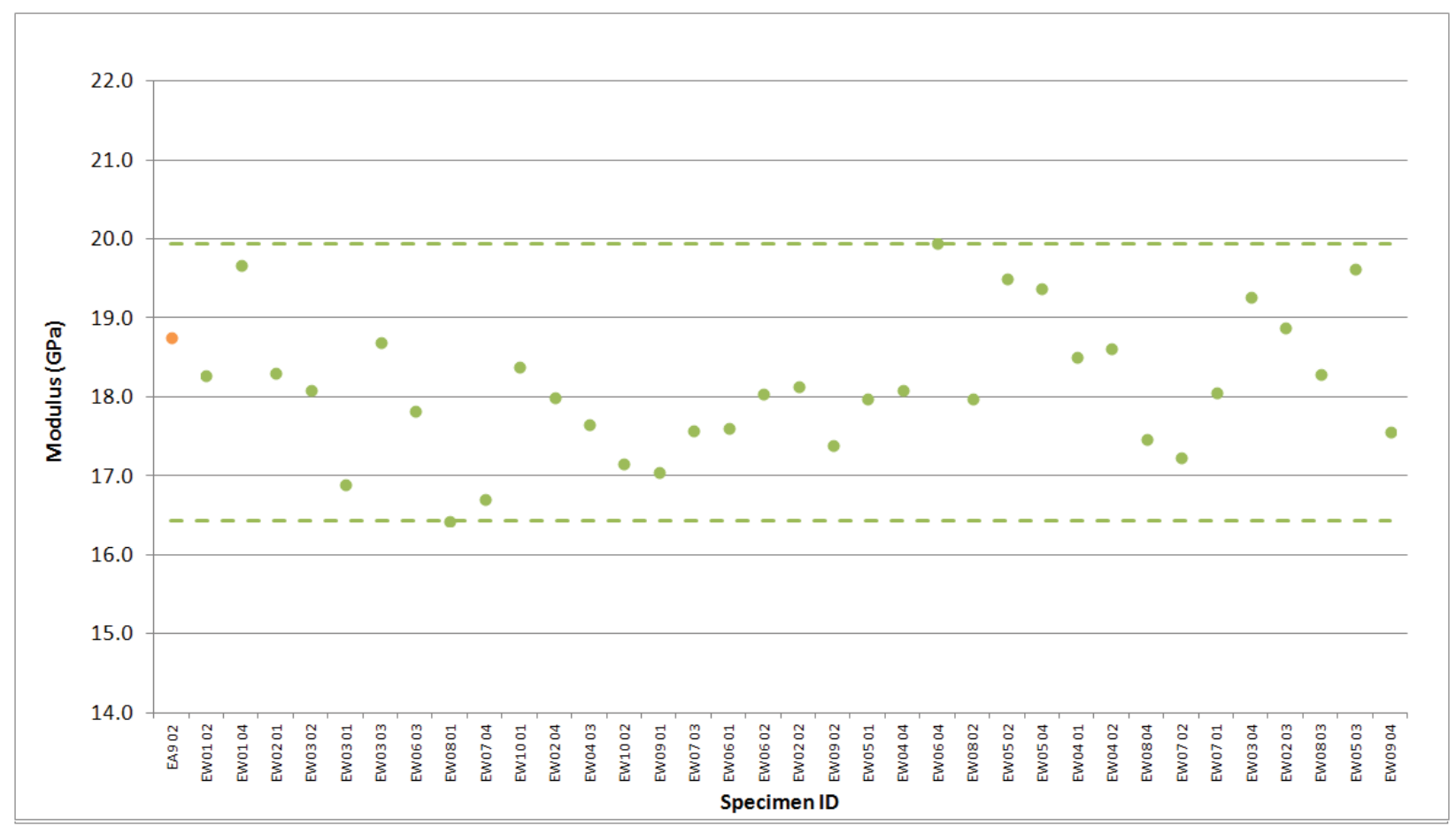

Figure A-110. IG-110 Creep Modulus by Sonic Resonance. 


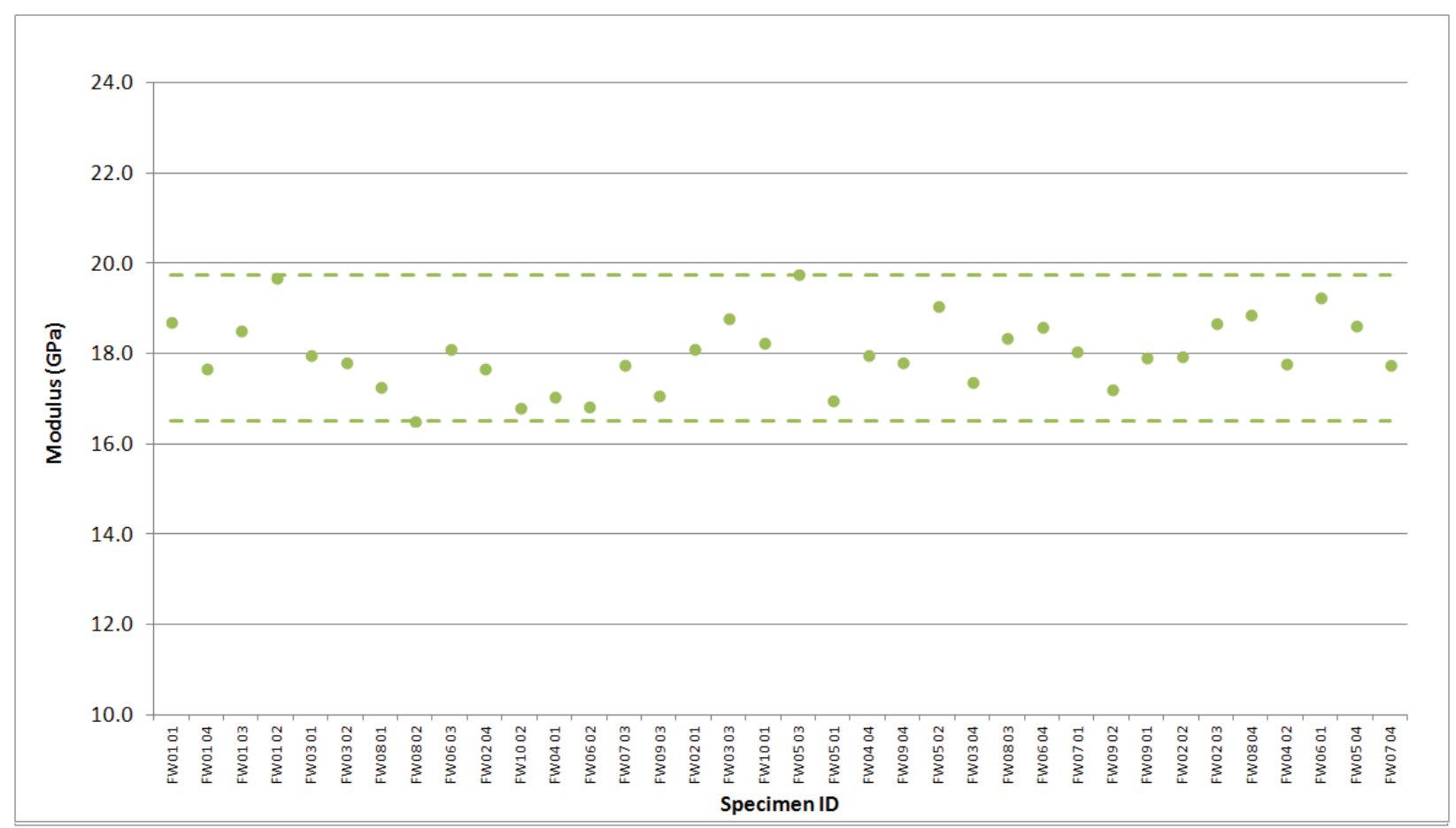

Figure A-111. IG-430 Creep Modulus by Sonic Resonance.

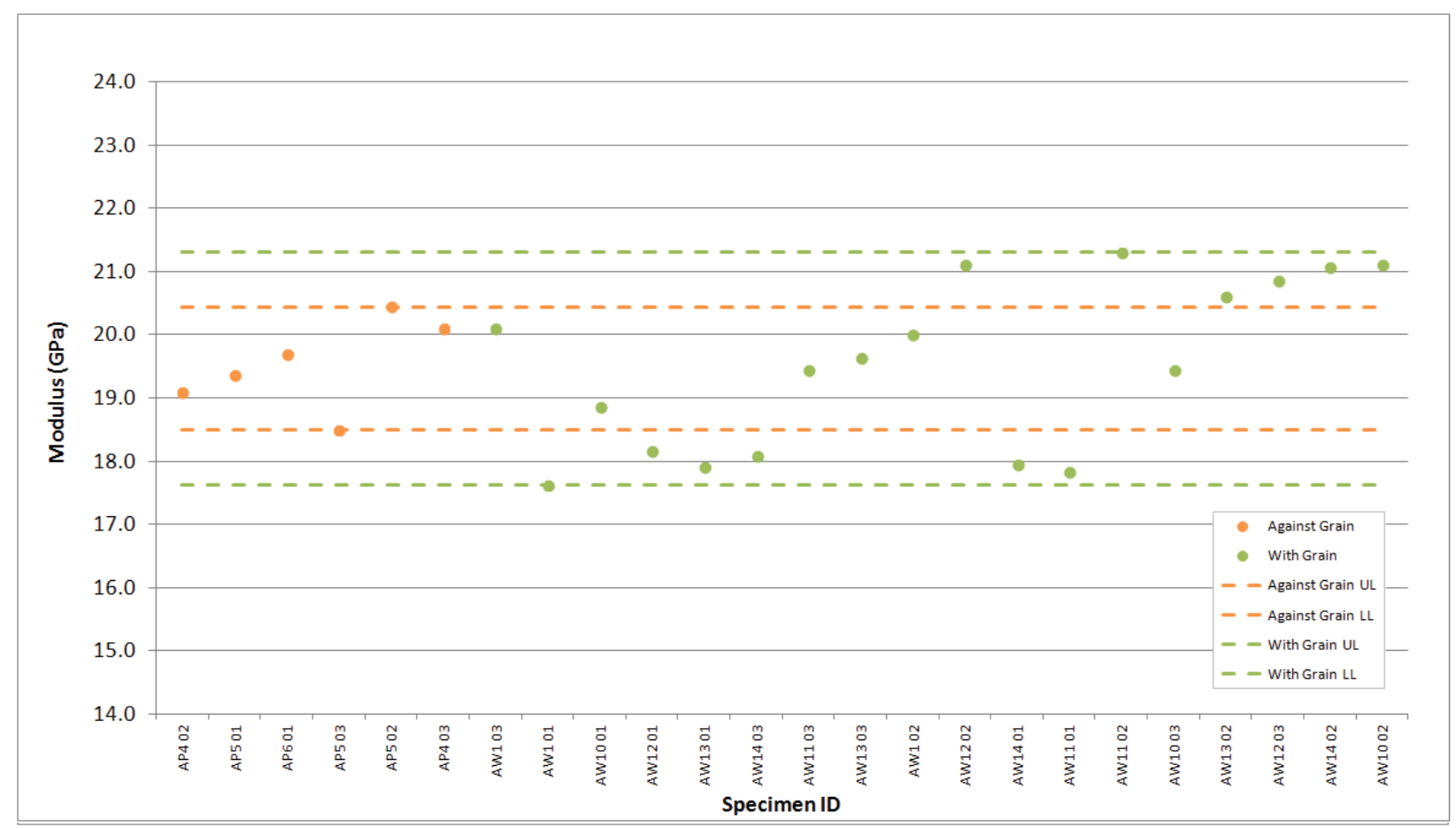

Figure A-112. NBG-17 Creep Modulus by Sonic Resonance. 


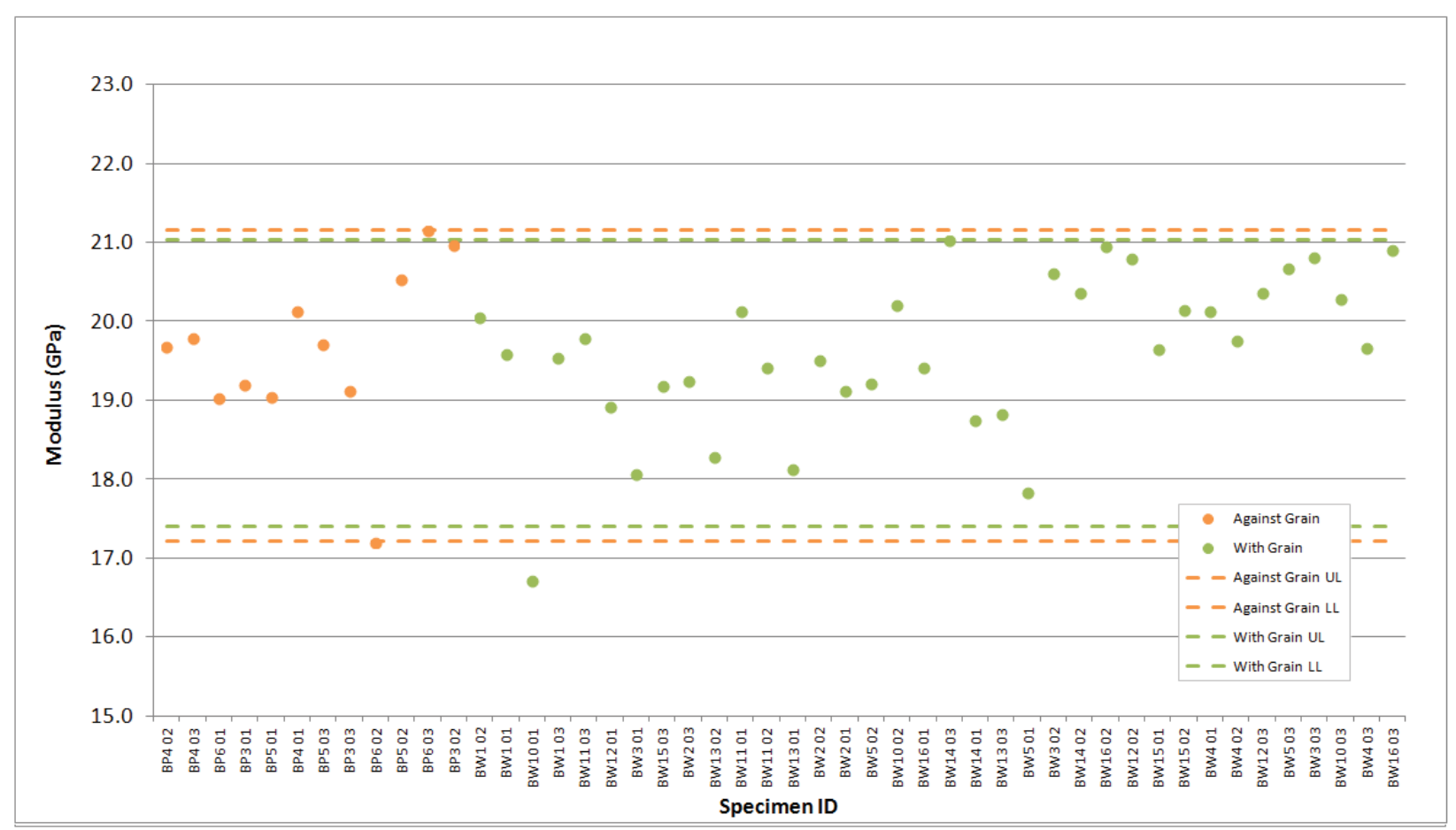

Figure A-113. NBG-18 Creep Modulus by Sonic Resonance.

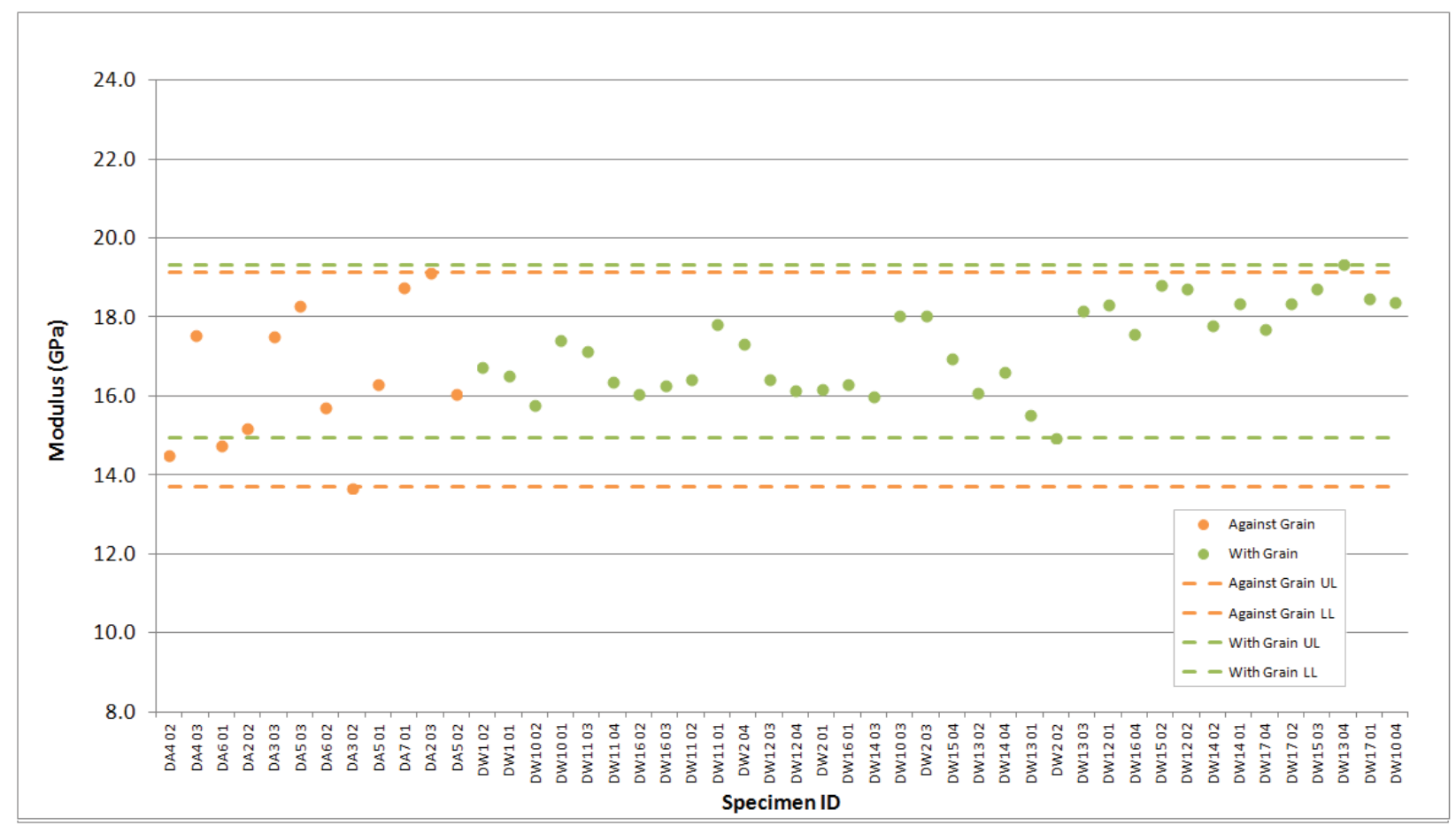

Figure A-114. PCEA Creep Modulus by Sonic Resonance. 


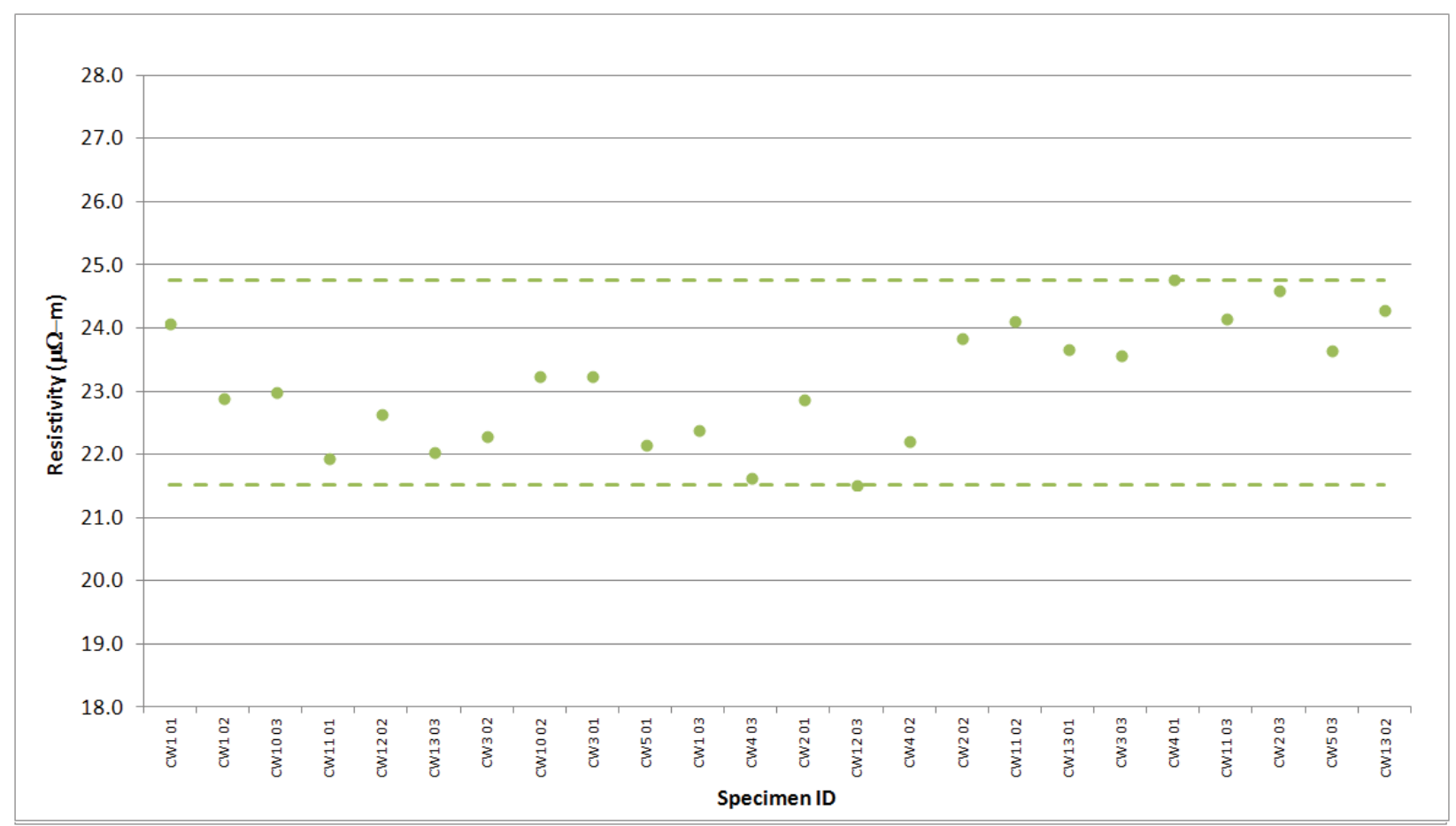

Figure A-115. H-451 Creep Resistivity.

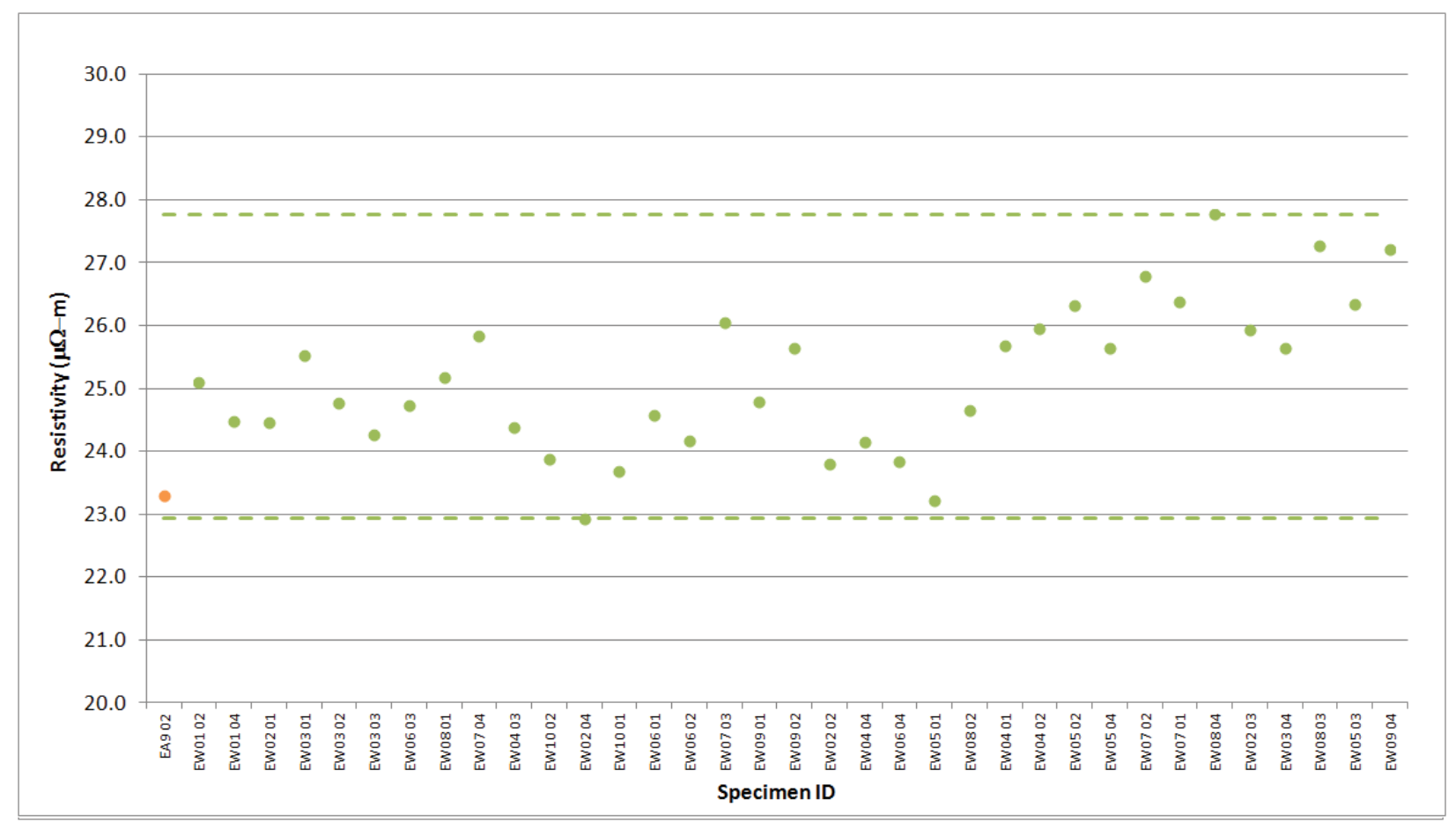

Figure A-116. IG-110 Creep Resistivity. 


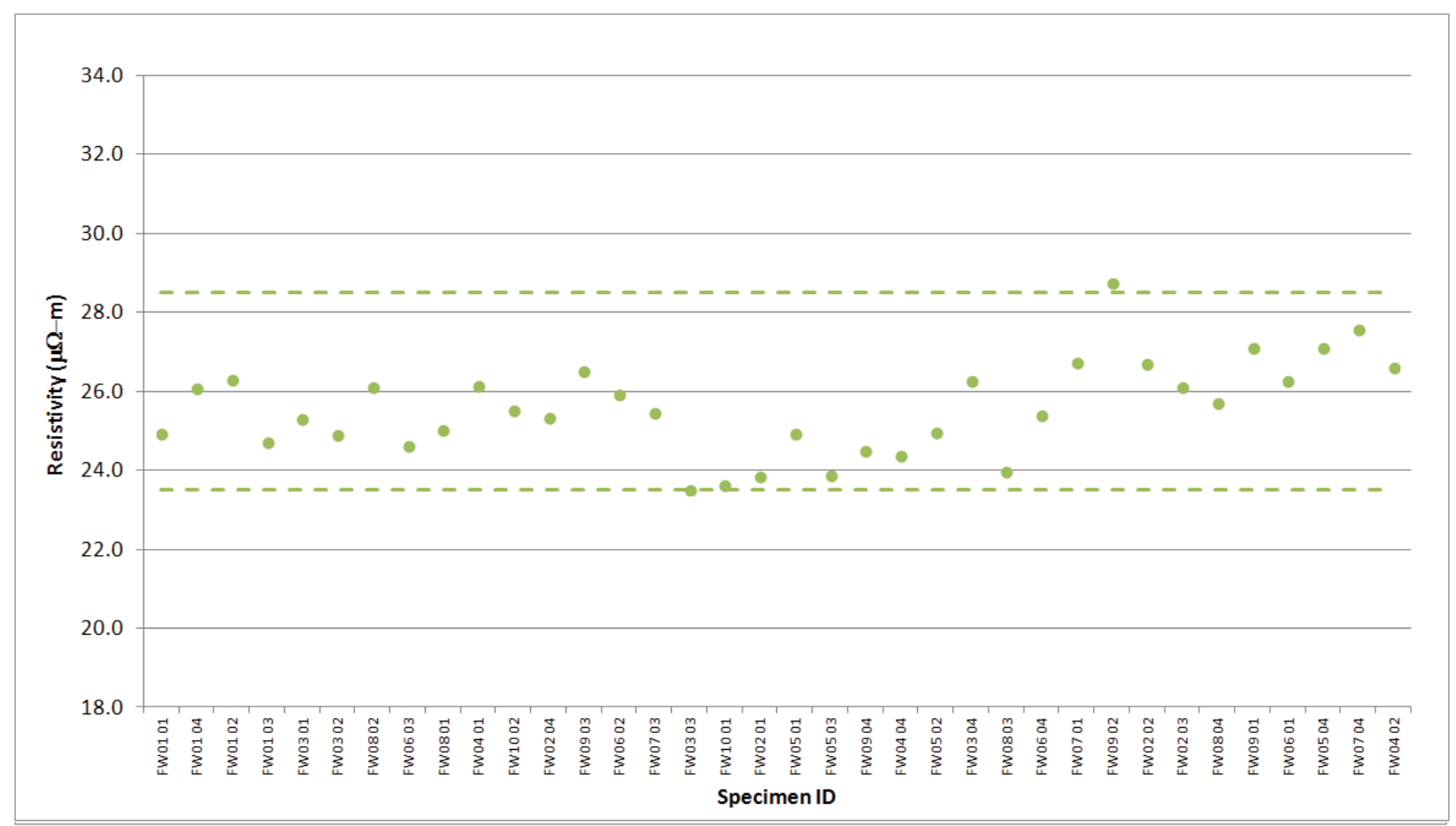

Figure A-117. IG-430 Creep Resistivity.

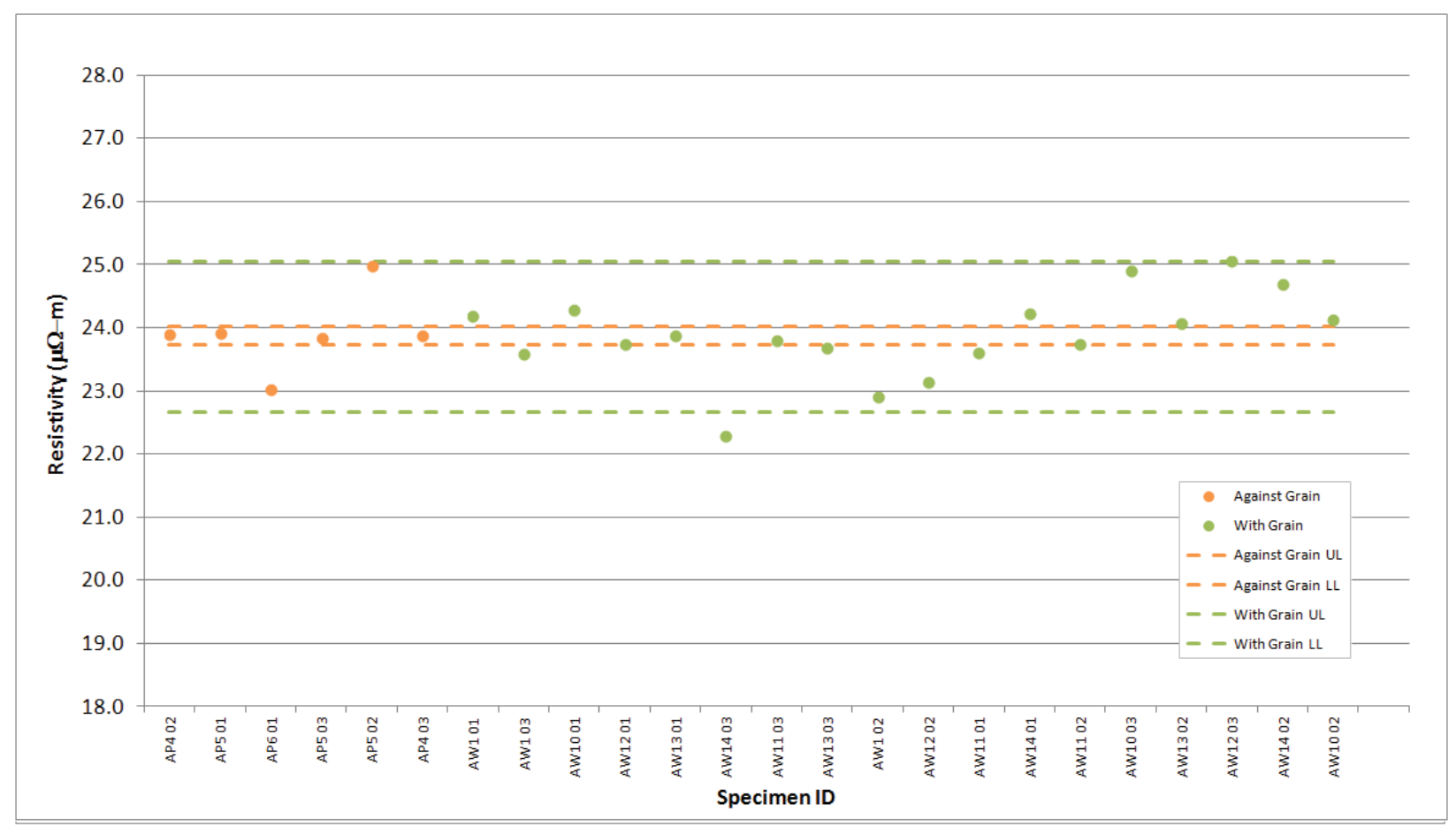

Figure A-118. NBG-17 Creep Resistivity. 


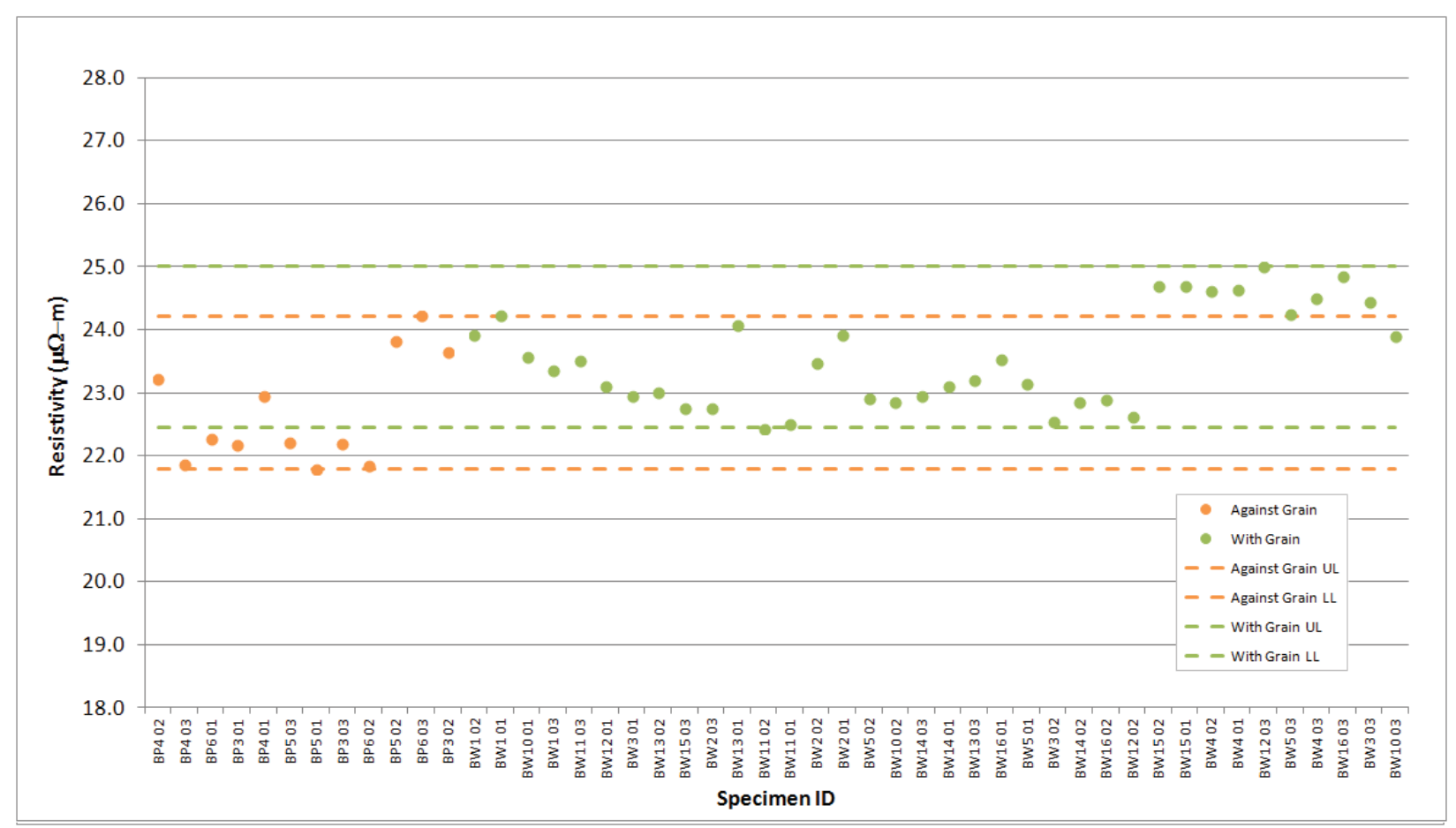

Figure A-119. NBG-18 Creep Resistivity.

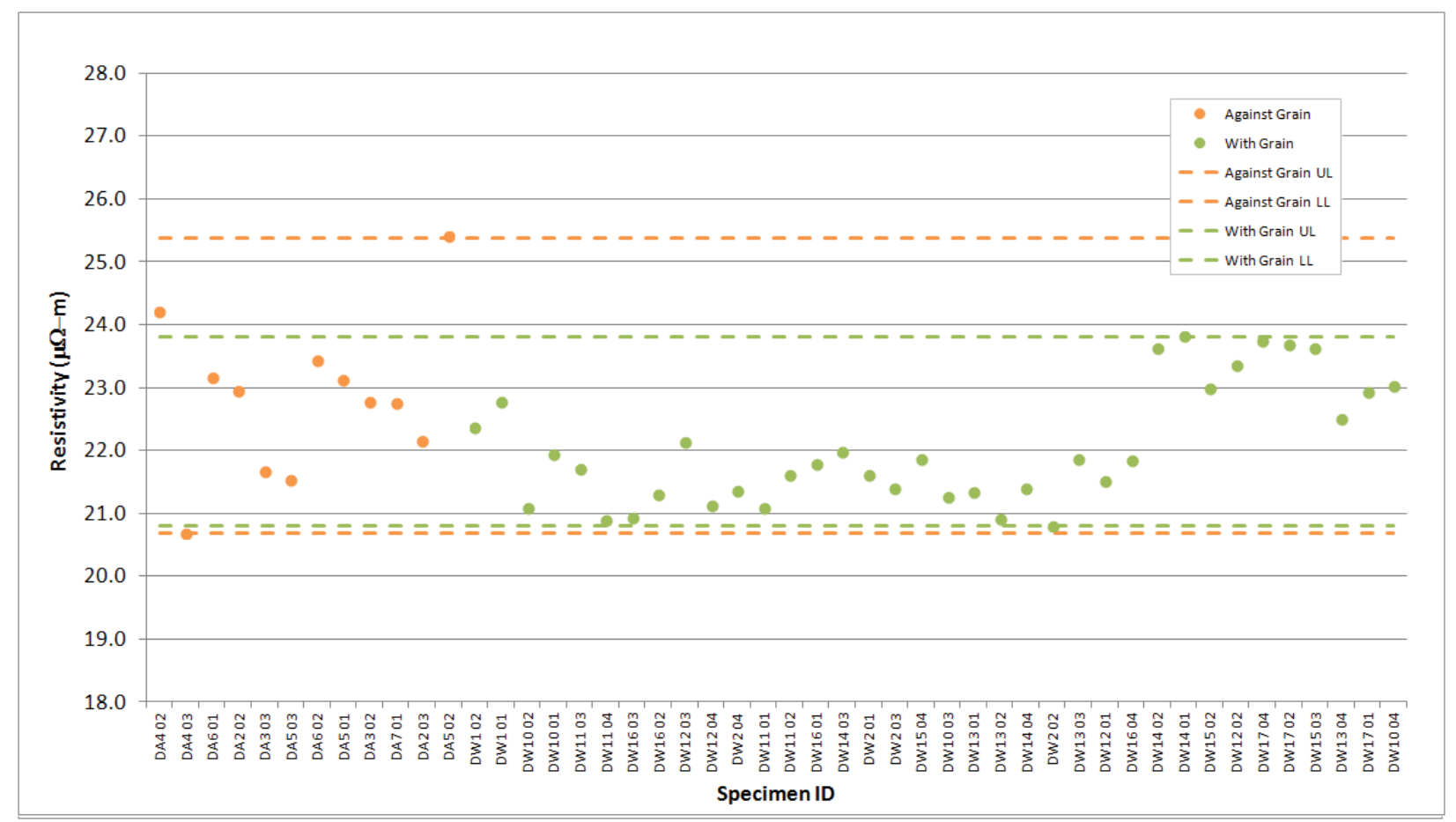

Figure A-120. PCEA Creep Resistivity. 


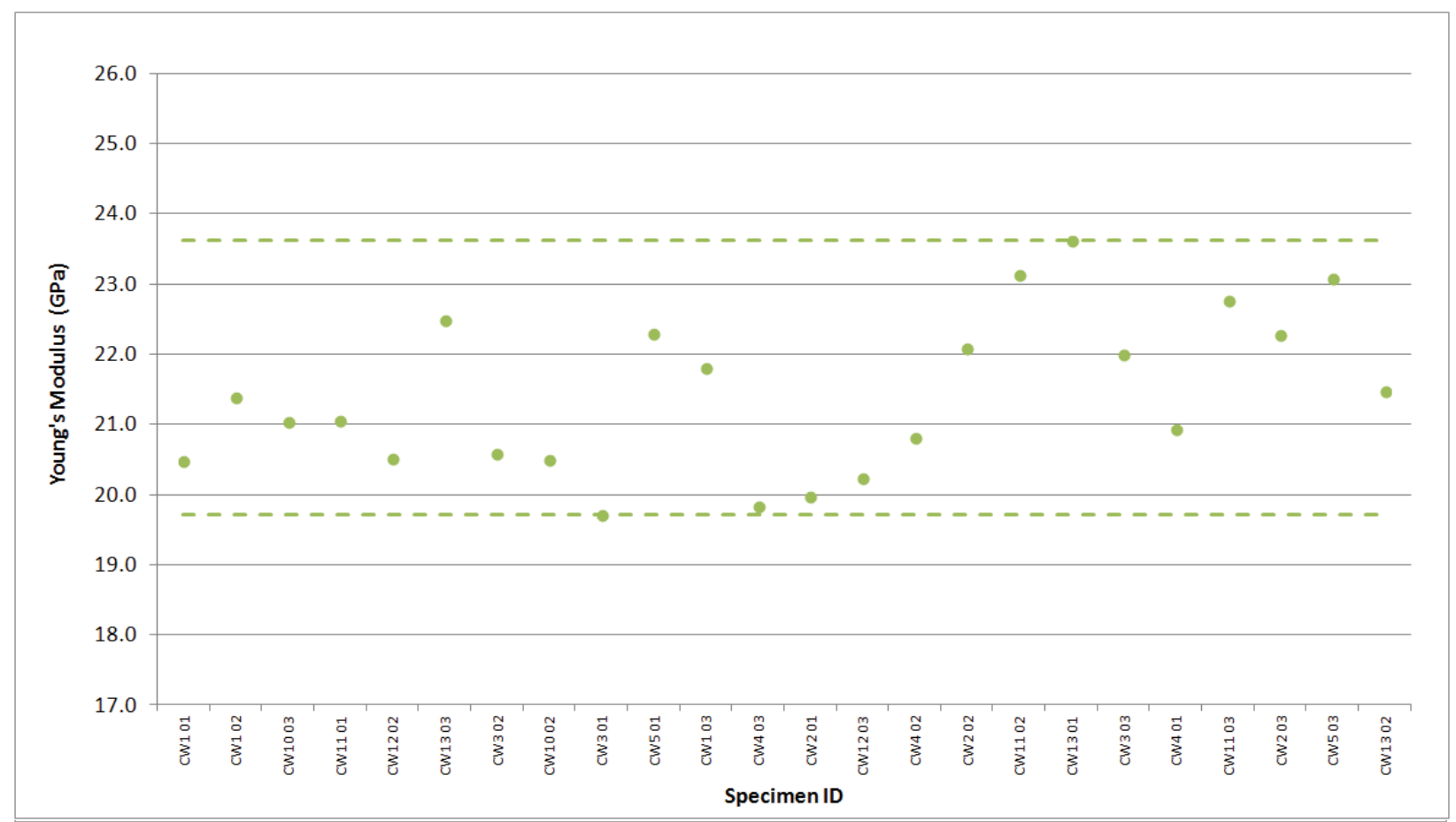

Figure A-121. H-451 Creep Young's Modulus by Sonic Velocity.

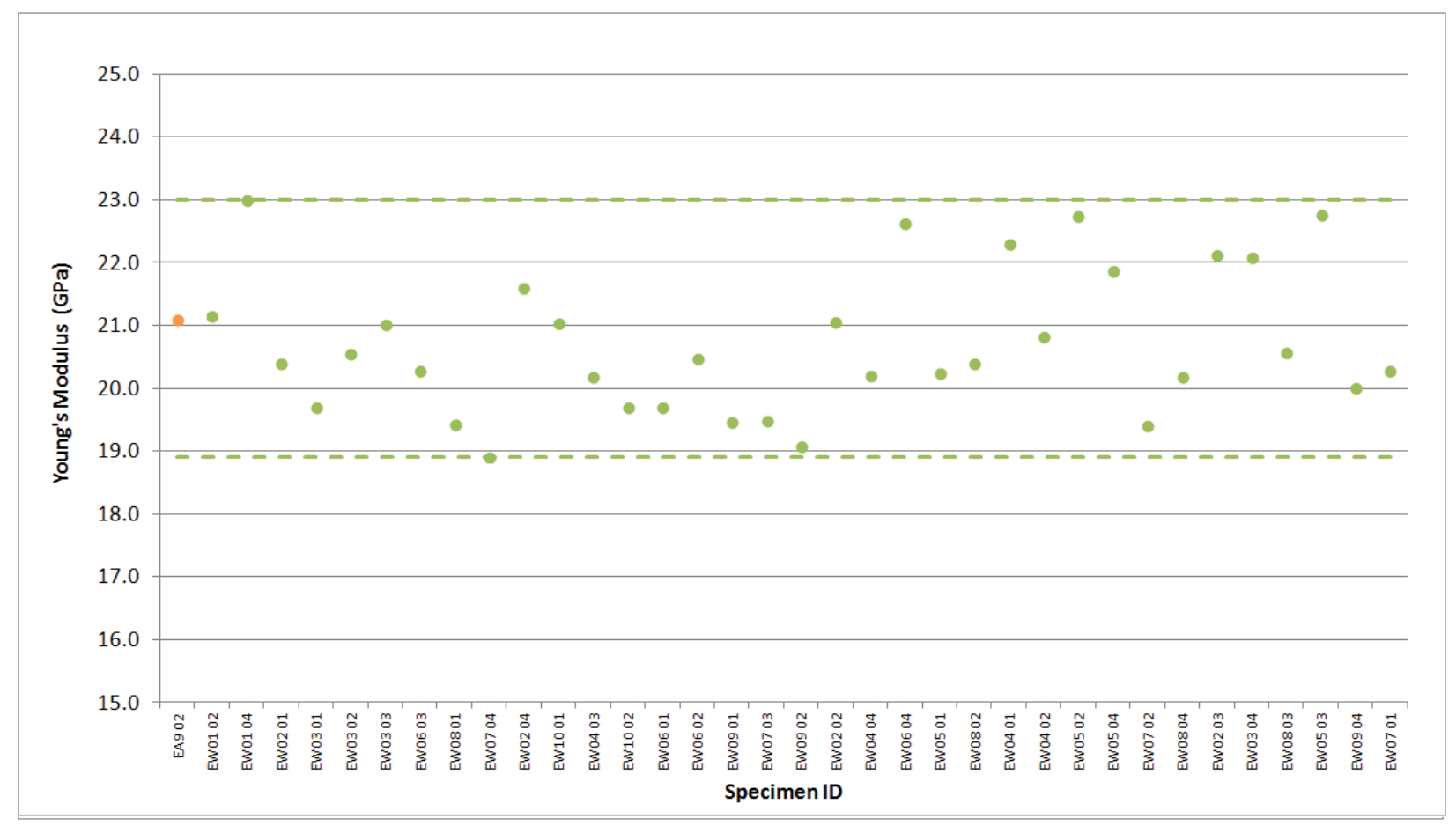

Figure A-122. IG-110 Creep Young's Modulus by Sonic Velocity. 


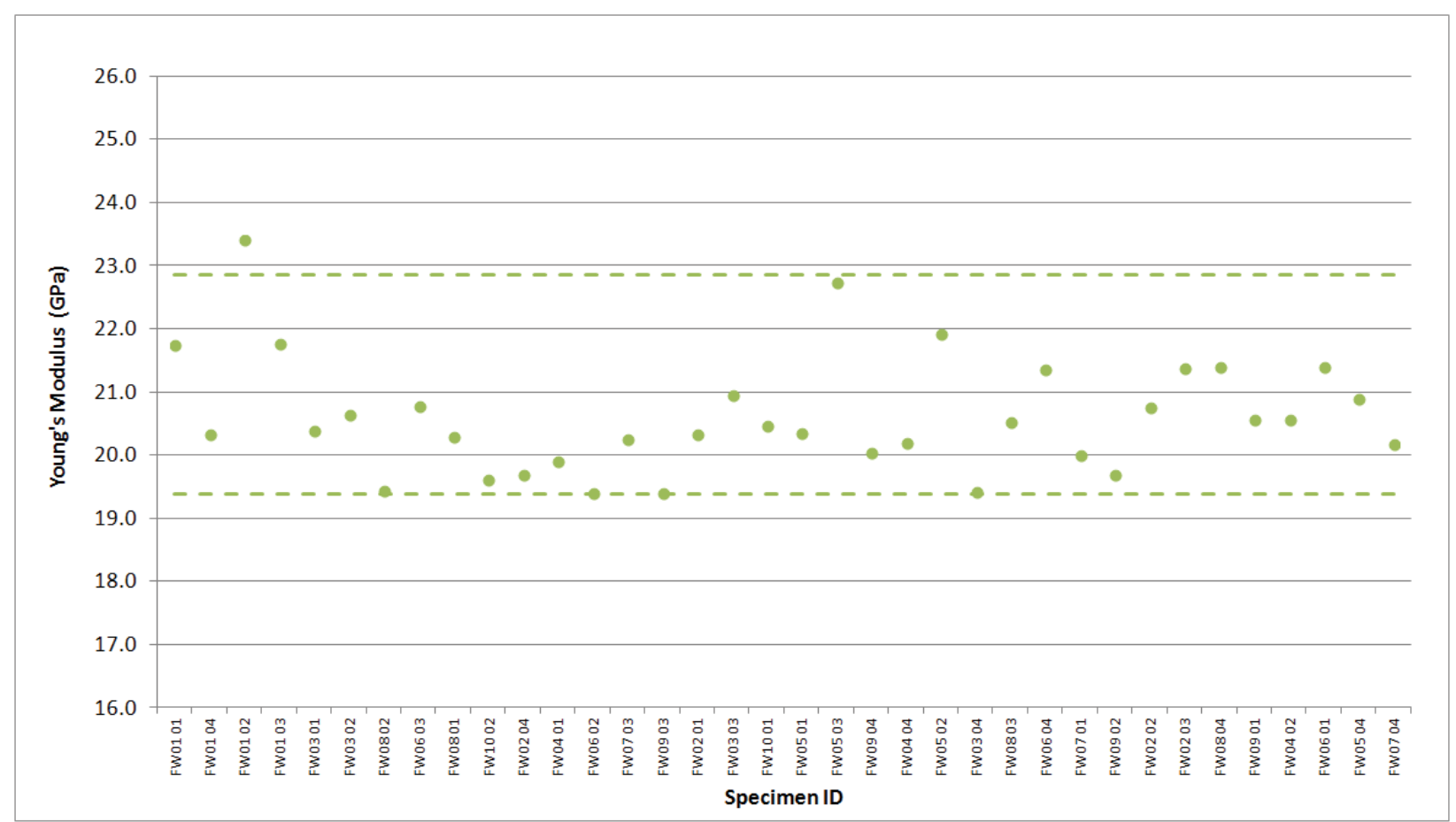

Figure A-123. IG-430 Creep Young's Modulus by Sonic Velocity.

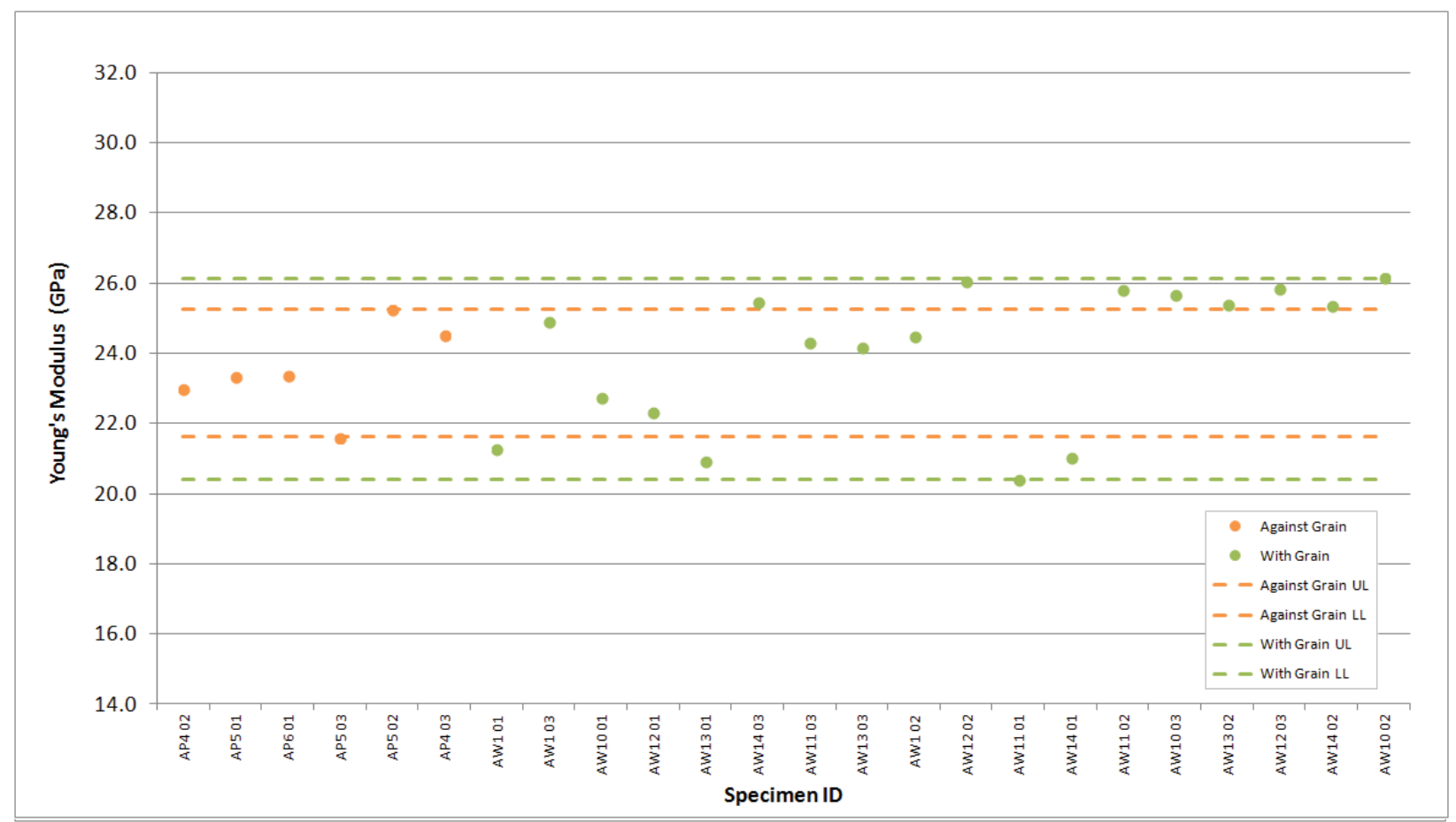

Figure A-124. NBG-17 Creep Young's Modulus by Sonic Velocity. 




Figure A-125. NBG-18 Creep Young's Modulus by Sonic Velocity.

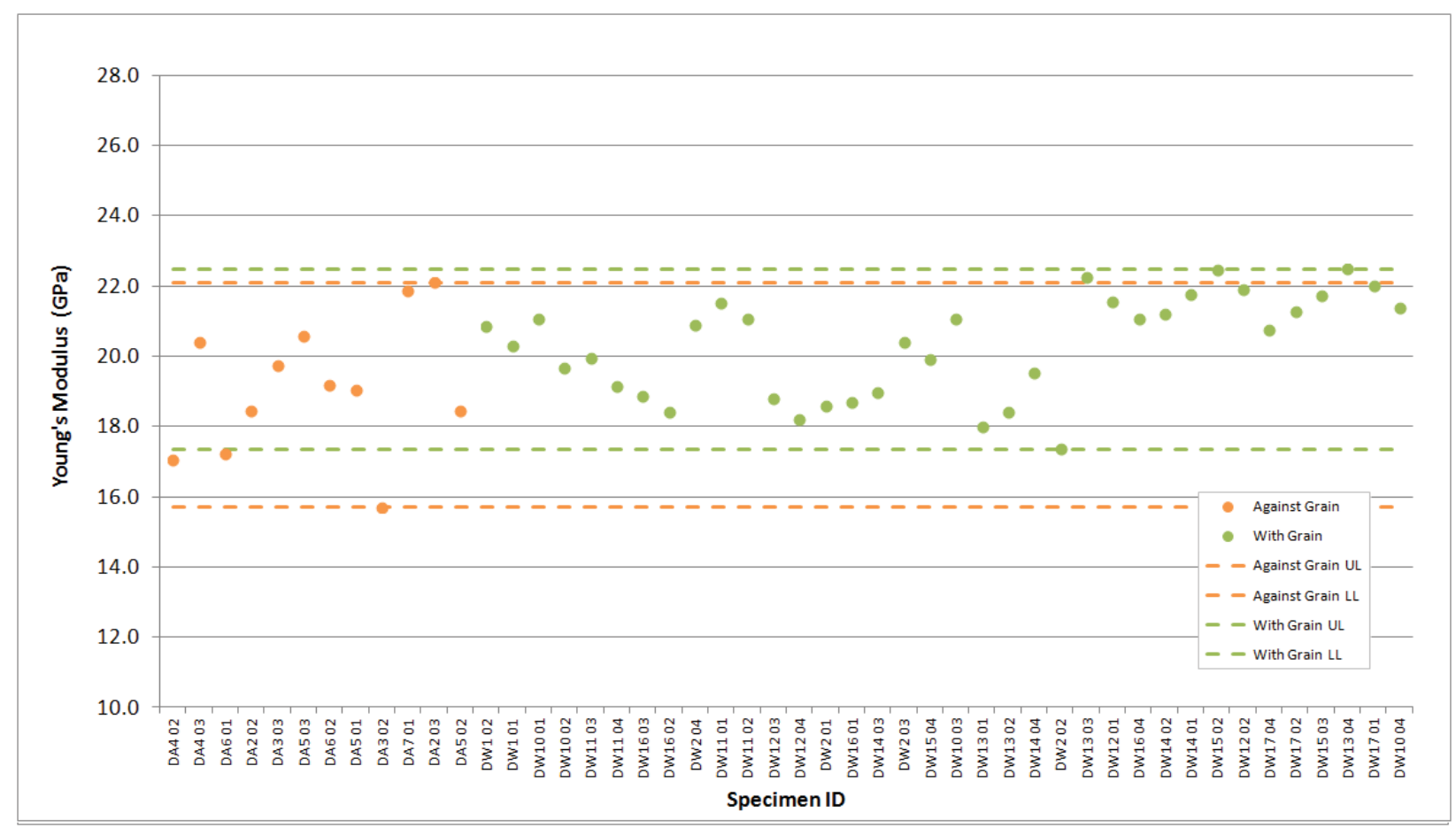

Figure A-126. PCEA Creep Young's Modulus by Sonic Velocity. 


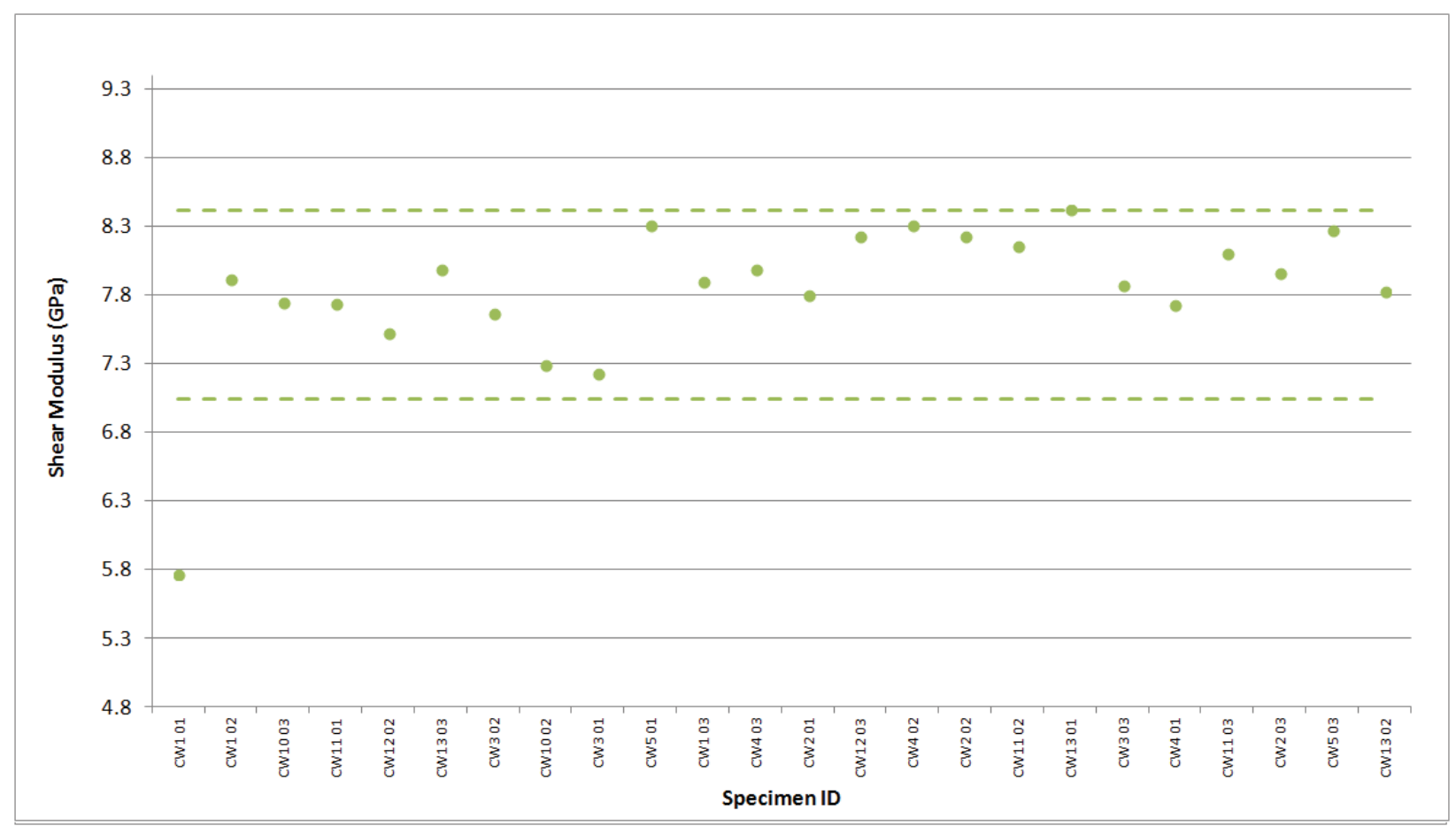

Figure A-127. H-451 Creep Shear Modulus by Sonic Velocity.

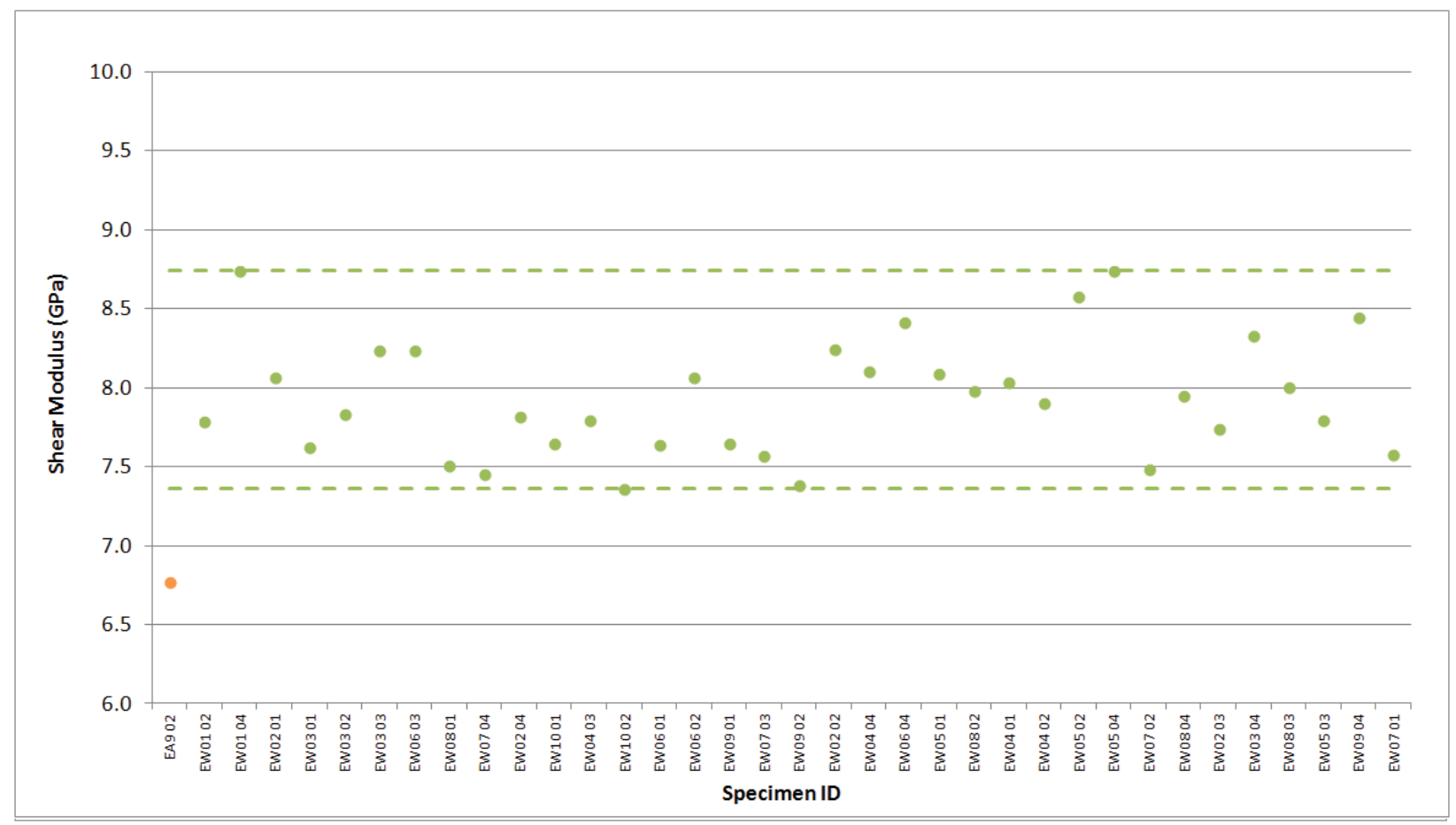

Figure A-128. IG-110 Creep Shear Modulus by Sonic Velocity. 


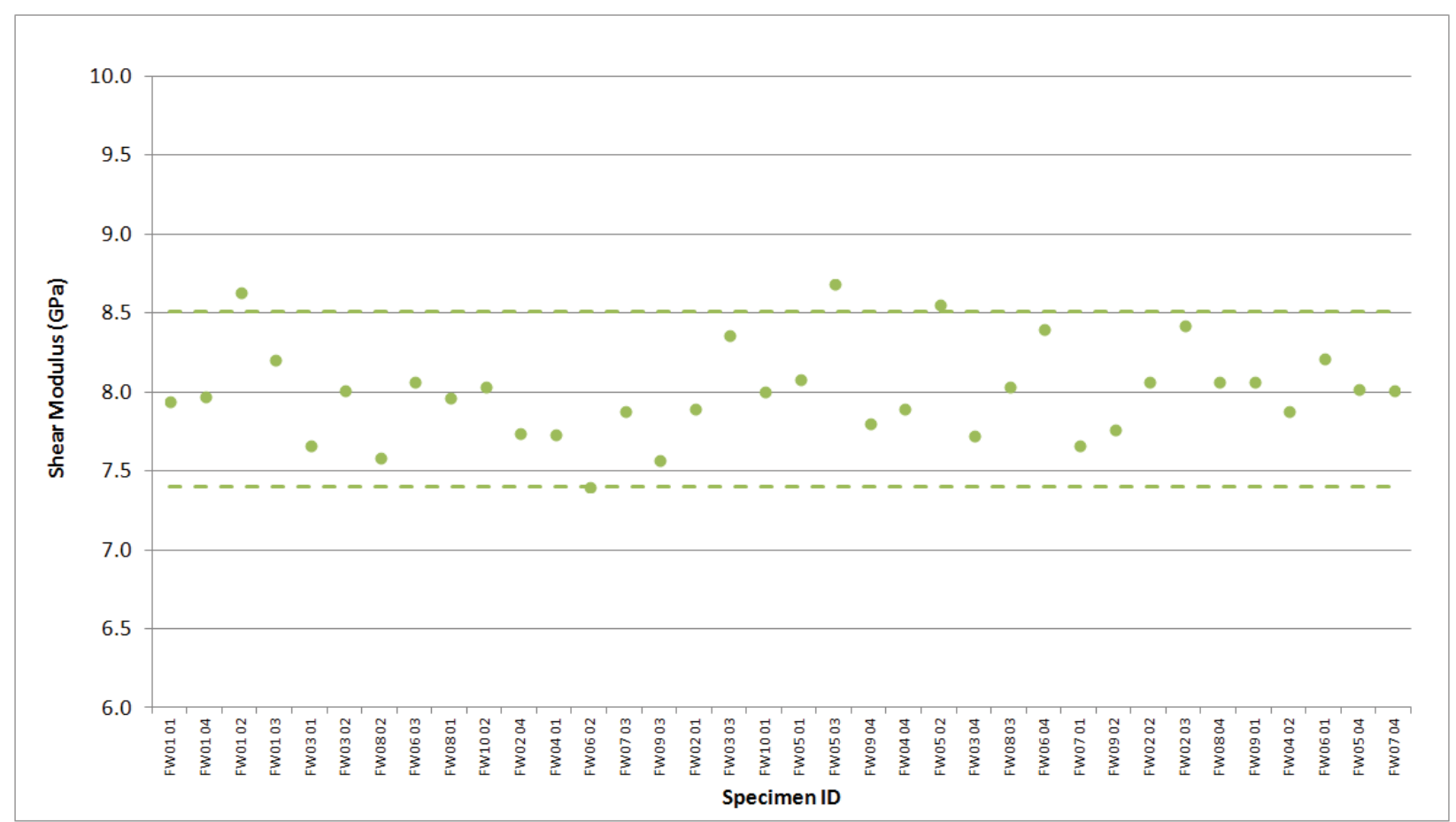

Figure A-129. IG-430 Creep Shear Modulus by Sonic Velocity.

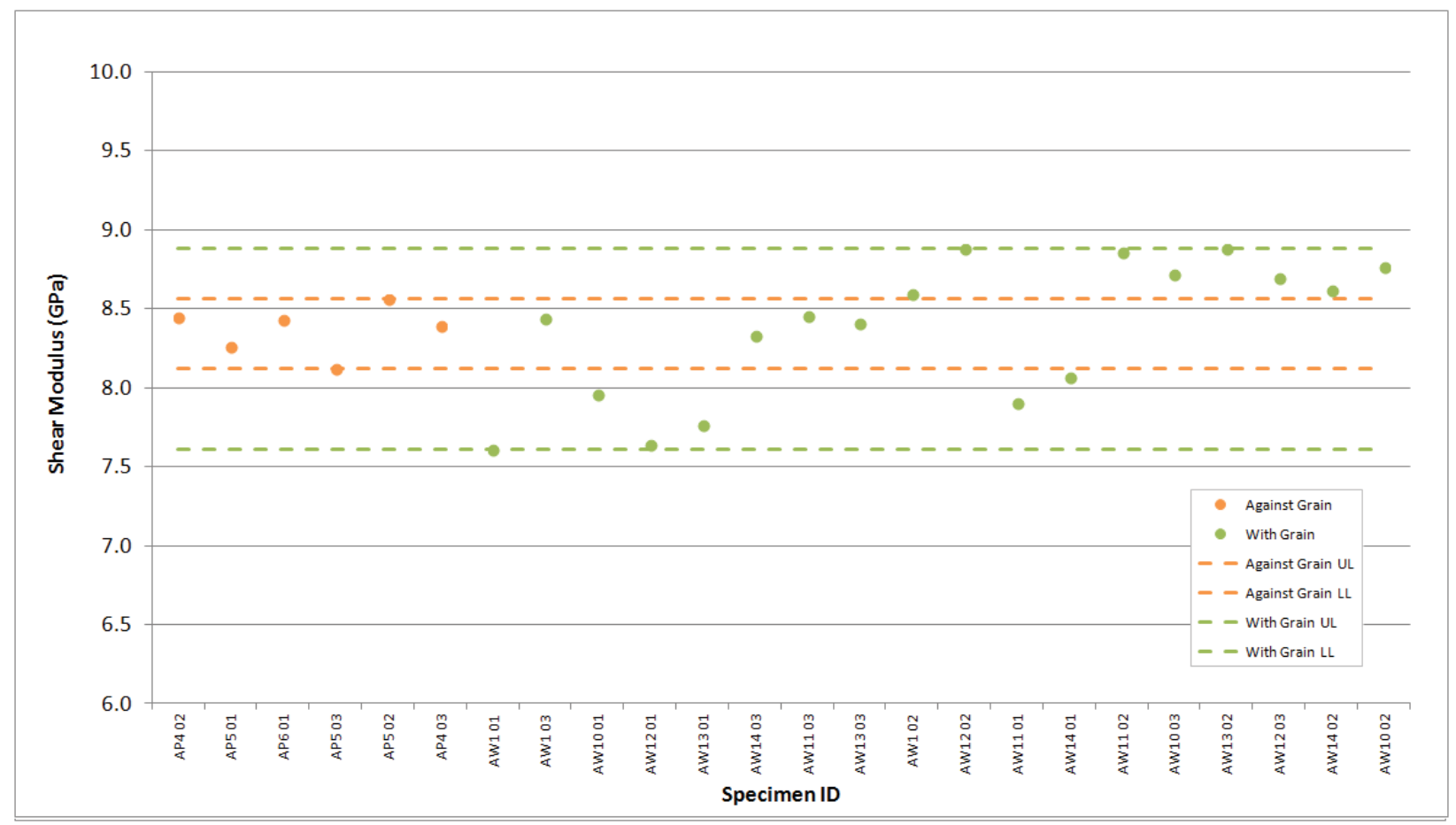

Figure A-130. NBG-17 Creep Shear Modulus by Sonic Velocity. 


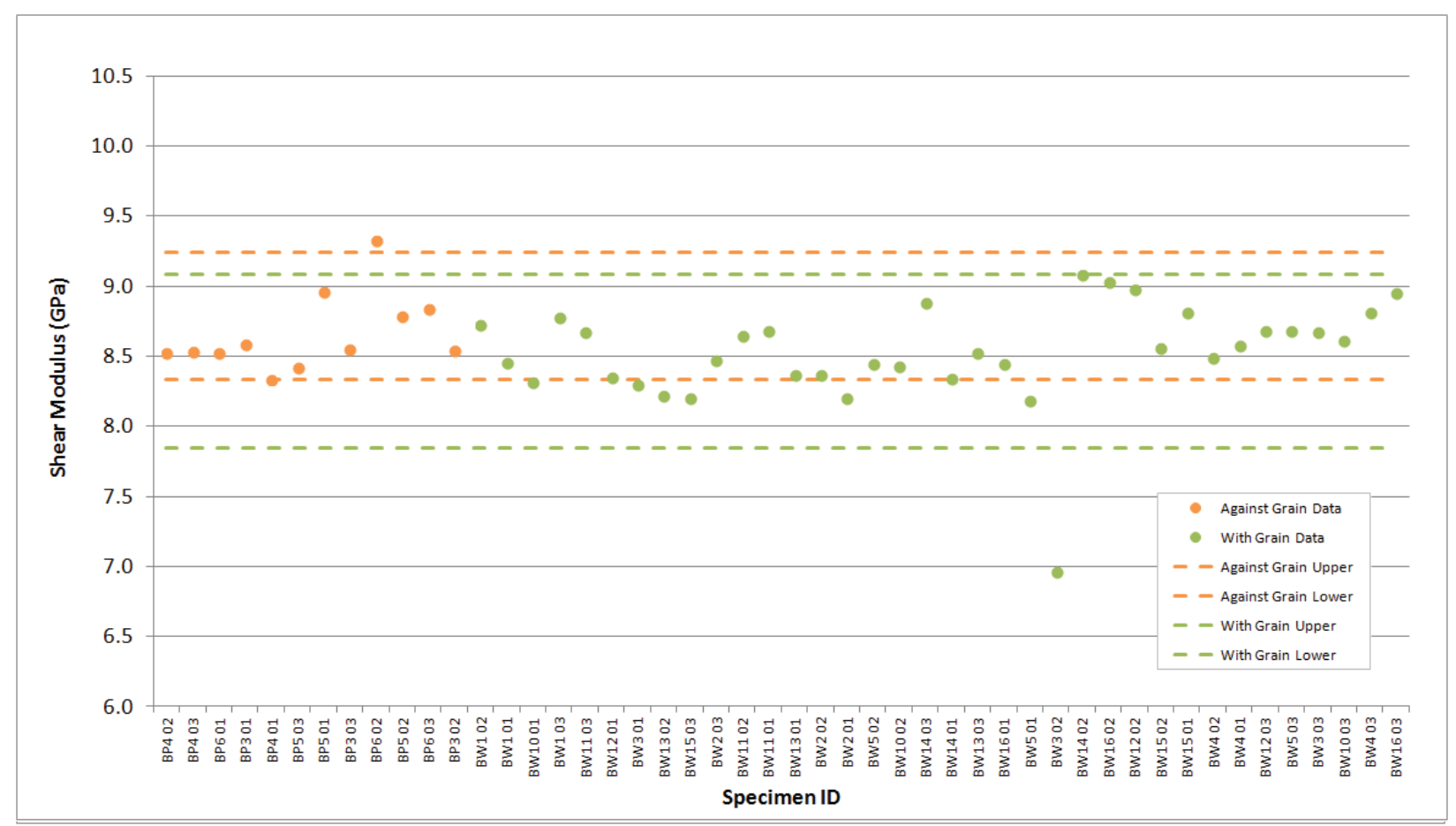

Figure A-131. NBG-18 Creep Shear Modulus by Sonic Velocity.

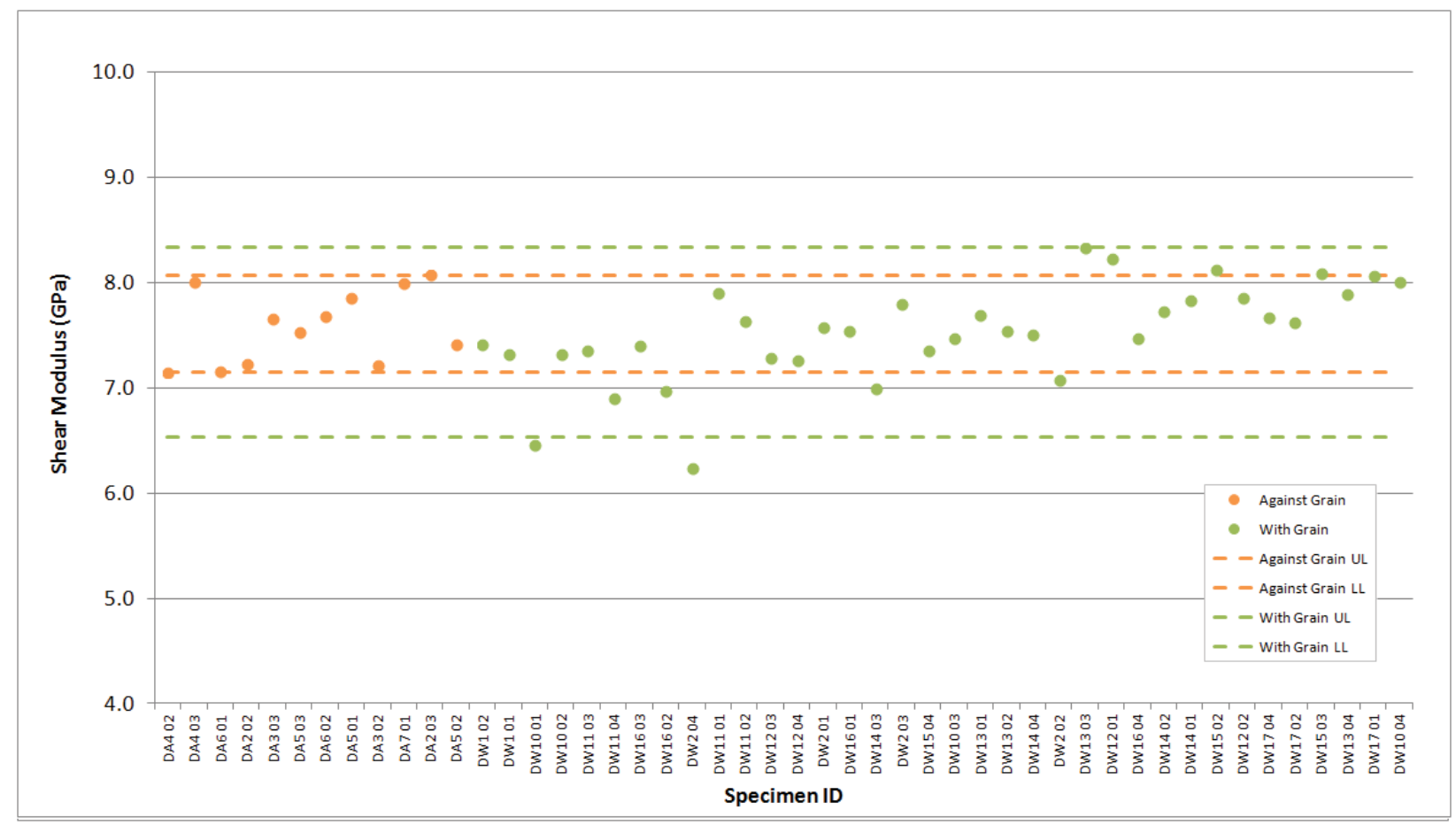

Figure A-132. PCEA Creep Shear Modulus by Sonic Velocity. 


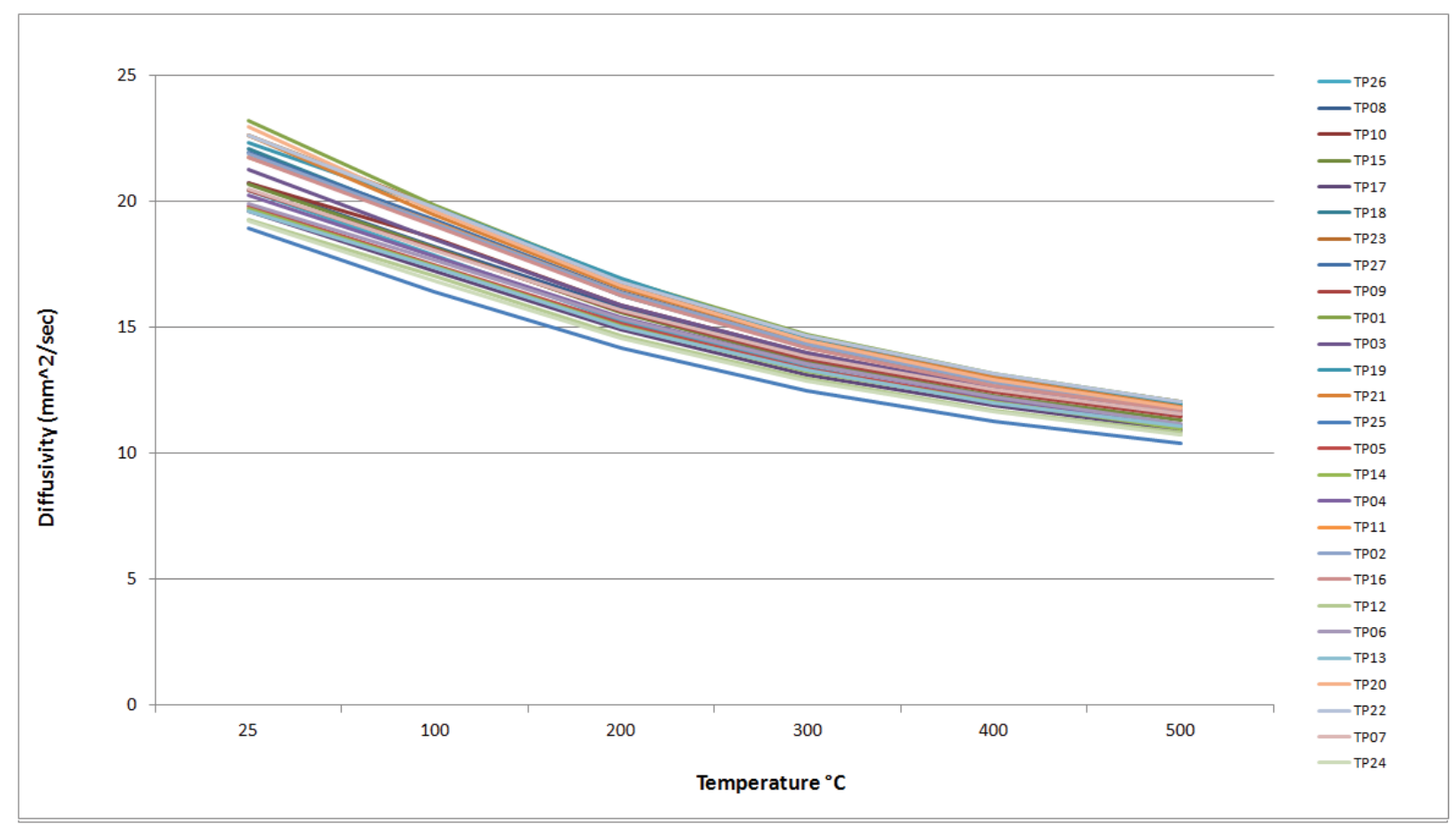

Figure A-133. 2114 Piggyback Diffusivity.

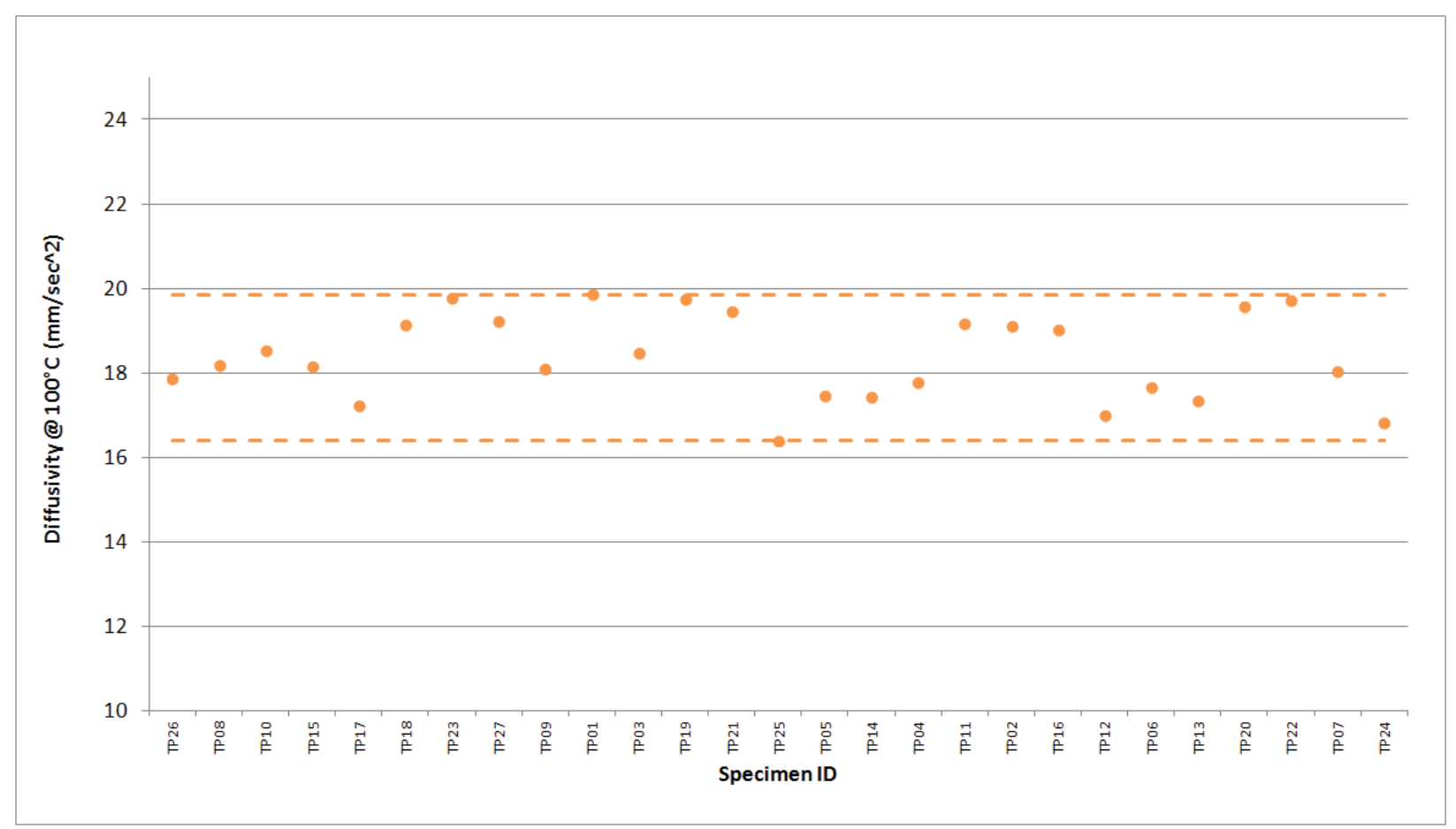

Figure A-134. 2114 Piggyback Diffusivity @ $100^{\circ} \mathrm{C}$. 


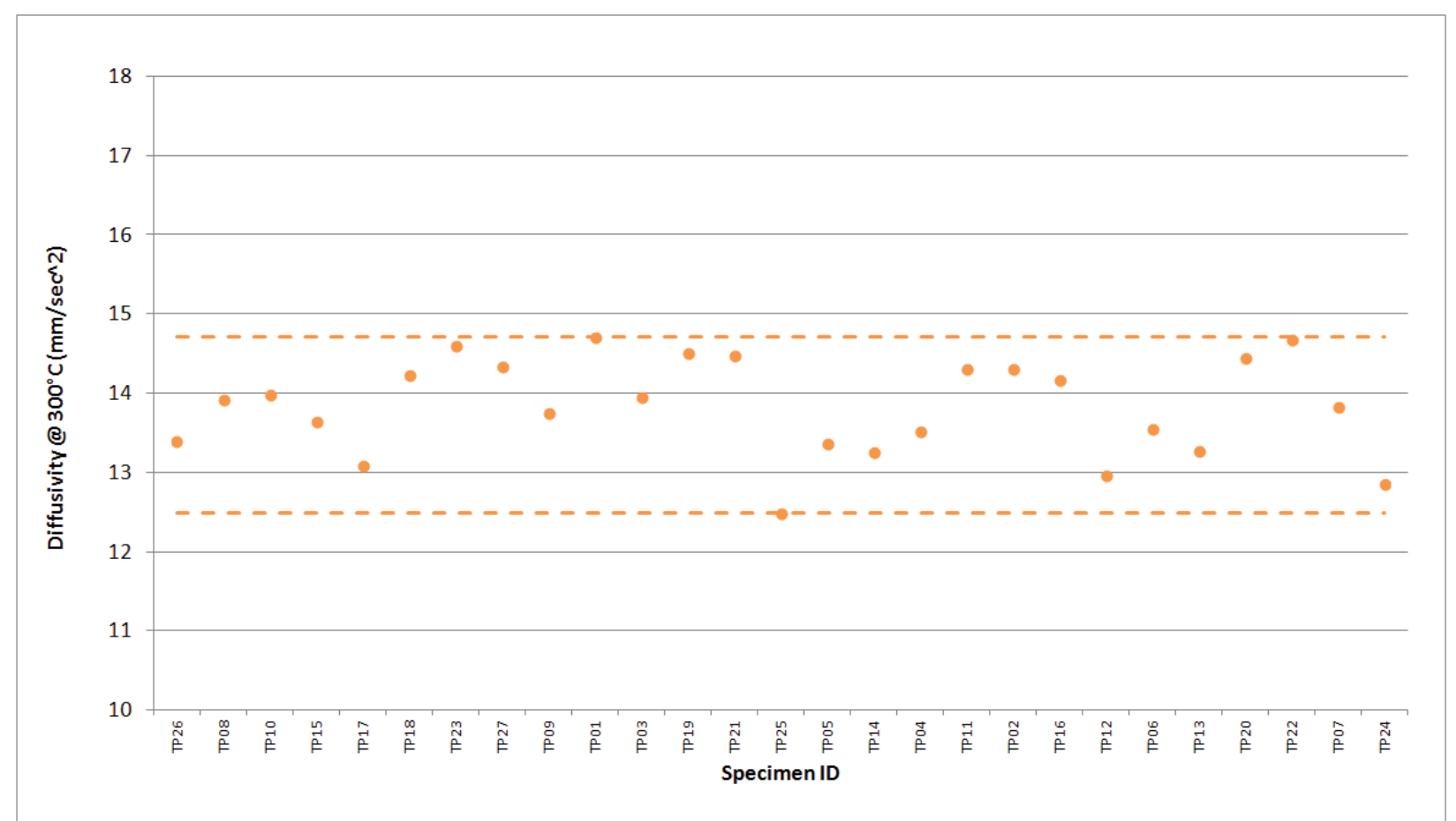

Figure A-135. 2114 Piggyback Diffusivity @ 300 C.

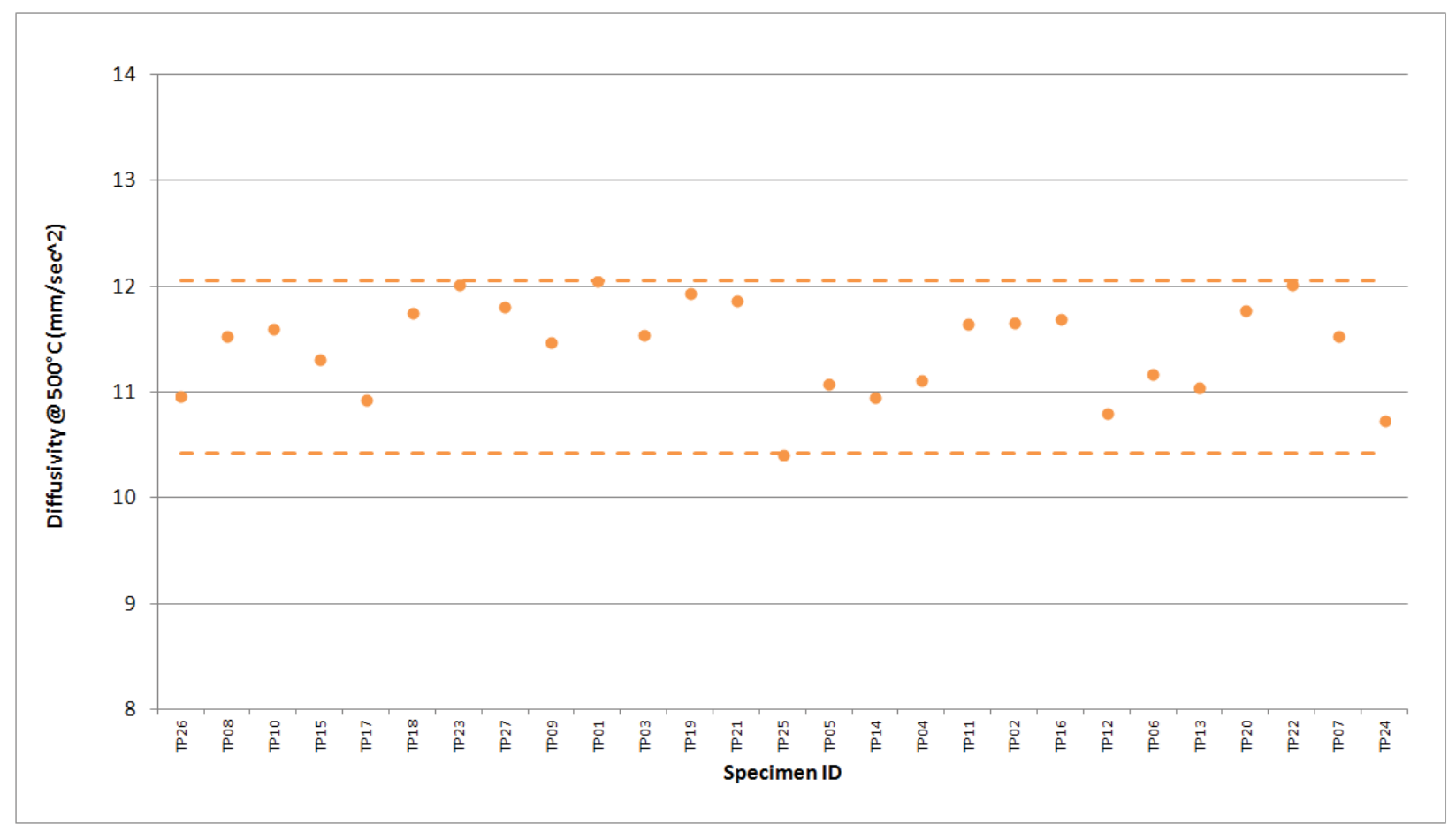

Figure A-136.2114 Piggyback Diffusivity@500². 


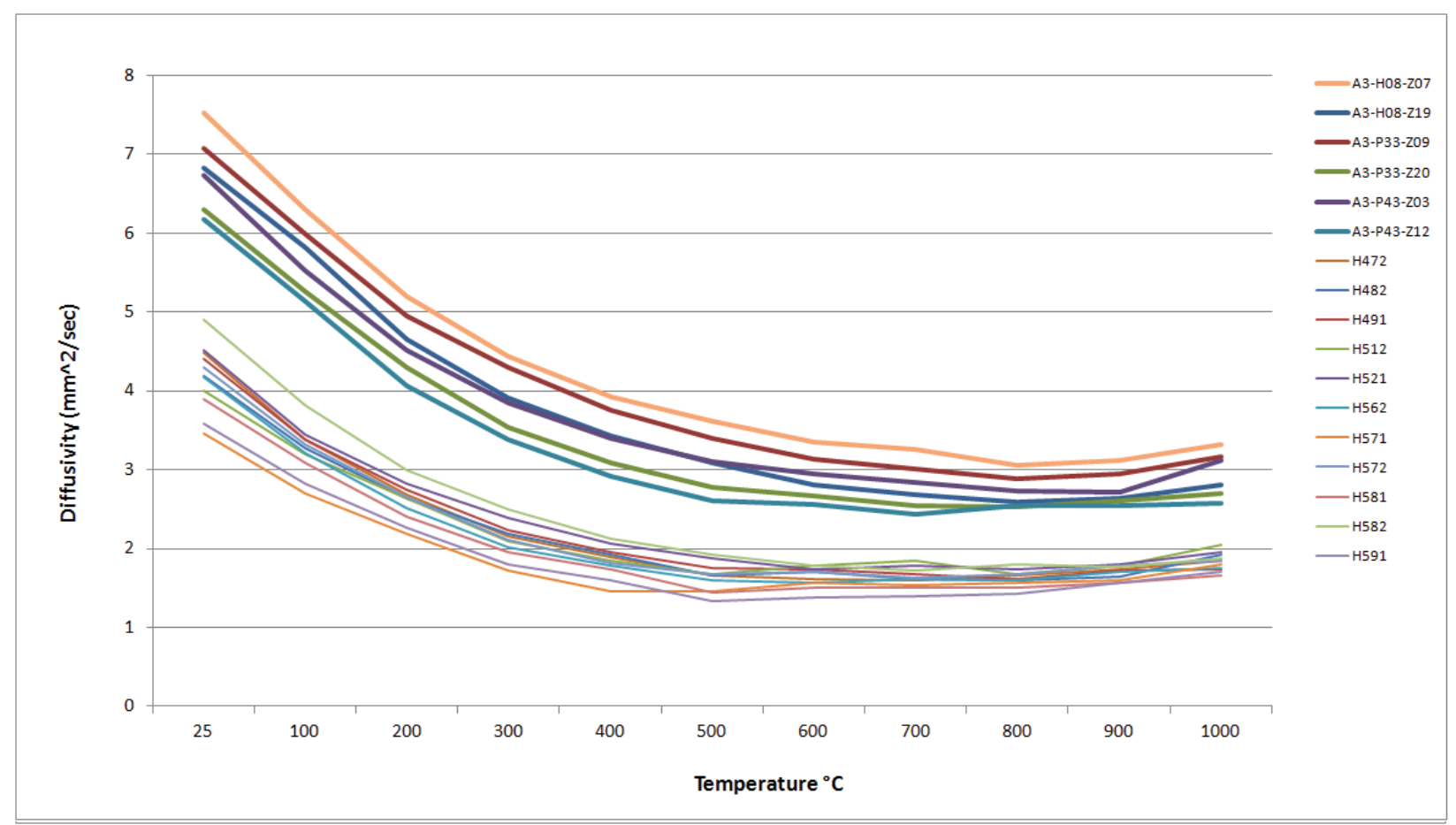

Figure A-137. A3 Piggyback Diffusivity.

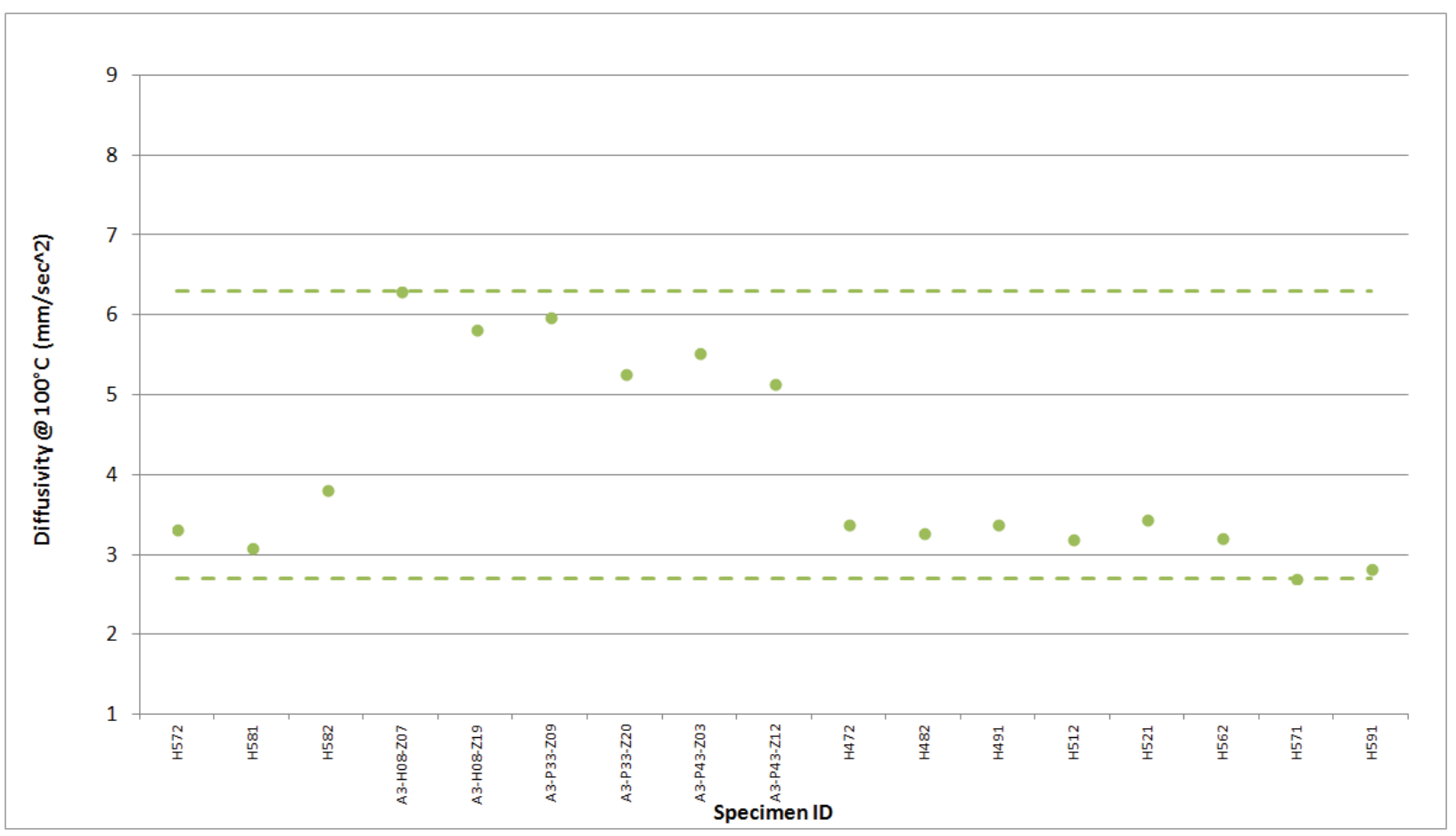

Figure A-138. A3 Piggyback Diffusivity@ $100^{\circ} \mathrm{C}$. 


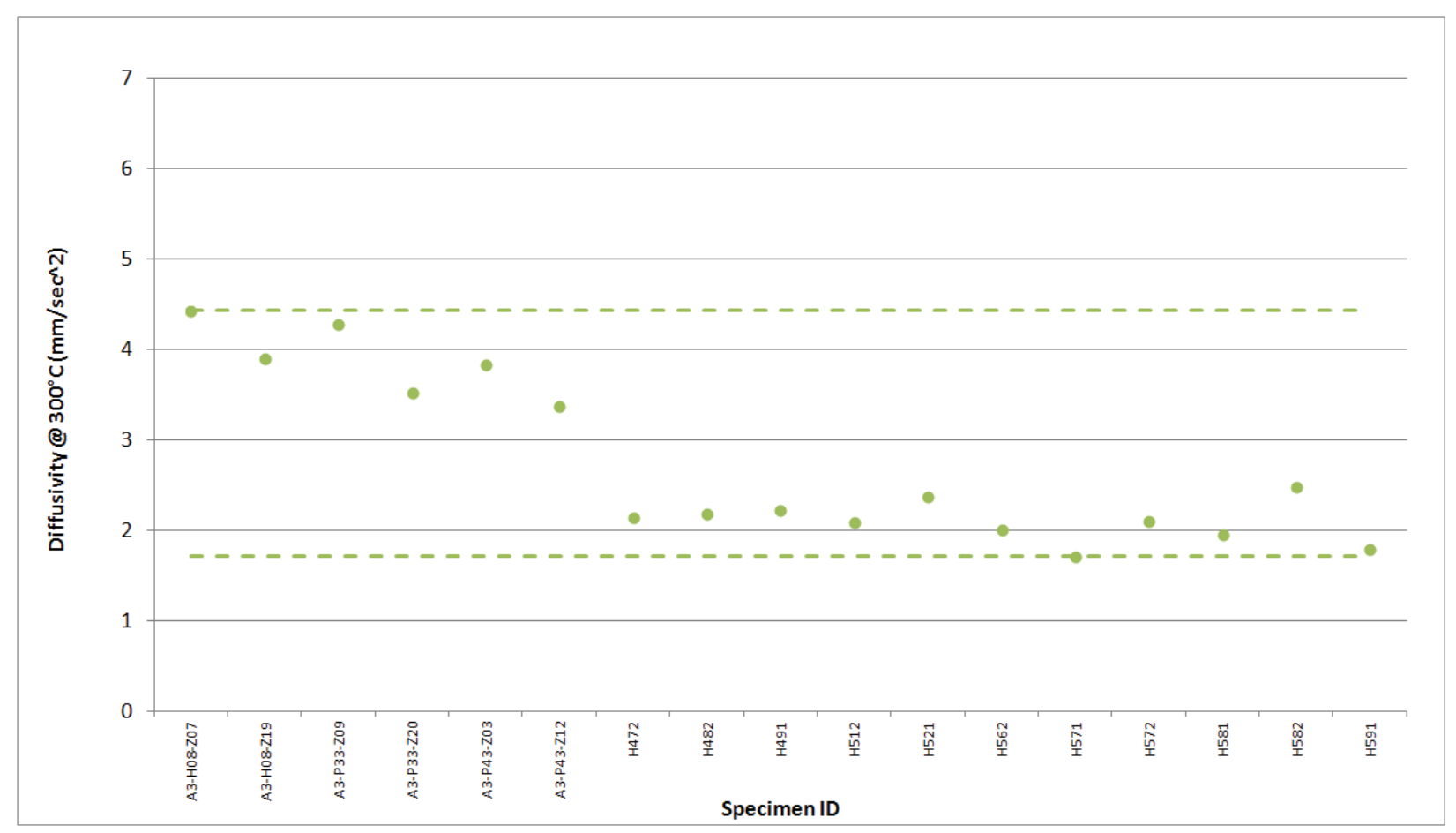

Figure A-139. A3 Piggyback Diffusivity@300C.

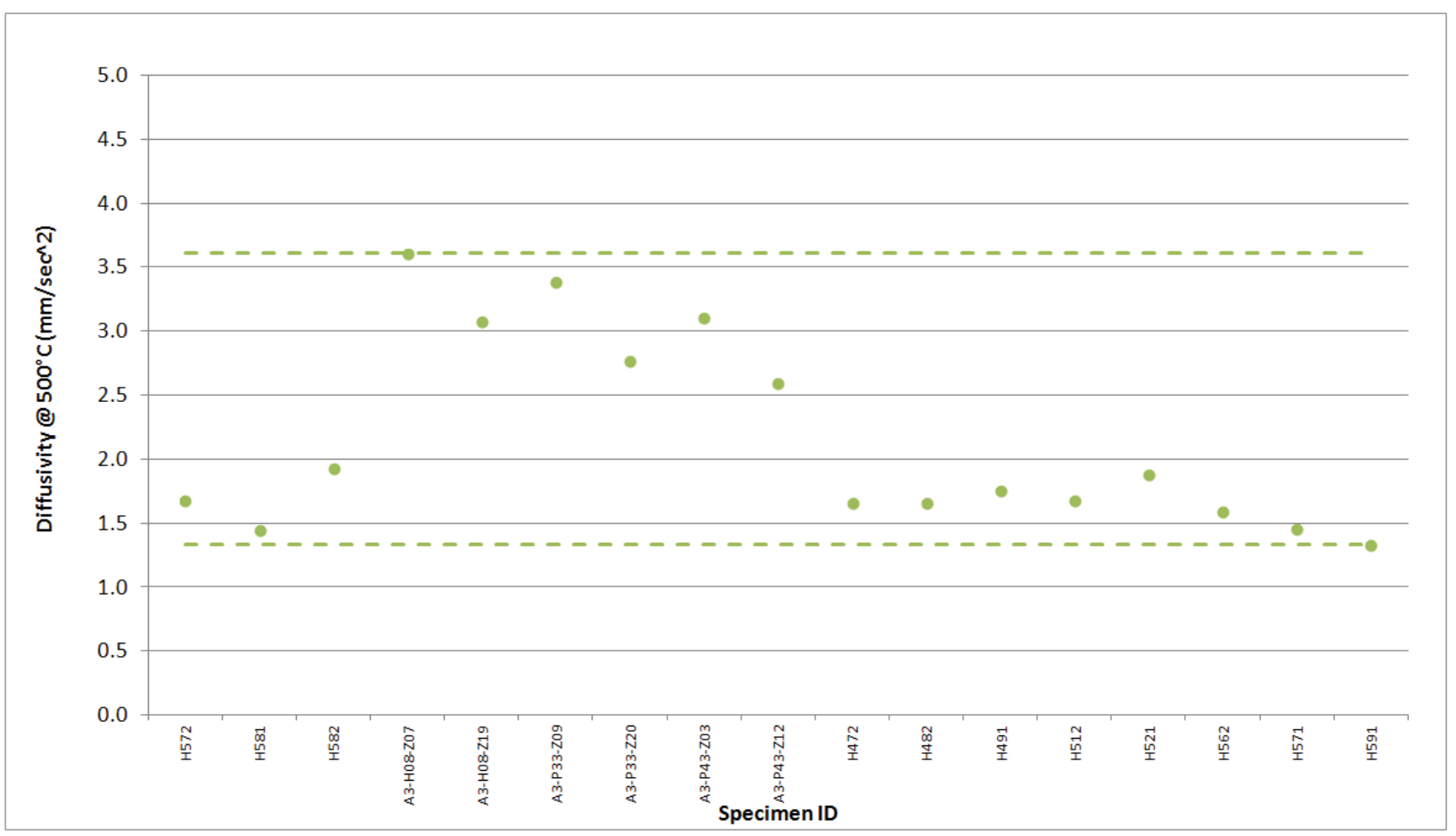

Figure A-140. A3 Piggyback Diffusivity@500C. 


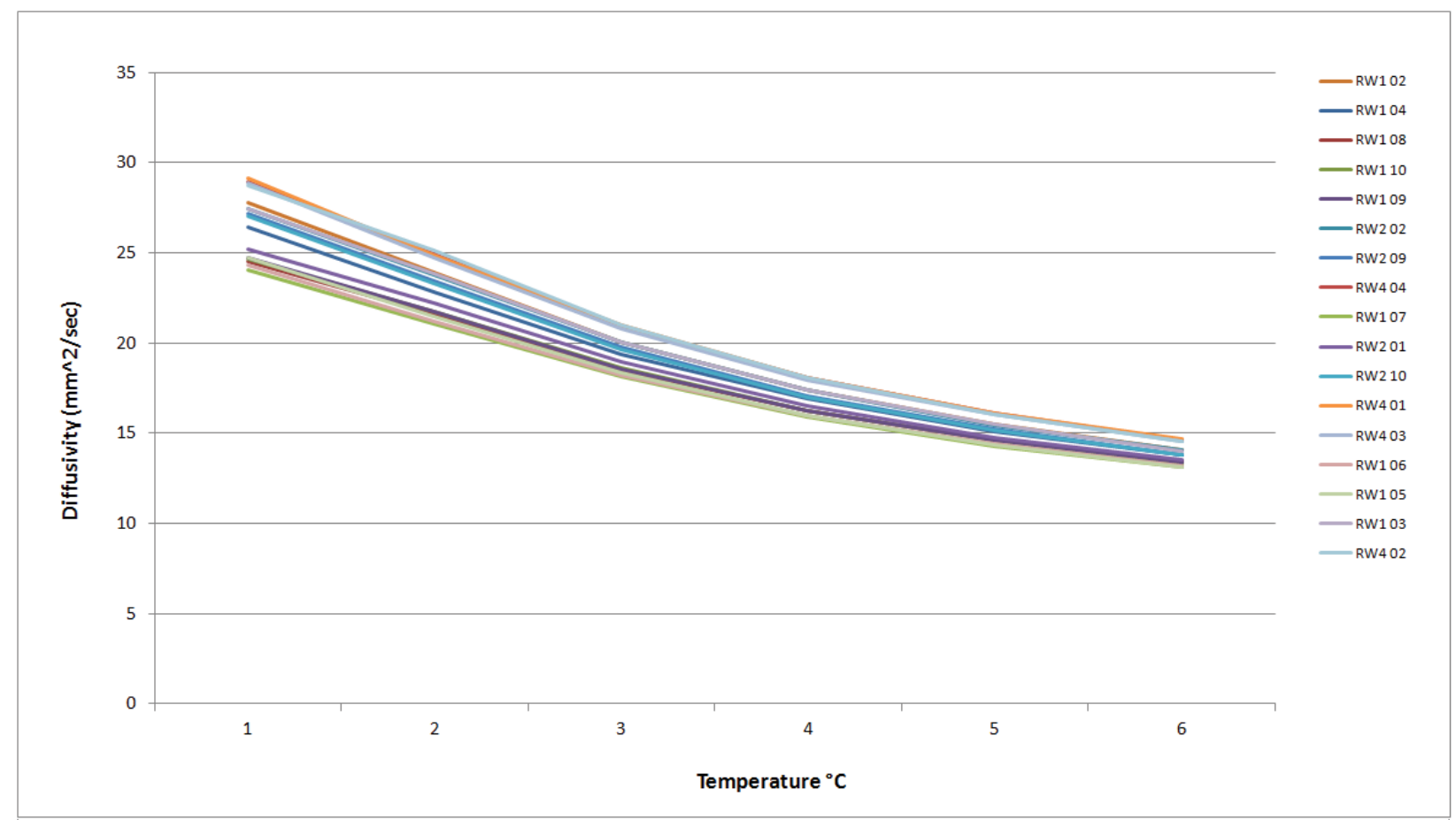

Figure A-141. BAN Piggyback Diffusivity.

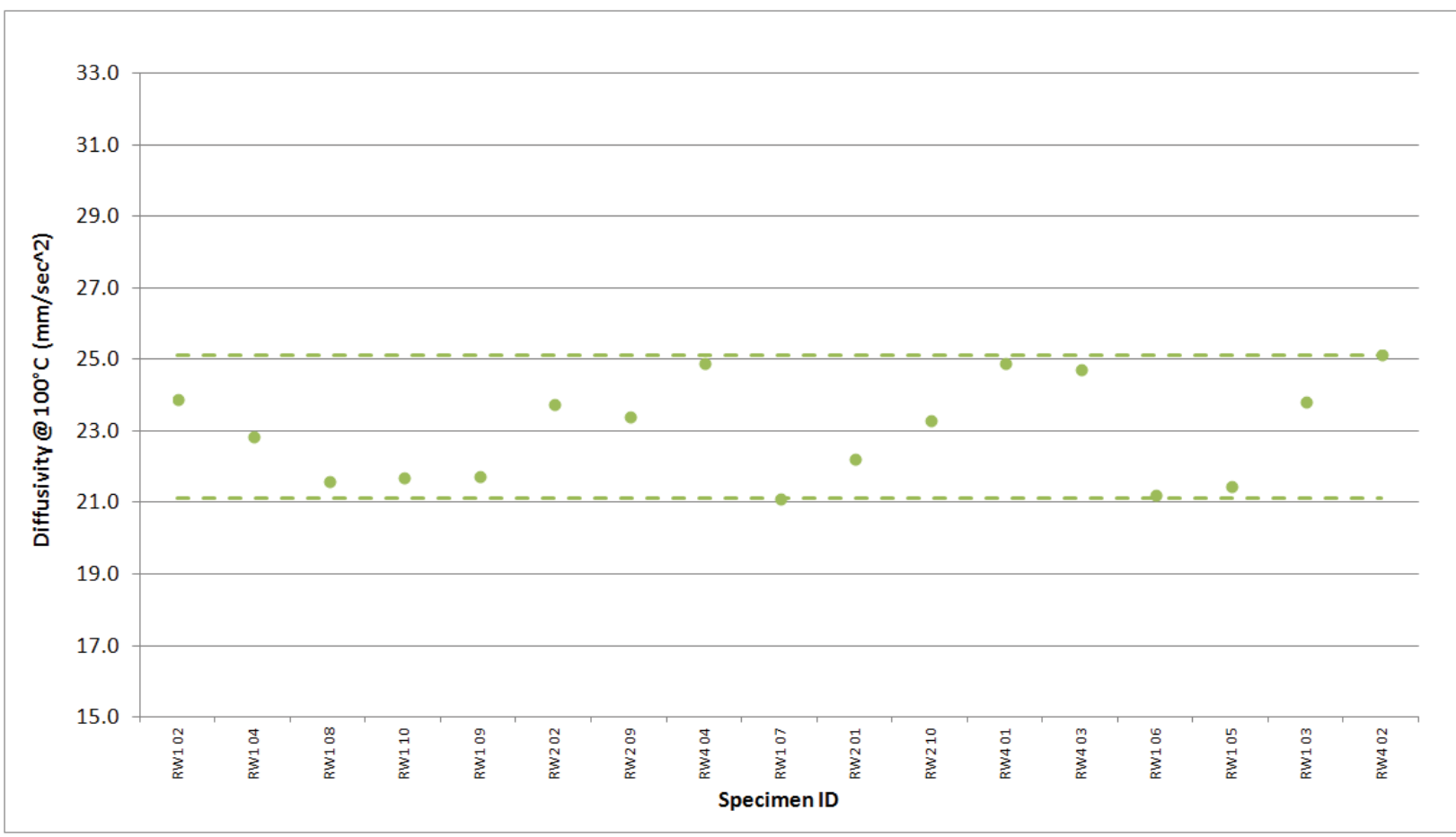

Figure A-142. BAN Piggyback Diffusivity @ $100^{\circ} \mathrm{C}$. 


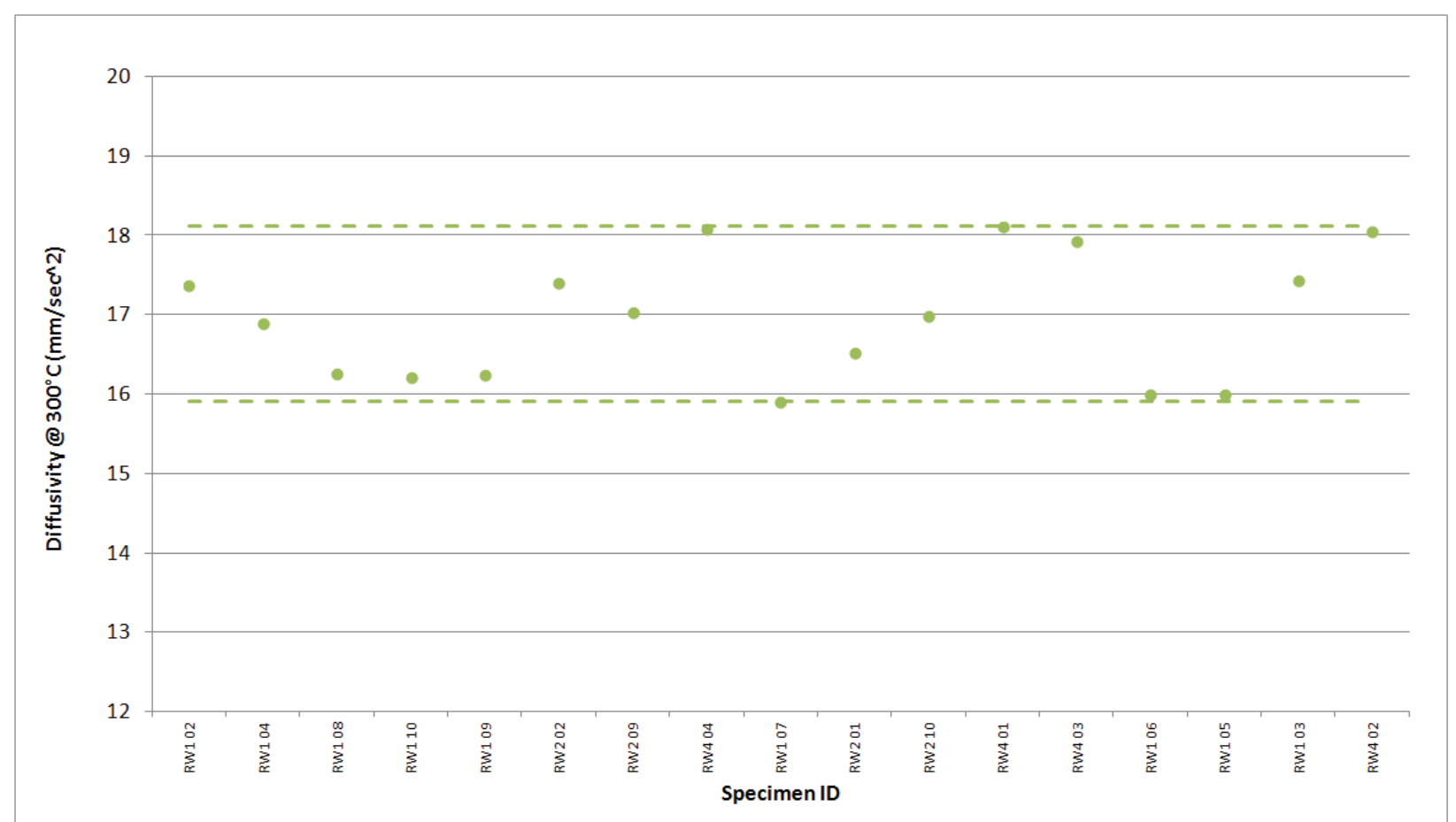

Figure A-143. BAN Piggyback Diffusivity @ 300 C.

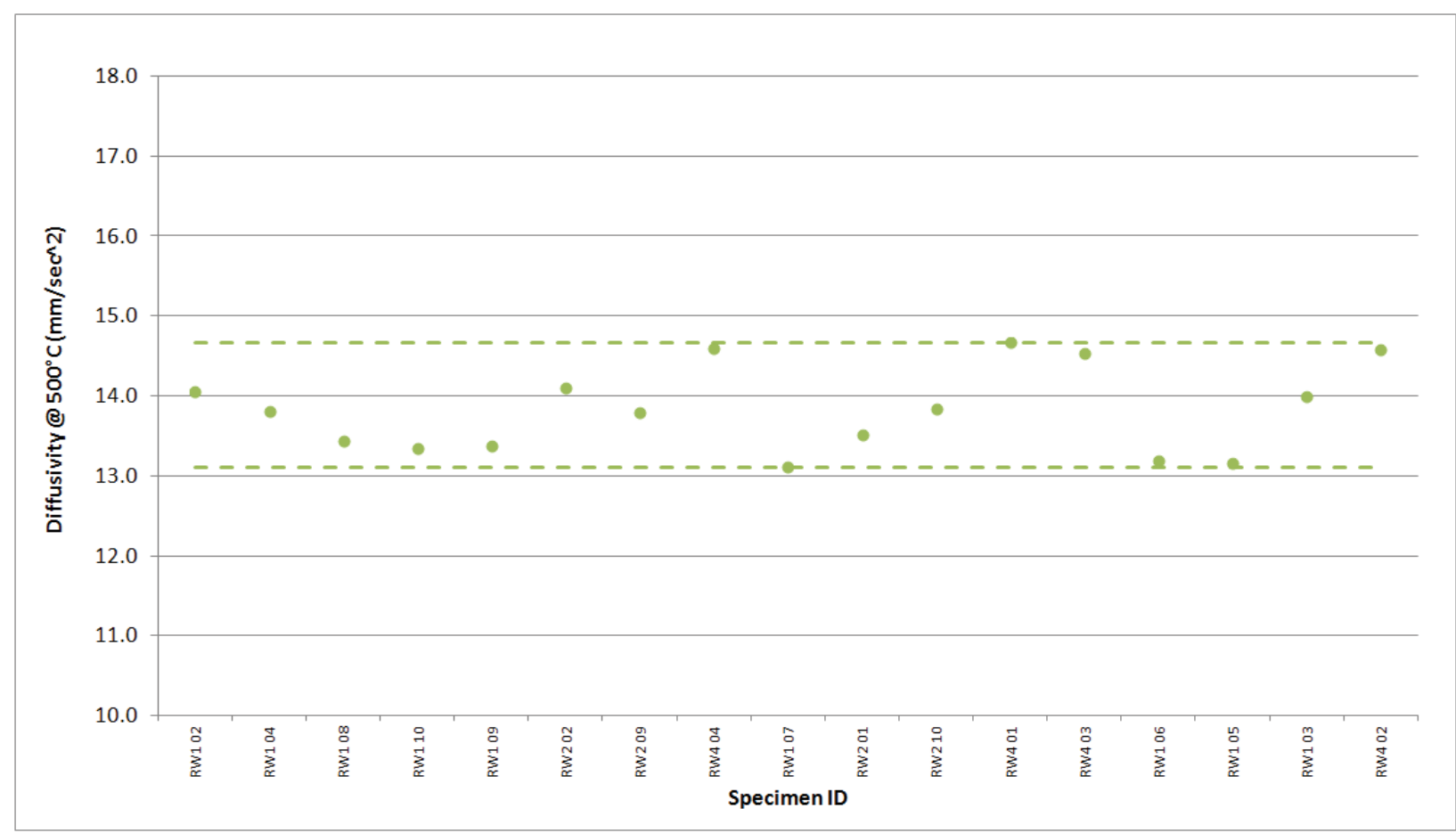

Figure A-144. BAN Piggyback Diffusivity@500². 


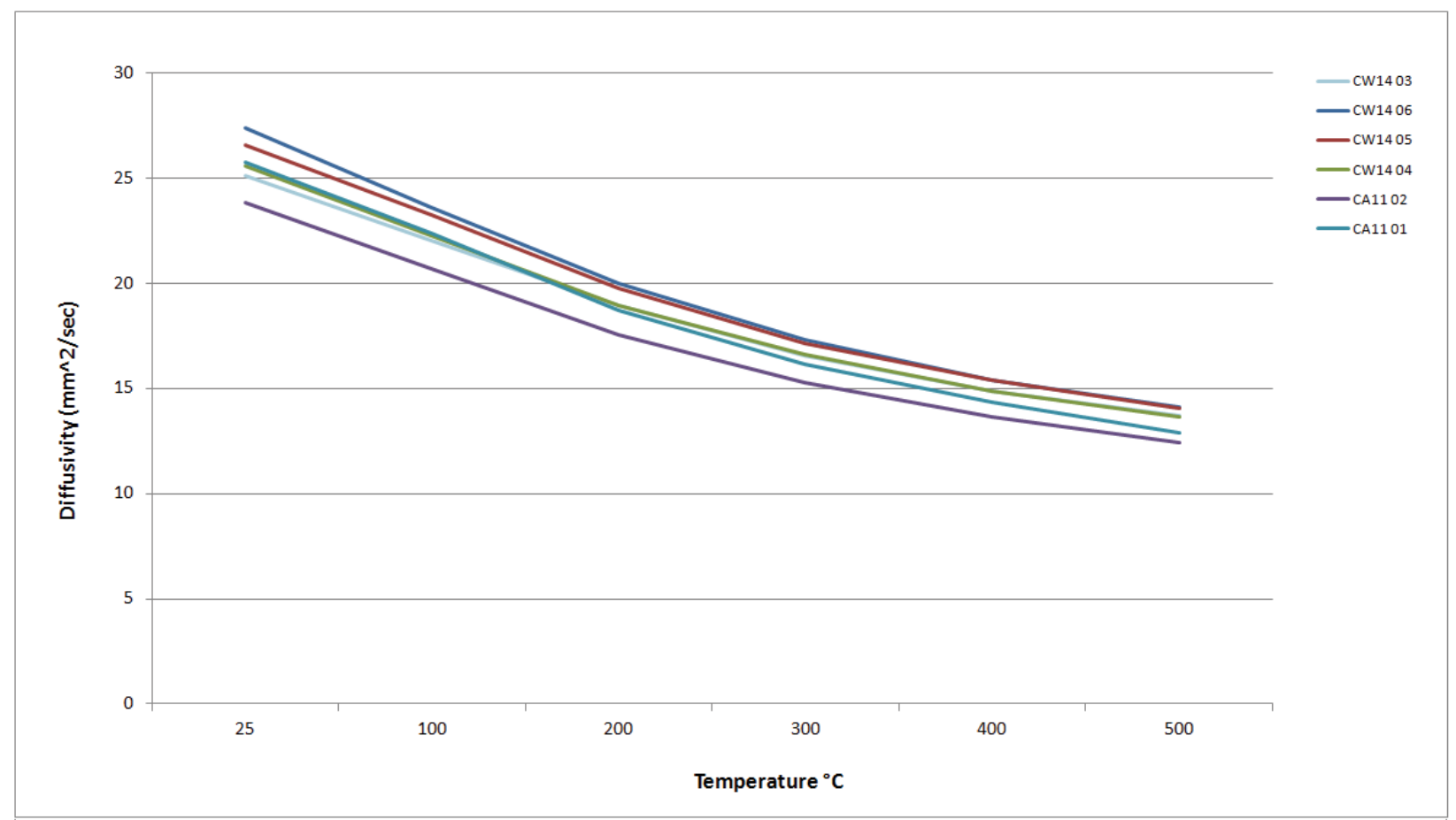

Figure A-145. H-451 Piggyback Diffusivity.

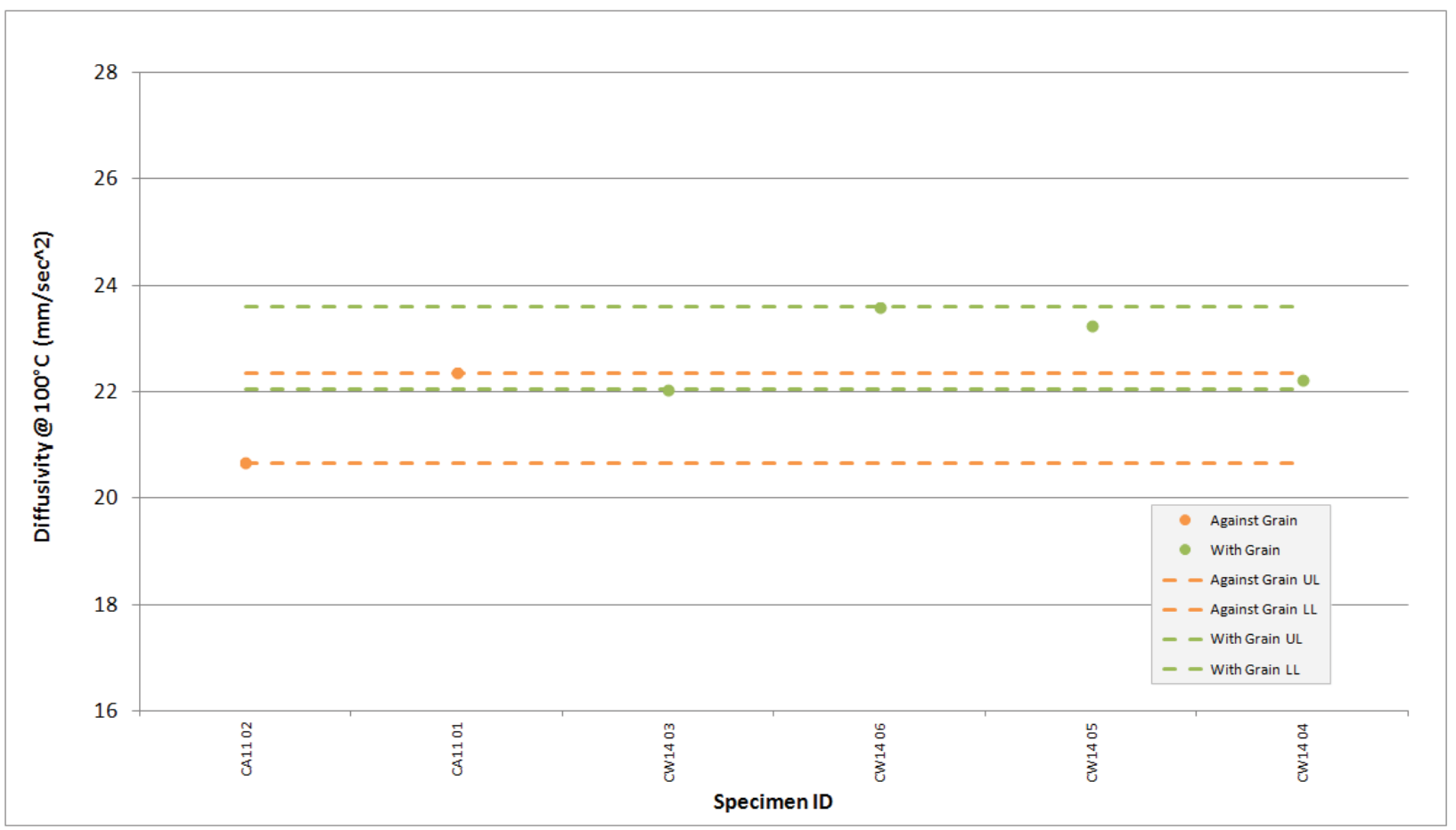

Figure A-146. H-451 Piggyback Diffusivity@ $100^{\circ} \mathrm{C}$. 


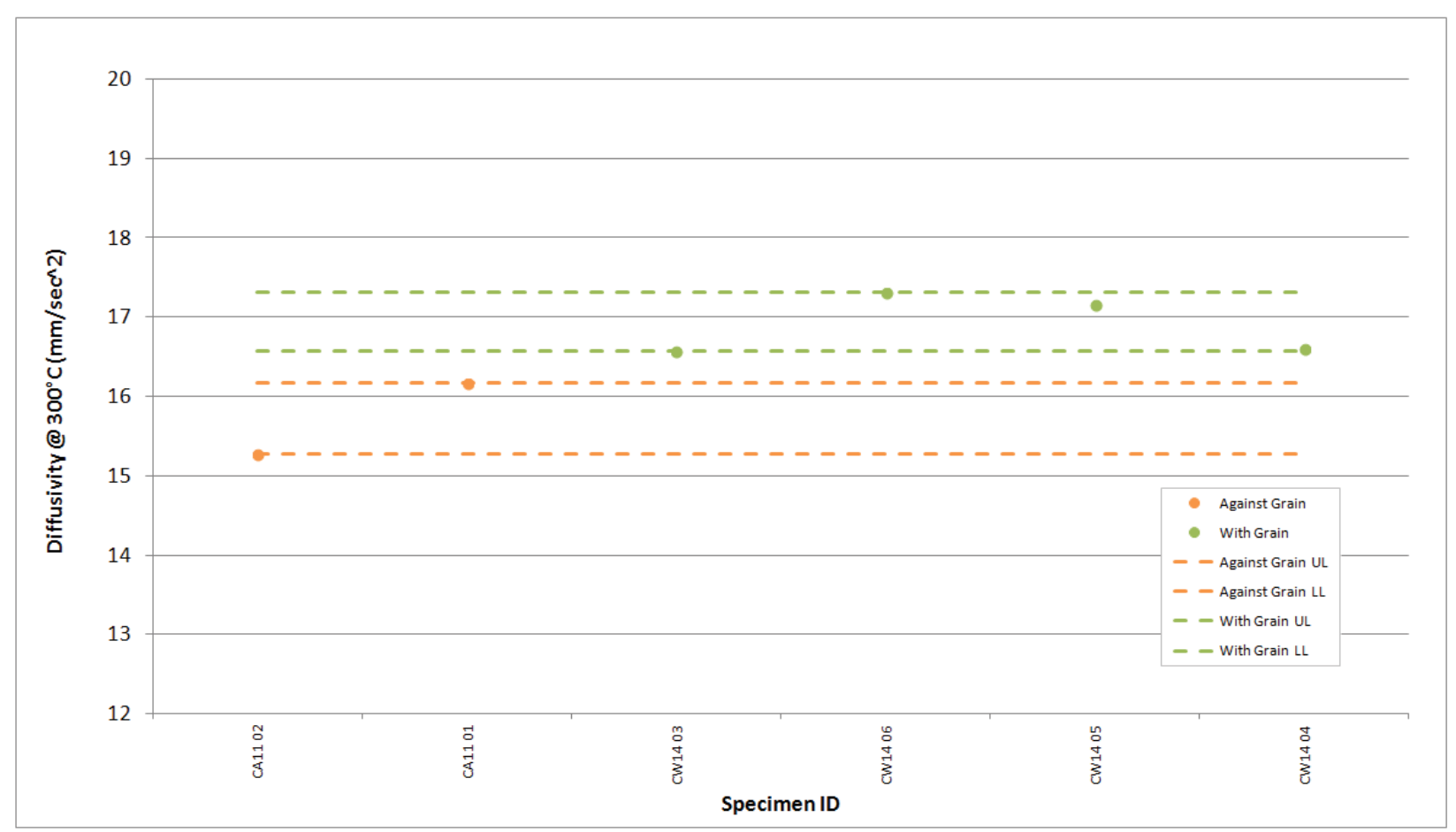

Figure A-147. H-451 Piggyback Diffusivity @ 300․

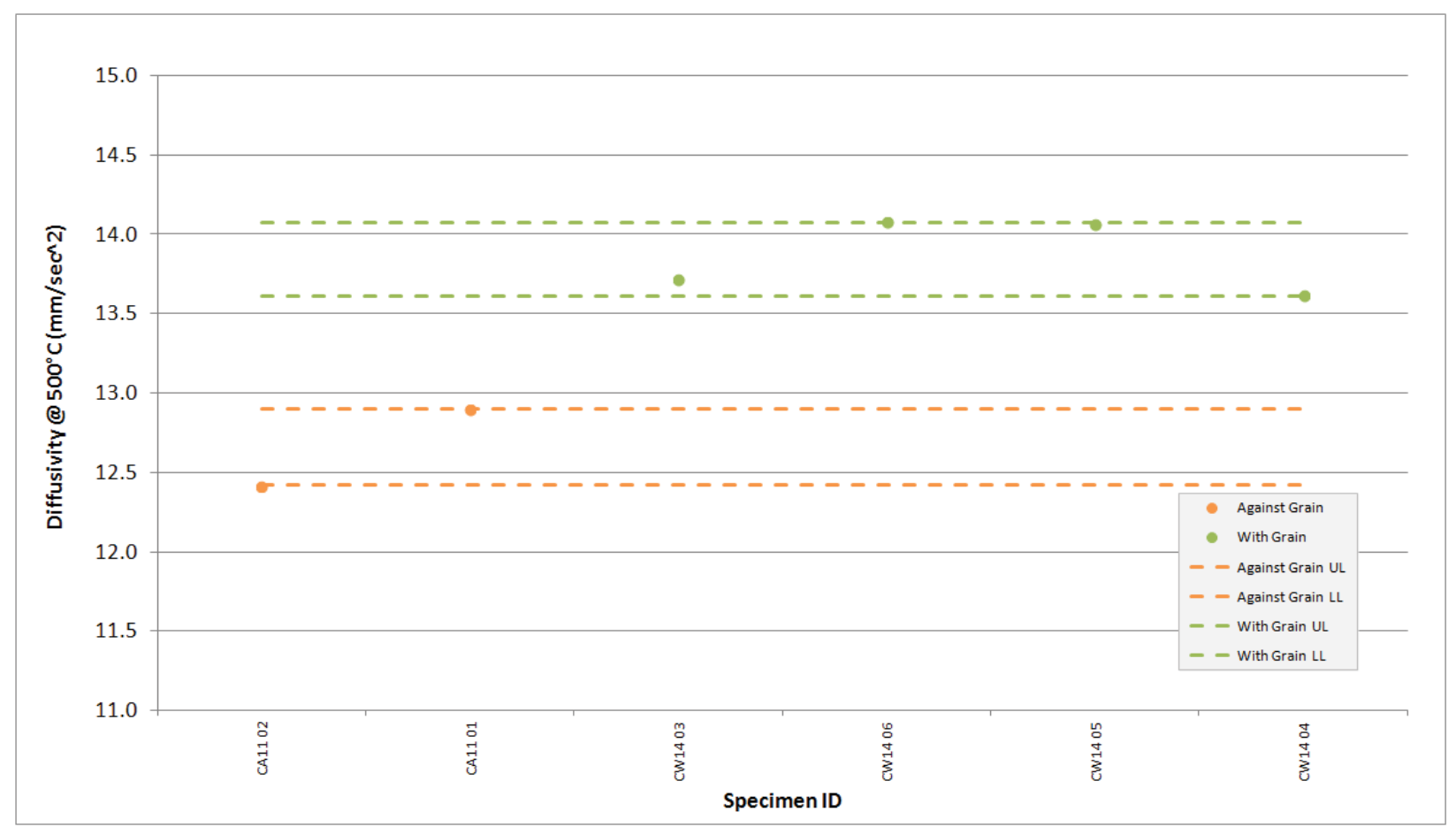

Figure A-148. H-451 Piggyback Diffusivity@500C. 


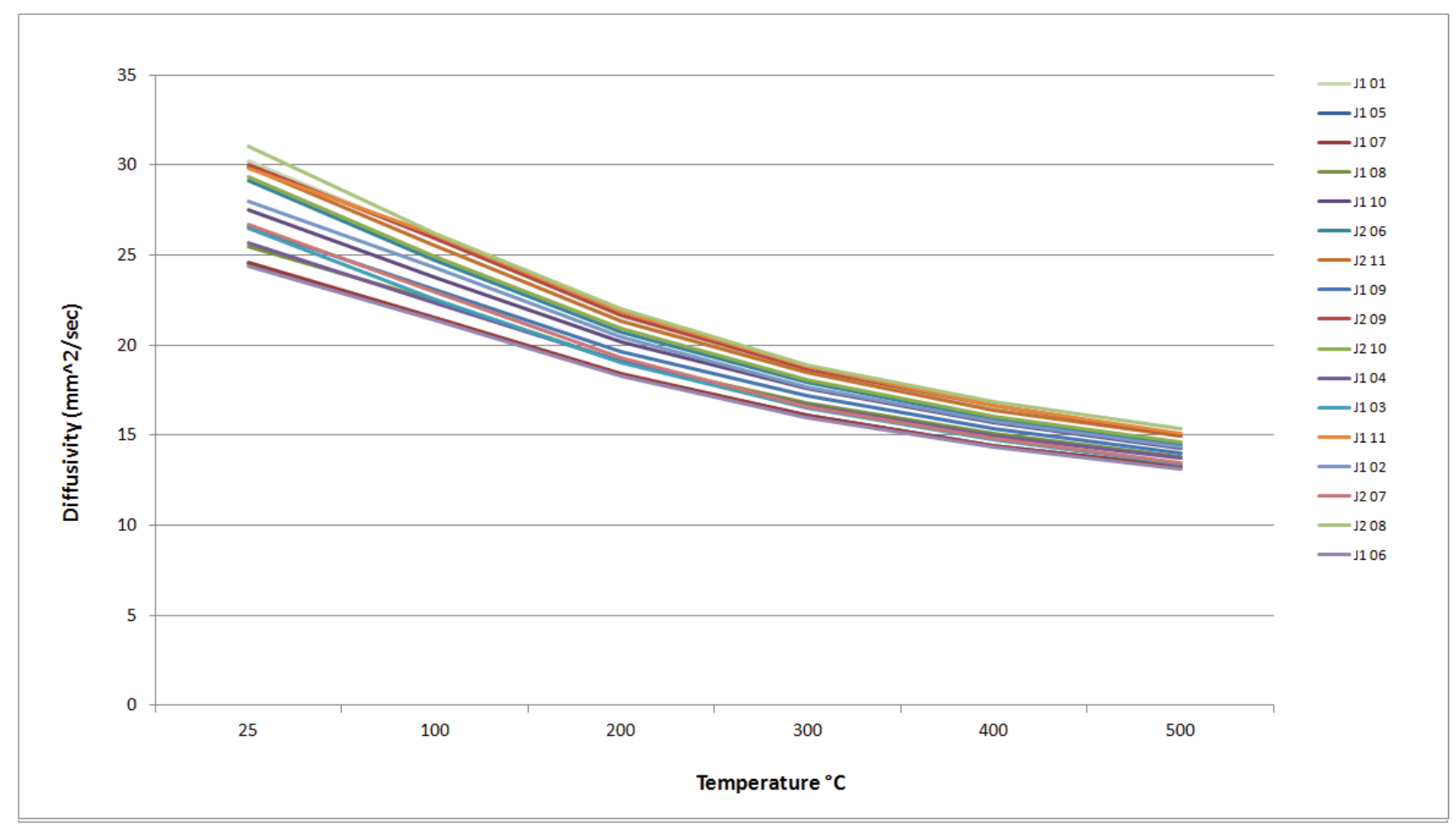

Figure A-149. HLM Piggyback Diffusivity.

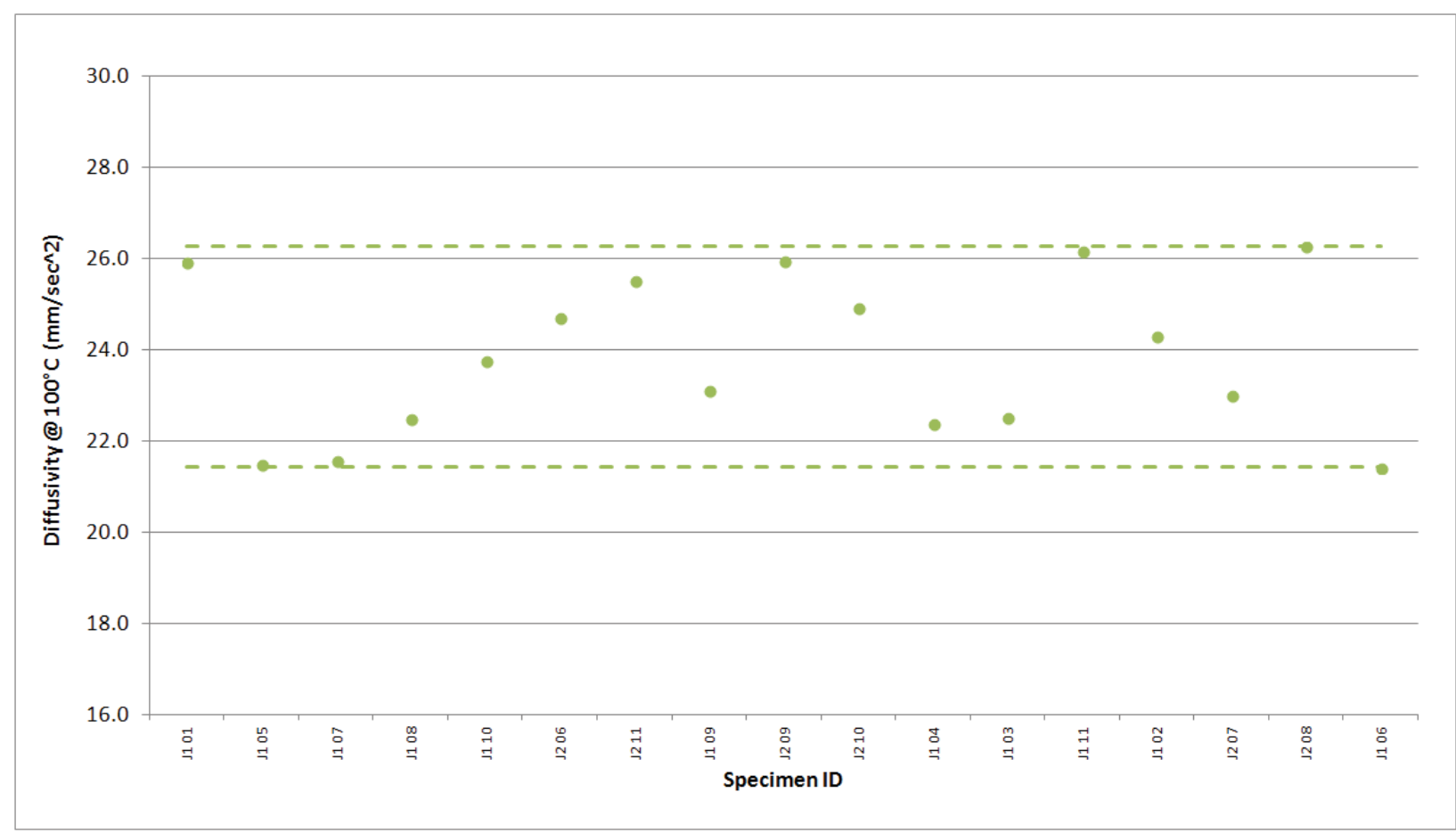

Figure A-150. HLM Piggyback Diffusivity@100C. 




Figure A-151. HLM Piggyback Diffusivity @ 300 C.

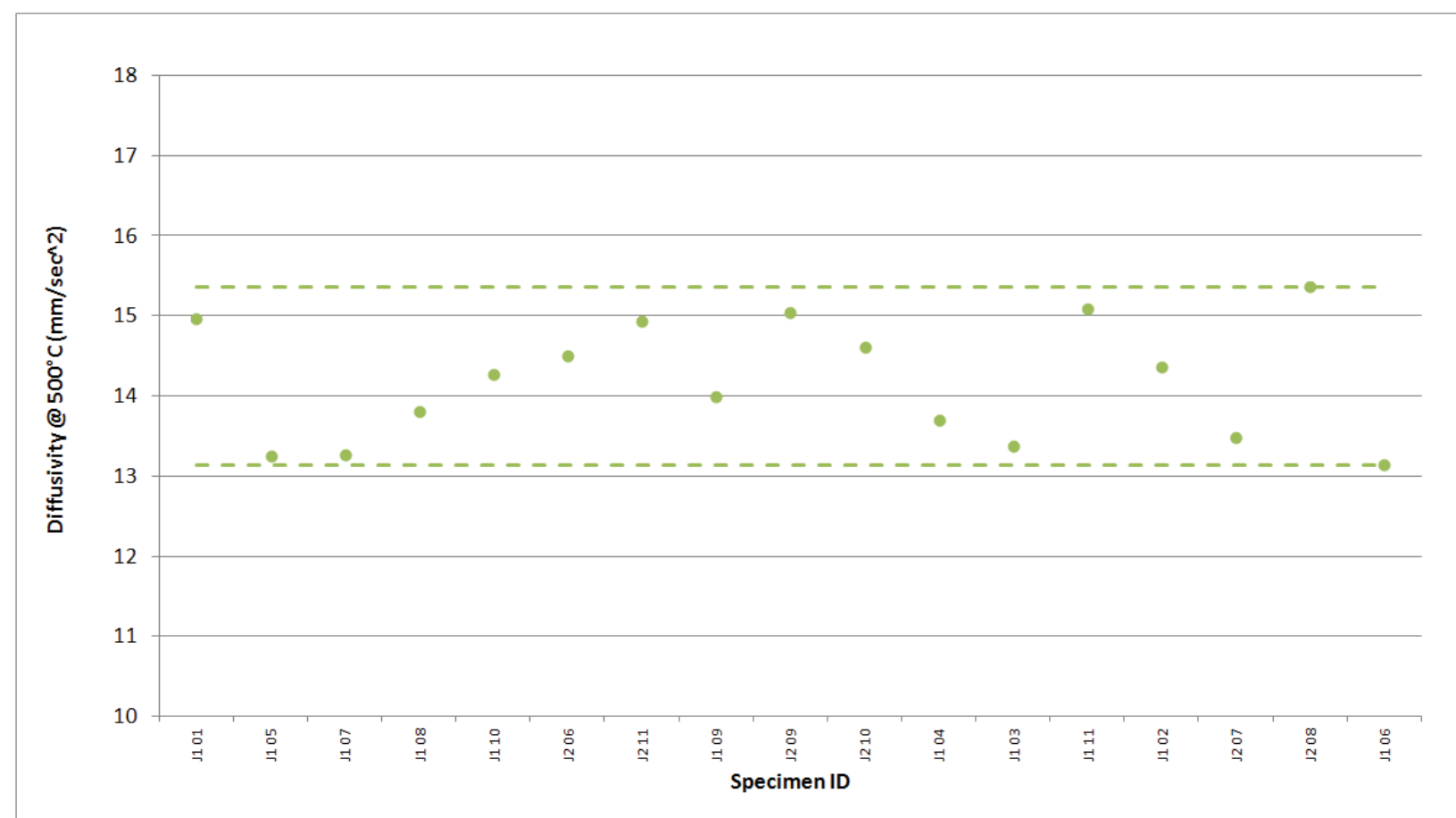

Figure A-152. HLM Piggyback Diffusivity @ 500 C. 


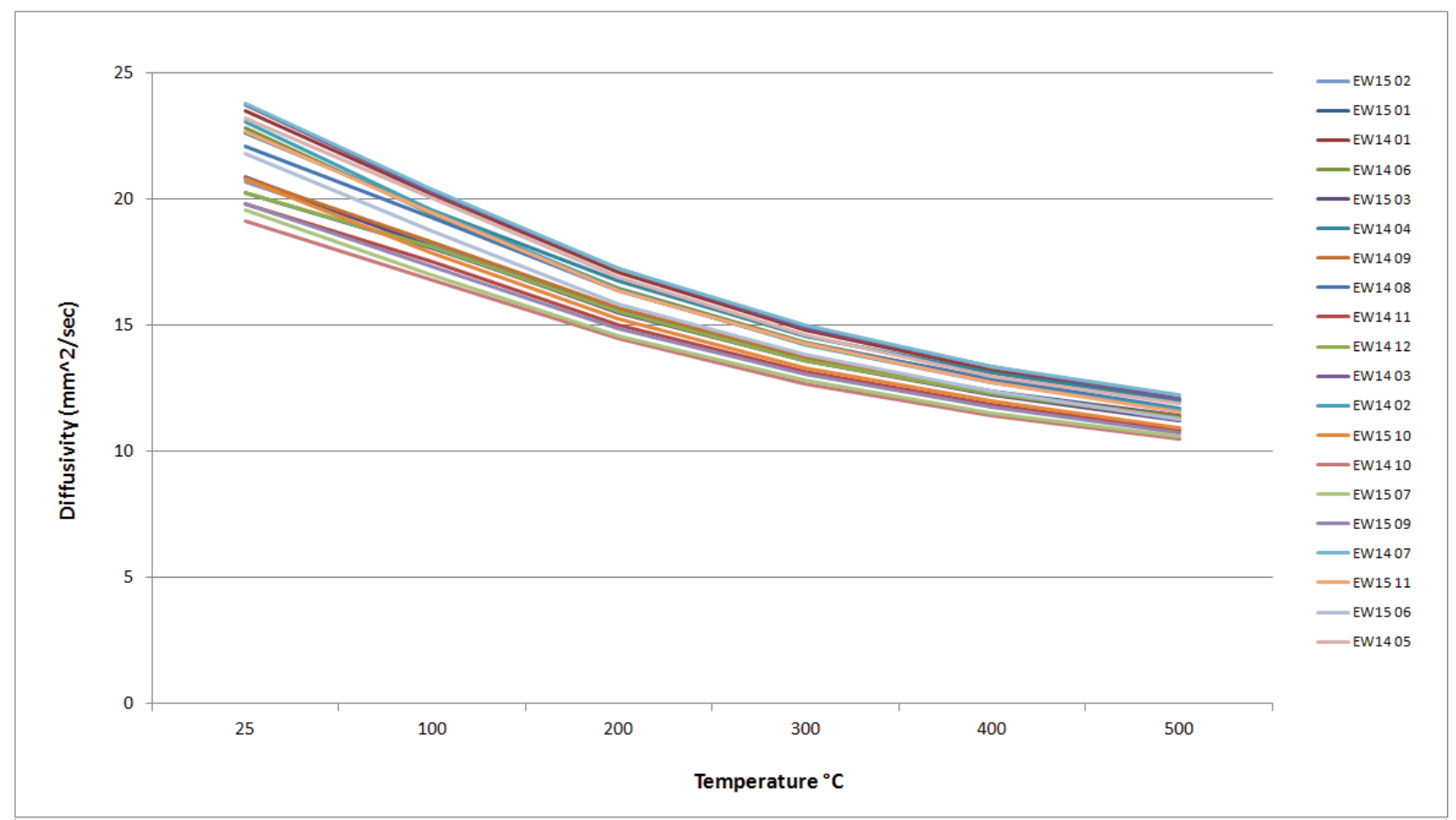

Figure A-153. IG-110 Piggyback Diffusivity.

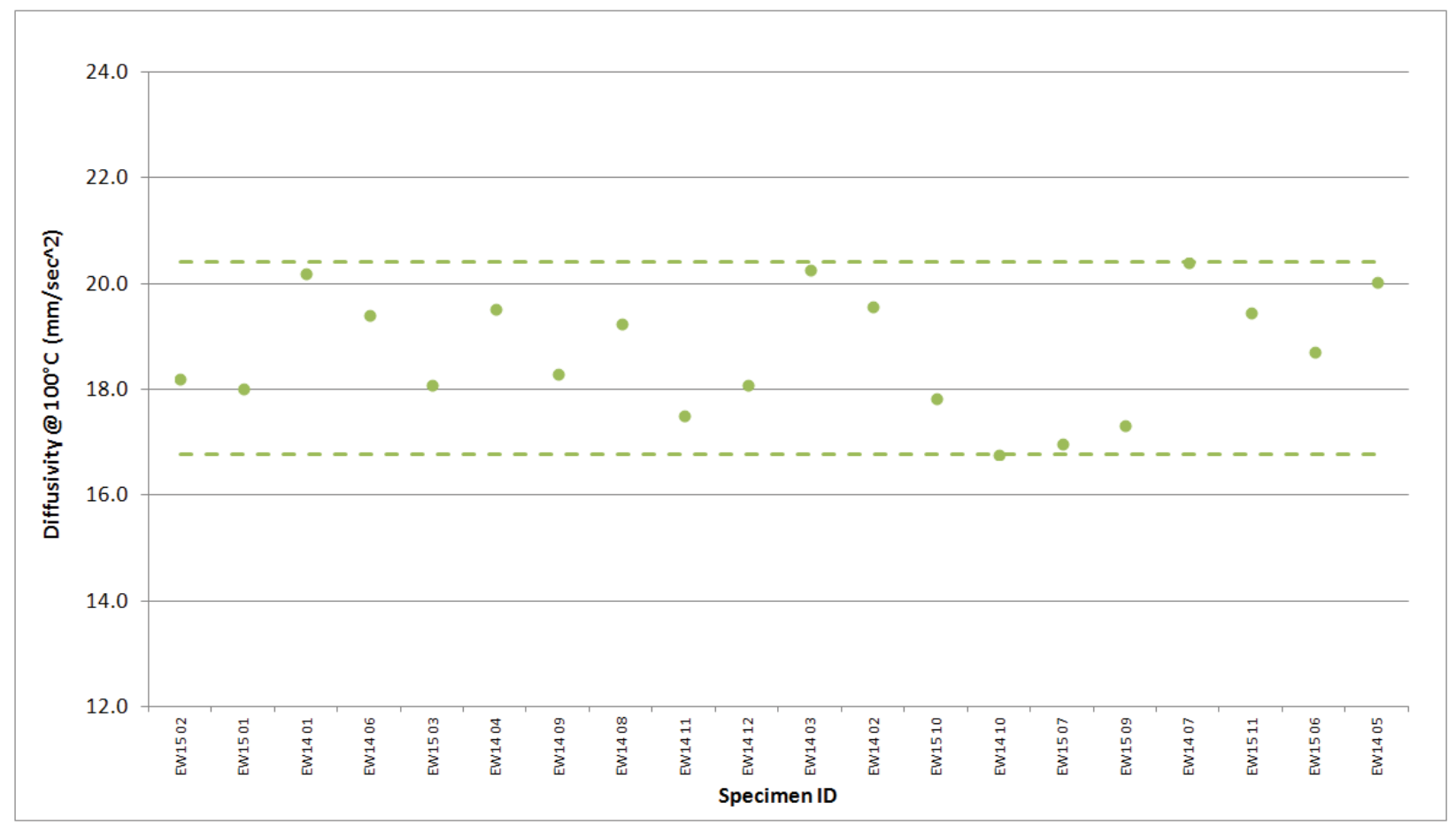

Figure A-154. IG-110 Piggyback Diffusivity@ $100^{\circ} \mathrm{C}$. 


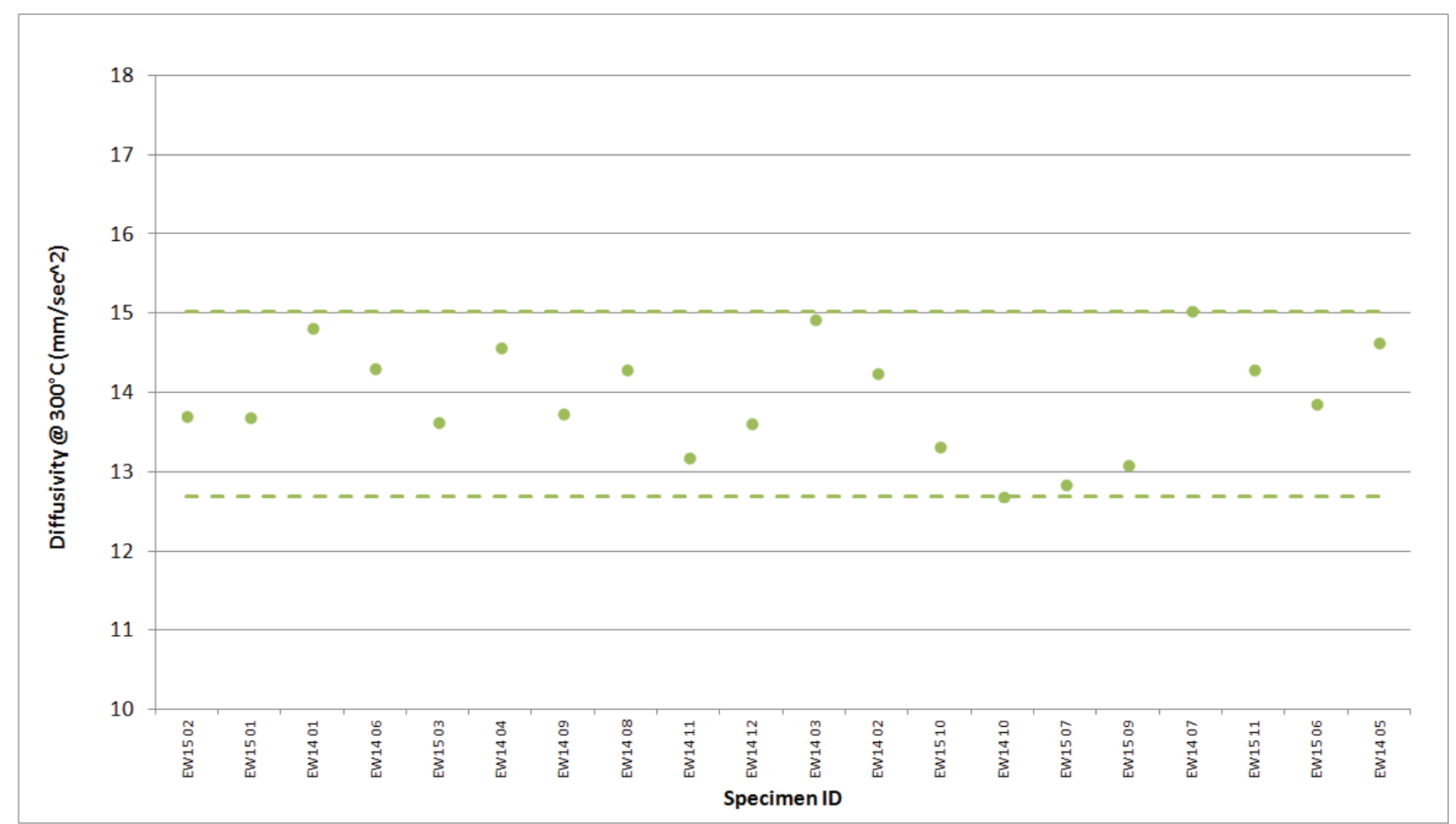

Figure A-155. IG-110 Piggyback Diffusivity@300C.



Figure A-156. IG-110 Piggyback Diffusivity@ $500^{\circ} \mathrm{C}$. 


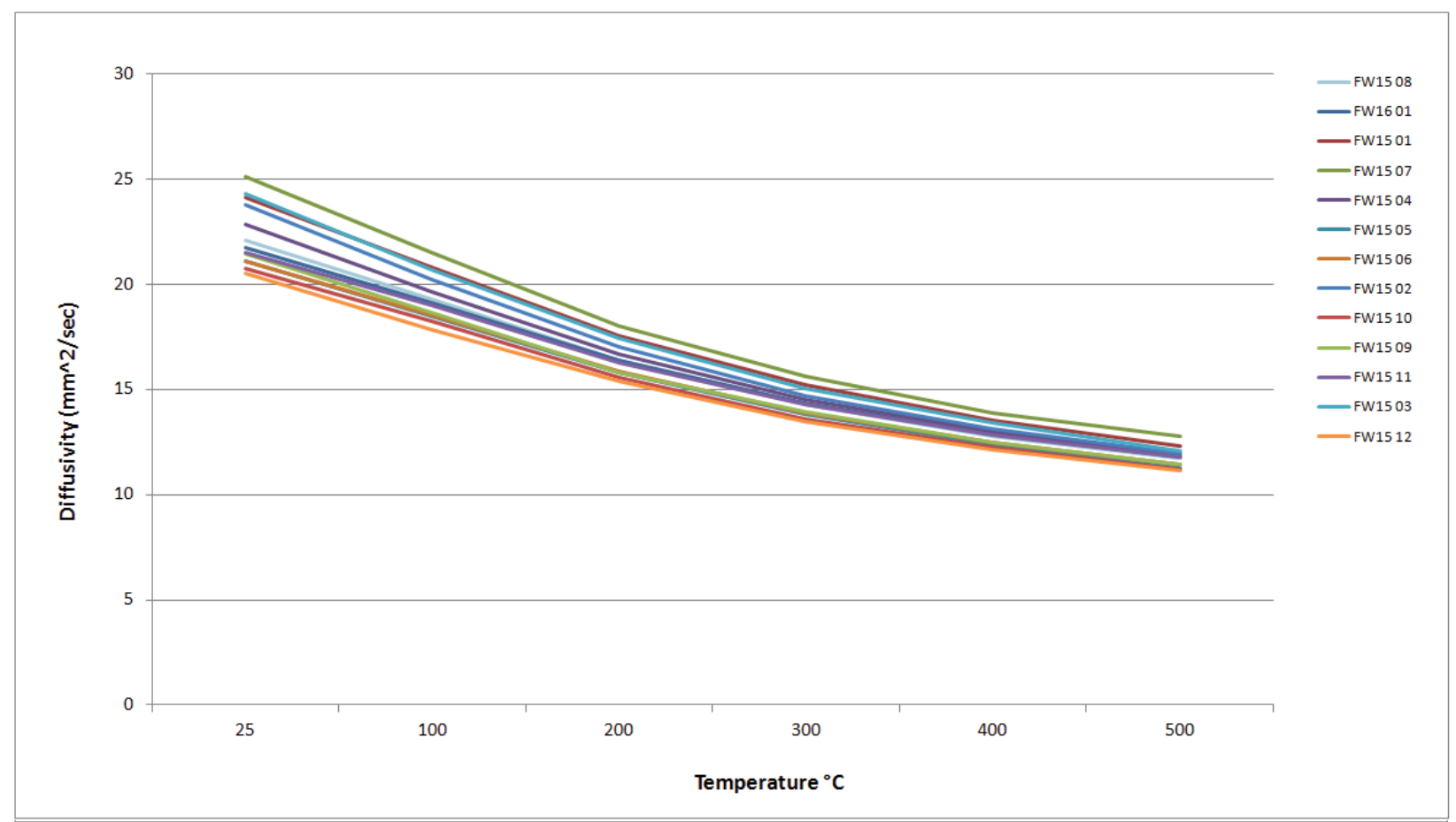

Figure A-157. IG-430 Piggyback Diffusivity.

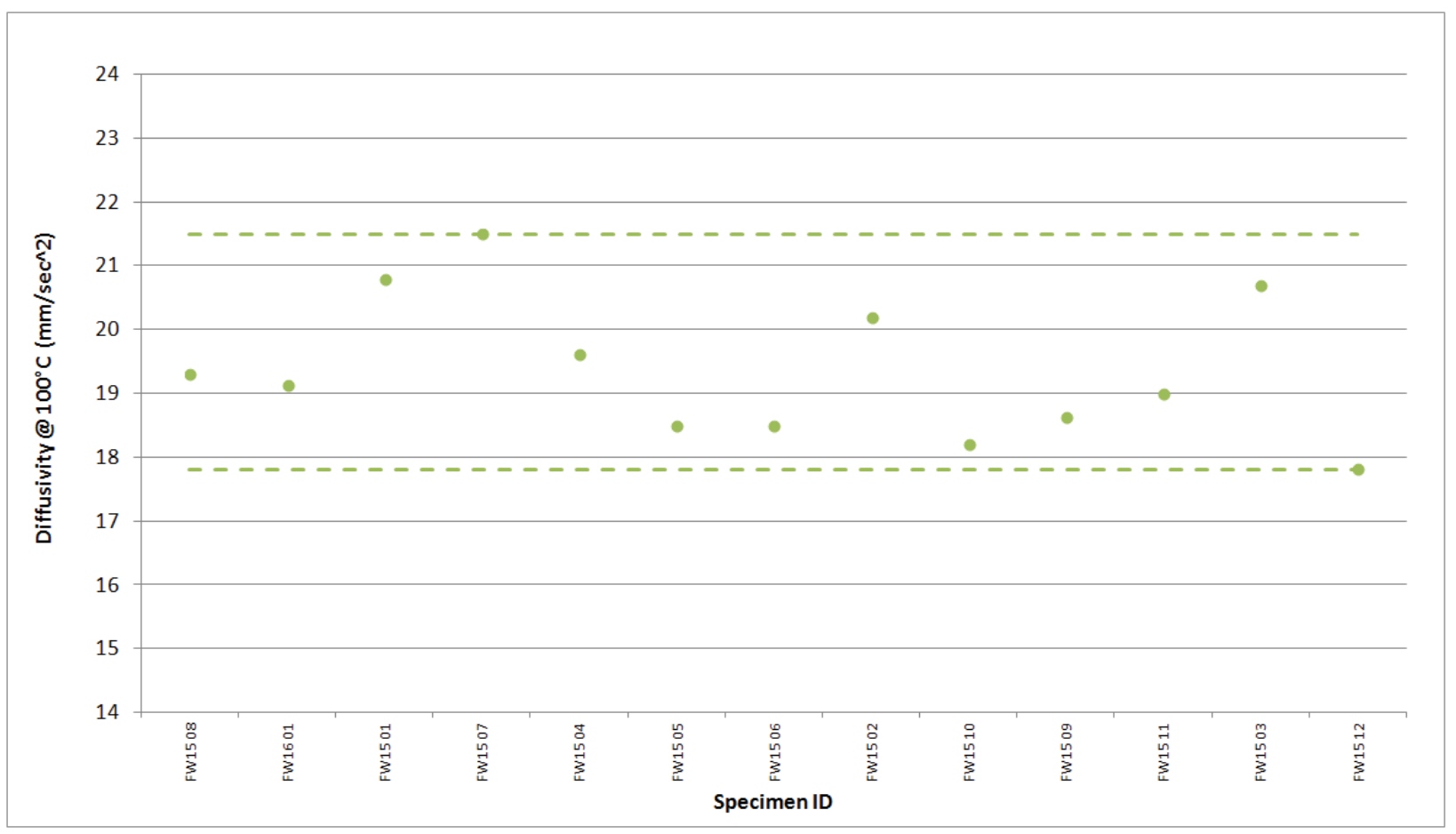

Figure A-158. IG-430 Piggyback Diffusivity@ $100^{\circ} \mathrm{C}$. 


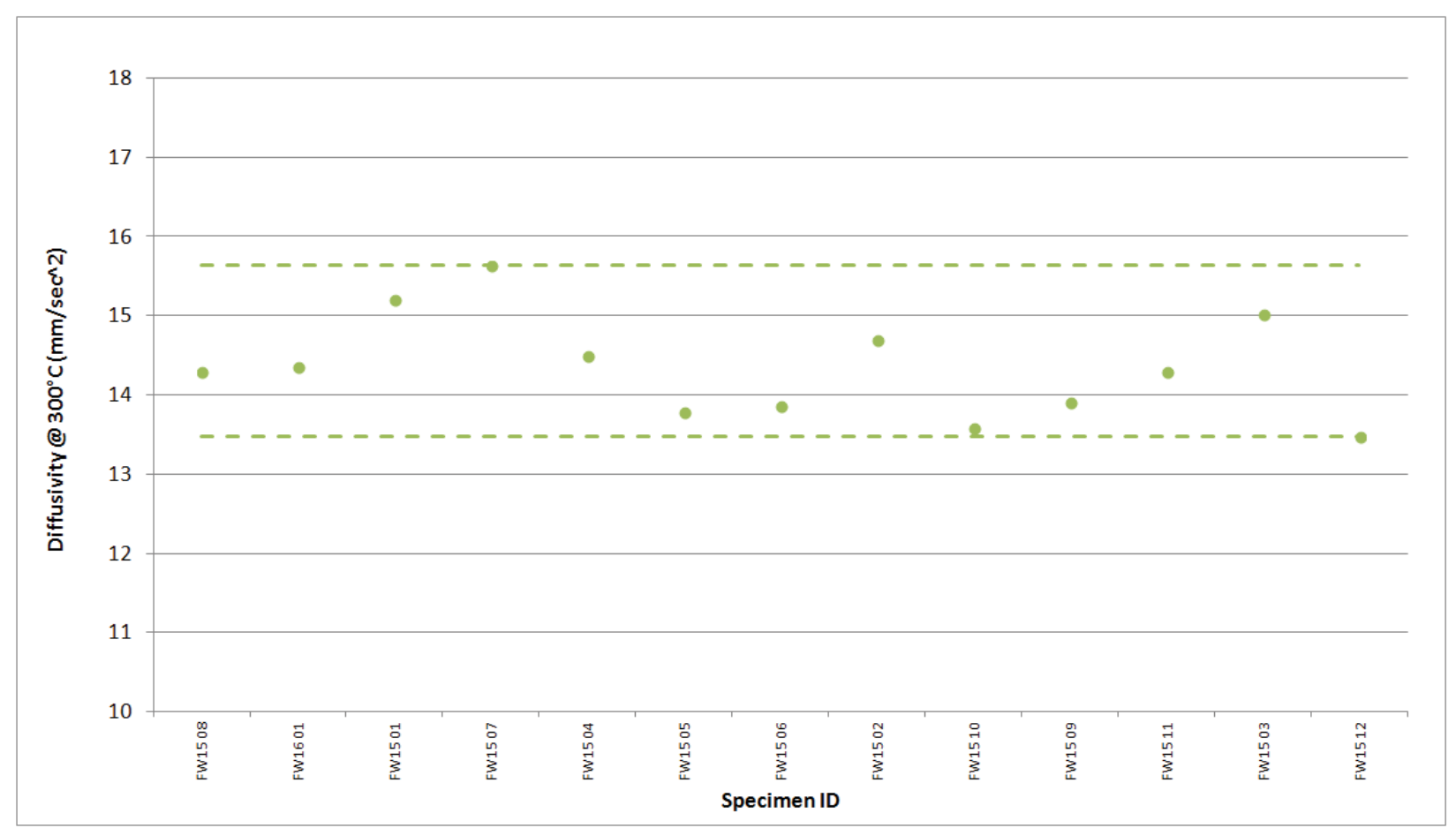

Figure A-159. IG-430 Piggyback Diffusivity@300C.

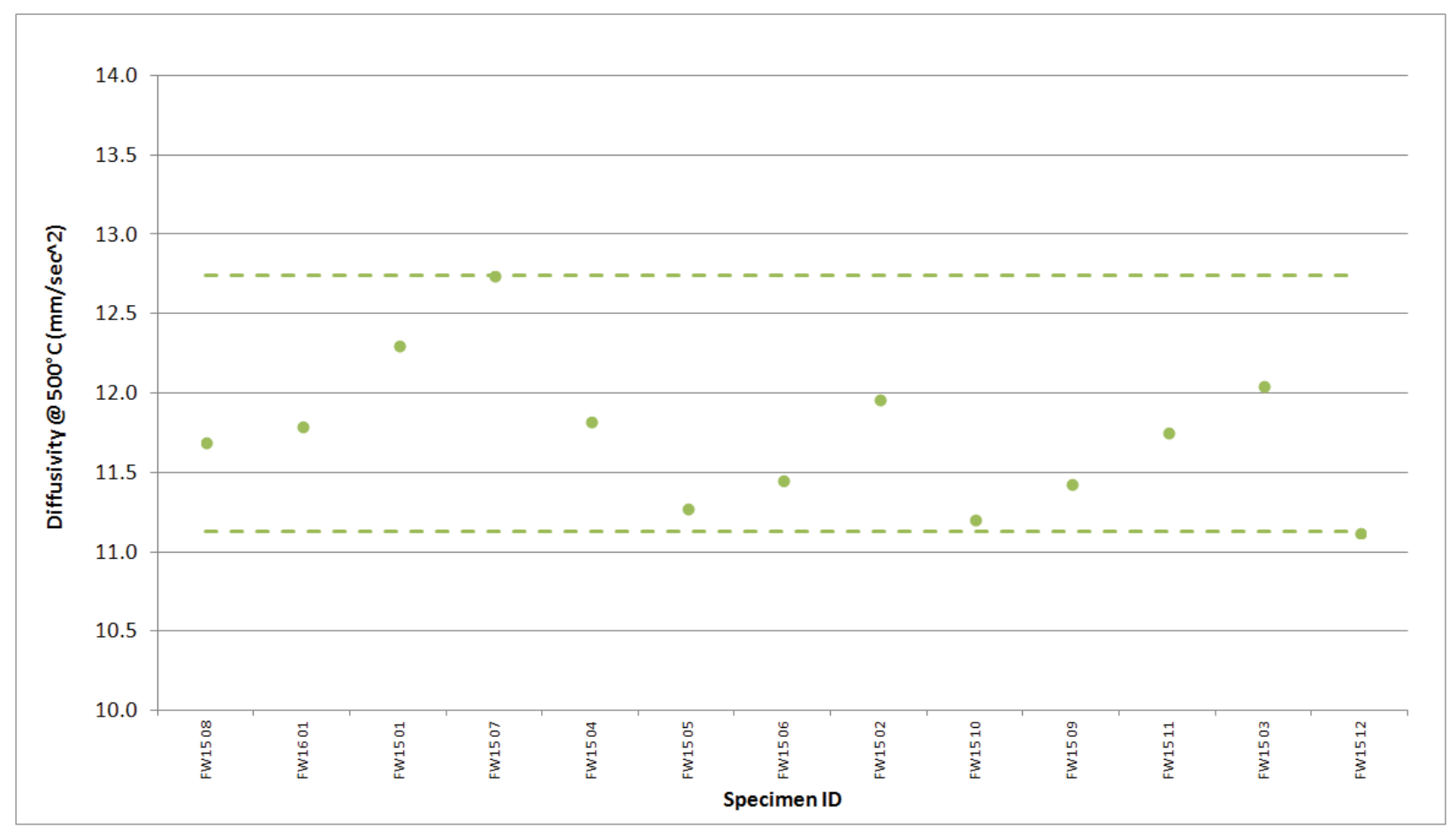

Figure A-160. IG-430 Piggyback Diffusivity@ $500^{\circ} \mathrm{C}$. 


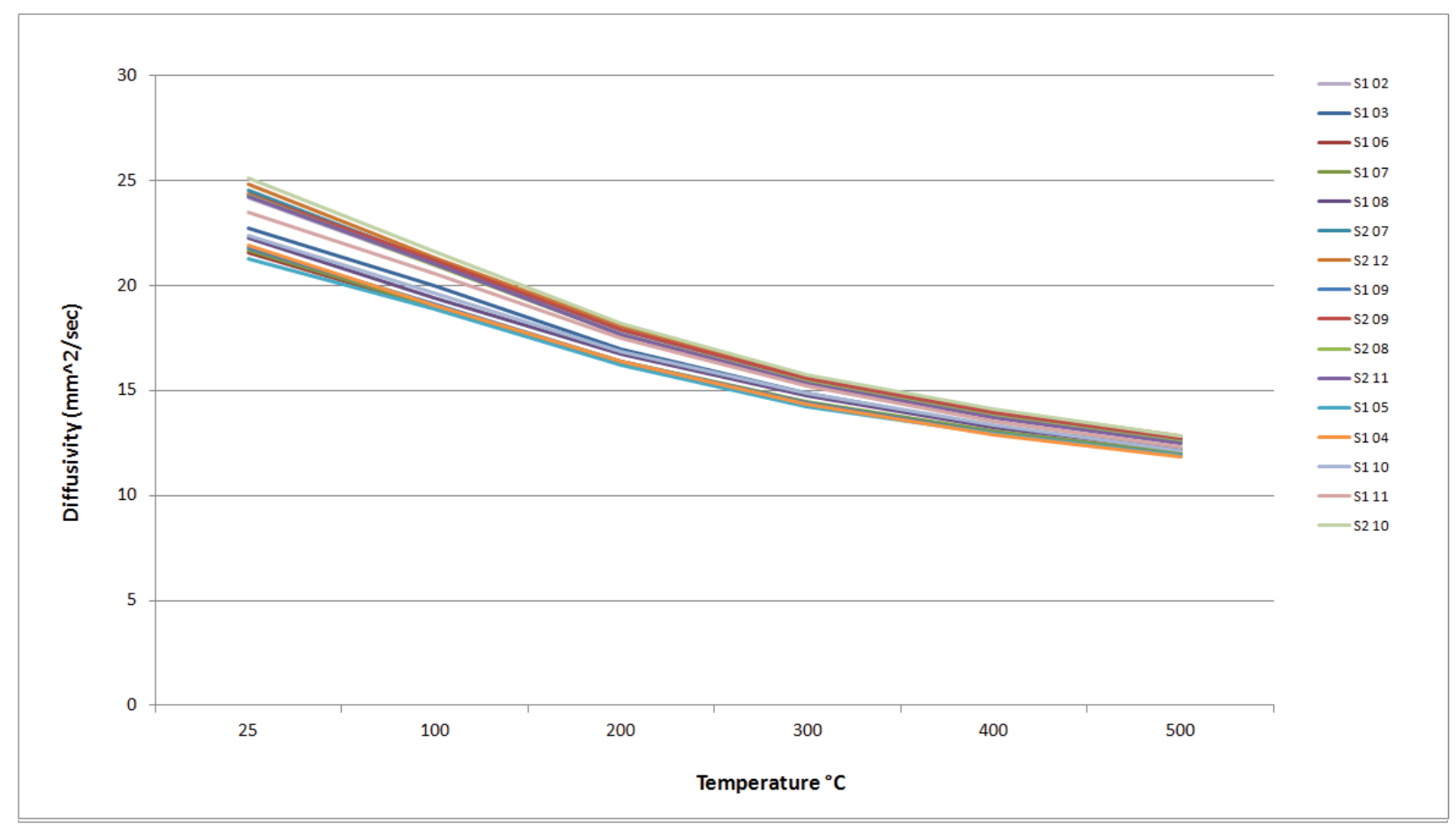

Figure A-161. NBG-10 Piggyback Diffusivity.



Figure A-162. NBG-10 Piggyback Diffusivity @ 100². 


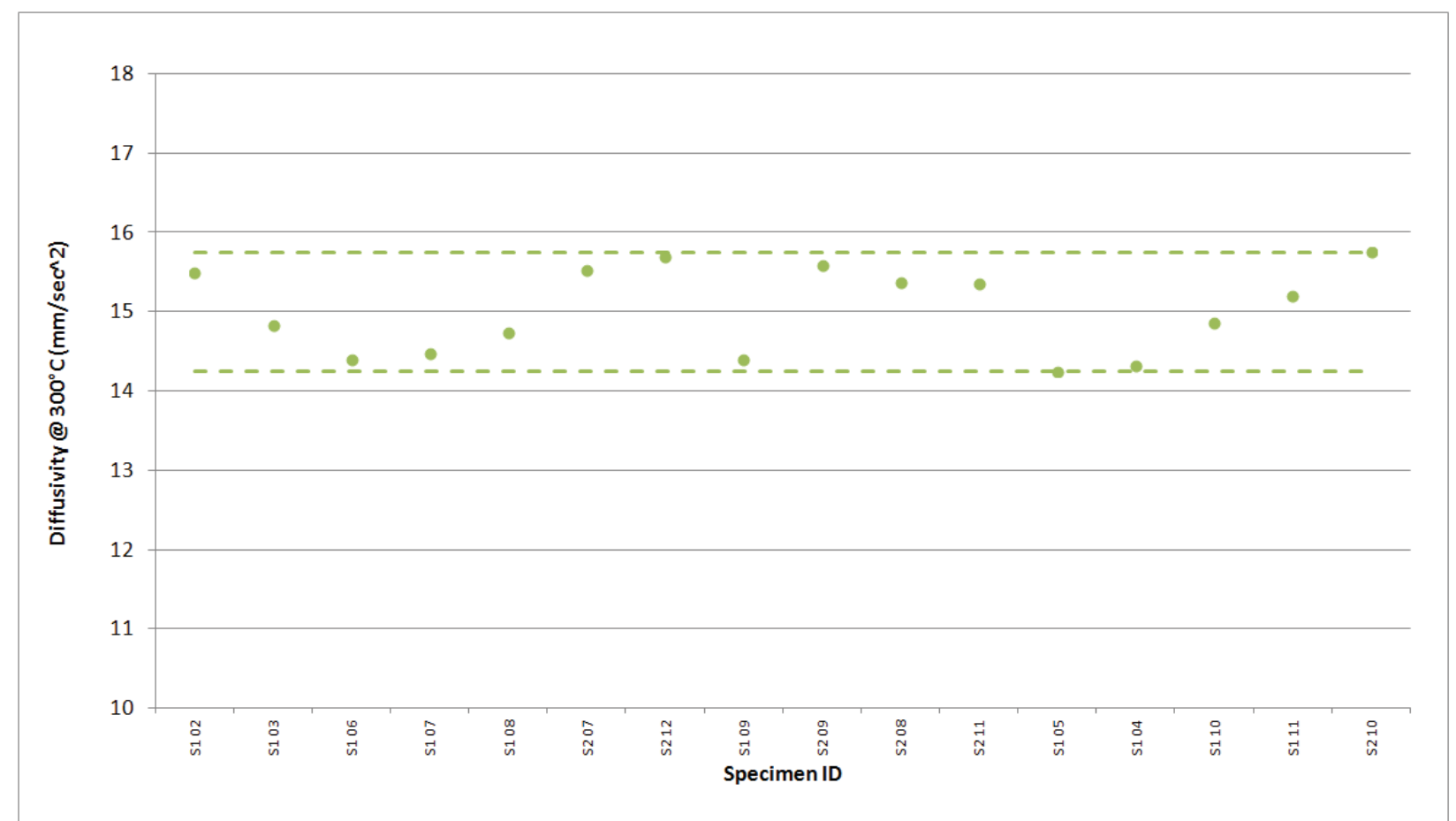

Figure A-163. NBG-10 Piggyback Diffusivity @ $300^{\circ} \mathrm{C}$.

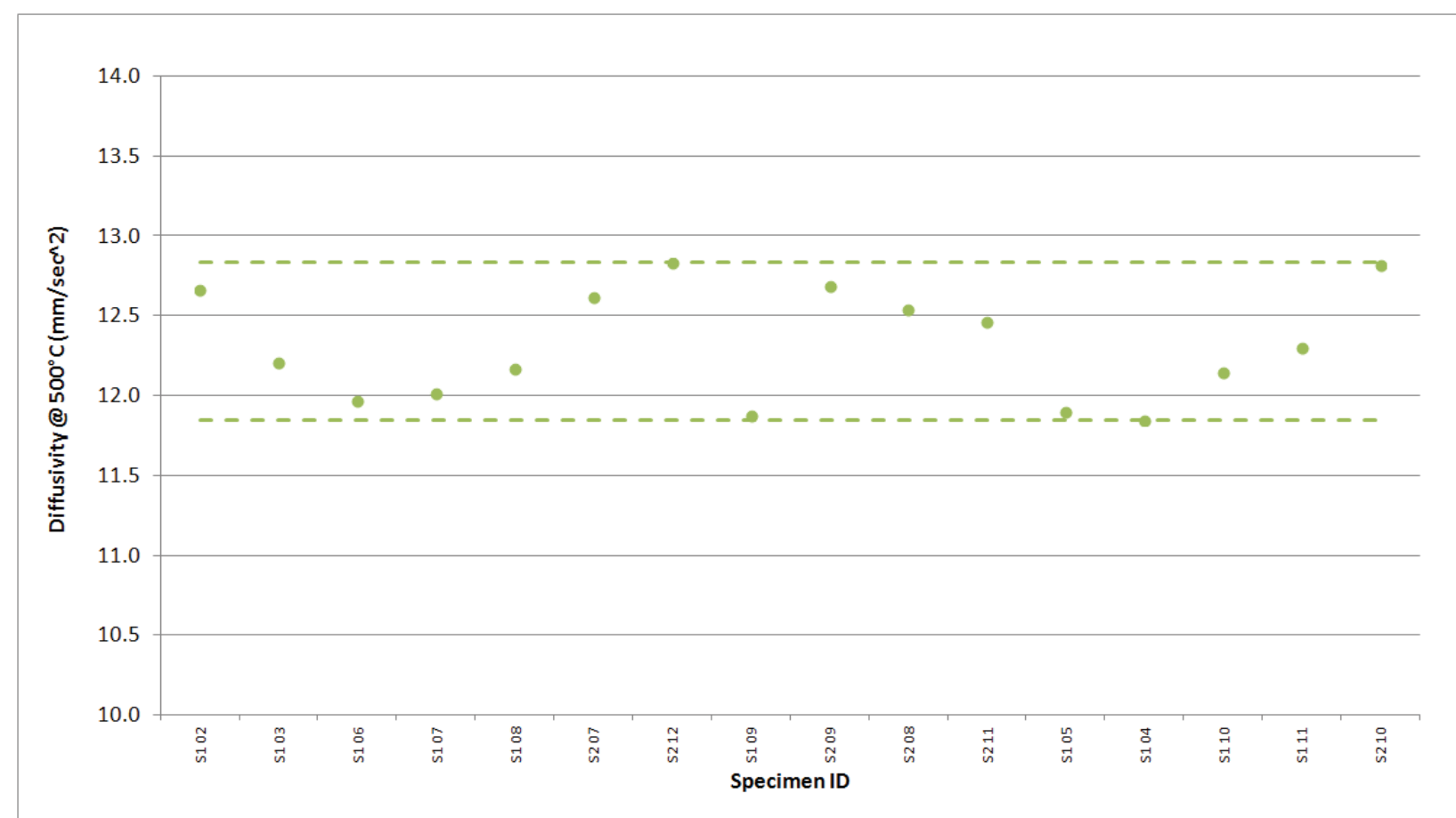

Figure A-164. NBG-10 Piggyback Diffusivity @ 500 ${ }^{\circ}$. 


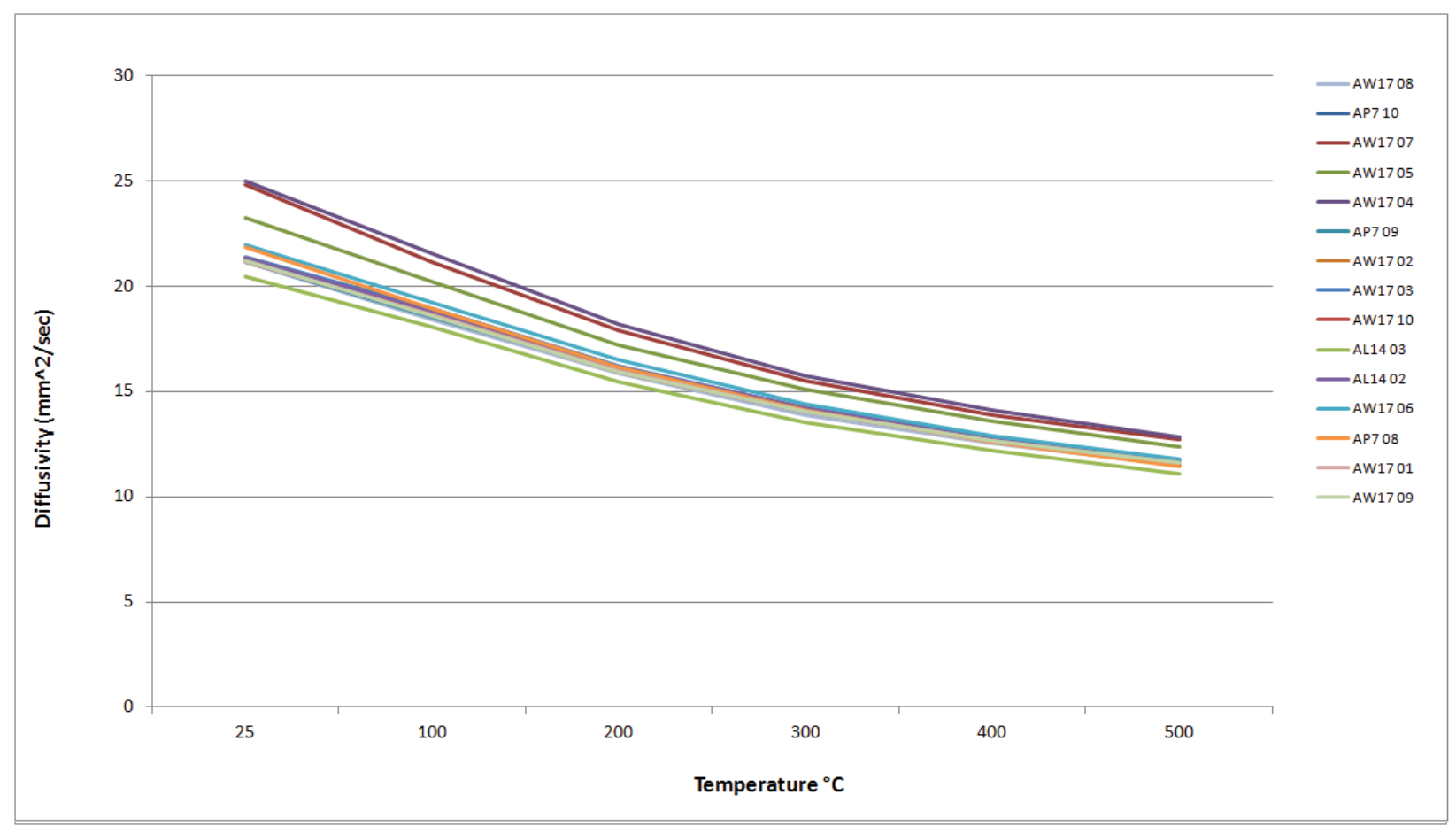

Figure A-165. NBG-17 Piggyback Diffusivity.

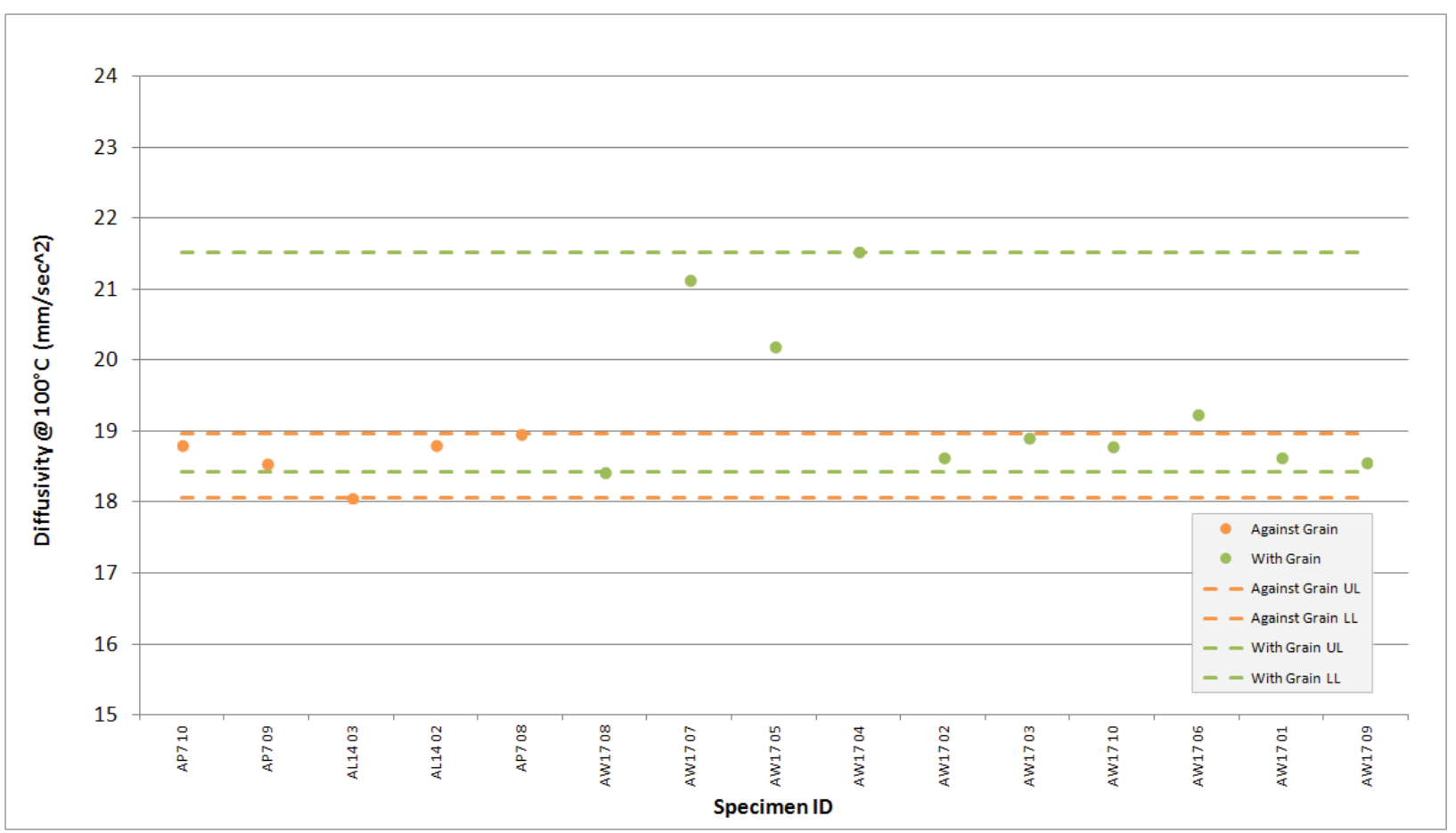

Figure A-166. NBG-17 Piggyback Diffusivity @ $100^{\circ} \mathrm{C}$. 


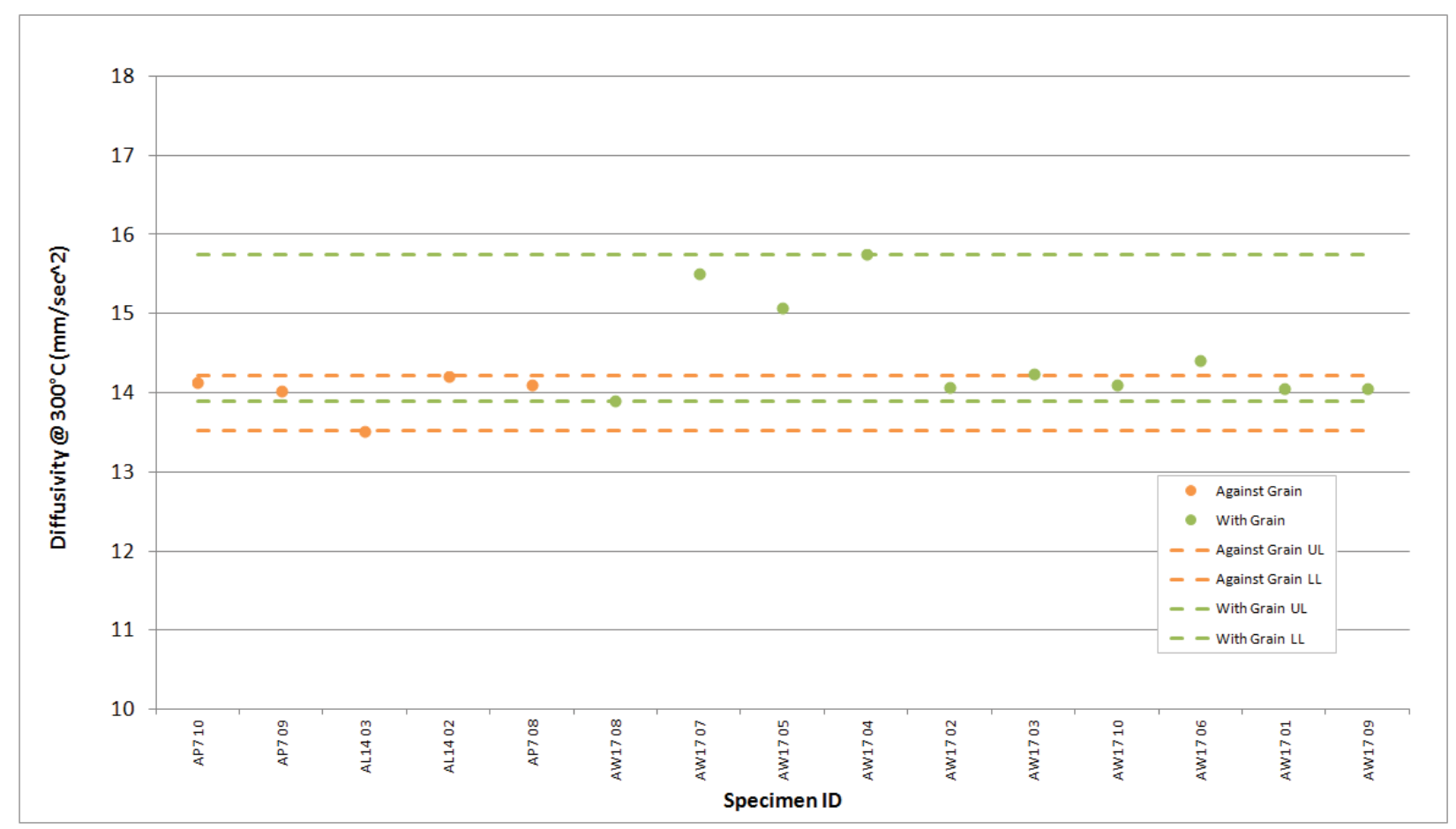

Figure A-167. NBG-17 Piggyback Diffusivity @ 300².

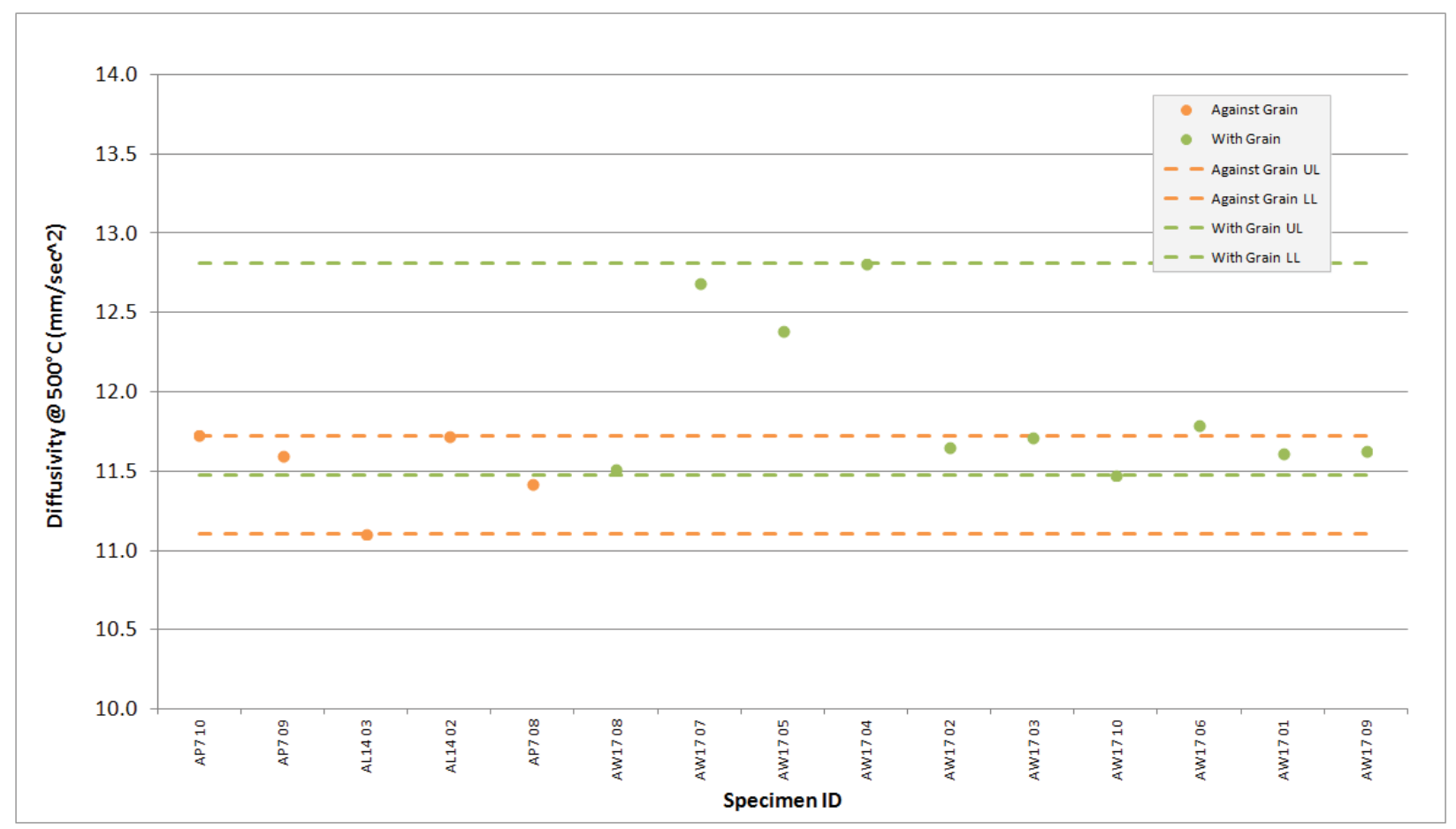

Figure A-168. NBG-17 Piggyback Diffusivity @ 500². 


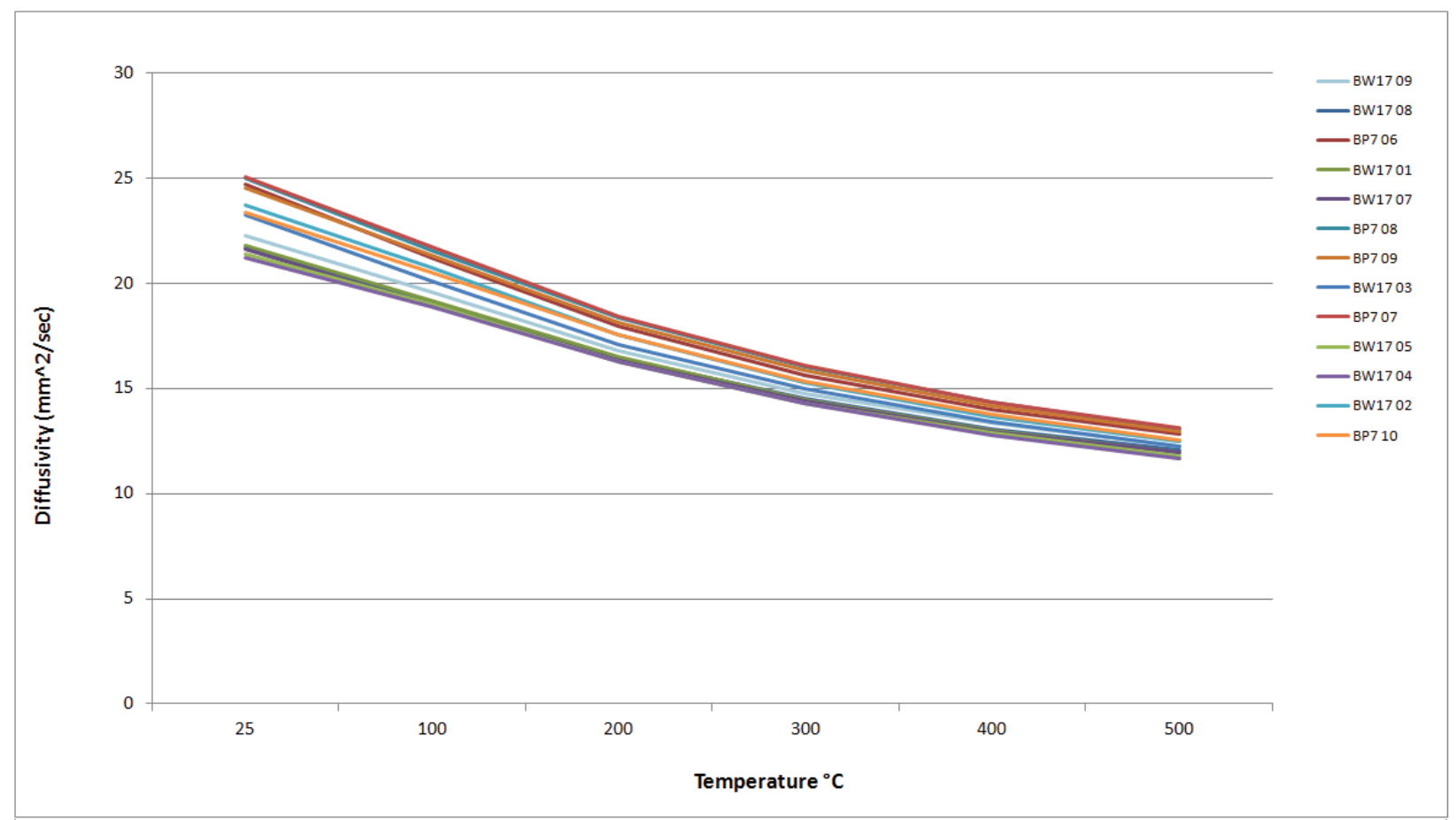

Figure A-169. NBG-18 Piggyback Diffusivity.

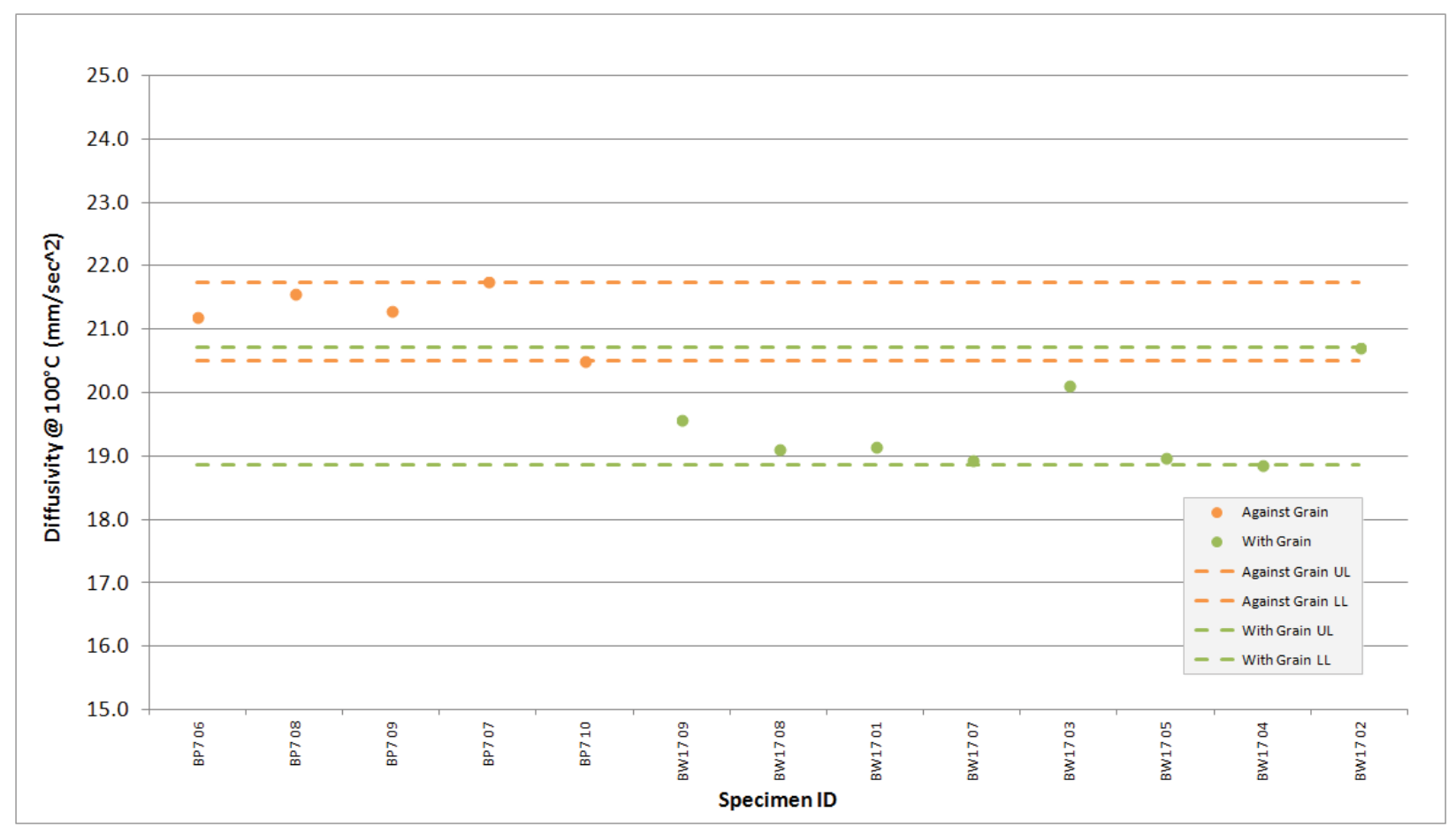

Figure A-170. NBG-18 Piggyback Diffusivity@100C. 


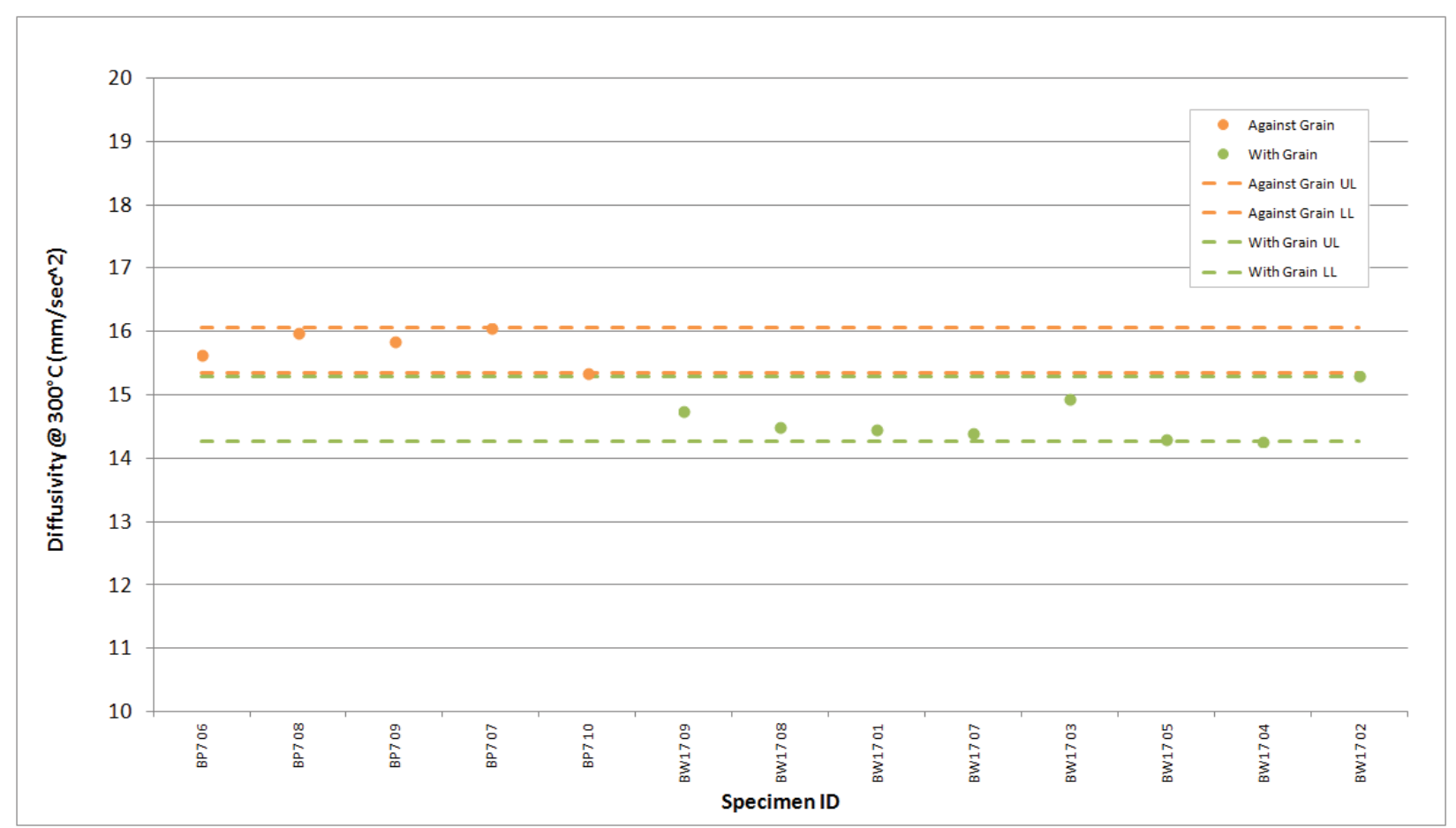

Figure A-171. NBG-18 Piggyback Diffusivity@300 C.

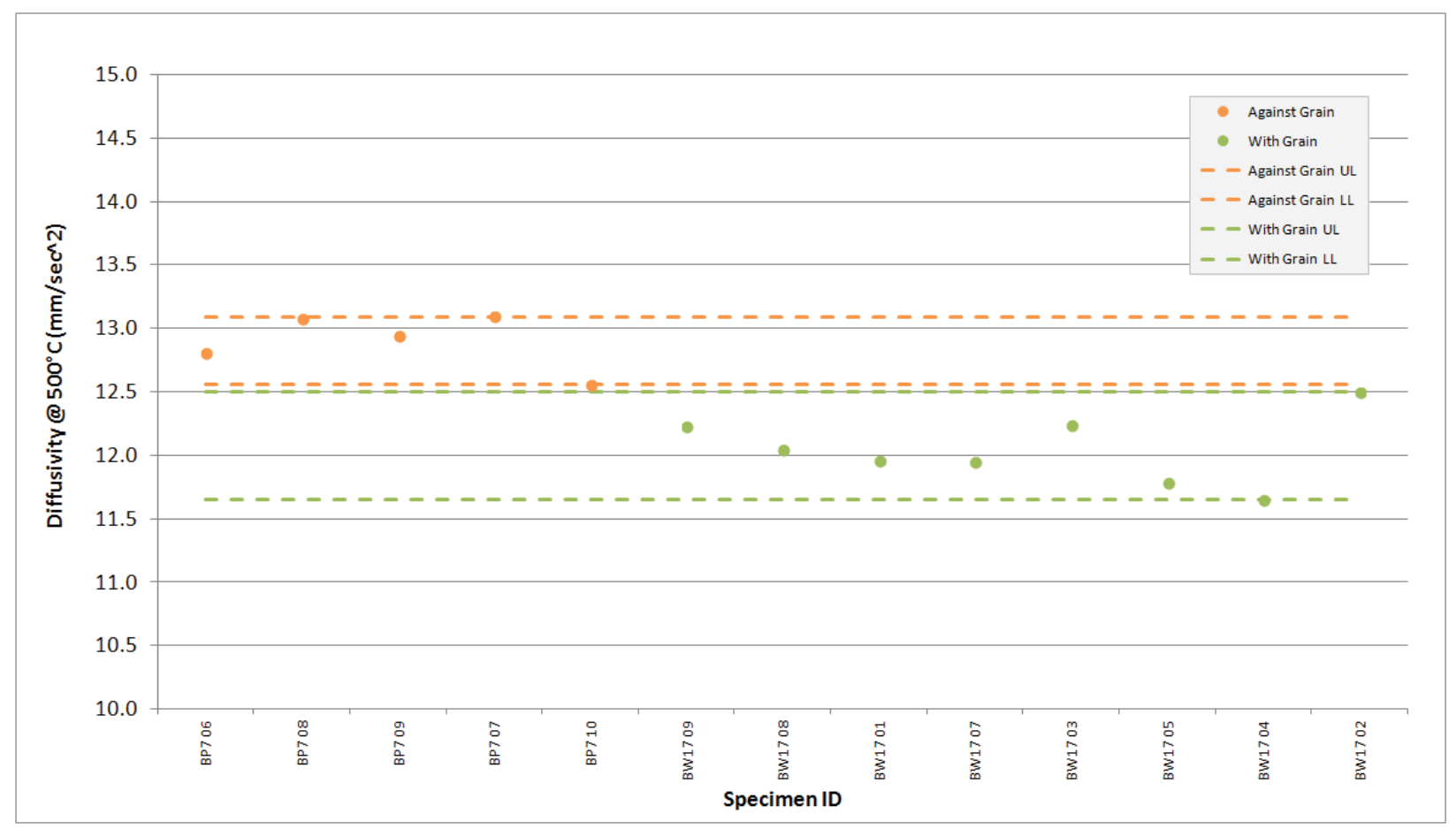

Figure A-172. NBG-18 Piggyback Diffusivity @ 500 ${ }^{\circ}$. 


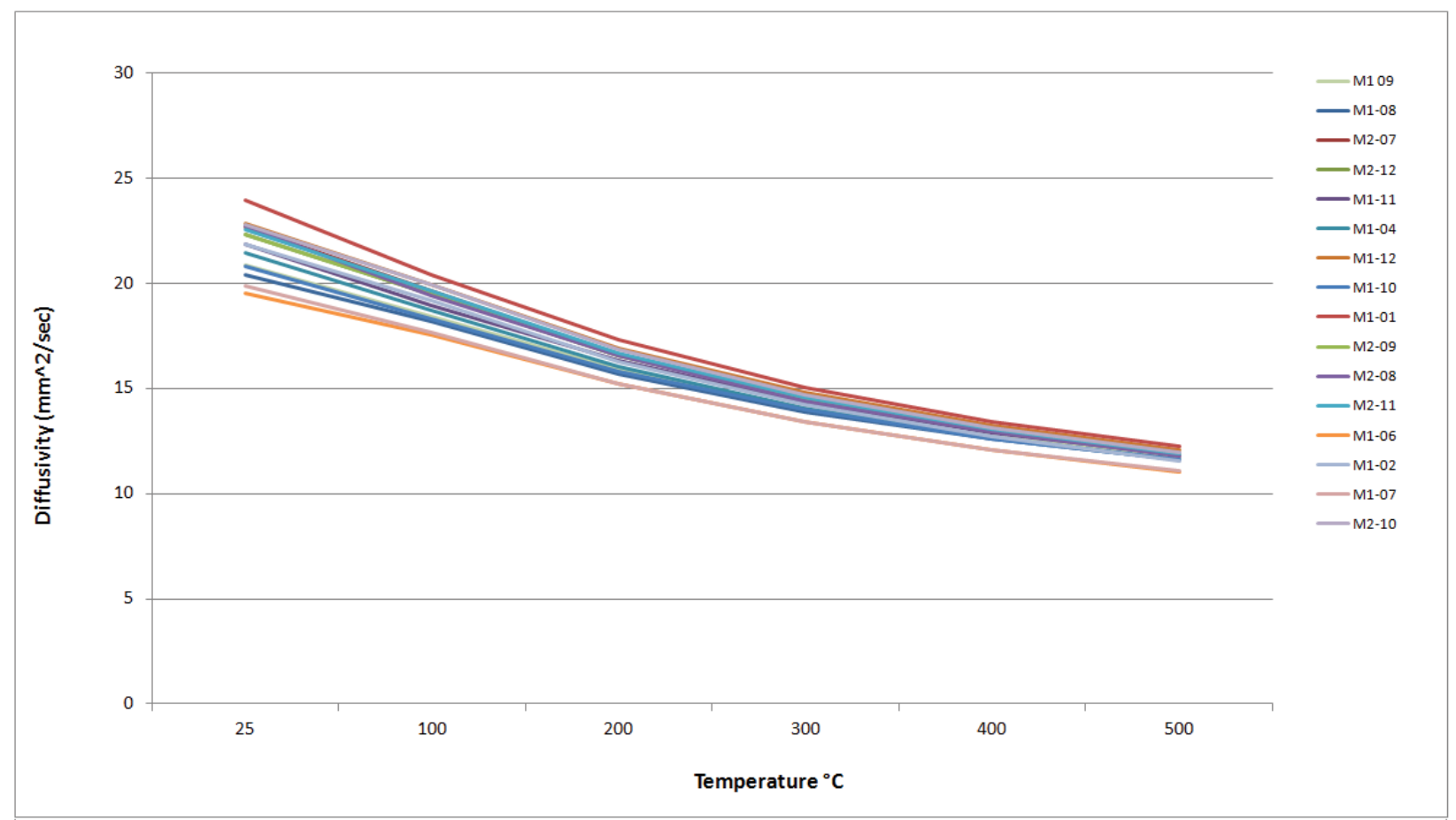

Figure A-173. NBG-25 Piggyback Diffusivity.

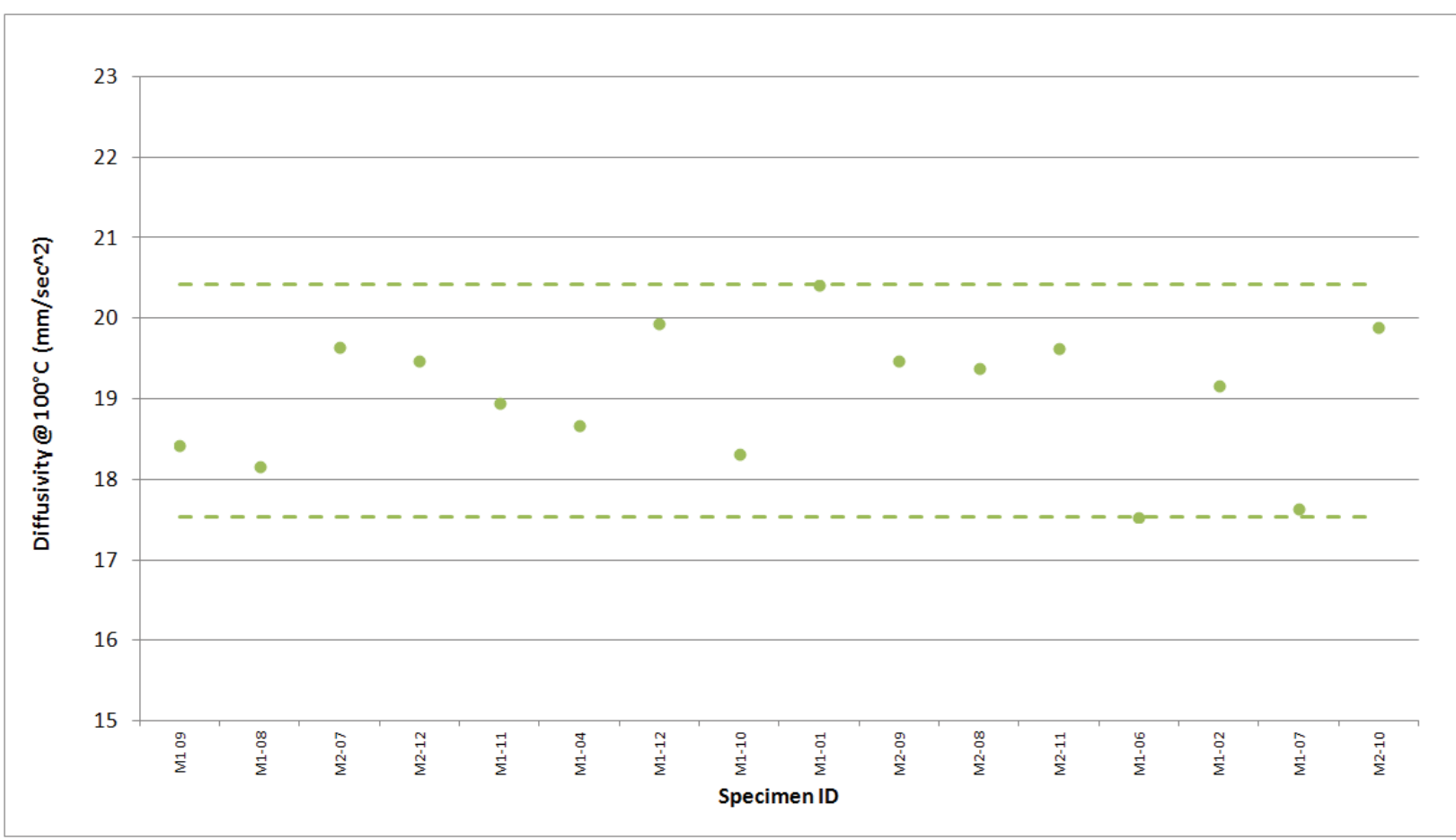

Figure A-174. NBG-25 Piggyback Diffusivity @ $100^{\circ} \mathrm{C}$. 


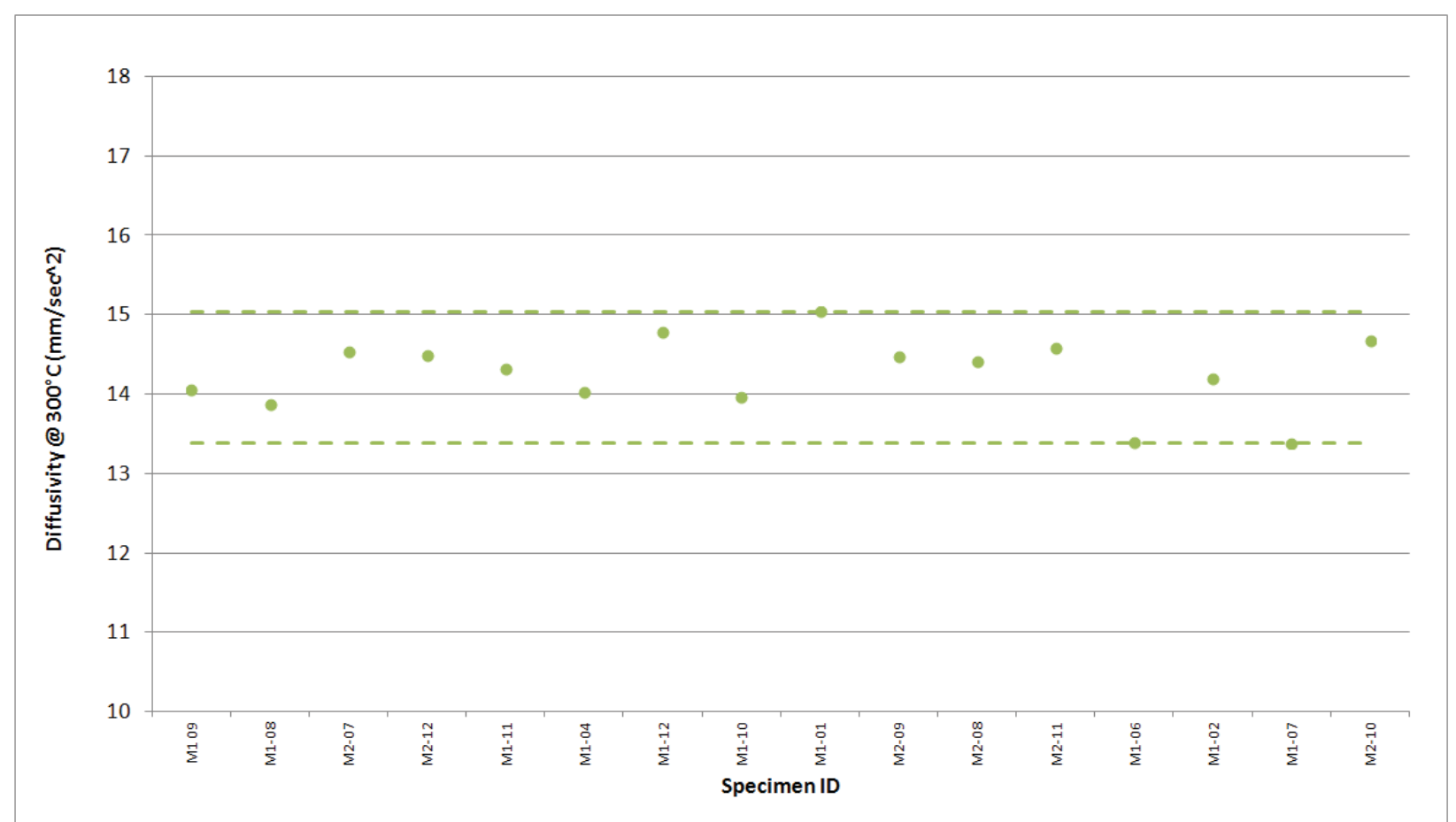

Figure A-175. NBG-25 Piggyback Diffusivity@300².

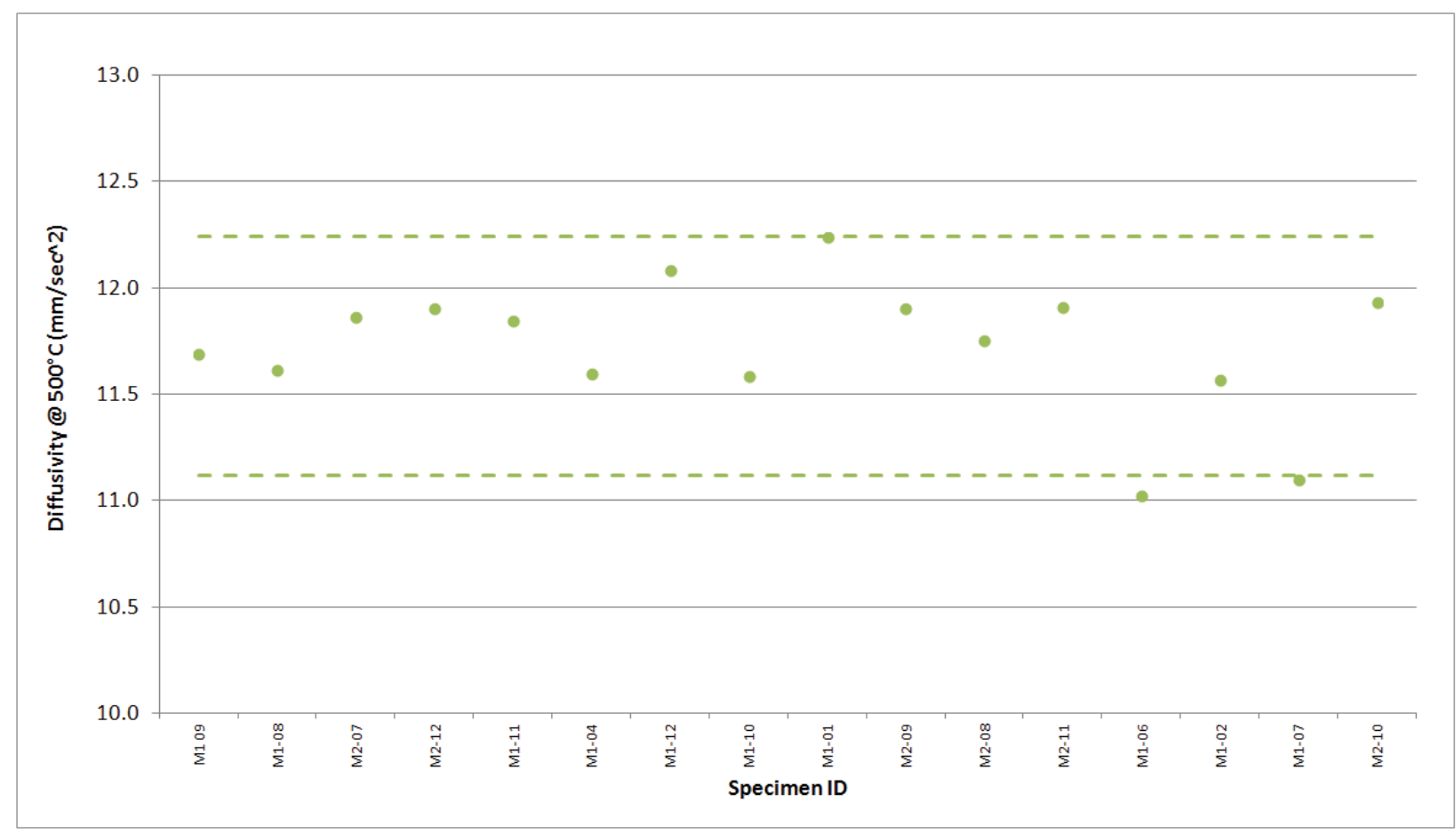

Figure A-176. NBG-25 Piggyback Diffusivity @ $500^{\circ} \mathrm{C}$. 


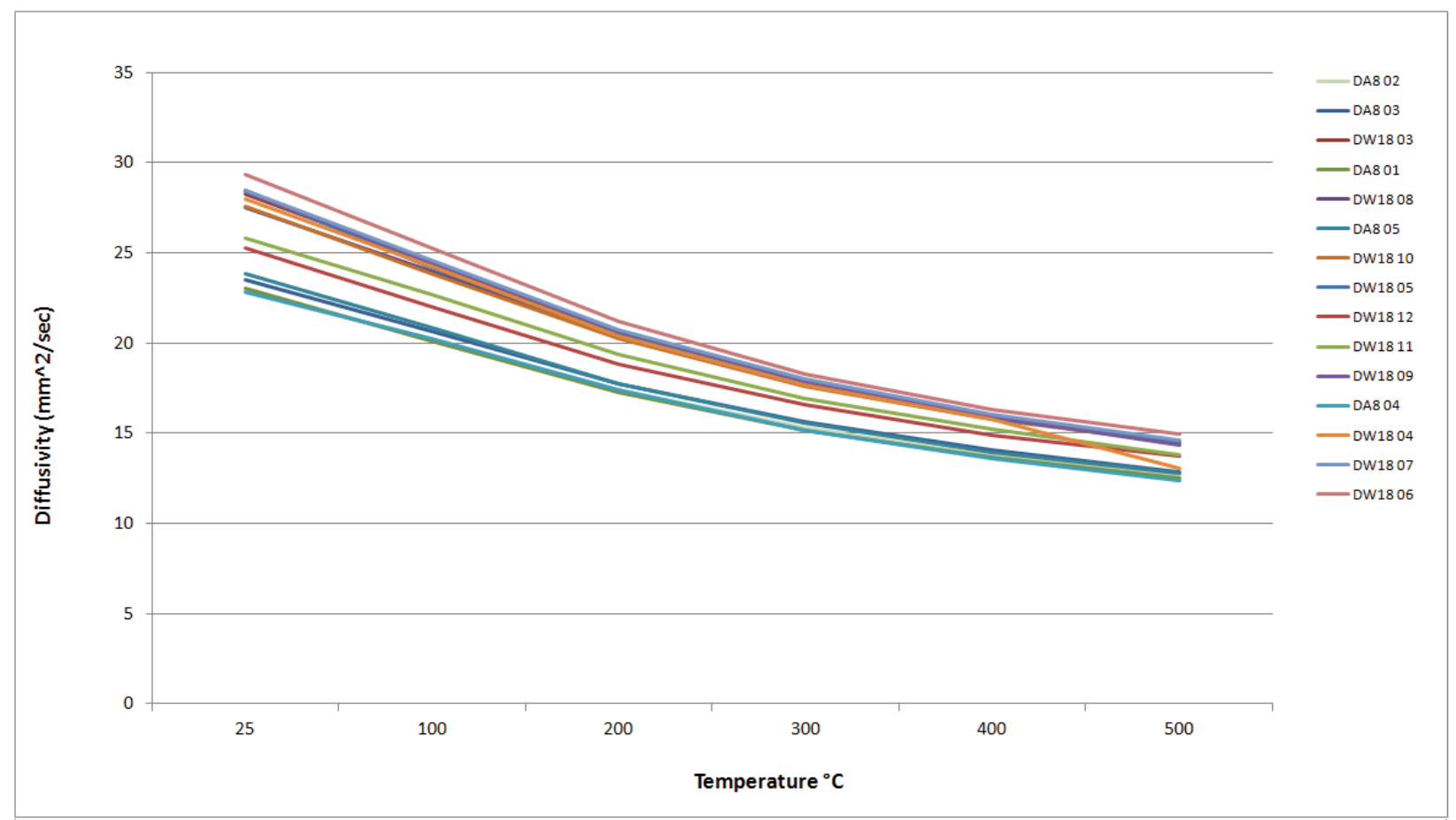

Figure A-177. PCEA Piggyback Diffusivity.

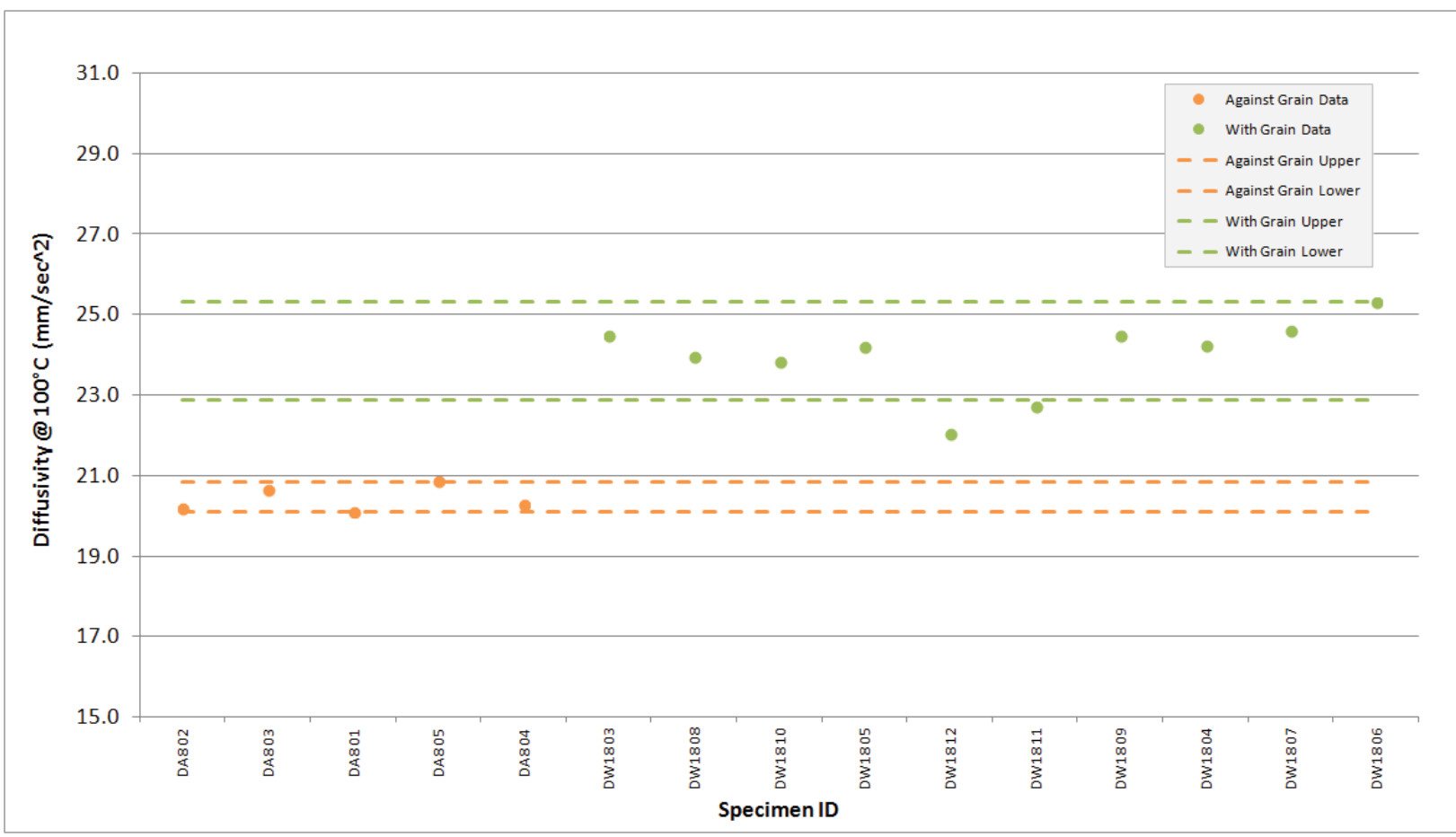

Figure A-178. PCEA Piggyback Diffusivity@ $100^{\circ} \mathrm{C}$. 


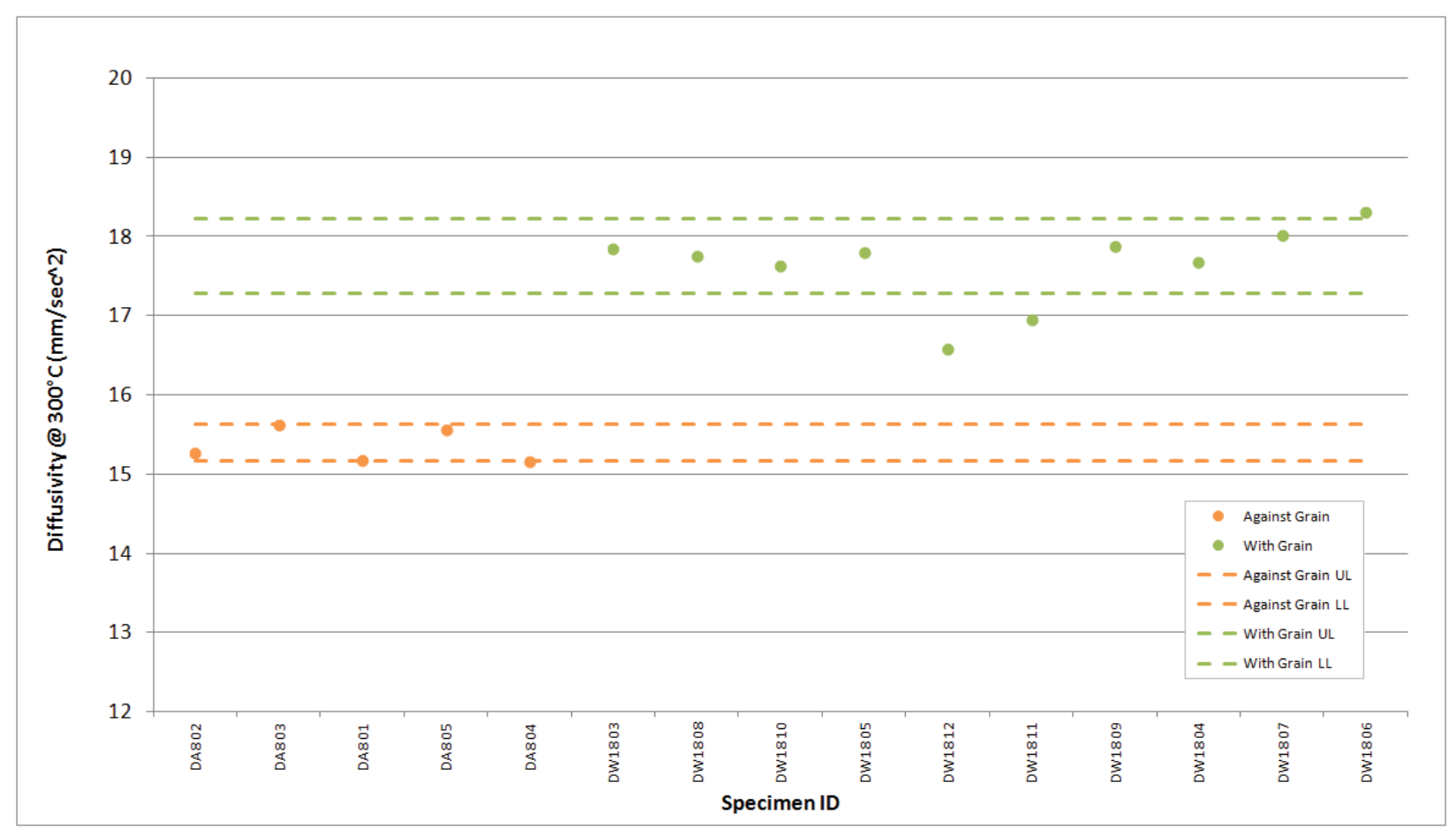

Figure A-179. PCEA Piggyback Diffusivity @ $300^{\circ} \mathrm{C}$.

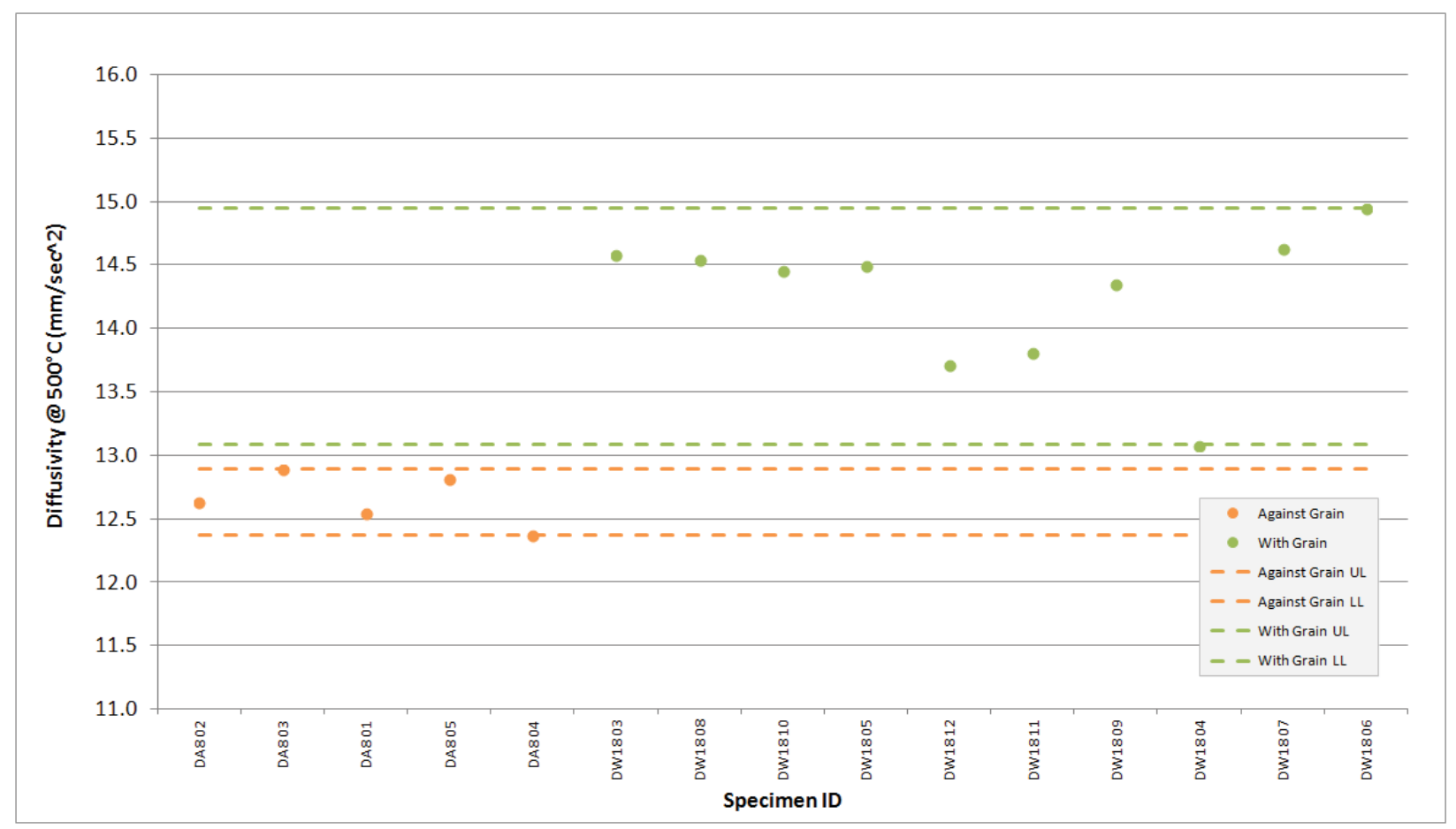

Figure A-180. PCEA Piggyback Diffusivity @ 500 ${ }^{\circ}$. 


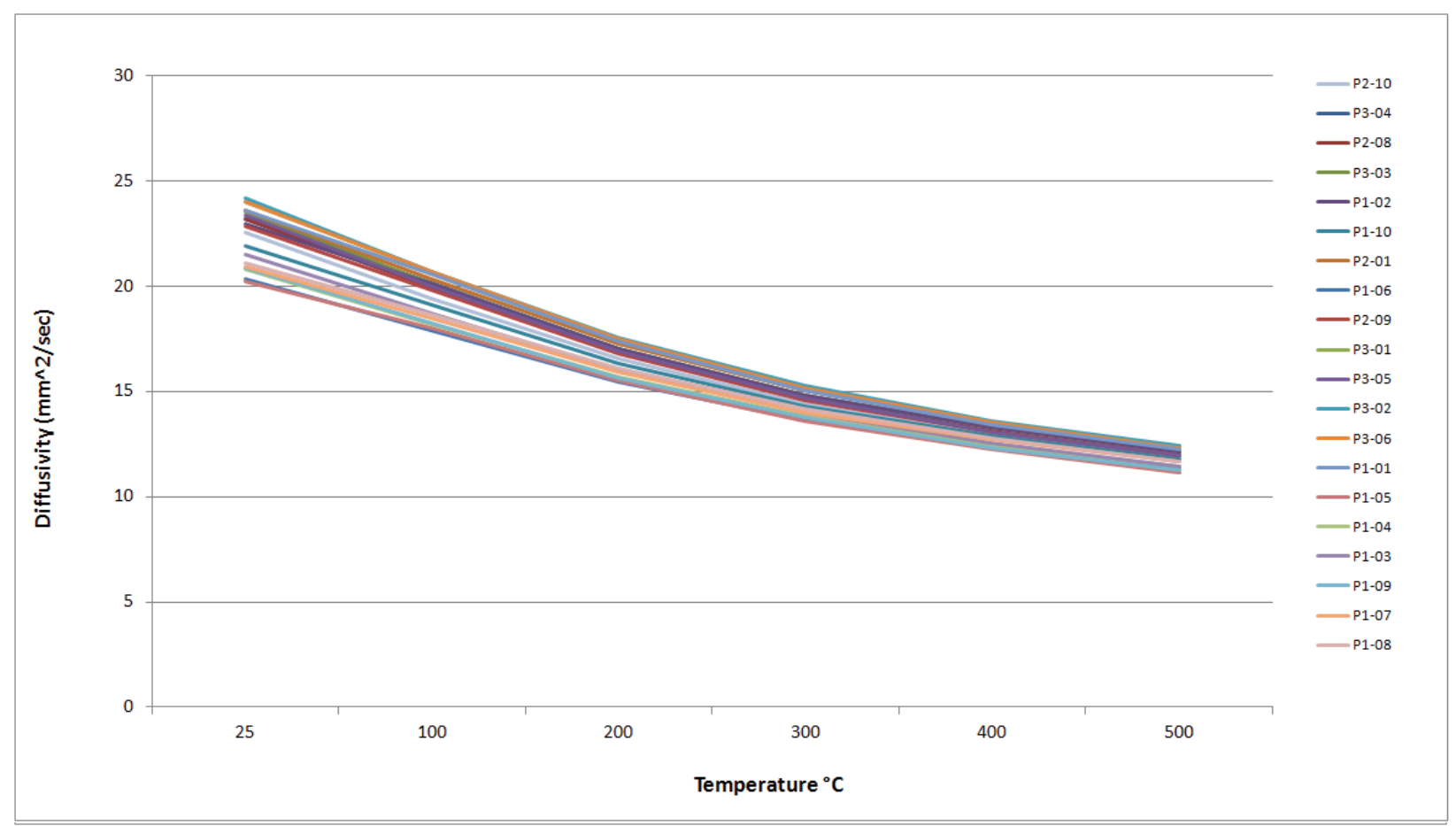

Figure A-181. PCIB Piggyback Diffusivity.



Figure A-182. PCIB Piggyback Diffusivity@100 . 


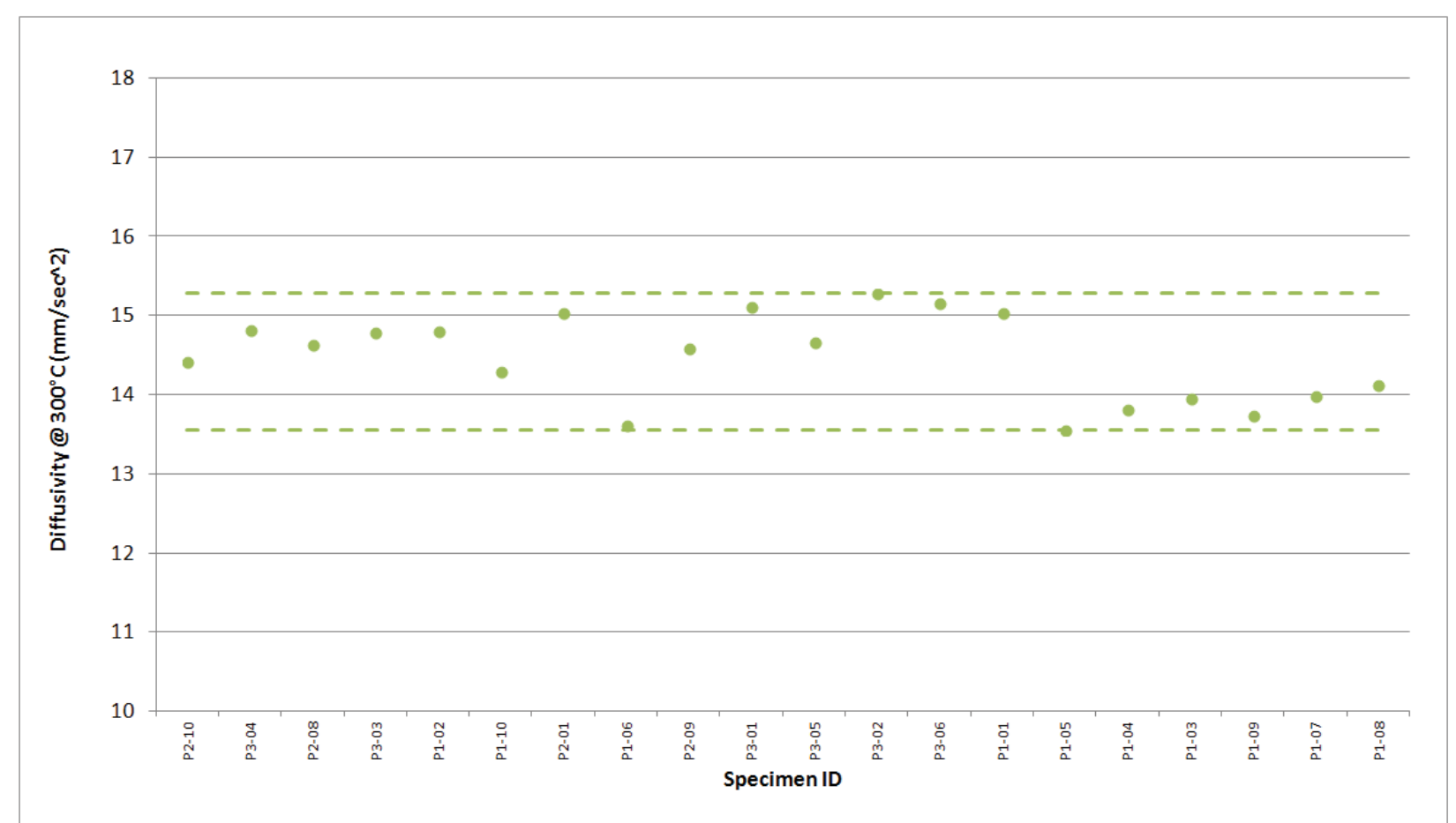

Figure A-183. PCIB Piggyback Diffusivity@300 C.



Figure A-184. PCIB Piggyback Diffusivity @ 500 C. 


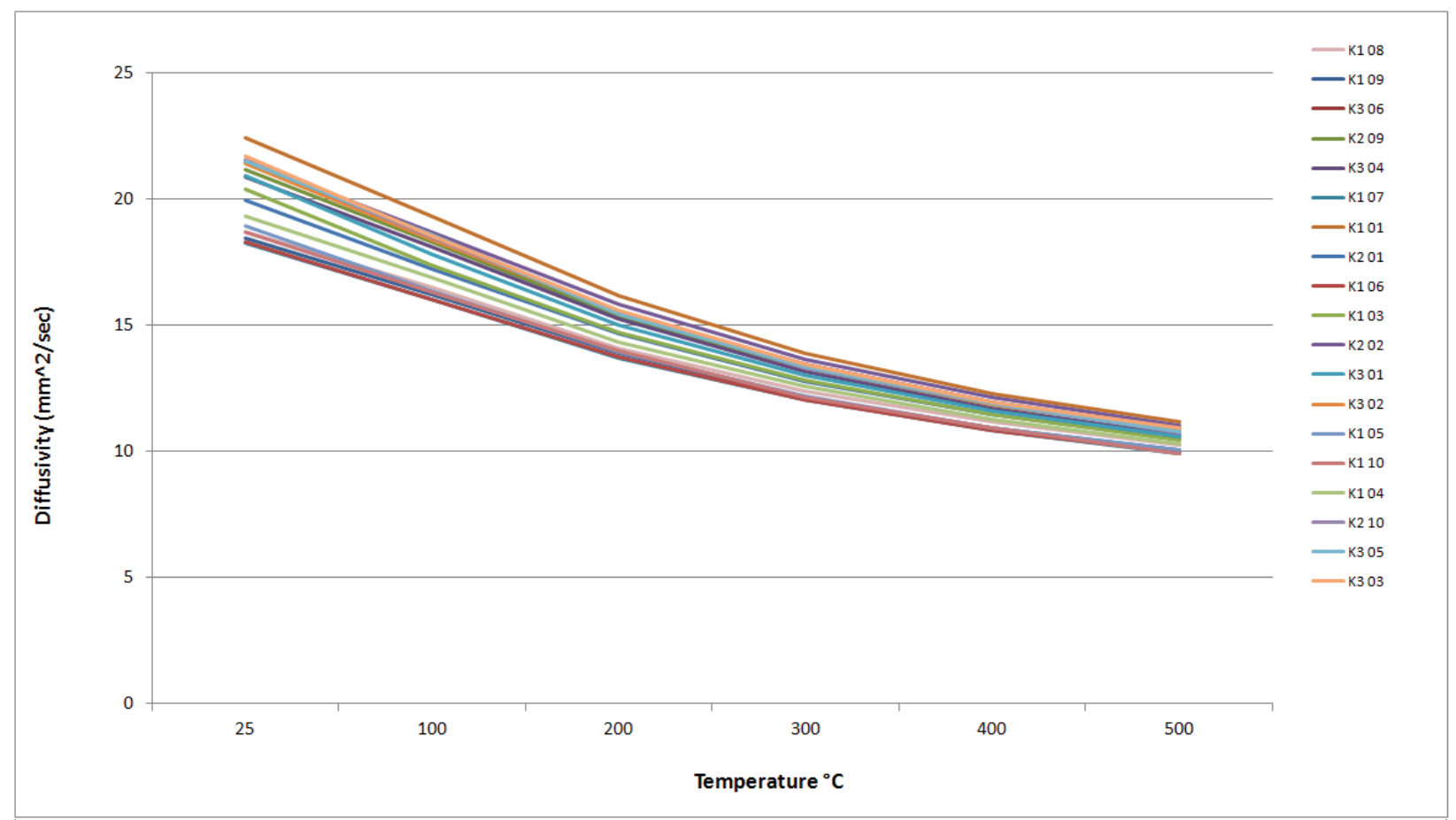

Figure A-185. PGX Piggyback Diffusivity.

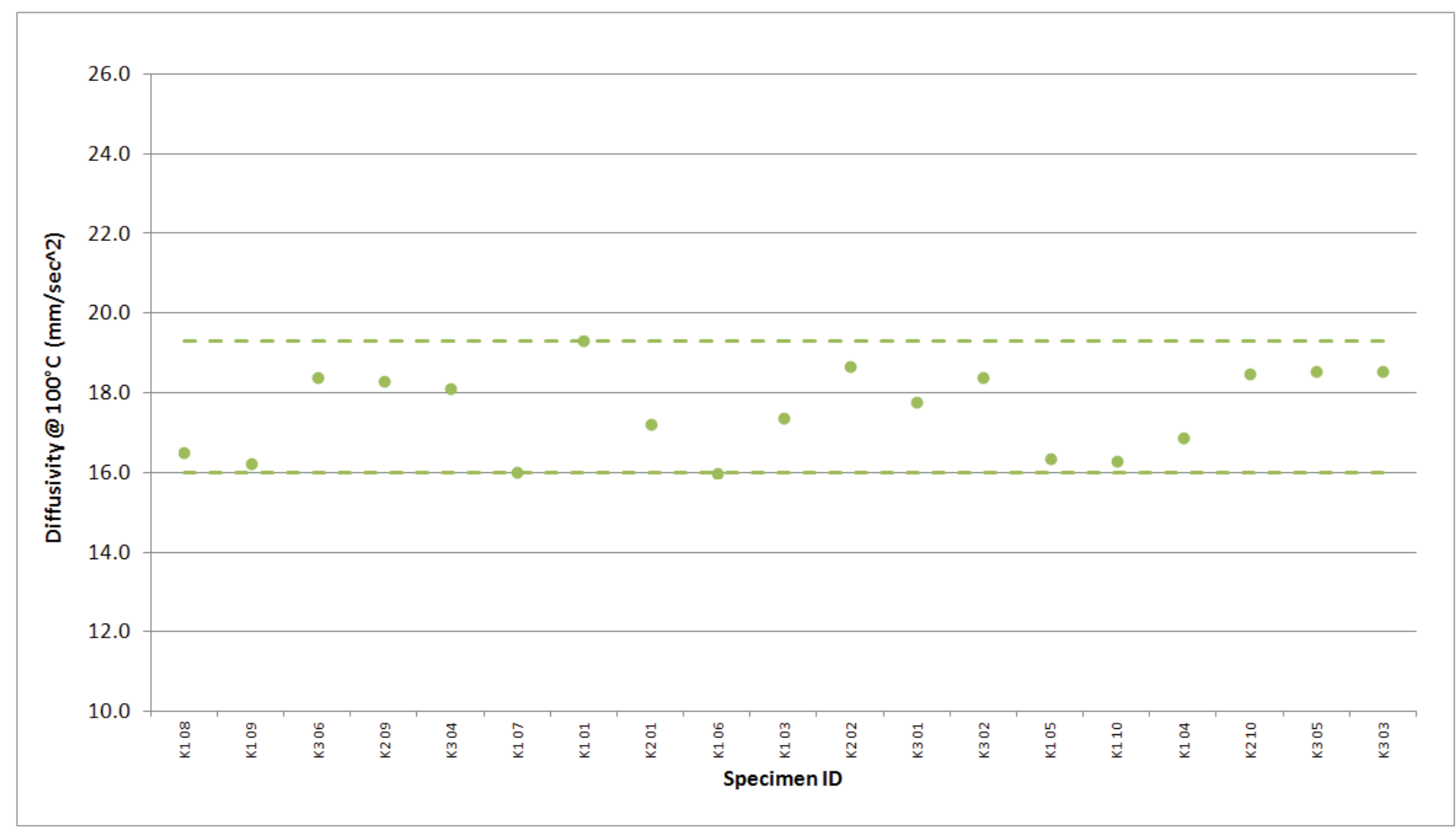

Figure A-186. PGX Piggyback Diffusivity@100² 


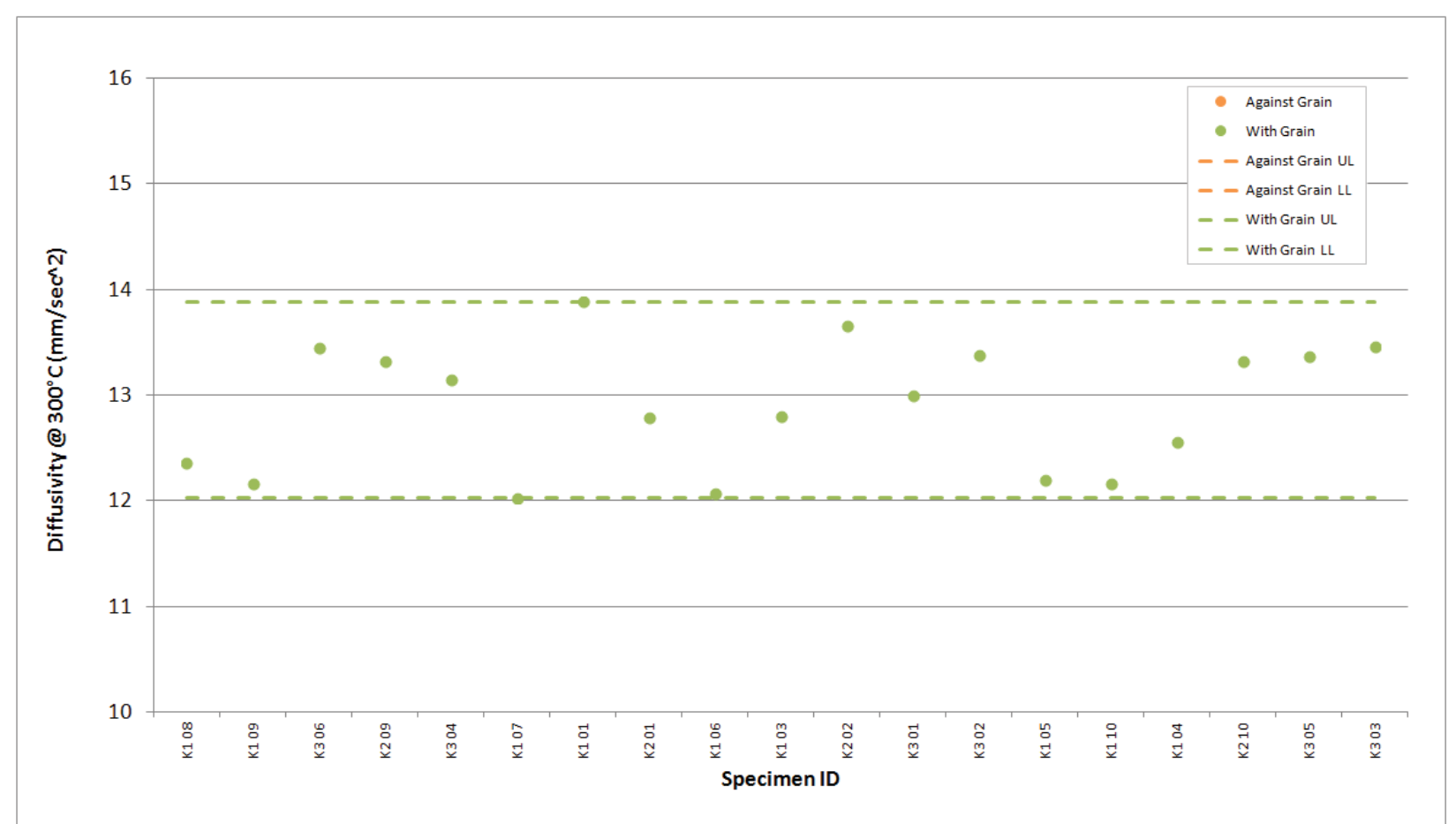

Figure A-187. PGX Piggyback Diffusivity @ 300 C.

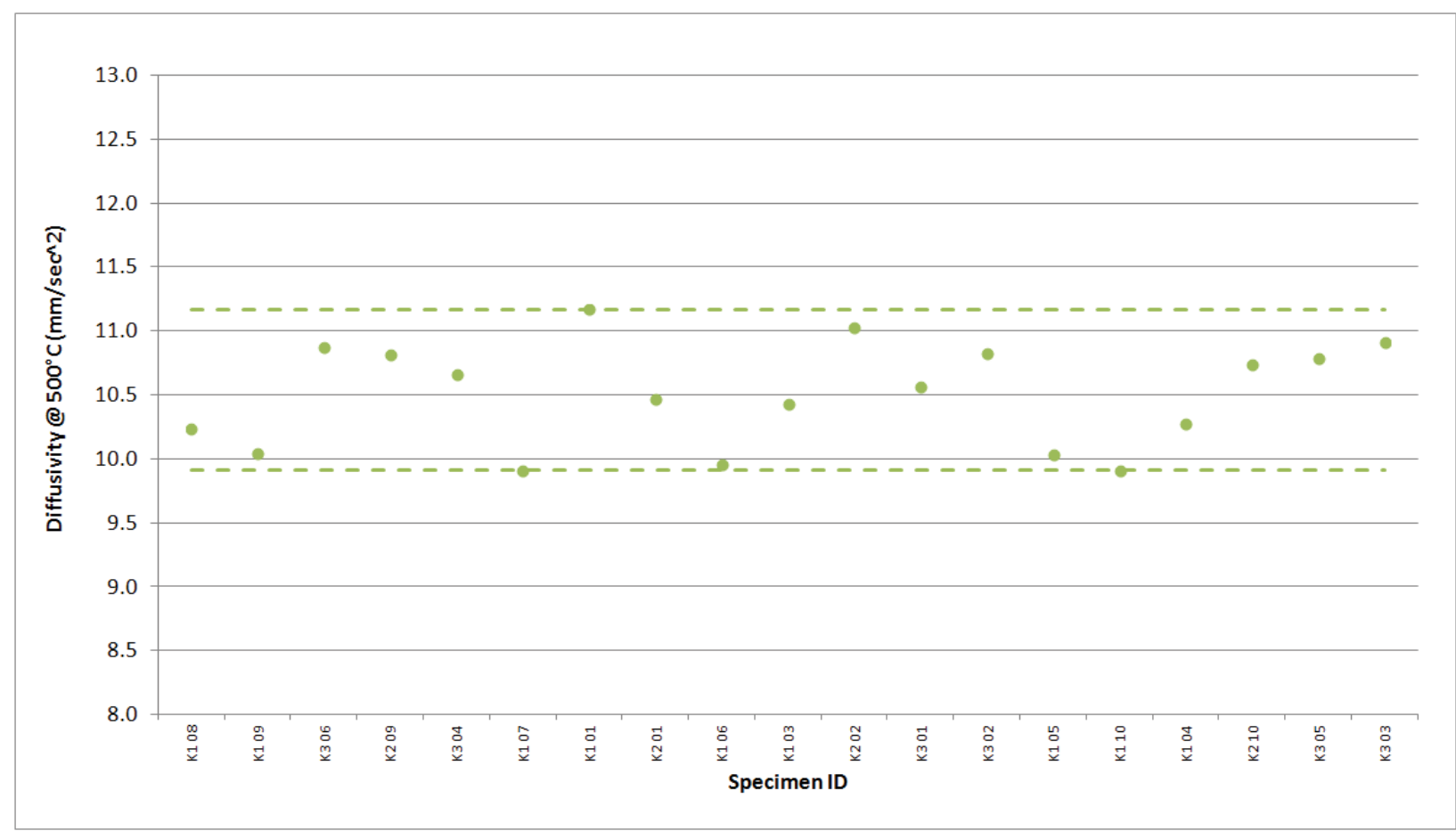

Figure A-188. PGX Piggyback Diffusivity@500² 


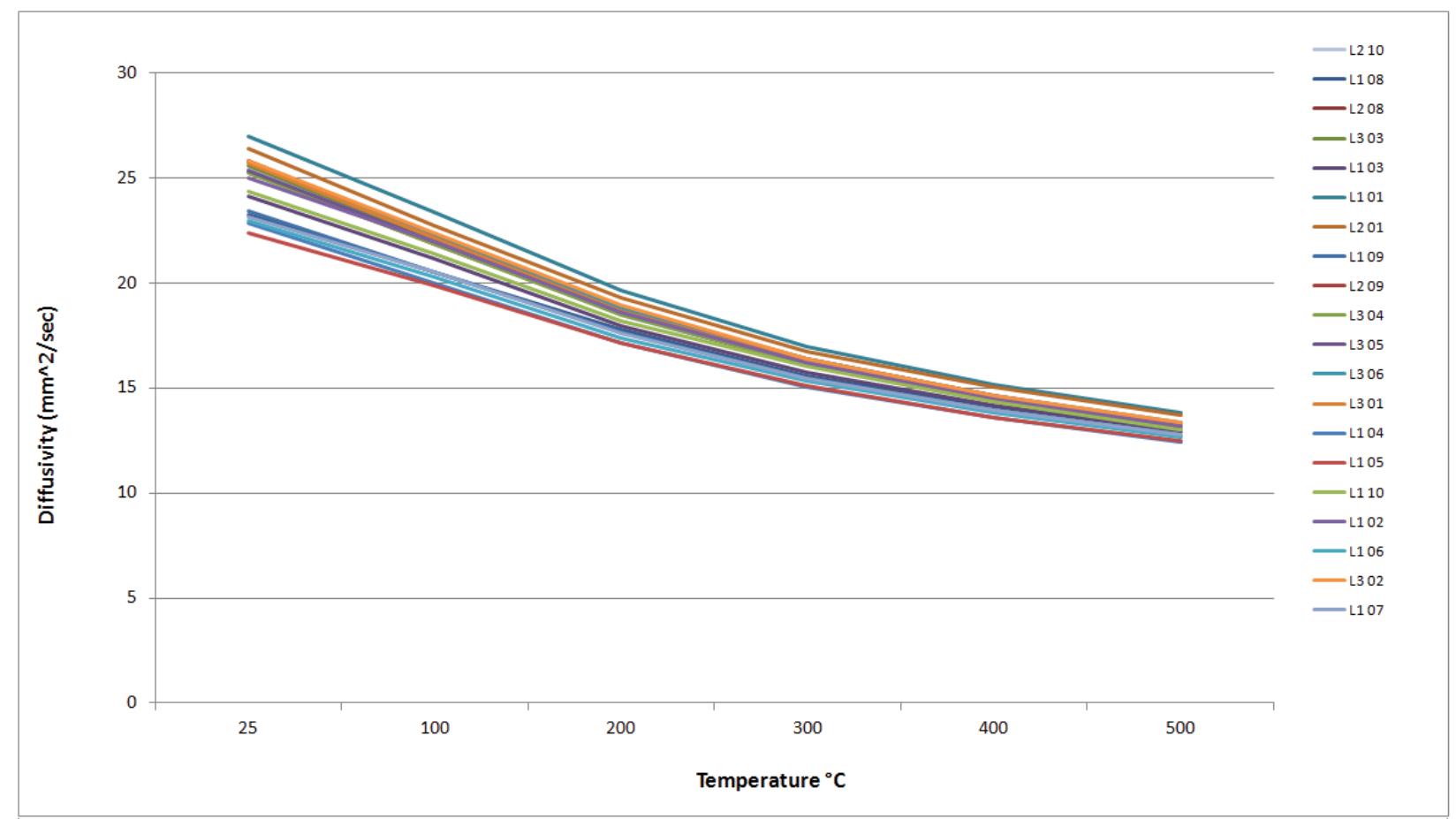

Figure A-189. PPEA Piggyback Diffusivity.

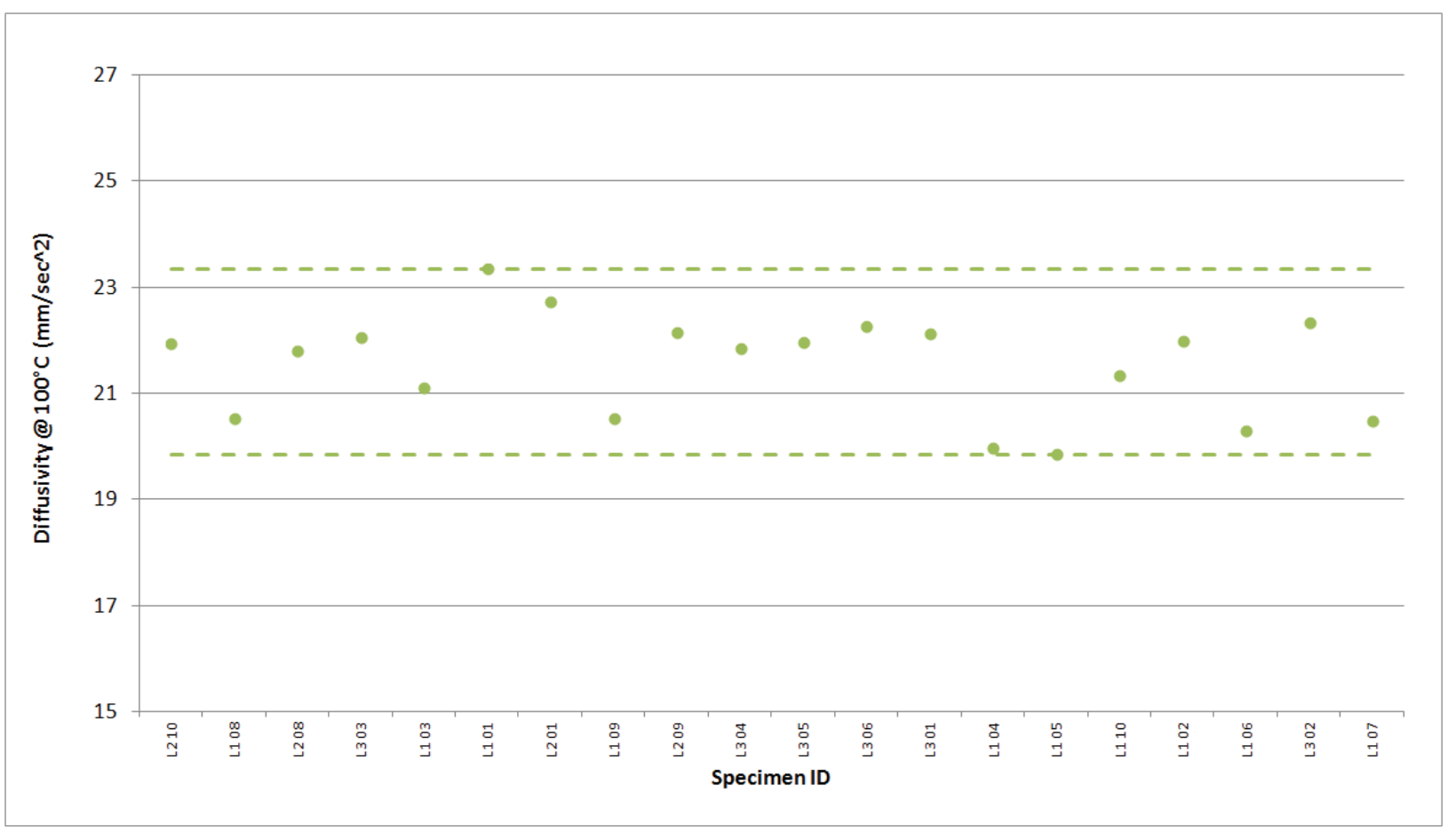

Figure A-190. PPEA Piggyback Diffusivity@100². 


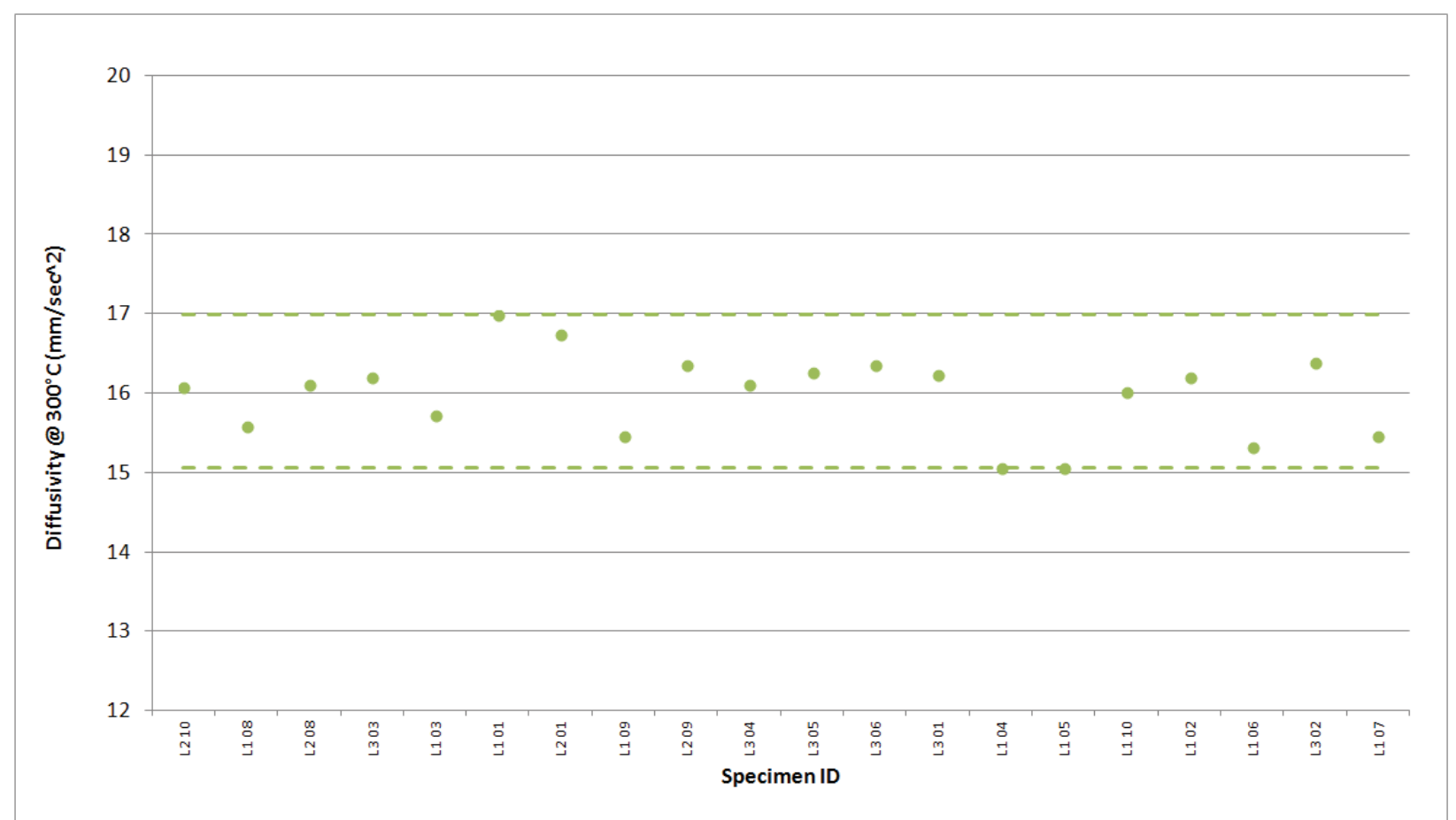

Figure A-191. PPEA Piggyback Diffusivity @300 C.



Figure A-192. PPEA Piggyback Diffusivity@500 C. 
Appendix B

Statistical Tables 


\section{Appendix B}

\section{Summary of Statistical Tables}

Table B-1. Creep specimen Length ( $\mathrm{mm}$ ) Summary Statistics.

\begin{tabular}{|l|c|c|c|c|c|c|}
\hline $\begin{array}{l}\text { Combined } \\
\text { Specimens }\end{array}$ & Mean & Std Dev & CoV (\%) & Median & $\begin{array}{c}\text { Upper } \\
\text { Limit }\end{array}$ & $\begin{array}{c}\text { Lower } \\
\text { Limit }\end{array}$ \\
\hline H-451 & 24.96 & 0.21 & 0.85 & 25.06 & 25.25 & 24.59 \\
\hline IG-110 & 25.02 & 0.19 & 0.76 & 25.12 & 25.25 & 24.61 \\
\hline IG-430 & 25.07 & 0.17 & 0.70 & 25.15 & 25.26 & 24.74 \\
\hline NBG-17 & 25.02 & 0.18 & 0.72 & 25.11 & 25.22 & 24.67 \\
\hline NBG-18 & 25.05 & 0.16 & 0.63 & 25.11 & 25.24 & 24.72 \\
\hline PCEA & 24.97 & 0.22 & 0.89 & 25.07 & 25.24 & 24.48 \\
\hline
\end{tabular}

\begin{tabular}{|l|c|c|c|c|c|c|}
\hline $\begin{array}{l}\text { Against } \\
\text { Grain } \\
\text { Specimens }\end{array}$ & Mean & Std Dev & CoV (\%) & Median & $\begin{array}{c}\text { Upper } \\
\text { Limit }\end{array}$ & $\begin{array}{c}\text { Lower } \\
\text { Limit }\end{array}$ \\
\hline H-451 & & & & & & \\
\hline IG-110 & 24.67 & & & 24.67 & 24.67 & 24.67 \\
\hline IG-430 & & & & & & \\
\hline NBG-17 & 25.01 & 0.21 & 0.82 & 25.05 & 25.21 & 24.73 \\
\hline NBG-18 & 25.05 & 0.16 & 0.64 & 25.11 & 25.23 & 24.74 \\
\hline PCEA & 25.01 & 0.24 & 0.95 & 25.11 & 25.24 & 24.51 \\
\hline
\end{tabular}

\begin{tabular}{|l|c|c|c|c|c|c|}
\hline $\begin{array}{l}\text { With Grain } \\
\text { Specimens }\end{array}$ & Mean & Std Dev & CoV (\%) & Median & $\begin{array}{c}\text { Upper } \\
\text { Limit }\end{array}$ & $\begin{array}{c}\text { Lower } \\
\text { Limit }\end{array}$ \\
\hline H-451 & 24.96 & 0.21 & 0.85 & 25.06 & 25.25 & 24.59 \\
\hline IG-110 & 25.03 & 0.18 & 0.74 & 25.13 & 25.25 & 24.61 \\
\hline IG-430 & 25.07 & 0.17 & 0.70 & 25.15 & 25.26 & 24.74 \\
\hline NBG-17 & 25.02 & 0.18 & 0.71 & 25.11 & 25.22 & 24.67 \\
\hline NBG-18 & 25.05 & 0.16 & 0.64 & 25.12 & 25.24 & 24.72 \\
\hline PCEA & 24.96 & 0.22 & 0.87 & 25.06 & 25.24 & 24.48 \\
\hline
\end{tabular}


Table B-2. Creep specimen Diameter (mm) Summary Statistics.

\begin{tabular}{|l|c|c|c|c|c|c|}
\hline $\begin{array}{l}\text { Combined } \\
\text { Specimens }\end{array}$ & Mean & Std Dev & CoV (\%) & Median & $\begin{array}{c}\text { Upper } \\
\text { Limit }\end{array}$ & $\begin{array}{c}\text { Lower } \\
\text { Limit }\end{array}$ \\
\hline H-451 & 12.67 & 0.04 & 0.28 & 12.68 & 12.73 & 12.62 \\
\hline IG-110 & 12.66 & 0.04 & 0.29 & 12.67 & 12.71 & 12.61 \\
\hline IG-430 & 12.66 & 0.04 & 0.28 & 12.66 & 12.71 & 12.60 \\
\hline NBG-17 & 12.66 & 0.03 & 0.25 & 12.67 & 12.71 & 12.61 \\
\hline NBG-18 & 12.66 & 0.03 & 0.26 & 12.67 & 12.72 & 12.61 \\
\hline PCEA & 12.66 & 0.04 & 0.31 & 12.67 & 12.73 & 12.59 \\
\hline
\end{tabular}

\begin{tabular}{|l|c|c|c|c|c|c|}
\hline $\begin{array}{l}\text { Against } \\
\text { Grain } \\
\text { Specimens }\end{array}$ & Mean & Std Dev & CoV (\%) & Median & $\begin{array}{c}\text { Upper } \\
\text { Limit }\end{array}$ & $\begin{array}{c}\text { Lower } \\
\text { Limit }\end{array}$ \\
\hline H-451 & & & & & & 12.70 \\
\hline IG-110 & 12.70 & & & & & 12.70 \\
\hline IG-430 & & & & 12.65 & 12.71 & 12.61 \\
\hline NBG-17 & 12.65 & 0.04 & 0.29 & 12.66 & 12.71 & 12.62 \\
\hline NBG-18 & 12.67 & 0.03 & 0.26 & 12.67 & 12.73 & 12.60 \\
\hline PCEA & 12.67 & 0.04 & 0.32 & & & \\
\hline
\end{tabular}

\begin{tabular}{|l|c|c|c|c|c|c|}
\hline $\begin{array}{l}\text { With Grain } \\
\text { Specimens }\end{array}$ & Mean & Std Dev & CoV (\%) & Median & $\begin{array}{c}\text { Upper } \\
\text { Limit }\end{array}$ & $\begin{array}{c}\text { Lower } \\
\text { Limit }\end{array}$ \\
\hline H-451 & 12.67 & 0.04 & 0.28 & 12.68 & 12.73 & 12.62 \\
\hline IG-110 & 12.66 & 0.04 & 0.29 & 12.67 & 12.71 & 12.61 \\
\hline IG-430 & 12.66 & 0.04 & 0.28 & 12.66 & 12.71 & 12.60 \\
\hline NBG-17 & 12.66 & 0.03 & 0.24 & 12.67 & 12.71 & 12.61 \\
\hline NBG-18 & 12.66 & 0.03 & 0.26 & 12.67 & 12.72 & 12.61 \\
\hline PCEA & 12.66 & 0.04 & 0.31 & 12.66 & 12.72 & 12.59 \\
\hline
\end{tabular}


Table B-3. Creep specimen Mass (g) Summary Statistics.

\begin{tabular}{|l|c|c|c|c|c|c|}
\hline $\begin{array}{l}\text { Combined } \\
\text { Specimens }\end{array}$ & Mean & Std Dev & CoV (\%) & Median & $\begin{array}{c}\text { Upper } \\
\text { Limit }\end{array}$ & $\begin{array}{c}\text { Lower } \\
\text { Limit }\end{array}$ \\
\hline H-451 & 5.52 & 0.03 & 0.49 & 5.51 & 5.56 & 5.47 \\
\hline IG-110 & 5.65 & 0.02 & 0.37 & 5.66 & 5.68 & 5.61 \\
\hline IG-430 & 5.75 & 0.02 & 0.34 & 5.75 & 5.79 & 5.70 \\
\hline NBG-17 & 5.89 & 0.05 & 0.88 & 5.92 & 5.93 & 5.79 \\
\hline NBG-18 & 5.94 & 0.01 & 0.15 & 5.94 & 5.96 & 5.92 \\
\hline PCEA & 5.66 & 0.03 & 0.46 & 5.65 & 5.69 & 5.61 \\
\hline
\end{tabular}

\begin{tabular}{|l|c|c|c|c|c|c|}
\hline $\begin{array}{l}\text { Against } \\
\text { Grain } \\
\text { Specimens }\end{array}$ & Mean & Std Dev & CoV (\%) & Median & $\begin{array}{c}\text { Upper } \\
\text { Limit }\end{array}$ & $\begin{array}{c}\text { Lower } \\
\text { Limit }\end{array}$ \\
\hline H-451 & & & & & & 5.67 \\
\hline IG-110 & 5.67 & & & & & 5.67 \\
\hline IG-430 & & & & 5.92 & 5.92 & 5.91 \\
\hline NBG-17 & 5.92 & 0.00 & 0.04 & 5.93 & 5.95 & 5.92 \\
\hline NBG-18 & 5.93 & 0.01 & 0.15 & 5.67 & 5.72 & 5.65 \\
\hline PCEA & 5.69 & 0.03 & 0.53 & & & \\
\hline
\end{tabular}

\begin{tabular}{|l|c|c|c|c|c|c|}
\hline $\begin{array}{l}\text { With Grain } \\
\text { Specimens }\end{array}$ & Mean & Std Dev & CoV (\%) & Median & $\begin{array}{c}\text { Upper } \\
\text { Limit }\end{array}$ & $\begin{array}{c}\text { Lower } \\
\text { Limit }\end{array}$ \\
\hline H-451 & 5.52 & 0.03 & 0.49 & 5.51 & 5.56 & 5.47 \\
\hline IG-110 & 5.65 & 0.02 & 0.37 & 5.66 & 5.68 & 5.61 \\
\hline IG-430 & 5.75 & 0.02 & 0.34 & 5.75 & 5.79 & 5.70 \\
\hline NBG-17 & 5.88 & 0.06 & 0.96 & 5.91 & 5.93 & 5.79 \\
\hline NBG-18 & 5.95 & 0.01 & 0.10 & 5.95 & 5.96 & 5.94 \\
\hline PCEA & 5.65 & 0.02 & 0.28 & 5.65 & 5.68 & 5.62 \\
\hline
\end{tabular}


Table B-4. Creep specimen Density (g/cm3) Summary Statistics.

\begin{tabular}{|l|c|c|c|c|c|c|}
\hline $\begin{array}{l}\text { Combined } \\
\text { Specimens }\end{array}$ & Mean & Std Dev & CoV (\%) & Median & $\begin{array}{c}\text { Upper } \\
\text { Limit }\end{array}$ & $\begin{array}{c}\text { Lower } \\
\text { Limit }\end{array}$ \\
\hline H-451 & 1.7801 & 0.0152 & 0.85 & 1.7807 & 1.8082 & 1.7527 \\
\hline IG-110 & 1.8226 & 0.0130 & 0.71 & 1.8216 & 1.8472 & 1.7921 \\
\hline IG-430 & 1.8543 & 0.0106 & 0.57 & 1.8555 & 1.8734 & 1.8282 \\
\hline NBG-17 & 1.9006 & 0.0130 & 0.68 & 1.9038 & 1.9195 & 1.8734 \\
\hline NBG-18 & 1.9141 & 0.0091 & 0.47 & 1.9154 & 1.9311 & 1.8987 \\
\hline PCEA & 1.8276 & 0.0131 & 0.72 & 1.8281 & 1.8605 & 1.8039 \\
\hline
\end{tabular}

\begin{tabular}{|l|c|c|c|c|c|c|}
\hline $\begin{array}{l}\text { Against } \\
\text { Grain } \\
\text { Specimens }\end{array}$ & Mean & Std Dev & CoV (\%) & Median & $\begin{array}{c}\text { Upper } \\
\text { Limit }\end{array}$ & $\begin{array}{c}\text { Lower } \\
\text { Limit }\end{array}$ \\
\hline H-451 & & & & & & \\
\hline IG-110 & 1.8414 & & & 1.8414 & 1.8414 & 1.8414 \\
\hline IG-430 & & & & & & \\
\hline NBG-17 & 1.9136 & 0.0050 & 0.26 & 1.9140 & 1.9195 & 1.9061 \\
\hline NBG-18 & 1.9102 & 0.0097 & 0.51 & 1.9094 & 1.9255 & 1.8987 \\
\hline PCEA & 1.8313 & 0.0154 & 0.84 & 1.8280 & 1.8605 & 1.8077 \\
\hline
\end{tabular}

\begin{tabular}{|l|c|c|c|c|c|c|}
\hline $\begin{array}{l}\text { With Grain } \\
\text { Specimens }\end{array}$ & Mean & Std Dev & CoV (\%) & Median & $\begin{array}{c}\text { Upper } \\
\text { Limit }\end{array}$ & $\begin{array}{c}\text { Lower } \\
\text { Limit }\end{array}$ \\
\hline H-451 & 1.7801 & 0.0152 & 0.85 & 1.7807 & 1.8082 & 1.7527 \\
\hline IG-110 & 1.8221 & 0.0128 & 0.70 & 1.8215 & 1.8472 & 1.7938 \\
\hline IG-430 & 1.8543 & 0.0106 & 0.57 & 1.8555 & 1.8734 & 1.8282 \\
\hline NBG-17 & 1.8962 & 0.0118 & 0.62 & 1.8986 & 1.9136 & 1.8734 \\
\hline NBG-18 & 1.9154 & 0.0086 & 0.45 & 1.9164 & 1.9311 & 1.8999 \\
\hline PCEA & 1.8263 & 0.0123 & 0.67 & 1.8281 & 1.8492 & 1.8039 \\
\hline
\end{tabular}


Table B-5. Creep specimen Coefficient of Thermal Expansion $(1 / \mathrm{K})$ at $100{ }^{\circ} \mathrm{C}$ Summary Statistics.

\begin{tabular}{|l|l|l|l|l|l|l|}
\hline $\begin{array}{l}\text { Combined } \\
\text { Specimens }\end{array}$ & Mean & Std Dev & CoV (\%) & Median & $\begin{array}{l}\text { Upper } \\
\text { Limit }\end{array}$ & $\begin{array}{l}\text { Lower } \\
\text { Limit }\end{array}$ \\
\hline H-451 & $4.26 \mathrm{E}-06$ & $5.61 \mathrm{E}-07$ & 13.18 & $4.08 \mathrm{E}-06$ & $5.13 \mathrm{E}-06$ & $3.55 \mathrm{E}-06$ \\
\hline IG-110 & $5.17 \mathrm{E}-06$ & $5.91 \mathrm{E}-07$ & 11.42 & $5.03 \mathrm{E}-06$ & $6.15 \mathrm{E}-06$ & $4.17 \mathrm{E}-06$ \\
\hline IG-430 & $5.78 \mathrm{E}-06$ & $6.91 \mathrm{E}-07$ & 11.96 & $5.62 \mathrm{E}-06$ & $6.90 \mathrm{E}-06$ & $4.50 \mathrm{E}-06$ \\
\hline NBG-17 & $5.52 \mathrm{E}-06$ & $4.62 \mathrm{E}-07$ & 8.37 & $5.46 \mathrm{E}-06$ & $6.22 \mathrm{E}-06$ & $4.99 \mathrm{E}-06$ \\
\hline NBG-18 & $5.70 \mathrm{E}-06$ & $4.32 \mathrm{E}-07$ & 7.58 & $5.64 \mathrm{E}-06$ & $6.37 \mathrm{E}-06$ & $4.87 \mathrm{E}-06$ \\
\hline PCEA & $4.85 \mathrm{E}-06$ & $5.25 \mathrm{E}-07$ & 10.82 & $4.68 \mathrm{E}-06$ & $6.05 \mathrm{E}-06$ & $4.12 \mathrm{E}-06$ \\
\hline
\end{tabular}

\begin{tabular}{|l|l|l|l|l|l|l|}
\hline $\begin{array}{l}\text { Against } \\
\text { Grain } \\
\text { Specimens }\end{array}$ & Mean & Std Dev & CoV (\%) & Median & $\begin{array}{l}\text { Upper } \\
\text { Limit }\end{array}$ & $\begin{array}{l}\text { Lower } \\
\text { Limit }\end{array}$ \\
\hline H-451 & & & & & & \\
\hline IG-110 & $6.09 \mathrm{E}-06$ & $\mathrm{NaN}$ & $\mathrm{NaN}$ & $6.09 \mathrm{E}-06$ & $6.09 \mathrm{E}-06$ & $6.09 \mathrm{E}-06$ \\
\hline IG-430 & & & & & & \\
\hline NBG-17 & $5.67 \mathrm{E}-06$ & $5.28 \mathrm{E}-07$ & 9.31 & $5.74 \mathrm{E}-06$ & $6.18 \mathrm{E}-06$ & $5.04 \mathrm{E}-06$ \\
\hline NBG-18 & $5.56 \mathrm{E}-06$ & $4.42 \mathrm{E}-07$ & 7.96 & $5.48 \mathrm{E}-06$ & $6.33 \mathrm{E}-06$ & $4.87 \mathrm{E}-06$ \\
\hline PCEA & $4.95 \mathrm{E}-06$ & $5.77 \mathrm{E}-07$ & 11.64 & $4.75 \mathrm{E}-06$ & $6.05 \mathrm{E}-06$ & $4.25 \mathrm{E}-06$ \\
\hline
\end{tabular}

\begin{tabular}{|l|l|l|l|l|l|l|}
\hline $\begin{array}{l}\text { With } \\
\text { Grain } \\
\text { Specimens }\end{array}$ & Mean & Std Dev & CoV (\%) & Median & $\begin{array}{l}\text { Upper } \\
\text { Limit }\end{array}$ & $\begin{array}{l}\text { Lower } \\
\text { Limit }\end{array}$ \\
\hline H-451 & $4.26 \mathrm{E}-06$ & $5.61 \mathrm{E}-07$ & 13.18 & $4.08 \mathrm{E}-06$ & $5.13 \mathrm{E}-06$ & $3.55 \mathrm{E}-06$ \\
\hline IG-110 & $5.15 \mathrm{E}-06$ & $5.77 \mathrm{E}-07$ & 11.22 & $4.93 \mathrm{E}-06$ & $6.15 \mathrm{E}-06$ & $4.17 \mathrm{E}-06$ \\
\hline IG-430 & $5.78 \mathrm{E}-06$ & $6.91 \mathrm{E}-07$ & 11.96 & $5.62 \mathrm{E}-06$ & $6.90 \mathrm{E}-06$ & $4.50 \mathrm{E}-06$ \\
\hline NBG-17 & $5.47 \mathrm{E}-06$ & $4.44 \mathrm{E}-07$ & 8.10 & $5.37 \mathrm{E}-06$ & $6.22 \mathrm{E}-06$ & $4.99 \mathrm{E}-06$ \\
\hline NBG-18 & $5.75 \mathrm{E}-06$ & $4.24 \mathrm{E}-07$ & 7.37 & $5.75 \mathrm{E}-06$ & $6.37 \mathrm{E}-06$ & $5.08 \mathrm{E}-06$ \\
\hline PCEA & $4.82 \mathrm{E}-06$ & $5.11 \mathrm{E}-07$ & 10.61 & $4.66 \mathrm{E}-06$ & $5.65 \mathrm{E}-06$ & $4.12 \mathrm{E}-06$ \\
\hline
\end{tabular}


Table B-6. Creep specimen Coefficient of Thermal Expansion $(1 / \mathrm{K})$ at $300{ }^{\circ} \mathrm{C}$ Summary Statistics.

\begin{tabular}{|l|l|l|l|l|l|l|}
\hline $\begin{array}{l}\text { Combined } \\
\text { Specimens }\end{array}$ & Mean & Std Dev & CoV (\%) & Median & $\begin{array}{l}\text { Upper } \\
\text { Limit }\end{array}$ & $\begin{array}{l}\text { Lower } \\
\text { Limit }\end{array}$ \\
\hline H-451 & $4.37 \mathrm{E}-06$ & $5.74 \mathrm{E}-07$ & 13.13 & $4.09 \mathrm{E}-06$ & $5.31 \mathrm{E}-06$ & $3.68 \mathrm{E}-06$ \\
\hline IG-110 & $5.32 \mathrm{E}-06$ & $5.83 \mathrm{E}-07$ & 10.97 & $5.17 \mathrm{E}-06$ & $6.29 \mathrm{E}-06$ & $4.42 \mathrm{E}-06$ \\
\hline IG-430 & $5.92 \mathrm{E}-06$ & $6.74 \mathrm{E}-07$ & 11.38 & $5.66 \mathrm{E}-06$ & $6.99 \mathrm{E}-06$ & $4.81 \mathrm{E}-06$ \\
\hline NBG-17 & $5.65 \mathrm{E}-06$ & $4.64 \mathrm{E}-07$ & 8.21 & $5.64 \mathrm{E}-06$ & $6.35 \mathrm{E}-06$ & $5.10 \mathrm{E}-06$ \\
\hline NBG-18 & $5.83 \mathrm{E}-06$ & $4.33 \mathrm{E}-07$ & 7.43 & $5.81 \mathrm{E}-06$ & $6.50 \mathrm{E}-06$ & $5.00 \mathrm{E}-06$ \\
\hline PCEA & $4.99 \mathrm{E}-06$ & $5.43 \mathrm{E}-07$ & 10.88 & $4.80 \mathrm{E}-06$ & $6.22 \mathrm{E}-06$ & $4.26 \mathrm{E}-06$ \\
\hline
\end{tabular}

\begin{tabular}{|l|l|l|l|l|l|l|}
\hline $\begin{array}{l}\text { Against } \\
\text { Grain } \\
\text { Specimens }\end{array}$ & Mean & Std Dev & CoV (\%) & Median & $\begin{array}{l}\text { Upper } \\
\text { Limit }\end{array}$ & $\begin{array}{l}\text { Lower } \\
\text { Limit }\end{array}$ \\
\hline H-451 & & & & & & \\
\hline IG-110 & $6.14 \mathrm{E}-06$ & NaN & NaN & $6.14 \mathrm{E}-06$ & $6.14 \mathrm{E}-06$ & $6.14 \mathrm{E}-06$ \\
\hline IG-430 & & & & & & \\
\hline NBG-17 & $5.79 \mathrm{E}-06$ & $5.31 \mathrm{E}-07$ & 9.16 & $5.86 \mathrm{E}-06$ & $6.33 \mathrm{E}-06$ & $5.19 \mathrm{E}-06$ \\
\hline NBG-18 & $5.67 \mathrm{E}-06$ & $4.52 \mathrm{E}-07$ & 7.98 & $5.61 \mathrm{E}-06$ & $6.46 \mathrm{E}-06$ & $5.00 \mathrm{E}-06$ \\
\hline PCEA & $5.11 \mathrm{E}-06$ & $5.84 \mathrm{E}-07$ & 11.42 & $4.91 \mathrm{E}-06$ & $6.22 \mathrm{E}-06$ & $4.39 \mathrm{E}-06$ \\
\hline
\end{tabular}

\begin{tabular}{|l|l|l|l|l|l|l|}
\hline $\begin{array}{l}\text { With } \\
\text { Grain } \\
\text { Specimens }\end{array}$ & Mean & Std Dev & CoV (\%) & Median & $\begin{array}{l}\text { Upper } \\
\text { Limit }\end{array}$ & $\begin{array}{l}\text { Lower } \\
\text { Limit }\end{array}$ \\
\hline H-451 & $4.37 \mathrm{E}-06$ & $5.74 \mathrm{E}-07$ & 13.13 & $4.09 \mathrm{E}-06$ & $5.31 \mathrm{E}-06$ & $3.68 \mathrm{E}-06$ \\
\hline IG-110 & $5.29 \mathrm{E}-06$ & $5.74 \mathrm{E}-07$ & 10.85 & $5.03 \mathrm{E}-06$ & $6.29 \mathrm{E}-06$ & $4.42 \mathrm{E}-06$ \\
\hline IG-430 & $5.92 \mathrm{E}-06$ & $6.74 \mathrm{E}-07$ & 11.38 & $5.66 \mathrm{E}-06$ & $6.99 \mathrm{E}-06$ & $4.81 \mathrm{E}-06$ \\
\hline NBG-17 & $5.60 \mathrm{E}-06$ & $4.46 \mathrm{E}-07$ & 7.95 & $5.54 \mathrm{E}-06$ & $6.35 \mathrm{E}-06$ & $5.10 \mathrm{E}-06$ \\
\hline NBG-18 & $5.88 \mathrm{E}-06$ & $4.20 \mathrm{E}-07$ & 7.14 & $5.94 \mathrm{E}-06$ & $6.50 \mathrm{E}-06$ & $5.20 \mathrm{E}-06$ \\
\hline PCEA & $4.95 \mathrm{E}-06$ & $5.31 \mathrm{E}-07$ & 10.73 & $4.79 \mathrm{E}-06$ & $5.82 \mathrm{E}-06$ & $4.26 \mathrm{E}-06$ \\
\hline
\end{tabular}


Table B-7. Creep specimen Coefficient of Thermal Expansion $(1 / \mathrm{K})$ at $500{ }^{\circ} \mathrm{C}$ Summary Statistics.

\begin{tabular}{|l|l|l|l|l|l|l|}
\hline $\begin{array}{l}\text { Combined } \\
\text { Specimens }\end{array}$ & Mean & Std Dev & CoV (\%) & Median & $\begin{array}{l}\text { Upper } \\
\text { Limit }\end{array}$ & $\begin{array}{l}\text { Lower } \\
\text { Limit }\end{array}$ \\
\hline H-451 & $4.72 \mathrm{E}-06$ & $5.61 \mathrm{E}-07$ & 11.87 & $4.45 \mathrm{E}-06$ & $5.57 \mathrm{E}-06$ & $4.04 \mathrm{E}-06$ \\
\hline IG-110 & $5.72 \mathrm{E}-06$ & $5.65 \mathrm{E}-07$ & 9.88 & $5.58 \mathrm{E}-06$ & $6.70 \mathrm{E}-06$ & $4.95 \mathrm{E}-06$ \\
\hline IG-430 & $6.37 \mathrm{E}-06$ & $6.47 \mathrm{E}-07$ & 10.15 & $6.10 \mathrm{E}-06$ & $7.39 \mathrm{E}-06$ & $5.38 \mathrm{E}-06$ \\
\hline NBG-17 & $5.99 \mathrm{E}-06$ & $4.66 \mathrm{E}-07$ & 7.78 & $5.99 \mathrm{E}-06$ & $6.71 \mathrm{E}-06$ & $5.41 \mathrm{E}-06$ \\
\hline NBG-18 & $6.16 \mathrm{E}-06$ & $4.35 \mathrm{E}-07$ & 7.06 & $6.17 \mathrm{E}-06$ & $6.82 \mathrm{E}-06$ & $5.31 \mathrm{E}-06$ \\
\hline PCEA & $5.33 \mathrm{E}-06$ & $5.43 \mathrm{E}-07$ & 10.19 & $5.08 \mathrm{E}-06$ & $6.61 \mathrm{E}-06$ & $4.68 \mathrm{E}-06$ \\
\hline
\end{tabular}

\begin{tabular}{|l|l|l|l|l|l|l|}
\hline $\begin{array}{l}\text { Against } \\
\text { Grain } \\
\text { Specimens }\end{array}$ & Mean & Std Dev & CoV (\%) & Median & $\begin{array}{l}\text { Upper } \\
\text { Limit }\end{array}$ & $\begin{array}{l}\text { Lower } \\
\text { Limit }\end{array}$ \\
\hline H-451 & & & & & & \\
\hline IG-110 & $6.41 \mathrm{E}-06$ & $\mathrm{NaN}$ & $\mathrm{NaN}$ & $6.41 \mathrm{E}-06$ & $6.41 \mathrm{E}-06$ & $6.41 \mathrm{E}-06$ \\
\hline IG-430 & & & & & & \\
\hline NBG-17 & $6.14 \mathrm{E}-06$ & $5.25 \mathrm{E}-07$ & 8.55 & $6.22 \mathrm{E}-06$ & $6.67 \mathrm{E}-06$ & $5.55 \mathrm{E}-06$ \\
\hline NBG-18 & $5.67 \mathrm{E}-06$ & $4.52 \mathrm{E}-07$ & 7.98 & $5.61 \mathrm{E}-06$ & $6.46 \mathrm{E}-06$ & $5.00 \mathrm{E}-06$ \\
\hline PCEA & $5.47 \mathrm{E}-06$ & $5.90 \mathrm{E}-07$ & 10.80 & $5.25 \mathrm{E}-06$ & $6.61 \mathrm{E}-06$ & $4.77 \mathrm{E}-06$ \\
\hline
\end{tabular}

\begin{tabular}{|l|l|l|l|l|l|l|}
\hline $\begin{array}{l}\text { With } \\
\text { Grain } \\
\text { Specimens }\end{array}$ & Mean & Std Dev & CoV (\%) & Median & $\begin{array}{l}\text { Upper } \\
\text { Limit }\end{array}$ & $\begin{array}{l}\text { Lower } \\
\text { Limit }\end{array}$ \\
\hline H-451 & $4.72 \mathrm{E}-06$ & $5.61 \mathrm{E}-07$ & 11.87 & $4.45 \mathrm{E}-06$ & $5.57 \mathrm{E}-06$ & $4.04 \mathrm{E}-06$ \\
\hline IG-110 & $5.70 \mathrm{E}-06$ & $5.60 \mathrm{E}-07$ & 9.83 & $5.39 \mathrm{E}-06$ & $6.70 \mathrm{E}-06$ & $4.95 \mathrm{E}-06$ \\
\hline IG-430 & $6.37 \mathrm{E}-06$ & $6.47 \mathrm{E}-07$ & 10.15 & $6.10 \mathrm{E}-06$ & $7.39 \mathrm{E}-06$ & $5.38 \mathrm{E}-06$ \\
\hline NBG-17 & $5.94 \mathrm{E}-06$ & $4.50 \mathrm{E}-07$ & 7.57 & $5.87 \mathrm{E}-06$ & $6.71 \mathrm{E}-06$ & $5.41 \mathrm{E}-06$ \\
\hline NBG-18 & $6.21 \mathrm{E}-06$ & $4.19 \mathrm{E}-07$ & 6.75 & $6.25 \mathrm{E}-06$ & $6.82 \mathrm{E}-06$ & $5.51 \mathrm{E}-06$ \\
\hline PCEA & $5.29 \mathrm{E}-06$ & $5.28 \mathrm{E}-07$ & 9.98 & $5.08 \mathrm{E}-06$ & $6.20 \mathrm{E}-06$ & $4.68 \mathrm{E}-06$ \\
\hline
\end{tabular}


Table B-8. Creep specimen Modulus (GPa) by Sonic Resonance Summary Statistics.

\begin{tabular}{|l|c|c|c|c|c|c|}
\hline $\begin{array}{l}\text { Combined } \\
\text { Specimens }\end{array}$ & Mean & Std Dev & CoV (\%) & Median & $\begin{array}{c}\text { Upper } \\
\text { Limit }\end{array}$ & $\begin{array}{c}\text { Lower } \\
\text { Limit }\end{array}$ \\
\hline H-451 & 17.85 & 0.81 & 4.52 & 17.91 & 18.91 & 16.12 \\
\hline IG-110 & 18.14 & 0.86 & 4.76 & 18.07 & 19.94 & 16.43 \\
\hline IG-430 & 18.01 & 0.79 & 4.40 & 17.96 & 19.75 & 16.51 \\
\hline NBG-17 & 19.52 & 1.19 & 6.10 & 19.54 & 21.30 & 17.63 \\
\hline NBG-18 & 19.63 & 0.99 & 5.05 & 19.68 & 21.15 & 17.31 \\
\hline PCEA & 17.03 & 1.33 & 7.78 & 17.06 & 19.33 & 13.69 \\
\hline
\end{tabular}

\begin{tabular}{|c|c|c|c|c|c|c|}
\hline $\begin{array}{l}\text { Against } \\
\text { Grain } \\
\text { Specimens }\end{array}$ & Mean & Std Dev & $\operatorname{CoV}(\%)$ & Median & $\begin{array}{l}\text { Upper } \\
\text { Limit }\end{array}$ & $\begin{array}{c}\text { Lower } \\
\text { Limit }\end{array}$ \\
\hline \multicolumn{7}{|l|}{ H-451 } \\
\hline IG-110 & 18.75 & & & 18.75 & 18.75 & 18.75 \\
\hline \multicolumn{7}{|l|}{ IG-430 } \\
\hline NBG-17 & 19.53 & 0.70 & 3.59 & 19.53 & 20.44 & 18.50 \\
\hline NBG-18 & 19.63 & 1.05 & 5.36 & 19.70 & 21.15 & 17.22 \\
\hline PCEA & 16.47 & 1.78 & 10.78 & 16.20 & 19.13 & 13.69 \\
\hline
\end{tabular}

\begin{tabular}{|l|c|c|c|c|c|c|}
\hline $\begin{array}{l}\text { With Grain } \\
\text { Specimens }\end{array}$ & Mean & Std Dev & CoV (\%) & Median & $\begin{array}{c}\text { Upper } \\
\text { Limit }\end{array}$ & $\begin{array}{c}\text { Lower } \\
\text { Limit }\end{array}$ \\
\hline H-451 & 17.85 & 0.81 & 4.52 & 17.91 & 18.91 & 16.12 \\
\hline IG-110 & 18.12 & 0.87 & 4.80 & 18.05 & 19.94 & 16.43 \\
\hline IG-430 & 18.01 & 0.79 & 4.40 & 17.96 & 19.75 & 16.51 \\
\hline NBG-17 & 19.51 & 1.33 & 6.82 & 19.54 & 21.30 & 17.63 \\
\hline NBG-18 & 19.62 & 0.98 & 5.02 & 19.67 & 21.03 & 17.40 \\
\hline PCEA & 17.22 & 1.11 & 6.42 & 17.24 & 19.33 & 14.95 \\
\hline
\end{tabular}


Table B-9. Creep specimen Resistivity (mW-m) Summary Statistics.

\begin{tabular}{|l|c|c|c|c|c|c|}
\hline $\begin{array}{l}\text { Combined } \\
\text { Specimens }\end{array}$ & Mean & Std Dev & CoV (\%) & Median & $\begin{array}{c}\text { Upper } \\
\text { Limit }\end{array}$ & $\begin{array}{c}\text { Lower } \\
\text { Limit }\end{array}$ \\
\hline H-451 & 23.11 & 0.96 & 4.16 & 23.11 & 24.76 & 21.52 \\
\hline IG-110 & 25.12 & 1.21 & 4.80 & 24.95 & 27.77 & 22.93 \\
\hline IG-430 & 25.57 & 1.18 & 4.61 & 25.49 & 28.51 & 23.51 \\
\hline NBG-17 & 23.89 & 0.65 & 2.73 & 23.88 & 25.05 & 22.79 \\
\hline NBG-18 & 23.34 & 0.87 & 3.74 & 23.18 & 25.00 & 21.79 \\
\hline PCEA & 22.23 & 1.07 & 4.81 & 21.90 & 25.40 & 20.68 \\
\hline
\end{tabular}

\begin{tabular}{|l|c|c|c|c|c|c|}
\hline $\begin{array}{l}\text { Against } \\
\text { Grain } \\
\text { Specimens }\end{array}$ & Mean & Std Dev & CoV (\%) & Median & $\begin{array}{c}\text { Upper } \\
\text { Limit }\end{array}$ & $\begin{array}{c}\text { Lower } \\
\text { Limit }\end{array}$ \\
\hline H-451 & & & & & & 23.29 \\
\hline IG-110 & 23.29 & & & 23.29 & 23.29 & \\
\hline IG-430 & & & & & & 23.02 \\
\hline NBG-17 & 23.92 & 0.62 & 2.59 & 23.89 & 22.74 \\
\hline NBG-18 & 22.68 & 0.85 & 3.76 & 22.25 & 22.79 \\
\hline PCEA & 22.82 & 1.25 & 5.48 & 22.87 & 25.39 & 20.68 \\
\hline
\end{tabular}

\begin{tabular}{|l|c|c|c|c|c|c|}
\hline $\begin{array}{l}\text { With Grain } \\
\text { Specimens }\end{array}$ & Mean & Std Dev & CoV (\%) & Median & $\begin{array}{c}\text { Upper } \\
\text { Limit }\end{array}$ & $\begin{array}{c}\text { Lower } \\
\text { Limit }\end{array}$ \\
\hline H-451 & 23.11 & 0.96 & 4.16 & 23.11 & 24.76 & 21.52 \\
\hline IG-110 & 25.18 & 1.18 & 4.70 & 25.10 & 27.77 & 22.93 \\
\hline IG-430 & 25.57 & 1.18 & 4.61 & 25.49 & 28.51 & 23.51 \\
\hline NBG-17 & 23.89 & 0.68 & 2.85 & 23.84 & 25.05 & 22.66 \\
\hline NBG-18 & 23.55 & 0.78 & 3.29 & 23.42 & 25.00 & 22.44 \\
\hline PCEA & 22.03 & 0.94 & 4.27 & 21.82 & 23.81 & 20.80 \\
\hline
\end{tabular}


Table B-10. Creep specimen Young's Modulus (GPa) by Sonic Velocity.

\begin{tabular}{|l|c|c|c|c|c|c|}
\hline $\begin{array}{l}\text { Combined } \\
\text { Specimens }\end{array}$ & Mean & Std Dev & CoV (\%) & Median & $\begin{array}{c}\text { Upper } \\
\text { Limit }\end{array}$ & $\begin{array}{c}\text { Lower } \\
\text { Limit }\end{array}$ \\
\hline H-451 & 21.43 & 1.12 & 5.23 & 21.23 & 23.62 & 19.72 \\
\hline IG-110 & 20.72 & 1.12 & 5.40 & 20.44 & 22.99 & 18.91 \\
\hline IG-430 & 20.61 & 0.92 & 4.49 & 20.43 & 22.85 & 19.39 \\
\hline NBG-17 & 23.89 & 1.84 & 7.70 & 24.40 & 26.14 & 20.39 \\
\hline NBG-18 & 24.63 & 1.27 & 5.15 & 24.92 & 26.93 & 21.23 \\
\hline PCEA & 20.04 & 1.63 & 8.15 & 20.36 & 22.49 & 15.70 \\
\hline
\end{tabular}

\begin{tabular}{|l|c|c|c|c|c|c|}
\hline $\begin{array}{l}\text { Against } \\
\text { Grain } \\
\text { Specimens }\end{array}$ & Mean & Std Dev & CoV (\%) & Median & $\begin{array}{c}\text { Upper } \\
\text { Limit }\end{array}$ & $\begin{array}{c}\text { Lower } \\
\text { Limit }\end{array}$ \\
\hline H-451 & & & & & & 21.09 \\
\hline IG-110 & 21.09 & & & 21.09 & 21.09 & \\
\hline IG-430 & & & & & & 21.61 \\
\hline NBG-17 & 23.51 & 1.26 & 5.35 & 23.35 & 25.24 & 23.42 \\
\hline NBG-18 & 25.23 & 1.05 & 4.18 & 25.33 & 26.93 & 15.70 \\
\hline PCEA & 19.15 & 1.93 & 10.08 & 19.11 & 22.10 & \\
\hline
\end{tabular}

\begin{tabular}{|l|c|c|c|c|c|c|}
\hline $\begin{array}{l}\text { With Grain } \\
\text { Specimens }\end{array}$ & Mean & Std Dev & CoV (\%) & Median & $\begin{array}{c}\text { Upper } \\
\text { Limit }\end{array}$ & $\begin{array}{c}\text { Lower } \\
\text { Limit }\end{array}$ \\
\hline H-451 & 21.43 & 1.12 & 5.23 & 21.23 & 23.62 & 19.72 \\
\hline IG-110 & 20.71 & 1.13 & 5.48 & 20.40 & 22.99 & 18.91 \\
\hline IG-430 & 20.61 & 0.92 & 4.49 & 20.43 & 22.85 & 19.39 \\
\hline NBG-17 & 24.02 & 2.01 & 8.38 & 24.69 & 26.14 & 20.39 \\
\hline NBG-18 & 24.43 & 1.28 & 5.25 & 24.78 & 26.66 & 21.23 \\
\hline PCEA & 20.33 & 1.43 & 7.04 & 20.82 & 22.49 & 17.36 \\
\hline
\end{tabular}


Table B-11. Creep specimen Shear Modulus (GPa) by Sonic Velocity.

\begin{tabular}{|l|c|c|c|c|c|c|}
\hline $\begin{array}{l}\text { Combined } \\
\text { Specimens }\end{array}$ & Mean & Std Dev & CoV (\%) & Median & $\begin{array}{c}\text { Upper } \\
\text { Limit }\end{array}$ & $\begin{array}{c}\text { Lower } \\
\text { Limit }\end{array}$ \\
\hline H-451 & 7.83 & 0.54 & 6.85 & 7.91 & 8.42 & 7.05 \\
\hline IG-110 & 7.91 & 0.42 & 5.26 & 7.88 & 8.74 & 6.83 \\
\hline IG-430 & 8.00 & 0.30 & 3.70 & 8.01 & 8.51 & 7.40 \\
\hline NBG-17 & 8.37 & 0.38 & 4.56 & 8.44 & 8.88 & 7.61 \\
\hline NBG-18 & 8.56 & 0.34 & 4.02 & 8.55 & 9.30 & 7.86 \\
\hline PCEA & 7.54 & 0.43 & 5.69 & 7.55 & 8.33 & 6.47 \\
\hline
\end{tabular}

\begin{tabular}{|c|c|c|c|c|c|c|}
\hline $\begin{array}{l}\text { Against } \\
\text { Grain } \\
\text { Specimens }\end{array}$ & Mean & Std Dev & CoV (\%) & Median & $\begin{array}{l}\text { Upper } \\
\text { Limit }\end{array}$ & $\begin{array}{c}\text { Lower } \\
\text { Limit }\end{array}$ \\
\hline \multicolumn{7}{|l|}{ H-451 } \\
\hline IG-110 & 6.77 & & & 6.77 & 6.77 & 6.77 \\
\hline \multicolumn{7}{|l|}{ IG-430 } \\
\hline NBG-17 & 8.37 & 0.16 & 1.86 & 8.41 & 8.56 & 8.12 \\
\hline NBG-18 & 8.66 & 0.27 & 3.17 & 8.55 & 9.25 & 8.33 \\
\hline PCEA & 7.58 & 0.35 & 4.61 & 7.60 & 8.07 & 7.15 \\
\hline
\end{tabular}

\begin{tabular}{|l|c|c|c|c|c|c|}
\hline $\begin{array}{l}\text { With Grain } \\
\text { Specimens }\end{array}$ & Mean & Std Dev & CoV (\%) & Median & $\begin{array}{c}\text { Upper } \\
\text { Limit }\end{array}$ & $\begin{array}{c}\text { Lower } \\
\text { Limit }\end{array}$ \\
\hline H-451 & 7.83 & 0.54 & 6.85 & 7.91 & 8.42 & 7.05 \\
\hline IG-110 & 7.94 & 0.37 & 4.69 & 7.91 & 8.74 & 7.36 \\
\hline IG-430 & 8.00 & 0.30 & 3.70 & 8.01 & 8.51 & 7.40 \\
\hline NBG-17 & 8.37 & 0.44 & 5.20 & 8.45 & 8.88 & 7.61 \\
\hline NBG-18 & 8.53 & 0.36 & 4.25 & 8.55 & 9.08 & 7.84 \\
\hline PCEA & 7.53 & 0.46 & 6.06 & 7.55 & 8.33 & 6.53 \\
\hline
\end{tabular}


Table B-12a. Piggyback specimen Length ( $\mathrm{mm}$ ) Summary Statistics.

\begin{tabular}{|l|c|c|c|c|c|c|}
\hline $\begin{array}{l}\text { Combined } \\
\text { Specimens }\end{array}$ & Mean & Std Dev & CoV (\%) & Median & $\begin{array}{c}\text { Upper } \\
\text { Limit }\end{array}$ & $\begin{array}{c}\text { Lower } \\
\text { Limit }\end{array}$ \\
\hline 2114 & 6.282 & 0.034 & 0.54 & 6.295 & 6.313 & 6.256 \\
\hline A3 Matrix & 6.291 & 0.047 & 0.75 & 6.272 & 6.375 & 6.248 \\
\hline BAN & 6.297 & 0.013 & 0.20 & 6.300 & 6.316 & 6.277 \\
\hline H-451 & 6.293 & 0.017 & 0.27 & 6.292 & 6.319 & 6.271 \\
\hline HLM & 6.275 & 0.020 & 0.32 & 6.278 & 6.302 & 6.244 \\
\hline IG-110 & 6.301 & 0.016 & 0.26 & 6.302 & 6.324 & 6.257 \\
\hline IG-430 & 6.301 & 0.010 & 0.15 & 6.300 & 6.321 & 6.288 \\
\hline NBG-10 & 6.292 & 0.017 & 0.26 & 6.292 & 6.318 & 6.268 \\
\hline NBG-17 & 6.264 & 0.035 & 0.55 & 6.268 & 6.303 & 6.205 \\
\hline NBG-18 & 6.284 & 0.008 & 0.12 & 6.283 & 6.297 & 6.272 \\
\hline NBG-25 & 6.293 & 0.010 & 0.16 & 6.297 & 6.305 & 6.275 \\
\hline PCEA & 6.295 & 0.016 & 0.25 & 6.298 & 6.317 & 6.272 \\
\hline PCIB & 6.302 & 0.009 & 0.15 & 6.303 & 6.316 & 6.289 \\
\hline PGX & 6.285 & 0.017 & 0.26 & 6.284 & 6.312 & 6.260 \\
\hline PPEA & 6.292 & 0.019 & 0.30 & 6.301 & 6.322 & 6.259 \\
\hline
\end{tabular}

Table B-12b. Piggyback specimen Length ( $\mathrm{mm}$ ) Summary Statistics.

\begin{tabular}{|l|l|l|l|l|l|l|}
\hline $\begin{array}{l}\text { Against } \\
\text { Grain } \\
\text { Specimens }\end{array}$ & Mean & Std Dev & CoV (\%) & Median & $\begin{array}{c}\text { Upper } \\
\text { Limit }\end{array}$ & $\begin{array}{c}\text { Lower } \\
\text { Limit }\end{array}$ \\
\hline 2114 & & & & & & \\
\hline A3 Matrix & & & & & & \\
\hline BAN & & & & & & \\
\hline H-451 & 6.308 & 0.019 & 0.31 & 6.308 & 6.322 & 6.294 \\
\hline HLM & & & & & & \\
\hline IG-110 & & & & & & \\
\hline IG-430 & & & & & & \\
\hline NBG-10 & & & & & & \\
\hline NBG-17 & 6.275 & 0.015 & 0.24 & 6.283 & 6.289 & 6.252 \\
\hline NBG-18 & 6.288 & 0.008 & 0.13 & 6.289 & 6.294 & 6.274 \\
\hline NBG-25 & & & & & & \\
\hline PCEA & 6.284 & 0.009 & 0.15 & 6.283 & 6.298 & 6.274 \\
\hline PCIB & & & & & & \\
\hline PGX & & & & & & \\
\hline PPEA & & & & & & \\
\hline
\end{tabular}


Table B-12c. Piggyback specimen Length ( $\mathrm{mm}$ ) Summary Statistics.

\begin{tabular}{|l|c|c|c|c|c|c|}
\hline $\begin{array}{l}\text { With Grain } \\
\text { Specimens }\end{array}$ & Mean & Std Dev & CoV (\%) & Median & $\begin{array}{c}\text { Upper } \\
\text { Limit }\end{array}$ & $\begin{array}{c}\text { Lower } \\
\text { Limit }\end{array}$ \\
\hline 2114 & 6.282 & 0.034 & 0.54 & 6.295 & 6.313 & 6.256 \\
\hline A3 Matrix & 6.291 & 0.047 & 0.75 & 6.272 & 6.375 & 6.248 \\
\hline BAN & 6.297 & 0.013 & 0.20 & 6.300 & 6.316 & 6.277 \\
\hline H-451 & 6.285 & 0.011 & 0.18 & 6.286 & 6.297 & 6.271 \\
\hline HLM & 6.275 & 0.020 & 0.32 & 6.278 & 6.302 & 6.244 \\
\hline IG-110 & 6.301 & 0.016 & 0.26 & 6.302 & 6.324 & 6.257 \\
\hline IG-430 & 6.301 & 0.010 & 0.15 & 6.300 & 6.321 & 6.288 \\
\hline NBG-10 & 6.292 & 0.017 & 0.26 & 6.292 & 6.318 & 6.268 \\
\hline NBG-17 & 6.258 & 0.041 & 0.65 & 6.265 & 6.303 & 6.199 \\
\hline NBG-18 & 6.282 & 0.007 & 0.11 & 6.282 & 6.291 & 6.272 \\
\hline NBG-25 & 6.293 & 0.010 & 0.16 & 6.297 & 6.305 & 6.275 \\
\hline PCEA & 6.300 & 0.016 & 0.25 & 6.307 & 6.317 & 6.272 \\
\hline PCIB & 6.302 & 0.009 & 0.15 & 6.303 & 6.316 & 6.289 \\
\hline PGX & 6.285 & 0.017 & 0.26 & 6.284 & 6.312 & 6.260 \\
\hline PPEA & 6.292 & 0.019 & 0.30 & 6.301 & 6.322 & 6.259 \\
\hline
\end{tabular}

Table B-13a. Piggyback specimen Diameter ( $\mathrm{mm}$ ) Summary Statistics.

\begin{tabular}{|l|c|c|c|c|c|c|}
\hline $\begin{array}{l}\text { Combined } \\
\text { Specimens }\end{array}$ & Mean & Std Dev & CoV (\%) & Median & $\begin{array}{c}\text { Upper } \\
\text { Limit }\end{array}$ & $\begin{array}{c}\text { Lower } \\
\text { Limit }\end{array}$ \\
\hline $\mathbf{2 1 1 4}$ & 12.679 & 0.026 & 0.21 & 12.674 & 12.734 & 12.638 \\
\hline A3 Matrix & 12.514 & 0.092 & 0.73 & 12.491 & 12.633 & 12.388 \\
\hline BAN & 12.673 & 0.019 & 0.15 & 12.673 & 12.707 & 12.649 \\
\hline H-451 & 12.667 & 0.019 & 0.15 & 12.666 & 12.689 & 12.646 \\
\hline HLM & 12.637 & 0.037 & 0.29 & 12.640 & 12.701 & 12.587 \\
\hline IG-110 & 12.654 & 0.036 & 0.28 & 12.658 & 12.720 & 12.606 \\
\hline IG-430 & 12.657 & 0.028 & 0.22 & 12.647 & 12.708 & 12.623 \\
\hline NBG-10 & 12.657 & 0.032 & 0.25 & 12.656 & 12.697 & 12.612 \\
\hline NBG-17 & 12.656 & 0.030 & 0.23 & 12.645 & 12.710 & 12.621 \\
\hline NBG-18 & 12.653 & 0.024 & 0.19 & 12.653 & 12.688 & 12.626 \\
\hline NBG-25 & 12.650 & 0.027 & 0.22 & 12.653 & 12.687 & 12.609 \\
\hline PCEA & 12.654 & 0.037 & 0.30 & 12.655 & 12.701 & 12.599 \\
\hline PCIB & 12.684 & 0.014 & 0.11 & 12.687 & 12.708 & 12.659 \\
\hline PGX & 12.640 & 0.039 & 0.31 & 12.658 & 12.688 & 12.578 \\
\hline PPEA & 12.667 & 0.029 & 0.23 & 12.681 & 12.711 & 12.621 \\
\hline
\end{tabular}


Table B-13b. Piggyback specimen Diameter (mm) Summary Statistics.

\begin{tabular}{|c|c|c|c|c|c|c|}
\hline $\begin{array}{l}\text { Against } \\
\text { Grain } \\
\text { Specimens }\end{array}$ & Mean & Std Dev & CoV (\%) & Median & $\begin{array}{l}\text { Upper } \\
\text { Limit }\end{array}$ & $\begin{array}{l}\text { Lower } \\
\text { Limit }\end{array}$ \\
\hline \multicolumn{7}{|l|}{2114} \\
\hline \multicolumn{7}{|l|}{ A3 Matrix } \\
\hline \multicolumn{7}{|l|}{ BAN } \\
\hline H-451 & 12.674 & 0.022 & 0.17 & 12.674 & 12.689 & 12.659 \\
\hline \multicolumn{7}{|l|}{ HLM } \\
\hline \multicolumn{7}{|l|}{ IG-110 } \\
\hline \multicolumn{7}{|l|}{ IG-430 } \\
\hline \multicolumn{7}{|l|}{ NBG-10 } \\
\hline NBG-17 & 12.634 & 0.012 & 0.09 & 12.642 & 12.645 & 12.621 \\
\hline NBG-18 & 12.675 & 0.014 & 0.11 & 12.675 & 12.688 & 12.654 \\
\hline \multicolumn{7}{|l|}{ NBG-25 } \\
\hline PCEA & 12.617 & 0.018 & 0.15 & 12.615 & 12.646 & 12.599 \\
\hline \multicolumn{7}{|l|}{ PCIB } \\
\hline \multicolumn{7}{|l|}{ PGX } \\
\hline PPEA & & & & & & \\
\hline
\end{tabular}

Table B-13c. Piggyback specimen Diameter ( $\mathrm{mm}$ ) Summary Statistics.

\begin{tabular}{|l|c|c|c|c|c|c|}
\hline $\begin{array}{l}\text { With Grain } \\
\text { Specimens }\end{array}$ & Mean & Std Dev & CoV (\%) & Median & $\begin{array}{c}\text { Upper } \\
\text { Limit }\end{array}$ & $\begin{array}{c}\text { Lower } \\
\text { Limit }\end{array}$ \\
\hline $\mathbf{2 1 1 4}$ & 12.679 & 0.026 & 0.21 & 12.674 & 12.734 & 12.638 \\
\hline A3 Matrix & 12.514 & 0.092 & 0.73 & 12.491 & 12.633 & 12.388 \\
\hline BAN & 12.673 & 0.019 & 0.15 & 12.673 & 12.707 & 12.649 \\
\hline H-451 & 12.663 & 0.020 & 0.16 & 12.660 & 12.687 & 12.646 \\
\hline HLM & 12.637 & 0.037 & 0.29 & 12.640 & 12.701 & 12.587 \\
\hline IG-110 & 12.654 & 0.036 & 0.28 & 12.658 & 12.720 & 12.606 \\
\hline IG-430 & 12.657 & 0.028 & 0.22 & 12.647 & 12.708 & 12.623 \\
\hline NBG-10 & 12.657 & 0.032 & 0.25 & 12.656 & 12.697 & 12.612 \\
\hline NBG-17 & 12.667 & 0.030 & 0.24 & 12.666 & 12.710 & 12.628 \\
\hline NBG-18 & 12.639 & 0.016 & 0.13 & 12.633 & 12.669 & 12.626 \\
\hline NBG-25 & 12.650 & 0.027 & 0.22 & 12.653 & 12.687 & 12.609 \\
\hline PCEA & 12.673 & 0.030 & 0.23 & 12.687 & 12.701 & 12.617 \\
\hline PCIB & 12.684 & 0.014 & 0.11 & 12.687 & 12.708 & 12.659 \\
\hline PGX & 12.640 & 0.039 & 0.31 & 12.658 & 12.688 & 12.578 \\
\hline PPEA & 12.667 & 0.029 & 0.23 & 12.681 & 12.711 & 12.621 \\
\hline
\end{tabular}


Table B-14a. Piggyback specimen Mass (g) Summary Statistics.

\begin{tabular}{|l|c|c|c|c|c|c|}
\hline $\begin{array}{l}\text { Combined } \\
\text { Specimens }\end{array}$ & Mean & Std Dev & CoV (\%) & Median & $\begin{array}{c}\text { Upper } \\
\text { Limit }\end{array}$ & $\begin{array}{c}\text { Lower } \\
\text { Limit }\end{array}$ \\
\hline $\mathbf{2 1 1 4}$ & 1.4551 & 0.0056 & 0.39 & 1.4552 & 1.4612 & 1.4489 \\
\hline A3 Matrix & 1.2361 & 0.1171 & 9.47 & 1.1729 & 1.4419 & 1.1288 \\
\hline BAN & 1.4753 & 0.0093 & 0.63 & 1.4716 & 1.4894 & 1.4669 \\
\hline H-451 & 1.3985 & 0.0148 & 1.06 & 1.4072 & 1.4099 & 1.3790 \\
\hline HLM & 1.4112 & 0.0025 & 0.18 & 1.4118 & 1.4144 & 1.4062 \\
\hline IG-110 & 1.4330 & 0.0047 & 0.33 & 1.4337 & 1.4404 & 1.4235 \\
\hline IG-430 & 1.4574 & 0.0032 & 0.22 & 1.4570 & 1.4628 & 1.4533 \\
\hline NBG-10 & 1.4361 & 0.0035 & 0.24 & 1.4356 & 1.4430 & 1.4307 \\
\hline NBG-17 & 1.4994 & 0.0077 & 0.52 & 1.5003 & 1.5046 & 1.4934 \\
\hline NBG-18 & 1.4993 & 0.0020 & 0.13 & 1.4998 & 1.5024 & 1.4962 \\
\hline NBG-25 & 1.4855 & 0.0018 & 0.12 & 1.4854 & 1.4887 & 1.4824 \\
\hline PCEA & 1.4253 & 0.0026 & 0.18 & 1.4245 & 1.4296 & 1.4212 \\
\hline PCIB & 1.4733 & 0.0030 & 0.21 & 1.4736 & 1.4783 & 1.4673 \\
\hline PGX & 1.4217 & 0.0037 & 0.26 & 1.4216 & 1.4286 & 1.4135 \\
\hline PPEA & 1.4760 & 0.0025 & 0.17 & 1.4760 & 1.4824 & 1.4720 \\
\hline
\end{tabular}

Table B-14b. Piggyback specimen Mass (g) Summary Statistics.

\begin{tabular}{|l|l|l|l|l|l|l|}
\hline $\begin{array}{l}\text { Against } \\
\text { Grain } \\
\text { Specimens }\end{array}$ & Mean & Std Dev & CoV (\%) & Median & $\begin{array}{c}\text { Upper } \\
\text { Limit }\end{array}$ & $\begin{array}{c}\text { Lower } \\
\text { Limit }\end{array}$ \\
\hline 2114 & & & & & & \\
\hline A3 Matrix & & & & & & \\
\hline BAN & & & & & & \\
\hline H-451 & 1.3795 & 0.0007 & 0.05 & 1.3795 & 1.3800 & 1.3790 \\
\hline HLM & & & & & & \\
\hline IG-110 & & & & & & \\
\hline IG-430 & & & & & & \\
\hline NBG-10 & & & & & & \\
\hline NBG-17 & 1.4997 & 0.0018 & 0.12 & 1.4997 & 1.5026 & 1.4974 \\
\hline NBG-18 & 1.5006 & 0.0011 & 0.07 & 1.5002 & 1.5024 & 1.4998 \\
\hline NBG-25 & & & & & & \\
\hline PCEA & 1.4248 & 0.0024 & 0.17 & 1.4245 & 1.4272 & 1.4212 \\
\hline PCIB & & & & & & \\
\hline PGX & & & & & & \\
\hline PPEA & & & & & & \\
\hline
\end{tabular}


Table B-14c. Piggyback specimen Mass (g) Summary Statistics.

\begin{tabular}{|l|c|c|c|c|c|c|}
\hline $\begin{array}{l}\text { With Grain } \\
\text { Specimens }\end{array}$ & Mean & Std Dev & CoV (\%) & Median & $\begin{array}{c}\text { Upper } \\
\text { Limit }\end{array}$ & $\begin{array}{c}\text { Lower } \\
\text { Limit }\end{array}$ \\
\hline $\mathbf{2 1 1 4}$ & 1.4551 & 0.0056 & 0.39 & 1.4552 & 1.4612 & 1.4489 \\
\hline A3 Matrix & 1.2361 & 0.1171 & 9.47 & 1.1729 & 1.4419 & 1.1288 \\
\hline BAN & 1.4753 & 0.0093 & 0.63 & 1.4716 & 1.4894 & 1.4669 \\
\hline H-451 & 1.4080 & 0.0014 & 0.10 & 1.4078 & 1.4099 & 1.4066 \\
\hline HLM & 1.4112 & 0.0025 & 0.18 & 1.4118 & 1.4144 & 1.4062 \\
\hline IG-110 & 1.4330 & 0.0047 & 0.33 & 1.4337 & 1.4404 & 1.4235 \\
\hline IG-430 & 1.4574 & 0.0032 & 0.22 & 1.4570 & 1.4628 & 1.4533 \\
\hline NBG-10 & 1.4361 & 0.0035 & 0.24 & 1.4356 & 1.4430 & 1.4307 \\
\hline NBG-17 & 1.4992 & 0.0096 & 0.64 & 1.5020 & 1.5046 & 1.4945 \\
\hline NBG-18 & 1.4985 & 0.0020 & 0.13 & 1.4984 & 1.5020 & 1.4962 \\
\hline NBG-25 & 1.4855 & 0.0018 & 0.12 & 1.4854 & 1.4887 & 1.4824 \\
\hline PCEA & 1.4255 & 0.0027 & 0.19 & 1.4248 & 1.4296 & 1.4216 \\
\hline PCIB & 1.4733 & 0.0030 & 0.21 & 1.4736 & 1.4783 & 1.4673 \\
\hline PGX & 1.4217 & 0.0037 & 0.26 & 1.4216 & 1.4286 & 1.4135 \\
\hline PPEA & 1.4760 & 0.0025 & 0.17 & 1.4760 & 1.4824 & 1.4720 \\
\hline
\end{tabular}

Table B-15a. Piggyback specimen Density (g/cm3) Summary Statistics.

\begin{tabular}{|l|c|c|c|c|c|c|}
\hline $\begin{array}{l}\text { Combined } \\
\text { Specimens }\end{array}$ & Mean & Std Dev & CoV (\%) & Median & $\begin{array}{c}\text { Upper } \\
\text { Limit }\end{array}$ & $\begin{array}{c}\text { Lower } \\
\text { Limit }\end{array}$ \\
\hline $\mathbf{2 1 1 4}$ & 1.8347 & 0.0116 & 0.63 & 1.8330 & 1.8595 & 1.8136 \\
\hline A3 Matrix & 1.5980 & 0.1549 & 9.70 & 1.5018 & 1.8441 & 1.4389 \\
\hline BAN & 1.8571 & 0.0106 & 0.57 & 1.8574 & 1.8793 & 1.8359 \\
\hline H-451 & 1.7637 & 0.0249 & 1.41 & 1.7720 & 1.7876 & 1.7249 \\
\hline HLM & 1.7932 & 0.0155 & 0.86 & 1.7876 & 1.8177 & 1.7701 \\
\hline IG-110 & 1.8083 & 0.0126 & 0.70 & 1.8083 & 1.8304 & 1.7855 \\
\hline IG-430 & 1.8382 & 0.0102 & 0.56 & 1.8406 & 1.8511 & 1.8200 \\
\hline NBG-10 & 1.8142 & 0.0126 & 0.69 & 1.8108 & 1.8386 & 1.7972 \\
\hline NBG-17 & 1.9028 & 0.0139 & 0.73 & 1.8998 & 1.9254 & 1.8783 \\
\hline NBG-18 & 1.8975 & 0.0088 & 0.46 & 1.9014 & 1.9108 & 1.8838 \\
\hline NBG-25 & 1.8783 & 0.0115 & 0.61 & 1.8793 & 1.8973 & 1.8633 \\
\hline PCEA & 1.8004 & 0.0165 & 0.92 & 1.8007 & 1.8232 & 1.7767 \\
\hline PCIB & 1.8503 & 0.0081 & 0.44 & 1.8501 & 1.8669 & 1.8370 \\
\hline PGX & 1.8029 & 0.0171 & 0.95 & 1.7943 & 1.8312 & 1.7821 \\
\hline PPEA & 1.8613 & 0.0149 & 0.80 & 1.8551 & 1.8856 & 1.8393 \\
\hline
\end{tabular}


Table B-15b. Piggyback specimen Density (g/cm3) Summary Statistics.

\begin{tabular}{|l|l|l|l|l|l|l|}
\hline $\begin{array}{l}\text { Against } \\
\text { Grain } \\
\text { Specimens }\end{array}$ & Mean & Std Dev & CoV (\%) & Median & $\begin{array}{c}\text { Upper } \\
\text { Limit }\end{array}$ & $\begin{array}{c}\text { Lower } \\
\text { Limit }\end{array}$ \\
\hline 2114 & & & & & & \\
\hline A3 Matrix & & & & & & \\
\hline BAN & & & & & & \\
\hline H-451 & 1.7335 & 0.0121 & 0.70 & 1.7335 & 1.7421 & 1.7249 \\
\hline HLM & & & & & & \\
\hline IG-110 & & & & & & \\
\hline IG-430 & & & & & & \\
\hline NBG-10 & & & & & & 1.9167 \\
\hline NBG-17 & 1.9063 & 0.0094 & 0.49 & 1.9011 & 1.9177 \\
\hline NBG-18 & 1.8914 & 0.0065 & 0.34 & 1.8900 & 1.9014 & 1.8852 \\
\hline NBG-25 & & & & & & \\
\hline PCEA & 1.8134 & 0.0081 & 0.45 & 1.8171 & 1.8214 & 1.8007 \\
\hline PCIB & & & & & & \\
\hline PGX & & & & & & \\
\hline PPEA & & & & & & \\
\hline
\end{tabular}

Table B-15c. Piggyback specimen Density (g/cm3) Summary Statistics.

\begin{tabular}{|l|c|c|c|c|c|c|}
\hline $\begin{array}{l}\text { With Grain } \\
\text { Specimens }\end{array}$ & Mean & Std Dev & CoV (\%) & Median & $\begin{array}{c}\text { Upper } \\
\text { Limit }\end{array}$ & $\begin{array}{c}\text { Lower } \\
\text { Limit }\end{array}$ \\
\hline $\mathbf{2 1 1 4}$ & 1.8347 & 0.0116 & 0.63 & 1.8330 & 1.8595 & 1.8136 \\
\hline A3 Matrix & 1.5980 & 0.1549 & 9.70 & 1.5018 & 1.8441 & 1.4389 \\
\hline BAN & 1.8571 & 0.0106 & 0.57 & 1.8574 & 1.8793 & 1.8359 \\
\hline H-451 & 1.7789 & 0.0084 & 0.47 & 1.7795 & 1.7876 & 1.7689 \\
\hline HLM & 1.7932 & 0.0155 & 0.86 & 1.7876 & 1.8177 & 1.7701 \\
\hline IG-110 & 1.8083 & 0.0126 & 0.70 & 1.8083 & 1.8304 & 1.7855 \\
\hline IG-430 & 1.8382 & 0.0102 & 0.56 & 1.8406 & 1.8511 & 1.8200 \\
\hline NBG-10 & 1.8142 & 0.0126 & 0.69 & 1.8108 & 1.8386 & 1.7972 \\
\hline NBG-17 & 1.9010 & 0.0159 & 0.83 & 1.8980 & 1.9254 & 1.8783 \\
\hline NBG-18 & 1.9013 & 0.0081 & 0.43 & 1.9038 & 1.9108 & 1.8914 \\
\hline NBG-25 & 1.8783 & 0.0115 & 0.61 & 1.8793 & 1.8973 & 1.8633 \\
\hline PCEA & 1.7939 & 0.0159 & 0.89 & 1.7877 & 1.8232 & 1.7767 \\
\hline PCIB & 1.8503 & 0.0081 & 0.44 & 1.8501 & 1.8669 & 1.8370 \\
\hline PGX & 1.8029 & 0.0171 & 0.95 & 1.7943 & 1.8312 & 1.7821 \\
\hline PPEA & 1.8613 & 0.0149 & 0.80 & 1.8551 & 1.8856 & 1.8393 \\
\hline
\end{tabular}


Table B-16a. Piggyback specimen Diffusivity $(\mathrm{mm} 2 / \mathrm{sec})$ at $100{ }^{\circ} \mathrm{C}$ Summary Statistics.

\begin{tabular}{|l|c|c|c|c|c|c|}
\hline $\begin{array}{l}\text { Combined } \\
\text { Specimens }\end{array}$ & Mean & Std Dev & CoV (\%) & Median & $\begin{array}{c}\text { Upper } \\
\text { Limit }\end{array}$ & $\begin{array}{c}\text { Lower } \\
\text { Limit }\end{array}$ \\
\hline $\mathbf{2 1 1 4}$ & 18.39 & 1.02 & 5.56 & 18.20 & 19.86 & 16.40 \\
\hline A3 Matrix & 4.10 & 1.24 & 30.37 & 3.38 & 6.29 & 2.70 \\
\hline BAN & 23.04 & 1.41 & 6.14 & 23.29 & 25.13 & 21.10 \\
\hline H-451 & 22.35 & 1.03 & 4.60 & 22.29 & 23.59 & 20.66 \\
\hline HLM & 23.86 & 1.74 & 7.31 & 23.76 & 26.26 & 21.43 \\
\hline IG-110 & 18.70 & 1.13 & 6.06 & 18.51 & 20.40 & 16.78 \\
\hline IG-430 & 19.37 & 1.12 & 5.78 & 19.13 & 21.49 & 17.81 \\
\hline NBG-10 & 20.18 & 1.00 & 4.98 & 20.29 & 21.61 & 18.90 \\
\hline NBG-17 & 19.14 & 1.00 & 5.24 & 18.80 & 20.04 & 18.06 \\
\hline NBG-18 & 20.14 & 1.09 & 5.39 & 20.12 & 21.74 & 18.86 \\
\hline NBG-25 & 19.05 & 0.84 & 4.42 & 19.28 & 20.41 & 17.54 \\
\hline PCEA & 22.80 & 1.92 & 8.41 & 23.85 & 25.31 & 20.10 \\
\hline PCIB & 19.48 & 0.98 & 5.06 & 19.87 & 20.67 & 17.90 \\
\hline PGX & 17.55 & 1.07 & 6.12 & 17.79 & 19.31 & 16.00 \\
\hline PPEA & 21.54 & 0.97 & 4.50 & 21.90 & 23.35 & 19.85 \\
\hline
\end{tabular}

Table B-16b. Piggyback specimen Diffusivity (mm2/sec) at $100{ }^{\circ} \mathrm{C}$ Summary Statistics.

\begin{tabular}{|c|c|c|c|c|c|c|}
\hline $\begin{array}{l}\text { Against } \\
\text { Grain } \\
\text { Specimens }\end{array}$ & Mean & Std Dev & CoV (\%) & Median & $\begin{array}{l}\text { Upper } \\
\text { Limit }\end{array}$ & $\begin{array}{c}\text { Lower } \\
\text { Limit }\end{array}$ \\
\hline \multicolumn{7}{|l|}{2114} \\
\hline \multicolumn{7}{|l|}{ A3 Matrix } \\
\hline \multicolumn{7}{|l|}{ BAN } \\
\hline H-451 & 21.51 & 1.19 & 5.55 & 21.51 & 22.35 & 20.66 \\
\hline \multicolumn{7}{|l|}{ HLM } \\
\hline \multicolumn{7}{|l|}{ IG-110 } \\
\hline \multicolumn{7}{|l|}{ IG-430 } \\
\hline \multicolumn{7}{|l|}{ NBG-10 } \\
\hline NBG-17 & 18.63 & 0.35 & 1.89 & 18.80 & 18.95 & 18.06 \\
\hline NBG-18 & 21.26 & 0.48 & 2.24 & 21.29 & 21.74 & 20.50 \\
\hline \multicolumn{7}{|l|}{ NBG-25 } \\
\hline PCEA & 20.41 & 0.32 & 1.57 & 20.26 & 20.84 & 20.10 \\
\hline \multicolumn{7}{|l|}{ PCIB } \\
\hline \multicolumn{7}{|l|}{ PGX } \\
\hline PPEA & & & & & & \\
\hline
\end{tabular}


Table B-16c. Piggyback specimen Diffusivity $(\mathrm{mm} 2 / \mathrm{sec})$ at $100{ }^{\circ} \mathrm{C}$ Summary Statistics.

\begin{tabular}{|l|c|c|c|c|c|c|}
\hline $\begin{array}{l}\text { With Grain } \\
\text { Specimens }\end{array}$ & Mean & Std Dev & CoV (\%) & Median & $\begin{array}{c}\text { Upper } \\
\text { Limit }\end{array}$ & $\begin{array}{c}\text { Lower } \\
\text { Limit }\end{array}$ \\
\hline 2114 & 18.39 & 1.02 & 5.56 & 18.20 & 19.86 & 16.40 \\
\hline A3 Matrix & 4.10 & 1.24 & 30.37 & 3.38 & 6.29 & 2.70 \\
\hline BAN & 23.04 & 1.41 & 6.14 & 23.29 & 25.13 & 21.10 \\
\hline H-451 & 22.78 & 0.76 & 3.33 & 22.73 & 23.59 & 22.04 \\
\hline HLM & 23.86 & 1.74 & 7.31 & 23.76 & 26.26 & 21.43 \\
\hline IG-110 & 18.70 & 1.13 & 6.06 & 18.51 & 20.40 & 16.78 \\
\hline IG-430 & 19.37 & 1.12 & 5.78 & 19.13 & 21.49 & 17.81 \\
\hline NBG-10 & 20.18 & 1.00 & 4.98 & 20.29 & 21.61 & 18.90 \\
\hline NBG-17 & 19.40 & 1.14 & 5.86 & 18.85 & 21.52 & 18.42 \\
\hline NBG-18 & 19.44 & 0.66 & 3.39 & 19.14 & 20.71 & 18.86 \\
\hline NBG-25 & 19.05 & 0.84 & 4.42 & 19.28 & 20.41 & 17.54 \\
\hline PCEA & 23.99 & 0.95 & 3.96 & 24.22 & 25.31 & 22.88 \\
\hline PCIB & 19.48 & 0.98 & 5.06 & 19.87 & 20.67 & 17.90 \\
\hline PGX & 17.55 & 1.07 & 6.12 & 17.79 & 19.31 & 16.00 \\
\hline PPEA & 21.54 & 0.97 & 4.50 & 21.90 & 23.35 & 19.85 \\
\hline
\end{tabular}

Table B-17a. Piggyback specimen Diffusivity $(\mathrm{mm} 2 / \mathrm{sec})$ at $300{ }^{\circ} \mathrm{C}$ Summary Statistics.

\begin{tabular}{|l|c|c|c|c|c|c|}
\hline $\begin{array}{l}\text { Combined } \\
\text { Specimens }\end{array}$ & Mean & Std Dev & CoV (\%) & Median & $\begin{array}{c}\text { Upper } \\
\text { Limit }\end{array}$ & $\begin{array}{c}\text { Lower } \\
\text { Limit }\end{array}$ \\
\hline $\mathbf{2 1 1 4}$ & 13.84 & 0.61 & 4.41 & 13.92 & 14.71 & 12.49 \\
\hline A3 Matrix & 2.74 & 0.93 & 33.93 & 2.23 & 4.43 & 1.72 \\
\hline BAN & 16.97 & 0.79 & 4.65 & 17.00 & 18.11 & 15.91 \\
\hline H-451 & 16.51 & 0.74 & 4.46 & 16.58 & 17.31 & 15.27 \\
\hline HLM & 17.46 & 1.04 & 5.93 & 17.59 & 18.92 & 15.99 \\
\hline IG-110 & 13.92 & 0.69 & 4.98 & 13.79 & 15.02 & 12.69 \\
\hline IG-430 & 14.35 & 0.65 & 4.52 & 14.30 & 15.63 & 13.47 \\
\hline NBG-10 & 15.01 & 0.54 & 3.58 & 15.03 & 15.75 & 14.25 \\
\hline NBG-17 & 14.35 & 0.61 & 4.27 & 14.11 & 14.84 & 13.58 \\
\hline NBG-18 & 15.06 & 0.67 & 4.47 & 14.95 & 16.06 & 14.27 \\
\hline NBG-25 & 14.27 & 0.46 & 3.24 & 14.36 & 15.04 & 13.39 \\
\hline PCEA & 16.88 & 1.19 & 7.05 & 17.64 & 18.30 & 15.16 \\
\hline PCIB & 14.48 & 0.55 & 3.81 & 14.62 & 15.28 & 13.56 \\
\hline PGX & 12.90 & 0.60 & 4.65 & 13.00 & 13.88 & 12.03 \\
\hline PPEA & 15.99 & 0.52 & 3.28 & 16.11 & 16.98 & 15.06 \\
\hline
\end{tabular}


Table B-17b. Piggyback specimen Diffusivity $(\mathrm{mm} 2 / \mathrm{sec})$ at $300{ }^{\circ} \mathrm{C}$ Summary Statistics.

\begin{tabular}{|c|c|c|c|c|c|c|}
\hline $\begin{array}{l}\text { Against } \\
\text { Grain } \\
\text { Specimens }\end{array}$ & Mean & Std Dev & CoV (\%) & Median & $\begin{array}{l}\text { Upper } \\
\text { Limit }\end{array}$ & $\begin{array}{l}\text { Lower } \\
\text { Limit }\end{array}$ \\
\hline \multicolumn{7}{|l|}{2114} \\
\hline \multicolumn{7}{|l|}{ A3 Matrix } \\
\hline \multicolumn{7}{|l|}{ BAN } \\
\hline H-451 & 15.72 & 0.63 & 4.01 & 15.72 & 16.16 & 15.27 \\
\hline \multicolumn{7}{|l|}{ HLM } \\
\hline \multicolumn{7}{|l|}{ IG-110 } \\
\hline \multicolumn{7}{|l|}{ IG-430 } \\
\hline \multicolumn{7}{|l|}{ NBG-10 } \\
\hline NBG-17 & 14.01 & 0.28 & 1.98 & 14.11 & 14.21 & 13.53 \\
\hline NBG-18 & 15.78 & 0.29 & 1.84 & 15.86 & 16.06 & 15.35 \\
\hline \multicolumn{7}{|l|}{ NBG-25 } \\
\hline PCEA & 15.36 & 0.22 & 1.44 & 15.28 & 15.62 & 15.16 \\
\hline \multicolumn{7}{|l|}{ PCIB } \\
\hline \multicolumn{7}{|l|}{ PGX } \\
\hline PPEA & & & & & & \\
\hline
\end{tabular}

Table B-17c. Piggyback specimen Diffusivity $(\mathrm{mm} 2 / \mathrm{sec})$ at $300{ }^{\circ} \mathrm{C}$ Summary Statistics.

\begin{tabular}{|l|c|c|c|c|c|c|}
\hline $\begin{array}{l}\text { With Grain } \\
\text { Specimens }\end{array}$ & Mean & Std Dev & CoV (\%) & Median & $\begin{array}{c}\text { Upper } \\
\text { Limit }\end{array}$ & $\begin{array}{c}\text { Lower } \\
\text { Limit }\end{array}$ \\
\hline $\mathbf{2 1 1 4}$ & 13.84 & 0.61 & 4.41 & 13.92 & 14.71 & 12.49 \\
\hline A3 Matrix & 2.74 & 0.93 & 33.93 & 2.23 & 4.43 & 1.72 \\
\hline BAN & 16.97 & 0.79 & 4.65 & 17.00 & 18.11 & 15.91 \\
\hline H-451 & 16.91 & 0.38 & 2.24 & 16.88 & 17.31 & 16.57 \\
\hline HLM & 17.46 & 1.04 & 5.93 & 17.59 & 18.92 & 15.99 \\
\hline IG-110 & 13.92 & 0.69 & 4.98 & 13.79 & 15.02 & 12.69 \\
\hline IG-430 & 14.35 & 0.65 & 4.52 & 14.30 & 15.63 & 13.47 \\
\hline NBG-10 & 15.01 & 0.54 & 3.58 & 15.03 & 15.75 & 14.25 \\
\hline NBG-17 & 14.51 & 0.67 & 4.64 & 14.16 & 15.75 & 13.90 \\
\hline NBG-18 & 14.61 & 0.36 & 2.45 & 14.48 & 15.30 & 14.27 \\
\hline NBG-25 & 14.27 & 0.46 & 3.24 & 14.36 & 15.04 & 13.39 \\
\hline PCEA & 17.64 & 0.51 & 2.87 & 17.78 & 18.23 & 17.28 \\
\hline PCIB & 14.48 & 0.55 & 3.81 & 14.62 & 15.28 & 13.56 \\
\hline PGX & 12.90 & 0.60 & 4.65 & 13.00 & 13.88 & 12.03 \\
\hline PPEA & 15.99 & 0.52 & 3.28 & 16.11 & 16.98 & 15.06 \\
\hline
\end{tabular}


Table B-18a. Piggyback specimen Diffusivity $(\mathrm{mm} 2 / \mathrm{sec})$ at $500{ }^{\circ} \mathrm{C}$ Summary Statistics.

\begin{tabular}{|l|c|c|c|c|c|c|}
\hline $\begin{array}{l}\text { Combined } \\
\text { Specimens }\end{array}$ & Mean & Std Dev & CoV (\%) & Median & $\begin{array}{c}\text { Upper } \\
\text { Limit }\end{array}$ & $\begin{array}{c}\text { Lower } \\
\text { Limit }\end{array}$ \\
\hline $\mathbf{2 1 1 4}$ & 11.43 & 0.45 & 3.91 & 11.54 & 12.05 & 10.42 \\
\hline A3 Matrix & 2.15 & 0.76 & 35.08 & 1.76 & 3.61 & 1.33 \\
\hline BAN & 13.83 & 0.53 & 3.86 & 13.81 & 14.66 & 13.11 \\
\hline H-451 & 13.47 & 0.67 & 4.97 & 13.67 & 14.08 & 12.42 \\
\hline HLM & 14.19 & 0.74 & 5.23 & 14.28 & 15.36 & 13.14 \\
\hline IG-110 & 11.42 & 0.51 & 4.46 & 11.40 & 12.22 & 10.50 \\
\hline IG-430 & 11.74 & 0.46 & 3.92 & 11.75 & 12.74 & 11.12 \\
\hline NBG-10 & 12.32 & 0.35 & 2.82 & 12.26 & 12.83 & 11.85 \\
\hline NBG-17 & 11.79 & 0.47 & 3.99 & 11.65 & 12.13 & 11.18 \\
\hline NBG-18 & 12.37 & 0.49 & 4.00 & 12.25 & 13.09 & 11.65 \\
\hline NBG-25 & 11.73 & 0.32 & 2.72 & 11.80 & 12.24 & 11.12 \\
\hline PCEA & 13.73 & 0.91 & 6.62 & 13.81 & 14.94 & 12.37 \\
\hline PCIB & 11.87 & 0.38 & 3.23 & 11.94 & 12.42 & 11.16 \\
\hline PGX & 10.51 & 0.40 & 3.85 & 10.57 & 11.17 & 9.91 \\
\hline PPEA & 13.08 & 0.36 & 2.73 & 13.14 & 13.80 & 12.45 \\
\hline
\end{tabular}

Table B-18b. Piggyback specimen Diffusivity (mm2/sec) at $500{ }^{\circ} \mathrm{C}$ Summary Statistics.

\begin{tabular}{|l|l|l|l|l|l|l|}
\hline $\begin{array}{l}\text { Against } \\
\text { Grain } \\
\text { Specimens }\end{array}$ & Mean & Std Dev & CoV (\%) & Median & $\begin{array}{c}\text { Upper } \\
\text { Limit }\end{array}$ & $\begin{array}{c}\text { Lower } \\
\text { Limit }\end{array}$ \\
\hline $\mathbf{2 1 1 4}$ & & & & & & \\
\hline A3 Matrix & & & & & & \\
\hline BAN & & & & & & \\
\hline H-451 & 12.66 & 0.34 & 2.68 & 12.66 & 12.90 & 12.42 \\
\hline HLM & & & & & & \\
\hline IG-110 & & & & & & \\
\hline IG-430 & & & & & & \\
\hline NBG-10 & & & & & & 11.73 \\
\hline NBG-17 & 11.51 & 0.26 & 2.26 & 11.60 & 12.10 \\
\hline NBG-18 & 12.90 & 0.22 & 1.72 & 12.94 & 13.09 & 12.56 \\
\hline NBG-25 & & & & & & \\
\hline PCEA & 12.65 & 0.21 & 1.66 & 12.64 & 12.89 & 12.37 \\
\hline PCIB & & & & & & \\
\hline PGX & & & & & & \\
\hline PPEA & & & & & & \\
\hline
\end{tabular}


Table B-18c. Piggyback specimen Diffusivity $(\mathrm{mm} 2 / \mathrm{sec})$ at $500{ }^{\circ} \mathrm{C}$ Summary Statistics.

\begin{tabular}{|l|c|c|c|c|c|c|}
\hline $\begin{array}{l}\text { With Grain } \\
\text { Specimens }\end{array}$ & Mean & Std Dev & CoV (\%) & Median & $\begin{array}{c}\text { Upper } \\
\text { Limit }\end{array}$ & $\begin{array}{c}\text { Lower } \\
\text { Limit }\end{array}$ \\
\hline $\mathbf{2 1 1 4}$ & 11.43 & 0.45 & 3.91 & 11.54 & 12.05 & 10.42 \\
\hline A3 Matrix & 2.15 & 0.76 & 35.08 & 1.76 & 3.61 & 1.33 \\
\hline BAN & 13.83 & 0.53 & 3.86 & 13.81 & 14.66 & 13.11 \\
\hline H-451 & 13.87 & 0.24 & 1.71 & 13.89 & 14.08 & 13.61 \\
\hline HLM & 14.19 & 0.74 & 5.23 & 14.28 & 15.36 & 13.14 \\
\hline IG-110 & 11.42 & 0.51 & 4.46 & 11.40 & 12.22 & 10.50 \\
\hline IG-430 & 11.74 & 0.46 & 3.92 & 11.75 & 12.74 & 11.12 \\
\hline NBG-10 & 12.32 & 0.35 & 2.82 & 12.26 & 12.83 & 11.85 \\
\hline NBG-17 & 11.93 & 0.50 & 4.20 & 11.68 & 12.81 & 11.48 \\
\hline NBG-18 & 12.05 & 0.27 & 2.27 & 12.00 & 12.50 & 11.65 \\
\hline NBG-25 & 11.73 & 0.32 & 2.72 & 11.80 & 12.24 & 11.12 \\
\hline PCEA & 14.26 & 0.56 & 3.90 & 14.48 & 14.94 & 13.08 \\
\hline PCIB & 11.87 & 0.38 & 3.23 & 11.94 & 12.42 & 11.16 \\
\hline PGX & 10.51 & 0.40 & 3.85 & 10.57 & 11.17 & 9.91 \\
\hline PPEA & 13.08 & 0.36 & 2.73 & 13.14 & 13.80 & 12.45 \\
\hline
\end{tabular}




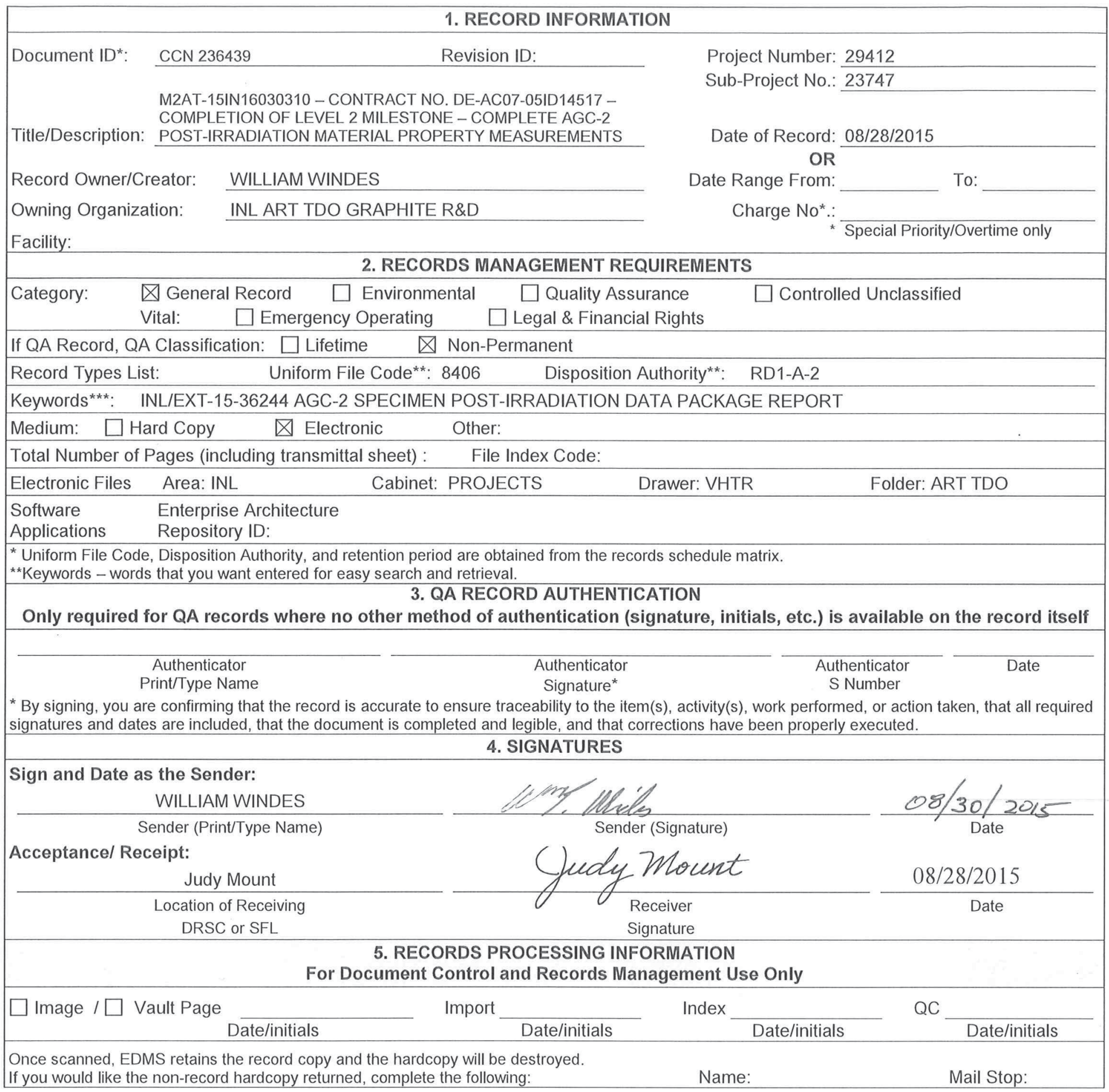

\section{INSTRUCTIONS}

* Document ID: Enter the unique identifier for the record if applicable. If transmitting multiple records with identifiers, attach a separate list. ** Uniform File Code/Disposition Authority: Enter UFC and disposition authority of the records as found in the Record Schedule Matrix and/or the organization's Record Types List. If transmitting multiple files on one form, all must have the same UFC and Disposition Authority.

${ }^{* * *}$ Keywords: Enter keywords that would be used for retrieval purposes.

NOTE: Completed Records Transmittal Forms that contain the QA Record Authentication Signature must be processed as part of the QA record. All other transmittal forms are maintained as:

UFC: 1206 Disposition Authority: A23-7 Retention Period: Cut off at the end of the month. Destroy when 3 months old. 